Pleage do not destroy or throw away this publication. If you have no further use for it write to the Geological Survey at Washington and ask for a frank to return it

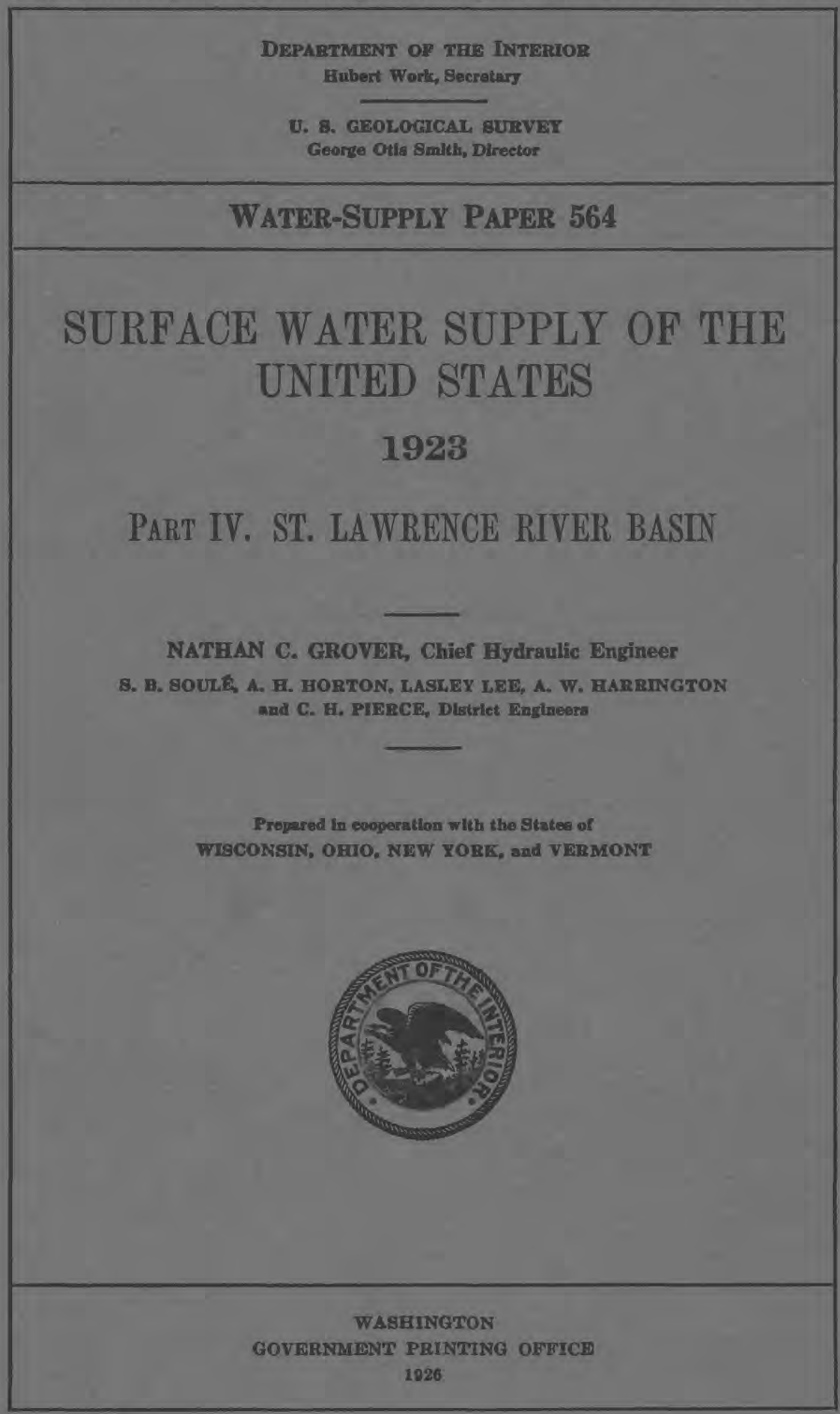


DEPaRTMENT OF THE INTERIOR

Hubert Work, Secretary

U. S. GEOLOGICAL SURVEY

George Otis Smith, Director

Water-Supply Paper 564

\section{SURFACE WATER SUPPLY OF THE UNITED STATES}

\section{3}

\section{PART IV. ST. LAWRENCE RIVER BASIN}

NATHAN C. GROVER, Chief Hydraulic Engineer

S. B. SOULE, A. H. HORTON, LASLEY LEE, A. W. HARRINGTON and C. H. PIERCE, District Engineers

Prepared in cooperation with the States of WISCONSIN, OHIO, NEW YORK, and VERMONT

WASHINGTON

Water Resources Branch, Geological Survey,

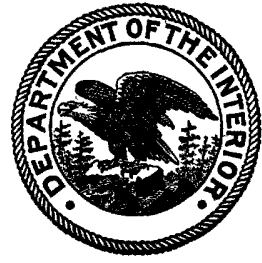
Box 3106, Capitol Station Oxlah-ra Ciy, Osla. GOVERNMENT PRINTING OFFICE 
ADDITIONAL COPIES

OF THIS PUBLICATION MAY BE PROCURED FROM THE SUPERINTENDENT OF DOCUMENTS GOVERNMENT PRINTING OFTICE WASHINGTON, D. C. AT

25 CENTS PER COPY

in:

$\therefore 2$ 


\section{CONTENTS}

Authorization and scope of work Page

Definition of terms... 2

Explanation of data..... 3

Accuracy of field data and computed results.

Cooperation... 5

Division of work 6

Gaging-station records 7

Streams tributary to Lake Superior

West Branch of Montreal River at Gile, Wis............. 7

Streams tributary to Lake Michigan ........... 8

Menominee River at Twin Falls, near Iron Mountain, Mich...- 8

Menominee River below Koss, Mich................. 10

Pine River near Florence, Wis........ 12

Pike River at Amberg, Wis._.......... 14

Peshtigo River at High Falls, near Crivitz, Wis........... 16

Oconto River near Gillett, Wis....................... 18

Fox River at Berlin, Wis._._.

Fox River at Rapide Croche dam, near Wrightstown, Wis..... 21

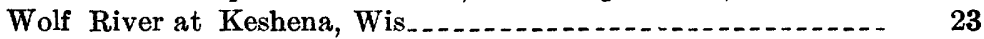

Wolf River at New London, Wis._.

Embarrass River near Embarrass, Wis_._.

Little Wolf River at Royalton, Wis........................ 29

Waupaca River near Waupaca, Wis.....

Sheboygan River near Sheboygan, Wis...... 33

Milwaukee River near Milwaukee, Wis................. 35

Little Calumet River at Harvey, Ill _...

Streams tributary to Lake Huron

Tittabawassee River at Freeland, Mich _. _ _ _......... 39

Streams tributary to Lake Erie....

Huron River at Barton, Mich........ 40

Maumee River at Antwerp, Ohio .....

Maumee River at Waterville, Ohio ........

Tiffin River near Stryker, Ohio ...

Auglaize River near Fort Jennings, Ohio .................. 48

Auglaize River near Defiance, Ohio ........... 50

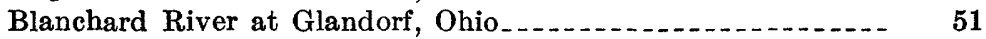

Miami and Erie Canal at Waterville, Ohio _.................. 53

Sandusky River near Upper Sandusky, Ohio ................ 54

Sandusky River near Mexico, Ohio _._.

East Branch of Black River at Elyria, Ohio................. 58

Cuyahoga River at Old Portage, Ohio....

Cuyahoga River at Brecksville, Ohio....................... 61

Cuyahoga River at Independence, Ohio................. 62

Ohio Canal feeder at Brecksville, Ohio

Ohio Canal at Independence, Ohịo .

Grand River near Madison, Ohio _... 67

Conneaut Creek at Amboy, Ohio

Cattaraugus Creek at Versailles, N. Y. 
Gaging-station records-Continued.

Streams tributary to Lake Ontario

Little Tonawanda Creek at Linden, N. Y.

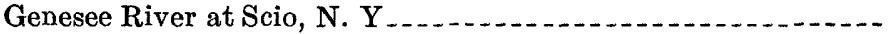

Genesee River at St. Helena, N. Y .

Genesee River at Jones Bridge, near Mount Morris, N. Y....

Genesee River at Driving Park Avenue, Rochester, N. Y .....

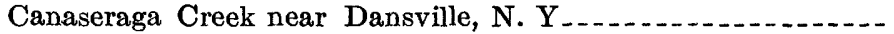

Keshequa Creek at Craig Colony, Sonyea, N. Y .........

Conesus Creek near Lakeville, N. Y

Canadice Lake outlet near Hemlock N. Y

Owasco Lake outlet near Auburn N. Y

East Branch of Fish Creek at Taberg, N. Y . .

Black River near Boonville, N. Y . . .

Black River at Watertown, N. Y

Forestport feeder near Boonville, N. Y

Black River canal (flowing south) near Boonville, N. Y......

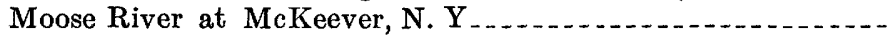

Moose River at Moose River, N. Y.

Middle Branch of Moose River at Old Forge, N. Y ........

Beaver River at State dam, near Beaver River, N. Y.........

Beaver River at Eagle Falls, near Number Four, N. Y .......

Streams tributary to St. Lawrence River. . . . . . . . . .

East Branch of Oswegatchie River at Cranberry Lake, N. Y ...-

East Branch of Oswegatchie River at Newton Falls, N. Y ......

Oswegatchie River near Heuvelton, N. Y ............................

West Branch of Oswegatchie River near Harrisville, N. Y....

Raquette River at Piercefield, N. Y ...

St. Regis River at Brasher Center, N. Y .

Richelieu River at Fort Montgomery, Rouses Point, N. Y...-

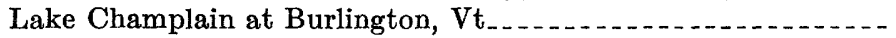

Saranac River near Plattsburg, N. Y ...

West Branch of Ausable River near Newman, N. Y . . . .

Ausable River at Ausable Forks, N. Y

Lake George at Rogers Rock, N. Y . . .

Lake George at Glen Island, near Bolton Landing, N. Y

Winooski River at Montpelier, Vt. . . . .

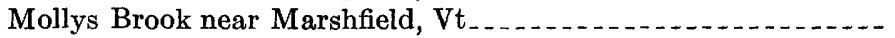

Jail Branch at East Barre, Vt.........

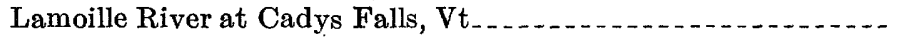

Green River at Garfield, Vt._.

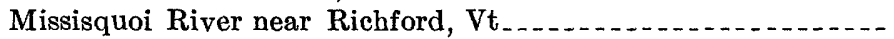

Clyde River at West Derby (Newport), Vt. .

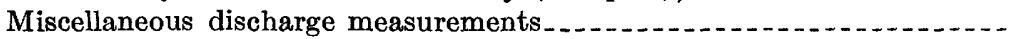

Appendix: Gaging stations and publications relating to water resources..

\section{ILLUSTRATIONS}

Plate I. A, Price current meters; $B$, Typical gaging station...........

II. Typical gaging stations: $A$, For wading measurement; $B$, For bridge measurement.

III. Water-stage recorders: $A, \mathrm{Au} ; B$, Gurley; $C$, Stevens....... 


\section{SURFACE WATER SUPPLY OF ST. LAWRENCE RIVER BASIN, 1923}

\section{AUTHORIZATION AND SCOPE OF WORK}

This volume is one of a series of 14 reports presenting records of measurements of flow made on streams in the United States during the year ending September 30, 1923.

The data presented in these reports were collected by the United States Geological Survey under the following authority contained in the organic law (20 Stat. L., p. 394):

Provided, That this officer [the Director] shall have the direction of the Geological Survey and the classification of public lands and examination of the geological structure, mineral resources, and products of the national domain.

The work was begun in 1888 in connection with special studies relating to irrigation in the arid West. Since the fiscal year ending June 30,1895 , successive sundry civil bills passed by Congress have carried the following item and appropriations:

For gaging the streams and determining the water supply of the United States, and for the investigation of underground currents and artesian wells, and for the preparation of reports upon the best methods of utilizing the water resources.

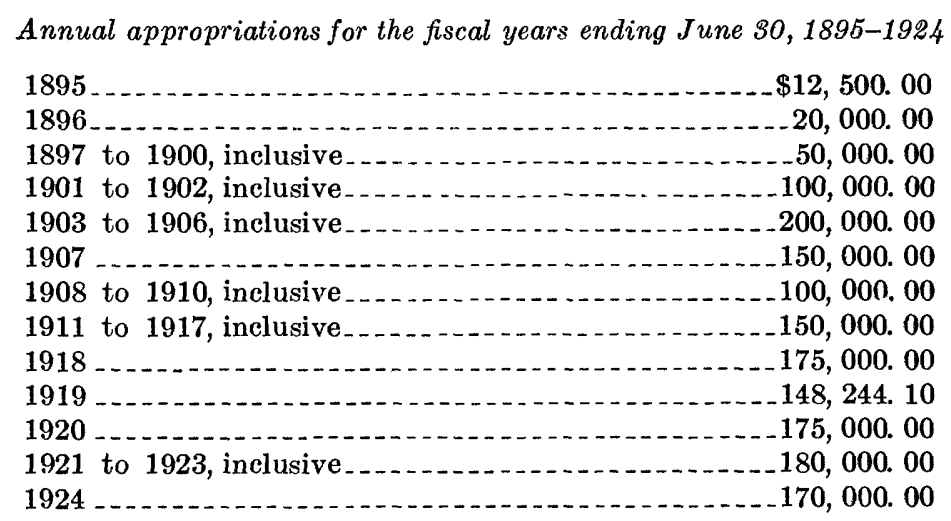

In the execution of the work many private and State organizations have cooperated, either by furnishing data or by assisting in collecting data. Acknowledgments for cooperation of the first kind are made in connection with the description of each station affected; cooperation of the second kind is acknowledged on pages 5 and 6 . 
Measurements of stream flow have been made at about 5,600 points in the United States and also at many points in Alaska and the Hawaiian Islands. In July, 1923, 1,590 gaging stations were being maintained by the Survey and the cooperating organizations. Many miscellaneous discharge measurements were made at other points. In connection with this work data were also collected in regard to precipitation, evaporation, storage reservoirs, river profiles, and water power in many sections of the country and will be made available in water-supply papers from time to time. Information in regard to publications relating to water resources is presented in the appendix to this report.

\section{DEFINITION OF TERMS}

The volume of water flowing in a stream-the "run-off" or "discharge"-is expressed in various terms, each of which has become associated with a certain class of work. These terms may be divided into two groups-(1) those that represent a rate of flow, as secondfeet, gallons per minute, miners' inches, and discharge in second-feet per square mile, and (2) those that represent the actual quantity of water as run-off in inches, acre-feet, and millions of cubic feet. The principal terms used in this series of reports are second-feet, secondfeet per square mile, run-off in inches, and acre-feet. They may be defined as follows:

"Second-feet" is an abbreviation for "cubic feet per second." A second-foot is the rate of discharge of water flowing in a channel of rectangular cross section 1 foot wide and 1 foot deep at an average velocity of one foot per second. It is generally used as a fundamental unit from which others are computed.

"Second-feet per square mile" is the average number of cubic feet of water flowing per second from each square mile of area drained, on the assumption that the run-off is distributed uniformly both as regards time and area.

"Run-off in inches" is the depth to which an area would be covered if all the water flowing from it in a given period were uniformly distributed on the surface. It is used for comparing run-off with rainfall, which is usually expressed in depth in inches.

An "acre-foot," equivalent to 43,560 cubic feet, is the quantity required to cover an acre to the depth of 1 foot. The term is commonly used in connection with storage for irrigation.

The following terms not in common use are here defined:

"Stage-discharge relation," an abbreviation for the term "relation of gage height to discharge."

"Control," a term used to designate the section or sections of the stream channel below the gage which determine the stage-discharge 


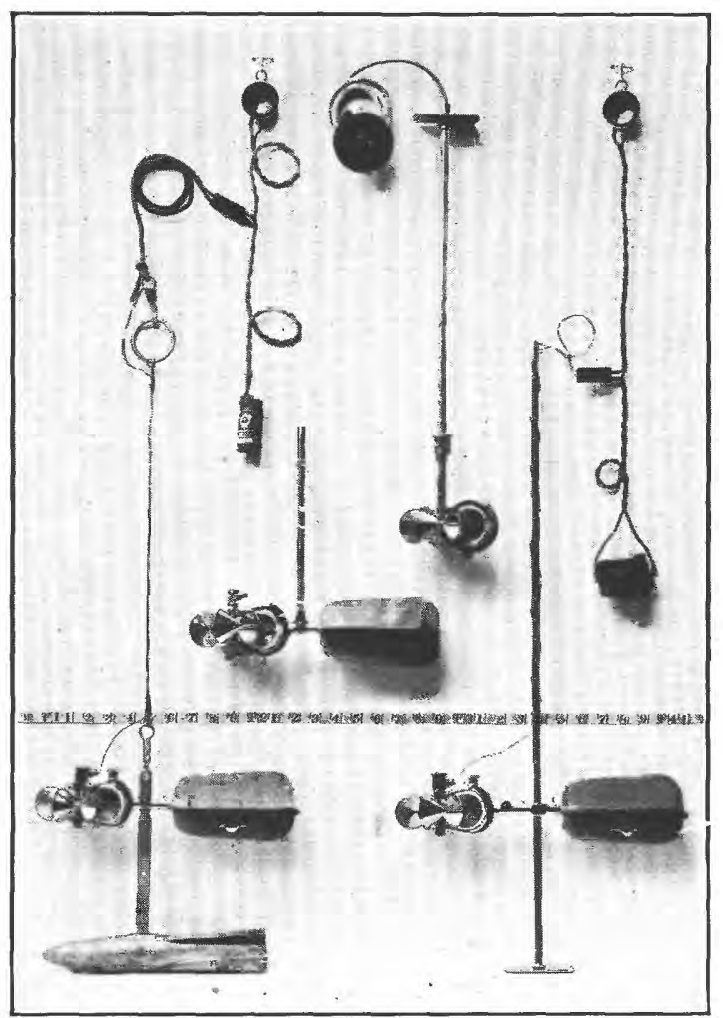

A, PRICE CURRENT METERS

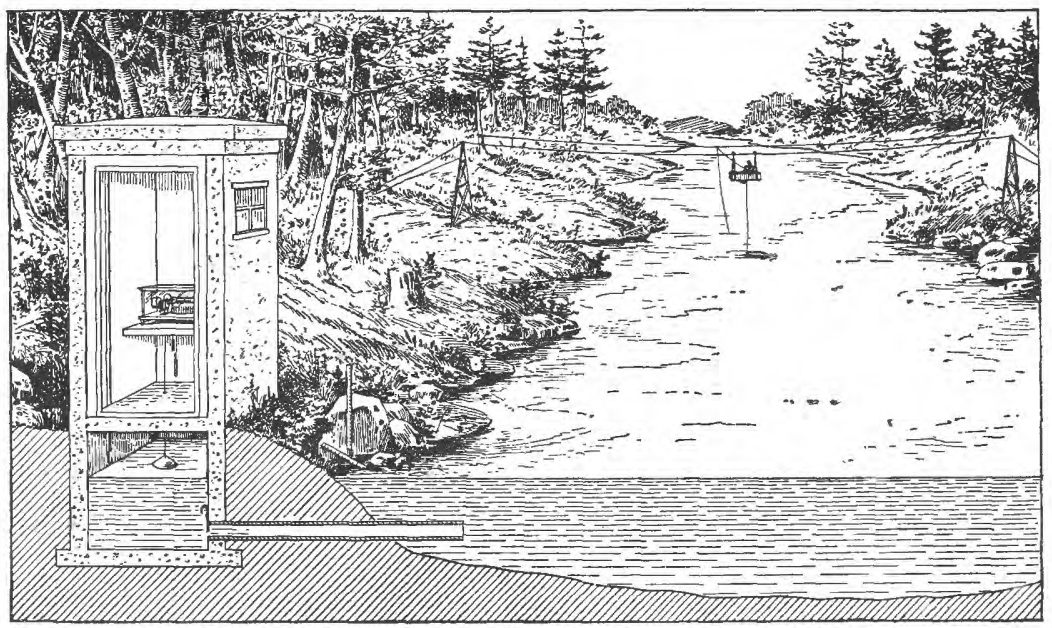

B. TYPICAL GAGING STATION 


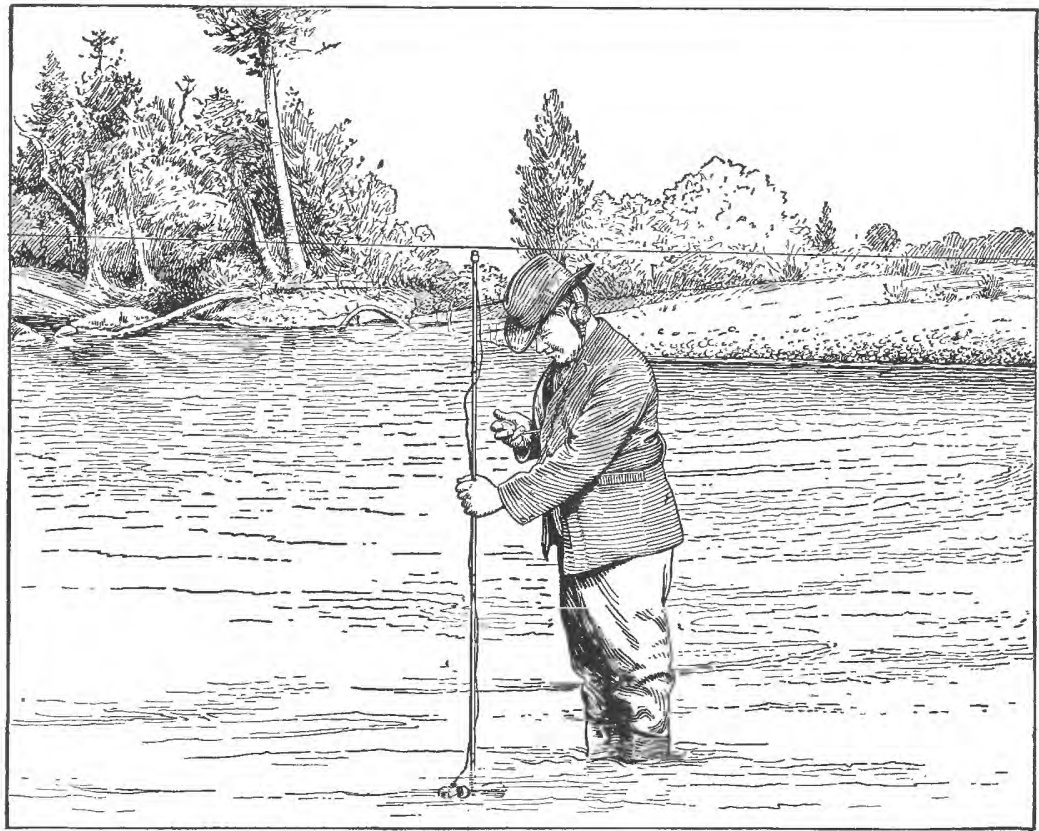

A

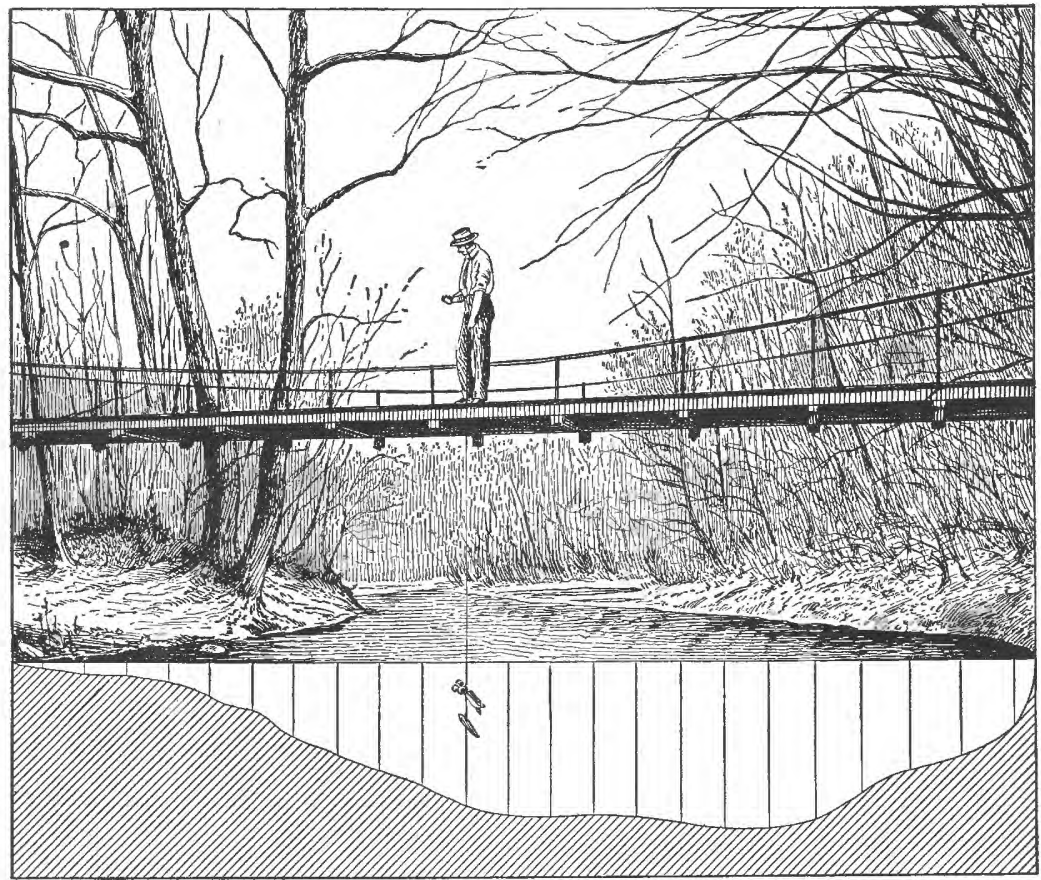

$B$

TYPICAL GAGING STATIONS

$A$, For wading measurement; $B$, for bridge measurement 

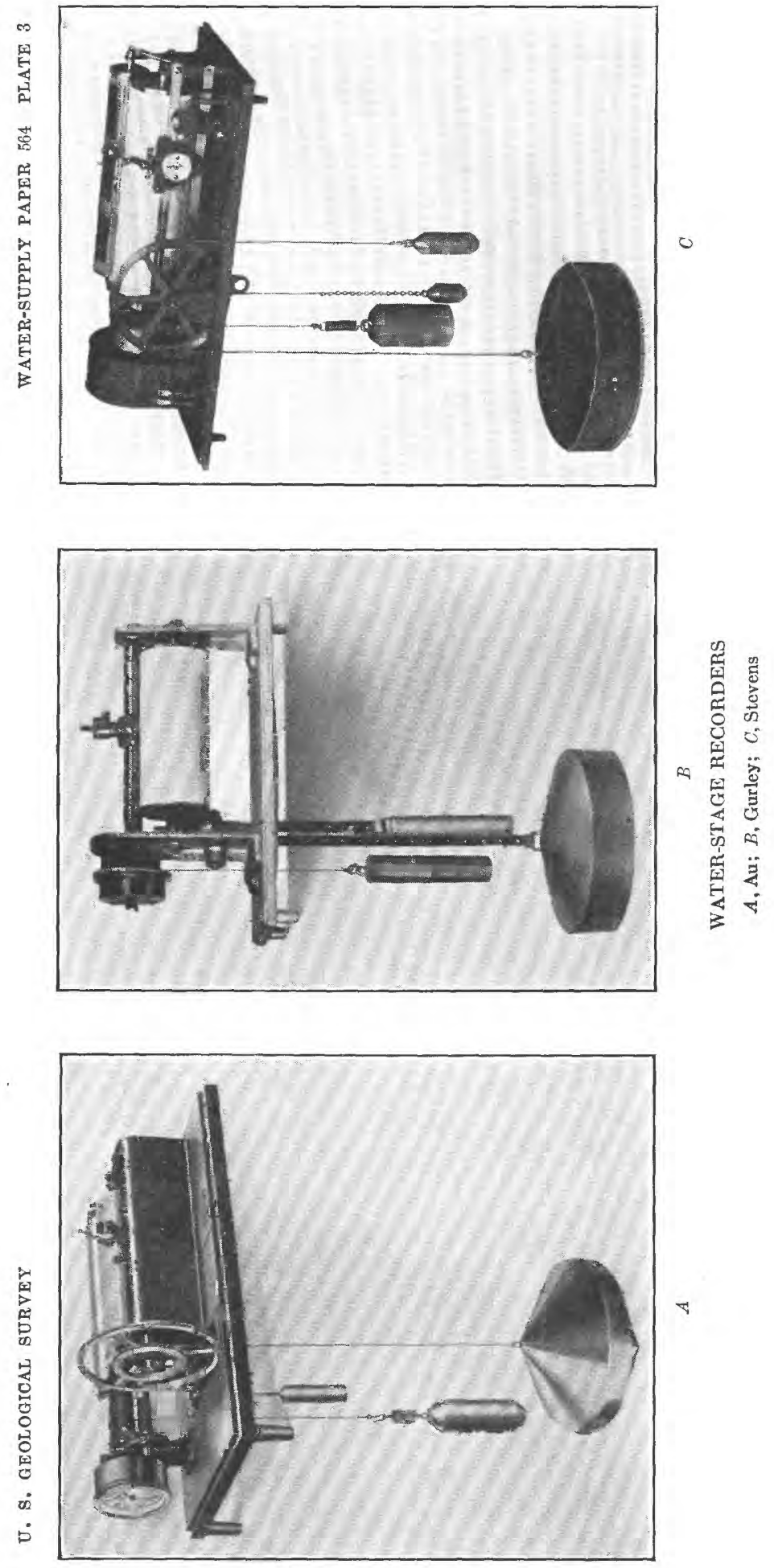
relation at the gage. It should be noted that the control may not be the same section or sections at all stages.

The "point of zero flow" for a gaging station is that point on the gage-the gage height-at which water ceases to flow over the control.

\section{EXPLANATION OF DATA}

The data presented in this report cover the year beginning October 1, 1922, and ending September 30, 1923. At the beginning of January in most parts of the United States much of the precipitation in the preceding three months is stored as ground water, in the form of snow or ice, or in ponds, lakes, and swamps, and this stored water passes off in the streams during the spring break-up. At the end of September, on the other hand, the only stored water available for run-off is possibly a small quantity in the ground; therefore the runoff for the year beginning October 1 is practically all derived from precipitation within that year.

The base data collected at gaging stations consist of records of stage, measurements of discharge, and general information used to supplement the gage heights and discharge measurements in determining the daily flow. The records of stage are obtained either from direct readings on a staff or chain gage or from water-stage recorder that gives a continuous record of the fluctuations. Measurements of discharge are made with a current meter. (See Pls. I-III.) The general methods are outlined in standard textbooks on the measurement of river discharge.

From the discharge measurements rating tables are prepared that give the discharge for any stage. The application of the daily gage heights to these rating tables gives the daily discharge from which the monthly and yearly mean discharge is computed.

The data presented for each gaging station in the area covered by this report comprise a description of the station, a table giving records of discharge measurements, a table showing the daily discharge of the stream, and a table of monthly and yearly discharge and run-off.

If the base data are insufficient to determine the daily discharge, tables giving daily gage height and records of discharge measurements are published.

The description of the station gives, in addition to statements regarding location and equipment, information in regard to any conditions that may effect the permanence of the stage-discharge relation, covering such subjects as the occurrence of ice, the use of the stream for log driving, shifting of control, and the cause and effect of backwater; it gives also information as to diversions that decrease the flow at the gage, artificial regulation, maximum and minimum recorded stages, and the accuracy of the records. 
The table of daily discharge gives, in general, the discharge in second-feet corresponding to the mean of the gage heights read each day. At stations on streams subject to sudden or rapid diurnal fluctuations the discharge obtained from the rating table and the mean daily gage height may not be the true mean discharge for the day. If such stations are equipped with water-stage recorders, the mean daily discharge may be obtained by averaging discharge at regular intervals during the day or by using the discharge integrator, an instrument operating on the principle of the planimeter and containing as an essential element the rating curve of the station.

In the table of monthly discharge the column headed "Maximum" gives the mean flow for the day when the mean gage height was highest. As the gage height is the mean for the day it does not indicate correctly the stage when the water surface was at crest height and the corresponding discharge was consequently larger than given in the maximum column. Likewise, in the column headed "Minimum" the quantity given is the mean flow for the day when the mean gage height was lowest. The column headed "Mean" is the average flow in cubic feet per second during the month. On this average flow computations recorded in the remaining columns, which are defined on page 2 , are based.

\section{ACCURACY OF FIELD DATA AND COMPUTED RESULTS}

The accuracy of stream-flow data depends primarily (1) on the permanency of the stage-discharge relation and (2) on the accuracy of observation of stage, measurements of flow, and interpretation of records.

A paragraph in the description of the station gives information regarding the (1) permanence of the stage-discharge relation, (2) precision with which the discharge rating curve is defined, (3) refinement of gage readings, (4) frequency of gage readings, and (5) methods of applying daily gage height to the rating table to obtain the daily discharge.

For the rating tables "well defined" indicates, in general, that the rating is probably accurate within 5 per cent; "fairly well defined," within 10 per cent; "poorly defined," within 15 to 25 per cent. These notes are very general and are based on the plotting of the individual measurements with reference to the mean rating curve.

The monthly means for any station may represent with high accuracy the quality of water flowing past the gage, but the figures showing discharge per square mile and run-off in inches may be subject to gross errors caused by the inclusion of large noncontributing districts in the measured drainage area, by lack of information concerning water diverted for irrigation or other use, or by inability to interpret the effect of artificial regulation of the flow of the river 
above the station. "Second-feet per square mile" and "run-off in inches" are therefore not computed if such errors appear probable. The computations are also omitted for stations on streams draining areas in which the annual rainfall is less than 20 inches. All figures representing "second-feet per square mile" and "run-off in inches" published in the earlier reports by the Survey, should be used with caution because of possible inherent sources of error not known to the Survey.

Many gaging stations on streams in the irrigated areas of the United States are situated above most of the diversions from those streams, and the discharge recorded does not show the water supply available for further development, as prior appropriations below the stations must first be satisfied. To give an idea of the amount of prior appropriations, a paragraph on diversions is presented in each station description. The figures given can not be considered exact but represent the best information available.

The tables of monthly discharge give only a general idea of the flow at the station and should not be used for other than preliminary estimates; the tables of daily discharge allow more detailed studies of the variation in flow. It should be borne in mind, however, that the observations in each succeeding year may be expected to throw new light on data previously published.

\section{COOPERATION}

The work in Wisconsin during the year ending September 30, 1923, was done in cooperation with the Railroad Commission of Wisconsin, C. M. Larson, chief engineer. The United States Engineer Corps cooperated in maintaining the stations on Fox River at Berlin and at Rapide Croche Dam and on Wolf River at New London.

The station on Little Calumet River at Harvey, Ill., was maintained in cooperation with the Illinois Department of Public Works and Buildings, Division of Waterways, W. L. Sackett, superintendent; gage reader paid by the Sanitary District of Chicago.

The work in Ohio was done in cooperation with the Ohio Cooperative Topographic Survey, C. E. Sherman, inspector.

The work in New York was carried on in cooperation with the State of New York, Frank M. Williams, State engineer and surveyor, prior to January 1, 1923, and Dwight B. La Du, State engineer and surveyor, after that date. The following organizations cooperated at certain other tations: Rochester Gas \& Electric Corporation (Genesee River at Driving Park Avenue, Rochester, N. Y.); the city of Rochester (Conesus Creek near Lakeville, N. Y., and Canadice Lake outlet near Hemlock, N. Y.); Utica Gas \& Electric Co. (East Branch of Fish Creek at Taberg, N. Y.); Black River Regu$72038-26 \dagger-$ wsP $564-2$ 
lating District (Black River at Watertown, N. Y., and Moose River at McKeever, N. Y.); Beaver River Power Corporation and Northern New York Utilities (Inc.) (Beaver River at Eagle Falls, near Number Four, N. Y.); the Commission for the Improvement of Oswegatchie River (East Branch of Oswegatchie River at Cranberry Lake, N. Y.); International Paper Co. (Raquette River at Piercefield, N. Y., and Lake George at Rogers Rock, N. Y.); New York \& Pennsylvania Co. (Bouquet River at Willsboro, N. Y.); Plattsburg Gas \& Electric Co.( Saranac River near Plattsburg, N. Y.).

The work in Vermont was carried on in cooperation with the State, the cooperating official being George A. Reed, State engineer. The following organizations and individuals cooperated in maintaining one or more gaging stations: Montpelier \& Barre Light \& Power Co. (Mollys Brook near Marshfield and Jail Branch at East Barre); Charles T. Middlebrook (Green River at Garfield); and Newport Electric Light Co. (Clyde River at West Derby).

\section{DIVISION OF WORK}

Data for stations in the Lake Superior and Lake Michigan drainage basins in Wisconsin and Michigan were collected and prepared for publication under the direction of S. B. Soule, district engineer, assisted by E. E. Foster and J. H. Olson.

Data for the station in. Illinois were collected and prepared for publication by $\mathrm{H}$. E. Grosbach, district engineer.

Data for stations on Huron River at Barton, Mich., and Tittabawassee River at Freeland, Mich., were prepared for publication by A. H. Horton, district engineer, assisted by W. C. Wiggins.

Data for stations in Ohio were collected and prepared for publication under the direction of Lasley Lee, district engineer, assisted by E. E. R. Dornbach, V. B. Lamoureux, F. R. Morgan, W. W. Perrin, W. A. Werner, and L. L. Dickson.

Data for stations in New York were collected and prepared for publication under the direction of $\mathrm{A}$. W. Harrington, district engineer, assisted by E. B. Shupe, J. L. Lamson, B. F. Howe, A. E. Johnson, and Agnes D. Buchanan.

Data for stations in Vermont were collected and prepared for publication under the direction of $\mathrm{C}$. $\mathrm{H}$. Pierce, district engineer, assisted by W. E. Armstrong, L. H. McCarthy, H. F. Hill, jr., and E. W. Downs.

The manuscript was assembled and reviewed by $O$. D. Mussey. 


\section{GAGING-STATION RECORDS}

\section{STREAMS TRIBUTARY TO LAKE SUPERIOR}

WEST BRANCH OF MONTREAL RIVER AT GIIE, WIS.

Location.-In sec. 27 , T. 46 N., R. 2 E., 800 feet upstream from highway bridge at Gile, Iron County, $21 / 2$ miles southwest of Hurley, Wis., and 4 miles upstream from junction of east and west branches.

Drainage area.-About 78 square miles ${ }^{1}$ (measured on map of Wisconsin Soil Survey; scale, 1 inch $=3$ miles).

Reconds aVailable.-April 26, 1918, to September 30, 1923.

GAGE.- Sloping gage bolted to rock ledge on left bank of river a few hundred feet upstream from pump house of Ottawa Mine; read by Carl Lang.

Discharge measurements.-Made from downstream side of highway bridge 800 feet below gage or by wading.

Channel and control.-Control formed by permanent rock ledge across narrow section of stream about 15 feet downstream from gage. Fall at control about 4 feet.

Extremes of Discharge.-Maximum stage recorded during year, 7.20 feet April 21 (discharge, 1,480 second-feet); minimum stage, 1.60 feet October 10 and 11 (discharge, 5.4 second-feet).

1918-1923: Maximum stage recorded, that of April 21, 1923; minimum stage, 1.32 feet July 23, 1918, and September 7, 1921 (discharge, about 2.4 second-feet).

Regulation.-None.

Accuracr.-Stage-discharge relation permanent; not seriously affected by ice. Rating curve used well defined below 710 second-feet. Gage read to hundredths once daily. Daily discharge ascertained by applying gage height to rating table. On dates when gage was not read (generally only Sundays) discharge was interpolated and is subject to small errors. Records good.

Discharge measurements of West Branch of Montreal River at Gile, Wis., during the year ending September 30,1923

[Made by E. E. Foster]

\begin{tabular}{l|l|l|l}
\hline Date & $\begin{array}{c}\text { Gage } \\
\text { height }\end{array}$ & $\begin{array}{c}\text { Dis } \\
\text { charge }\end{array}$ \\
\hline $\begin{array}{l}\text { Oct. } 21 \\
\text { July } 20\end{array}$ & $\begin{array}{r}\text { Feet } \\
1.82\end{array}$ & $\begin{array}{r}\text { Sec.-ft. } \\
\mathbf{9 . 3}\end{array}$ \\
\hline
\end{tabular}

1 Supersedes figure published in previous reports 
Daily discharge, in second-feet, of West Branch of Montreal River at Gile, Wis., for the year ending September 30, 1923

\begin{tabular}{|c|c|c|c|c|c|c|c|c|c|c|c|c|}
\hline Day & Oct. & Nov. & Dec. & Jan. & Feb. & Mar. & Apr. & May & June & July & Aug. & Sept. \\
\hline $\begin{array}{l}1-- \\
2-- \\
3-\end{array}$ & $\begin{array}{l}7.8 \\
9.4 \\
9.4 \\
8.3 \\
7.2\end{array}$ & $\begin{array}{l}14 \\
14 \\
14 \\
14 \\
16\end{array}$ & $\begin{array}{l}60 \\
66 \\
58 \\
50 \\
34\end{array}$ & $\begin{array}{l}8.8 \\
8.5 \\
8.3 \\
8.1 \\
7.6\end{array}$ & $\begin{array}{l}7.9 \\
8.1 \\
7.9 \\
8.0 \\
8.1\end{array}$ & $\begin{array}{l}6.1 \\
6.7 \\
7.0 \\
7.1 \\
7.2\end{array}$ & $\begin{array}{l}7.1 \\
7.2 \\
7.4 \\
7.6 \\
7.9\end{array}$ & $\begin{array}{l}214 \\
198 \\
184 \\
170 \\
158\end{array}$ & $\begin{array}{l}30 \\
28 \\
31 \\
34 \\
45\end{array}$ & $\begin{array}{l}59 \\
52 \\
50 \\
63 \\
76\end{array}$ & $\begin{array}{l}38 \\
34 \\
31 \\
26 \\
22\end{array}$ & $\begin{array}{l}11 \\
18 \\
26 \\
34 \\
28\end{array}$ \\
\hline $\begin{array}{r}6 \\
7 \\
8 \\
9 \\
9 \\
10\end{array}$ & $\begin{array}{l}7.2 \\
6.3 \\
6.3 \\
6.3 \\
5.4\end{array}$ & $\begin{array}{l}17 \\
19 \\
23 \\
28 \\
31\end{array}$ & $\begin{array}{l}26 \\
26 \\
24 \\
23 \\
22\end{array}$ & $\begin{array}{l}7.4 \\
7.3 \\
7.2 \\
7.2 \\
7.2\end{array}$ & $\begin{array}{l}8.3 \\
8.1 \\
8.1 \\
8.1 \\
8.1\end{array}$ & $\begin{array}{l}7.2 \\
7.2 \\
7.2 \\
7.0 \\
7.0\end{array}$ & $\begin{array}{r}7.9 \\
8.1 \\
8.6 \\
9.2 \\
12\end{array}$ & $\begin{array}{l}158 \\
158 \\
184 \\
184 \\
184\end{array}$ & $\begin{array}{r}60 \\
92 \\
110 \\
125 \\
130\end{array}$ & $\begin{array}{l}125 \\
250 \\
292 \\
335 \\
290\end{array}$ & $\begin{array}{l}18 \\
15 \\
14 \\
13 \\
11\end{array}$ & $\begin{array}{l}26 \\
23 \\
21 \\
20 \\
18\end{array}$ \\
\hline $\begin{array}{l}1 \ldots \\
\cdots \cdots \\
\cdots \\
\cdots\end{array}$ & $\begin{array}{l}5.4 \\
7.2 \\
6.3 \\
5.4 \\
6.3\end{array}$ & $\begin{array}{l}36 \\
39 \\
42 \\
42 \\
36\end{array}$ & $\begin{array}{l}20 \\
18 \\
16 \\
16 \\
15\end{array}$ & $\begin{array}{l}7.0 \\
7.0 \\
7.0 \\
7.3 \\
7.6\end{array}$ & $\begin{array}{l}7.8 \\
7.4 \\
7.2 \\
7.2 \\
7.0\end{array}$ & $\begin{array}{l}7.0 \\
7.0 \\
7.0 \\
7.2 \\
7.2\end{array}$ & $\begin{array}{l}16 \\
24 \\
34 \\
42 \\
48\end{array}$ & $\begin{array}{l}184 \\
184 \\
184 \\
184 \\
158\end{array}$ & $\begin{array}{r}135 \\
105 \\
87 \\
63 \\
52\end{array}$ & $\begin{array}{l}250 \\
214 \\
214 \\
170 \\
145\end{array}$ & $\begin{array}{l}14 \\
21 \\
28 \\
24 \\
20\end{array}$ & $\begin{array}{l}17 \\
16 \\
15 \\
14 \\
14\end{array}$ \\
\hline 19 & $\begin{array}{l}7.2 \\
8.3 \\
8.3 \\
9.4 \\
9.4\end{array}$ & $\begin{array}{l}34 \\
36 \\
38 \\
37 \\
36\end{array}$ & $\begin{array}{l}14 \\
13 \\
12 \\
10 \\
9.4\end{array}$ & $\begin{array}{l}8.1 \\
8.5 \\
9.0 \\
8.5 \\
8.1\end{array}$ & $\begin{array}{l}7.0 \\
6.8 \\
6.8 \\
6.7 \\
6.7\end{array}$ & $\begin{array}{l}7.0 \\
7.0 \\
7.1 \\
7.2 \\
7.2\end{array}$ & $\begin{array}{r}55 \\
115 \\
135 \\
250 \\
1,130\end{array}$ & $\begin{array}{l}135 \\
130 \\
125\end{array}$ & $\begin{array}{l}42 \\
36 \\
30 \\
28 \\
32\end{array}$ & $\begin{array}{r}120 \\
87 \\
63 \\
48 \\
40\end{array}$ & $\begin{array}{l}15 \\
15 \\
14 \\
21 \\
28\end{array}$ & $\begin{array}{l}13 \\
12 \\
12 \\
14 \\
14\end{array}$ \\
\hline $\begin{array}{l}23 \\
25 \\
25\end{array}$ & $\begin{array}{l}11.0 \\
13.0 \\
15.0 \\
20 \\
19\end{array}$ & $\begin{array}{l}36 \\
45 \\
42 \\
42 \\
42\end{array}$ & $\begin{array}{l}9.4 \\
9.9 \\
10 \\
10 \\
10\end{array}$ & $\begin{array}{l}8.1 \\
8.1 \\
8.3 \\
8.3 \\
8.1\end{array}$ & $\begin{array}{l}6.5 \\
6.5 \\
6.5 \\
6.3 \\
6.3\end{array}$ & $\begin{array}{l}7.2 \\
7.2 \\
7.2 \\
7.2 \\
7.2\end{array}$ & $\begin{array}{r}1,480 \\
985 \\
490 \\
415 \\
385\end{array}$ & $\begin{array}{r}135 \\
125 \\
110 \\
92 \\
76\end{array}$ & $\begin{array}{l}158 \\
310 \\
290 \\
237 \\
184\end{array}$ & $\begin{array}{l}36 \\
39 \\
42 \\
96 \\
79\end{array}$ & $\begin{array}{l}36 \\
32 \\
28 \\
22 \\
18\end{array}$ & $\begin{array}{l}14 \\
15 \\
14 \\
14 \\
15\end{array}$ \\
\hline $\begin{array}{l}26 \\
27 \\
28 \\
29 \\
30 \\
31\end{array}$ & $\begin{array}{l}20 \\
18 \\
18 \\
17 \\
16 \\
15\end{array}$ & $\begin{array}{l}40 \\
38 \\
38 \\
30 \\
45\end{array}$ & $\begin{array}{l}10 \\
9.9 \\
9.7 \\
9.4 \\
9.4 \\
9.1\end{array}$ & $\begin{array}{l}8.1 \\
8.3 \\
8.3 \\
8.3 \\
8.1 \\
7.9\end{array}$ & $\begin{array}{l}6.3 \\
6.1 \\
5.9\end{array}$ & $\begin{array}{l}7.2 \\
7.0 \\
7.0 \\
7.0 \\
7.0 \\
7.0\end{array}$ & $\begin{array}{l}335 \\
310 \\
290 \\
261 \\
232\end{array}$ & $\begin{array}{l}60 \\
56 \\
52 \\
48 \\
40 \\
32\end{array}$ & $\begin{array}{r}170 \\
170 \\
125 \\
83 \\
66\end{array}$ & $\begin{array}{l}66 \\
48 \\
40 \\
46 \\
52 \\
48\end{array}$ & $\begin{array}{c}16 \\
15 \\
14 \\
14 \\
12 \\
9.4\end{array}$ & $\begin{array}{c}13 \\
10 \\
9.4 \\
9.4 \\
11\end{array}$ \\
\hline
\end{tabular}

Note.-Gage not read Sundays; discharge interpolated.

Monthly discharge of West Branch at Montreal River at Gile, Wis., for the year ending September 30,1923

[Drainage area, 78 square miles]

\begin{tabular}{|c|c|c|c|c|c|}
\hline \multirow{2}{*}{ Month } & \multicolumn{4}{|c|}{ Discharge in second-feet } & \multirow{2}{*}{$\begin{array}{c}\text { Run-off } \\
\text { in inches }\end{array}$} \\
\hline & Maximum & Minimum & Mean & $\underset{\text { mile }}{\text { Per square }}$ & \\
\hline $\begin{array}{l}\text { October } \\
\text { November } \\
\text { Derember } \\
\text { January } \\
\text { February } \\
\text { March } \\
\text { April } \\
\text { May } \\
\text { June } \\
\text { July } \\
\text { August } \\
\text { September }\end{array}$ & $\begin{array}{c}20 \\
45 \\
66 \\
9.0 \\
8.3 \\
7.2 \\
1,480 \\
214 \\
310 \\
335 \\
38 \\
34\end{array}$ & $\begin{array}{r}5.4 \\
14.0 \\
9.1 \\
7.0 \\
5.9 \\
6.1 \\
7.1 \\
32 \\
28 \\
36 \\
9.4 \\
9.4\end{array}$ & $\begin{array}{l}10.6 \\
32.1 \\
20.6 \\
7.92 \\
7.20 \\
7.06 \\
237 \\
134 \\
103 \\
114 \\
20.6 \\
16.4\end{array}$ & $\begin{array}{l}0.136 \\
.412 \\
.264 \\
.102 \\
.0924 \\
.0905 \\
3.04 \\
1.72 \\
1.32 \\
1.46 \\
.264 \\
.210\end{array}$ & $\begin{array}{r}0.16 \\
.46 \\
.30 \\
.12 \\
.10 \\
.10 \\
3.39 \\
1.98 \\
1.47 \\
1.68 \\
.30 \\
.23\end{array}$ \\
\hline The year... & 1,480 & 5.4 & 59.2 & .759 & 10.29 \\
\hline
\end{tabular}

STREAMS TRIBUTARY TO LAKE MICHIGAN

MENOMINEE RIVER AT TWIN FALLS, NEAR IRON MOUNTAIN, MICH.

Location.-In sec. 12, T. 40 N., R. 31 W., at power plant of Peninsular Power Co., $3 \frac{1}{2}$ miles north of city of Iron Mountain, Mich. Pine River enters from right 3 miles below station. 
Drainage area.-1,790 square miles.

Records Available.-January 1, 1914, to September 30, 1923.

Gagrs.-Staff and float gages used to determine effective head on water wheels.

Discharge. - The daily discharge was computed from hourly determinations of the flow through the turbines computed from a record of the number of wheels in operation, the kilowatt output, and the effective head. To the average flow through the turbines is added the water passing over the spillway, through the gates, down the log sluice, and leakage through the idle wheels and through the dam.

Extremes of Discharge.- Maximum mean daily discharge recorded during the year, 10,000 second-feet April 23; minimum mean daily discharge, 618 secondfeet August 23.

1914-1923: Maximum mean daily discharge recorded, 16,700 second-feet April 23 and 24, 1916; minimum mean daily discharge, 274 second-feet August 10, 1919.

Regdlation.-Besides the regulation at this power plant, the flow is subject to the regulation of a power plant on Brule River about 5 miles above this point, owned by the same company. Owing to variations in demand, the daily discharge bears no relation to the natural flow, but the mean monthly discharge probably corresponds closely to the natural flow.

AccurAcx.-Discharge records published in the following tables were obtained by adding 10 per cent to discharge as computed from power-plant records. This correction is based upon the results of five current-meter measurements made in 1919 and 1922 by the United States Geological Survey at a point about 1 mile downstream from power plant.

Cooperation.-Daily-discharge records furnished by Mead and Seastone, consulting engineers, Madison, Wis.

Daily discharge, in second-feet, of Menominee River at Twin Falls, near Iron Mountain, Mich., for the year ending September 30, 1923

\begin{tabular}{|c|c|c|c|c|c|c|c|c|c|c|c|c|}
\hline & ct. & ov. & Dec. & Jan. & Feb. & Mar. & Apr. & May & June & July & Aug. & Sept. \\
\hline$\ldots$ & $\begin{array}{r}1,020 \\
1,040 \\
943 \\
1,050 \\
1,070\end{array}$ & $\begin{array}{l}937 \\
806 \\
917 \\
991 \\
968\end{array}$ & $\begin{array}{r}1,310 \\
1,320 \\
823 \\
1,220\end{array}$ & $\begin{array}{l}988.8 \\
942 \\
800 \\
902\end{array}$ & $\begin{array}{l}771 \\
805 \\
915 \\
912\end{array}$ & $\begin{array}{r}780 \\
861 \\
906 \\
1,170 \\
972\end{array}$ & $\begin{array}{l}726 \\
845 \\
887 \\
772\end{array}$ & $\begin{array}{l}000 \\
300 \\
170 \\
650 \\
380\end{array}$ & $\begin{array}{r}1,220 \\
1,150 \\
904 \\
1,260 \\
1,180\end{array}$ & $\begin{array}{r}1,200 \\
1,230 \\
1,330 \\
974 \\
1,030\end{array}$ & $\begin{array}{l}020 \\
130 \\
060 \\
160 \\
040\end{array}$ & $\begin{array}{r}705 \\
647 \\
1,030 \\
1,130\end{array}$ \\
\hline & $\begin{array}{l}962 \\
932 \\
895 \\
824 \\
796\end{array}$ & $\begin{array}{r}922 \\
960 \\
932 \\
938 \\
1,020\end{array}$ & $\begin{array}{l}1,080 \\
1,180 \\
958 \\
1,040 \\
1,020\end{array}$ & $\begin{array}{l}925 \\
965 \\
953 \\
908\end{array}$ & $\begin{array}{l}927 \\
938 \\
996 \\
981 \\
927\end{array}$ & $\begin{array}{l}956 \\
824 \\
744 \\
725 \\
713\end{array}$ & $\begin{array}{l}666 \\
660 \\
896 \\
729 \\
691\end{array}$ & $\begin{array}{l}4,500 \\
4,230 \\
3,450 \\
3,220 \\
2,880\end{array}$ & 1,960 & $\begin{array}{l}1,110 \\
1,230 \\
1,980 \\
2,180 \\
3,000\end{array}$ & $\begin{array}{r}989 \\
1,100 \\
1,000 \\
992 \\
957\end{array}$ & $\begin{array}{r}987 \\
1,150 \\
1,120 \\
957 \\
998\end{array}$ \\
\hline & $\begin{array}{r}764 \\
850 \\
949 \\
952 \\
1,010\end{array}$ & $\begin{array}{r}1,180 \\
847 \\
1,100 \\
1,150 \\
1,600\end{array}$ & $\begin{array}{l}947 \\
952 \\
874 \\
904 \\
931\end{array}$ & $\begin{array}{r}1,010 \\
940 \\
956 \\
949 \\
837\end{array}$ & 732 & $\begin{array}{l}696 \\
860 \\
795 \\
844 \\
896\end{array}$ & $\begin{array}{r}864 \\
893 \\
1,030 \\
768 \\
740\end{array}$ & & 2,700 & & 846 & $\begin{array}{l}1,070 \\
1,140 \\
1,170 \\
1,150 \\
1,130\end{array}$ \\
\hline & $\begin{array}{l}965 \\
896 \\
778 \\
864 \\
901\end{array}$ & $\begin{array}{l}1,410 \\
1,520 \\
1,600 \\
1,280 \\
2,040\end{array}$ & $\begin{array}{r}970 \\
1,040 \\
969 \\
954 \\
890\end{array}$ & 729 & 619 & $\begin{array}{l}917 \\
902 \\
780\end{array}$ & $\begin{array}{r}798 \\
866 \\
1,240 \\
1,040 \\
2,350\end{array}$ & & 1,420 & 1,160 & 630 & $\begin{array}{r}932 \\
1,110 \\
1,110 \\
1,040 \\
1,020\end{array}$ \\
\hline & $\begin{array}{r}910 \\
954 \\
923 \\
1,040 \\
1,040\end{array}$ & $\begin{array}{r}1,870 \\
1,370 \\
1,300 \\
1,340 \\
946\end{array}$ & $\begin{array}{l}739 \\
774\end{array}$ & 970 & 689 & $\begin{array}{r}867 \\
1,040 \\
902 \\
892 \\
723\end{array}$ & $\begin{array}{r}5,000 \\
7,940 \\
10,000 \\
9,170 \\
7,150\end{array}$ & & & & 773 & $\begin{array}{r}1,060 \\
949 \\
1,100 \\
1,068 \\
1,000\end{array}$ \\
\hline 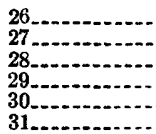 & $\begin{array}{r}1,070 \\
965 \\
966 \\
1,020 \\
980 \\
937\end{array}$ & $\begin{array}{r}820 \\
1,170 \\
1,110 \\
1,270 \\
1,140\end{array}$ & $\begin{array}{r}981 \\
956 \\
773 \\
732 \\
815 \\
1,020\end{array}$ & $\begin{array}{l}865 \\
759 \\
692 \\
688 \\
749 \\
755\end{array}$ & $\begin{array}{l}893 \\
825 \\
790\end{array}$ & $\begin{array}{l}866 \\
867 \\
870 \\
876 \\
806 \\
928\end{array}$ & $\begin{array}{l}7,740 \\
7,130 \\
6,460 \\
6,310 \\
5,940\end{array}$ & $\begin{array}{l}1,210 \\
1,390 \\
1,340\end{array}$ & $\begin{array}{l}1, \\
1,4 \\
1,7\end{array}$ & $\begin{array}{l}1,130 \\
849 \\
1,160 \\
1,050\end{array}$ & 763 & $\begin{array}{r}1,030 \\
1,060 \\
908 \\
817 \\
971\end{array}$ \\
\hline
\end{tabular}


Monthly discharge of Menominee River at Twin Falls, near Iron Mountain, Mich., for the year ending September 30, 1923

[Drainage area, 1,790 square miles]

\begin{tabular}{|c|c|c|c|c|c|}
\hline \multirow{2}{*}{ Month } & \multicolumn{4}{|c|}{ Discharge in second-feet } & \multirow{2}{*}{$\begin{array}{l}\text { Run-off } \\
\text { in inches }\end{array}$} \\
\hline & Maximum & Minimum & Mean & $\underset{\text { mile }}{\text { Per square }}$ & \\
\hline $\begin{array}{l}\text { October } \\
\text { November } \\
\text { December } \\
\text { January } \\
\text { February } \\
\text { March } \\
\text { April } \\
\text { May } \\
\text { June } \\
\text { July } \\
\text { August } \\
\text { September }\end{array}$ & $\begin{array}{r}1,070 \\
2,040 \\
1,320 \\
1,010 \\
996 \\
1,170 \\
10,000 \\
5,300 \\
3,760 \\
3,050 \\
1,160 \\
1,170\end{array}$ & $\begin{array}{r}764 \\
806 \\
732 \\
688 \\
619 \\
696 \\
660 \\
1,160 \\
904 \\
745 \\
618 \\
647\end{array}$ & $\begin{array}{r}944 \\
1,180 \\
962 \\
852 \\
790 \\
854 \\
3,040 \\
2,680 \\
1,880 \\
1,440 \\
819 \\
1,010\end{array}$ & $\begin{array}{l}0.527 \\
.659 \\
.537 \\
.476 \\
.441 \\
.477 \\
1.70 \\
1.50 \\
1.05 \\
.804 \\
.458 \\
.564\end{array}$ & $\begin{array}{r}0.61 \\
.74 \\
.62 \\
.55 \\
.46 \\
.55 \\
1.90 \\
1.73 \\
1.17 \\
.93 \\
.53 \\
.63\end{array}$ \\
\hline The year & 10,000 & 618 & 1,370 & .765 & 10.42 \\
\hline
\end{tabular}

MENOMINEE RIVER BELOW KOSS, MICH.

Location.-In sec. 9, T. 34 N., R. 27 W., at power plant of Menominee \& Marinette Light \& Traction Co., 4 miles below Koss, Marinette County, Mich., and 3 miles west of Ingalls, Mich. Little Cedar River, draining an area entirely in Michigan, enters from left half a mile below station.

Drainage area.- $-3,790$ square miles.

Records available.-July 1, 1913, to September 30, 1923.

Discharge.-Daily discharge was computed from hourly determinations of the flow through the turbines (from kilowatt output and effective head) plus discharge through the gates and over the spillway. No account was taken of the water passing through the exciter turbine, nor waste over the "trash gate" at the power house. This amount was, however, relatively small.

Extremes of DischARge.-Maximum mean daily discharge during year, 18,700 second-feet April 23; minimum mean daily discharge, 938 second-feet August 20.

1913-1923: Maximum mean daily discharge recorded, 23,200 second-feet April 23 and 25, 1916; minimum mean daily discharge, 926 second-feet November 24, 1922.

Regulation.-Above the station are the following power plants: Sturgeon Falls, owned by Pennsylvania Iron Mining Co., 50 miles; Little Quinnesec, owned by Kimberly Clark, 57 miles; Upper Quinnesec, owned by Oliver Iron Mining Co., 62 miles; Twin Falls, owned by Peninsular Power Co. With the exception of the Kimberly Clark dam at Little Quinnesec, the dams furnish power for utility and mining uses, so that the flow past the dams is compartively uniform. The Kimberly Clark dam is used for paper mills and regulates the flow on Sundays and holidays. The effect of this regulation generally is felt at the station on Tuesdays. The monthly flow probably represents the natural flow.

Accuracr.-A discharge measurement was made September 12, 1922, at highway bridge about 4 miles below station which checks the discharge as computed from the power-plant records within 4 per cent. See Water-Supply Paper 524 for statement regarding earlier measurements. Records good.

Cooperation.-Daily discharge records furnished by Edward Daniell, general manager of the Menominee \& Marinette Light \& Traction Co. 
Daily discharge, in second-feet, of Menominee River below Koss, Mich., for the year ending September 30, 1923

\begin{tabular}{c|r|r|r|r|r|r|r|r|r|r|r|r|r|r}
\hline Day & Oct. & Nov. & Dec. & Jan. & Feb. & Mar. & Apr. & May & June & July & Aug. & Sept. \\
\hline & & & & & & & & & \\
\hline & & & & & & & & & \\
\hline
\end{tabular}

Monthly discharge of Menominee River below Koss, Mich., for the year ending September 30, 1923

[Drainage area, 3,790 square miles]

\begin{tabular}{|c|c|c|c|c|c|}
\hline \multirow{2}{*}{ Month } & \multicolumn{4}{|c|}{ Discharge in second-feet } & \multirow{2}{*}{$\begin{array}{l}\text { Run-off } \\
\text { in inches }\end{array}$} \\
\hline & Maximum & Minimum & Mean & $\begin{array}{c}\text { Per square } \\
\text { mile }\end{array}$ & \\
\hline $\begin{array}{l}\text { October } \\
\text { November } \\
\text { December } \\
\text { January } \\
\text { February } \\
\text { March } \\
\text { April } \\
\text { May } \\
\text { June } \\
\text { July } \\
\text { August } \\
\text { September }\end{array}$ & $\begin{array}{r}1,880 \\
3,360 \\
2,510 \\
1,780 \\
1,640 \\
1,710 \\
18,700 \\
12,300 \\
10,100 \\
4,840 \\
2,250 \\
2,440\end{array}$ & $\begin{array}{l}1,300 \\
1,640 \\
1,280 \\
1,190 \\
1,995 \\
1,100 \\
1,130 \\
2,660 \\
2,340 \\
1,860 \\
938 \\
1,440\end{array}$ & $\begin{array}{l}1,700 \\
2,190 \\
1,680 \\
1,530 \\
1,350 \\
1.520 \\
6,930 \\
5,820 \\
4,520 \\
2,920 \\
1,510 \\
1,880\end{array}$ & $\begin{array}{l}0.449 \\
.578 \\
.443 \\
.404 \\
.356 \\
.401 \\
1.83 \\
1.54 \\
1.19 \\
.770 \\
.398 \\
.496\end{array}$ & $\begin{array}{r}0.52 \\
.64 \\
.51 \\
.47 \\
.37 \\
.46 \\
2.04 \\
1.78 \\
1.33 \\
.85 \\
.46 \\
.55\end{array}$ \\
\hline The year... & 18,700 & 938 & 2,800 & .739 & 10.02 \\
\hline
\end{tabular}


PINE RIVER IEAR FLORENCE, WIS.

Locatron.-In secs. 23 and 26, T. 39 N., R. 17 E., at highway bridge 8 miles southwest of Florence, Florence County, and 12 miles above mouth of river. Popple River enters from right 200 feet above station.

Drainage area.-488 square miles (measured on map issued by Wisconsin Geological and Natural History Survey, edition of 1911; scale, 1 inch $=6$ miles).

Records available.-January 22, 1914, to September 30, 1923.

GAGE.-Chain gage fastened to guardrail on upstream side of bridge; read by William Taft.

Discharge measurements.--Made from upstream side of bridge or by wading.

Channel and control.-Coarse gravel and stones; left bank high and not subject to overflow; extremely high water may overflow right bank around approach to bridge.

Extremes of discharge.--Maximum mean daily discharge recorded during year, 2,220 second-feet, April 23; minimum mean daily discharge, 112 second-feet, March 31 . Both maximum and minimum occurred during the period when discharge was based on power-house records and were partly the result of regulation.

1914-1923: Maximum stage recorded, 9.25 feet at noon April 23, 1916 (discharge, approximately 4,520 second-feet); minimum discharge, that of March 31, 1923.

IcE.-Stage-discharge relation seriously affected by ice.

Regulation.-None except during period December 1 to April 27, for which period discharge is based on records of power plant about 3 miles below station and is affected by loss and gain in storage in the service reservoir at that plant.

Accoracy. - Stage-discharge relation permanent. Rating curve well defined between 144 and 1,710 second-feet. Gage read to half-tenths once daily. Daily discharge ascertained by applying daily gage height to rating table except for period December 1 to April 27, when no gage heights were available and the greater part of the period the stage-discharge relation was affected by ice. For this period the records are based on power-house records and are fair. For the remainder of the year, the records are good.

The following discharge measurement was made by E. E. Foster:

July 28, 1923: Gage height, 1.96 feet; discharge, 298 second-feet. 
Daily discharge, in second-feet, of Pine River near Florence, Wis., for the year ending September 30, 1923

\begin{tabular}{|c|c|c|c|c|c|c|c|c|c|c|c|c|}
\hline Day & Oct. & Nov. & Dec. & Jan. & Feb. & Mar. & Apr. & May & June & July & Aug. & Sept. \\
\hline$\cdots$ & $\begin{array}{l}204 \\
204 \\
193 \\
182 \\
182\end{array}$ & $\begin{array}{l}193 \\
193 \\
182 \\
182 \\
193\end{array}$ & $\begin{array}{l}272 \\
272 \\
222 \\
220 \\
275\end{array}$ & $\begin{array}{l}211 \\
221 \\
218 \\
203 \\
218\end{array}$ & $\begin{array}{l}209 \\
209 \\
209 \\
206 \\
202\end{array}$ & $\begin{array}{l}173 \\
190 \\
199 \\
124 \\
197\end{array}$ & $\begin{array}{l}204 \\
198 \\
154 \\
174 \\
173\end{array}$ & $\begin{array}{l}1,410 \\
1,410 \\
1,360 \\
1,360 \\
1,360\end{array}$ & & $\begin{array}{l}610 \\
576 \\
508\end{array}$ & $\begin{array}{l}262 \\
262 \\
262 \\
250 \\
238\end{array}$ & $\begin{array}{l}226 \\
226 \\
215 \\
215 \\
215\end{array}$ \\
\hline 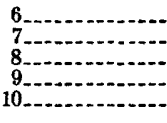 & $\begin{array}{l}172 \\
162 \\
162 \\
162 \\
162\end{array}$ & $\begin{array}{l}204 \\
204 \\
215 \\
226 \\
226\end{array}$ & $\begin{array}{l}255 \\
236 \\
233 \\
234 \\
168\end{array}$ & $\begin{array}{l}203 \\
170 \\
202 \\
202 \\
202\end{array}$ & $\begin{array}{l}193 \\
182 \\
182 \\
182 \\
182\end{array}$ & $\begin{array}{l}201 \\
142 \\
173 \\
221 \\
136\end{array}$ & $\begin{array}{l}244 \\
227 \\
173 \\
237 \\
216\end{array}$ & $\begin{array}{r}1,260 \\
1,220 \\
1,120 \\
990 \\
945\end{array}$ & $\begin{array}{r}576 \\
945 \\
1,760 \\
1,760 \\
1,660\end{array}$ & $\begin{array}{l}444 \\
382 \\
382 \\
382 \\
444\end{array}$ & $\begin{array}{l}238 \\
226 \\
215 \\
215 \\
215\end{array}$ & $\begin{array}{l}238 \\
311 \\
367 \\
367 \\
367\end{array}$ \\
\hline & $\begin{array}{l}172 \\
172 \\
182 \\
182 \\
182\end{array}$ & $\begin{array}{l}238 \\
250 \\
250\end{array}$ & $\begin{array}{l}238 \\
253 \\
220\end{array}$ & $\begin{array}{l}199 \\
204 \\
199\end{array}$ & $\begin{array}{l}166 \\
194 \\
187 \\
177 \\
160\end{array}$ & $\begin{array}{l}228 \\
201 \\
190\end{array}$ & $\begin{array}{l}155 \\
151 \\
234\end{array}$ & $\begin{array}{l}900 \\
815 \\
815 \\
780 \\
780\end{array}$ & & & $\begin{array}{l}204 \\
193 \\
193 \\
193 \\
193\end{array}$ & $\begin{array}{l}338 \\
311 \\
286 \\
262 \\
250\end{array}$ \\
\hline & $\begin{array}{l}182 \\
182 \\
193 \\
204 \\
204\end{array}$ & $\begin{array}{l}262 \\
274\end{array}$ & $\begin{array}{l}193 \\
185 \\
170\end{array}$ & $\begin{array}{l}199 \\
207 \\
201 \\
185 \\
198\end{array}$ & $\begin{array}{l}160 \\
139 \\
151 \\
186 \\
173\end{array}$ & $\begin{array}{l}209 \\
145 \\
148\end{array}$ & $\begin{array}{r}265 \\
270 \\
272 \\
462 \\
1,310\end{array}$ & $\begin{array}{l}746 \\
746 \\
746 \\
746 \\
815\end{array}$ & & & $\begin{array}{l}193 \\
215 \\
215 \\
215\end{array}$ & 250 \\
\hline & $\begin{array}{l}215 \\
215 \\
215 \\
204 \\
204\end{array}$ & $\begin{array}{l}298 \\
324 \\
338 \\
315 \\
274\end{array}$ & 194 & $\begin{array}{l}\mathbf{1 4 6} \\
211 \\
234 \\
191 \\
201\end{array}$ & $\begin{array}{l}140 \\
142 \\
136 \\
149 \\
175\end{array}$ & $\begin{array}{l}153 \\
122\end{array}$ & & $\begin{array}{l}815 \\
815 \\
746 \\
678 \\
610\end{array}$ & & & & 215 \\
\hline$\ldots$ & $\begin{array}{l}204 \\
204 \\
204 \\
204 \\
204\end{array}$ & $\begin{array}{l}238 \\
261 \\
272 \\
273\end{array}$ & $\begin{array}{l}196 \\
213 \\
221 \\
218 \\
221 \\
193\end{array}$ & $\begin{array}{l}191 \\
199 \\
166 \\
204 \\
225 \\
214\end{array}$ & $\begin{array}{l}13 \\
13 \\
21\end{array}$ & $\begin{array}{l}196 \\
194 \\
156\end{array}$ & $\begin{array}{l}1,8 \\
1,8 \\
1,5\end{array}$ & $\begin{array}{l}770 \\
176 \\
444\end{array}$ & $\stackrel{9}{9}$ & 262 & $\begin{array}{l}204 \\
204 \\
193 \\
193 \\
204\end{array}$ & $\begin{array}{l}93 \\
93 \\
93 \\
93\end{array}$ \\
\hline
\end{tabular}

Monthly discharge of Pine River near Florence, Wis., for the year ending September 30,1923

[Drainage area, 488 square miles]

\begin{tabular}{|c|c|c|c|c|c|}
\hline \multirow{2}{*}{ Month } & \multicolumn{4}{|c|}{ Discharge in second-feet } & \multirow{2}{*}{$\begin{array}{c}\text { Run-off in } \\
\text { inches }\end{array}$} \\
\hline & Maximum & Minimum & Mean & $\begin{array}{c}\text { Per square } \\
\text { mile }\end{array}$ & \\
\hline $\begin{array}{l}\text { October } \\
\text { November } \\
\text { December } \\
\text { January } \\
\text { February } \\
\text { March } \\
\text { April } \\
\text { May } \\
\text { June } \\
\text { July } \\
\text { August } \\
\text { September. }\end{array}$ & $\begin{array}{r}215 \\
338 \\
275 \\
234 \\
217 \\
228 \\
2.220 \\
1,410 \\
1,760 \\
678 \\
262 \\
367\end{array}$ & $\begin{array}{l}162 \\
182 \\
166 \\
146 \\
133 \\
112 \\
151 \\
444 \\
352 \\
262 \\
193 \\
182\end{array}$ & $\begin{array}{l}191 \\
251 \\
215 \\
200 \\
174 \\
171 \\
794 \\
871 \\
970 \\
435 \\
216 \\
253\end{array}$ & $\begin{array}{r}0.391 \\
.514 \\
.441 \\
.410 \\
.357 \\
.350 \\
1.63 \\
1.78 \\
1.99 \\
.891 \\
.443 \\
.518\end{array}$ & $\begin{array}{r}0.45 \\
.57 \\
.51 \\
.47 \\
.37 \\
.40 \\
1.82 \\
2.05 \\
2.22 \\
1.03 \\
.51 \\
.58\end{array}$ \\
\hline The year .... & 2,220 & 112 & 395 & .809 & 10.98 \\
\hline
\end{tabular}


PIKE RIVER AT AMBERG, WIS.

Location.-In sec. 15, T. 35 N., R. 21 E., at Chicago, Milwaukee \& St. Paul Railway bridge half a mile south of Amberg, Marinette County, 1 mile below junction of two branches of the Pike River, and 11 miles above mouth.

Drainage area.-240 square miles (measured on map issued by Wisconsin Geological and Natural History Survey, edition of 1911 ; scale, 1 inch $=6$ miles).

Records aVailable.-February 26, 1914, to September 30, 1923.

GAGE.-Chain gage fastened to guardrail on upstream side of bridge; read by Frank Bunce.

Discharge measurements.-Made from a highway bridge a quarter of a mile downstream from the bridge to which the gage is attached or by wading.

Channel and control.- Solid rock and some loose granite boulders; channel permanent but very rough at gage. Banks medium high; not subject to overflow.

Extremes of discharge.-Maximum stage recorded during year, 6.04 feet at 7.45 a. $\mathrm{m}$. April 21 (discharge, 1,870 second-feet); minimum mean daily discharge, 90 second-feet January 30 (stage-discharge relation affected by ice).

1914-1923: Maximum stage recorded, 7.68 feet at 5 p. m. April 10, 1922 (discharge, 2,730 second-feet); minimum mean daily discharge, 65 secondfeet January 27, 1922 (stage-discharge relation affected by ice).

Regulation.-None.

ACCURACY.-Stage-discharge relation permanent except when affected by ice. Rating curve well defined between 120 and 1,120 second-feet. Gage read to quarter-tenths once daily. Daily discharge ascertained by applying daily gage height to rating curve, except when stage-discharge relation was affected by ice, for which period it was ascertained by applying to the rating table daily gage heights corrected for ice effect by means of discharge measurements, observer's notes, and weather records. Open-water records good except during high stages a few days in April, for which they are fair; winter records fair.

Discharge measurements of Pike River at Amberg, Wis., during the year ending September 30, 192S

\begin{tabular}{|c|c|c|c|c|c|c|c|}
\hline Date & Made by- & $\begin{array}{c}\text { Gage } \\
\text { height }\end{array}$ & $\begin{array}{c}\text { Dis- } \\
\text { charge }\end{array}$ & Date & Made by- & $\begin{array}{c}\text { Gage } \\
\text { height }\end{array}$ & $\begin{array}{l}\text { Dis- } \\
\text { charge }\end{array}$ \\
\hline $\begin{array}{ll}\text { Oct. } & 19 \\
\text { Jan. } & 16\end{array}$ & $\begin{array}{l}\text { E. E. Foster } \\
\text { J. H. Olson }\end{array}$ & $\begin{array}{c}\text { Feet } \\
1.91 \\
a 2.05\end{array}$ & $\begin{array}{r}\text { Sec.-ft. } \\
170 \\
130\end{array}$ & $\begin{array}{l}\text { Feb. } 26 \\
\text { July } 24\end{array}$ & $\begin{array}{l}\text { J. H. Olson } \\
\text { E. E. Foster.... }\end{array}$ & $\begin{array}{c}\text { Feet } \\
a 2.40 \\
1.83\end{array}$ & $\begin{array}{r}\text { Sec.-ft. } \\
104 \\
160\end{array}$ \\
\hline
\end{tabular}

- Stage-discharge relation affected by ice. 
Daily discharge, in second-feet, of Pike River at Amberg, Wis., for the year ending September 30, 1923

\begin{tabular}{|c|c|c|c|c|c|c|c|c|c|c|c|c|}
\hline D & Oct. & Nov. & Dec. & Jan. & Feb. & Mar. & Apr. & May & June & July & Aug. & Sept. \\
\hline $\begin{array}{l}1 \ldots \ldots \\
2 \ldots \\
3 \ldots \ldots \\
4 \ldots \\
5 \ldots\end{array}$ & $\begin{array}{l}158 \\
154 \\
148 \\
148 \\
142\end{array}$ & $\begin{array}{l}169 \\
176 \\
169 \\
162 \\
169\end{array}$ & $\begin{array}{l}244 \\
148 \\
204 \\
303 \\
148\end{array}$ & $\begin{array}{l}120 \\
130 \\
125 \\
125 \\
120\end{array}$ & $\begin{array}{l}110 \\
110 \\
110 \\
110 \\
110\end{array}$ & $\begin{array}{l}110 \\
120 \\
130 \\
125 \\
120\end{array}$ & $\begin{array}{l}150 \\
160 \\
170 \\
180 \\
205\end{array}$ & $\begin{array}{l}493 \\
444 \\
412 \\
380 \\
364\end{array}$ & $\begin{array}{l}217 \\
217 \\
192 \\
192 \\
258\end{array}$ & $\begin{array}{l}202 \\
204 \\
187 \\
204 \\
273\end{array}$ & $\begin{array}{l}138 \\
134 \\
131 \\
128 \\
122\end{array}$ & $\begin{array}{l}118 \\
122 \\
142 \\
150 \\
158\end{array}$ \\
\hline $\begin{array}{l}6 \\
7 \\
9\end{array}$ & $\begin{array}{l}142 \\
142 \\
169 \\
176 \\
169\end{array}$ & $\begin{array}{l}192 \\
199 \\
185 \\
199 \\
204\end{array}$ & $\begin{array}{l}142 \\
215 \\
160 \\
120 \\
150\end{array}$ & $\begin{array}{l}110 \\
115 \\
120 \\
125 \\
130\end{array}$ & $\begin{array}{l}110 \\
110 \\
115 \\
120 \\
125\end{array}$ & $\begin{array}{l}120 \\
120 \\
120 \\
120 \\
125\end{array}$ & $\begin{array}{l}230 \\
260 \\
290 \\
320 \\
260\end{array}$ & $\begin{array}{l}333 \\
333 \\
333 \\
348 \\
380\end{array}$ & $\begin{array}{l}288 \\
444\end{array}$ & $\begin{array}{l}303 \\
288 \\
258 \\
230 \\
230\end{array}$ & $\begin{array}{l}118 \\
118 \\
122 \\
118 \\
118\end{array}$ & $\begin{array}{l}162 \\
192 \\
204 \\
192 \\
169\end{array}$ \\
\hline $\begin{array}{l}11 \ldots \\
12 \\
14 \\
15\end{array}$ & $\begin{array}{l}162 \\
162 \\
158 \\
154 \\
158\end{array}$ & $\begin{array}{l}204 \\
204 \\
204 \\
217 \\
210\end{array}$ & $\begin{array}{l}170 \\
160 \\
170 \\
180 \\
160\end{array}$ & $\begin{array}{l}125 \\
120 \\
120 \\
120 \\
125\end{array}$ & $\begin{array}{l}130 \\
130 \\
130 \\
120 \\
110\end{array}$ & $\begin{array}{l}140 \\
135 \\
130\end{array}$ & $\begin{array}{l}200 \\
150 \\
180 \\
205 \\
260\end{array}$ & $\begin{array}{l}412 \\
364 \\
341 \\
318 \\
318\end{array}$ & $\begin{array}{l}582 \\
476 \\
444 \\
348 \\
318\end{array}$ & $\begin{array}{l}217 \\
202 \\
187 \\
182 \\
194\end{array}$ & $\begin{array}{l}118 \\
118 \\
118 \\
118 \\
118\end{array}$ & $\begin{array}{l}148 \\
142 \\
134 \\
122 \\
122\end{array}$ \\
\hline - & $\begin{array}{l}169 \\
180 \\
182 \\
185 \\
185\end{array}$ & $\begin{array}{l}204 \\
217 \\
230 \\
244 \\
244\end{array}$ & $\begin{array}{l}120 \\
110 \\
120 \\
110 \\
120\end{array}$ & $\begin{array}{l}130 \\
120 \\
110 \\
105 \\
100\end{array}$ & $\begin{array}{r}110 \\
.115 \\
120 \\
120 \\
120\end{array}$ & $\begin{array}{l}125 \\
120 \\
120 \\
120 \\
130\end{array}$ & $\begin{array}{r}320 \\
395 \\
546 \\
990 \\
1,400\end{array}$ & $\begin{array}{l}348 \\
380 \\
364 \\
348 \\
460\end{array}$ & $\begin{array}{l}288 \\
258 \\
244 \\
217 \\
217\end{array}$ & $\begin{array}{l}217 \\
200 \\
182 \\
167 \\
165\end{array}$ & $\begin{array}{l}114 \\
109 \\
104 \\
109 \\
109\end{array}$ & $\begin{array}{l}118 \\
118 \\
138 \\
142 \\
148\end{array}$ \\
\hline 25 & $\begin{array}{l}192 \\
185 \\
185 \\
180 \\
180\end{array}$ & $\begin{array}{l}258 \\
273 \\
273 \\
204 \\
169\end{array}$ & $\begin{array}{l}110 \\
120 \\
120 \\
120 \\
120\end{array}$ & $\begin{array}{l}110 \\
120 \\
120 \\
120 \\
120\end{array}$ & $\begin{array}{l}120 \\
130 \\
130 \\
120 \\
110\end{array}$ & $\begin{array}{l}140 \\
160 \\
180 \\
150 \\
120\end{array}$ & $\begin{array}{r}1,800 \\
1,460 \\
1,120 \\
862\end{array}$ & $\begin{array}{l}582 \\
546 \\
476 \\
396 \\
364\end{array}$ & $\begin{array}{l}305 \\
396 \\
348 \\
303 \\
288\end{array}$ & $\begin{array}{l}160 \\
150 \\
140 \\
160 \\
158\end{array}$ & $\begin{array}{l}128 \\
138 \\
133 \\
128 \\
122\end{array}$ & $\begin{array}{l}158 \\
148 \\
142 \\
138 \\
134\end{array}$ \\
\hline $\begin{array}{l}26 \\
27 \\
28 \\
29 \\
30 \\
31\end{array}$ & $\begin{array}{l}176 \\
169 \\
162 \\
162 \\
162 \\
169\end{array}$ & $\begin{array}{l}193 \\
217 \\
273 \\
185 \\
192\end{array}$ & $\begin{array}{l}135 \\
150 \\
130 \\
110 \\
120 \\
120\end{array}$ & $\begin{array}{r}120 \\
120 \\
120 \\
105 \\
90 \\
100\end{array}$ & $\begin{array}{l}100 \\
100 \\
105 \\
\end{array}$ & $\begin{array}{l}115 \\
110 \\
105 \\
100 \\
120 \\
140\end{array}$ & $\begin{array}{l}739 \\
698 \\
620 \\
582 \\
546\end{array}$ & $\begin{array}{l}333 \\
303 \\
273 \\
244 \\
217 \\
217\end{array}$ & $\begin{array}{l}318 \\
217 \\
204 \\
197 \\
190\end{array}$ & $\begin{array}{l}148 \\
148 \\
148 \\
158 \\
158 \\
154\end{array}$ & $\begin{array}{l}118 \\
122 \\
134 \\
128 \\
122 \\
122\end{array}$ & $\begin{array}{l}128 \\
122 \\
122 \\
122 \\
118\end{array}$ \\
\hline
\end{tabular}

Note-Stage-discharge relation affected by ice Dec. 7 to Apr. 17. Gage not read and discharged interpolated Oct. 6, 18, Nov. 15, 26, Apr. 23, May 13, 18, July 17, 27, Aug. 3, 10, 23, and Sept. 4.

\section{$M$ onthly discharge of Pike River at Amberg, Wis., for the year ending September 30,1923}

[Drainage area, 240 square miles ]

\begin{tabular}{|c|c|c|c|c|c|}
\hline \multirow{2}{*}{ Month } & \multicolumn{4}{|c|}{ Discharge in second-feet } & \multirow{2}{*}{$\begin{array}{c}\text { Run-off in } \\
\text { inches }\end{array}$} \\
\hline & Maximum & Minimum & Mean & $\underset{\text { mile }}{\text { Per square }}$ & \\
\hline $\begin{array}{l}\text { October } \\
\text { November } \\
\text { December } \\
\text { January } \\
\text { February } \\
\text { March } \\
\text { April } \\
\text { May } \\
\text { June } \\
\text { July } \\
\text { August } \\
\text { September }\end{array}$ & $\begin{array}{r}192 \\
273 \\
303 \\
130 \\
130 \\
180 \\
1,850 \\
582 \\
904 \\
303 \\
138 \\
204\end{array}$ & $\begin{array}{r}142 \\
162 \\
110 \\
90 \\
100 \\
100 \\
150 \\
217 \\
190 \\
140 \\
104 \\
118\end{array}$ & $\begin{array}{l}167 \\
208 \\
149 \\
117 \\
116 \\
127 \\
572 \\
369 \\
337 \\
193 \\
122 \\
142\end{array}$ & $\begin{array}{l}0.696 \\
.867 \\
.621 \\
.488 \\
.483 \\
.529 \\
2.38 \\
1.54 \\
1.40 \\
.804 \\
.508 \\
.592\end{array}$ & $\begin{array}{r}0.80 \\
.97 \\
.72 \\
.56 \\
.50 \\
.61 \\
2.66 \\
1.78 \\
1.56 \\
.93 \\
.59 \\
.66\end{array}$ \\
\hline The year.. & 1.850 & 90 & 218 & .908 & 12.34 \\
\hline
\end{tabular}




\section{PESHTIGO RIVER AT HIGH FALIS, NEAR CRIVITZ, WIS.}

Location.-In sec. 1, T. 32 N., R. 18 E., at High Falls, near Crivitz, Marinette County, at power house of Wisconsin Public Service Corporation, 1 mile upstream from Thunder River (coming in from right), and 15 miles by road northwest of Crivitz.

Drainage area.-520 square miles (measured on Wisconsin Geological and Natural History Survey map, edition of 1911 ; scale, 1 inch $=6$ miles).

Reconds available.-August 3, 1912, to September 30, 1923.

Discharge. - Owing to backwater caused by construction of the power plant at Johnson Falls, 3 miles downstream from High Falls, the gage-height record from the water-stage recorder formerly used at a site one-fourth mile downstream from the power house at High Falls could not be used for determination of discharge. The daily discharge after September 30, 1922, was, · therefore, computed from hourly determinations of flow through the turbines (from kilowatt output and effective head) plus discharge through the gates and over the spillway.

Extrepies of DISCHARGE.-Maximum mean daily dicharge recorded during year, 2,330 second-feet April 26; minimum mean daily discharge, 51 second-feet October 29, December 17, and January 1.

1912-1923: Maximum stage from water-stage recorder, 7.80 feet at 4.30 p. m. April 11, 1922 (discharge, 3,860 second-feet); minimum stage, 0.97 foot from midnight to $7.20 \mathrm{a}$. $\mathrm{m}$. October 27,1919 (discharge, 43 secondfeet). Owing to artificial regulation, extremes given do not represent the natural flow.

Regulation.-Considerable diurnal fluctuation caused by operation of powe plant and during log-driving season by manipulation of gates. Mean monthly flow does not represent the natural flow because of storage in the service reservoir.

Accuracy.-Discharge is computed from hourly readings at the power plant and records are fair.

Cooperation.-Records of daily discharge furnished by the Wisconsin Public Service Corporation.

The following discharge measurement was made by S. B. Soulé:

October 19, 1922: Gage height, 2.51 feet; discharge, 488 second-feet. 
laily discharge, in second-feet, of Peshtigo River at High Falls, near Crivitz, Wis., for the year ending September 30,1928

\begin{tabular}{|c|c|c|c|c|c|c|c|c|c|c|c|c|}
\hline Da & Oct. & Nov. & Dec. & Jan. & Feb. & Mar. & Apr. & May & June & July & Aug. & Sept. \\
\hline $\begin{array}{l}1- \\
2- \\
3 . \\
4- \\
5\end{array}$ & $\begin{array}{l}120 \\
224 \\
233 \\
316 \\
309\end{array}$ & $\begin{array}{r}588 \\
406 \\
260 \\
185 \\
52\end{array}$ & $\begin{array}{r}344 \\
293 \\
71 \\
480 \\
761\end{array}$ & $\begin{array}{r}51 \\
141 \\
167\end{array}$ & $\begin{array}{r}286 \\
289 \\
221 \\
84 \\
259\end{array}$ & $\begin{array}{l}365 \\
384 \\
651 \\
143\end{array}$ & $\begin{array}{r}52 \\
577 \\
542 \\
287 \\
277\end{array}$ & $\begin{array}{r}1,380 \\
1,490 \\
1,180 \\
977 \\
1,080\end{array}$ & $\begin{array}{l}748 \\
482 \\
285 \\
468 \\
680\end{array}$ & $\begin{array}{r}52 \\
273 \\
493 \\
244\end{array}$ & $\begin{array}{l}268 \\
255 \\
274 \\
269 \\
153\end{array}$ & $\begin{array}{l}164 \\
209 \\
190 \\
259 \\
385\end{array}$ \\
\hline $\begin{array}{r}6 \\
7 \ldots \\
8 \ldots \\
9 \\
10 \ldots\end{array}$ & $\begin{array}{l}270 \\
299 \\
257 \\
269 \\
289\end{array}$ & $\begin{array}{l}408 \\
438 \\
430 \\
393 \\
412\end{array}$ & $\begin{array}{l}327 \\
294 \\
465 \\
553 \\
247\end{array}$ & $\begin{array}{r}182 \\
78 \\
343 \\
216 \\
234\end{array}$ & $\begin{array}{l}292 \\
269 \\
290 \\
221 \\
174\end{array}$ & $\begin{array}{l}501 \\
536 \\
620 \\
520 \\
492\end{array}$ & $\begin{array}{l}611 \\
500 \\
281 \\
229 \\
230\end{array}$ & $\begin{array}{r}1,070 \\
937 \\
885 \\
891 \\
. \quad 916\end{array}$ & $\begin{array}{r}473 \\
607 \\
772 \\
1,410 \\
1,690\end{array}$ & & $\begin{array}{l}312 \\
284 \\
240 \\
243 \\
187\end{array}$ & $\begin{array}{l}348 \\
562 \\
659 \\
222 \\
318\end{array}$ \\
\hline & $\begin{array}{r}281 \\
272 \\
251 \\
439 \\
86\end{array}$ & $\begin{array}{l}201 \\
231 \\
299 \\
291 \\
250\end{array}$ & $\begin{array}{l}240 \\
295 \\
217 \\
275 \\
216\end{array}$ & $\begin{array}{l}228 \\
186 \\
170 \\
302\end{array}$ & $\begin{array}{r}80 \\
262 \\
283 \\
156 \\
139\end{array}$ & $\begin{array}{l}112 \\
538 \\
341 \\
328 \\
316\end{array}$ & $\begin{array}{l}219 \\
324 \\
483 \\
472 \\
375\end{array}$ & $\begin{array}{l}911 \\
916 \\
511 \\
852 \\
951\end{array}$ & & & $\begin{array}{r}119 \\
52 \\
266 \\
335 \\
290\end{array}$ & $\begin{array}{l}428 \\
431 \\
373 \\
248 \\
194\end{array}$ \\
\hline S... & $\begin{array}{l}291 \\
291 \\
281 \\
261 \\
267\end{array}$ & $\begin{array}{r}131 \\
236 \\
250 \\
52 \\
284\end{array}$ & $\begin{array}{r}206 \\
51 \\
231 \\
234 \\
223\end{array}$ & & $\begin{array}{r}155 \\
142 \\
64 \\
193 \\
208\end{array}$ & $\begin{array}{l}361 \\
557 \\
314 \\
421 \\
316\end{array}$ & $\begin{array}{l}613 \\
668 \\
719 \\
871 \\
964\end{array}$ & $\begin{array}{l}943 \\
941 \\
816 \\
765 \\
\mathbf{4 3 6}\end{array}$ & $\begin{array}{r}1,130 \\
980 \\
888 \\
825 \\
850\end{array}$ & 617 & $\begin{array}{r}288 \\
267 \\
247 \\
52 \\
276\end{array}$ & $\begin{array}{l}190 \\
297 \\
301 \\
266 \\
469\end{array}$ \\
\hline . & $\begin{array}{l}217 \\
188 \\
492 \\
454 \\
440\end{array}$ & $\begin{array}{l}504 \\
427 \\
300 \\
267 \\
290\end{array}$ & $\begin{array}{r}168 \\
208 \\
124 \\
52 \\
52\end{array}$ & $\begin{array}{r}52 \\
285 \\
316 \\
287 \\
287\end{array}$ & $\begin{array}{r}202 \\
239 \\
388 \\
122 \\
79\end{array}$ & $\begin{array}{l}499 \\
504 \\
571 \\
270 \\
228\end{array}$ & $\begin{array}{r}1,020 \\
684 \\
1,090 \\
1,670 \\
2,270\end{array}$ & $\begin{array}{l}568 \\
747 \\
781 \\
833 \\
784\end{array}$ & $\begin{array}{l}351 \\
778\end{array}$ & $\begin{array}{l}431 \\
261 \\
410 \\
462 \\
357\end{array}$ & $\begin{array}{l}319 \\
223 \\
225 \\
338 \\
199\end{array}$ & $\begin{array}{r}465 \\
161 \\
99 \\
316 \\
357\end{array}$ \\
\hline $\begin{array}{l}26 \ldots \\
27 \\
28 \\
29 \\
30\end{array}$ & $\begin{array}{r}445 \\
433 \\
282 \\
51 \\
356 \\
472\end{array}$ & $\begin{array}{l}418 \\
340 \\
280 \\
314 \\
338\end{array}$ & $\begin{array}{l}317 \\
417 \\
348 \\
314 \\
219 \\
120\end{array}$ & $\begin{array}{l}277 \\
261 \\
185 \\
290 \\
317 \\
311\end{array}$ & $\begin{array}{r}316 \\
397 \\
371 \\
-.\end{array}$ & $\begin{array}{l}291 \\
308 \\
291 \\
292 \\
291 \\
205\end{array}$ & $\begin{array}{l}2,330 \\
2,180 \\
2,120 \\
1,840 \\
1,670\end{array}$ & $\begin{array}{l}823 \\
569 \\
739 \\
899 \\
345 \\
692\end{array}$ & $\begin{array}{l}886 \\
905 \\
867 \\
742 \\
356\end{array}$ & $\begin{array}{r}317 \\
263 \\
\cdot 188 \\
122 \\
254 \\
294\end{array}$ & $\begin{array}{r}52 \\
235 \\
341 \\
\mathbf{2 3 1} \\
214 \\
244\end{array}$ & $\begin{array}{r}400 \\
266 \\
268 \\
162 \\
72\end{array}$ \\
\hline
\end{tabular}

Monthly discharge of Peshtigo River at High Falls, near Crivitz, Wis., for the year ending September 30, 1923

[Drainage area, 520 square miles]

\begin{tabular}{|c|c|c|c|c|c|}
\hline \multirow{2}{*}{ Month } & \multicolumn{4}{|c|}{ Discharge in second-feet } & \multirow{2}{*}{$\begin{array}{l}\text { Run-off } \\
\text { in inches }\end{array}$} \\
\hline & Maximum & Minimum & Mean & $\underset{\text { mile }}{\text { Per square }}$ & \\
\hline $\begin{array}{l}\text { October. } \\
\text { November } \\
\text { December. } \\
\text { January } \\
\text { February } \\
\text { March } \\
\text { April } \\
\text { May } \\
\text { June } \\
\text { July } \\
\text { August } \\
\text { September }\end{array}$ & $\begin{array}{r}492 \\
588 \\
761 \\
343 \\
397 \\
651 \\
2,330 \\
1,490 \\
1,920 \\
871 \\
341 \\
659\end{array}$ & $\begin{array}{r}51 \\
52 \\
51 \\
51 \\
64 \\
112 \\
52 \\
345 \\
285 \\
52 \\
52 \\
72\end{array}$ & $\begin{array}{l}295 \\
309 \\
270 \\
227 \\
221 \\
387 \\
872 \\
859 \\
899 \\
458 \\
235 \\
303\end{array}$ & $\begin{array}{c}0.567 \\
.594 \\
.519 \\
.437 \\
.425 \\
.744 \\
1.68 \\
1.65 \\
1.73 \\
.881 \\
.452 \\
.583\end{array}$ & $\begin{array}{r}0.65 \\
.66 \\
.60 \\
.50 \\
.44 \\
.86 \\
1.87 \\
1.90 \\
1.93 \\
1.02 \\
.52 \\
.65\end{array}$ \\
\hline The year.... & 2,330 & 51 & 445 & .856 & 11.60 \\
\hline
\end{tabular}


OCONTO RIVER NEAR GILLETT, WIS.

Location.-In sec. 34, T. 28 N., R. 18 E., at highway bridge $2 \frac{1}{2}$ miles southeast of Gillett, Oconto County.

Drainage area.-678 square miles (measured on Wisconsin Geological and Natural History Survey map, edition of 1911; scale, 1 inch $=6$ miles).

Records available.-June 7, 1906, to March 30, 1909; January 6, 1914, to September 30, 1923.

GAGE.- Chain gage attached to iron railing on upstream side of bridge; read by Harvey Gilbertson. Zero of gage was raised 4.0 feet January 6, 1914.

Discharge meastrements.-Made from upstream side of bridge.

ChanNel and control.-Gravel; permanent. Left bank of medium height and not subject to overflow. During extremely high stages water may overflow around right end of bridge.

Extremes of discharge.-Maximum discharge recorded during year, 3,930 second-feet April 20-22; minimum discharge, about 195 second-feet Janu ary 10.

1906-1923: Maximum stage recorded, 9.1 feet at 3 p. m. April 11, 1922, caused by failure of a dam at Pulcifer, 4 miles upstream (discharge, 6,470 second-feet). Minimum open-water discharge, 95 second-feet June 3 and $6,1907$.

ICE.-Stage-discharge relation seriously affected by ice.

Regulation.- None.

ACCURACY.- Stage-discharge relation practically permanent, except as affected by ice. Rating curve well defined between 300 and 1,850 second-feet and fairly well defined between 1,850 and 6,500 second-feet. Gage read to quarter-tenths once daily. Daily discharge obtained by applying daily gage height to rating table, except for period when stage-discharge relation was affected by ice, for which it was obtained by applying to rating table daily gage height corrected for ice effect by means of two discharge measurements observer's notes, and weather records. Open-water records excellent, except. for extremely high stages, for which they are good; records for winter period are fair.

Discharge measurements of Oconto River near Gillett, Wis., during the year ending September 30, 1923

\begin{tabular}{|c|c|c|c|}
\hline Date & Made by- & $\begin{array}{c}\text { Gage } \\
\text { height }\end{array}$ & $\begin{array}{c}\text { Dis- } \\
\text { charge }\end{array}$ \\
\hline $\begin{array}{ll}\text { Jan. } & 17 \\
\text { Feb. } & 27 \\
\text { July } & 29\end{array}$ & $\begin{array}{l}\text { J. H. Olson } \\
\text { E. E. Foster }\end{array}$ & $\begin{array}{l}\text { Feet } \\
2.52 \\
2.78 \\
1.22\end{array}$ & $\begin{array}{r}\text { Sec. }-f t . \\
338 \\
\mathbf{3 4 5} \\
\mathbf{3 8 2}\end{array}$ \\
\hline
\end{tabular}

- Stage-discharge relation affected by ice. 
Daily discharge, in second-feet, of Oconto River near Gillett, Wis., for the year ending September 30, 1923

\begin{tabular}{|c|c|c|c|c|c|c|c|c|c|c|c|c|}
\hline Day & Oct. & Nov. & Dec. & Jan. & Feb. & Mar. & Apr. & May & June & July & Aug. & Sept. \\
\hline & $\begin{array}{l}415 \\
438 \\
438 \\
438 \\
438\end{array}$ & $\begin{array}{l}510 \\
510 \\
510 \\
560 \\
585\end{array}$ & $\begin{array}{l}560 \\
610 \\
560 \\
585 \\
590\end{array}$ & $\begin{array}{l}305 \\
305 \\
300 \\
290 \\
300\end{array}$ & $\begin{array}{l}325 \\
325 \\
325 \\
325 \\
325\end{array}$ & $\begin{array}{l}380 \\
380 \\
380 \\
380 \\
380\end{array}$ & $\begin{array}{l}340 \\
340 \\
320 \\
405 \\
450\end{array}$ & $\begin{array}{l}1,770 \\
1,530 \\
1,450 \\
1,150 \\
1,290\end{array}$ & 485 & 350 & $\begin{array}{l}370 \\
350 \\
330 \\
330 \\
312\end{array}$ & $\begin{array}{l}350 \\
350 \\
370 \\
392 \\
392\end{array}$ \\
\hline $\begin{array}{c}6 \\
7 \\
8 \\
8 \\
9 \\
10 \\
10 \\
-. .\end{array}$ & $\begin{array}{l}370 \\
370 \\
415 \\
415 \\
438\end{array}$ & $\begin{array}{l}585 \\
510 \\
510 \\
510 \\
510\end{array}$ & $\begin{array}{l}470 \\
445 \\
425 \\
565 \\
540\end{array}$ & $\begin{array}{l}305 \\
290 \\
270 \\
230 \\
195\end{array}$ & $\begin{array}{l}315 \\
305 \\
305 \\
305 \\
305\end{array}$ & $\begin{array}{l}380 \\
380 \\
380 \\
380 \\
380\end{array}$ & $\begin{array}{l}490 \\
540 \\
590 \\
660 \\
725\end{array}$ & $\begin{array}{r}1,150 \\
792 \\
975 \\
1,040 \\
1,040\end{array}$ & $\begin{array}{r}635 \\
738 \\
1,150 \\
792 \\
635\end{array}$ & 510 & $\begin{array}{l}295 \\
312 \\
312 \\
312 \\
312\end{array}$ & $\begin{array}{l}370 \\
392 \\
460 \\
460 \\
438\end{array}$ \\
\hline & $\begin{array}{l}460 \\
510 \\
370 \\
350 \\
330\end{array}$ & $\begin{array}{l}560 \\
415 \\
460 \\
560 \\
560\end{array}$ & $\begin{array}{l}515 \\
515\end{array}$ & $\begin{array}{l}240 \\
270 \\
290 \\
305 \\
330\end{array}$ & $\begin{array}{l}305 \\
305 \\
305 \\
305 \\
305\end{array}$ & $\begin{array}{l}380 \\
400 \\
425 \\
400 \\
380\end{array}$ & $\begin{array}{r}940 \\
1,160 \\
1,300\end{array}$ & $\begin{array}{r}1,010 \\
940 \\
910 \\
880 \\
792\end{array}$ & $\begin{array}{r}1,610 \\
685 \\
660\end{array}$ & 050 & $\begin{array}{l}312 \\
312 \\
295 \\
263 \\
312\end{array}$ & 350 \\
\hline & $\begin{array}{l}460 \\
660 \\
560 \\
510 \\
510\end{array}$ & $\begin{array}{l}510 \\
560 \\
560 \\
535 \\
850\end{array}$ & & 360 & 305 & $\begin{array}{l}380 \\
380 \\
380 \\
380 \\
390\end{array}$ & & $\begin{array}{r}1,290 \\
370 \\
370 \\
975 \\
975\end{array}$ & 2,410 & 400 & & 415 \\
\hline (a) & $\begin{array}{l}510 \\
312 \\
370 \\
685 \\
610\end{array}$ & $\begin{array}{l}820 \\
820 \\
792 \\
710 \\
685\end{array}$ & $\begin{array}{l}\mathbf{4 2 5} \\
\mathbf{4 1 5} \\
405 \\
370 \\
340\end{array}$ & $\begin{array}{l}320 \\
305 \\
320 \\
340 \\
330\end{array}$ & $\begin{array}{l}290 \\
300 \\
325 \\
325 \\
325\end{array}$ & $\begin{array}{l}405 \\
425 \\
445 \\
425 \\
405\end{array}$ & & $\begin{array}{l}710 \\
910 \\
940 \\
880 \\
792\end{array}$ & $\begin{array}{l}1,9 \\
1,2\end{array}$ & & $\begin{array}{l}370 \\
392 \\
350 \\
350\end{array}$ & 460 \\
\hline 0. & $\begin{array}{l}510 \\
460 \\
485 \\
370 \\
585 \\
510\end{array}$ & $\begin{array}{l}510 \\
485 \\
535 \\
535 \\
535\end{array}$ & $\begin{array}{l}340 \\
340 \\
330 \\
325 \\
315 \\
305\end{array}$ & $\begin{array}{l}325 \\
325 \\
325 \\
330 \\
340 \\
330\end{array}$ & $\begin{array}{l}340 \\
360 \\
370\end{array}$ & $\begin{array}{l}390 \\
380 \\
380 \\
380 \\
360 \\
340\end{array}$ & $\begin{array}{l}3,130 \\
2,410 \\
2,410 \\
2,170 \\
1,930\end{array}$ & $\begin{array}{l}765 \\
710 \\
685 \\
585\end{array}$ & 392 & 370 & $\begin{array}{l}370 \\
370\end{array}$ & $\begin{array}{l}438 \\
415 \\
415 \\
392 \\
370\end{array}$ \\
\hline
\end{tabular}

Noтe.-Stage-discharge relation affected by ice Dec. 5 to Apr. 21.

Monthly discharge of Oconto River near Gillett, Wis., for the year ending September 30,1923

[Drainage area, 678 square miles]

\begin{tabular}{|c|c|c|c|c|c|}
\hline \multirow{2}{*}{ Month } & \multicolumn{4}{|c|}{ Discharge in second-feet } & \multirow{2}{*}{$\underset{\text { Run-off in }}{\text { inches }}$} \\
\hline & Maximum & Minimum & Mean & $\underset{\text { mile }}{\text { Per square }}$ & \\
\hline $\begin{array}{l}\text { October } \\
\text { November } \\
\text { December } \\
\text { January } \\
\text { February } \\
\text { March } \\
\text { April } \\
\text { Magy } \\
\text { June } \\
\text { July } \\
\text { August } \\
\text { Suptember }\end{array}$ & $\begin{array}{r}685 \\
850 \\
610 \\
360 \\
370 \\
445 \\
3,930 \\
1,770 \\
2,410 \\
585 \\
392 \\
460\end{array}$ & $\begin{array}{l}312 \\
415 \\
305 \\
195 \\
290 \\
340 \\
320 \\
370 \\
312 \\
350 \\
263 \\
330\end{array}$ & $\begin{array}{r}461 \\
577 \\
460 \\
308 \\
318 \\
388 \\
1,860 \\
990 \\
952 \\
475 \\
333 \\
393\end{array}$ & $\begin{array}{l}0.680 \\
.851 \\
.678 \\
.454 \\
.469 \\
.572 \\
.74 \\
1.39 \\
1.40 \\
.701 \\
.491 \\
.580\end{array}$ & $\begin{array}{r}0.78 \\
.95 \\
.78 \\
.52 \\
.49 \\
.66 \\
3.06 \\
1.60 \\
1.56 \\
.81 \\
.57 \\
.65\end{array}$ \\
\hline The year....................... & 3,030 & 195 & 621 & .916 & 12.43 \\
\hline
\end{tabular}

FOX RIVER AT BERLIN, WIS.

Location.-In sec. 16, T. 17 N., R. 13 E., at Government lock and dam, 21/3 miles upstream from Berlin, Green Lake County.

Drainage AREA:-1,430 square miles (measured on map issued by Wisconsin Geological and Natural History Survey, edition of 1911 ; scale, 1 inch $=6$ miles).

Records available.--January 1, 1898, to September 30, 1923. 
GAGE.-Staff gage located in pool immediately below dam; read by lock tender for United States Engineer Corps.

Channel and control.- Sand and gravel, one channel at all stages; banks low and subject to overflow.

Discharge measurements.-Made from downstream side of Huron Street highway bridge in city of Berlin, $2 \frac{1}{3}$ miles downstream from gage. Rating curves for gage corrected for any small inflow between the gage and measuring section.

ExtREMES OF DISCHARGE.-Maximum mean daily discharge recorded during year, 6,050 second-feet, April 12; minimum mean daily discharge, 465 second-feet, September 20 and 21.

1898-1923: Maximum mean daily discharge, 6,400 second-feet, March 28, 30, 1916; minimum mean daily discharge, 250 second-feet, February 1-4, 1900.

ICE.-Stage-discharge relation affected by ice.

ACCURACY. - Stage-discharge relation practically permanent except for effect of ice. Rating curve well defined between 800 and 6,000 second-feet. Gage read three times daily; in general, however, noon reading alone is used in determination of daily discharge. Daily discharge ascertained by applying mean daily gage height to rating table, corrected for period of ice effect by means of curves hased on discharge measurements and observer's notes. Open-water records good; winter records roughly approximate.

Cooperation.--Records have been collected and computations of daily discharge made by United States Engineer Corps. Open-water records obtained from rating curves based on discharge measurements made by United States Geological Survey.

Daily discharge, in second-feet, of Fox River at Berlin, Wis., for the year ending September 30, 1923

\begin{tabular}{r|r|r|r|r|r|r|r|r|r|r|r|r}
\hline Day & Oct. & Nov. & Dec. & Jan. & Feb. & Mar. & Apr. & May & June & July & Aug. & Sept. \\
\hline & & & & & & & & \\
\hline & & & & & & & & \\
\hline
\end{tabular}


Monthly discharge of Fox River at Berlin, Wis., for the year ending September 30. 1923

[Drainage area, 1,430 square miles]

\begin{tabular}{|c|c|c|c|c|c|}
\hline \multirow{2}{*}{ Month } & \multicolumn{4}{|c|}{ Discharge in second-feet } & \multirow{2}{*}{$\begin{array}{c}\text { Run-off in } \\
\text { inches }\end{array}$} \\
\hline & Maximum & Minimum & Mean & $\underset{\text { mile }}{\text { Per square }}$ & \\
\hline $\begin{array}{l}\text { October } \\
\text { November } \\
\text { December } \\
\text { January } \\
\text { February } \\
\text { March } \\
\text { April } \\
\text { May } \\
\text { June } \\
\text { July } \\
\text { August } \\
\text { September }\end{array}$ & $\begin{array}{r}865 \\
1,270 \\
1,060 \\
735 \\
765 \\
1,680 \\
6,050 \\
2,750 \\
905 \\
705 \\
560 \\
675\end{array}$ & $\begin{array}{r}705 \\
735 \\
615 \\
590 \\
560 \\
830 \\
1,520 \\
940 \\
615 \\
535 \\
490 \\
465\end{array}$ & $\begin{array}{r}777 \\
1,010 \\
757 \\
673 \\
628 \\
1,490 \\
3,950 \\
1,530 \\
722 \\
599 \\
517 \\
583\end{array}$ & $\begin{array}{c}0.543 \\
.706 \\
.529 \\
.471 \\
.439 \\
1.04 \\
2.76 \\
1.07 \\
.505 \\
.419 \\
.362 \\
.408\end{array}$ & $\begin{array}{r}0.63 \\
.78 \\
.61 \\
.54 \\
.46 \\
1.20 \\
3.08 \\
1.23 \\
.56 \\
.48 \\
.42 \\
.46\end{array}$ \\
\hline The year. & 6,050 & 465 & 1,100 & .769 & 10.46 \\
\hline
\end{tabular}

FOX RIVER AT RAPIDE CROCHE DAM, NEAR WRIGHTSTOWN, WIS.

Location.- At Rapide Croche dam, in sec. 4, T. 21 N., R. 19 E., 2 miles from Wrightstown, Brown County, and 19 miles downstream from Lake Winnebago.

Records avallable.-March 3, 1896, to September 30, 1923.

DraINAGE AREA.-6,150 square miles (measured on map issued by Wisconsin Geological and Natural History Survey, edition of 1911 ; scale, 1 inch $=6$ miles).

Determination of pischarge.-The dam owned by the United States Government and operated by the United States Engineer Corps to aid navigation is made of timber and is equipped with four needle sluice gates which are used only in times of high water. A vertical staff gage at the lower end of the canal leading to the lock and about a quarter of a mile below the dam is read five times daily at 7 a. m., 9 a. m., noon, 3 p. m., and 6 p. m. The mean flow for the day is computed from a formula, using the five gage heights for the day, assuming gradual changes in gage height between the readings and weighting the different gage heights by elapsed time.

Extremes of pischarge.-Maximum mean daily discharge during year, 13,700 second-feet May 1-3; minimum mean daily discharge, 1,180 second-feet September 3.

1918-1923: Maximum mean daily discharge, 20,100 second-feet April 23, 1922; minimum mean daily discharge, 742 second-feet August 15, 1921.

Regulation.-The flow past the station is controlled by regulation in Lake Winnebago, which has an area of 215 square miles, and to some extent by dams between the outlet of Lake Winnebago and the station. The dams are operated for the development of power and in the interests of navigation. Throughout the period covered by the records the same storage conditions have existed.

ACCURACY.-Records good.

Cooperation.-The records were collected and computation of daily discharge made by the United States Engineer Corps, based on curves which were developed by current-meter measurements made by engineers of the United States Geological Survey.

No discharge measurements were made at this station during the year. 
Daily discharge, in second-feet, of Fox River at Rapide Croche dam, near Wrightstown, Wis., for the year ending September 30, 1923

\begin{tabular}{|c|c|c|c|c|c|c|c|c|c|c|c|c|}
\hline D & ct. & ov. & ec. & an. & eb. & Lar. & Apr. & Iay & une & July & Aug. & Sept. \\
\hline & $\begin{array}{l}1,950 \\
2,940 \\
2,870 \\
2,830\end{array}$ & $\begin{array}{l}5,100 \\
3,290 \\
3,270 \\
2,980\end{array}$ & & & & & & & & & & 2,000 \\
\hline & $\begin{array}{l}2,610 \\
2,620 \\
2,010 \\
2,760 \\
2,940\end{array}$ & $\begin{array}{l}920 \\
380 \\
290 \\
320 \\
600\end{array}$ & & $\begin{array}{l}510 \\
520 \\
890 \\
560 \\
580\end{array}$ & & & & & & & & $\begin{array}{l}2,140 \\
2,100 \\
2,010 \\
1,600 \\
1,760\end{array}$ \\
\hline & $\begin{array}{l}2,920 \\
2,840 \\
2,850 \\
2,900 \\
2,680\end{array}$ & $\begin{array}{l}3,060 \\
2,740 \\
2,760 \\
3,120 \\
3,110\end{array}$ & & $\begin{array}{l}4,670 \\
4,770 \\
4,920 \\
4,200 \\
4,460\end{array}$ & & & & & & & $\begin{array}{l}1,400 \\
1,260 \\
1,620 \\
2,060 \\
1,890\end{array}$ & $\begin{array}{l}2,120 \\
2,100 \\
2,020\end{array}$ \\
\hline & $\begin{array}{l}3,520 \\
3,690 \\
3,670 \\
3,720 \\
3,740\end{array}$ & $\begin{array}{l}2,950 \\
2,940 \\
3,060 \\
2,860 \\
2,580\end{array}$ & & $\begin{array}{l}4,920 \\
4,660 \\
5,050\end{array}$ & & & & & & & & 2,250 \\
\hline & $\begin{array}{l}3,810 \\
2,620 \\
2,570 \\
3,710 \\
3,480\end{array}$ & $\begin{array}{l}3,120 \\
3,280 \\
3,270 \\
3,160 \\
3,200\end{array}$ & $\begin{array}{l}4,190 \\
4,160 \\
3,130 \\
2,890\end{array}$ & $\begin{array}{l}4,490 \\
4,510 \\
4,810 \\
4,880 \\
5,020\end{array}$ & $\begin{array}{l}5,7 \\
5,8\end{array}$ & & & & & & & $\begin{array}{l}1,790 \\
2,000 \\
2,160\end{array}$ \\
\hline$\ldots$ & $\begin{array}{l}3,450 \\
3,390 \\
3,330 \\
2,790 \\
2,780 \\
3,230\end{array}$ & $\begin{array}{l}2,890 \\
2,730 \\
3,120 \\
3,180 \\
3,240\end{array}$ & $\begin{array}{l}3,990 \\
3,990 \\
3,980 \\
4,120 \\
3,910 \\
3,110\end{array}$ & $\begin{array}{l}5,010 \\
4,810 \\
4,060 \\
4,480 \\
4,800 \\
4,820\end{array}$ & $\begin{array}{l}5,23 \\
5,48 \\
5,38\end{array}$ & $\begin{array}{l}5,5 \\
5,4 \\
5,3 \\
5,5 \\
5,6\end{array}$ & $\begin{array}{l}13, \\
13, \\
13, \\
13,\end{array}$ & $\begin{array}{l}7,0 \\
5, \\
5, \\
5,5 \\
5,2 \\
5,\end{array}$ & $\begin{array}{l}4,7 \\
4,6 \\
4,7 \\
4,6 \\
4,6\end{array}$ & $\begin{array}{l}2,440 \\
2,410 \\
2,440 \\
1,850 \\
1,920 \\
2,110\end{array}$ & $\begin{array}{l}1,330 \\
1,570 \\
1,960 \\
1,840 \\
1,920 \\
1,980\end{array}$ & $\begin{array}{l}2,130 \\
2,240 \\
1,960 \\
1,490\end{array}$ \\
\hline
\end{tabular}

Monthly discharge of Fox River at Rapide Croche dam, near Wrightstown, Wis., for the year ending September 30, 1923

[Drainage area, 6,150 square miles]

\begin{tabular}{|c|c|c|c|c|c|}
\hline \multirow{2}{*}{ Month } & \multicolumn{4}{|c|}{ Discharge in second-feet } & \multirow{2}{*}{$\begin{array}{l}\text { Run-off } \\
\text { in inches }\end{array}$} \\
\hline & Maximum & Minimum & Mean & $\begin{array}{c}\text { Per square } \\
\text { mile }\end{array}$ & \\
\hline $\begin{array}{l}\text { October } \\
\text { November } \\
\text { December } \\
\text { January } \\
\text { February } \\
\text { March } \\
\text { April } \\
\text { May } \\
\text { June } \\
\text { July } \\
\text { August } \\
\text { September... }\end{array}$ & $\begin{array}{r}3,810 \\
3,600 \\
4,230 \\
5,050 \\
5,850 \\
6,760 \\
13,400 \\
13,700 \\
5,240 \\
4,670 \\
2,460 \\
2,410\end{array}$ & $\begin{array}{l}1,910 \\
2,580 \\
2,080 \\
3,520 \\
3,320 \\
4,970 \\
4,590 \\
4,630 \\
3,370 \\
1,850 \\
1,260 \\
1,180\end{array}$ & $\begin{array}{l}3,000 \\
3,100 \\
3,440 \\
4,530 \\
4,750 \\
5,800 \\
9,150 \\
8,250 \\
4,580 \\
3,170 \\
1,860 \\
1,970\end{array}$ & $\begin{array}{r}0.488 \\
.504 \\
.560 \\
.737 \\
.772 \\
.943 \\
1.49 \\
1.34 \\
.745 \\
.515 \\
.302 \\
.320\end{array}$ & $\begin{array}{r}0.56 \\
.56 \\
.65 \\
.85 \\
.80 \\
1.09 \\
1.66 \\
1.54 \\
.83 \\
.59 \\
.35 \\
.36\end{array}$ \\
\hline The year & 13,700 & 1,180 & 4,460 & .725 & 9.84 \\
\hline
\end{tabular}




\section{WOLF RIVER AT KESHENA, WIS.}

Location.-In sec. 26, T. 28 N., R. 15 E., at highway bridge at Keshena, Shawano County, 3 miles below junction with West Branch of Wolf River.

Drainage AREa.- 840 square miles.

Records available.-May 9, 1907, to March 31, 1909; February 10, 1911, to September 30, 1923.

GAGE.-Chain gage fastened to downstream side of bridge December 9, 1914; May 9, 1907, to November 29, 1914, vertical staff gage fastened to downstream abutment; both gages at same datum. Read by G. Sloniker.

Discharge measurements.-Made from bridge to which gage is attached.

Channel and control.-Gravel; smooth and practically permanent. Banks of medium height, overflow improbable.

Extremes of discharge.-Maximum stage recorded during year, 5.6 feet April 21 and 22 (discharge, 3,180 second-feet); minimum discharge, about 365 second-feet April 2 (stage-discharge relation affected by ice).

1907-1909; 1911-1923: Maximum stage recorded, 7.30 feet at 6.30 p. m. April 10, 1922 (discharge, 4,390 second-feet) ; minimum discharge during openwater periods, 275 second-feet September 26, 1908.

IcE.-Stage-discharge relation seriously affected by ice.

Regulation.-The river and its main tributaries above Keshena are controlled to some extent by logging dams.

ACCURACY.-Stage-discharge relation permanent except as affected by ice. Rating curve well defined throughout. Gage read to hundredths twice daily. Daily discharge ascertained by applying mean daily gage height to rating table, except for period when stage-discharge relation was affected by ice, for which it was ascertained by applying to rating table mean daily gage height corrected for ice effect by means of two discharge measurements, observer's notes, and weather records. Open-water records excellent; winter records fair.

Discharge measurements of Wolf River at Keshena, Wis., during the year ending September 30, 1929

\begin{tabular}{|c|c|c|c|c|c|c|c|}
\hline Date & Made by- & $\begin{array}{c}\text { Gage } \\
\text { height }\end{array}$ & $\begin{array}{l}\text { Dis- } \\
\text { charge }\end{array}$ & Date & Made by- & $\begin{array}{c}\text { Gage } \\
\text { height }\end{array}$ & $\begin{array}{l}\text { Dis- } \\
\text { charge }\end{array}$ \\
\hline $\begin{array}{l}\text { Oct, } 19 \\
\text { Jan } 18 \\
\text { Feb, 28 }\end{array}$ & $\begin{array}{l}\text { Soulé and Fosterr. } \\
\text { J. H, Olson }\end{array}$ & $\begin{array}{c}\text { Feet } \\
1.84 \\
22.97 \\
-3.05\end{array}$ & $\begin{array}{r}\text { Sec. } f t . \\
646 \\
540 \\
473\end{array}$ & $\begin{array}{l}\text { Apr. } 23 \\
\text { June } 24\end{array}$ & $\begin{array}{l}\text { S. B. Souls } \\
\text { E. E. Foster }\end{array}$ & $\begin{array}{l}\text { Feet } \\
5.48 \\
2.71\end{array}$ & $\begin{array}{r}\text { Sec.-ft. } \\
3,090 \\
1,070\end{array}$ \\
\hline
\end{tabular}

- Stage-discharge relation affected by ice. 
Daily discharge, in second-feet, of Wolf River at Keshena, Wis., for the year ending September 30,1923

\begin{tabular}{|c|c|c|c|c|c|c|c|c|c|c|c|c|}
\hline Day & Oct. & Nov. & Dec. & Jan. & Feb. & Mar. & Apr. & May & June & July & Aug. & Sept. \\
\hline $\begin{array}{l}1 \\
2 \ldots \\
3 \\
4 \\
5 \\
5\end{array}$ & $\begin{array}{l}600 \\
600 \\
600 \\
600 \\
600\end{array}$ & $\begin{array}{l}600 \\
640 \\
640 \\
600 \\
640\end{array}$ & $\begin{array}{l}770 \\
680 \\
600 \\
640\end{array}$ & & $\begin{array}{l}535 \\
515 \\
515 \\
445 \\
445\end{array}$ & $\begin{array}{l}460 \\
460 \\
480 \\
460 \\
480\end{array}$ & $\begin{array}{l}380 \\
365 \\
410 \\
445 \\
445\end{array}$ & $\begin{array}{l}2,000 \\
1,930 \\
1,790 \\
1,650 \\
1,580\end{array}$ & $\begin{array}{r}770 \\
815 \\
815 \\
725 \\
1,130\end{array}$ & $\begin{array}{l}860 \\
815 \\
860 \\
910 \\
910\end{array}$ & $\begin{array}{l}600 \\
600 \\
600 \\
565 \\
565\end{array}$ & $\begin{array}{l}565 \\
640 \\
725 \\
725 \\
725\end{array}$ \\
\hline $\begin{array}{r}6 \\
7 \\
8 \\
9 \\
9 \\
10\end{array}$ & $\begin{array}{l}600 \\
600 \\
600 \\
600 \\
600\end{array}$ & $\begin{array}{l}725 \\
640 \\
600 \\
640 \\
640\end{array}$ & & & $\begin{array}{l}445 \\
460 \\
460 \\
460 \\
460\end{array}$ & $\begin{array}{l}480 \\
480 \\
445 \\
445 \\
445\end{array}$ & $\begin{array}{l}480 \\
550 \\
495 \\
535 \\
610\end{array}$ & $\begin{array}{l}1,440 \\
1,370 \\
1,370 \\
1,370 \\
1,370\end{array}$ & $\begin{array}{l}1,130 \\
1,250 \\
1,720 \\
\mathbf{1}, 720 \\
\mathbf{1}, 650\end{array}$ & $\begin{array}{l}960 \\
960 \\
910 \\
770 \\
860\end{array}$ & $\begin{array}{l}565 \\
600 \\
600 \\
565 \\
530\end{array}$ & $\begin{array}{l}725 \\
725 \\
725 \\
680 \\
565\end{array}$ \\
\hline 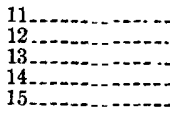 & $\begin{array}{l}600 \\
600 \\
600 \\
600 \\
600\end{array}$ & $\begin{array}{l}640 \\
600 \\
640 \\
815 \\
640\end{array}$ & , & $\begin{array}{l}550 \\
535 \\
515 \\
550 \\
550\end{array}$ & $\begin{array}{l}445 \\
445 \\
460 \\
460 \\
445\end{array}$ & $\begin{array}{l}425 \\
445 \\
445 \\
445 \\
445\end{array}$ & $\begin{array}{r}695 \\
805 \\
900 \\
1,050 \\
1,000\end{array}$ & $\begin{array}{r}1,370 \\
1,310 \\
1,250 \\
960 \\
1,250\end{array}$ & $\begin{array}{l}1,650 \\
1,720 \\
1,650 \\
1,580 \\
1,510\end{array}$ & $\begin{array}{r}910 \\
1,070 \\
1,010 \\
910 \\
860\end{array}$ & $\begin{array}{l}530 \\
530 \\
530 \\
530 \\
530\end{array}$ & $\begin{array}{l}680 \\
640 \\
640 \\
600 \\
565\end{array}$ \\
\hline (n) & $\begin{array}{l}600 \\
640 \\
680 \\
640 \\
640\end{array}$ & $\begin{array}{r}600 \\
600 \\
815 \\
910 \\
1,130\end{array}$ & 635 & $\begin{array}{l}\mathbf{5 5 0} \\
\mathbf{5 3 5} \\
\mathbf{5 5 0} \\
\mathbf{5 5 0} \\
\mathbf{5 3 5}\end{array}$ & $\begin{array}{l}480 \\
460 \\
460 \\
445 \\
460\end{array}$ & $\begin{array}{l}445 \\
445 \\
445 \\
445 \\
425\end{array}$ & $\begin{array}{l}1,160 \\
1,280 \\
1,400 \\
1,790 \\
2,540\end{array}$ & $\begin{array}{r}1,250 \\
1,250 \\
1,130 \\
1,960 \\
1,310\end{array}$ & $\begin{array}{l}1,440 \\
1,310 \\
1,250 \\
1,250 \\
1,250\end{array}$ & $\begin{array}{l}815 \\
770 \\
770 \\
725 \\
680\end{array}$ & $\begin{array}{l}530 \\
530 \\
565 \\
565 \\
565\end{array}$ & $\begin{array}{l}530 \\
530 \\
565 \\
600 \\
600\end{array}$ \\
\hline 3 & $\begin{array}{l}640 \\
640 \\
640 \\
640 \\
640\end{array}$ & $\begin{array}{l}910 \\
815 \\
565 \\
725 \\
640\end{array}$ & & $\begin{array}{l}515 \\
495 \\
515 \\
495 \\
495\end{array}$ & $\begin{array}{l}445 \\
460 \\
460 \\
460 \\
460\end{array}$ & $\begin{array}{l}445 \\
480 \\
480 \\
480 \\
460\end{array}$ & $\begin{array}{l}3,100 \\
3,180 \\
3,100 \\
3,020 \\
2,860\end{array}$ & $\begin{array}{l}1,720 \\
1,440 \\
1,250 \\
1,250 \\
1,070\end{array}$ & $\begin{array}{l}1,310 \\
1,310 \\
1,190 \\
1,070 \\
1,010\end{array}$ & $\begin{array}{l}640 \\
640 \\
640 \\
680 \\
640\end{array}$ & $\begin{array}{l}680 \\
680 \\
640 \\
600 \\
565\end{array}$ & $\begin{array}{l}640 \\
640 \\
640 \\
600 \\
640\end{array}$ \\
\hline (3) & $\begin{array}{l}600 \\
565 \\
565 \\
565 \\
565 \\
600\end{array}$ & $\begin{array}{l}600 \\
640 \\
640 \\
680 \\
725\end{array}$ & & $\begin{array}{l}480 \\
480 \\
495 \\
495 \\
515 \\
515\end{array}$ & $\begin{array}{r}425 \\
460 \\
460 \\
- \\
-\end{array}$ & $\begin{array}{l}445 \\
425 \\
410 \\
410 \\
410 \\
380\end{array}$ & $\begin{array}{l}2,780 \\
2,700 \\
2,540 \\
2,380 \\
2,220\end{array}$ & $\begin{array}{r}\mathbf{1}, 130 \\
1,130 \\
\mathbf{9 1 0} \\
770 \\
\mathbf{9 1 0} \\
\mathbf{9 6 0}\end{array}$ & $\begin{array}{r}1,010 \\
960 \\
910 \\
910 \\
860\end{array}$ & $\begin{array}{l}640 \\
640 \\
640 \\
600 \\
600 \\
600\end{array}$ & $\begin{array}{l}565 \\
565 \\
600 \\
600 \\
565 \\
565\end{array}$ & $\begin{array}{l}600 \\
600 \\
565 \\
565 \\
565\end{array}$ \\
\hline
\end{tabular}

Noте.-Stage-discharge relation affected by ice Dec. 5 to Apr. 19.

Monthly discharge of Wolf River at Keshena, Wis., for the year ending September 30,1928

[Drainage area, 840 square miles]

\begin{tabular}{|c|c|c|c|c|c|}
\hline \multirow{2}{*}{ Month } & \multicolumn{4}{|c|}{ Discharge in second-feet } & \multirow{2}{*}{$\begin{array}{c}\text { Run-off in } \\
\text { inches }\end{array}$} \\
\hline & Maximum & Minimum & Mean & $\begin{array}{c}\text { Per square } \\
\text { mile }\end{array}$ & \\
\hline $\begin{array}{l}\text { October } \\
\text { November } \\
\text { December } \\
\text { January } \\
\text { February } \\
\text { March } \\
\text { April } \\
\text { May } \\
\text { June } \\
\text { July } \\
\text { August } \\
\text { September }\end{array}$ & $\begin{array}{r}680 \\
1,130 \\
770 \\
535 \\
480 \\
3,180 \\
2,000 \\
1,720 \\
1,070 \\
680 \\
725 \\
\end{array}$ & $\begin{array}{r}565 \\
565 \\
480 \\
425 \\
380 \\
365 \\
770 \\
725 \\
600 \\
530 \\
530 \\
\end{array}$ & $\begin{array}{r}608 \\
690 \\
640 \\
542 \\
462 \\
447 \\
1,510 \\
1,300 \\
1,230 \\
784 \\
575 \\
627 \\
\end{array}$ & $\begin{array}{r}0.724 \\
.821 \\
.762 \\
.645 \\
.550 \\
.532 \\
1.80 \\
1.55 \\
1.46 \\
.933 \\
.685 \\
.746 \\
\end{array}$ & $\begin{array}{r}0.83 \\
.92 \\
.88 \\
.74 \\
.57 \\
.61 \\
2.01 \\
1.79 \\
1.63 \\
1.08 \\
.79 \\
.83 \\
\end{array}$ \\
\hline The year.......... & 3,180 & 365 & 785 & .935 & 12.68 \\
\hline
\end{tabular}


WOLF RIVER AT NEW LONDON, WIS.

Location.-In sec. 12, T. 22 N., R. 14 E., at Pearl Street highway bridge, New London, Waupaca County. Embarrass River enters from right threefourths of a mile above station, and Little Wolf River, also from right, 5 miles below.

Drainage area.-2,240 square miles (measured on map issued by Wisconsin Geological and Natural History Survey, edition of 1911 ; scale, 1 inch $=6$ miles).

Records available.-October 1, 1913, to September 30, 1923 . Unpublished gage heights March 1, 1899, to September 30,1913, are in files of the office of the United States Engineer Corps, Milwaukee, Wis.

GAGE.-Staff gage fastened to right hand downstream pier of Pearl Street Bridge. Datum of gage raised 0.641 foot on March 1, 1911, according to information of the United States Engineer Corps. Zero of gage is at elevation 748.874 feet above mean sea level, New York City datum.

Discharge meastrements.-Made from Shawano Street Bridge four blocks below gage.

Channel and control.-Sand, hardpan, and mud; not permanent. Control not well defined. Banks at the gage fairly high. During flood stages the water from Embarrass River flows across the city of New London into the channel of Wolf River below the gage.

EXTREMES OF DISCHARGE.--Maximum stage recorded during year, 10.2 feet at 8 a. m. April 24 (discharge, 10,100 second-feet); minimum stage, 1.0 foot at 8 a. m. August 17, 20, and 21 (discharge, 780 second-feet).

1914-1923: Maximum stage recorded, 11.4 feet at 8 a. m. April 13, 1922 (discharge, 15,500 second-feet) ; minimum discharge, 700 second-feet February 6-9, 1918. The office of the United States Engineer Corps reports a stage of 11.6 feet on April 16, 1888.

ICE.-Stage-discharge relation affected by ice.

REGULATIon.-Little, if any, diurnal fluctuation, owing to operation of power plant above the station, has been observed at the gage; monthly flow natural.

ACCURACY.- Stage-discharge relation not permanent. Rating curves used before and after period of ice effect fairly well defined. Gage read to tenths once daily. Daily discharge ascertained by applying daily gage height to rating table, except for period when stage-discharge relation was affected by ice, for which it was obtained by applying to rating table mean daily gage height corrected for ice effect by means of two discharge measurements, observẹr's notes, and weather records. Open-water records fair; winter records poor.

Discharge measurements of Wolf River at New London, Wis., during the year ending September 30,1923

\begin{tabular}{|c|c|c|c|c|c|c|c|}
\hline Date & Made by- & $\begin{array}{c}\text { Gage } \\
\text { height }\end{array}$ & $\begin{array}{l}\text { Dis- } \\
\text { charge }\end{array}$ & Date & Made by- & $\begin{array}{c}\text { Gage } \\
\text { height }\end{array}$ & $\begin{array}{l}\text { Dis- } \\
\text { charge }\end{array}$ \\
\hline $\begin{array}{l}\text { Oct. } 18 \\
\text { Jan. } 20\end{array}$ & $\begin{array}{l}\text { E. E. Foster. } \\
\text { J. H. Olson. }\end{array}$ & $\begin{array}{c}\text { Feet } \\
2.08 \\
3.00\end{array}$ & $\begin{array}{r}\text { Sec.fft } \\
1,120 \\
999\end{array}$ & $\begin{array}{l}\text { Mar. } 2 \\
\text { June } 22\end{array}$ & $\begin{array}{l}\text { J. H. Olson } \\
\text { E. E. Foster }\end{array}$ & $\begin{array}{c}\text { Feet } \\
-3.20 \\
4.65\end{array}$ & $\begin{array}{r}\text { Sec. } f t . \\
923 \\
2,510\end{array}$ \\
\hline
\end{tabular}

- Stage-discharge relation affected by ice. 
Daily discharge, in second-feet, of Wolf River at New London, Wis., for the year ending September 30, 1923

\begin{tabular}{|c|c|c|c|c|c|c|c|c|c|c|c|c|}
\hline Day & et. & Nov. & Dec. & Jan. & Feb. & Mar. & Apr. & Lay & June & July & lug. & ept. \\
\hline & $\begin{array}{l}1,090 \\
1,170 \\
1,130\end{array}$ & & 1,530 & & & $\begin{array}{l}1,020 \\
1,210 \\
1,250\end{array}$ & & & & & $\begin{array}{l}920 \\
980 \\
945 \\
910 \\
910\end{array}$ & 1,02 \\
\hline & $\begin{array}{l}1,130 \\
1,090 \\
1,090 \\
1,090 \\
1,130\end{array}$ & $\begin{array}{l}1,290 \\
1,330 \\
1,410 \\
1,410 \\
1,330\end{array}$ & & 985 & & & & & & & $\begin{array}{l}910 \\
910 \\
910 \\
910 \\
910\end{array}$ & $\begin{array}{l}1,20 \\
1,24\end{array}$ \\
\hline & $\begin{array}{l}1,090 \\
1,020 \\
1,060 \\
1,060 \\
1,060\end{array}$ & $\begin{array}{l}1,290 \\
1,210 \\
1,330 \\
1,330 \\
1,370\end{array}$ & & $\begin{array}{l}985 \\
985 \\
985 \\
985 \\
985\end{array}$ & & $\begin{array}{l}1,290 \\
1,290 \\
1,290 \\
1,330 \\
1,330\end{array}$ & & & & & $\begin{array}{l}840 \\
875 \\
875 \\
840 \\
840\end{array}$ & $\begin{array}{r}1,20 \\
1,20 \\
1,05 \\
98 \\
98\end{array}$ \\
\hline & $\begin{array}{l}1,090 \\
1,090 \\
1,130 \\
1,130 \\
1,130\end{array}$ & $\begin{array}{l}1,410 \\
1,410 \\
1,370 \\
1,410 \\
1,650\end{array}$ & $\begin{array}{l}1,06 \\
1,02 \\
1,02 \\
1,02 \\
98\end{array}$ & & $\begin{array}{l}915 \\
915 \\
915\end{array}$ & & & & & & & $\begin{array}{l}98 \\
94 ! \\
94 ! \\
910 \\
875\end{array}$ \\
\hline & $\begin{array}{l}1,130 \\
1,130 \\
1,170 \\
1,170 \\
1,130\end{array}$ & $\begin{array}{l}1,770 \\
1,900 \\
2,000 \\
2,050 \\
1,850\end{array}$ & & & & $\begin{array}{l}1,330 \\
1,370 \\
1,370 \\
1,410 \\
1,410\end{array}$ & $\begin{array}{r}8,380 \\
9,350 \\
9,710 \\
10,100 \\
9,710\end{array}$ & & & & & $\begin{array}{l}94 \\
1,02 \\
1,05 \\
1,05 \\
1,05\end{array}$ \\
\hline 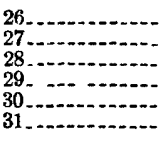 & $\begin{array}{l}1,130 \\
1,060 \\
1,060 \\
1,060 \\
1,130 \\
1,090\end{array}$ & $\begin{array}{l}1,730 \\
1,530 \\
1,450 \\
1,450 \\
1,610\end{array}$ & $\begin{array}{r}98 \\
98 \\
98 \\
98 \\
98 \\
1,06\end{array}$ & $\begin{array}{l}985 \\
985 \\
985 \\
985 \\
985\end{array}$ & $\begin{array}{l}880 \\
880 \\
880\end{array}$ & $\begin{array}{l}1,490 \\
1,530 \\
1,530 \\
1,490\end{array}$ & $\begin{array}{l}8,690 \\
8,380 \\
7,540 \\
7,030 \\
6,560\end{array}$ & & & & 910 & $\begin{array}{l}1,050 \\
1,020 \\
1,020 \\
1,020 \\
1,020\end{array}$ \\
\hline
\end{tabular}

Note.-Stage-discharge relation affected by ice Dec. 5 to Apr. 6.

Monthly discharge of Wolf River at New London, Wis., for the year ending September 30,1923

[Drainage area, 2,240 square miles]

\begin{tabular}{|c|c|c|c|c|c|}
\hline \multirow{2}{*}{ Month } & \multicolumn{4}{|c|}{ Discharge in second-feet } & \multirow{2}{*}{$\begin{array}{c}\text { Run-off } \\
\text { in inches }\end{array}$} \\
\hline & Maximum & Minimum & Mean & $\begin{array}{c}\text { Per square } \\
\text { mile }\end{array}$ & \\
\hline $\begin{array}{l}\text { October } \\
\text { November } \\
\text { December } \\
\text { January } \\
\text { February } \\
\text { March } \\
\text { April } \\
\text { May } \\
\text { June } \\
\text { July } \\
\text { Auguist } \\
\text { September }\end{array}$ & $\begin{array}{r}1,210 \\
2,050 \\
1,610 \\
1,020 \\
985 \\
1,530 \\
10,100 \\
5,950 \\
2,660 \\
2,210 \\
1,020 \\
1,280\end{array}$ & $\begin{array}{r}1,020 \\
1,060 \\
915 \\
985 \\
880 \\
880 \\
1,530 \\
2,160 \\
1,620 \\
1,020 \\
780 \\
875\end{array}$ & $\begin{array}{r}1,110 \\
1,450 \\
1,190 \\
990 \\
929 \\
1,320 \\
5,960 \\
3,390 \\
2,240 \\
1,470 \\
890 \\
1,040\end{array}$ & $\begin{array}{l}0.496 \\
.647 \\
.531 \\
.442 \\
.415 \\
.590 \\
2.66 \\
1.51 \\
1.00 \\
.656 \\
.397 \\
.464\end{array}$ & $\begin{array}{r}0.57 \\
.72 \\
.61 \\
.51 \\
.43 \\
.68 \\
2.97 \\
1.74 \\
1.12 \\
.76 \\
.46 \\
.52\end{array}$ \\
\hline The year.-- & 10,100 & 780 & 1,830 & .817 & 11.09 \\
\hline
\end{tabular}


GMBARRASS RIVIR NRAR EMBARRASS, WIS.

Location.-At highway bridge on line between T. 26 N., R. 14 E., and T. 26 N., R. 15 E., 1 mile downstream from mouth of Mill Creek and 4 miles upstream from Embarrass, Waupaca County.

Drainage AREA.-395 square miles (measured on map issued by Wisconsin Geological and Natural History Survey, edition of 1911 ; scale, 1 inch $=6$ miles).

Records avallable.-June 5, 1919, to September 30, 1923.

GAGE.-Chain gage fastened to downstream handrail; read by Charles Murawski.

Channel and control.-Bed of channel at gage and downstream heavy gravel. Riffle 100 feet downstream forms control. Right bank not subject to overflow; left bank of medium height and will be overflowed at stage of about 9 feet.

Discharge measurements.- Made from downstream side of bridge.

EXTREMES OF DISCHARGE.-Maximum stage recorded during year, 8.70 feet at 8 a. m. April 22 (discharge, 3,500 second-feet); minimum stage, 2.38 feet at 7 a. m. July 23 (discharge, about 34 second-feet).

1919-1923: Maximum stage recorded, 11.50 feet at 4 p. m. April 10,1922 (discharge, about 6,760 second-feet); minimum stage, that of July 23, 1923. ICE.-Stage-discharge relation seriously affected by ice.

REgulation.-Several dams above station create head for development of power, but they do not have enough storage to cause any but slight daily fluctuation.

ACCURACY.-Stage-discharge relation permanent. Rating curve well defined between 114 and 2,800 second-feet; extended beyond these limits. Gage read to hundredths twice daily. Daily discharge ascertained by applying mean daily gage height to rating table except during period when stage-discharge relation was affected by ice, for which it was obtained by applying to rating table mean daily gage height corrected for ice effect by means of two discharge measurements, observer's notes, and weather records. Openwater records good except at low stages, for which they are fair; winter records fair.

Discharge measurements of Embarrass River near Embarrass, Wis., during the year ending September 30, 1923

\begin{tabular}{|c|c|c|c|c|c|c|c|}
\hline Date & Made by- & $\begin{array}{c}\text { Gage } \\
\text { height }\end{array}$ & $\begin{array}{l}\text { Dis- } \\
\text { charge }\end{array}$ & Date & Made by- & $\begin{array}{l}\text { Gage } \\
\text { height }\end{array}$ & $\begin{array}{l}\text { Dis- } \\
\text { charge }\end{array}$ \\
\hline $\begin{array}{l}\text { Jan. } 19 \\
\text { Mar. } 1\end{array}$ & J. H. Olson & $\begin{array}{l}\text { Feet } \\
\text { a } 3.52 \\
\text { a } 3.90\end{array}$ & $\begin{array}{r}\text { Sec. } f t . \\
138 \\
138\end{array}$ & $\begin{array}{l}\text { Apr. } 23 \\
\text { June } 22\end{array}$ & $\begin{array}{l}\text { S. B. Soulé, } \\
\text { E. E. Foster........... }\end{array}$ & $\begin{array}{l}\text { Feet } \\
7.45 \\
3.14\end{array}$ & $\begin{array}{r}\text { Sec. ft } \\
2,720 \\
216\end{array}$ \\
\hline
\end{tabular}

a Stage-discharge relation affected by ice. 
Daily discharge, in second-feet, of Embarrass River near Embarrass, Wis., for the year ending September 30, 1923

\begin{tabular}{|c|c|c|c|c|c|c|c|c|c|c|c|c|}
\hline Day & Oct. & Nov. & Dec. & Jan. & Feb. & Mar. & Apr. & May & June & July & Aug. & Sept. \\
\hline $\begin{array}{l}1 \ldots \\
2 \ldots \\
3 \ldots \\
4 \ldots \\
5 \ldots\end{array}$ & $\begin{array}{l}183 \\
180 \\
173 \\
170 \\
173\end{array}$ & $\begin{array}{l}200 \\
270 \\
217 \\
232 \\
262\end{array}$ & $\begin{array}{l}299 \\
342 \\
303 \\
278 \\
135\end{array}$ & $\begin{array}{r}100 \\
100 \\
115 \\
115 \\
70\end{array}$ & $\begin{array}{r}140 \\
125 \\
80 \\
140 \\
155\end{array}$ & $\begin{array}{l}140 \\
140 \\
140 \\
125 \\
115\end{array}$ & $\begin{array}{r}70 \\
80 \\
80 \\
100 \\
100\end{array}$ & $\begin{array}{l}550 \\
598 \\
526 \\
502 \\
455\end{array}$ & $\begin{array}{l}196 \\
217 \\
478 \\
455 \\
478\end{array}$ & $\begin{array}{l}135 \\
152 \\
235 \\
574 \\
303\end{array}$ & $\begin{array}{l}135 \\
143 \\
132 \\
124 \\
122\end{array}$ & $\begin{array}{l}149 \\
152 \\
158 \\
203 \\
210\end{array}$ \\
\hline $\begin{array}{r}6 \\
7 \\
8 \\
9 \\
9 \\
10 . \\
\ldots\end{array}$ & $\begin{array}{l}170 \\
164 \\
167 \\
167 \\
164\end{array}$ & $\begin{array}{l}295 \\
255 \\
307 \\
243 \\
117\end{array}$ & $\begin{array}{l}365 \\
340 \\
260 \\
240 \\
220\end{array}$ & $\begin{array}{r}80 \\
125 \\
115 \\
140 \\
155\end{array}$ & $\begin{array}{r}185 \\
90 \\
90 \\
170 \\
90\end{array}$ & $\begin{array}{l}115 \\
115 \\
80 \\
90 \\
80\end{array}$ & $\begin{array}{r}90 \\
155 \\
205 \\
303 \\
299\end{array}$ & $\begin{array}{l}432 \\
386 \\
386 \\
432 \\
455\end{array}$ & $\begin{array}{l}432 \\
526 \\
622 \\
697 \\
697\end{array}$ & $\begin{array}{l}342 \\
364 \\
574 \\
502 \\
432\end{array}$ & $\begin{array}{r}132 \\
130 \\
143 \\
104 \\
83\end{array}$ & $\begin{array}{l}190 \\
155 \\
193 \\
214 \\
221\end{array}$ \\
\hline 4. & $\begin{array}{l}167 \\
167 \\
167 \\
167 \\
170\end{array}$ & $\begin{array}{l}278 \\
255 \\
295 \\
251 \\
262\end{array}$ & $\begin{array}{l}185 \\
170 \\
185 \\
155 \\
155\end{array}$ & $\begin{array}{l}140 \\
100 \\
100 \\
140 \\
125\end{array}$ & $\begin{array}{l}170 \\
155 \\
170 \\
100 \\
125\end{array}$ & $\begin{array}{r}70 \\
115 \\
125 \\
90 \\
100\end{array}$ & $\begin{array}{r}364 \\
647 \\
1,010 \\
1,060 \\
1,010\end{array}$ & $\begin{array}{l}409 \\
409 \\
342 \\
320 \\
303\end{array}$ & $\begin{array}{l}502 \\
432 \\
346 \\
364 \\
320\end{array}$ & $\begin{array}{l}299 \\
207 \\
247 \\
278 \\
266\end{array}$ & $\begin{array}{r}107 \\
86 \\
109 \\
77 \\
109\end{array}$ & $\begin{array}{l}180 \\
155 \\
152 \\
140 \\
140\end{array}$ \\
\hline 9 & $\begin{array}{l}167 \\
170 \\
193 \\
173 \\
173\end{array}$ & $\begin{array}{l}251 \\
274 \\
364 \\
364 \\
598\end{array}$ & $\begin{array}{r}155 \\
80 \\
125 \\
90 \\
140\end{array}$ & $\begin{array}{r}155 \\
140 \\
140 \\
125 \\
60\end{array}$ & $\begin{array}{l}170 \\
205 \\
100 \\
170 \\
100\end{array}$ & $\begin{array}{r}100 \\
90 \\
100 \\
115 \\
115\end{array}$ & $\begin{array}{l}1,120 \\
1,300 \\
1,960 \\
2,590 \\
3,220\end{array}$ & $\begin{array}{l}346 \\
386 \\
386 \\
364 \\
478\end{array}$ & $\begin{array}{l}282 \\
243 \\
217 \\
186 \\
180\end{array}$ & $\begin{array}{l}210 \\
183 \\
140 \\
143 \\
140\end{array}$ & $\begin{array}{r}132 \\
122 \\
130 \\
95 \\
114\end{array}$ & $\begin{array}{l}140 \\
130 \\
155 \\
164 \\
177\end{array}$ \\
\hline 24 & $\begin{array}{l}203 \\
196 \\
214 \\
207 \\
183\end{array}$ & $\begin{array}{l}574 \\
598 \\
478 \\
386 \\
320\end{array}$ & $\begin{array}{l}115 \\
100 \\
125 \\
125 \\
125\end{array}$ & $\begin{array}{r}115 \\
115 \\
125 \\
125 \\
70\end{array}$ & $\begin{array}{r}90 \\
185 \\
170 \\
80 \\
115\end{array}$ & $\begin{array}{r}140 \\
115 \\
100 \\
80 \\
80\end{array}$ & $\begin{array}{l}3,430 \\
3,430 \\
2,730 \\
2,020 \\
1,600\end{array}$ & $\begin{array}{l}598 \\
622 \\
574 \\
455 \\
386\end{array}$ & $\begin{array}{l}255 \\
247 \\
196 \\
193 \\
161\end{array}$ & $\begin{array}{r}217 \\
140 \\
39 \\
86 \\
132\end{array}$ & $\begin{array}{l}100 \\
100 \\
127 \\
164 \\
140\end{array}$ & $\begin{array}{l}180 \\
200 \\
200 \\
196 \\
170\end{array}$ \\
\hline 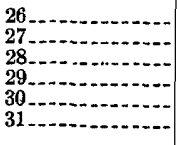 & $\begin{array}{l}167 \\
164 \\
170 \\
177 \\
190 \\
186\end{array}$ & $\begin{array}{l}342 \\
303 \\
307 \\
255 \\
225\end{array}$ & $\begin{array}{l}140 \\
125 \\
115 \\
115 \\
115 \\
115\end{array}$ & $\begin{array}{r}70 \\
140 \\
140 \\
140 \\
155 \\
80\end{array}$ & \begin{tabular}{r}
140 \\
140 \\
80 \\
\hdashline... \\
\end{tabular} & $\begin{array}{l}90 \\
90 \\
90 \\
90 \\
80 \\
80\end{array}$ & $\begin{array}{r}1,300 \\
1,120 \\
955 \\
824 \\
747\end{array}$ & $\begin{array}{l}324 \\
278 \\
278 \\
207 \\
235 \\
196\end{array}$ & $\begin{array}{l}137 \\
143 \\
152 \\
167 \\
158\end{array}$ & $\begin{array}{l}170 \\
132 \\
132 \\
140 \\
132 \\
149\end{array}$ & $\begin{array}{l}119 \\
146 \\
143 \\
119 \\
203 \\
190\end{array}$ & $\begin{array}{l}177 \\
164 \\
158 \\
143 \\
152\end{array}$ \\
\hline
\end{tabular}

Nore.-Stage-discharge relation affected by ice Dec. 6 to Apr. 8 .

Monthly discharge of Embarrass River near Embarrass, Wis., for the year ending September 30, 1923

[Drainage area, 395 square miles]

\begin{tabular}{|c|c|c|c|c|c|}
\hline \multirow{2}{*}{ Month } & \multicolumn{4}{|c|}{ Discharge in second-feet } & \multirow{2}{*}{$\begin{array}{l}\text { Run-off } \\
\text { in inches }\end{array}$} \\
\hline & Maximum & Minimum & Mean & $\underset{\text { mile }}{\text { Per square }}$ & \\
\hline $\begin{array}{l}\text { October } \\
\text { November } \\
\text { December } \\
\text { January } \\
\text { February } \\
\text { March } \\
\text { April } \\
\text { May } \\
\text { June } \\
\text { July } \\
\text { August } \\
\text { September }\end{array}$ & $\begin{array}{r}214 \\
598 \\
365 \\
155 \\
205 \\
140 \\
3,430 \\
622 \\
697 \\
574 \\
203 \\
221\end{array}$ & \begin{tabular}{r|}
164 \\
117 \\
80 \\
60 \\
80 \\
70 \\
70 \\
196 \\
137 \\
39 \\
77 \\
130
\end{tabular} & $\begin{array}{r}177 \\
313 \\
179 \\
117 \\
133 \\
103 \\
1,130 \\
407 \\
323 \\
232 \\
125 \\
171\end{array}$ & $\begin{array}{l}0.448 \\
.792 \\
.453 \\
.296 \\
.337 \\
.261 \\
2.86 \\
1.03 \\
.818 \\
.587 \\
.316 \\
.433\end{array}$ & $\begin{array}{r}0.52 \\
.88 \\
.52 \\
.34 \\
.35 \\
.30 \\
3.19 \\
1.19 \\
.91 \\
.68 \\
.36 \\
.48\end{array}$ \\
\hline The year......... & 3,430 & 39 & 283 & .716 & 9.72 \\
\hline
\end{tabular}




\section{LITTLE WOLF RIVER AT ROYALTON, WIS.}

Location.-In sec. 1, T. 22 N., R. 13 E., at highway bridge at Royalton, Waupaca County, 4 miles above mouth of river.

Drainage AREA. -485 square miles (measured on Wisconsin Geological and Natural History Survey map, edition of 1911 ; scale, 1 inch $=6$ miles).

Records aVatlable.--January 13, 1914, to September 30, 1923.

GAGE.--SIoping gage on left bank 150 feet upstream from highway bridge; read by J. C. Jensen. Prior to August 20, 1915, a chain gage fastened to upstream side of highway bridge was used. Datum of sloping gage is 0.75 foot higher than that of the chain gage; owing to change in slope, however, difference between the readings on the slope gage and chain gage is not constant.

Discharge measurements.-Made from cable 500 feet upstream from gage or by wading.

Channel and control.-Stream bed gage section consists of heavy gravel and rock; fairly permanent. At measuring section bed is fine, smooth gravel. Neither bank is overflowed to any extent at flood stages.

Extremes of Discharge.-Maximum open-water stage during year, 5.4 feet at 8 a. m. April 15 (discharge, 3,700 second-feet); minimum discharge, 160 second-feet several days in January.

1914-1923: Maximum discharge recorded, 5,780 second-feet at 7 a. $\mathrm{m}$. April 10 and 5 p. m. April 11, 1922; minimum discharge, about 120 secondfeet January 20, 1922.

ICE.-Stage-discharge relation affected by ice.

REgULATION.-The few power plants above the station have little storage; no diurnal fluctuation has been observed at the gage.

ACCURACY.- Stage-discharge relation permanent throughout the year. Rating curve well defined below 3,220 second-feet; gage read to quarter-tenths twice daily. Daily discharge obtained by applying mean daily gage height to rating table, except for period when stage-discharge relation was affected by ice, for which it was obtained by applying to rating table mean daily gage height corrected for ice effect by means of two discharge measurements, observer's notes, and weather records. Open-water records good; winter records fair.

Discharge measurements of Little Wolf River at Royalton, Wis., during the year ending September 30, 1923

\begin{tabular}{|c|c|c|c|c|c|c|c|}
\hline Date & Made by- & $\begin{array}{c}\text { Gage } \\
\text { height }\end{array}$ & $\begin{array}{c}\text { Dis- } \\
\text { charge }\end{array}$ & Date & Made by- & $\begin{array}{c}\text { Gage } \\
\text { height }\end{array}$ & $\begin{array}{c}\text { Dis- } \\
\text { charge }\end{array}$ \\
\hline $\begin{array}{l}\text { Oct. } 18 \\
\text { Jan. } 22\end{array}$ & $\begin{array}{l}\text { s. B. Soules } \\
\text { J. H. Olson }\end{array}$ & $\begin{array}{c}\text { Feet } \\
1.59 \\
01.87\end{array}$ & $\begin{array}{r}\text { Sec. } f t . \\
283 \\
212\end{array}$ & $\begin{array}{l}\text { Mar. } 5 \\
\text { June } 22\end{array}$ & $\begin{array}{l}\text { J. H. Olson } \\
\text { E. E. Foster..... }\end{array}$ & $\begin{array}{r}\text { Feet } \\
02.58 \\
1.53\end{array}$ & $\begin{array}{r}\text { Sec. } f t . \\
285 \\
265\end{array}$ \\
\hline
\end{tabular}

a Stage-discharge relation affected by ice. $72038-26 \dagger-w s P$ 564-3 
Daily discharge, in second-feet, of Little Wolf River at Royalton, Wis., for the year ending September 30, 1923

\begin{tabular}{|c|c|c|c|c|c|c|c|c|c|c|c|c|}
\hline Day & Oct. & Nov. & Dec. & Jan. & Feb. & Mar. & Apr. & May & June & July & Aug. & Sept. \\
\hline $\begin{array}{l}1 \ldots \\
2 \ldots \\
3 \ldots \\
4 \ldots \\
5 \ldots\end{array}$ & $\begin{array}{l}257 \\
248 \\
280 \\
273 \\
267\end{array}$ & $\begin{array}{l}248 \\
238 \\
267 \\
273 \\
305\end{array}$ & $\begin{array}{l}501 \\
466 \\
501 \\
466 \\
560\end{array}$ & $\begin{array}{l}170 \\
170 \\
180 \\
170 \\
160\end{array}$ & $\begin{array}{l}215 \\
225 \\
205 \\
195 \\
185\end{array}$ & $\begin{array}{l}245 \\
275 \\
260 \\
340 \\
295\end{array}$ & $\begin{array}{l}340 \\
560 \\
710 \\
770 \\
830\end{array}$ & $\begin{array}{l}800 \\
740 \\
770 \\
740 \\
740\end{array}$ & $\begin{array}{l}276 \\
310 \\
472 \\
310 \\
276\end{array}$ & $\begin{array}{l}241 \\
227 \\
284 \\
310 \\
392\end{array}$ & $\begin{array}{l}234 \\
234 \\
241 \\
227 \\
219\end{array}$ & $\begin{array}{l}227 \\
227 \\
223 \\
251 \\
241\end{array}$ \\
\hline $\begin{array}{r}6 \ldots \\
7 \ldots \\
8 \ldots \\
9 \\
10 \ldots\end{array}$ & $\begin{array}{l}248 \\
267 \\
267 \\
243 \\
248\end{array}$ & $\begin{array}{l}293 \\
305 \\
305 \\
280 \\
273\end{array}$ & $\begin{array}{l}590 \\
560 \\
590 \\
445 \\
445\end{array}$ & $\begin{array}{l}170 \\
170 \\
170 \\
170 \\
160\end{array}$ & $\begin{array}{l}195 \\
205 \\
185 \\
185 \\
195\end{array}$ & $\begin{array}{l}320 \\
340 \\
340 \\
390 \\
320\end{array}$ & $\begin{array}{r}935 \\
900 \\
970 \\
1,210 \\
\mathbf{1}, 570\end{array}$ & $\begin{array}{l}620 \\
590 \\
560 \\
530 \\
472\end{array}$ & $\begin{array}{l}310 \\
530 \\
472 \\
407 \\
328\end{array}$ & $\begin{array}{l}501 \\
444 \\
680 \\
900 \\
865\end{array}$ & $\begin{array}{l}213 \\
234 \\
227 \\
234 \\
241\end{array}$ & $\begin{array}{l}251 \\
342 \\
461 \\
328 \\
310\end{array}$ \\
\hline $\begin{array}{l}13 \ldots \\
14 \\
15\end{array}$ & $\begin{array}{l}267 \\
232 \\
211 \\
238 \\
280\end{array}$ & $\begin{array}{l}280 \\
323 \\
352 \\
305 \\
280\end{array}$ & $\begin{array}{l}445 \\
365 \\
340 \\
.275 \\
245\end{array}$ & $\begin{array}{l}160 \\
170 \\
170 \\
160 \\
170\end{array}$ & $\begin{array}{l}185 \\
205 \\
205 \\
200 \\
195\end{array}$ & $\begin{array}{l}295 \\
275 \\
260 \\
275 \\
275\end{array}$ & $\begin{array}{l}1,870 \\
2,290 \\
2,740 \\
3,100 \\
3,700\end{array}$ & $\begin{array}{l}461 \\
461 \\
417 \\
428 \\
461\end{array}$ & $\begin{array}{l}366 \\
328 \\
276 \\
310 \\
284\end{array}$ & $\begin{array}{l}\mathbf{7 7 0} \\
\mathbf{5 9 0} \\
\mathbf{4 6 1} \\
\mathbf{3 1 0} \\
\mathbf{3 4 2}\end{array}$ & $\begin{array}{l}234 \\
241 \\
227 \\
219 \\
207\end{array}$ & $\begin{array}{l}260 \\
270 \\
241 \\
241 \\
241\end{array}$ \\
\hline $\begin{array}{l}16 \ldots \\
17 \\
18 \\
19 \\
20 \ldots \ldots\end{array}$ & $\begin{array}{l}257 \\
201 \\
243 \\
314 \\
323\end{array}$ & $\begin{array}{l}293 \\
472 \\
501 \\
530 \\
620\end{array}$ & $\begin{array}{l}215 \\
205 \\
180 \\
180 \\
180\end{array}$ & $\begin{array}{l}185 \\
185 \\
180 \\
185 \\
180\end{array}$ & $\begin{array}{l}215 \\
195 \\
195 \\
185 \\
205\end{array}$ & $\begin{array}{l}260 \\
245 \\
260 \\
275 \\
260\end{array}$ & $\begin{array}{l}3,460 \\
2,860 \\
2,740 \\
2,740 \\
2,620\end{array}$ & $\begin{array}{l}444 \\
461 \\
444 \\
800 \\
680\end{array}$ & $\begin{array}{l}297 \\
284 \\
284 \\
310 \\
276\end{array}$ & $\begin{array}{l}310 \\
284 \\
318 \\
284 \\
284\end{array}$ & $\begin{array}{l}186 \\
207 \\
207 \\
203 \\
207\end{array}$ & $\begin{array}{l}270 \\
270 \\
260 \\
260 \\
251\end{array}$ \\
\hline $\begin{array}{l}21 \\
22 \\
23\end{array} 2 \ldots$ & $\begin{array}{l}386 \\
352 \\
386 \\
181 \\
217\end{array}$ & $\begin{array}{l}620 \\
650 \\
590 \\
\mathbf{5 9 0} \\
\mathbf{5 3 0}\end{array}$ & $\begin{array}{l}170 \\
180 \\
170 \\
180 \\
170\end{array}$ & $\begin{array}{l}180 \\
185 \\
215 \\
235 \\
235\end{array}$ & $\begin{array}{l}205 \\
215 \\
245 \\
260 \\
215\end{array}$ & $\begin{array}{l}260 \\
245 \\
235 \\
235 \\
245\end{array}$ & $\begin{array}{l}2,740 \\
2,740 \\
2,620 \\
2,510 \\
2,510\end{array}$ & $\begin{array}{l}560 \\
620 \\
590 \\
530 \\
444\end{array}$ & $\begin{array}{l}270 \\
270 \\
276 \\
260 \\
270\end{array}$ & $\begin{array}{l}276 \\
284 \\
270 \\
241 \\
234\end{array}$ & $\begin{array}{l}234 \\
227 \\
219 \\
207 \\
207\end{array}$ & $\begin{array}{l}276 \\
376 \\
407 \\
376 \\
328\end{array}$ \\
\hline $\begin{array}{l}26 \\
27 \\
28 \\
29 \\
30 \\
31\end{array}$ & $\begin{array}{l}217 \\
232 \\
238 \\
248 \\
238 \\
243\end{array}$ & $\begin{array}{l}439 \\
\mathbf{3 6 1} \\
\mathbf{3 7 1} \\
501 \\
501\end{array}$ & $\begin{array}{l}185 \\
185 \\
170 \\
180 \\
170 \\
170\end{array}$ & $\begin{array}{l}245 \\
235 \\
235 \\
225 \\
205 \\
215\end{array}$ & $\begin{array}{r}205 \\
195 \\
205 \\
- \\
\hdashline \\
\hdashline\end{array}$ & $\begin{array}{l}260 \\
260 \\
295 \\
260 \\
245 \\
260\end{array}$ & $\begin{array}{r}1,770 \\
1,300 \\
1,300 \\
1, \mathbf{1 3 0} \\
\mathbf{8 6 5}\end{array}$ & $\begin{array}{l}392 \\
310 \\
342 \\
310 \\
310 \\
\mathbf{3 1 0}\end{array}$ & $\begin{array}{r}310 \\
284 \\
260 \\
270 \\
251 \\
\end{array}$ & $\begin{array}{l}245 \\
241 \\
245 \\
245 \\
241 \\
241\end{array}$ & $\begin{array}{r}213 \\
227 \\
234 \\
207 \\
219 \\
227\end{array}$ & $\begin{array}{r}356 \\
328 \\
310 \\
270 \\
297 \\
\end{array}$ \\
\hline
\end{tabular}

Note.-Stage-discharge relation affected by ice Dec. 9 to Apr. 14.

Monthly discharge of Little Wolf River at Royalton, Wis., for the year ending September 30, 1923

[Drainage area, 485 square miles]

\begin{tabular}{|c|c|c|c|c|c|}
\hline \multirow{2}{*}{ Month } & \multicolumn{4}{|c|}{ Discharge in second-feet } & \multirow{2}{*}{$\begin{array}{l}\text { Run-off } \\
\text { in inches }\end{array}$} \\
\hline & Maximum & Minimum & Mean & $\underset{\text { mile }}{\text { Persquare }}$ & \\
\hline $\begin{array}{l}\text { October } \\
\text { November } \\
\text { December } \\
\text { January } \\
\text { February } \\
\text { March } \\
\text { April } \\
\text { May } \\
\text { June } \\
\text { July } \\
\text { August } \\
\text { September }\end{array}$ & $\begin{array}{r}386 \\
650 \\
590 \\
245 \\
260 \\
390 \\
3,700 \\
800 \\
530 \\
900 \\
241 \\
461\end{array}$ & $\begin{array}{l}181 \\
238 \\
170 \\
160 \\
185 \\
235 \\
340 \\
310 \\
251 \\
227 \\
186 \\
223 \\
\end{array}$ & $\begin{array}{r}261 \\
397 \\
312 \\
189 \\
204 \\
278 \\
1,880 \\
527 \\
314 \\
373 \\
221 \\
292\end{array}$ & \begin{tabular}{|c|}
0.538 \\
.819 \\
.643 \\
.390 \\
.421 \\
.573 \\
3.88 \\
1.09 \\
.647 \\
.769 \\
.456 \\
.602 \\
\end{tabular} & $\begin{array}{r}0.62 \\
.91 \\
.74 \\
.45 \\
.44 \\
.66 \\
4.33 \\
1.26 \\
.72 \\
.89 \\
.53 \\
.67\end{array}$ \\
\hline The year.... & 3,700 & 160 & 436 & .899 & 12.22 \\
\hline
\end{tabular}


WAUPACA RIVER NEAR WAUPACA, WIS.

Location.-Near north line of sec. 1, T. 21 N., R. 12 E., at highway bridge 4 miles downstream from Waupaca, Waupaca County.

Drainage area.-305 square miles (measured on Wisconsin Geological and Natural History Survey map, edition of 1911; scale, 1 inch $=6$ miles).

Records available.-October 18, 1917, to September 30, 1923. June 28, 1916, to October 18,1917 , records were obtained at a station near Weyauwega, 1 mile downstream from present site.

GAGE.-Chain gage, bolted to upstream handrail of bridge; read by George Radtke.

Discharge measurements.-Made from upstream side of bridge or by wading.

Channel and control.-Bed consists of fine gravel and clay; clean and free from vegetation. Control not well defined and is not permanent. Right bank high and seldom overflowed; left bank of medium height and is overflowed at a stage of about 6 feet.

ICE.-Stage-discharge relation seriously affected by ice.

EXTREMEs of DISCHARGE.-Maximum stage recorded during year, 5.4 feet at 9 a. m. April 13 (discharge, 2,410 second-feet); minimum discharge, 115 secondfeet January 6 and February 24 (stage-discharge relation affected by ice).

1918-1923: Maximum open-water stage recorded, 5.6 feet March 17, 1919 (discharge, 2,600 second-feet); minimum stage, 1.28 feet November 21, 1920 (discharge, 96 second-feet).

Regulation.-Power plants at Waupaca and above on the main stream and also several on Crystal River may cause slight fluctuation during low stages. Pondage at the various plants is small and mean monthly discharge is believed to represent closely the natural flow.

ACCURACY.-Stage-discharge relation probally permanent during year. Rating curve fairly well defined between 180 and 700 second-feet. Gage read to hundredths once daily. Daily discharge ascertained by applying daily gage height to rating table, except for period when stage-discharge relation was affected by ice, for which it was obtained by applying to rating table daily gage height corrected for ice effect by means of two discharge measurements, observer's notes, and weather records. Open-water records fair; winter records poor.

Discharge measurements of Waupaca River near Waupaca, Wis., during the year ending September 30, 1923

\begin{tabular}{|c|c|c|c|c|c|c|c|}
\hline Date & Made by- & $\begin{array}{c}\text { Gage: } \\
\text { height }\end{array}$ & $\begin{array}{c}\text { Dis- } \\
\text { charge }\end{array}$ & Date & Made by- & $\begin{array}{c}\text { Gage } \\
\text { height }\end{array}$ & $\begin{array}{c}\text { Dis. } \\
\text { ehargo }\end{array}$ \\
\hline $\begin{array}{ll}\text { Oct. } & 18 \\
\text { Jan, } & 21\end{array}$ & $\begin{array}{l}\text { s. B. Soule } \\
\text { J. H. Olson }\end{array}$ & $\begin{array}{c}\text { Feet } \\
1.80 \\
a 2.77\end{array}$ & $\begin{array}{r}\text { Sec. } . f t . \\
203 \\
176\end{array}$ & $\begin{array}{l}\text { Mar. } 3 \\
\text { June } 20\end{array}$ & $\begin{array}{l}\text { J. H. Olson } \\
\text { E. } \text { E. Foster }\end{array}$ & $\begin{array}{l}\text { Feet } \\
-3.80 \\
1.98\end{array}$ & $\begin{array}{r}\text { Sec. } \\
280 \\
260\end{array}$ \\
\hline
\end{tabular}

- Stage-discharge relation affected by ice. 
Daily discharge, in second-feet, of Waupaca River near Waupaca, Wis., for the year ending September 30, 1923

\begin{tabular}{|c|c|c|c|c|c|c|c|c|c|c|c|c|}
\hline Day & Oct. & Nov. & Dec. & Jan. & Feb. & Mar. & Apr. & May & June & July & Aug. & Sept. \\
\hline $\begin{array}{l}1 \ldots \\
2 \ldots \\
3 \ldots \\
4 \ldots \\
5 . . .\end{array}$ & $\begin{array}{l}236 \\
236 \\
209 \\
209 \\
209\end{array}$ & $\begin{array}{l}209 \\
209 \\
222 \\
222 \\
222\end{array}$ & $\begin{array}{l}296 \\
295 \\
265 \\
250 \\
250\end{array}$ & $\begin{array}{l}150 \\
150 \\
150 \\
135 \\
150\end{array}$ & $\begin{array}{l}150 \\
150 \\
160 \\
150 \\
150\end{array}$ & $\begin{array}{l}150 \\
150 \\
220 \\
265 \\
310\end{array}$ & $\begin{array}{l}210 \\
280 \\
680 \\
960 \\
840\end{array}$ & $\begin{array}{l}280 \\
280 \\
265 \\
265 \\
265\end{array}$ & $\begin{array}{l}183 \\
196 \\
222 \\
222 \\
196\end{array}$ & $\begin{array}{l}209 \\
209 \\
183 \\
183 \\
222\end{array}$ & $\begin{array}{l}183 \\
183 \\
196 \\
170 \\
196\end{array}$ & $\begin{array}{l}196 \\
209 \\
236 \\
209 \\
209\end{array}$ \\
\hline $\begin{array}{r}6 \ldots \\
7 \ldots \\
8 \ldots \\
9 \ldots \\
10 . .\end{array}$ & $\begin{array}{l}209 \\
209 \\
209 \\
209 \\
209\end{array}$ & $\begin{array}{l}296 \\
265 \\
236 \\
250 \\
209\end{array}$ & $\begin{array}{l}250 \\
250 \\
250 \\
235 \\
235\end{array}$ & $\begin{array}{l}115 \\
160 \\
170 \\
160 \\
150\end{array}$ & $\begin{array}{l}160 \\
150 \\
160 \\
150 \\
135\end{array}$ & $\begin{array}{l}265 \\
280 \\
265 \\
259 \\
185\end{array}$ & $\begin{array}{l}330 \\
345 \\
345 \\
400 \\
400\end{array}$ & $\begin{array}{l}250 \\
250 \\
250 \\
265 \\
250\end{array}$ & $\begin{array}{l}196 \\
209 \\
196 \\
196 \\
209\end{array}$ & $\begin{array}{l}574 \\
574 \\
265 \\
222 \\
296\end{array}$ & $\begin{array}{l}183 \\
170 \\
170 \\
170 \\
170\end{array}$ & $\begin{array}{l}196 \\
236 \\
222 \\
222 \\
222\end{array}$ \\
\hline ........ & $\begin{array}{l}209 \\
209 \\
209 \\
209 \\
209\end{array}$ & $\begin{array}{l}222 \\
222 \\
236 \\
222 \\
209\end{array}$ & $\begin{array}{l}235 \\
220 \\
220 \\
220 \\
195\end{array}$ & $\begin{array}{l}125 \\
150 \\
150 \\
160 \\
170\end{array}$ & $\begin{array}{l}135 \\
140 \\
150 \\
140 \\
130\end{array}$ & $\begin{array}{l}210 \\
210 \\
210 \\
235 \\
220\end{array}$ & $\begin{array}{l}1,020 \\
1,610 \\
2,410 \\
2,050 \\
1,230\end{array}$ & $\begin{array}{l}250 \\
250 \\
250 \\
250 \\
250\end{array}$ & $\begin{array}{l}222 \\
250 \\
222 \\
158 \\
196\end{array}$ & $\begin{array}{l}250 \\
222 \\
222\end{array}$ & $\begin{array}{l}236 \\
209 \\
209 \\
170 \\
170\end{array}$ & $\begin{array}{l}196 \\
170 \\
183 \\
170 \\
183\end{array}$ \\
\hline - & $\begin{array}{l}196 \\
209 \\
209 \\
209 \\
196\end{array}$ & $\begin{array}{l}209 \\
222 \\
236 \\
329 \\
296\end{array}$ & $\begin{array}{l}195 \\
170 \\
170 \\
160 \\
160\end{array}$ & $\begin{array}{l}135 \\
150 \\
160 \\
160 \\
160\end{array}$ & $\begin{array}{l}125 \\
150 \\
160 \\
160 \\
135\end{array}$ & $\begin{array}{l}220 \\
220 \\
330 \\
310 \\
310\end{array}$ & $\begin{array}{l}780 \\
636 \\
680 \\
680 \\
636\end{array}$ & $\begin{array}{l}296 \\
250 \\
265 \\
250 \\
312\end{array}$ & $\begin{array}{l}209 \\
196 \\
209 \\
183 \\
222\end{array}$ & $\begin{array}{l}209 \\
222 \\
196 \\
183 \\
183\end{array}$ & $\begin{array}{l}170 \\
170 \\
170 \\
183 \\
196\end{array}$ & $\begin{array}{l}183 \\
183 \\
183 \\
183 \\
209\end{array}$ \\
\hline 4 & $\begin{array}{l}222 \\
209 \\
196 \\
209 \\
209\end{array}$ & $\begin{array}{l}280 \\
280 \\
265 \\
236 \\
236\end{array}$ & $\begin{array}{l}160 \\
170 \\
135 \\
150 \\
160\end{array}$ & $\begin{array}{l}155 \\
150 \\
150 \\
185 \\
185\end{array}$ & $\begin{array}{l}170 \\
160 \\
160 \\
115 \\
150\end{array}$ & $\begin{array}{l}280 \\
310 \\
295 \\
280 \\
250\end{array}$ & $\begin{array}{l}636 \\
680 \\
595 \\
456 \\
382\end{array}$ & $\begin{array}{l}364 \\
280 \\
280 \\
265 \\
250\end{array}$ & $\begin{array}{l}236 \\
265 \\
222 \\
222 \\
222\end{array}$ & $\begin{array}{l}195 \\
196 \\
209 \\
209 \\
236\end{array}$ & $\begin{array}{l}196 \\
183\end{array}$ & $\begin{array}{l}209 \\
209 \\
209 \\
183 \\
170\end{array}$ \\
\hline $\begin{array}{l}6 \\
7 \\
8 \\
8 \\
9 \\
0 \\
-\end{array}$ & $\begin{array}{l}209 \\
196 \\
196 \\
209 \\
222 \\
209\end{array}$ & $\begin{array}{l}222 \\
209 \\
196 \\
196 \\
236\end{array}$ & $\begin{array}{l}160 \\
170 \\
195 \\
195 \\
170 \\
160\end{array}$ & $\begin{array}{l}150 \\
160 \\
160 \\
160 \\
170 \\
170\end{array}$ & \begin{tabular}{c}
160 \\
160 \\
150 \\
\hdashline- \\
.--
\end{tabular} & $\begin{array}{l}220 \\
310 \\
210 \\
310 \\
280 \\
265\end{array}$ & $\begin{array}{l}382 \\
346 \\
296 \\
312 \\
312\end{array}$ & $\begin{array}{l}250 \\
222 \\
209 \\
196 \\
196 \\
209\end{array}$ & $\begin{array}{l}312 \\
280 \\
250 \\
236 \\
222\end{array}$ & $\begin{array}{l}183 \\
196 \\
209 \\
196 \\
209 \\
196\end{array}$ & $\begin{array}{l}183 \\
183 \\
183 \\
196 \\
183 \\
196\end{array}$ & $\begin{array}{l}183 \\
183 \\
196 \\
209 \\
209\end{array}$ \\
\hline
\end{tabular}

,Notr.-Stage-discharge relation affected by ice Dec. 5 to Apr. 8.

Monthly. discharge of Waupaca River near Waupaca, Wis., for the year ending September 30, 1923

[Drainage area, 305 square miles]

\begin{tabular}{|c|c|c|c|c|c|}
\hline \multirow{2}{*}{ Month } & \multicolumn{4}{|c|}{ Discharge in second-feet } & \multirow{2}{*}{$\begin{array}{l}\text { Run-off } \\
\text { in inches }\end{array}$} \\
\hline & Maximum & Minimum & Mean & $\underset{\text { mile }}{\text { Per square }}$ & \\
\hline $\begin{array}{l}\text { October } \\
\text { November } \\
\text { December } \\
\text { January } \\
\text { February } \\
\text { March } \\
\text { April } \\
\text { May } \\
\text { June } \\
\text { July } \\
\text { August } \\
\text { September }\end{array}$ & $\begin{array}{r}236 \\
296 \\
296 \\
185 \\
170 \\
330 \\
2,410 \\
364 \\
312 \\
574 \\
236 \\
236\end{array}$ & $\begin{array}{l}196 \\
196 \\
135 \\
115 \\
115 \\
150 \\
210 \\
196 \\
158 \\
183 \\
170 \\
170\end{array}$ & $\begin{array}{l}209 \\
237 \\
205 \\
155 \\
149 \\
252 \\
697 \\
257 \\
219 \\
239 \\
185 \\
199\end{array}$ & $\begin{array}{r}0.685 \\
.777 \\
.672 \\
.508 \\
.489 \\
.826 \\
2.29 \\
.843 \\
.718 \\
.784 \\
.607 \\
.652\end{array}$ & $\begin{array}{r}0.79 \\
.87 \\
.77 \\
.59 \\
.51 \\
.95 \\
2.56 \\
.97 \\
.80 \\
.90 \\
.70 \\
.73\end{array}$ \\
\hline The year.... & 2,410 & 115 & 250 & .820 & 11.14 \\
\hline
\end{tabular}


SHEBOYGAN RIVER NEAR SHEBOYGAN, WIS.

Location.-In sec. 28 , T. 15 N., R. 23 E., 2 miles west of Sheboygan, Sheboygan County, and $21 / 2$ miles above mouth.

Drainage area.-403 square miles (measured on Wisconsin Geological and Natural History Survey map, edition of 1911 ; scale, 1 inch $=6$ miles).

Records available.-June 30, 1916, to September 30, 1923.

GAGE.-Chain gage fastened to upstream side of bridge; read by Wilma Opgenorth.

Discharge measurements.-From highway bridge or by wading. At extreme flood stages measurement may be made from Chicago \& North Western Railway Bridge, one-third mile downstream.

Channel and control.-Control is a well-defined riffle 200 feet below bridge. Stream bed composed of heavy gravel, free from aquatic growth. Banks are of medium height and are rarely overflowed.

EXTREMEs OF Discharge.-Maximum stage recorded during year, 7.5 feet at 8.30 a. m. April 5 (discharge, 3,800 second-feet). Minimum discharge, 5 second-feet January 5 and 6 (stage-discharge relation affected by ice).

1916-1923: Maximum stage recorded, 9.40 feet at 7 a. m. March 26, 1920 (discharge, 7,140 second-feet); minimum stage, 1.48 feet at $4.30 \mathrm{p}$. m. August 27,1922 , caused by shutdown of power plants (discharge, about 1 second-foot).

IcE.-Stage-discharge relation affected by ice.

REGULATION.-Operation of small power plants upstream causes a small amount of diurnal fluctuation at low stages.

Accuracy.-Stage-discharge relation fairly permanent. Rating curve well defined throughout the range of stage occurring during the year. Gage read to hundredths twice daily; slight diurnal fluctuation during low-water periods probably impairs the accuracy of the mean daily gage height. Daily discharge ascertained by applying mean daily gage height to rating table except as indicated in footnote to table of daily discharge. Open-water records good; winter records poor.

Discharge measurements of Sheboygan River near Sheboygan, Wis., during the year ending September 30,1923

\begin{tabular}{|c|c|c|c|c|c|c|c|}
\hline Date & Made by- & $\begin{array}{c}\text { Gage } \\
\text { height }\end{array}$ & $\begin{array}{c}\text { Dis- } \\
\text { charge }\end{array}$ & Date & Made by- & $\begin{array}{c}\text { Gage } \\
\text { height }\end{array}$ & $\begin{array}{c}\text { Dis- } \\
\text { charge }\end{array}$ \\
\hline 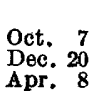 & $\begin{array}{c}\text { S. B. Soule } \\
\text { do }\end{array}$ & $\begin{array}{r}\text { Feet } \\
2.05 \\
-2.26 \\
5.63\end{array}$ & $\begin{array}{r}\text { Sec. } f t . \\
49.5 \\
23.0 \\
2,010\end{array}$ & $\begin{array}{l}\text { June } 17 \\
\text { Aug. } 18\end{array}$ & $\begin{array}{l}\text { E. E. Foster } \\
\text { S. B. Soulé }\end{array}$ & $\begin{array}{c}\text { Feet } \\
2.10 \\
1.87\end{array}$ & $\begin{array}{c}\text { Sec.-ft. } \\
59.8 \\
16\end{array}$ \\
\hline
\end{tabular}

- Stage-discharge relation affected by ice. 
Daily discharge, in second-feet, of Sheboygan River near Sheboygan, Wis., for the year ending September 30, 1928

\begin{tabular}{|c|c|c|c|c|c|c|c|c|c|c|c|c|}
\hline Day & Oct. & Nov. & Dec. & Jan. & Feb. & Mar. & Apr. & May & June & July & Aug. & Sept. \\
\hline $\begin{array}{l}1 \\
2 \ldots \\
3 \ldots \ldots \\
4 \\
5\end{array}$ & $\begin{array}{l}60 \\
46 \\
52 \\
58 \\
70\end{array}$ & $\begin{array}{r}70 \\
38 \\
109 \\
93 \\
68\end{array}$ & $\begin{array}{r}48 \\
72 \\
48 \\
70 \\
100\end{array}$ & $\begin{array}{r}55 \\
65 \\
75 \\
55 \\
5\end{array}$ & $\begin{array}{r}85 \\
55 \\
55 \\
85 \\
100\end{array}$ & & $\begin{array}{r}610 \\
2,400 \\
3,800\end{array}$ & $\begin{array}{l}165 \\
148 \\
145 \\
126 \\
126\end{array}$ & $\begin{array}{l}66 \\
58 \\
70 \\
44 \\
46\end{array}$ & $\begin{array}{l}35 \\
41 \\
35 \\
62 \\
46\end{array}$ & $\begin{array}{l}37 \\
32 \\
51 \\
41 \\
33\end{array}$ & $\begin{array}{l}40 \\
58 \\
43 \\
38 \\
41\end{array}$ \\
\hline $\begin{array}{r}6 \\
70 \\
9 \\
9\end{array}$ & $\begin{array}{l}58 \\
48 \\
70 \\
66 \\
46\end{array}$ & $\begin{array}{l}81 \\
74 \\
72 \\
66 \\
66\end{array}$ & $\begin{array}{l}85 \\
75 \\
40 \\
20 \\
40\end{array}$ & $\begin{array}{r}5 \\
20 \\
15 \\
20 \\
30\end{array}$ & $\begin{array}{l}45 \\
20 \\
30 \\
40 \\
25\end{array}$ & & $\begin{array}{l}3,700 \\
3,300 \\
2,760 \\
2,760 \\
2,850\end{array}$ & $\begin{array}{r}118 \\
93 \\
96 \\
115 \\
120\end{array}$ & $\begin{array}{l}52 \\
62 \\
66 \\
84 \\
41\end{array}$ & $\begin{array}{l}38 \\
46 \\
51 \\
52 \\
51\end{array}$ & $\begin{array}{l}33 \\
29 \\
37 \\
32 \\
51\end{array}$ & $\begin{array}{l}38 \\
\mathbf{4 3} \\
51 \\
32 \\
35\end{array}$ \\
\hline $\begin{array}{l}11 \\
12 \\
14 \\
15\end{array}$ & $\begin{array}{l}74 \\
74 \\
84 \\
60 \\
68\end{array}$ & $\begin{array}{r}74 \\
72 \\
72 \\
84 \\
104 \\
104\end{array}$ & $\begin{array}{l}30 \\
30 \\
20 \\
15 \\
25\end{array}$ & $\begin{array}{l}30 \\
25 \\
10 \\
20 \\
15\end{array}$ & $\begin{array}{l}40 \\
25 \\
40 \\
40 \\
40\end{array}$ & & $\begin{array}{l}3,030 \\
2,760 \\
2,760 \\
1,950 \\
1,150\end{array}$ & $\begin{array}{l}74 \\
79 \\
62 \\
66 \\
88\end{array}$ & $\begin{array}{l}46 \\
43 \\
46 \\
40 \\
46\end{array}$ & $\begin{array}{l}44 \\
51 \\
44 \\
33 \\
41\end{array}$ & $\begin{array}{l}41 \\
40 \\
30 \\
29 \\
32\end{array}$ & $\begin{array}{l}33 \\
37 \\
32 \\
34 \\
24\end{array}$ \\
\hline $\begin{array}{l}16 \\
17 \\
18 \\
19\end{array}$ & $\begin{array}{l}66 \\
76 \\
62 \\
66 \\
62\end{array}$ & $\begin{array}{l}68 \\
56 \\
68 \\
66 \\
76\end{array}$ & $\begin{array}{l}25 \\
20 \\
25 \\
40 \\
25\end{array}$ & $\begin{array}{l}10 \\
25 \\
15 \\
25 \\
20\end{array}$ & $\begin{array}{l}25 \\
30 \\
25 \\
30 \\
40\end{array}$ & 610 & $\begin{array}{r}1,150 \\
905 \\
795 \\
690 \\
690\end{array}$ & $\begin{array}{r}120 \\
88 \\
79 \\
112 \\
204\end{array}$ & $\begin{array}{l}43 \\
49 \\
64 \\
46 \\
68\end{array}$ & $\begin{array}{l}37 \\
26 \\
43 \\
43 \\
43\end{array}$ & $\begin{array}{l}26 \\
40 \\
23 \\
30 \\
24\end{array}$ & $\begin{array}{l}44 \\
21 \\
62 \\
44 \\
52\end{array}$ \\
\hline $\begin{array}{l}21 \\
22 \\
25 \\
25\end{array}$ & $\begin{array}{l}62 \\
49 \\
64 \\
48 \\
54\end{array}$ & $\begin{array}{r}101 \\
74 \\
79 \\
35 \\
46\end{array}$ & $\begin{array}{l}25 \\
40 \\
55 \\
55 \\
15\end{array}$ & $\begin{array}{l}\mathbf{2 5} \\
\mathbf{5 5} \\
\mathbf{5 5} \\
40 \\
30\end{array}$ & $\begin{array}{l}40 \\
30 \\
55 \\
45 \\
40\end{array}$ & & $\begin{array}{l}590 \\
640 \\
540 \\
518 \\
428\end{array}$ & $\begin{array}{l}201 \\
186 \\
168 \\
152 \\
148\end{array}$ & $\begin{array}{l}60 \\
43 \\
43 \\
28 \\
44\end{array}$ & $\begin{array}{l}33 \\
26 \\
28 \\
37 \\
34\end{array}$ & $\begin{array}{r}58 \\
18 \\
35 \\
26 \\
123\end{array}$ & $\begin{array}{l}68 \\
68 \\
41 \\
48 \\
51\end{array}$ \\
\hline $\begin{array}{l}26 \\
27 \\
28 \\
29 \\
31 \\
31\end{array}$ & $\begin{array}{l}44 \\
56 \\
58 \\
44 \\
72 \\
64\end{array}$ & $\begin{array}{l}48 \\
44 \\
58 \\
62 \\
66\end{array}$ & $\begin{array}{l}20 \\
10 \\
40 \\
45 \\
40 \\
40\end{array}$ & $\begin{array}{r}40 \\
40 \\
40 \\
40 \\
40 \\
100\end{array}$ & $\begin{array}{r}75 \\
125 \\
45 \\
\\
\hdashline\end{array}$ & & $\begin{array}{l}640 \\
193 \\
158 \\
216 \\
201\end{array}$ & $\begin{array}{r}115 \\
101 \\
86 \\
84 \\
79 \\
60\end{array}$ & $\begin{array}{l}41 \\
88 \\
56 \\
40 \\
46\end{array}$ & $\begin{array}{l}37 \\
33 \\
26 \\
33 \\
29 \\
29\end{array}$ & $\begin{array}{l}\mathbf{5 4} \\
\mathbf{3 4} \\
\mathbf{3 7} \\
68 \\
\mathbf{4 0} \\
\mathbf{3 7}\end{array}$ & $\begin{array}{l}44 \\
44 \\
37 \\
56 \\
54\end{array}$ \\
\hline
\end{tabular}

Note.-Stage-discharge relation affected by ice Dec. 5 to Apr. 3. Gage not read Mar. 1 to Apr. 3. Daily discharge, Dec. 5 to Feb. 28, determined from gage heights corrected for effect of ice by means of one discharge measurement, observer's notes, and weather records. Mean discharge, Mar. 1 to Apr. 3, estimated by comparison with discharge of Milwaukee River. Braced figures show mean discharge for periods indicated.

Monthly discharge of Sheboygan River near Sheboygan, Wis., for the year ending September 30, 1928

[Drainage area, 403 square miles]

\begin{tabular}{|c|c|c|c|c|c|}
\hline \multirow{2}{*}{ Month } & \multicolumn{4}{|c|}{ Discharge in second-feet } & \multirow{2}{*}{$\begin{array}{c}\text { Run-off in } \\
\text { inches }\end{array}$} \\
\hline & Maximum & Minimum & Mean & $\underset{\text { mitile }}{\text { Per square }}$ & \\
\hline $\begin{array}{l}\text { October } \\
\text { November } \\
\text { December } \\
\text { January } \\
\text { February } \\
\text { March } \\
\text { April } \\
\text { May } \\
\text { June } \\
\text { July } \\
\text { August } \\
\text { September }\end{array}$ & $\begin{array}{r}84 \\
109 \\
100 \\
100 \\
125 \\
3,800 \\
204 \\
88 \\
62 \\
123 \\
68\end{array}$ & \begin{tabular}{r}
44 \\
35 \\
10 \\
5 \\
20 \\
\hdashline 158 \\
60 \\
28 \\
26 \\
18 \\
21
\end{tabular} & $\begin{array}{c}60.7 \\
69.7 \\
39.9 \\
33.7 \\
47.5 \\
610 \\
1,450 \\
116 \\
52.3 \\
38.9 \\
39.4 \\
43.8\end{array}$ & $\begin{array}{l}0.151 \\
.173 \\
.099 \\
.084 \\
.118 \\
1.51 \\
3.60 \\
.288 \\
.130 \\
.097 \\
.098 \\
.109\end{array}$ & $\begin{array}{r}0.17 \\
.19 \\
.11 \\
.10 \\
.12 \\
1.74 \\
4.02 \\
.33 \\
.14 \\
.11 \\
.11 \\
.12\end{array}$ \\
\hline The year... & 3,800 & 5 & 216 & .536 & 7.26 \\
\hline
\end{tabular}




\section{MILWAUKEE RIVER NEAR MUTWUKEE, WIS.}

Location.-In NW. $1 / 4$ sec. 5, T. 7 N., R. 22 E., immediately above an old quarry near north limits of Milwaukee, Milwaukee County, half a mile below concrete highway bridge, 1 mile above Mineral Spring road, and 51/2 miles above confluence of Milwaukee and Menominee Rivers.

Drainage area.-661 square miles (measured on Wisconsin Geological and Natural History Survey map, edition of 1911 ; scale, 1 inch $=6$ miles).

Records available.-April 30, 1914, to September 30, 1923.

GAGE.-Slope gage set in concrete foundations on left bank of river; prior to April 18, 1918, chain gage fastened to cantilever arms supported by posts. Both gages at same datum. Gage read by Mrs. Richard Kuehl.

Channel and control.-Bed of channel at gage heavy gravel. About 200 feet below gage is a rock outcrop, affording a 4-foot fall which forms the control and is permanent. Below the control the river flows in an artificial channel, which at one time was a quarry. Left bank above and below the control high and not subject to overflow; right bank above control of medium height; below the control the right bank is artificial and of such height as to be seldom overflowed.

Discharge measorements.-Made by wading immediateiy above gage; athigh stages from a railroad bridge one-fourth mile below gage.

Extremes of Discharge.-Maximum stage recorded during year, 5.7 feet at 3 p. $m$. April 7 (discharge, 6,820 second-feet); minimum stage recorded, 0.39 foot several times August 18-20 (discharge, 38 second-feet).

1914-1923: Maximum stage recorded, 9 feet March 20, 1918 (discharge, 15,100 second-feet, revised); minimum discharge recorded, about 26 secondfeet August 2, 1916.

ICE.-Stage-discharge relation affected by ice.

Regulation.-No diurnal fluctuation at gage resulting from operation of small plants above.

Accuracr.-Dtage-discharge relation permanent. Rating curve well defined throughout range of stage which occurred during the year. Gage read to quarter-tenths twice daily. Daily discharge ascertained by applying mean daily gage height to rating table except as shown in footnote to table of daily discharge. Open-water records excellent; winter records fair.

Discharge measurements of Milwaukee River near Milwaukee, Wis., during the year ending September 30, 1923

\begin{tabular}{|c|c|c|c|c|c|c|c|}
\hline Date & Made by- & $\begin{array}{c}\text { Gage } \\
\text { height }\end{array}$ & $\begin{array}{c}\text { Dis- } \\
\text { charge }\end{array}$ & Date & Made by- & $\begin{array}{l}\text { Gage } \\
\text { height }\end{array}$ & $\begin{array}{l}\text { Dis- } \\
\text { charge }\end{array}$ \\
\hline $\begin{array}{l}\text { Oct. } 8 \\
\text { Dec. } \\
\text { A pr. } 8\end{array}$ & $\begin{array}{l}\text { S. B. Soulé...... } \\
\text { do } \\
\text { do }\end{array}$ & $\begin{array}{l}\text { Feet } \\
0.66 \\
.87 \\
4.86\end{array}$ & $\begin{array}{r}\text { Sec. } \cdot f t . \\
88 \\
62 \\
5,240\end{array}$ & $\begin{array}{l}\text { June } 15 \\
\text { Aug. } 17\end{array}$ & $\begin{array}{l}\text { E. E. Foster } \\
\text { S. B. Soule. }\end{array}$ & $\begin{array}{l}\text { Freet } \\
0.80 \\
.45\end{array}$ & $\begin{array}{l}\text { Sec.-ft. } \\
121 \\
45.4\end{array}$ \\
\hline
\end{tabular}

\footnotetext{
- Stage-discharge relation affected by ice.
} 
Daily discharge, in second-feet, of Milwaukee River near Milwaukee, Wis., for the year ending September 30, 1923

\begin{tabular}{|c|c|c|c|c|c|c|c|c|c|c|c|c|}
\hline Day & Oct. & Nov. & Dec. & Jan. & Feb. & Mar. & Apr. & May & June & July & Aug. & Sept. \\
\hline $\begin{array}{l}1 \ldots \ldots \\
3 \ldots \\
4 \\
5\end{array}$ & $\begin{array}{r}99 \\
89 \\
73 \\
148 \\
114\end{array}$ & $\begin{array}{l}188 \\
130 \\
105 \\
167 \\
175\end{array}$ & $\begin{array}{r}175 \\
208 \\
243 \\
208 \\
87\end{array}$ & $\begin{array}{r}150 \\
130 \\
85 \\
130 \\
115\end{array}$ & $\begin{array}{l}250 \\
115 \\
115 \\
115 \\
100\end{array}$ & $\begin{array}{l}1,100 \\
1,540 \\
1,720 \\
2,410 \\
2,680\end{array}$ & $\begin{array}{r}552 \\
585 \\
2,830 \\
5,200 \\
4,990\end{array}$ & $\begin{array}{l}280 \\
289 \\
280 \\
243 \\
230\end{array}$ & $\begin{array}{r}121 \\
92 \\
124 \\
152 \\
171\end{array}$ & $\begin{array}{r}118 \\
92 \\
97 \\
92 \\
82\end{array}$ & $\begin{array}{l}75 \\
71 \\
97 \\
89 \\
71\end{array}$ & $\begin{array}{l}75 \\
73 \\
71 \\
71 \\
80\end{array}$ \\
\hline $\begin{array}{r}6 \ldots \\
7 \ldots \\
8 \ldots \\
9 \ldots \\
10 . .\end{array}$ & $\begin{array}{r}67 \\
175 \\
87 \\
80 \\
148\end{array}$ & $\begin{array}{l}167 \\
167 \\
217 \\
208 \\
188\end{array}$ & $\begin{array}{r}167 \\
188 \\
130 \\
190 \\
85\end{array}$ & $\begin{array}{l}100 \\
130 \\
130 \\
115 \\
115\end{array}$ & $\begin{array}{r}100 \\
75 \\
75 \\
165 \\
130\end{array}$ & $\begin{array}{l}2,410 \\
2,150 \\
1,540 \\
1,320 \\
1,430\end{array}$ & $\begin{array}{l}5,200 \\
6,820 \\
4,990 \\
3,640 \\
2,680\end{array}$ & $\begin{array}{l}226 \\
226 \\
221 \\
212 \\
208\end{array}$ & $\begin{array}{l}270 \\
338 \\
432 \\
409 \\
392\end{array}$ & $\begin{array}{l}92 \\
92 \\
80\end{array}$ & $\begin{array}{l}69 \\
61 \\
52\end{array}$ & $\begin{array}{r}89 \\
102 \\
118 \\
118 \\
121\end{array}$ \\
\hline . & $\begin{array}{l}145 \\
167 \\
137 \\
114 \\
145\end{array}$ & $\begin{array}{l}188 \\
188 \\
208 \\
230 \\
323\end{array}$ & $\begin{array}{l}55 \\
75 \\
65 \\
85 \\
75\end{array}$ & $\begin{array}{l}115 \\
115 \\
115 \\
130 \\
250\end{array}$ & $\begin{array}{r}130 \\
115 \\
85 \\
100 \\
10 p\end{array}$ & $\begin{array}{r}1,320 \\
900 \\
490 \\
520 \\
318\end{array}$ & $\begin{array}{l}2,410 \\
2,280 \\
2,020 \\
1,600 \\
1,430\end{array}$ & $\begin{array}{l}208 \\
188 \\
167 \\
160 \\
118\end{array}$ & $\begin{array}{l}234 \\
188 \\
152 \\
145 \\
\text { I34 }\end{array}$ & $\begin{array}{r}105 \\
94 \\
82 \\
71 \\
71\end{array}$ & $\begin{array}{l}61 \\
73 \\
73\end{array}$ & $\begin{array}{l}97 \\
75 \\
75 \\
92 \\
94\end{array}$ \\
\hline 18 & $\begin{array}{l}137 \\
137 \\
148 \\
137 \\
130\end{array}$ & $\begin{array}{l}275 \\
252 \\
252 \\
323 \\
298\end{array}$ & $\begin{array}{l}75 \\
75 \\
40 \\
75 \\
65\end{array}$ & $\begin{array}{l}250 \\
115 \\
150 \\
165 \\
190\end{array}$ & $\begin{array}{l}130 \\
130 \\
130 \\
150 \\
130\end{array}$ & $\begin{array}{l}270 \\
248 \\
270 \\
270 \\
318\end{array}$ & $\begin{array}{r}2,280 \\
2,410 \\
1,600 \\
1,320 \\
900\end{array}$ & $\begin{array}{l}364 \\
280 \\
338 \\
432 \\
730\end{array}$ & $\begin{array}{r}92 \\
77 \\
71 \\
1,160 \\
392\end{array}$ & 71 & $\begin{array}{l}38 \\
41 \\
38\end{array}$ & $\begin{array}{r}67 \\
52 \\
82 \\
63 \\
239\end{array}$ \\
\hline $24 \ldots \ldots$ & $\begin{array}{r}105 \\
99 \\
118 \\
105 \\
118\end{array}$ & $\begin{array}{l}230 \\
230 \\
208 \\
275 \\
252\end{array}$ & $\begin{array}{l}55 \\
55 \\
55 \\
55 \\
85\end{array}$ & $\begin{array}{l}130 \\
150 \\
130 \\
130 \\
150\end{array}$ & $\begin{array}{l}130 \\
100 \\
115 \\
150 \\
230\end{array}$ & $\begin{array}{l}270 \\
248 \\
270 \\
855 \\
900\end{array}$ & $\begin{array}{l}810 \\
810 \\
770 \\
730 \\
655\end{array}$ & $\begin{array}{l}810 \\
692 \\
420 \\
392 \\
270\end{array}$ & $\begin{array}{r}308 \\
270 \\
226 \\
105 \\
87\end{array}$ & $\begin{array}{l}45 \\
97 \\
80\end{array}$ & $\begin{array}{l}55 \\
52 \\
45\end{array}$ & $\begin{array}{l}318 \\
208 \\
188 \\
188 \\
145\end{array}$ \\
\hline $\begin{array}{l}26 \\
27 \\
29 \\
30\end{array}$ & $\begin{array}{r}114 \\
99 \\
99 \\
105 \\
114 \\
175\end{array}$ & \begin{tabular}{c}
175 \\
208 \\
167 \\
252 \\
208 \\
\hdashline-
\end{tabular} & $\begin{array}{r}100 \\
75 \\
115 \\
100 \\
100 \\
115\end{array}$ & $\begin{array}{l}150 \\
130 \\
150 \\
150 \\
130 \\
130\end{array}$ & \begin{tabular}{r}
550 \\
855 \\
1,100 \\
\hdashline$\ldots .$. \\
\end{tabular} & $\begin{array}{l}900 \\
730 \\
655 \\
364 \\
810 \\
655\end{array}$ & $\begin{array}{l}520 \\
520 \\
460 \\
420 \\
376\end{array}$ & $\begin{array}{l}226 \\
208 \\
234 \\
196 \\
160 \\
145\end{array}$ & \begin{tabular}{r|r|r}
71 \\
134 \\
167 \\
134 \\
124
\end{tabular} & $\begin{array}{r}75 \\
105 \\
105 \\
97 \\
75 \\
75\end{array}$ & $\begin{array}{l}52 \\
71 \\
87 \\
80 \\
75 \\
71\end{array}$ & $\begin{array}{r}118 \\
111 \\
118 \\
108 \\
92\end{array}$ \\
\hline
\end{tabular}

NoTe-Stage-discharge relation affected by ice Dec. 8 to Feb. 26. Daily discharge determined from gage heights correated for effect of ice by means of one discharge measurement, observer's notes, and weather records.

Monthly discharge of Milwaukee River near Milwaukee, Wis., for the year ending September 30, 1923

[Drainage area, 661 square miles]

\begin{tabular}{|c|c|c|c|c|c|}
\hline \multirow{2}{*}{ Month } & \multicolumn{4}{|c|}{ Discharge in second-feet } & \multirow{2}{*}{$\underset{\text { in inches }}{\text { Run-off }}$} \\
\hline & Maximum & Minimum & ea & $\begin{array}{l}\text { Per square } \\
\text { mile }\end{array}$ & \\
\hline $\begin{array}{l}\text { October } \\
\text { November } \\
\text { December } \\
\text { January } \\
\text { February } \\
\text { March } \\
\text { April } \\
\text { May } \\
\text { June } \\
\text { July } \\
\text { August }\end{array}$ & $\begin{array}{r}175 \\
323 \\
243 \\
250 \\
1,100 \\
2,680 \\
6,820 \\
810 \\
1,160 \\
118 \\
97 \\
318\end{array}$ & $\begin{array}{r}67 \\
105 \\
40 \\
85 \\
75 \\
248 \\
376 \\
118 \\
71 \\
45 \\
38 \\
52\end{array}$ & $\begin{array}{c}120 \\
215 \\
106 \\
140 \\
202 \\
904 \\
2,060 \\
295 \\
226 \\
81.8 \\
64.5 \\
115\end{array}$ & $\begin{array}{l}0.182 \\
.325 \\
.160 \\
.212 \\
.306 \\
1.46 \\
3.12 \\
.446 \\
.342 \\
.124 \\
.098 \\
.174\end{array}$ & $\begin{array}{r}0.21 \\
.36 \\
.18 \\
.24 \\
.32 \\
1.68 \\
3.48 \\
.51 \\
.38 \\
.14 \\
.11 \\
.19\end{array}$ \\
\hline The year. & 6,820 & 38 & 381 & .576 & 7.80 \\
\hline
\end{tabular}




\section{LITTLE CALUMET RIVER AT HARVET, IIL.}

Location.-In NW. $1 / 4$ sec. 9, T. 36 N., R. 14 E., at Illinois Central Railroad bridge, 800 feet north of railroad station at One hundred and forty-seventh Street, Harvey, Cook County, and 11 miles above mouth of river.

Drainage AREA. - 570 square miles (measured on map issued by United States Geological Survey; scale, 1:500,000).

Riecords available.-Daily discharge, October 1, 1916, to September 30, 1923. Daily gage heights collected by Sanitary District of Chicago, June 10, 1907, to September 30, 1916.

GAGE.-Vertical staff gage attached to bridge pier; read by Mrs. H. Wurtman. Discharge measurements.-Made from highway bridge 2,000 feet below gage or by wading.

Channel and control.-Bed of river composed of clay and gravel. Lowwater control gravel and boulders; shifts occasionally. Banks not subject to overflow.

Extremes of Discharge.-Maximum stage recorded during year, 7.35 feet at 8 a. m. September 3 (discharge, 1,840 second-feet); minimum stage, 2.96 feet July 24-26 and August 2 and 3 (discharge, 51 second-feet).

1907-1923: Maximum stage recorded, 13.4 feet March 6, 1908 (discharge not determined). Minimum discharge from 1917 to 1922 , estimated at less than 25 second-feet in January, 1918.

ACC URACY. - Stage-discharge relation practically permanent during year except as affected by ice. Rating curve well defined. Gage read to hundredths once daily. Daily discharge ascertained by applying daily gage height to rating table except as shown in footnote to table of daily discharge. Openwater records good; winter records fair.

Discharge measurements of Little Calumet River at Harvey, Ill., during the year ending September 30, 1923

[Made by H. E. Grosbach]

\begin{tabular}{|c|c|c|}
\hline Date & $\begin{array}{c}\text { Gage } \\
\text { height }\end{array}$ & $\begin{array}{c}\text { Dis- } \\
\text { charge }\end{array}$ \\
\hline 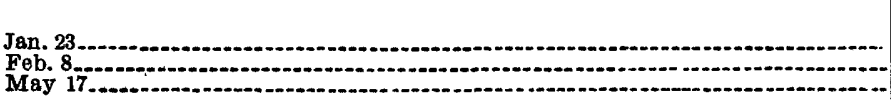 & $\begin{array}{l}\text { Feet } \\
: 3.29 \\
-3.40 \\
5.58\end{array}$ & $\begin{array}{l}\text { Sec. }-f t . \\
\quad 102 \\
87.1 \\
011\end{array}$ \\
\hline
\end{tabular}

a Stage-discharge relation affected by ice. $72038-26 \dagger-$ wsP $564-4$ 
Daily discharge, in second-feet, of Little Calumet River at Harvey, Ill., for the year ending September, 30, 1923

\begin{tabular}{|c|c|c|c|c|c|c|c|c|c|c|c|c|}
\hline Day & Oct. & Nov. & Dec. & Jan. & Feb. & Mar. & Apr. & May & June & July & Aug. & Sept. \\
\hline $\begin{array}{l}1 \\
2 \\
3 \\
4 \\
5\end{array}$ & $\begin{array}{l}56 \\
56 \\
56 \\
56 \\
56\end{array}$ & $\begin{array}{r}88 \\
100 \\
92 \\
96 \\
100\end{array}$ & $\begin{array}{l}104 \\
125 \\
116 \\
112 \\
146\end{array}$ & & & $\begin{array}{l}341 \\
468 \\
431 \\
358 \\
324\end{array}$ & $\begin{array}{l}590 \\
590 \\
590 \\
590 \\
548\end{array}$ & $\begin{array}{r}123 \\
112 \\
102 \\
96 \\
96\end{array}$ & $\begin{array}{r}590 \\
548 \\
508 \\
508 \\
468\end{array}$ & $\begin{array}{r}84 \\
84 \\
77 \\
81 \\
146\end{array}$ & $\begin{array}{l}53 \\
51 \\
51 \\
59 \\
70\end{array}$ & $\begin{array}{r}207 \\
220 \\
1,840 \\
1,040 \\
680\end{array}$ \\
\hline $\begin{array}{r}6 \\
7 \\
8 \\
9 \\
10 \ldots \ldots \ldots\end{array}$ & $\begin{array}{l}56 \\
63 \\
70 \\
73 \\
81\end{array}$ & $\begin{array}{l}108 \\
100 \\
100 \\
104 \\
108\end{array}$ & $\begin{array}{l}139 \\
146 \\
157 \\
169 \\
146\end{array}$ & & 100 & $\begin{array}{l}341 \\
376 \\
394 \\
376 \\
431\end{array}$ & $\begin{array}{l}508 \\
508 \\
508 \\
468 \\
468\end{array}$ & $\begin{array}{l}92 \\
84 \\
84 \\
84 \\
92\end{array}$ & $\begin{array}{l}431 \\
815 \\
680 \\
590 \\
508\end{array}$ & $\begin{array}{l}112 \\
134 \\
123 \\
116 \\
112\end{array}$ & $\begin{array}{r}77 \\
88 \\
134 \\
181 \\
146\end{array}$ & $\begin{array}{l}548 \\
635 \\
590 \\
548 \\
508\end{array}$ \\
\hline $\begin{array}{l}11 \\
12 \\
13 \\
14 \\
15\end{array}$ & $\begin{array}{l}146 \\
134 \\
123 \\
110 \\
123\end{array}$ & $\begin{array}{l}104 \\
100 \\
123 \\
152 \\
157\end{array}$ & 139 & & & $\begin{array}{r}431 \\
1,130 \\
1,420 \\
815 \\
770\end{array}$ & $\begin{array}{l}431 \\
431 \\
412 \\
394 \\
376\end{array}$ & $\begin{array}{r}92 \\
141 \\
292 \\
262 \\
262\end{array}$ & $\begin{array}{l}468 \\
431 \\
412 \\
376 \\
358\end{array}$ & $\begin{array}{r}108 \\
104 \\
92 \\
88 \\
82\end{array}$ & $\begin{array}{r}815 \\
1,130 \\
680 \\
508 \\
431\end{array}$ & $\begin{array}{l}508 \\
590 \\
548 \\
508 \\
508\end{array}$ \\
\hline $\begin{array}{l}16 \ldots \\
17 \ldots \ldots \\
18 \ldots \ldots \\
19 \ldots \ldots \\
20 \ldots \ldots\end{array}$ & $\begin{array}{l}123 \\
112 \\
104 \\
104 \\
100\end{array}$ & $\begin{array}{l}146 \\
139 \\
139 \\
139 \\
139\end{array}$ & & 110 & & $\begin{array}{r}1,570 \\
1,220 \\
1,040 \\
680 \\
1,040\end{array}$ & $\begin{array}{l}341 \\
324 \\
308 \\
262 \\
234\end{array}$ & $\begin{array}{r}770 \\
1,040 \\
770 \\
680 \\
770\end{array}$ & $\begin{array}{l}324 \\
292 \\
277 \\
248 \\
220\end{array}$ & $\begin{array}{l}73 \\
73 \\
70 \\
66 \\
63\end{array}$ & $\begin{array}{l}341 \\
308 \\
277 \\
248 \\
234\end{array}$ & $\begin{array}{l}468 \\
431 \\
431 \\
431 \\
431\end{array}$ \\
\hline $\begin{array}{l}21 \\
22 \\
23 \\
24 \\
25\end{array}$ & $\begin{array}{r}100 \\
92 \\
100 \\
100 \\
92\end{array}$ & $\begin{array}{l}125 \\
125 \\
123 \\
123 \\
123\end{array}$ & 115 & & & $\begin{array}{l}860 \\
860 \\
905 \\
860 \\
815\end{array}$ & $\begin{array}{l}234 \\
220 \\
207 \\
194 \\
194\end{array}$ & $\begin{array}{l}815 \\
860 \\
860 \\
815 \\
770\end{array}$ & $\begin{array}{r}181 \\
157 \\
123 \\
108 \\
88\end{array}$ & $\begin{array}{l}59 \\
53 \\
52 \\
51 \\
51\end{array}$ & $\begin{array}{l}234 \\
181 \\
157 \\
139 \\
123\end{array}$ & $\begin{array}{l}431 \\
412 \\
394 \\
376 \\
341\end{array}$ \\
\hline $\begin{array}{l}26 \\
27 \\
28 \\
29 \\
30 \\
31\end{array}$ & $\begin{array}{r}92 \\
100 \\
96 \\
92 \\
92 \\
88\end{array}$ & $\begin{array}{l}100 \\
108 \\
112 \\
100 \\
112 \\
-\end{array}$ & & & $\mid \begin{array}{ll}-\ldots-n \\
-\ldots-n\end{array}$ & $\begin{array}{l}770 \\
770 \\
725 \\
680 \\
680 \\
635\end{array}$ & $\begin{array}{r}181 \\
157 \\
146 \\
134 \\
123 \\
- \\
-\end{array}$ & $\begin{array}{l}770 \\
725 \\
680 \\
680 \\
635 \\
590\end{array}$ & $\begin{array}{r}81 \\
73 \\
88 \\
92 \\
88 \\
-0\end{array}$ & $\begin{array}{l}51 \\
56 \\
59 \\
56 \\
53 \\
53\end{array}$ & $\begin{array}{l}108 \\
130 \\
207 \\
194 \\
181 \\
194\end{array}$ & $\begin{array}{l}324 \\
303 \\
292 \\
277 \\
262\end{array}$ \\
\hline
\end{tabular}

NotE.-Discharge estimated Dec. 12 to Feb. 28, on account of ice, from gage-height record, discharge measurements, observer's notes, and weather records. Braced figures show mean discharge for periods indicated.

Monthly discharge of Little Calumet River at Harvey, Ill., for the year ending September 30, 1923

[Drainage area, 570 square miles]

\begin{tabular}{|c|c|c|c|c|c|}
\hline \multirow{2}{*}{ Month } & \multicolumn{4}{|c|}{ Discharge in second-feet } & \multirow{2}{*}{$\underset{\text { Run-inches }}{\text { Run }}$} \\
\hline & Maximum & Minimum & Mean & $\begin{array}{c}\text { Per square } \\
\text { mile }\end{array}$ & \\
\hline $\begin{array}{l}\text { October } \\
\text { November } \\
\text { December } \\
\text { January } \\
\text { February } \\
\text { March } \\
\text { April } \\
\text { May } \\
\text { June } \\
\text { July } \\
\text { August } \\
\text { September }\end{array}$ & \begin{tabular}{r}
146 \\
157 \\
\hdashline 1,570 \\
1,040 \\
590 \\
815 \\
146 \\
1,130 \\
1,840 \\
\end{tabular} & $\begin{array}{r}56 \\
88 \\
\\
\\
324 \\
123 \\
84 \\
73 \\
51 \\
51 \\
207\end{array}$ & $\begin{array}{l}91.8 \\
116 \\
123 \\
110 \\
170 \\
726 \\
356 \\
459 \\
338 \\
80.1 \\
249 \\
503\end{array}$ & $\begin{array}{r}0.161 \\
.204 \\
.216 \\
.193 \\
.298 \\
1.27 \\
.625 \\
.805 \\
.593 \\
.141 \\
.437 \\
.882\end{array}$ & $\begin{array}{l}0.19 \\
.23 \\
.25 \\
.22 \\
.31 \\
1.46 \\
.70 \\
.93 \\
.66 \\
.16 \\
.50 \\
.98\end{array}$ \\
\hline The year ........ & 1,840 & 51 & 277 & .486 & 6.59 \\
\hline
\end{tabular}




\section{STREAMS TRIBUTARY TO LAKE HUंRON}

\section{TITTABAWASSEE RIVER AT FREELAND, MICH.}

LOCATION.-At highway bridge at Freeland.

Drainage AREA. $-2,530$ square miles.

Records available.-August 22, 1903, to December 31, 1909; January 1, 1912 , to September 30, 1923.

Cooperation-Daily-discharge record furnished by G. S. Williams, consulting engineer, Ann Arbor, Mich.

Daily discharge, in second-feet, of Tittabawassee River at Freeland, Mich., for the year ending September 30, 1923

\begin{tabular}{|c|c|c|c|c|c|c|c|c|c|c|c|c|}
\hline & Oct. & Nov. & Dec. & Jan. & Feb. & Mar. & Apr. & May & June & July & Aug. & Sept. \\
\hline & $\begin{array}{l}646 \\
786 \\
930 \\
700 \\
675\end{array}$ & $\begin{array}{r}990 \\
930 \\
1,020 \\
1,050 \\
1,270\end{array}$ & $\begin{array}{l}1,110 \\
1,200 \\
1,240 \\
1,240 \\
1,270\end{array}$ & $\begin{array}{l}787 \\
838 \\
804 \\
787 \\
750\end{array}$ & $\begin{array}{l}750 \\
858 \\
804 \\
750 \\
700\end{array}$ & $\begin{array}{r}1,600 \\
1,740 \\
3,480 \\
6,360 \\
10,900\end{array}$ & & & & $\begin{array}{l}870 \\
870 \\
845 \\
785 \\
930\end{array}$ & $\begin{array}{l}489 \\
513 \\
489 \\
513 \\
489\end{array}$ & $\begin{array}{r}646 \\
620 \\
700 \\
900 \\
1,170\end{array}$ \\
\hline $\begin{array}{l}6 \\
7 \\
8 \\
9 \\
9\end{array}$ & $\begin{array}{r}675 \\
700 \\
930 \\
1,240 \\
1,600\end{array}$ & $\begin{array}{l}1,410 \\
1,300 \\
1,270 \\
1,240 \\
1,200\end{array}$ & $\begin{array}{l}1,600 \\
1,760 \\
1,760 \\
1,660 \\
1,520\end{array}$ & $\begin{array}{l}750 \\
967 \\
928 \\
858 \\
838\end{array}$ & $\begin{array}{l}718 \\
750 \\
770 \\
750 \\
770\end{array}$ & $\begin{array}{r}11,400 \\
9,760 \\
8,700 \\
8,100 \\
6,360\end{array}$ & $\begin{array}{r}9,140 \\
9,320 \\
13,200 \\
14,400 \\
13,700\end{array}$ & & $\begin{array}{l}1,820 \\
1,860 \\
2,320 \\
2,270 \\
2,230\end{array}$ & $\begin{array}{r}1,240 \\
1,240 \\
1,080 \\
990 \\
870\end{array}$ & $\begin{array}{l}489 \\
489 \\
465 \\
416 \\
416\end{array}$ & $\begin{array}{r}930 \\
990 \\
1,080 \\
930 \\
900\end{array}$ \\
\hline & $\begin{array}{l}1,600 \\
1,560 \\
1,520 \\
1,600 \\
1,600\end{array}$ & $\begin{array}{l}1,200 \\
1,240 \\
1,200 \\
1,170 \\
1,110\end{array}$ & $\begin{array}{l}1,300 \\
1,240 \\
1,130 \\
1,110 \\
1,110\end{array}$ & $\begin{array}{l}804 \\
787 \\
750 \\
750 \\
770\end{array}$ & $\begin{array}{l}770 \\
750 \\
750 \\
750 \\
718\end{array}$ & $\begin{array}{l}5,480 \\
5,800 \\
6,360 \\
6,590 \\
6,360\end{array}$ & $\begin{array}{r}12,500 \\
11,400 \\
9,660 \\
8,700 \\
7,500\end{array}$ & & & $\begin{array}{l}760 \\
700 \\
646 \\
592 \\
646\end{array}$ & $\begin{array}{l}416 \\
234 \\
234 \\
310 \\
128\end{array}$ & $\begin{array}{l}870 \\
815 \\
760 \\
730 \\
700\end{array}$ \\
\hline 9 & $\begin{array}{r}1,520 \\
1,450 \\
1,340 \\
1,110 \\
960\end{array}$ & $\begin{array}{l}1,080 \\
1,050 \\
1,080 \\
1,110 \\
1,140\end{array}$ & $\begin{array}{l}1,110 \\
1,110 \\
1,080 \\
1,020 \\
1,000\end{array}$ & $\begin{array}{l}77 \\
78 \\
80 \\
80 \\
78\end{array}$ & $\begin{array}{l}66 \\
64 \\
64 \\
71 \\
73\end{array}$ & & $\begin{array}{l}6, \\
6, \\
6, \\
6, \\
6,\end{array}$ & $\begin{array}{r}4,250 \\
8,100 \\
16,800 \\
12,500 \\
9,660\end{array}$ & & $\begin{array}{l}730 \\
700 \\
646\end{array}$ & $\begin{array}{l}128 \\
154\end{array}$ & $\begin{array}{l}700 \\
646 \\
620 \\
646 \\
675\end{array}$ \\
\hline 34 & $\begin{array}{l}930 \\
900 \\
870 \\
900 \\
870\end{array}$ & $\begin{array}{l}1,140 \\
1,140 \\
1,170 \\
1,140 \\
1,140 .\end{array}$ & $\begin{array}{l}983 \\
967 \\
928 \\
910 \\
866\end{array}$ & $\begin{array}{l}821 \\
866 \\
928 \\
866 \\
838\end{array}$ & $\begin{array}{l}735 \\
750 \\
770 \\
770 \\
787\end{array}$ & $\begin{array}{r}8,520 \\
8,700 \\
8,700 \\
9,320 \\
10,700\end{array}$ & & $\begin{array}{r}14,400 \\
16,800 \\
9,320 \\
8,460 \\
5,280\end{array}$ & & $\begin{array}{l}64 \\
56 \\
54 \\
56 \\
56\end{array}$ & $\begin{array}{l}234 \\
358 \\
529 \\
588 \\
588\end{array}$ & $\begin{array}{l}700 \\
675 \\
646 \\
646 \\
646\end{array}$ \\
\hline $\begin{array}{l}26 \\
27 \\
28 \\
29 \\
30\end{array}$ & $\begin{array}{l}845 \\
815 \\
815 \\
930 \\
960 \\
990\end{array}$ & $\begin{array}{l}1,110 \\
1,110 \\
1,100 \\
1,080 \\
1,080\end{array}$ & $\begin{array}{l}858 \\
787 \\
866 \\
838 \\
787 \\
750\end{array}$ & $\begin{array}{l}787 . \\
750 \\
770 \\
770 \\
750 \\
750\end{array}$ & $\begin{array}{r}804 \\
804 \\
821 \\
\end{array}$ & $\begin{array}{l}9,760 \\
7,500 \\
6,700 \\
6,140 \\
5,280 \\
4,750\end{array}$ & $\begin{array}{l}3,760 \\
3,150 \\
2,840 \\
\mathbf{2}, 660 \\
\mathbf{2}, 570\end{array}$ & $\begin{array}{l}4,750 \\
4,150 \\
3,760 \\
3,480 \\
3,150 \\
2,400\end{array}$ & $\begin{array}{r}1,240 \\
1,140 \\
930 \\
900 \\
900\end{array}$ & $\begin{array}{l}592 \\
592 \\
620 \\
566 \\
540 \\
465\end{array}$ & $\begin{array}{l}529 \\
481 \\
566 \\
646 \\
700 \\
675\end{array}$ & $\begin{array}{l}646 \\
646 \\
620 \\
620 \\
592\end{array}$ \\
\hline
\end{tabular}


Monthly discharge of Tittabawassee River at Freeland, Mich., for the year ending September 30, 1923

[Drainage area, 2,530 square miles]

\begin{tabular}{|c|c|c|c|c|c|}
\hline \multirow{2}{*}{ Month } & \multicolumn{4}{|c|}{ Discharge in second-feet } & \multirow{2}{*}{$\begin{array}{l}\text { Run-off in } \\
\text { inches }\end{array}$} \\
\hline & Maximum & Minimum & Mean & $\begin{array}{c}\text { Per square } \\
\text { mille }\end{array}$ & \\
\hline $\begin{array}{l}\text { October } \\
\text { November } \\
\text { December } \\
\text { January } \\
\text { February } \\
\text { March } \\
\text { April } \\
\text { May } \\
\text { June } \\
\text { July } \\
\text { August } \\
\text { September }\end{array}$ & $\begin{array}{r}1,600 \\
1,410 \\
1,760 \\
967 \\
858 \\
11,400 \\
14,400 \\
16,800 \\
2,320 \\
1,240 \\
700 \\
1,170\end{array}$ & $\begin{array}{r}646 \\
930 \\
750 \\
750 \\
648 \\
1,600 \\
2,570 \\
1,900 \\
900 \\
465 \\
128 \\
592\end{array}$ & $\begin{array}{r}1,060 \\
1,140 \\
1,130 \\
807 \\
750 \\
7,140 \\
7,250 \\
5,260 \\
1,660 \\
741 \\
408 \\
749\end{array}$ & $\begin{array}{r}0.419 \\
.451 \\
.447 \\
.319 \\
.296 \\
2.82 \\
2.87 \\
2.08 \\
.656 \\
.293 \\
.161 \\
.296\end{array}$ & $\begin{array}{r}0.48 \\
.50 \\
.52 \\
.37 \\
.31 \\
3.25 \\
3,20 \\
2.40 \\
.73 \\
.34 \\
.19 \\
.33\end{array}$ \\
\hline The year & 16,800 & 128 & 2,350 & .929 & 12.62 \\
\hline
\end{tabular}

NotE.-Monthly and yearly discharge computed by engineers of U. S. Geological Survey from dailydischarge record furnished by G. S. Williams, consulting engineer, Ann Arbor, Mich.

\section{STREAMS TRIBUTARY TO LAKE ERIE}

\section{HORON RIVER AT BARTON, MICH.}

Location.- At dam and power plant of Eastern Michigan Edison Co. at Barton near Ann Arbor.

Drainage area. - 723 square miles.

Records available.- January 1, 1914, to September 30, 1923.

Determination of Discharge.- Flow computed from records of operation of power plant, the flow through undersluice during floods, and the depth of flow over dam. The flow through the power house is determined from a calibration of the turbines by means of a specially constructed weir, the crest of which was formed by a $1 / 4$ by 5 inch milled plate, the discharge over the weir being computed by Bazin's formula for free overflow. The greater part of the flood water passes through undersluices in the power-house foundations, and this flow is determined from a weir calibration of the sluices. Water flows over crest of dam only a few days during year.

Cooperation,- Daily-discharge record furnished by G. S. Williams, consulting engineer, Ann Arbor, Mich. 
Daily discharge, in second-feet, of Huron River at Barton, Mich., for the year ending September 30, 1923

\begin{tabular}{|c|c|c|c|c|c|c|c|c|c|c|c|c|}
\hline Day & Oct. & Nov. & Dec. & Jan. & Feb. & Mar. & Apr. & May & June & July & Aug. & Sept. \\
\hline- & $\begin{array}{l}146 \\
142 \\
140 \\
146 \\
141\end{array}$ & $\begin{array}{l}171 \\
162 \\
160 \\
196 \\
151\end{array}$ & $\begin{array}{l}181 \\
199 \\
167\end{array}$ & $\begin{array}{l}198 \\
204 \\
216 \\
194 \\
212\end{array}$ & $\begin{array}{l}213 \\
195 \\
252 \\
147 \\
207\end{array}$ & $\begin{array}{l}444 \\
499 \\
839 \\
849 \\
903\end{array}$ & $\begin{array}{l}493 \\
457 \\
428 \\
528 \\
582\end{array}$ & & $\begin{array}{l}232 \\
244\end{array}$ & $\begin{array}{r}6 \\
128 \\
87 \\
51 \\
115\end{array}$ & $\begin{array}{r}147 \\
143 \\
140 \\
155 \\
6\end{array}$ & $\begin{array}{r}70 \\
6 \\
6 \\
87 \\
78\end{array}$ \\
\hline $\begin{array}{l}6 .- \\
7 \cdots \\
8 \cdots \\
9 \cdots \\
10-\cdots\end{array}$ & $\begin{array}{l}163 \\
215 \\
156 \\
139 \\
161\end{array}$ & $\begin{array}{l}208 \\
186 \\
183 \\
185 \\
186\end{array}$ & $\begin{array}{l}185 \\
184 \\
185 \\
188 \\
186\end{array}$ & $\begin{array}{l}195 \\
157 \\
212 \\
212 \\
197\end{array}$ & $\begin{array}{l}195 \\
182 \\
182 \\
185 \\
191\end{array}$ & $\begin{array}{l}831 \\
849 \\
787 \\
789 \\
745\end{array}$ & $\begin{array}{l}614 \\
640 \\
647 \\
\end{array}$ & 198 & 164 & $\begin{array}{r}104 \\
114 \\
100 \\
92 \\
194\end{array}$ & $\begin{array}{l}131 \\
142 \\
121 \\
132 \\
108\end{array}$ & $\begin{array}{l}80 \\
90 \\
98 \\
72 \\
90\end{array}$ \\
\hline & $\begin{array}{l}207 \\
148 \\
185 \\
182 \\
184\end{array}$ & $\begin{array}{l}182 \\
164 \\
190 \\
212 \\
195\end{array}$ & $\begin{array}{l}187 \\
187 \\
134 \\
169 \\
188\end{array}$ & $\begin{array}{l}153 \\
190 \\
294 \\
178 \\
203\end{array}$ & $\begin{array}{l}114 \\
181 \\
193 \\
174 \\
153\end{array}$ & $\begin{array}{r}780 \\
994 \\
1,010 \\
930 \\
921\end{array}$ & $\begin{array}{l}400 \\
457 \\
471\end{array}$ & 242 & 210 & $\begin{array}{l}135 \\
152 \\
141 \\
141 \\
165\end{array}$ & $\begin{array}{r}73 \\
6 \\
130 \\
121 \\
122\end{array}$ & $\begin{array}{l}104 \\
103 \\
102 \\
101 \\
111\end{array}$ \\
\hline & $\begin{array}{l}185 \\
192 \\
178 \\
170 \\
162\end{array}$ & $\begin{array}{l}201 \\
197 \\
259 \\
136 \\
199\end{array}$ & $\begin{array}{l}182 \\
167 \\
172 \\
167 \\
184\end{array}$ & 212 & $\begin{array}{r}179 \\
189 \\
34 \\
205 \\
186\end{array}$ & $\begin{array}{r}1,050 \\
986 \\
937\end{array}$ & 370 & 540 & 147 & 144 & $\begin{array}{r}121 \\
112 \\
55 \\
6 \\
110\end{array}$ & $\begin{array}{r}6 \\
102 \\
130 \\
107 \\
168\end{array}$ \\
\hline 5 & $\begin{array}{l}197 \\
151 \\
174 \\
159 \\
160\end{array}$ & $\begin{array}{l}185 \\
200\end{array}$ & $\begin{array}{l}183 \\
178\end{array}$ & $\begin{array}{l}199 \\
203 \\
192 \\
226 \\
261\end{array}$ & $\begin{array}{l}169 \\
153\end{array}$ & $\begin{array}{l}856 \\
88 \\
911\end{array}$ & 349 & 411 & 21 & $\begin{array}{r}211 \\
53\end{array}$ & $\begin{array}{l}85 \\
94 \\
84 \\
83 \\
47\end{array}$ & $\begin{array}{l}250 \\
246 \\
214 \\
226 \\
169\end{array}$ \\
\hline $\begin{array}{l}26 \\
27 \\
28 \\
30\end{array}$ & $\begin{array}{l}171 \\
173 \\
197 \\
109 \\
197 \\
174\end{array}$ & $\begin{array}{l}155 \\
201 \\
199 \\
187 \\
161\end{array}$ & $\begin{array}{l}191 \\
204 \\
191 \\
183 \\
200 \\
195\end{array}$ & $\begin{array}{l}197 \\
192 \\
186 \\
206 \\
202 \\
207\end{array}$ & $\begin{array}{l}240 \\
312 \\
453\end{array}$ & $\begin{array}{l}736 \\
673 \\
688 \\
575 \\
497 \\
542\end{array}$ & $\begin{array}{l}336 \\
332 \\
347 \\
283 \\
326\end{array}$ & 373 & $\begin{array}{l}127 \\
130 \\
149 \\
130 \\
122\end{array}$ & $\begin{array}{r}115 \\
138 \\
106 \\
51 \\
153 \\
153\end{array}$ & $\begin{array}{r}6 \\
96 \\
78 \\
77 \\
85 \\
79\end{array}$ & $\begin{array}{l}183 \\
204 \\
185 \\
208 \\
162\end{array}$ \\
\hline
\end{tabular}

Monthly discharge of Huron River at Barton, Mich., for the year ending September 30,1923

[Drainage area, 723 square miles]

\begin{tabular}{|c|c|c|c|c|c|}
\hline \multirow{2}{*}{ Month } & \multicolumn{4}{|c|}{ Discharge in second-feet } & \multirow{2}{*}{$\begin{array}{l}\text { Run-off in } \\
\text { inches }\end{array}$} \\
\hline & Maximum & Minimum & Mean & $\begin{array}{c}\text { Per square } \\
\text { mile }\end{array}$ & \\
\hline $\begin{array}{l}\text { October } \\
\text { November } \\
\text { December } \\
\text { January } \\
\text { February } \\
\text { March } \\
\text { April } \\
\text { May } \\
\text { June } \\
\text { July } \\
\text { August } \\
\text { September }\end{array}$ & $\begin{array}{r}215 \\
259 \\
218 \\
294 \\
453 \\
1,230 \\
647 \\
545 \\
255 \\
211 \\
155 \\
250\end{array}$ & $\begin{array}{r}109 \\
136 \\
134 \\
124 \\
34 \\
444 \\
283 \\
198 \\
67 \\
6 \\
6 \\
6\end{array}$ & $\begin{array}{l}168 \\
186 \\
183 \\
201 \\
188 \\
824 \\
458 \\
337 \\
167 \\
125 \\
93.4 \\
125\end{array}$ & $\begin{array}{r}0.232 \\
.257 \\
.253 \\
.278 \\
.260 \\
1.14 \\
.633 \\
.466 \\
.231 \\
.173 \\
.129 \\
.173\end{array}$ & $\begin{array}{r}0.27 \\
.29 \\
.29 \\
.32 \\
.27 \\
1.31 \\
.71 \\
.54 \\
.26 \\
.20 \\
.15 \\
.19\end{array}$ \\
\hline The year & 1,230 & 6 & 255 & .353 & 4.80 \\
\hline
\end{tabular}

Nore.-Monthly and yearly discharge computed by engineers of U. S. Geological Survey from dailydischarge record furnished by $G$. S. Williams, consulting engineer, Ann Arbor, Mich. 
MAUMEE RIVER AT ANTWERP, OHIO

Location.-At highway bridge 1 mile north of Antwerp, Paulding County.

Drainage AREa.-2,130 square miles (area in Ohio measured on topographic maps, area in Michigan on United States Geological Survey map of Michigan, and area in Indiana on General Land Office map).

Records AVAILABLE.-September 1, 1921, to September 30, 1923.

GAGE.-Chain gage on highway bridge; read by H. G. Carr.

Discharge measurements.-Made from bridge at gage or by wading.

Channel and control.-Channel straight for 500 feet above and below gage. One channel at all stages. Left bank high; right bank fairly high. Control for low water is rock and gravel riflle about half a mile below gage; control for high water is long stretch of river below gage. Zero flow would occur at zero gage height.

EXTREMEs of DISCHARGe.-Maximum stage recorded during the year, 16.5 feet at 7.40 a. m. March 18 (discharge, 14,400 second-feet); minimum stage, 1.10 feet October 3-6 (discharge, 145 second-feet).

1921-1923: Maximum stage recorded, 16.8 feet at 7.30 a. m. April 2, 1922 (discharge, 14,700 second-feet); minimum stage, 1.08 feet at $6 \mathrm{p}$. m. September 9, 1921 (discharge, 133 second-feet).

ICE.-Stage-discharge relation affected by ice.

Diversions.-None.

Regulation.-None.

ACCURACY.- Stage-discharge relation permanent except when affected by ice. Rating curve well defined. Gage read to hundredths twice daily. Daily discharge ascertained by applying mean daily gage height to rating table except as shown in footnote to table of daily discharge. Open-water records good; winter records fair.

The following discharge measurement was made by F. R. Morgan: August 20, 1923: Gage height, 1.77 feet; discharge, 344 second-feet. 
Daily discharge, in second-feet, of Maumee River at Antwerp, Ohio, for the year ending September 80, 1923

\begin{tabular}{r|r|r|r|r|r|r|r|r|r|r|r|r}
\hline Day & Oct. & Nov. & Dec. & Jan. & Feb. & Mar. & Apr. & May & June & July & Aug. & Sept. \\
\hline & & & & & & & & \\
\hline
\end{tabular}

Nore.-Stage-discharge relation affected by ice Feb. 5 to Mar. 3; discharge estimated from study of observer's notes, weather records, and records of flow at near-by points. Braced figures show mean discbarge for periods indicated. Monthly discharge of Maumee River at Antwerp, Ohio, for the year ending September
30,1923

[Drainage area, 2,130 square miles]

\begin{tabular}{|c|c|c|c|c|c|}
\hline \multirow{2}{*}{ Month } & \multicolumn{4}{|c|}{ Discharge in second-feet } & \multirow{2}{*}{$\begin{array}{c}\text { Run-off in } \\
\text { inches }\end{array}$} \\
\hline & Maximum & Mínimum & Mean & $\underset{\text { mile }}{\text { Per square }}$ & \\
\hline $\begin{array}{l}\text { October } \\
\text { November } \\
\text { December } \\
\text { January } \\
\text { February } \\
\text { March } \\
\text { April } \\
\text { May } \\
\text { June } \\
\text { July } \\
\text { August } \\
\text { September }\end{array}$ & $\begin{array}{r}248 \\
234 \\
2,370 \\
4,400 \\
3,230 \\
14,200 \\
3,860 \\
11,900 \\
445 \\
2,020 \\
655 \\
1,040 \\
\end{array}$ & $\begin{array}{r}145 \\
182 \\
182 \\
655 \\
1,040 \\
445 \\
308 \\
182 \\
194 \\
169 \\
169\end{array}$ & $\begin{array}{r}191 \\
201 \\
436 \\
1,960 \\
1,590 \\
5,720 \\
1,230 \\
2,790 \\
304 \\
568 \\
325 \\
400\end{array}$ & $\begin{array}{r}0.089 \\
.094 \\
.205 \\
.920 \\
.746 \\
2.69 \\
.577 \\
1.31 \\
.143 \\
.267 \\
.153 \\
.188\end{array}$ & $\begin{array}{r}0.10 \\
.10 \\
.24 \\
1.06 \\
.78 \\
3.10 \\
.64 \\
1.51 \\
.16 \\
.31 \\
.18 \\
.21\end{array}$ \\
\hline The year...... & 14,200 & 145 & 1,320 & C. 620 & 8.39 \\
\hline
\end{tabular}




\section{MAUMEE RIVER AT WATERVILLE, OHIO}

Location.-At highway bridge at Waterville, Lucas County.

Drainage area. - 6,310 square miles (area in Ohio measured on topographic maps, area in Michigan on United States Geological Survey map of Michigan, and area in Indiana on General Land Office map).

Records available.--November 19, 1898, to December 31, 1901, and August 26, 1921, to September 30, 1923.

GaGE.-Chain gage on highway bridge; read by John Rhodes.

Discharge Measurements.-Made from bridge at gage or by wading.

Channel and control.-Stream bed is rock ledge. One channel at all stages. Channel straight for half a mile above and below gage. Island one-eighth mile above gage. Control permanent. Zero flow would occur at gage height 1 foot.

ICE.-Stage-discharge relation affected by ice during severe winters.

Extremes of DISCHARge.- Maximum combined discharge of river and canal during year, 39,000 second-feet March 18; minimum combined daily discharge, 345 second-feet December 5.

1921-1923: Maximum combined discharge of river and canal, 41,700 second-feet April 2, 1922; minimum combined discharge, 299 second-feet September 8, 1921 .

REgulation.-Flow at extremely low water may be affected by regulation of Auglaize River at dam of Defiance Gas \& Electric Co., near Defiance.

Diversions.-Water is diverted into Miami and Erie Canal at Grand Rapids and carried past station. For record of this diversion, see page 53.

AccoraCY.--Stage-discharge relation permanent; not seriously affected by ice. Rating curve well defined up to 25,000 second-feet. Gage read to hundredths once daily. Daily discharge ascertained by applying daily gage height to rating table. Records good except those for winter which are fair owing to possible ice effect.

Discharge measurements of Maumee River at Waterville, Ohio, during the year ending September 30, 1923

\begin{tabular}{|c|c|c|c|c|c|c|c|}
\hline Date & Made by- & $\begin{array}{c}\text { Gage } \\
\text { height }\end{array}$ & $\begin{array}{c}\text { Dis- } \\
\text { charge }\end{array}$ & Date & Made by- & $\begin{array}{c}\text { Gage } \\
\text { height }\end{array}$ & $\begin{array}{c}\text { Dis- } \\
\text { chargo }\end{array}$ \\
\hline $\begin{array}{lr}\text { Oct. } & 3 \\
\text { May } & 12\end{array}$ & $\begin{array}{l}\text { E. E. R. Dornbach. } \\
- \text { do } \\
\text { do }\end{array}$ & $\begin{array}{c}\text { Feet } \\
1.52 \\
1.46 \\
4.31\end{array}$ & $\begin{array}{r}\text { Sec. } f t . \\
75.9 \\
59.2 \\
6,220\end{array}$ & Aug. $\begin{array}{r}1 \\
2 \\
15\end{array}$ & $\begin{array}{l}\text { Lee and Dickson........ } \\
\text { F. R. Morgan }\end{array}$ & $\begin{array}{l}\text { Feet } \\
2.04 \\
1.94 \\
1.92\end{array}$ & $\begin{array}{r}\text { Sec.-ft. } \\
596 \\
473 \\
397\end{array}$ \\
\hline
\end{tabular}


Daily discharge, in second-feet, of Maumee River at Waterville, Ohio, for the year ending September, 30, 1928

\begin{tabular}{r|r|r|r|r|r|r|r|r|r|r|r|r}
\hline Day & Oct. & Nov. & Dec. & Jan. & Feb. & Mar. & Apr. & May & June & July & Aug. & Sept. \\
\hline & & & & & & & & & \\
\hline
\end{tabular}

Monthly discharge, in second-feet, of Maumee River and Miami and Erie Canal at Waterville, Ohio, for the year ending September 30, 1923

\begin{tabular}{|c|c|c|c|c|c|}
\hline \multirow{2}{*}{$\bullet$} & \multirow{2}{*}{$\begin{array}{l}\text { Maximum } \\
\text { (com- } \\
\text { bined) }\end{array}$} & \multirow{2}{*}{$\begin{array}{c}\text { Minimum } \\
\text { (com- } \\
\text { bined) }\end{array}$} & \multicolumn{3}{|c|}{ Mean } \\
\hline & & & River & Canal & Combined \\
\hline $\begin{array}{l}\text { October } \\
\text { November } \\
\text { December } \\
\text { January } \\
\text { February } \\
\text { March } \\
\text { April } \\
\text { May } \\
\text { June } \\
\text { July } \\
\text { August }\end{array}$ & $\begin{array}{r}643 \\
500 \\
8,650 \\
22,500 \\
13,200 \\
39,000 \\
18,000 \\
37,100 \\
1,400 \\
4,320 \\
1.440 \\
2,090\end{array}$ & $\begin{array}{r}377 \\
354 \\
345 \\
2,570 \\
1,620 \\
2,580 \\
860 \\
531 \\
476 \\
435 \\
488 \\
\mathbf{4 6 4}\end{array}$ & $\begin{array}{r}81.7 \\
68.0 \\
804 \\
8,280 \\
5,160 \\
15,400 \\
3,690 \\
\mathbf{7}, 840 \\
429 \\
667 \\
319 \\
501\end{array}$ & $\begin{array}{l}359 \\
328 \\
400 \\
472 \\
441 \\
281 \\
386 \\
410 \\
428 \\
438 \\
448 \\
420\end{array}$ & $\begin{array}{r}441 \\
396 \\
1,200 \\
8,750 \\
5,610 \\
15,700 \\
4,070 \\
8,250 \\
857 \\
1,100 \\
767 \\
921\end{array}$ \\
\hline The year.... & 39,000 & 345 & 3,620 & 401 & 4,020 \\
\hline
\end{tabular}




\section{TIFFIN RIVER NEAR STRYKER, OHIO}

Location.-In sec. 17, T. 6 N., R. 4 E., at highway bridge 2 miles southwest of Stryker, Williams County.

Drainage ARea.-450 square miles; revised (area in Ohio measured on topographic maps; area in Michigan on United States Geological Survey map, scale $1: 1,000,000$ ).

Records available.-September 1, 1921, to September 30, 1923.

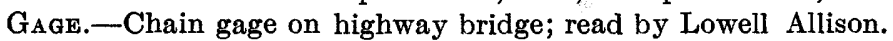

Discharge MEasurements.-Made from bridge or by wading.

Channer and control.-Channel straight for 400 feet above and below gage. One channel at all stages. Banks high and brushy. Control for low water is ruin of old timber milldam half a mile below gage; control for high water is long stretch of river below gage. Zero flow would occur at zero gage height.

Extremes of discharge.-Maximum stage recorded during year, 12.2 feet at 5.10 p.m. March 17 (discharge, 1,840 second-feet); minimum stage, 1.13 feet August 3 and 4, and 7.a. m. August 10 (discharge, 16 second-feet).

1921-1923: Maximum stage recorded, 13 feet at 5.30 p. m. April 1, 1922 (discharge, 1,990 second-feet); minimum stage, 1.10 feet at 8.35 a.m. August 16, 1922 (discharge, 15 second-feet).

ICE.--Stage-discharge relation affected by ice.

Accuracy.-Stage-discharge relation permanent except as affected by ice. Rating curve well defined. Gage read to hundredths twice daily. Daily discharge ascertained by applying mean daily gage height to rating table. Records good except for period of ice effect, for which they are fair.

The following discharge measurement was made by F. R. Morgan: August 18, 1923: Gage height, 1.69 feet; discharge, 64.4 second-feet. 
Daily discharge, in second-feet, of Tiffin River near Stryker, Ohio, for the year ending September 30,1923

\begin{tabular}{|c|c|c|c|c|c|c|c|c|c|c|c|c|}
\hline Day & Oct. & Nov. & Dec. & Jan. & Feb. & Mar. & Apr. & May & June & July & Aug. & Sept. \\
\hline $\begin{array}{l}1 \\
2 \\
3 \\
4 \\
5\end{array}$ & $\begin{array}{l}22 \\
24 \\
24 \\
22 \\
27\end{array}$ & $\begin{array}{l}46 \\
61 \\
72 \\
78 \\
78\end{array}$ & $\begin{array}{l}61 \\
56 \\
62 \\
72 \\
83\end{array}$ & $\begin{array}{l}588 \\
556 \\
305 \\
187 \\
140\end{array}$ & $\begin{array}{l}187 \\
380 \\
556 \\
508\end{array}$ & $\begin{array}{r}722 \\
756 \\
773 \\
988 \\
1,170\end{array}$ & $\begin{array}{l}133 \\
152 \\
152 \\
173 \\
275\end{array}$ & $\begin{array}{l}78 \\
78 \\
72 \\
72 \\
66\end{array}$ & $\begin{array}{l}72 \\
66 \\
59 \\
60 \\
47\end{array}$ & $\begin{array}{l}31 \\
31 \\
28 \\
29 \\
32\end{array}$ & $\begin{array}{r}18 \\
21 \\
16 \\
16 \\
120\end{array}$ & $\begin{array}{l}31 \\
24 \\
30 \\
28 \\
25\end{array}$ \\
\hline $\begin{array}{r}6 \\
6 \\
8 \\
8 \\
9\end{array}$ & $\begin{array}{l}26 \\
39 \\
51 \\
44 \\
44\end{array}$ & $\begin{array}{l}78 \\
72 \\
72 \\
72 \\
72\end{array}$ & $\begin{array}{r}95 \\
78 \\
107 \\
101 \\
66\end{array}$ & $\begin{array}{r}173 \\
126 \\
83 \\
89 \\
89\end{array}$ & 200 & $\begin{array}{r}1,200 \\
722 \\
476 \\
320 \\
444\end{array}$ & $\begin{array}{l}654 \\
826 \\
671 \\
492 \\
365\end{array}$ & $\begin{array}{l}72 \\
66 \\
59 \\
66 \\
66\end{array}$ & $\begin{array}{r}89 \\
215 \\
245 \\
215 \\
187\end{array}$ & $\begin{array}{l}27 \\
30 \\
40 \\
38 \\
40\end{array}$ & $\begin{array}{l}83 \\
39 \\
25 \\
22 \\
18\end{array}$ & $\begin{array}{l}34 \\
72 \\
95 \\
66 \\
55\end{array}$ \\
\hline $\begin{array}{l}11 \\
12 \\
13 \\
14 \\
15\end{array}$ & $\begin{array}{l}39 \\
38 \\
47 \\
57 \\
59\end{array}$ & $\begin{array}{l}66 \\
66 \\
66 \\
66 \\
72\end{array}$ & $\begin{array}{l}61 \\
60 \\
66 \\
66 \\
58\end{array}$ & $\begin{array}{r}101 \\
66 \\
72 \\
89 \\
126\end{array}$ & & $\begin{array}{r}756 \\
1,310 \\
1,590 \\
1,760 \\
1,650\end{array}$ & $\begin{array}{l}320 \\
275 \\
215 \\
201 \\
201\end{array}$ & $\begin{array}{r}78 \\
95 \\
140 \\
133 \\
572\end{array}$ & $\begin{array}{r}126 \\
101 \\
89 \\
78 \\
72\end{array}$ & $\begin{array}{l}50 \\
78 \\
66 \\
61 \\
47\end{array}$ & $\begin{array}{r}19 \\
556 \\
773 \\
844 \\
396\end{array}$ & $\begin{array}{l}46 \\
40 \\
36 \\
26 \\
28\end{array}$ \\
\hline $\begin{array}{l}16 \\
17 \\
18 \\
19 \\
20\end{array}$ & $\begin{array}{l}52 \\
49 \\
40 \\
46 \\
49\end{array}$ & $\begin{array}{l}83 \\
78 \\
78 \\
83 \\
83\end{array}$ & $\begin{array}{l}51 \\
54 \\
52 \\
48 \\
46\end{array}$ & $\begin{array}{l}290 \\
396 \\
201 \\
335 \\
380\end{array}$ & 150 & $\begin{array}{l}1,700 \\
1,780 \\
1,740 \\
1,590 \\
1,370\end{array}$ & $\begin{array}{l}230 \\
260 \\
230 \\
187 \\
159\end{array}$ & $\begin{array}{r}1,020 \\
1,170 \\
1,020 \\
722 \\
671\end{array}$ & $\begin{array}{r}78 \\
215 \\
72 \\
53 \\
52\end{array}$ & $\begin{array}{l}33 \\
32 \\
28 \\
25 \\
21\end{array}$ & $\begin{array}{r}187 \\
83 \\
61 \\
53 \\
42\end{array}$ & $\begin{array}{l}24 \\
20 \\
19 \\
22 \\
51\end{array}$ \\
\hline 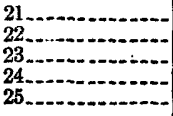 & $\begin{array}{l}48 \\
49 \\
58 \\
58 \\
51\end{array}$ & $\begin{array}{l}78 \\
72 \\
72 \\
66 \\
66\end{array}$ & $\begin{array}{l}44 \\
54 \\
57 \\
58 \\
61\end{array}$ & $\begin{array}{l}412 \\
396 \\
350 \\
166 \\
140\end{array}$ & 72 & $\begin{array}{l}916 \\
620 \\
790 \\
540 \\
476\end{array}$ & $\begin{array}{l}146 \\
140 \\
133 \\
120 \\
107\end{array}$ & $\begin{array}{l}688 \\
492 \\
320 \\
215 \\
166\end{array}$ & $\begin{array}{l}48 \\
38 \\
43 \\
36 \\
34\end{array}$ & $\begin{array}{l}24 \\
22 \\
24 \\
24 \\
25\end{array}$ & $\begin{array}{l}34 \\
29 \\
28 \\
26 \\
24\end{array}$ & $\begin{array}{l}350 \\
808 \\
492 \\
215 \\
114\end{array}$ \\
\hline $\begin{array}{l}26 \\
27 \\
28 \\
28 \\
30 \\
31\end{array}$ & $\begin{array}{l}53 \\
53 \\
51 \\
55 \\
57 \\
61\end{array}$ & $\begin{array}{c}66 \\
66 \\
56 \\
60 \\
61 \\
- \\
--\end{array}$ & $\begin{array}{r}61 \\
72 \\
126 \\
140 \\
120 \\
173\end{array}$ & $\begin{array}{r}120 \\
107 \\
107 \\
101 \\
95 \\
107\end{array}$ & \begin{tabular}{r}
201 \\
588 \\
705 \\
\hdashline \\
\hdashline$-2 .-$ \\
\end{tabular} & $\begin{array}{l}412 \\
350 \\
290 \\
230 \\
201 \\
173\end{array}$ & $\begin{array}{r}107 \\
95 \\
101 \\
95 \\
89 \\
\end{array}$ & $\begin{array}{r}146 \\
120 \\
107 \\
95 \\
95 \\
78\end{array}$ & $\begin{array}{r}31 \\
36 \\
37 \\
40 \\
42 \\
-\end{array}$ & $\begin{array}{l}26 \\
25 \\
31 \\
28 \\
25 \\
24\end{array}$ & $\begin{array}{l}22 \\
28 \\
30 \\
29 \\
37 \\
39\end{array}$ & $\begin{array}{r}83 \\
78 \\
72 \\
89 \\
95 \\
\end{array}$ \\
\hline
\end{tabular}

Nork. - Stage-discharge relation affected by ice Feb. 5-24; discharge estimated from study of observer's notes, weather records, and records of flow of near-by streams. Braced figures indicate mean discharge for period indicated.

Monthly discharge of Tiffin River near Stryker, Ohio, for the year ending September 30,1923

[Drainage area, 450 square miles]

\begin{tabular}{|c|c|c|c|c|c|}
\hline \multirow{2}{*}{ Month } & \multicolumn{4}{|c|}{ Discharge in second-feet } & \multirow{2}{*}{$\begin{array}{l}\text { Run-ofif } \\
\text { in inches }\end{array}$} \\
\hline & Maximum & Minimum & Mean & $\begin{array}{c}\text { Per square } \\
\text { mile }\end{array}$ & \\
\hline $\begin{array}{l}\text { October } \\
\text { November. } \\
\text { December. } \\
\text { January } \\
\text { February } \\
\text { March } \\
\text { April } \\
\text { June } \\
\text { July } \\
\text { August } \\
\text { September }\end{array}$ & $\begin{array}{r}61 \\
83 \\
173 \\
588 \\
1,780 \\
826 \\
1,170 \\
245 \\
78 \\
844 \\
808\end{array}$ & $\begin{array}{r}22 \\
46 \\
44 \\
66 \\
173 \\
89 \\
59 \\
31 \\
21 \\
16 \\
19\end{array}$ & $\begin{array}{l}44.9 \\
70.2 \\
74.5 \\
209 \\
239 \\
897 \\
243 \\
285 \\
85.9 \\
33.7 \\
120 \\
106\end{array}$ & $\begin{array}{c}0.100 \\
.156 \\
.166 \\
.464 \\
.531 \\
1.99 \\
.540 \\
.633 \\
.191 \\
.075 \\
.267 \\
.236\end{array}$ & $\begin{array}{r}0.12 \\
.17 \\
.19 \\
.53 \\
.55 \\
2.29 \\
.60 \\
.73 \\
.21 \\
.09 \\
.31 \\
.26\end{array}$ \\
\hline The year ............. & 1,780 & 16 & 201 & .447 & $\therefore \quad 6.05$ \\
\hline
\end{tabular}




\section{AUGLAIZE RIVER NEAR FORT JENNINGS, OHIO}

Locatron.-SE. $1 / 4$ sec. 15, T. 1 S., R. 5 E., at highway bridge $31 / 2$ miles northeast of Fort Jennings, Putnam County, and 6 miles by river above mouth of Ottawa River.

Drainage area.-333 square miles (measured on topographic maps).

Records avairable.-August 31, 1921, to September 30, 1923.

GAGE.-Chain gage on highway bridge; read by G. S. Hedrick.

Discharge measurements.-Made from bridge at gage or by wading.

ChanNel AND control.-Channel straight for 300 feet above and 1,000 feet below gage. Right bank high; left bank fairly high, subject to overflow at extremely high stages. One channel at all stages. Prior to October 29, 1922, control for low water was loose rock dam 800 feet below gage. On that date an opening was made in the center of the loose rock dam down to bedrock in order to lower pool at the gage and allow submerged quarry coffer dam 500 feet below the gage to form contol for low water. Zero flow would occur at gage height 0.3 foot, from measurements made after pool was lowered.

Extremes of STAGE.-Maximum stage recorded during period of record, 15.6 feet April 19, 1922; minimum discharge probably occurred October 4, 1921.

Regulation.- None.

Diversions.- None.

ACCURACY.--Gage read to hundredths once daily. Record reliable. Rating curve not yet developed for high water. Stage-discharge relation very unstable prior to October 29, 1922.

Discharge measurements of Auglaize River near Fort Jennings, Ohio, during the years ending September 30, 1921-1923

\begin{tabular}{|c|c|c|c|c|c|c|c|}
\hline Date & Made by- & $\begin{array}{c}\text { Gage } \\
\text { height }\end{array}$ & $\begin{array}{l}\text { Dis- } \\
\text { charge }\end{array}$ & Date & Made by- & $\begin{array}{c}\text { Gàge } \\
\text { height }\end{array}$ & $\begin{array}{c}\text { Dis- } \\
\text { charge }\end{array}$ \\
\hline $\begin{array}{l}1921 \\
\text { Aug. } 31 \\
\text { Oct. } 25 \\
1922 \\
\text { Mar. } 13 \\
\text { May } 17 \\
1 \\
\text { May } 29\end{array}$ & 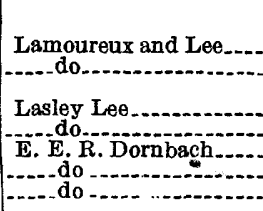 & $\begin{array}{l}\text { Feet } \\
2.40 \\
2.87 \\
\\
4.38 \\
4.56 \\
2.86 \\
3.48 \\
4.50\end{array}$ & $\begin{array}{c}\text { Sec.-ft. } \\
50.5 \\
51.3 \\
536 \\
596 \\
161 \\
314 \\
515\end{array}$ & $\begin{array}{l}\text { 1922 } \\
\text { June 3 } \\
\text { July } 10 \\
15 \\
\text { Aug. } 23 \\
\text { Oet. 29 } \\
1923 \\
\text { May } 8 \\
\text { Aug. 20 }\end{array}$ & $\begin{array}{l}\text { E. E. R. Dornbach } \\
\text { Lasley Lee do } \\
\text { E. E. R. Dornbach } \\
\text { F. R. Morgan }\end{array}$ & $\begin{array}{c}\text { Feet } \\
3.06 \\
2.98 \\
4.73 \\
2.20 \\
1.55 \\
1.81 \\
1.39\end{array}$ & \begin{tabular}{|c} 
Sec. $f t$. \\
185 \\
189 \\
505 \\
43.6 \\
41.5 \\
62.5 \\
31.4
\end{tabular} \\
\hline
\end{tabular}

Daily gage height, in feet, of Auglaize River near Fort Jennings, Ohio, for the period August 31, 1921, to September 30, 1923

\begin{tabular}{|c|c|c|c|c|c|c|c|c|}
\hline Day & Aug. & Sept. & Day & Aug. & Sept. & Day & Aug. & Sept. \\
\hline $\begin{array}{l}1921 \\
2 \\
3 \\
5\end{array}$ & 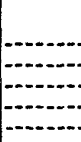 & $\begin{array}{l}2.40 \\
2.30 \\
2.28 \\
2.08 \\
2.10\end{array}$ & $\begin{array}{l}11 \ldots \ldots \\
12 \ldots \ldots \\
13 \\
14 \ldots \ldots \\
15 \ldots \ldots\end{array}$ & & $\begin{array}{l}2.50 \\
2.40 \\
2.48 \\
2.26 \\
2.04\end{array}$ & $\begin{array}{l}21 \ldots \\
22 \\
23 \\
24 \\
25\end{array}$ & $\ldots$ & $\begin{array}{l}2.04 \\
2.30 \\
2.28 \\
2.24 \\
2.30\end{array}$ \\
\hline $\begin{array}{c}6 \\
7 \\
8 \\
8\end{array}$ & 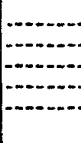 & $\begin{array}{l}2.20 \\
2.30 \\
2.12 \\
2.18 \\
2.32\end{array}$ & $\begin{array}{l}16 \\
17 \\
18 \\
19\end{array}$ & & $\begin{array}{l}2.46 \\
2.50 \\
2.24 \\
2.16 \\
2.10\end{array}$ & $\begin{array}{l}26 \ldots \ldots \\
27 \\
28\end{array}$ & (2. & $\begin{array}{l}2.26 \\
2.22 \\
2.44 \\
2.42 \\
2.44\end{array}$ \\
\hline
\end{tabular}


Daily gage height, in feet, of A uglaize Rivernear Fort Jennings, Ohio, for the period A ugust 31, 1921, to September 30, 1923-Continued

\begin{tabular}{|c|c|c|c|c|c|c|c|c|c|c|c|c|}
\hline D & Det. & rov. & Dec. & Jan. & Feb. & Mar. & Apr. & May & June & July & Aug. & Sept, \\
\hline $\begin{array}{l}1 \ldots \\
2 . . \\
3 .- \\
4 \ldots \\
5 .-\end{array}$ & $\begin{array}{l}2.46 \\
2.22 \\
2.00 \\
1.94 \\
2.22\end{array}$ & $\begin{array}{l}3.1 \\
4.3 \\
4.1 \\
3.3 \\
2.98\end{array}$ & $\begin{array}{l}3.4 \\
3.3 \\
4.8 \\
3.8 \\
3.4\end{array}$ & $\begin{array}{l}2.68 \\
2.92 \\
2.98 \\
2.96 \\
7.3\end{array}$ & $\begin{array}{l}2.80 \\
6.3 \\
8.5 \\
7.4 \\
5.0\end{array}$ & $\begin{array}{l}\text { 3. } 2 \\
3.0 \\
3.0 \\
3.0 \\
2.86\end{array}$ & \begin{tabular}{|r}
13.5 \\
10.2 \\
6.1 \\
4.9 \\
4.3
\end{tabular} & $\begin{array}{l}2.86 \\
2.86 \\
3.0 \\
4.8 \\
4.1\end{array}$ & $\begin{array}{l}3.1 \\
3.1 \\
3.0 \\
2.92 \\
2.86\end{array}$ & & $\begin{array}{l}2.40 \\
2.36 \\
2.40 \\
2.36 \\
2.46\end{array}$ & $\begin{array}{l}2.48 \\
2.62 \\
6.0 \\
8.8 \\
6.8\end{array}$ \\
\hline $\begin{array}{r}6 \\
7 \ldots \\
8 \\
9 \\
10\end{array}$ & $\begin{array}{l}2.22 \\
2.46 \\
2.54 \\
2.52 \\
2.40\end{array}$ & $\begin{array}{l}2.82 \\
2.76 \\
2472 \\
2.70 \\
2.66\end{array}$ & $\begin{array}{l}3.2 \\
3.1 \\
3.0 \\
3.0 \\
2.98\end{array}$ & $\begin{array}{l}7.3 \\
4.5 \\
3.7 \\
3.4 \\
3.2\end{array}$ & $\begin{array}{l}4.3 \\
3.7 \\
3.5 \\
3.3 \\
3.2\end{array}$ & $\begin{array}{l}2.88 \\
3.7 \\
4.0 \\
3.6 \\
3.5\end{array}$ & $\begin{array}{l}3.9 \\
3.6 \\
5.2 \\
7.6 \\
5.3\end{array}$ & $\begin{array}{l}3.4 \\
3.2 \\
3.1 \\
3.0 \\
3.5\end{array}$ & $\begin{array}{l}2.86 \\
2.82 \\
2.76 \\
2.68 \\
3.0\end{array}$ & $\begin{array}{l}2.46 \\
2.38 \\
2.84 \\
4.1 \\
3.0\end{array}$ & $\begin{array}{l}2,40 \\
2.36 \\
2.32 \\
2.48 \\
2.48\end{array}$ & $\begin{array}{l}4.0 \\
3.2 \\
2.93 \\
2.82 \\
3.5\end{array}$ \\
\hline$\because$ & $\begin{array}{l}2.34 \\
2.36 \\
2.40 \\
2.28 \\
2.40\end{array}$ & $\begin{array}{l}2.80 \\
3.2 \\
3.4 \\
3.4 \\
4.4\end{array}$ & $\begin{array}{l}2.94 \\
2.94 \\
3.0 \\
3.2 \\
3.3\end{array}$ & $\begin{array}{l}3.1 \\
3.2 \\
3.0 \\
2.92 \\
2.92\end{array}$ & \begin{tabular}{l|}
3.2 \\
3.4 \\
3.4 \\
3.1 \\
2.88
\end{tabular} & $\begin{array}{l}5.0 \\
5.8 \\
4.4 \\
4.8 \\
9.6\end{array}$ & $\begin{array}{r}9.1 \\
10.6 \\
6.8 \\
5.8 \\
11.3\end{array}$ & $\begin{array}{l}5.4 \\
4.7 \\
7.2 \\
4.5 \\
3.4\end{array}$ & $\begin{array}{l}3.7 \\
3.7 \\
3.1 \\
2.88 \\
2.76\end{array}$ & & $\begin{array}{l}2.42 \\
2.48 \\
2.40 \\
2.40 \\
2.40\end{array}$ & $\begin{array}{l}7.1 \\
6.4 \\
4.4 \\
3.4 \\
3.0\end{array}$ \\
\hline 9 & $\begin{array}{l}2.44 \\
2.30 \\
2.24 \\
2.26 \\
2.50\end{array}$ & $\begin{array}{l}4.0 \\
4.1 \\
6.4 \\
8.3 \\
9.8\end{array}$ & $\begin{array}{l}3.3 \\
3.4 \\
8.0 \\
8.8 \\
5.7\end{array}$ & $\begin{array}{l}2.72 \\
2.66 \\
2.82 \\
4.1 \\
4.7\end{array}$ & $\begin{array}{l}2.68 \\
2.78 \\
2.90 \\
2.92 \\
5.4\end{array}$ & $\begin{array}{l}4.8 \\
4.3 \\
3.5 \\
3.5 \\
4.2\end{array}$ & $\begin{array}{r}13.0 \\
8.2 \\
12.3 \\
15.6 \\
11.1\end{array}$ & $\begin{array}{r}3.1 \\
3.0 \\
3.1 \\
5.9 \\
11.2\end{array}$ & & & $\begin{array}{l}2.44 \\
2.50 \\
; 2.40 \\
2.44 \\
2.14\end{array}$ & $\begin{array}{l}2.8 \\
2.7 \\
2.6 \\
2.6 \\
2.5\end{array}$ \\
\hline 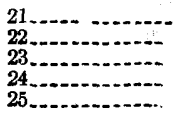 & $\begin{array}{l}2.36 \\
2.50 \\
2.60 \\
2.60 \\
2.88\end{array}$ & $\begin{array}{l}7.0 \\
4.4 \\
3.8 \\
3.5 \\
4.0\end{array}$ & $\begin{array}{l}4.3 \\
3.5 \\
3.4 \\
4.2 \\
6.0\end{array}$ & $\begin{array}{l}3.6 \\
3.3 \\
3.1\end{array}$ & $\begin{array}{l}5.3 \\
7.3 \\
7.7 \\
7.5 \\
4.8\end{array}$ & $\begin{array}{l}4.3 \\
3.8 \\
4.1 \\
5.6 \\
4.6\end{array}$ & $\begin{array}{l}5.2 \\
4.4 \\
3.9 \\
3.6 \\
3.3\end{array}$ & $\begin{array}{r}11.0 \\
7.8 \\
4.9 \\
4.0 \\
6.0\end{array}$ & $\begin{array}{l}2.52 \\
2.50 \\
2.50 \\
2.56 \\
2.48\end{array}$ & $\begin{array}{r}2.70 \\
2.66 \\
2.62 \\
2.58 \\
2.52\end{array}$ & $\begin{array}{l}2.12 \\
2.42 \\
2.44\end{array}$ & $\begin{array}{l}2.5 \\
2.4 \\
2.3 \\
2.4\end{array}$ \\
\hline $\begin{array}{l}28 \ldots \\
29 \\
30 \ldots \\
31 \ldots\end{array}$ & $\begin{array}{l}2.82 \\
2.90 \\
2.84 \\
2.82 \\
3.0 \\
3.1\end{array}$ & $\begin{array}{l}3.9 \\
4.2 \\
4.4 \\
4.2 \\
3.6 \\
\end{array}$ & $\begin{array}{l}4.4 \\
3.4 \\
3.1 \\
3.1 \\
2.82 \\
2.88\end{array}$ & $\begin{array}{l}3.2 \\
2.88 \\
2.88 \\
2.78 \\
2.78 \\
2.76\end{array}$ & $\begin{array}{l}3.7 \\
3.3 \\
3.3 \\
-\end{array}$ & $\begin{array}{r}4.1 \\
6.6 \\
9.0 \\
8.4 \\
7.4 \\
12.4\end{array}$ & \begin{tabular}{l}
3.1 \\
3.1 \\
3.0 \\
2.94 \\
2.88 \\
\hdashline
\end{tabular} & $\begin{array}{l}6.5 \\
7.4 \\
6.2 \\
4.6 \\
3.6 \\
3.3\end{array}$ & $\begin{array}{l}2.60 \\
2.56 \\
2.38 \\
2,52 \\
2.74 \\
-\end{array}$ & & $\begin{array}{l}2.58 \\
2.66 \\
2.56 \\
2.62 \\
2.62 \\
2.56\end{array}$ & $\begin{array}{l}2.46 \\
2.48 \\
2.46 \\
2.42 \\
2.44\end{array}$ \\
\hline & $\begin{array}{l}2.42 \\
2.50 \\
2.48 \\
2.50 \\
2.52\end{array}$ & $\begin{array}{l}1.48 \\
1.42 \\
1.34 \\
1.24 \\
1.36\end{array}$ & $\begin{array}{l}1.62 \\
1.54 \\
1.62 \\
1.60 \\
1.78\end{array}$ & $\begin{array}{l}9.8 \\
9.2 \\
6.2 \\
5.5 \\
4.4\end{array}$ & $\begin{array}{l}4.6 \\
5.4 \\
5.6 \\
4.4 \\
3.45\end{array}$ & $\begin{array}{l}4.4 \\
3.50 \\
3.12 \\
4.6 \\
5.8\end{array}$ & $\begin{array}{l}2.52 \\
2.42 \\
2.42 \\
2.52 \\
5.4\end{array}$ & $\begin{array}{l}2.12 \\
1.98 \\
1.82 \\
1.74 \\
1.76\end{array}$ & $\begin{array}{l}2.10 \\
2.10 \\
2.06 \\
1.82 \\
1.88\end{array}$ & & $\begin{array}{l}1.56 \\
1.68 \\
1.70 \\
1.80 \\
2,00\end{array}$ & $\begin{array}{l}1.92 \\
2.00 \\
1.66 \\
1.76 \\
1.92\end{array}$ \\
\hline $\begin{array}{l}6 . \\
7 \\
8 \\
9\end{array}$ & $\begin{array}{l}2.50 \\
2.56 \\
2.62 \\
2.56 \\
2.60\end{array}$ & $\begin{array}{l}1.26 \\
1.20 \\
1.30 \\
1.28 \\
1.16\end{array}$ & $\begin{array}{l}1.70 \\
1.78 \\
1.86 \\
1.80 \\
1.82\end{array}$ & $\begin{array}{l}\text { 3. } 6 \\
3.45 \\
3.22 \\
4.1 \\
5.1\end{array}$ & $\begin{array}{l}4.4 \\
4.2 \\
3.60 \\
3.60 \\
2.96\end{array}$ & $\begin{array}{l}4.6 \\
4.0 \\
3.50 \\
3.28 \\
5.1\end{array}$ & $\begin{array}{l}9.6 \\
7.2 \\
5.0 \\
3.80 \\
3.32\end{array}$ & & $\begin{array}{l}2.10 \\
1.98 \\
2.08 \\
2.24\end{array}$ & & $\begin{array}{l}1.72 \\
1.78 \\
1.50 \\
1.64 \\
1.86\end{array}$ & $\begin{array}{l}1,82 \\
1.72 \\
1.80 \\
1.80 \\
1.44\end{array}$ \\
\hline & $\begin{array}{l}2.62 \\
2.70 \\
2.68 \\
2.66 \\
2.62\end{array}$ & $\begin{array}{l}1.32 \\
1.20 \\
1.28 \\
1.30 \\
1.26\end{array}$ & $\begin{array}{l}2.26 \\
2.14 \\
2.92 \\
2.08 \\
2.04\end{array}$ & $\begin{array}{r}4.2 \\
3.75 \\
3.45 \\
5.1 \\
11.7\end{array}$ & $\begin{array}{l}2.68 \\
2.34 \\
7.0 \\
6.4 \\
4.0\end{array}$ & $\begin{array}{l}6.8 \\
8.8 \\
9.4 \\
6.2 \\
4.6\end{array}$ & $\begin{array}{l}3.14 \\
2.20 \\
2.82 \\
2.76 \\
2.94\end{array}$ & $\begin{array}{r}1 . \\
3 . \\
10 . \\
12 . \\
9 .\end{array}$ & $\begin{array}{l}2.18 \\
2.16 \\
2.26 \\
2.24 \\
2.60\end{array}$ & & $\begin{array}{l}1.30 \\
1.64 \\
1.56\end{array}$ & $\begin{array}{l}1.7 \\
1.6 \\
1.7 \\
1.7\end{array}$ \\
\hline & $\begin{array}{l}2.64 \\
2.54 \\
2.52 \\
2.50 \\
2.62\end{array}$ & $\begin{array}{l}1.20 \\
1.58 \\
1.62 \\
1.72 \\
1.62\end{array}$ & $\begin{array}{l}2.00 \\
1.96 \\
1.76 \\
1.82 \\
1.80\end{array}$ & $\begin{array}{l}9.8 \\
5.7 \\
4.3 \\
3.55 \\
2.28\end{array}$ & $\begin{array}{l}4.4 \\
4.8 \\
4.4 \\
4.4 \\
3.60\end{array}$ & $\begin{array}{c}11.7 \\
11.9 \\
7.0 \\
5.0 \\
3.65\end{array}$ & $\begin{array}{l}2.82 \\
2.72 \\
2.52 \\
2.54 \\
2.48\end{array}$ & $\begin{array}{c}12.2 \\
10.1 \\
6.0 \\
4.6 \\
3.95\end{array}$ & $\begin{array}{l}2 . \\
2 . \\
2 . \\
2 .\end{array}$ & & $\begin{array}{l}1.72 \\
1.40 \\
1.64 \\
1.74 \\
1.38\end{array}$ & $\begin{array}{l}1.0 \\
1.4 \\
1.5 \\
1.6 \\
1.7\end{array}$ \\
\hline 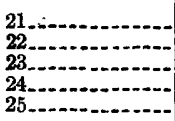 & $\begin{array}{l}2.66 \\
2.66 \\
2.62 \\
2.56 \\
2.56\end{array}$ & $\begin{array}{l}1.78 \\
1.76 \\
1.76 \\
1.72 \\
1.74\end{array}$ & $\begin{array}{l}1.88 \\
1.94 \\
1.90 \\
1.76 \\
1.68\end{array}$ & $\begin{array}{l}5.3 \\
7.8 \\
5.4 \\
4.1 \\
3.45\end{array}$ & $\begin{array}{l}3.24 \\
3.04 \\
2.82 \\
2.70 \\
2.70\end{array}$ & $\begin{array}{l}3.22 \\
3.40 \\
8.4 \\
5.6 \\
4.4\end{array}$ & $\begin{array}{l}2.40 \\
2.40 \\
2.22 \\
2.20 \\
2.12\end{array}$ & $\begin{array}{l}\text { 3. } 5 \\
3.2 \\
2.9 \\
2.8 \\
2.6\end{array}$ & $\begin{array}{l}1.98 \\
1.90 \\
1.86 \\
1.96 \\
1.58\end{array}$ & $\begin{array}{l}1.70 \\
1.62 \\
1.44 \\
1.70 \\
1.66\end{array}$ & $\begin{array}{l}1.66 \\
1.40 \\
1.68 \\
1.60 \\
1.54\end{array}$ & $\begin{array}{l}1.8 \\
1 . \\
1 . \\
1 .\end{array}$ \\
\hline $\begin{array}{l}26 \\
27 \\
28\end{array}$ & $\begin{array}{l}2.64 \\
2.60 \\
2.60 \\
1.56 \\
1.80 \\
1.44\end{array}$ & $\begin{array}{l}1.62 \\
1.56 \\
1.56 \\
1.60 \\
1.64\end{array}$ & $\begin{array}{l}1.58 \\
1.62 \\
7.1 \\
8.8 \\
7.0 \\
5.7\end{array}$ & $\begin{array}{l}3.20 \\
3.12 \\
6.4 \\
7.6 \\
5.2 \\
4.2\end{array}$ & $\begin{array}{c}2.60 \\
3.50 \\
5.2 \\
\end{array}$ & $\begin{array}{l}3.80 \\
3.40 \\
3.06 \\
2.86 \\
2.80 \\
2.60\end{array}$ & $\begin{array}{l}2.12 \\
2.16 \\
2.16 \\
2.06 \\
1.92\end{array}$ & $\begin{array}{l}2.56 \\
2.50 \\
2.48 \\
2.32 \\
2.20 \\
2.06\end{array}$ & $\begin{array}{l}1.74 \\
1.70 \\
1.82 \\
1.70 \\
1.92\end{array}$ & $\begin{array}{l}1.66 \\
1.60 \\
1.67 \\
1.72 \\
1.52 \\
1.62\end{array}$ & $\begin{array}{l}1.72 \\
1.34 \\
1.74 \\
2.06 \\
1.60 \\
1.80\end{array}$ & $\begin{array}{l}1.48 \\
1.40 \\
1.52 \\
1.50 \\
1.50\end{array}$ \\
\hline
\end{tabular}


AUGLAIZE RIVER NEAR DEFIANCE, OHIO

Location.-In NE. $1 / 4$ sec. 9 , T. 3 N., R. 4 E. at dam and power plant of Defiance Gas \& Electric Co., 3 miles south of Defiance, Defiance County.

Drainage area.-2,320 square miles (measured on topographic maps).

Records avallable.-May 20 to October 24, 1903; April 13, 1915, to September 30, 1923.

GAGES.-Vertical staff gage on upstream side of power plant at right end of dam. Auxiliary staff gage in tailwater. Gages set to mean sea level datum. Crest of dam is 688 feet and top of flashing 689.75 feet above mean sea level. Gages read hourly by power-plant attendants.

Discharge Measurements.-Made from highway bridge $13 / 4$ miles below dam or by wading.

Discharge.-Daily discharge ascertained by power company from hourly readings on head and tail gages, log sheets of power plant, and ratings of crest of dam, Taintor gates, and turbines.

EXTREMES OF DISCHARGE.--Maximum mean daily discharge during year, 20,800 second-feet May 17; minimum mean daily discharge, 18 second-feet August 31.

1915-1923: Maximum mean daily discharge, 36,100 second-feet March 18, 1919; minimum mean daily discharge, 7 second-feet June 30, 1918.

ICE.-Determination of discharge over dam and through plant not seriously affected by ice.

Drversions.- None.

Regulation.-Flow regulated by Defiance Gas \& Electric Co. Record of discharge not corrected for storage.

ACCURACY.-Discharge ascertained by power company has been checked by current-meter measurements made below dam at various stages and found accurate. The leakage through dam and power plant has been determined for various stages below crest level by current-meter measurements made by wading below dam when power plant was shut down. The leakage varies from 7 second-feet at headwater elevation 679 feet to 41 second-feet at headwater elevation 688 feet. All daily discharge figures below 300 secondfeet have been corrected for leakage by engineers of the United States Geological Survey. Records good.

Cooperation.-Record of daily discharge, not cơrected for leakage, furnished by Defiance Gas \& Electric Co.

Discharge measurements of Auglaize River near Defiance, Ohio, during the year ending September 30, 1923

\begin{tabular}{|c|c|c|c|}
\hline Date & Made by- & $\begin{array}{c}\text { Gage } \\
\text { height }\end{array}$ & Discharge \\
\hline $\begin{array}{l}\text { Oct. } 5 \\
\text { May } 10 \\
\text { Aug. } 17\end{array}$ & $\begin{array}{l}\text { E. E. R. Dornbach } \\
\text { F. R. Morgan }\end{array}$ & $\begin{array}{l}\text { Feet } \\
80.50 \\
86.90 \\
86.20\end{array}$ & $\begin{array}{r}\text { Sec. } f t . \\
12.8 \\
\mathbf{3 6 . 3} \\
33.6\end{array}$ \\
\hline
\end{tabular}

Note.-Add 600 feet to gage heights to reduce to mean sea level datum. 
Daily. discharge, in second-feet, of A uglaize River near Defiance, Ohio, for the year ending September 30, 1923

\begin{tabular}{|c|c|c|c|c|c|c|c|c|c|c|c|c|}
\hline Day & Oct. & Nor. & Dec. & Jan. & Feb. & Mar. & Apr. & May & June & July & Aug. & Sept. \\
\hline 2. & $\begin{array}{l}26 \\
27 \\
27 \\
35 \\
28\end{array}$ & $\begin{array}{l}23 \\
24 \\
24 \\
98 \\
25\end{array}$ & $\begin{array}{r}46 \\
30 \\
47 \\
149 \\
432\end{array}$ & $\begin{array}{l}7,430 \\
8,950 \\
5,330 \\
3,560 \\
2,630\end{array}$ & \begin{tabular}{|l}
3,330 \\
4,190 \\
4,860 \\
3,990 \\
2,290
\end{tabular} & $\begin{array}{r}763 \\
813 \\
1,400 \\
2,200 \\
4,200\end{array}$ & $\begin{array}{r}454 \\
7701 \\
686 \\
700 \\
1,110\end{array}$ & $\begin{array}{l}660 \\
417 \\
242 \\
281 \\
299\end{array}$ & $\begin{array}{l}650 \\
777 \\
772 \\
721 \\
468\end{array}$ & $\begin{array}{c}343 \\
36 \\
153 \\
725 \\
135\end{array}$ & 167 & $\begin{array}{l}3 \\
2 \\
2 \\
2 \\
3\end{array}$ \\
\hline & $\begin{array}{r}29 \\
29 \\
30 \\
30 \\
459\end{array}$ & $\begin{array}{r}25 \\
143 \\
70 \\
44 \\
25\end{array}$ & $\begin{array}{r}118 \\
129 \\
129 \\
31 \\
47\end{array}$ & $\begin{array}{l}1,880 \\
1,090 \\
1,390 \\
1,220 \\
1,060\end{array}$ & $\begin{array}{l}1,420 \\
1,080 \\
1,020 \\
1,070 \\
1,010\end{array}$ & $\begin{array}{l}4,060 \\
2,940 \\
2,030 \\
1,420 \\
2,340\end{array}$ & $\begin{array}{l}7,450 \\
8,470\end{array}$ & $\begin{array}{r}72 \\
302 \\
109 \\
112 \\
119\end{array}$ & $\begin{array}{l}158 \\
224 \\
227 \\
231 \\
862\end{array}$ & $\begin{array}{r}141 \\
119 \\
90 \\
38 \\
104\end{array}$ & $\begin{array}{r}37 \\
35 \\
196 \\
368 \\
31\end{array}$ & $\begin{array}{r}28 \\
436 \\
600 \\
77 \\
284\end{array}$ \\
\hline$\ldots$ & $\begin{array}{r}50 \\
80 \\
29 \\
29 \\
312\end{array}$ & $\begin{array}{l}25 \\
25 \\
26 \\
59 \\
59\end{array}$ & $\begin{array}{r}44 \\
657 \\
143 \\
98 \\
74\end{array}$ & $\begin{array}{l}1,800 \\
1,620 \\
1,310 \\
1,420 \\
8,740\end{array}$ & $\begin{array}{r}611 \\
981 \\
2,090 \\
4,200 \\
2,970\end{array}$ & $\begin{array}{r}6,480 \\
10,600 \\
13,200 \\
12,600 \\
8,570\end{array}$ & $\begin{array}{r}1,250 \\
1,120 \\
896 \\
747 \\
692\end{array}$ & $\begin{array}{r}244 \\
531 \\
6,350 \\
11,600 \\
12,200\end{array}$ & $\begin{array}{l}301 \\
646 \\
688 \\
681 \\
256\end{array}$ & 509 & $\begin{array}{r}49 \\
211 \\
213 \\
38 \\
32\end{array}$ & $\begin{array}{l}24 \\
22 \\
20 \\
19 \\
20\end{array}$ \\
\hline 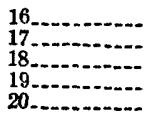 & $\begin{array}{r}444 \\
123 \\
26 \\
26 \\
27\end{array}$ & $\begin{array}{r}42 \\
127 \\
27 \\
28 \\
98\end{array}$ & $\begin{array}{r}33 \\
307 \\
55 \\
34 \\
34\end{array}$ & $\begin{array}{r}12,500 \\
9,140 \\
6,480 \\
4,580 \\
2,610\end{array}$ & $\begin{array}{l}1,920 \\
1,440 \\
1,120 \\
1,180 \\
1,030\end{array}$ & $\begin{array}{r}13,600 \\
20,600 \\
19,900 \\
10,700 \\
4,160\end{array}$ & $\begin{array}{l}815 \\
750 \\
771 \\
624 \\
606\end{array}$ & $\begin{array}{r}18,200 \\
20,800 \\
13,600 \\
8,180 \\
4,630\end{array}$ & $\begin{array}{l}252 \\
244\end{array}$ & & 33 & $\begin{array}{l}25 \\
31 \\
28 \\
23\end{array}$ \\
\hline 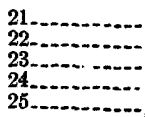 & $\begin{array}{l}27 \\
79 \\
79 \\
80 \\
86\end{array}$ & $\begin{array}{r}99 \\
93 \\
218 \\
245 \\
53\end{array}$ & $\begin{array}{r}102 \\
64 \\
36 \\
37 \\
37\end{array}$ & $\begin{array}{r}2,320 \\
5,240 \\
5,450 \\
.3,550 \\
2,270\end{array}$ & $\begin{array}{r}1,020 \\
852 \\
875 \\
817 \\
570\end{array}$ & $\begin{array}{r}4,990 \\
4,020\end{array}$ & $\begin{array}{l}514 \\
342 \\
709 \\
769 \\
685\end{array}$ & $\begin{array}{r}3,340 \\
2,440 \\
646 \\
606 \\
561\end{array}$ & 3 & 58 & $\begin{array}{l}290 \\
2913\end{array}$ & $\begin{array}{l}287 \\
237 \\
85 \\
260 \\
278\end{array}$ \\
\hline 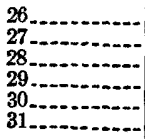 & $\begin{array}{r}100 \\
49 \\
141 \\
164 \\
705 \\
121\end{array}$ & $\begin{array}{r}337 \\
28 \\
28 \\
103 \\
29\end{array}$ & $\begin{array}{r}38 \\
403 \\
698 \\
3,120 \\
4,510 \\
3,920\end{array}$ & $\begin{array}{l}2,240 \\
1,030 \\
1,100 \\
3,610 \\
3,970 \\
3,310\end{array}$ & $\begin{array}{l}1,090 \\
1,040 \\
790\end{array}$ & $\begin{array}{r}2,730 \\
1,720 \\
1,380 \\
1,190 \\
946 \\
600\end{array}$ & $\begin{array}{l}678 \\
616 \\
475 \\
84 \\
524\end{array}$ & $\begin{array}{r}1,300 \\
341 \\
6550 \\
683 \\
219 \\
434\end{array}$ & $\begin{array}{l}146 \\
182 \\
168 \\
108 \\
106\end{array}$ & $\begin{array}{l}499 \\
223 \\
185 \\
162\end{array}$ & $\begin{array}{r}41 \\
268 \\
313 \\
312 \\
61 \\
18\end{array}$ & $\begin{array}{r}245 \\
185 \\
239 \\
263 \\
75\end{array}$ \\
\hline
\end{tabular}

Monthly discharge, in second-feet, of A uglaize River near Defiance, Ohio, for the year ending September 30,1923

\begin{tabular}{|c|c|c|c|c|c|c|c|}
\hline Month & Maximum & Minimum & Mean & Month & Maximum & Minimum & Mean \\
\hline $\begin{array}{l}\text { October. } \\
\text { November } \\
\text { December } \\
\text { January } \\
\text { February } \\
\text { March }\end{array}$ & $\begin{array}{r}705 \\
337 \\
4,510 \\
12,500 \\
4,860 \\
20,600\end{array}$ & $\begin{array}{r}26 \\
23 \\
30 \\
1,030 \\
570 \\
600\end{array}$ & $\begin{array}{r}113 \\
75 \\
504 \\
3,830 \\
1,740 \\
5,400\end{array}$ & $\begin{array}{l}\text { May } \\
\text { June } \\
\text { July } \\
\text { August } \\
\text { September }\end{array}$ & $\begin{array}{r}20,800 \\
862 \\
806 \\
641 \\
602 \\
\end{array}$ & $\begin{array}{l}72 \\
37 \\
36 \\
18 \\
21 \\
\end{array}$ & $\begin{array}{r}3,550 \\
355 \\
362 \\
198 \\
199 \\
\end{array}$ \\
\hline April & 8,470 & & 1,500 & The year... & 20,800 & 18 & 1,490 \\
\hline
\end{tabular}

\section{BLANCHARD RIVER AT GLANDORF, OHIO}

Locamon.-In NE. $1 / 4$ sec. 17, T. 1 N., R. 7 E., at highway bridge three-fourths mile northeast of Glandorf, Putnam County, and 11/4 miles above mouth of Cranberry Creek.

Drainage AREa.-643 square miles (measured on topographic maps).

Reconds available.-August 30, 1921, to September 30, 1923.

GAGE.-Chain gage on highway bridge, read to hundredths once daily by Victor Unterbrink.

Discharge measurements.--Made from bridge at gage or by wading.

Channel and control.-Channel straight for 500 feet above and below gage. Banks fairly high and wooded. One channel at all stages. Control is stretch of channel below gage; practically permanent. Zero flow would occur at gage height 0.7 foot. 
Extremes of stage.-Maximum stage recorded during year, 19.1 feet at 6 a. m. March 17; minimum stage, 1.68 feet at 6 a. m. October 6 and 7 .

1921-1923: Maximum stage recorded, 22.4 feet at 1 p. m. April 1, 1922; minimum stage, 1.58 feet at 11 a. m. and 3 p. m. August 30 , and 6 p. m. August 31, 1921.

ICE.-Stage-discharge relation seriously affected by ice.

Drversions:-None.

Regulation.- None.

Data inadequate for determination of discharge.

Discharge measurements of Blanchard River at Glandorf, Ohio, during the year ending September 30, 1925

\begin{tabular}{|c|c|c|c|}
\hline Date & Made by- & $\begin{array}{l}\text { Gage } \\
\text { height }\end{array}$ & Discharg!? \\
\hline $\begin{array}{l}\text { May } 7 \\
\text { Aug. 21 }\end{array}$ & $\begin{array}{l}\text { E. E. R. Dornbach. } \\
\text { F. R. Morgan }\end{array}$ & $\begin{array}{l}\text { Feet } \\
2.14 \\
1.78\end{array}$ & $\begin{array}{l}\text { Sec.fft. } \\
\text { 34. } 10 \\
15 .:\end{array}$ \\
\hline
\end{tabular}

Daily gage height, in feet, of Blanchard River at Glandorf, Ohio, for the year ending September 30, 1923

\begin{tabular}{|c|c|c|c|c|c|c|c|c|c|c|c|c|}
\hline & ct. & Nov. & Dec. & Jan. & Feb. & Mar. & Apr. & May & June & July & Aug. & Sept. \\
\hline & $\begin{array}{l}1.76 \\
1.78 \\
1.76 \\
1.74 \\
1.72\end{array}$ & $\begin{array}{l}1.90 \\
1.90 \\
1.90 \\
1.90 \\
1.88\end{array}$ & $\begin{array}{l}1.92 \\
1.90 \\
1.90 \\
1.90 \\
2.02\end{array}$ & $\begin{array}{r}11 . \\
8 . \\
7 . \\
6 .\end{array}$ & $\begin{array}{r}7.3 \\
8.7 \\
10.0 \\
8.4\end{array}$ & & $\begin{array}{l}45 \\
45 \\
45 \\
48\end{array}$ & $\begin{array}{l}2.70 \\
2.64 \\
2.58 \\
2.48 \\
2.58\end{array}$ & & & $2 . \%$ & $\begin{array}{l}2.1 \\
2.2 \\
2 . \\
2.3 \\
2.3\end{array}$ \\
\hline $6 \ldots$ & $\begin{array}{l}1.68 \\
1.68 \\
1.72 \\
1.78 \\
2.08\end{array}$ & $\begin{array}{l}1.90 \\
1.90 \\
1.90 \\
1.90 \\
1.90\end{array}$ & $\begin{array}{l}1.98 \\
2.06 \\
2.25 \\
2.30 \\
2.26\end{array}$ & $\begin{array}{l}4.8 \\
4.4 \\
3.98 \\
4.0 \\
4.4\end{array}$ & $\begin{array}{l}6.7 \\
6.0 \\
5.4 \\
5.0 \\
4.6\end{array}$ & $\begin{array}{l}7.0 \\
6.4 \\
5.4 \\
4.8 \\
6.5\end{array}$ & & $\begin{array}{l}2.48 \\
2.25 \\
2.18 \\
2.56 \\
2.88\end{array}$ & & & & $\begin{array}{l}3 . \\
2 . \\
2 .\end{array}$ \\
\hline & $\begin{array}{l}2.02 \\
1.90 \\
1.94 \\
1.94 \\
1.94\end{array}$ & $\begin{array}{l}1.90 \\
1.90 \\
1.90 \\
1.90 \\
1.94\end{array}$ & $\begin{array}{l}2.20 \\
2.20 \\
2.14 \\
2.14 \\
2.12\end{array}$ & $\begin{array}{r}4 \\
74 \\
14\end{array}$ & $\begin{array}{r}4.5 \\
4.4 \\
8.4 \\
13.7 \\
12.8\end{array}$ & & & & & & & 1. \\
\hline & $\begin{array}{l}1.94 \\
1.92 \\
1.90 \\
1.86 \\
1.84\end{array}$ & $\begin{array}{l}1.96 \\
2.00 \\
2.46 \\
2.20 \\
2.02\end{array}$ & $\begin{array}{l}2.08 \\
2.00 \\
2.00 \\
1.96 \\
1.96 .\end{array}$ & $\begin{array}{r}17.7 \\
15.5 \\
12.4 \\
7.7 \\
5.9\end{array}$ & $\begin{array}{l}8.7 \\
7.4 \\
7.4 \\
7.2 \\
7.0\end{array}$ & & & $\begin{array}{r}12.3 \\
8.4\end{array}$ & & & & $\begin{array}{l}1.8 \\
1.8 \\
1.8 \\
3.8\end{array}$ \\
\hline 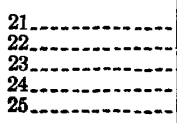 & $\begin{array}{l}1.82 \\
1.82 \\
1.92 \\
1.95 \\
1.90\end{array}$ & $\begin{array}{l}2.00 \\
1.96 \\
1.92 \\
1.86 \\
1.80\end{array}$ & $\begin{array}{l}1.96 \\
2.00 \\
2.00 \\
2.00 \\
2.12\end{array}$ & $\begin{array}{r}7.7 \\
12.4 \\
14.8 \\
7.6 \\
6.0\end{array}$ & $\begin{array}{l}6.6 \\
6.3 \\
6.1 \\
5.8 \\
5.8\end{array}$ & $\begin{array}{r}7.4 \\
5.1 \\
10.0 \\
12.1 \\
10.8\end{array}$ & & $\begin{array}{l}4.6 \\
4.0 \\
3.67\end{array}$ & & & & $\begin{array}{l}3.05 \\
2.87 \\
2.67 \\
2.45 \\
2.27\end{array}$ \\
\hline 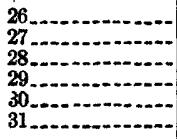 & $\begin{array}{l}1.90 \\
1.90 \\
1.90 \\
1.90 \\
1.90 \\
1.90\end{array}$ & $\begin{array}{l}1.80 \\
1.82 \\
1.82 \\
1.84 \\
1.84\end{array}$ & $\begin{array}{l}3.00 \\
4.5 \\
6.7 \\
7.7 \\
7.0 \\
8.8\end{array}$ & $\begin{array}{l}5.1 \\
4.6 \\
4.8 \\
8.3 \\
9.0 \\
8.1\end{array}$ & $\begin{array}{l}5 \\
5 . \\
7 .\end{array}$ & $\begin{array}{l}7.6 \\
5.4 \\
5.0 \\
4.8 \\
4.4 \\
3.9\end{array}$ & & $\begin{array}{l}3.57 \\
3.38 \\
3.16 \\
2.90 \\
2.84 \\
2.78\end{array}$ & & $\begin{array}{r}2.10 \\
2.72 \\
2.72 \\
2.56\end{array}$ & $\begin{array}{l}1.73 \\
1.73 \\
2.01 \\
2.43 \\
2.53 \\
2.25\end{array}$ & $\begin{array}{l}2 . \\
2 . \\
2 . \\
2 . \\
3 .\end{array}$ \\
\hline
\end{tabular}

Note.-Stage-discharge relation affected by ice Feb. 13 to Mar. 1 
MIAMI AND ERIE CANAL AT WATERVILIE, OHIO

Location.-At highway bridge at Waterville, Lucas County, opposite gaging station on Maumee River at Waterville.

Records avaILable.-August 26, 1921, September 30, 1923.

GAGE.-Vertical staff gage on downstream wing wall of left abutment; read by John Rhodes. Prior to September 13, 1922, chain gage on downstream side of highway bridge. Both gages at same datum.

Discharge MEASUREMENTs.-Made from footbridge 500 feet below gage.

Channel and control.-Channel straight for a quarter of a mile above and below gage. One channel at all stages. Control is long stretch of channel below gage; shifting. Zero flow occurs at gage height 0.86 foot.

EXTREMes of DISCHARGE.-Maximum stage recorded during year, 6.95 feet January 8 (discharge, 580 second-feet). No flow in canal March 15, when there was break in bank above gage.

1921-1923: Maximum stage recorded, 7.07 feet March 2, 1922 (discharge, 610 second-feet). No flow in canal March 15, 1923.

ICE.--Stage-discharge relation may be affected by ice during severe winters.

REgulation.-The flow in the canal is regulated at the head gate at Grand Rapids, 10 miles upstream.

ACCURACY.- Stage-discharge relation not permanent; not seriously affected by ice. Gage read to half-tenths once daily. Daily discharge ascertained by shifting-control method. Records fair.

The canal diverts from Maumee River at Grand Rapids, 10 miles above Waterville. The water is used for power at Maumee and Toledo.

Discharge measurements of Miamı and Erie Canal at Waterville, Ohio; during the year ending September 30, 1923

\begin{tabular}{|c|c|c|c|c|c|c|c|}
\hline Date & Made by一 & $\begin{array}{c}\text { Gage } \\
\text { height }\end{array}$ & $\begin{array}{c}\text { Dis- } \\
\text { charge }\end{array}$ & Date & Made by- & $\begin{array}{r}\text { Gage } \\
\text { height }\end{array}$ & $\begin{array}{c}\text { Dis- } \\
\text { charge }\end{array}$ \\
\hline $\begin{array}{l}\text { Oet. } 2 \\
\text { May } 12\end{array}$ & E.E.R. Dornbach & $\begin{array}{c}\text { Feet } \\
5.98 \\
6.32\end{array}$ & $\begin{array}{r}\text { Sec. } f t \text {. } \\
467 \\
461\end{array}$ & Aug. $\frac{2}{15}$ & $\begin{array}{l}\text { Dickson and Lee..... } \\
\text { F.R.Morgan }\end{array}$ & $\begin{array}{c}\text { Feet } \\
6.15 \\
5.97\end{array}$ & $\begin{array}{r}\text { Sec.ft. } \\
477 \\
440\end{array}$ \\
\hline
\end{tabular}


Daily discharge, in second-feet, of Miami and Erie Canal at Waterville, Ohio, for the year ending September 30, 1923

\begin{tabular}{c|r|r|r|r|r|r|r|r|r|r|r|r}
\hline Day & Oct. & Nov. & Dec. & Jan. & Feb. & Mar. & Apr. & May & June & July & Aug. & Sept. \\
\hline & & & & & & & & & & & \\
\hline & & & & & & & & & & \\
\hline
\end{tabular}

Monthly discharge, in second-feet, of Miami and Erie Canal at Waterville, Ohio, for the year ending September 30, 1923

\begin{tabular}{|c|c|c|c|c|c|c|c|}
\hline Month & Maximum & Minimum & Mean & Month & Maximum & Minimum & Mean \\
\hline $\begin{array}{l}\text { October } \\
\text { November } \\
\text { December } \\
\text { January } \\
\text { February } \\
\text { March }\end{array}$ & $\begin{array}{l}492 \\
450 \\
520 \\
580 \\
550 \\
506\end{array}$ & $\begin{array}{r}303 \\
292 \\
292 \\
314 \\
397 \\
0\end{array}$ & $\begin{array}{l}359 \\
328 \\
400 \\
472 \\
441 \\
281\end{array}$ & $\begin{array}{l}\text { May } \\
\text { June } \\
\text { July } \\
\text { August } \\
\text { September.... }\end{array}$ & $\begin{array}{l}464 \\
478 \\
506 \\
478 \\
450\end{array}$ & $\begin{array}{l}372 \\
384 \\
372 \\
384 \\
384\end{array}$ & $\begin{array}{l}410 \\
428 \\
438 \\
448 \\
420\end{array}$ \\
\hline April & 436 & 303 & 386 & The year... & 580 & 0 & 401 \\
\hline
\end{tabular}

\section{SANDUSKY RIVER NEAR UPPER SANDUSKY, OHIO}

Location.-In sec. 21 , T. 2 S., R. 14 E., at highway bridge 2 miles northeast of Upper Sandusky, Wyandot County.

Drainage area.-299 square miles (measured on topographic maps).

Records ayailable.-October 20, 1921, to September 30, 1923.

GAGE.-Chain gage on downstream side of highway bridge.

Discharge MeASUREMENTs.-Made from bridge at gage or by wading.

Channel and control.-Channel is straight for 400 feet above gage but is divided by island; straight for 1,000 feet below gage. One channel at all stages. Banks are low and wooded. All water flows under bridge up to gage height 11.3 feet, when road leading to bridge on right bank is overflowed. Control for low water is riffle 200 feet below gage, composed of rock ledge and gravel. At high stages control is long stretch of channel below gage. Zero flow would occur at gage height 0.1 foot, as determined August 14, 1923. 
Extremes of Discharge.-Maximum stage recorded during year, 6.6 feet at 4.30 p. m. January 15 (discharge, 2,850 second-feet); minimum stage, 1.06 feet at 9 a. $\mathrm{m}$. October 6 (discharge, 7.5 second-feet).

1921-1923: Maximum stage recorded, 8 feet April 18, 1922 (discharge, 4,060 second-feet); minimum stage, that of October 6, 1922.

ICE.-Stage-discharge relation seriously affected by ice.

ACCURACY.-Stage-discharge relation practically permanent except as affected by leaves on control during October, November, and December and by ice during February and March. Rating curve well defined. Gage read to hundredths once daily. Daily discharge ascertained by applying daily gage height to rating table. Records good except during period of ice effect, for which they are fair.

Discharge measurements of Sandusky River near Upper Sandusky, Ohio, during the year ending September 30, 1928

\begin{tabular}{|c|c|c|c|c|c|c|c|}
\hline Date & Made by - & $\begin{array}{l}\text { Gage } \\
\text { height }\end{array}$ & $\begin{array}{c}\text { Dis- } \\
\text { charge }\end{array}$ & Date & Made by- & $\begin{array}{c}\text { Gage } \\
\text { height }\end{array}$ & $\begin{array}{l}\text { Dis- } \\
\text { charge }\end{array}$ \\
\hline $\begin{array}{l}\text { Oct. } 29 \\
\text { Nov. } 21 \\
\text { Apr. } 10\end{array}$ & $\begin{array}{l}\text { Lasley Lee } \\
\text { E. E. R. Dornbach }\end{array}$ & $\begin{array}{c}\text { Feet } \\
1.27 \\
1.35 \\
2.18\end{array}$ & $\begin{array}{r}\text { Sec. } f t . \\
10.4 \\
18.4 \\
217\end{array}$ & $\begin{array}{l}\text { Apr. } 10 \\
\text { May } 15 \\
\text { Aug. } 14\end{array}$ & $\begin{array}{l}\text { E. E. R. Dornbach } \\
\text { F. R. Morgan }\end{array}$ & $\begin{array}{c}\text { Feet } \\
2.09 \\
4.16 \\
1.08\end{array}$ & $\begin{array}{c}\text { Sec.-ft. } \\
188 \\
1,160 \\
10.8\end{array}$ \\
\hline
\end{tabular}

Daily discharge, in second-feet, of Sandusky River near Upper Sandusky, Ohio, for the year ending September 30, 1923

\begin{tabular}{r|r|r|r|r|r|r|r|r|r|r|r|r}
\hline Day & Oct. & Nov. & Dec. & Jan. & Feb. & Mar. & A pr. & May & June & July & Aug. & Sept. \\
\hline & & & & & & & & \\
\hline
\end{tabular}

Nork.-Stage-discharge relation afiected by leaves matted on control Oct. 13 to Dec. 29. Method for shifting control used Oct. 13-28. Stage-discharge relation affected by ice Feb. 15 to Mar. 3, flow estimated from study of observer's notes, weather records, and records of flow of near-by streams. 
Monthly discharge of Sandusky River near Upper Sandusky, Ohio, for the year ending September 30, 1923

[Drainage area, 299 square miles]

\begin{tabular}{|c|c|c|c|c|c|}
\hline \multirow{2}{*}{ Month } & \multicolumn{4}{|c|}{ Discharge in second-feet } & \multirow{2}{*}{$\begin{array}{l}\text { Run-off } \\
\text { in inches }\end{array}$} \\
\hline & Maximum & Minimum & Mean & $\underset{\text { mile }}{\text { Per square }}$ & \\
\hline $\begin{array}{l}\text { October } \\
\text { November } \\
\text { December } \\
\text { January } \\
\text { February } \\
\text { March } \\
\text { April } \\
\text { May }\end{array}$ & \begin{tabular}{r}
32 \\
32 \\
1,060 \\
2,850 \\
\hdashline 2,460 \\
1,950 \\
2,460 \\
102 \\
528 \\
54 \\
373
\end{tabular} & \begin{tabular}{c}
7.5 \\
11 \\
12 \\
132 \\
\hdashline 107 \\
33 \\
22 \\
13 \\
12 \\
9.4 \\
11
\end{tabular} & $\begin{array}{l}14.3 \\
18.2 \\
91.3 \\
606 \\
588 \\
604 \\
288 \\
339 \\
28.9 \\
62.8 \\
16.5 \\
59.4\end{array}$ & $\begin{array}{c}0.048 \\
.061 \\
.310 \\
2.03 \\
1.97 \\
2.02 \\
.963 \\
1.14 \\
.097 \\
.021 \\
.055 \\
.199\end{array}$ & $\begin{array}{r}0.06 \\
.07 \\
.36 \\
2.34 \\
2.05 \\
2.33 \\
1.07 \\
1.31 \\
.11 \\
.02 \\
.06 \\
.22\end{array}$ \\
\hline The year ...... & 2,850 & 7.5 & 225 & .753 & 10.23 \\
\hline
\end{tabular}

SANDUSKY RIVER NEAR MEXICO, OHIO

Location.-In sec. 13, T. 1 N., R. 14 E., at highway bridge $41 / 4$ miles north of. Mexico, Wyandot County. Honey Creek enters on right 3 miles below station.

Drainage area.-776 square miles (measured on topographic maps).

Records avallable.-March 1 to September 30, 1923, at present site; November 17,1898 , to November 16,1900 , at highway bridge at Mexico.

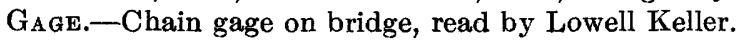

DischaRge MEASUREMENTS. - Made from bridge at gage or by wading:

Cinannel and control.-Channel straight for 800 feet above and below gage. Right bank high and wooded; left bank fairly high, wooded, subject to overflow at extremely high water. One channel at all stages. Control for low water is riffle of boulders on rock ledge 100 feet below gage; fairly permanent. Control at high stages is long stretch of channel below gage. Zero flow would occur at gage height 0.7 foot.

Extremes of Discharge.- Maximum stage recorded during period of record, 11.45 feet at time of discharge measurement May 13 (discharge, 4,900 second-feet) ; minimum stage recorded, 1.62 feet at 3.35 p. m. Augușt 14 (discharge, 29 second-feet).

Diversions.-None.

Regulation.-None.

Accuracr.-Stage-discharge relation for low water changed during high water on May 13. Rating curves well defined. Gage read to hundredths once daily. Daily discharge ascertained by applying daily gage height to rating table. Records good. 
Discharge measurements of Sandusky River near Mexico, Ohio, during the years ending September 30, 1921-1923

\begin{tabular}{|c|c|c|c|c|c|c|c|}
\hline Date & Made by- & $\begin{array}{l}\text { Gage } \\
\text { height }\end{array}$ & $\begin{array}{l}\text { Dis- } \\
\text { charge }\end{array}$ & Date & Made by- & $\begin{array}{c}\text { Gage } \\
\text { height }\end{array}$ & $\begin{array}{l}\text { Dis- } \\
\text { charge }\end{array}$ \\
\hline $\begin{array}{c}1921 \\
\text { Sept. } 8 \\
1922\end{array}$ & Lee and Lamoureux & $\begin{array}{l}\text { Feet } \\
1.90\end{array}$ & $\begin{array}{r}\text { Sec. }-f t . \\
38.8\end{array}$ & $\begin{array}{r}1922 \\
\text { Aug. } 18 \\
29\end{array}$ & $\begin{array}{l}\text { E. E. R. Dornbach } \\
\text { Lee and Sherman }\end{array}$ & $\begin{array}{l}\text { Feet } \\
1.89 \\
2.09\end{array}$ & $\begin{aligned} \text { Sec.ft. } \\
34.9 \\
52.3\end{aligned}$ \\
\hline $\begin{array}{l}\text { Mar. } 14 \\
\text { Apr. } 12 \\
\text { May } 20\end{array}$ & \begin{tabular}{|l} 
E. E. R. Dornbach \\
do
\end{tabular} & $\begin{array}{r}5.87 \\
8.92 \\
12.90\end{array}$ & $\begin{array}{l}1,270 \\
2,960 \\
6,130\end{array}$ & $\begin{array}{l}1923 \\
\text { May } 13 \\
\text { Aug. } 14\end{array}$ & $\begin{array}{l}\text { E. E. R. Dornbach } \\
\text { F. R. Morgan }\end{array}$ & $\begin{array}{r}11.45 \\
1.63\end{array}$ & $\begin{array}{l}4,900 \\
29.8\end{array}$ \\
\hline
\end{tabular}

Daily discharge, in second-feet, of Sandusky River near Mexico, Ohio, for the period March 1 to September 30, 1923

\begin{tabular}{|c|c|c|c|c|c|c|c|}
\hline Day & Mar. & Apr. & May & June & July & Aug. & Sept. \\
\hline $\begin{array}{l}1 \\
2 \\
3 \\
4 \\
5\end{array}$ & $\begin{array}{r}1,100 \\
600 \\
495 \\
530 \\
1,280\end{array}$ & $\begin{array}{r}170 \\
160 \\
180 \\
249 \\
2,880\end{array}$ & $\begin{array}{l}73 \\
71 \\
65 \\
63 \\
60\end{array}$ & $\begin{array}{l}93 \\
87 \\
81 \\
81 \\
87\end{array}$ & $\begin{array}{l}56 \\
55 \\
55 \\
81 \\
81\end{array}$ & $\begin{array}{r}40 \\
35 \\
107 \\
130 \\
93\end{array}$ & $\begin{array}{r}107 \\
87 \\
68 \\
93 \\
230\end{array}$ \\
\hline $\begin{array}{r}6 \\
7 \\
7 \\
9 \\
9\end{array}$ & $\begin{array}{l}980 \\
820 \\
530 \\
356 \\
565\end{array}$ & $\begin{array}{r}3,960 \\
3,580 \\
1,580 \\
860 \\
600\end{array}$ & $\begin{array}{l}60 \\
58 \\
54 \\
51 \\
53\end{array}$ & $\begin{array}{r}76 \\
230 \\
255 \\
282 \\
206\end{array}$ & $\begin{array}{r}87 \\
565 \\
860 \\
820 \\
705\end{array}$ & $\begin{array}{l}70 \\
76 \\
70 \\
68 \\
65\end{array}$ & $\begin{array}{r}122 \\
1,020 \\
740 \\
390 \\
195\end{array}$ \\
\hline (n) & $\begin{array}{l}2,280 \\
3,090 \\
2,760 \\
1,880 \\
1,060\end{array}$ & $\begin{array}{l}408 \\
356 \\
276 \\
249 \\
236\end{array}$ & $\begin{array}{r}60 \\
236 \\
4,840 \\
4,840 \\
4,360\end{array}$ & $\begin{array}{r}130 \\
114 \\
93 \\
87 \\
93\end{array}$ & $\begin{array}{l}670 \\
740 \\
460 \\
268 \\
174\end{array}$ & $\begin{array}{l}68 \\
60 \\
53 \\
30 \\
48\end{array}$ & $\begin{array}{r}192 \\
87 \\
68 \\
64 \\
56\end{array}$ \\
\hline $\begin{array}{l}16 \\
17 \\
19\end{array}$ & $\begin{array}{l}2,160 \\
3,160 \\
2,040 \\
1,140 \\
670\end{array}$ & $\begin{array}{l}224 \\
201 \\
190 \\
160 \\
150\end{array}$ & $\begin{array}{l}4,840 \\
3,800 \\
1,930 \\
1,060 \\
\mathbf{6 7 0}\end{array}$ & $\begin{array}{l}87 \\
81 \\
76 \\
70 \\
64\end{array}$ & $\begin{array}{r}174 \\
130 \\
100 \\
.87 \\
81\end{array}$ & $\begin{array}{l}48 \\
46 \\
43 \\
45 \\
46\end{array}$ & $\begin{array}{l}53 \\
55 \\
51 \\
49 \\
60\end{array}$ \\
\hline (2) & $\begin{array}{r}495 \\
1,100 \\
3,580 \\
3.960 \\
2,880\end{array}$ & $\begin{array}{r}140 \\
121 \\
109 \\
109 \\
95\end{array}$ & $\begin{array}{l}600 \\
740 \\
530 \\
311 \\
242\end{array}$ & $\begin{array}{l}64 \\
60 \\
56 \\
53 \\
49\end{array}$ & $\begin{array}{l}70 \\
66 \\
60 \\
51 \\
55\end{array}$ & $\begin{array}{l}45 \\
41 \\
45 \\
48 \\
41\end{array}$ & $\begin{array}{r}81 \\
100 \\
122 \\
138 \\
100\end{array}$ \\
\hline $\begin{array}{l}26 \\
27 \\
28\end{array} 29$ & $\begin{array}{r}1,100 \\
705 \\
495 \\
408 \\
290 \\
236\end{array}$ & $\begin{array}{l}92 \\
85 \\
89 \\
85 \\
82\end{array}$ & $\begin{array}{l}184 \\
164 \\
146 \\
130 \\
122 \\
107\end{array}$ & $\begin{array}{l}43 \\
53 \\
58 \\
66 \\
64\end{array}$ & $\begin{array}{l}64 \\
70 \\
70 \\
66 \\
55 \\
48\end{array}$ & $\begin{array}{r}41 \\
56 \\
58 \\
122 \\
195 \\
164\end{array}$ & $\begin{array}{r}76 \\
70 \\
93 \\
155 \\
155\end{array}$ \\
\hline
\end{tabular}

Monthly discharge of Sandusky River near Mexico, Ohio, for the period March 1 to September 30, 1923

[Drainage area, 776 square miles]

\begin{tabular}{|c|c|c|c|c|c|}
\hline \multirow{2}{*}{ Month } & \multicolumn{4}{|c|}{ Discharge in second-feet } & \multirow{2}{*}{$\begin{array}{l}\text { Run-off in } \\
\text { inches }\end{array}$} \\
\hline & Maximum & Minimum & Mean & $\begin{array}{c}\text { Per square } \\
\text { mile }\end{array}$ & \\
\hline 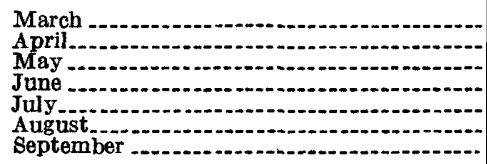 & $\begin{array}{r}3,960 \\
3,960 \\
4,840 \\
282 \\
860 \\
195 \\
1,020\end{array}$ & $\begin{array}{r}236 \\
82 \\
51 \\
43 \\
48 \\
30 \\
49\end{array}$ & $\begin{array}{c}1,380 \\
589 \\
985 \\
97.9 \\
223 \\
67.6 \\
160\end{array}$ & $\begin{array}{l}1.78 \\
.759 \\
1.27 \\
.126 \\
.288 \\
.087 \\
.206\end{array}$ & $\begin{array}{r}2.05 \\
.85 \\
1.46 \\
.14 \\
.38 \\
.10 \\
.22\end{array}$ \\
\hline
\end{tabular}




\section{EAST BRANCH OF BLACK RIVER AT ELYRIA; OHIO.}

Location.--At Fuller Street Bridge, 11/4 miles southeast of center of Elyria, Lorain County, and 3 miles by river above junction with West Branch.

Drainage ARea.-211 square miles (measured on topographic maps).

Records avajlable.-July 8, 1922, to September 30, 1923.

GAGE.-Chain gage on bridge, read to hundredths twice daily by Mrs. A. B. Coon and Mrs. Joe Wojcik.

Discharge measurements.-Made from bridge at gage or by wading.

Channel and control.-Channel straight for 1,000 feet above and 700 feet below gage. Banks high and clean, not subject to overflow. One channel at all stages. Low-water control is a solid rock ledge extending diagonally across channel 25 feet below gage. Control for high stages is long stretch of channel below gage. Zero flow would occur at gage height 0.52 foot.

ExTremes of STAGE.-Maximum stage recorded during period of record, 4.5 feet at 5.40 p. m. April 6, 1923; minimum stage recorded, 0.57 foot at 7.30 a. m. and 5.30 p. m. October 5 and 9.30 a. m. October 6, 1922 .

IcE.-Stage-discharge relation affected by ice.

Diversions.-None.

Regulation.-None.

Data inadequate for determination of discharge.

Discharge measurements of East Branch of Black River at Elyria, Ohio, during the years ending September 30, 1922 and 1923

\begin{tabular}{|c|c|c|c|c|c|c|c|}
\hline Date & Made by- & $\begin{array}{c}\text { Gage } \\
\text { height }\end{array}$ & $\begin{array}{c}\text { Dis- } \\
\text { charge }\end{array}$ & Date & Made by- & $\underset{\text { height }}{\text { Gage }}$ & $\begin{array}{c}\text { Dis- } \\
\text { charge }\end{array}$ \\
\hline $\begin{array}{r}\text { July }_{27}^{1922} \\
\\
\begin{array}{c}1923 \\
\text { Aug. } 6\end{array}\end{array}$ & $\begin{array}{l}\text { Lee and Sherman } \\
\text { E. E. R. Dornbach... } \\
\text { L. I. Dickson ........... }\end{array}$ & $\begin{array}{r}\text { Feet } \\
1.28 \\
.89 \\
\\
1.42\end{array}$ & $\begin{array}{r}\text { Sec. }-f t . \\
71.6 \\
12.2 \\
\\
97.5\end{array}$ & $\begin{array}{l}1923 \\
\text { Aug. } 30 \\
\text { Sept. } 11 \\
11\end{array}$ & $\begin{array}{l}\text { I. L. Diekson } \\
\text { E. E. R. Dornbach. } \\
\text { I. L. Dickson }\end{array}$ & $\begin{array}{c}\text { Feet } \\
1.54 \\
.82 \\
.80\end{array}$ & $\begin{array}{r}\text { Sec. } f t . \\
133 \\
7.6 \\
8.1\end{array}$ \\
\hline
\end{tabular}

Daily gage height, in feet, of East Branch of Black River at Elyria, Ohio, for the period July 8, 1922, to September 30, 1923

\begin{tabular}{|c|c|c|c|c|c|c|c|c|c|c|c|}
\hline Day & July & Aug. & Sept. & Day & July & Aug. & Sept. & Day & July & Aug. & Sept. \\
\hline $\begin{array}{ll} & 1922 \\
1 & \ldots \\
2 & \ldots \\
3 & \ldots \\
4 & \ldots \\
5 & \ldots\end{array}$ & $\cdots$ & $\begin{array}{l}0.74 \\
.74 \\
.74 \\
.70 \\
.71\end{array}$ & $\begin{array}{r}0.76 \\
.76 \\
.79 \\
.98 \\
1.28\end{array}$ & \begin{tabular}{ll} 
& 1922 \\
11 & \\
12 & \\
13 & \\
14 & - \\
15 & \\
\hdashline & -
\end{tabular} & $\begin{array}{l}0.98 \\
1.09 \\
.97 \\
.96 \\
.92\end{array}$ & $\begin{array}{l}0.74 \\
.72 \\
.72 \\
.74 \\
.73\end{array}$ & $\begin{array}{l}0.80 \\
.76 \\
.74 \\
.72 \\
.72\end{array}$ & $\begin{array}{ll} & 1922 \\
21 & -\ldots \\
22 & -\ldots \\
23 & \\
24 & -\ldots \\
25 & -\ldots . \\
\end{array}$ & $\begin{array}{l}0.84 \\
.82 \\
.81 \\
.80 \\
.88\end{array}$ & $\begin{array}{l}0.68 \\
.66 \\
.66 \\
.68 \\
.69\end{array}$ & $\begin{array}{r}0.74 \\
.72 \\
.72 \\
.70 \\
.70\end{array}$ \\
\hline $\begin{array}{r}6 \\
7 \\
7\end{array}$ & $\begin{array}{l}1.27 \\
1.43 \\
1.10\end{array}$ & $\begin{array}{l}.71 \\
.70 \\
.73 \\
.78 \\
.80\end{array}$ & $\begin{array}{l}1.10 \\
.94 \\
.88 \\
.84 \\
.80\end{array}$ & $\begin{array}{l}16 \\
17 \\
18 \\
19\end{array}$ & $\begin{array}{l}.87 \\
.82 \\
.82 \\
.87 \\
.87\end{array}$ & $\begin{array}{l}.72 \\
.72 \\
.71 \\
.70 \\
.69\end{array}$ & $\begin{array}{l}.72 \\
.71 \\
.71 \\
.74 \\
.72\end{array}$ & $\begin{array}{l}26 \\
27 \\
28\end{array}$ & $\begin{array}{l}.90 \\
.88 \\
.81 \\
.76 \\
.76 \\
.74\end{array}$ & $\begin{array}{l}.69 \\
.68 \\
.72 \\
.75 \\
.74 \\
.78\end{array}$ & $\begin{array}{r}.67 \\
.62 \\
.60 \\
.62 \\
.66 \\
-.-\end{array}$ \\
\hline
\end{tabular}


Daily gage height, in feet, of East Branch of Black River at Elyria, Ohio, for the period July 8, 1922, to September.30, 1923-Continued

\begin{tabular}{c|r|r|r|r|r|r|r|r|r|r|r|r}
\hline Day & Oct. & Nov. & Dec. & Jan. & Feb. & Mar. & Apr. & May & June & July & Aug. & Sept. \\
\hline $1922-23$ & & & & & & & & & & & & \\
\hline
\end{tabular}

\section{CUYAHOGA RIVER AT OLD PORTAGE, OHIO}

Location.-At highway bridge at Old Portage, also known as Cranmer, Summit County, 4 miles northwest of Akron. Little Cuyahoga River enters on left $11 / 4$ miles above station.

Drainage area.-405 square miles (measured on topographic maps).

Records AVAILABLe.--September 27, 1921, to September 30, 1923.

GAGE.-Chain gage on highway bridge; read by N. A. Bucklin.

Discharge measurements.- - Made from bridge at gage or by wading.

Channel and control.-Channel straight 300 feet above and below gage.

Banks fairly high, wooded. At extremely high stages water flows through second channel on right bank. Bed of stream composed of sand and gravel. Control for low water is riffle $\mathbf{5 0}$ feet below gage; control for high water is long stretch of channel below gage. Zero flow would occur at zero gage height. EXTREMEs of DISCHARGE.-Maximum stage recorded during year, 6.60 feet at

4 p. m. January 21 (discharge, 1,840 second-feet); minimum stage, 0.98 foot at 7.30 a. $\mathrm{m}$. September 1 (discharge, 51 second-feet).

1921-1923: Maximum stage recorded, 7.2 feet on March 31, 1922 (discharge,

2,130 second-feet); minimum stage, that of September 1, 1923.

ICE.-Stage-discharge relation not affected by ice.

Diversions.-Municipal water supply for Akron is diverted from headwaters of this stream. A small amount of water is diverted into this stream from

Tuscarawas River by the Ohio Canal.

Regulation.-Flow regulated at reservoir above Akron. 
ACCURACr.-Stage-discharge relation for low water changed during high water on January 21; not affected by ice. Rating curves well defined. Gage read to hundredths once daily up to November 9 , twice daily thereafter. Daily discharge ascertained by applying mean daily gage height to rating table. Records good.

Discharge measurements of Cuyahoga River at Old Portage, Ohio, during the year ending September 30, 1923

\begin{tabular}{|c|c|c|c|c|c|c|c|}
\hline Date & Made by- & $\begin{array}{c}\text { Gage } \\
\text { height }\end{array}$ & $\begin{array}{l}\text { Dis- } \\
\text { charge }\end{array}$ & Date & Made by- & $\begin{array}{c}\text { Gage } \\
\text { height }\end{array}$ & $\begin{array}{c}\text { Dis- } \\
\text { charge }\end{array}$ \\
\hline $\begin{array}{ll}\text { Oct. } & 12 \\
\text { Nov. } & 10 \\
\text { May } 26\end{array}$ & $\begin{array}{l}\text { E. E. R. Dornbach } \\
\text { Lasley Lee } \\
\text { Co o e r t, Le e, a n } \mathrm{d} \\
\text { Dornbach............ }\end{array}$ & $\begin{array}{l}\text { Feet } \\
1.98 \\
1.10 \\
2.09\end{array}$ & $\begin{array}{c}\text { Sec.-ft. } \\
246 \\
76.2 \\
279\end{array}$ & $\begin{array}{r}\text { Aug. } 29 \\
\text { Sept. } 8 \\
10 \\
16\end{array}$ & $\begin{array}{l}\text { L. L. Dickson } \\
\text { Morgan and Ansiey.-. } \\
\text { Morgan and Byrnes.-. } \\
\text { Dornbach and Ansley. }\end{array}$ & $\begin{array}{l}\text { Feet } \\
1.84 \\
1.61 \\
1.13 \\
1.10\end{array}$ & $\begin{array}{c}\text { Sec.-ft. } \\
200 \\
148 \\
72.3 \\
66.6\end{array}$ \\
\hline
\end{tabular}

Daily discharge, in second-feet, of Cuyahoga River at Old Portage, Ohio, for the year ending September, 30, 1923

\begin{tabular}{|c|c|c|c|c|c|c|c|c|c|c|c|c|}
\hline Day & Oct. & Nov. & Dec. & Jan. & Feb. & Mar. & Apr. & May & June & July & Aug. & Sept. \\
\hline $\begin{array}{l}1 \ldots \\
2 \ldots \\
3 \ldots \\
4 \ldots \\
5 \ldots\end{array}$ & $\begin{array}{r}69 \\
176 \\
122 \\
91 \\
98\end{array}$ & 90 & $\begin{array}{l}197 \\
165 \\
119 \\
197 \\
176\end{array}$ & $\begin{array}{l}590 \\
501 \\
501 \\
472 \\
415\end{array}$ & $\begin{array}{l}530 \\
590 \\
710 \\
620 \\
560\end{array}$ & $\begin{array}{r}446 \\
446 \\
620 \\
805 \\
1,020\end{array}$ & $\begin{array}{l}272 \\
336\end{array}$ & $\begin{array}{l}189 \\
189 \\
113 \\
147 \\
138\end{array}$ & $\begin{array}{l}178 \\
121 \\
100 \\
102 \\
138\end{array}$ & $\begin{array}{r}94 \\
121 \\
94 \\
108 \\
178\end{array}$ & $\begin{array}{l}129 \\
121 \\
260 \\
260 \\
121\end{array}$ & $\begin{array}{r}129 \\
78 \\
73 \\
113 \\
100\end{array}$ \\
\hline $\begin{array}{r}6 \\
7 \\
8 \\
9 \\
10\end{array}$ & $\begin{array}{r}94 \\
79 \\
145\end{array}$ & $\begin{array}{l}186 \\
186 \\
208 \\
103\end{array}$ & $\begin{array}{r}176 \\
186 \\
243 \\
306 \\
84\end{array}$ & $\begin{array}{l}359 \\
306 \\
346 \\
359 \\
346\end{array}$ & $\begin{array}{l}560 \\
530 \\
474 \\
446 \\
323\end{array}$ & $\begin{array}{r}1,020 \\
1,050 \\
945 \\
740 \\
945\end{array}$ & $\begin{array}{r}1,160 \\
945 \\
1,020 \\
1,080 \\
945\end{array}$ & $\begin{array}{l}113 \\
138 \\
157 \\
200 \\
189\end{array}$ & $\begin{array}{l}111 \\
323 \\
167 \\
147 \\
212\end{array}$ & $\begin{array}{l}167 \\
129 \\
108 \\
121 \\
138\end{array}$ & $\begin{array}{l}138 \\
147 \\
167 \\
129 \\
121\end{array}$ & $\begin{array}{r}94 \\
138 \\
147 \\
108 \\
121\end{array}$ \\
\hline $15 \ldots$ & $\begin{array}{l}85 \\
76\end{array}$ & $\begin{array}{r}136 \\
94 \\
155 \\
165 \\
319\end{array}$ & $\begin{array}{r}255 \\
220 \\
186 \\
96 \\
165\end{array}$ & $\begin{array}{l}415 \\
176 \\
243 \\
319 \\
980\end{array}$ & $\begin{array}{l}272 \\
297 \\
446 \\
502 \\
362\end{array}$ & $\begin{array}{l}1,160 \\
1,240 \\
1,320 \\
1,280 \\
1,200\end{array}$ & $\begin{array}{l}740 \\
530 \\
474 \\
446 \\
390\end{array}$ & $\begin{array}{r}236 \\
770 \\
1,280 \\
945 \\
980\end{array}$ & $\begin{array}{l}272 \\
297 \\
248 \\
284 \\
248\end{array}$ & $\begin{array}{r}200 \\
178 \\
99 \\
121 \\
88\end{array}$ & $\begin{array}{l}121 \\
138 \\
121 \\
129 \\
121\end{array}$ & $\begin{array}{l}113 \\
129 \\
121 \\
121 \\
113\end{array}$ \\
\hline $\begin{array}{l}18 \\
19 \\
20\end{array}$ & 100 & $\begin{array}{r}319 \\
280 \\
231 \\
86 \\
155\end{array}$ & $\begin{array}{r}136 \\
88 \\
165 \\
155 \\
231\end{array}$ & $\begin{array}{l}980 \\
620 \\
710 \\
980 \\
910\end{array}$ & $\begin{array}{l}390 \\
390 \\
310 \\
323 \\
272\end{array}$ & $\begin{array}{r}1,400 \\
1,320 \\
945 \\
875 \\
805\end{array}$ & $\begin{array}{l}390 \\
390 \\
446 \\
418 \\
390\end{array}$ & $\begin{array}{l}945 \\
805 \\
620 \\
560 \\
530\end{array}$ & $\begin{array}{l}129 \\
284 \\
297 \\
260 \\
236\end{array}$ & $\begin{array}{l}129 \\
157 \\
157 \\
121 \\
121\end{array}$ & $\begin{array}{r}121 \\
121 \\
121 \\
84 \\
113\end{array}$ & $\begin{array}{r}70 \\
85 \\
121 \\
129 \\
310\end{array}$ \\
\hline $\begin{array}{l}24 \ldots \ldots \\
25 \ldots \ldots\end{array}$ & $\begin{array}{r}82 \\
119 \\
280 \\
280 \\
186\end{array}$ & $\begin{array}{r}136 \\
94 \\
122 \\
136 \\
124\end{array}$ & $\begin{array}{r}208 \\
136 \\
145 \\
155 \\
98\end{array}$ & $\begin{array}{l}1,760 \\
1,600 \\
1,320 \\
1,200 \\
1,160\end{array}$ & $\begin{array}{l}272 \\
284 \\
272 \\
260 \\
138\end{array}$ & $\begin{array}{l}680 \\
740 \\
875 \\
770 \\
710\end{array}$ & $\begin{array}{l}336 \\
362 \\
323 \\
310 \\
362\end{array}$ & $\begin{array}{l}560 \\
502 \\
390 \\
362 \\
336\end{array}$ & $\begin{array}{r}100 \\
94 \\
99 \\
82 \\
103\end{array}$ & $\begin{array}{r}121 \\
68 \\
108 \\
138 \\
129\end{array}$ & $\begin{array}{l}121 \\
138 \\
129 \\
129 \\
113\end{array}$ & $\begin{array}{l}284 \\
147 \\
113 \\
167 \\
167\end{array}$ \\
\hline $\begin{array}{l}26 \\
27 \\
28 \\
29 \\
30 \\
31\end{array}$ & $\begin{array}{r}186 \\
186 \\
79 \\
76 \\
165 \\
108\end{array}$ & $\begin{array}{r}71 \\
145 \\
106 \\
165 \\
74\end{array}$ & $\begin{array}{l}186 \\
165 \\
501 \\
620 \\
443 \\
472\end{array}$ & $\begin{array}{l}945 \\
710 \\
770 \\
710 \\
560 \\
560\end{array}$ & $\begin{array}{r}224 \\
390 \\
446 \\
\end{array}$ & $\begin{array}{l}740 \\
620 \\
560 \\
446 \\
390 \\
362\end{array}$ & $\begin{array}{l}297 \\
310 \\
362 . \\
272 \\
310\end{array}$ & $\begin{array}{l}260 \\
224 \\
297 \\
297 \\
178 \\
212\end{array}$ & $\begin{array}{r}88 \\
90 \\
138 \\
113 \\
85\end{array}$ & $\begin{array}{r}121 \\
113 \\
129 \\
79 \\
113 \\
138\end{array}$ & $\begin{array}{r}61 \\
113 \\
284 \\
157 \\
147 \\
138\end{array}$ & $\begin{array}{l}167 \\
157 \\
167 \\
147 \\
167\end{array}$ \\
\hline
\end{tabular}

Nore.-Discharge Oct. 9-13,16-20, and Nov. 1-6, estimated by comparison of record of combined flow o r.ver and canal at Independence. Braced figures indicate mean discharge for periods indicated. 
Monthly discharge, in second-feet, of Cuyahoga River at Old Portage, Ohio, for the year ending September 30, 1928

\begin{tabular}{|c|c|c|c|c|c|c|c|}
\hline Month & Maximum & Minimum & Mean & Month & Maximum & Minimum & Mean \\
\hline $\begin{array}{l}\text { October } \\
\text { November } \\
\text { December } \\
\text { January } \\
\text { Februay } \\
\text { March }\end{array}$ & $\begin{array}{r}280 \\
319 \\
620 \\
1,760 \\
710 \\
1,400\end{array}$ & \begin{tabular}{r|}
69 \\
71 \\
84 \\
176 \\
138 \\
362
\end{tabular} & $\begin{array}{l}128 \\
145 \\
215 \\
704 \\
400 \\
854\end{array}$ & $\begin{array}{l}\text { May } \\
\text { June............... } \\
\text { July } \\
\text { August. } \\
\text { September..... }\end{array}$ & $\begin{array}{r}1,280 \\
323 \\
200 \\
284 \\
310\end{array}$ & $\begin{array}{r}113 \\
82 \\
68 \\
61 \\
70\end{array}$ & $\begin{array}{l}415 \\
172 \\
125 \\
139 \\
136\end{array}$ \\
\hline April & $\begin{array}{l}1,400 \\
1,160\end{array}$ & $\begin{array}{l}362 \\
272\end{array}$ & $\begin{array}{l}854 \\
507\end{array}$ & The year.- & 1,760 & 61 & 329 \\
\hline
\end{tabular}

\section{CUYAHOGA RIVER AT BRECKSVILLE, OHIO}

Location.-At highway bridge at Brecksville railroad station, 2 miles east of Brecksville, Cuyahoga County. Chippewa Creek enters on right 800 feet above gage.

Drainage area. - 584 square miles (measured on topographic maps).

Reconds available.-March 1 to September 30, 1923.

GAGE.-Foxboro water-stage recorder of the pressure type and auxiliary vertical staff gage on highway bridge, read by Leonard Brown.

Discharge MEASUREMENTS.-Made from bridge at gage or by wading.

Channel and control.-Channel slightly curved above and below gage. Left bank high; right bank fairly high; subject to overflow at extremely high stages. Control is timber dam with steel crest, 300 feet below gage. Leakage negligible.

Extremes of Discharge.-Maximum combined discharge of river and canal feeder during period of record, 3,070 second-feet April 5; minimum combined discharge, 92 second-feet August 20 and 21.

IcE.-Stage-discharge relation not affected by ice during period of record.

Diversions. - Water is diverted into the Ohio Canal feeder at the dam 300 feet below gage. For a record of this diversion see page 64. A small amount of water is diverted into this stream from Tuscarawas River by the Ohio Canal.

Regulation.-Flow is regulated at reservoir above Akron.

Accoracr.-Stage-discharge relation permanent. Rating curve fairly well defined. Daily discharge ascertained by applying mean daily gage height to rating table. Records good.

Cooperation.-Gage-height record furnished by American Steel \& Wire Co.

Discharge measurements of Cuyahoga River at Brecksville, Ohio, during the year ending September 30,1928

\begin{tabular}{|c|c|c|c|c|c|c|c|}
\hline Date & Made by- & $\begin{array}{c}\text { Gage } \\
\text { height }\end{array}$ & $\begin{array}{c}\text { Dis- } \\
\text { charge }\end{array}$ & Date & Made by- & $\begin{array}{c}\text { Gage } \\
\text { height }\end{array}$ & $\begin{array}{c}\text { Dis- } \\
\text { charge }\end{array}$ \\
\hline $\begin{array}{l}\text { Mar. } 12 \\
13 \\
\text { May } 26\end{array}$ & $\begin{array}{l}\text { E. E. R. Dornbach.... } \\
\text { Lee and Covert..... }\end{array}$ & $\begin{array}{l}\text { Feet } \\
2.05 \\
1.80 \\
.57\end{array}$ & $\begin{array}{r}\text { Sec.-ft. } \\
2,380 \\
1,800 \\
377\end{array}$ & $\begin{array}{lr}\text { Aug. } & 7 \\
30 \\
\text { Sept. } 11\end{array}$ & L. L. Dickson. & $\begin{array}{c}\text { Feet } \\
0.25 \\
.13 \\
.05\end{array}$ & $\begin{array}{r}\text { Sec. }-f t . \\
203 \\
159 \\
146\end{array}$ \\
\hline
\end{tabular}

72038-26†-wSP 564-5 
Combined daily discharge, in second-feet, of Cuyahoga River and Ohio Canal feeder at Brecksville, Ohio, for the period March 1 to September 30, 1923

\begin{tabular}{|c|c|c|c|c|c|c|c|}
\hline Day & Mar. & A pr. & May & June & July & Aug. & Sept. \\
\hline $\begin{array}{l}1 \\
2 \\
3 \\
4 \\
\end{array}$ & $\begin{array}{r}737 \\
793 \\
1,080 \\
1,460 \\
1,260\end{array}$ & $\begin{array}{r}340 \\
391 \\
393 \\
616 \\
3,070\end{array}$ & $\begin{array}{l}271 \\
225 \\
312 \\
169 \\
193\end{array}$ & $\begin{array}{l}220 \\
174 \\
150 \\
150 \\
169\end{array}$ & $\begin{array}{l}122 \\
122 \\
145 \\
210 \\
197\end{array}$ & $\begin{array}{l}169 \\
144 \\
361 \\
299 \\
221\end{array}$ & $\begin{array}{l}144 \\
115 \\
122 \\
100 \\
219\end{array}$ \\
\hline $\begin{array}{r}6 \\
7 \\
8\end{array}$ & $\begin{array}{r}1,220 \\
1,230 \\
1,110 \\
911 \\
2,090\end{array}$ & $\begin{array}{l}1,940 \\
1,460 \\
1,320 \\
1,270 \\
1,110\end{array}$ & $\begin{array}{l}193 \\
186 \\
271 \\
325 \\
287\end{array}$ & $\begin{array}{l}178 \\
304 \\
272 \\
238 \\
302\end{array}$ & $\begin{array}{l}205 \\
201 \\
183 \\
156 \\
178\end{array}$ & $\begin{array}{l}188 \\
240 \\
221 \\
162 \\
132\end{array}$ & $\begin{array}{l}158 \\
152 \\
225 \\
132 \\
120\end{array}$ \\
\hline 11 12 13 & $\begin{array}{l}1,730 \\
1,980 \\
1,830 \\
1,650 \\
1,470\end{array}$ & $\begin{array}{l}898 \\
737 \\
538 \\
585 \\
674\end{array}$ & $\begin{array}{r}354 \\
2,470 \\
1,740 \\
1,180 \\
1,240\end{array}$ & $\begin{array}{l}306 \\
316 \\
293 \\
289 \\
266\end{array}$ & $\begin{array}{l}244 \\
205 \\
148 \\
145 \\
136\end{array}$ & $\begin{array}{l}131 \\
134 \\
141 \\
142 \\
141\end{array}$ & $\begin{array}{l}123 \\
120 \\
129 \\
113 \\
113\end{array}$ \\
\hline 16 18 18 & $\begin{array}{l}2,300 \\
1,710 \\
1,400 \\
1,220 \\
1,060\end{array}$ & $\begin{array}{l}582 \\
531 \\
631 \\
518 \\
477\end{array}$ & $\begin{array}{r}1,040 \\
926 \\
784 \\
646 \\
772\end{array}$ & $\begin{array}{l}215 \\
159 \\
232 \\
349 \\
282\end{array}$ & $\begin{array}{l}132 \\
149 \\
142 \\
147 \\
142\end{array}$ & $\begin{array}{r}108 \\
113 \\
104 \\
108 \\
92\end{array}$ & $\begin{array}{l}113 \\
122 \\
122 \\
122 \\
299\end{array}$ \\
\hline $\begin{array}{l}21 \\
22 \\
24\end{array} 25$ & $\begin{array}{r}873 \\
1,620 \\
1,490 \\
1,080 \\
937\end{array}$ & $\begin{array}{l}457 \\
413 \\
403 \\
380 \\
373\end{array}$ & $\begin{array}{l}885 \\
645 \\
506 \\
455 \\
409\end{array}$ & $\begin{array}{l}201 \\
192 \\
170 \\
137 \\
170\end{array}$ & $\begin{array}{l}129 \\
116 \\
100 \\
134 \\
141\end{array}$ & $\begin{array}{r}92 \\
138 \\
116 \\
125 \\
113\end{array}$ & $\begin{array}{l}416 \\
268 \\
174 \\
192 \\
183\end{array}$ \\
\hline $\begin{array}{l}26 \\
27 \\
28\end{array}$ & $\begin{array}{l}716 \\
640 \\
650 \\
544 \\
470 \\
422\end{array}$ & $\begin{array}{l}312 \\
296 \\
277 \\
290 \\
351\end{array}$ & $\begin{array}{l}386 \\
357 \\
401 \\
371 \\
283 \\
220\end{array}$ & $\begin{array}{l}183 \\
187 \\
192 \\
165 \\
138\end{array}$ & $\begin{array}{l}148 \\
138 \\
157 \\
122 \\
147 \\
167\end{array}$ & $\begin{array}{l}104 \\
104 \\
302 \\
174 \\
132 \\
140\end{array}$ & $\begin{array}{l}183 \\
202 \\
223 \\
160 \\
174\end{array}$ \\
\hline
\end{tabular}

Combined monthly discharge, in second-feet, of Cuyahoga River and Ohio Canal feeder at Brecksville, Ohio, for the period March 1 to September 30, 1923

\begin{tabular}{|c|c|c|c|c|c|}
\hline \multirow{2}{*}{ Month } & \multirow{2}{*}{$\begin{array}{c}\text { Maximum } \\
\text { (com- } \\
\text { bined) }\end{array}$} & \multirow{2}{*}{$\begin{array}{c}\text { Minimum } \\
\text { (com- } \\
\text { bined) }\end{array}$} & \multicolumn{3}{|c|}{ Mean } \\
\hline & & & River & Canal & Combined \\
\hline $\begin{array}{l}\text { March } \\
\text { April } \\
\text { May } \\
\text { June } \\
\text { July } \\
\text { August } \\
\text { September }-\end{array}$ & $\begin{array}{r}2,300 \\
3,070 \\
2,470 \\
349 \\
244 \\
361 \\
416\end{array}$ & $\begin{array}{r}422 \\
277 \\
169 \\
137 \\
100 \\
92 \\
100\end{array}$ & $\begin{array}{r}1,150 \\
649 \\
522 \\
121 \\
29.5 \\
41,1 \\
53.9\end{array}$ & $\begin{array}{r}68.0 \\
71.6 \\
75.2 \\
99.2 \\
126 \\
117 \\
114\end{array}$ & $\begin{array}{r}1,220 \\
721 \\
597 \\
220 \\
155 \\
158 \\
168\end{array}$ \\
\hline
\end{tabular}

CUYAHOGA RIVER AT INDEPENDENCE, OHIO

Locatron.-In T. 6 N., R. 12 W., at highway bridge three-eighths mile northwest of Thornburg and 1 mile north of Independence, Cuyahoga County.

Drainage area. - 709 square miles (measured on topographic maps).

Records available.-September 21, 1903, to July 21, 1906, and September 28, 1921 , to May 31, 1923, when station was discontinued.

GAGE.-Chain gage on highway bridge, read by John Zimmerman.

Discharge measurements.- Made from bridge at gage or by wading.

Channel and control.-Channel straight for 500 feet above and 3,000 feet below gage. Banks fairly high. One channel at all stages. Bed of stream composed of gravel and small boulders. Control for low water is riffle $\mathbf{7 0 0}$ feet below gage. Control for high water is long stretch of channel. Zero flow would occur at 0.00 foot gage height. 
EXTREMES OF DISCHARGE.-Maximum combined daily discharge of river and canal during period, October 1, 1922, to May 31, 1923, 6,200 second-feet January 21; minimum combined daily discharge, 117 second-feet October 30.

1921-1923: Maximum combined daily discharge, that of January 21, 1923; minimum combined daily discharge, 116 second-feet on October 3, 1921.

IcE.-Stage-discharge relation affected by ice during severe winters.

Diversions.- Water is diverted into the Ohio Canal at Brecksville about 6 miles upstream and carried past station. For a record of this diversion, see page 65. A small amount of water is diverted into this stream from Tuscarawas River by the Ohio Canal.

Regulation.--Flow is regulated at reservoir above Akron.

Accuracy.-Stage-discharge relation for low water changed during high water of January 21, 1923; not affected by ice. Rating curve used prior to the change well defined up to 3,500 second-feet. Rating curve used thereafter fairly well defined. Gage read to hundredths twice daily. Daily discharge ascertained by applying mean daily gage height to rating table. Records good.

Discharge measurements of Cuyahoga River at Independence, Ohio, during the year ending September 30, 1923

\begin{tabular}{|c|c|c|c|c|c|c|c|}
\hline Date & Made by- & $\begin{array}{c}\text { Gage } \\
\text { beight }\end{array}$ & $\begin{array}{c}\text { Dis- } \\
\text { charge }\end{array}$ & Date & Made by- & $\begin{array}{c}\text { Gage } \\
\text { height }\end{array}$ & $\begin{array}{l}\text { Dis- } \\
\text { charge }\end{array}$ \\
\hline $\begin{array}{l}\text { Oct. } 13 \\
\text { Nov. } 9\end{array}$ & $\begin{array}{l}\text { E. E. R. Dornbach. } \\
\text { Lasley Lee }\end{array}$ & $\begin{array}{c}\text { Feet } \\
1.96 \\
1.76\end{array}$ & $\begin{array}{r}\text { Sec. } f t . \\
206 \\
140\end{array}$ & $\begin{array}{l}\text { Mar. } 13 \\
\text { May } 27\end{array}$ & $\begin{array}{l}\text { E. E. R. Dornbach. } \\
\text { Lasley Lee... }\end{array}$ & $\begin{array}{c}\text { Feet } \\
5.88 \\
2.30\end{array}$ & $\begin{array}{r}\text { Sec.-ft. } \\
2,150 \\
354\end{array}$ \\
\hline
\end{tabular}

Daily discharge, in second-feet, of Cuyahoga River at Independence, Ohio, for the period October 1, 1922, to May 31,1923

\begin{tabular}{|c|c|c|c|c|c|c|c|c|}
\hline Day & Oct. & Nov. & Dec. & Jan. & Feb. & Mar. & Apr. & May \\
\hline $\begin{array}{l}1 \\
3 \\
3\end{array}$ & $\begin{array}{r}70 \\
86 \\
134 \\
97 \\
94\end{array}$ & $\begin{array}{r}90 \\
126 \\
116 \\
82 \\
55\end{array}$ & $\begin{array}{r}71 \\
97 \\
166 \\
116 \\
\cdot 177\end{array}$ & $\begin{array}{r}1,220 \\
745 \\
700 \\
575 \\
495\end{array}$ & $\begin{array}{r}770 \\
1,080 \\
1,690 \\
985 \\
810\end{array}$ & $\begin{array}{r}610 \\
650 \\
1,230 \\
2,170 \\
1,690\end{array}$ & $\begin{array}{r}338 \\
355 \\
372 \\
460 \\
3,640\end{array}$ & $\begin{array}{l}\mathbf{1 8 7} \\
\mathbf{1 7 3} \\
\mathbf{1 6 8} \\
106 \\
132\end{array}$ \\
\hline 90 & $\begin{array}{r}73 \\
81 \\
122 \\
116 \\
160\end{array}$ & $\begin{array}{r}60 \\
129 \\
114 \\
126 \\
136\end{array}$ & $\begin{array}{l}149 \\
163 \\
380 \\
380 \\
292\end{array}$ & $\begin{array}{l}415 \\
328 \\
362 \\
328 \\
310\end{array}$ & $\begin{array}{l}690 \\
610 \\
570 \\
530 \\
460\end{array}$ & $\begin{array}{l}1,330 \\
1,230 \\
1,080 \\
895 \\
3,000\end{array}$ & $\begin{array}{l}3,160 \\
1,630 \\
1,330 \\
1,230 \\
1,080\end{array}$ & $\begin{array}{l}126 \\
106 \\
163 \\
221 \\
236\end{array}$ \\
\hline 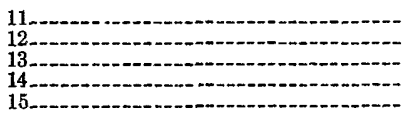 & $\begin{array}{l}292 \\
142 \\
149 \\
180 \\
118\end{array}$ & $\begin{array}{r}124 \\
120 \\
70 \\
132 \\
455\end{array}$ & $\begin{array}{l}114 \\
183 \\
180 \\
136 \\
132\end{array}$ & $\begin{array}{r}275 \\
292 \\
189 \\
328 \\
2,520\end{array}$ & $\begin{array}{l}390 \\
338 \\
895 \\
770 \\
530\end{array}$ & $\begin{array}{l}2,380 \\
3,400 \\
2,100 \\
1,690 \\
1,450\end{array}$ & $\begin{array}{l}850 \\
730 \\
610 \\
530 \\
610\end{array}$ & $\begin{array}{r}236 \\
4,120 \\
2,760 \\
1,690 \\
1,450\end{array}$ \\
\hline 3 & $\begin{array}{r}61 \\
132 \\
129 \\
103 \\
118\end{array}$ & $\begin{array}{r}258 \\
199 \\
132 \\
109 \\
70\end{array}$ & $\begin{array}{r}149 \\
103 \\
70 \\
186 \\
224\end{array}$ & $\begin{array}{r}1,690 \\
1,070 \\
970 \\
2,100 \\
1,450\end{array}$ & $\begin{array}{r}690 \\
985 \\
1,130 \\
1,080 \\
985\end{array}$ & $\begin{array}{l}3,000 \\
1,960 \\
1,330 \\
1,130 \\
940\end{array}$ & $\begin{array}{l}650 \\
570 \\
530 \\
495 \\
460\end{array}$ & $\begin{array}{r}1,230 \\
1,030 \\
810 \\
690 \\
610\end{array}$ \\
\hline 20 & $\begin{array}{r}99 \\
71 \\
99 \\
171 \\
157\end{array}$ & $\begin{array}{r}120 \\
116 \\
78 \\
101 \\
111\end{array}$ & $\begin{array}{r}189 \\
129 \\
129 \\
99 \\
84\end{array}$ & $\begin{array}{l}6,130 \\
3,400 \\
2,030 \\
1,510 \\
1,390\end{array}$ & $\begin{array}{l}850 \\
268 \\
268 \\
236 \\
143\end{array}$ & $\begin{array}{r}895 \\
1,450 \\
1,690 \\
1,230 \\
940\end{array}$ & $\begin{array}{l}425 \\
390 \\
338 \\
302 \\
268\end{array}$ & $\begin{array}{l}940 \\
730 \\
530 \\
460 \\
338\end{array}$ \\
\hline $\begin{array}{l}26 \\
28 \\
28 \\
31 \\
31\end{array}$ & $\begin{array}{r}122 \\
82 \\
105 \\
61 \\
50 \\
107\end{array}$ & $\begin{array}{r}103 \\
58 \\
139 \\
111 \\
146\end{array}$ & $\begin{array}{r}101 \\
241 \\
2,240 \\
1,170 \\
745 \\
655\end{array}$ & $\begin{array}{r}1,130 \\
940 \\
1,330 \\
1,130 \\
850 \\
\mathbf{7 7 0}\end{array}$ & $\begin{array}{r}236 \\
810 \\
770 \\
\\
\hdashline\end{array}$ & $\begin{array}{l}895 \\
770 \\
690 \\
530 \\
460 \\
390\end{array}$ & $\begin{array}{l}302 \\
268 \\
252 \\
268 \\
302\end{array}$ & $\begin{array}{l}302 \\
355\end{array}$ \\
\hline
\end{tabular}

Notr.-Mean discharge May 28-31 estimated by comparison with record of flow of Cuyahoga River at Brecksville. Braced figure gives mean discharge for period indicated. 
Monthly discharge of Cuyahoga River and Ohio Canal at Independence, Ohio, for the period October 1, 1922, to May 31, 1923

\begin{tabular}{|c|c|c|c|c|c|}
\hline \multirow{3}{*}{ Month } & \multicolumn{5}{|c|}{ Discharge in second-feet } \\
\hline & \multirow{2}{*}{$\begin{array}{c}\text { Maximum } \\
\text { (com- } \\
\text { bined) }\end{array}$} & \multirow{2}{*}{$\underset{\substack{\text { (com- } \\
\text { bined) }}}{\operatorname{Minimum}}$} & \multicolumn{3}{|c|}{ Mean } \\
\hline & & & River & Canal & Combined \\
\hline $\begin{array}{l}\text { October } \\
\text { November } \\
\text { December } \\
\text { January } \\
\text { February } \\
\text { March } \\
\text { April } \\
\text { May }\end{array}$ & $\begin{array}{r}357 \\
533 \\
2,310 \\
6,200 \\
1,760 \\
3,470 \\
3,700 \\
4,180\end{array}$ & $\begin{array}{l}117 \\
126 \\
135 \\
258 \\
212 \\
452 \\
314 \\
168\end{array}$ & $\begin{array}{r}116 \\
126 \\
298 \\
1,120 \\
699 \\
1,390 \\
758 \\
675\end{array}$ & $\begin{array}{l}65.6 \\
74.1 \\
67.7 \\
68.2 \\
66.9 \\
66.9 \\
62.1 \\
63.3\end{array}$ & $\begin{array}{r}181 \\
200 \\
366 \\
1,260 \\
\mathbf{7 6 6} \\
\mathbf{1 , 4 6 0} \\
\mathbf{8 2 0} \\
\mathbf{7 3 9}\end{array}$ \\
\hline
\end{tabular}

OHIO CANAL FEEDER AT BRECKSVILLE, OHIO

Locatron.-300 feet below head gate at dam on Cuyahoga River at Brecksville railroad station, 2 miles east of Brecksville, Cuyahoga County.

Records avallable.-March 1 to September 30, 1923.

GAGE.- Vertical staff on right bank 300 feet below head gate; read by Leonard Brown.

Discharge Meastrements.-Made from footbridge at gage or by wading.

Channel and control.-Channel straight for 200 feet above and below gage. Control is stretch of channel below gage; shifting.

Extremes of DIscharge.-Maximum mean daily discharge during period of record, 140 second-feet July 28 and 31; minimum mean daily discharge, 44 second-feet March 14 and 15.

Accuracy.-Stage-discharge relation not permanent; not affected by ice. Gage read to hundredths twice daily. Daily discharge ascertained by method for shifting control. Records fair.

Cooperation.-Gage-height record furnished by American Steel \& Wire Co.

The Ohio Canal feeder diverts water from Cuyahoga River at the dam at Brecksville. The water is used for industrial purposes at Cleveland by the American Steel \& Wire Co.

Discharge measurements of Ohio Canal feeder at Brecksville, Ohio, during the year ending September 30, 1928

\begin{tabular}{|c|c|c|c|c|c|c|c|}
\hline Date & Made by- & $\begin{array}{c}\text { Gage } \\
\text { height }\end{array}$ & $\begin{array}{c}\text { Dis- } \\
\text { charge }\end{array}$ & Date & Made by- & $\begin{array}{c}\text { Gage } \\
\text { height }\end{array}$ & $\begin{array}{c}\text { Dis- } \\
\text { charge }\end{array}$ \\
\hline $\begin{array}{l}\text { Mar. } 12 \\
13 \\
\text { May } 26\end{array}$ & $\begin{array}{l}\text { E. E. R. Dornbach.-. } \\
\text { do }\end{array}$ & $\begin{array}{c}\text { Feet } \\
0.99 \\
.98 \\
1.40\end{array}$ & $\begin{array}{r}\text { Sec. }-f t . \\
64.4 \\
63.7 \\
75.7\end{array}$ & $\begin{array}{l}\text { Aug. } \mathbf{7} \\
\mathbf{3 0} \\
\text { Sept. } \mathbf{1 1}\end{array}$ & $\begin{array}{l}\text { L. L. Dickson } \\
\text { do }\end{array}$ & $\begin{array}{l}\text { Feet } \\
1.76 \\
1.68 \\
1.80\end{array}$ & $\begin{array}{r}\text { Sec.oft. } \\
116 \\
121 \\
120\end{array}$ \\
\hline
\end{tabular}


Daily discharge, in second-feet, of Ohio Canal feeder at Brecksville, Ohio, for the period March 1 to September 30, 1923

\begin{tabular}{rr|r|r|r|r|r|r|r}
\hline & Mar. & Apr. & May & June & July & Aug. & Sept. \\
\hline 1 & & & & \\
\hline
\end{tabular}

Note.-Daily discharge Mar. 1-7 estimated by comparison with record of flow of Ohio Canal at Independence.

Monthly discharge, in second-feet, of Ohio Canal feeder at Brecksville, Ohio, for the period March 1 to September 30, 1923

\begin{tabular}{|c|c|c|c|c|c|c|c|}
\hline Month & Maximum & Minimum & Mean & Month & Maximum & Minimum & Mean \\
\hline $\begin{array}{l}\text { March } \\
\text { April } \\
\text { May-................... } \\
\text { June. }\end{array}$ & $\begin{array}{r}96 \\
84 \\
84 \\
131\end{array}$ & $\begin{array}{l}44 \\
66 \\
70 \\
76\end{array}$ & $\begin{array}{l}68.0 \\
71.6 \\
75.2 \\
99.2\end{array}$ & $\begin{array}{l}\text { July } \\
\text { August } \\
\text { September }\end{array}$ & $\begin{array}{l}140 \\
131 \\
131\end{array}$ & $\begin{array}{r}100 \\
92 \\
88\end{array}$ & $\begin{array}{l}-126 \\
117 \\
114\end{array}$ \\
\hline
\end{tabular}

\section{OHIO Canal aT INDEPENDENCE, oHIO}

Location.-At highway bridge 1 mile north of Independence, Cuyahoga County, and 7 miles southeast of Cleveland.

Records Avarlable.-September 28, 1921, to May 31, 1923, when station was discontinued.

GAGE.-Chain gage on highway bridge, read by John Zimmerman.

Discharge measurements.-Made from bridge at gage.

Channel and control.-Channel straight for 600 feet above and 2,000 feet below gage. One channel at all stages. Bed of stream silt and fine gravel. Control is long stretch of channel below gage. Considerable aquatic growth in canal during summer. Zero flow would occur at gage height 0.3 foot.

EXTREMES OF DISCHARge.-Maximum mean daily discharge during period October 1, 1922, to May 31, 1923, 83 second-feet January 17; minimum mean daily discharge, 60 second-feet several days in December, April, and May. 
1921-1923: Maximum mean daily discharge, 92 second-feet June 13 and 15-17, 1922; minimum mean daily discharge, 54 second-feet October 9, 1921. ICE.-Stage-discharge relation affected by ice.

ACCURACY.- Stage-discharge relation affected by aquatic growth during summer and by ice for short period. Rating curve fairly well defined. Gage read to half-tenths twice daily. Daily discharge ascertained by applying mean daily gage height to rating table; shifting-control method used October 1 to November 9 and March 21 to May 27. Records fair.

Water is diverted into the canal from Cuyahoga River by the Ohio Canal feeder at the dam at Brecksville. The water is used for industrial purposes at Cleveland by the American Steel \& Wire Co.

Discharge measurements of Ohio Canal at Independence, Ohio, during the year ending September 30, 1923

\begin{tabular}{|c|c|c|c|c|c|c|c|}
\hline Date & Made by- & $\begin{array}{c}\text { Gage } \\
\text { height }\end{array}$ & $\begin{array}{l}\text { Dis- } \\
\text { charge }\end{array}$ & Date & Made by- & $\begin{array}{c}\text { Gage } \\
\text { height }\end{array}$ & $\begin{array}{l}\text { Dis- } \\
\text { charge }\end{array}$ \\
\hline $\begin{array}{lr}\text { Oct. } & 13 \\
\text { Nov. } & 9\end{array}$ & $\begin{array}{l}\text { E. E. R. Dornbach } \\
\text { Lasley Lee.... }\end{array}$ & $\begin{array}{l}\text { Feet } \\
4.60 \\
4.40\end{array}$ & $\begin{array}{r}\text { Sec.-ft. } \\
64.9 \\
78.0\end{array}$ & $\begin{array}{l}\text { Mar. } 13 \\
\text { May } 27\end{array}$ & E. E. R. Dornbach & $\begin{array}{c}\text { Feet } \\
3.88 \\
3.78\end{array}$ & $\begin{array}{r}\text { Sec. ft. } \\
66.4 \\
62.7\end{array}$ \\
\hline
\end{tabular}

Daily discharge, in second-feet, of Ohio Canal at Independence, Ohio, for the period October 1, 1922, to May 31, 1923

\begin{tabular}{|c|c|c|c|c|c|c|c|c|}
\hline Day & Oct. & Nov. & Dec. & Jan. & Feb. & Mar. & Apr. & May \\
\hline (12. & $\begin{array}{l}65 \\
67 \\
69 \\
65 \\
62\end{array}$ & $\begin{array}{l}69 \\
74 \\
76 \\
74 \\
71\end{array}$ & $\begin{array}{l}71 \\
71 \\
74 \\
74 \\
74\end{array}$ & $\begin{array}{l}69 \\
69 \\
69 \\
69 \\
69\end{array}$ & $\begin{array}{l}65 \\
67 \\
69 \\
69 \\
69\end{array}$ & $\begin{array}{l}69 \\
69 \\
69 \\
69 \\
69\end{array}$ & $\begin{array}{l}60 \\
60 \\
65 \\
62 \\
65\end{array}$ & $\begin{array}{l}62 \\
60 \\
62 \\
62 \\
62\end{array}$ \\
\hline $\begin{array}{r}6 \\
70 \\
9 \\
9\end{array}$ & $\begin{array}{l}62 \\
62 \\
62 \\
65 \\
65\end{array}$ & $\begin{array}{l}74 \\
76 \\
76 \\
78 \\
76\end{array}$ & $\begin{array}{l}71 \\
65 \\
69 \\
65 \\
62\end{array}$ & $\begin{array}{l}69 \\
69 \\
69 \\
69 \\
67\end{array}$ & $\begin{array}{l}69 \\
69 \\
69 \\
67 \\
65\end{array}$ & $\begin{array}{l}69 \\
69 \\
69 \\
69 \\
69\end{array}$ & $\begin{array}{l}62 \\
62 \\
62 \\
62 \\
65\end{array}$ & $\begin{array}{l}62 \\
62 \\
65 \\
62 \\
65\end{array}$ \\
\hline 5 & $\begin{array}{l}65 \\
65 \\
65 \\
62 \\
62\end{array}$ & $\begin{array}{l}78 \\
76 \\
76 \\
76 \\
78\end{array}$ & $\begin{array}{l}60 \\
60 \\
60 \\
74 \\
74\end{array}$ & $\begin{array}{l}67 \\
69 \\
69 \\
69 \\
69\end{array}$ & $\begin{array}{l}67 \\
69 \\
69 \\
69\end{array}$ & $\begin{array}{l}67 \\
67 \\
67 \\
67 \\
65\end{array}$ & $\begin{array}{l}62 \\
62 \\
62 \\
62 \\
62\end{array}$ & $\begin{array}{l}65 \\
62 \\
60 \\
62 \\
65\end{array}$ \\
\hline 8 & $\begin{array}{l}62 \\
65 \\
65 \\
65 \\
65\end{array}$ & $\begin{array}{l}76 \\
74 \\
74 \\
74 \\
74\end{array}$ & $\begin{array}{l}65 \\
65 \\
65 \\
69 \\
69\end{array}$ & $\begin{array}{l}69 \\
83 \\
69 \\
67 \\
65\end{array}$ & 65 & $\begin{array}{l}71 \\
74 \\
62 \\
67 \\
67\end{array}$ & $\begin{array}{l}62 \\
62 \\
62 \\
62 \\
65\end{array}$ & $\begin{array}{l}65 \\
65 \\
65 \\
65 \\
65\end{array}$ \\
\hline 21 & $\begin{array}{l}67 \\
67 \\
67 \\
69 \\
69\end{array}$ & $\begin{array}{l}74 \\
74 \\
74 \\
74 \\
74\end{array}$ & $\begin{array}{l}67 \\
67 \\
69 \\
69 \\
67\end{array}$ & $\begin{array}{l}69 \\
65 \\
69 \\
67 \\
65\end{array}$ & 69 & $\begin{array}{l}65 \\
65 \\
67 \\
65 \\
67\end{array}$ & $\begin{array}{l}60 \\
60 \\
62 \\
62 \\
62\end{array}$ & $\begin{array}{l}65 \\
65 \\
65 \\
65 \\
65\end{array}$ \\
\hline 30 & $\begin{array}{l}69 \\
69 \\
69 \\
69 \\
67 \\
67\end{array}$ & $\begin{array}{l}71 \\
69 \\
71 \\
71 \\
71\end{array}$ & $\begin{array}{l}69 \\
69 \\
69 \\
60 \\
67 \\
69\end{array}$ & $\begin{array}{l}67 \\
65 \\
67 \\
67 \\
65 \\
65\end{array}$ & 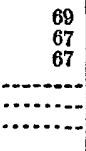 & $\begin{array}{l}62 \\
65 \\
65 \\
62 \\
65 \\
62\end{array}$ & $\begin{array}{r}62 \\
60 \\
62 \\
60 \\
65 \\
\cdots\end{array}$ & $\begin{array}{l}65 \\
62 \\
62 \\
62 \\
62 \\
62\end{array}$ \\
\hline
\end{tabular}

Note-Mean discharge Feb. 15-24, estimated because of ice from observer's notes and weather records. Gage not read A pr. 13-18; discharge interpolated. Discharge May 28-31 estimated by comparison with record of Ohio Canal feeder at Brecksville. 
Monthly discharge, in second-feet, of Ohio Canal at Independence, Ohio, for the period October 1, 1922, to May 31,1928

\begin{tabular}{|c|c|c|c|c|c|c|c|}
\hline Month & Maximum & Minimum & Mean & Month & Maximum & Minimum & Mean \\
\hline $\begin{array}{l}\text { October } \\
\text { November } \\
\text { December } \\
\text { January }\end{array}$ & $\begin{array}{l}69 \\
78 \\
74 \\
83\end{array}$ & $\begin{array}{l}62 \\
69 \\
60 \\
65\end{array}$ & $\begin{array}{l}65.6 \\
74.1 \\
67.7 \\
68.2\end{array}$ & $\begin{array}{l}\text { February } \\
\text { March...-. } \\
\text { April } \\
\text { May...- }\end{array}$ & $\begin{array}{l}69 \\
74 \\
65 \\
65\end{array}$ & $\begin{array}{l}65 \\
62 \\
60 \\
60\end{array}$ & $\begin{array}{l}66.9 \\
66.9 \\
62.1 \\
63.3\end{array}$ \\
\hline
\end{tabular}

GRAND RIVER NEAR MADISON, OHIO

Location.-At highway bridge 2 miles south of Madison, Lake County. Griswold Creek enters from left, half a mile below station.

Drainage area.-587 square miles (measured on topographic maps).

Records available.-July 7, 1922, to September 30, 1923.

GAGE.-Chain gage on highway bridge, read by G. C. Wenger and E. H. Horton.

Discharge MEASUREMENTs.-Made from bridge at gage or by wading.

Channel and control. - Channel straight for 500 feet above and below gage. Left bank high and clean; right bank fairly high and brushy. One channel at all stages. Control for low water is riffle 150 feet below gage; for high stages is long stretch of channel below gage; shifts during high water. Zero flow would occur at gage height 0.7 foot.

Extremes of Discharge.-Maximum stage recorded during period of record, 7.56 feet at 8.30 a. m. April 6, 1923 (discharge, 5,500 second-feet); minimum stage, 1.10 feet at 5.30 p. m. August 27, 1923 (discharge, 1.5 second-feet). .

Ice. -Stage-discharge relation seriously affected by ice.

Accoracr.- Stage-discharge relation for low water changed during high water on April 6, 1923; affected by ice during December, February, and March. Rating curves fairly well defined. Gage read to hundredths twice daily. Daily discharge ascertained by applying mean daily gage height to rating table. Records good except for periods of ice effect, for which they are fair.

Discharge measurements of Grand River near Madison, Ohio, during the years ending September 30, 1922 and 1923

\begin{tabular}{|c|c|c|c|c|c|c|c|}
\hline Date & Made by- & $\begin{array}{c}\text { Gage } \\
\text { height }\end{array}$ & $\begin{array}{c}\text { Dis- } \\
\text { charge }\end{array}$ & Date & Made by- & $\begin{array}{c}\text { Gage } \\
\text { height }\end{array}$ & $\begin{array}{c}\text { Dis- } \\
\text { charge }\end{array}$ \\
\hline${ }_{26}^{1922} 7$ & $\begin{array}{l}\text { Lee and Sherman } \\
\text { E. E. R. Dornbach }\end{array}$ & $\begin{array}{l}\text { Feet } \\
1.89 \\
1.66\end{array}$ & $\begin{array}{r}S e c . f t . \\
41.4 \\
22.0\end{array}$ & $\begin{array}{l}1923 \\
\text { Aug. } 8 \\
28 \\
\text { Sept. } 12\end{array}$ & $\begin{array}{l}\text { L. L. Dickson } \\
\text { do }\end{array}$ & $\begin{array}{r}\text { Feet } \\
1.41 \\
1.52 \\
1.64\end{array}$ & $\begin{array}{r}\text { Sec. }-f t . \\
7.2 \\
14.7 \\
22.9\end{array}$ \\
\hline May 28 & E. E. R. Dornbach ...- & 1.96 & 59.5 & & 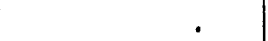 & & \\
\hline
\end{tabular}


Daily discharge, in second-feet, of Grand River near Madison, Ohio, for the period July, 7, 1922, to September 30, 1923

\begin{tabular}{|c|c|c|c|c|c|c|c|c|c|c|c|c|c|}
\hline Day & July & Aug. & Sep & & Day & July & Aug. & Sept. & & ay & July & Aug. & Bept. \\
\hline $\begin{array}{ll} & 1 \\
1 & = \\
2 & - \\
3 & - \\
4 & \ldots\end{array}$ & 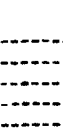 & $\begin{array}{r}24 \\
471 \\
213 \\
106 \\
61\end{array}$ & & \begin{tabular}{l||l}
10 & 11 \\
$\mathbf{1 7}$ & 12 \\
68 & 13 \\
17 & 14 \\
33
\end{tabular}. & \begin{tabular}{l}
1922 \\
\\
\hdashline
\end{tabular} & $\begin{array}{l}30 \\
24 \\
49 \\
83 \\
66\end{array}$ & $\begin{array}{l}28 \\
17 \\
20 \\
15 \\
13\end{array}$ & $\begin{array}{l}26 \\
26 \\
49 \\
20 \\
20\end{array}$ & $\begin{array}{l}21 \\
22- \\
23- \\
24- \\
25=\end{array}$ & 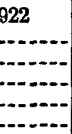 & $\begin{array}{r}156 \\
130 \\
88 \\
64 \\
37\end{array}$ & $\begin{array}{r}7 \\
7 \\
8 \\
9 \\
13\end{array}$ & $\begin{array}{l}6 \\
6 \\
8\end{array}$ \\
\hline $\begin{array}{r}6 \\
7 \\
8 \\
8 \\
9 \\
10\end{array}$ & $\begin{array}{l}38 \\
47 \\
61 \\
49\end{array}$ & $\begin{array}{l}39 \\
22 \\
88 \\
41 \\
18\end{array}$ & & $\begin{array}{l}15 \\
10 \\
16 \\
10 \\
15\end{array}$ & (ב-n & $\begin{array}{r}39 \\
33 \\
98 \\
150 \\
138\end{array}$ & $\begin{array}{r}9 \\
21 \\
9 \\
9 \\
7\end{array}$ & $\begin{array}{r}35 \\
25 \\
10 \\
13 \\
7\end{array}$ & $\begin{array}{l}26 . \\
27 . \\
28= \\
29- \\
30 . \\
31 .\end{array}$ & - & $\begin{array}{l}33 \\
28 \\
18 \\
10 \\
18 \\
17\end{array}$ & $\begin{array}{r}9 \\
10 \\
8 \\
8 \\
8 \\
9\end{array}$ & $\begin{array}{r}8 \\
8 \\
11 \\
8 \\
10 \\
-\end{array}$ \\
\hline Day & & Oct. & Nor. & Dec. & Jan. & Feb. & Mar. & A pr. & May & June & July & Aug. & Sept. \\
\hline $\begin{array}{l}1922 \\
1 \\
3 \\
4 \\
5\end{array}$ & & $\begin{array}{r}10 \\
7 \\
7 \\
7 \\
5\end{array}$ & $\begin{array}{r}10 \\
10 \\
9 \\
8 \\
9\end{array}$ & $\begin{array}{l}15 \\
23 \\
20 \\
22 \\
21\end{array}$ & $\begin{array}{r}1,020 \\
1,020 \\
730 \\
645 \\
572\end{array}$ & $\begin{array}{r}880 \\
1,250 \\
2,360 \\
3,360 \\
2,240\end{array}$ & $\begin{array}{r}600 \\
900 \\
2,480 \\
4,040 \\
4,220\end{array}$ & $\begin{array}{r}175 \\
133 \\
299 \\
645 \\
3,520\end{array}$ & $\begin{array}{l}67 \\
76 \\
59 \\
45 \\
45\end{array}$ & $\begin{array}{l}29 \\
35 \\
42 \\
28 \\
20\end{array}$ & $\begin{array}{l}10 \\
14 \\
15 \\
13 \\
16\end{array}$ & $\begin{array}{r}10 \\
9 \\
11 \\
13 \\
12\end{array}$ & $\begin{array}{l}28 \\
24 \\
19 \\
16 \\
19\end{array}$ \\
\hline 6. & & $\begin{array}{l}5 \\
5 \\
6 \\
7 \\
6\end{array}$ & $\begin{array}{l}17 \\
11 \\
13 \\
10 \\
11\end{array}$ & $\begin{array}{l}13 \\
92 \\
27 \\
18 \\
16\end{array}$ & $\begin{array}{l}393 \\
299 \\
264 \\
230 \\
264\end{array}$ & $\begin{array}{r}1,090 \\
820 \\
490 \\
213 \\
83\end{array}$ & $\begin{array}{r}2,360 \\
1,420 \\
1,090 \\
595 \\
1,420\end{array}$ & $\begin{array}{r}4,980 \\
2,480 \\
1,900 \\
1,250 \\
700\end{array}$ & $\begin{array}{l}39 \\
29 \\
25 \\
34 \\
39\end{array}$ & $\begin{array}{r}44 \\
58 \\
136 \\
412 \\
342\end{array}$ & $\begin{array}{l}18 \\
21 \\
28 \\
42 \\
28\end{array}$ & $\begin{array}{l}16 \\
11 \\
11 \\
12 \\
12\end{array}$ & $\begin{array}{l}19 \\
21 \\
39 \\
49 \\
38\end{array}$ \\
\hline $\begin{array}{l}11 \\
12 \\
13 \\
15\end{array}$ & & $\begin{array}{r}6 \\
10 \\
9 \\
6 \\
6\end{array}$ & $\begin{array}{l}15 \\
17 \\
16 \\
11 \\
20\end{array}$ & $\begin{array}{l}39 \\
75\end{array}$ & $\begin{array}{r}230 \\
133 \\
281 \\
510 \\
1,020\end{array}$ & $\begin{array}{r}210 \\
230 \\
393 \\
950 \\
1,250\end{array}$ & $\begin{array}{l}3,200 \\
4,780 \\
4,040 \\
2,240 \\
1,7 C 0\end{array}$ & $\begin{array}{l}450 \\
290 \\
220 \\
193 \\
220\end{array}$ & $\begin{array}{r}108 \\
308 \\
1,250 \\
1,250 \\
1,250\end{array}$ & $\begin{array}{r}205 \\
160 \\
142 \\
94 \\
44\end{array}$ & $\begin{array}{l}58 \\
44 \\
40 \\
34 \\
28\end{array}$ & $\begin{array}{r}12 \\
14 \\
8 \\
9 \\
10\end{array}$ & $\begin{array}{l}34 \\
30 \\
26 \\
13 \\
16\end{array}$ \\
\hline $\begin{array}{l}16 \\
17 \\
18 \\
19 \\
20\end{array}$ & & $\begin{array}{l}6 \\
6 \\
7 \\
6 \\
9\end{array}$ & $\begin{array}{l}18 \\
18 \\
23 \\
25 \\
30\end{array}$ & 20 & $\begin{array}{l}1,600 \\
2,000 \\
2,120 \\
2,480 \\
2,000\end{array}$ & 2,480 & $\begin{array}{r}2,120 \\
1,900 \\
1,420 \\
1,170 \\
760\end{array} \mid$ & $\begin{array}{l}395 \\
550 \\
450 \\
325 \\
220\end{array}$ & $\begin{array}{r}1,600 \\
1,330 \\
880 \\
595 \\
412\end{array}$ & $\begin{array}{l}38 \\
28 \\
20 \\
20 \\
20\end{array}$ & $\begin{array}{l}26 \\
24 \\
22 \\
83 \\
22\end{array}$ & $\begin{array}{l}7 \\
8 \\
7 \\
8 \\
7\end{array}$ & $\begin{array}{r}15 \\
11 \\
\cdot 9 \\
9 \\
12\end{array}$ \\
\hline $\begin{array}{l}21 \\
22 \\
24 \\
25\end{array}$ & & $\begin{array}{r}10 \\
9 \\
10 \\
10 \\
9\end{array}$ & $\begin{array}{l}26 \\
24 \\
21 \\
37 \\
29\end{array}$ & 77 & $\begin{array}{l}3,200 \\
4,220 \\
2,480 \\
1,900 \\
1,600\end{array}$ & 600 & $\begin{array}{r}550 \\
760 \\
1,250 \\
1,420 \\
1,170\end{array}$ & $\begin{array}{r}148 \\
120 \\
133 \\
96 \\
85\end{array}$ & $\begin{array}{l}308 \\
220 \\
163 \\
154 \\
139\end{array}$ & $\begin{array}{r}16 \\
14 \\
12 \\
7 \\
10\end{array}$ & $\begin{array}{l}13 \\
24 \\
24 \\
19 \\
12\end{array}$ & $\begin{array}{l}7 \\
7 \\
5 \\
5 \\
4\end{array}$ & $\begin{array}{l}22 \\
22 \\
27 \\
42 \\
49\end{array}$ \\
\hline $\begin{array}{l}26 \\
27 \\
28 \\
29 \\
30\end{array}$ & $\ldots$ & $\begin{array}{r}8 \\
7 \\
21 \\
12 \\
13 \\
10\end{array}$ & $\begin{array}{l}20 \\
43 \\
42 \\
33 \\
20\end{array}$ & $\begin{array}{r}200 \\
393 \\
510 \\
1,170 \\
1,020 \\
950\end{array}$ & $\begin{array}{l}950 \\
393 \\
490 \\
490 \\
530 \\
760\end{array}$ & 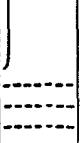 & $\begin{array}{l}820 \\
471 \\
393 \\
281 \\
162 \\
127\end{array}$ & $\begin{array}{l}76 \\
67 \\
85 \\
59 \\
59 \\
-\end{array}$ & $\begin{array}{r}108 \\
85 \\
58 \\
50 \\
48 \\
35\end{array}$ & $\begin{array}{l}16 \\
13 \\
19 \\
12 \\
12\end{array}$ & $\begin{array}{r}8 \\
10 \\
13 \\
10\end{array}$ & $\begin{array}{r}3 \\
2 \\
9 \\
\mathbf{9} \\
27 \\
22\end{array}$ & $\begin{array}{r}48 \\
26 \\
112 \\
74 \\
33\end{array}$ \\
\hline
\end{tabular}

Notn.-Stage-discharge relation affected by ice Dec. 13-24 and Feb. 17 to Mar. 2; discharge estimated from study of observer's notes, weather records, and records of flow of near-by streams. Braced figures give mean discharge for periods indicated. 
Monthly discharge of Grand River near Madison, Ohio, for the period July 7, 1922, to September 30,1923

[Drainage area, 587 square miles]

\begin{tabular}{|c|c|c|c|c|c|}
\hline \multirow{2}{*}{ Month } & \multicolumn{4}{|c|}{ Discharge in second-feet } & \multirow{2}{*}{$\begin{array}{c}\text { Run-off in } \\
\text { inches }\end{array}$} \\
\hline & Maximum & Minimum & Mean & $\begin{array}{c}\text { Per square } \\
\text { mille }\end{array}$ & \\
\hline $\begin{array}{l}\text { July } 7-31 \ldots \\
\text { August } \\
\text { September }\end{array}$ & $\begin{array}{r}156 \\
471 \\
68\end{array}$ & $\begin{array}{r}10 \\
7 \\
6\end{array}$ & $\begin{array}{l}60.2 \\
42.8 \\
17.5\end{array}$ & $\begin{array}{r}0.103 \\
.073 \\
.030\end{array}$ & $\begin{array}{l}0.10 \\
.08 \\
.03\end{array}$ \\
\hline $\begin{array}{l}\text { October } \\
\text { November } \\
\text { December } \\
\text { January } \\
\text { February } \\
\text { March } \\
\text { April } \\
\text { May } \\
\text { June } \\
\text { July } \\
\text { August } \\
\text { September }\end{array}$ & $\begin{array}{r}21 \\
43 \\
1,170 \\
4,220 \\
3,360 \\
4,780 \\
4,980 \\
1,600 \\
412 \\
58 \\
27 \\
112\end{array}$ & $\begin{array}{r}5 \\
8 \\
133 \\
127 \\
59 \\
25 \\
7 \\
7 \\
2 \\
9\end{array}$ & $\begin{array}{c}8.23 \\
19.9 \\
159 \\
1,120 \\
911 \\
1,610 \\
677 \\
349 \\
68.3 \\
22.1 \\
10.4 \\
30.1\end{array}$ & $\begin{array}{l}.014 \\
.034 \\
.271 \\
1.91 \\
1.55 \\
2.74 \\
1.15 \\
.595 \\
.116 \\
.038 \\
.018 \\
.051\end{array}$ & $\begin{array}{l}.02 \\
.04 \\
.31 \\
2.20 \\
1.61 \\
3.16 \\
1.28 \\
.69 \\
.13 \\
.04 \\
.02 \\
.06\end{array}$ \\
\hline The year & 4,980 & 2 & 414 & .705 & 9.56 \\
\hline
\end{tabular}

CONNEAUT CRERK AT AMBOY, OHIO

Location.-At highway bridge half a mile east of Amboy, Ashtabula County, and 3 miles southwest of Conneaut.

Drainage area.-178 square miles (measured on topographic maps).

Records available.-July 1, 1922, to September 30, 1923.

GAGE.-Chain gage on highway bridge; read by J. L. Evans.

Discharge MEASUREMENTs.-Made from bridge at gage or by wading.

Channel and control.-Channel straight for 300 feet above and 1,000 feet below gage. Left bank high and clean; right bank fairly high and brushy. The flood of March, 1913, flowed over right bank and across the road leading to bridge at a point some distance from bridge. Control for low-water is rock ledge 75 feet below gage. Control for high water is long stretch of channel below gage. Zero flow would occur at gage height 0.6 foot.

Extremes of DIscharge.-Maximum stage recorded during period of record, 5.6 feet at 5 p. m. March 4, 1923 (discharge, 2,970 second-feet); minimum stage, 1.06 feet at 6 p. m. August 20, 1923 (discharge, 1.6 second-feet).

Diversions.-None.

Regulation.-None.

AcCuracr.-Stage-discharge relation permanent except as affected by ice. Rating curve well defined below 1,200 second-feet. Gage read to hundredths twice daily. Daily discharge ascertained by applying mean daily gage height to rating table. Records good except for periods of ice effect, for which they are fair.

$72038-26 \dagger-$ wsP $564-6$ 
Discharge measurements of Conneaut Creek at Amboy, Ohio, during the year ending September 30, 1923

\begin{tabular}{|c|c|c|c|c|c|c|c|}
\hline Date & Made by- & $\begin{array}{l}\text { Gage } \\
\text { height }\end{array}$ & $\begin{array}{c}\text { Dis- } \\
\text { charge }\end{array}$ & Date & Made by - & $\begin{array}{r}\text { Gage } \\
\text { height }\end{array}$ & $\begin{array}{c}\text { Dis- } \\
\text { charge }\end{array}$ \\
\hline $\begin{array}{l}\text { May } 28 \\
\text { Aug. } 8\end{array}$ & $\begin{array}{l}\text { E. E. R. Dornbach } \\
\text { L. L. Dickson }\end{array}$ & $\begin{array}{c}\text { Feet } \\
1.48 \\
1.25\end{array}$ & $\begin{array}{r}S e c-f t \\
21.6 \\
4.3\end{array}$ & $\begin{array}{l}\text { Aug. } 28 \\
\text { Sept. } 12\end{array}$ & LL. L. Dickson... & $\begin{array}{c}\text { Feet } \\
1.43 \\
1.38\end{array}$ & \begin{tabular}{|r} 
Sec-ft \\
10.2 \\
8.6
\end{tabular} \\
\hline
\end{tabular}

Daily discharge, in second-feet, of Conneaut Creek" at Amboy, Ohio, for the year ending September 30, 1923

\begin{tabular}{|c|c|c|c|c|c|c|c|c|c|c|c|c|}
\hline Da & Oct. & Nov. & Dec. & Jan. & Feb. & Mar. & Apr. & May & June & July & Aug. & Sept. \\
\hline $\begin{array}{l}2 .- \\
3- \\
4- \\
5\end{array}$ & $\begin{array}{l}5.7 \\
5.2 \\
4.5 \\
5.7 \\
5.7\end{array}$ & $\begin{array}{c}10.6 \\
8.9 \\
24 \\
40 \\
16.2\end{array}$ & $\begin{array}{r}43 \\
64 \\
132 \\
88 \\
91\end{array}$ & $\begin{array}{r}655 \\
1,130 \\
590 \\
475 \\
400\end{array}$ & $\begin{array}{r}224 \\
352 \\
400 \\
1.310 \\
655\end{array}$ & $\begin{array}{r}300 \\
2.530 \\
2,310\end{array}$ & $\begin{array}{r}122 \\
110 \\
102 \\
231 \\
1,600\end{array}$ & $\begin{array}{l}43 \\
38 \\
35 \\
20\end{array}$ & $\begin{array}{r}11.6 \\
22 \\
18.9 \\
6.2 \\
13.7\end{array}$ & $\begin{array}{c}5.2 \\
6.2 \\
3.3 \\
20 \\
6.2\end{array}$ & $\begin{array}{l}2.8 \\
3.5 \\
2.9 \\
2.8 \\
3.2\end{array}$ & $\begin{array}{r}3.7 \\
3.2 \\
4.2 \\
3.9 \\
11.6\end{array}$ \\
\hline $\begin{array}{r}6 \\
7- \\
8- \\
9- \\
10\end{array}$ & $\begin{array}{r}3.9 \\
3.9 \\
5.2 \\
10.6 \\
14.9\end{array}$ & $\begin{array}{l}36 \\
32 \\
38 \\
32 \\
32\end{array}$ & $\begin{array}{r}75 \\
77 \\
77 \\
75 \\
168\end{array}$ & $\begin{array}{l}231 \\
132 \\
159 \\
210 \\
132\end{array}$ & $\begin{array}{r}330 \\
1,130 \\
955 \\
530 \\
425\end{array}$ & $\begin{array}{l}800 \\
352 \\
231 \\
220 \\
352\end{array}$ & $\begin{array}{r}1,800 \\
690 \\
375 \\
249 \\
198\end{array}$ & $\begin{array}{r}36 \\
33 \\
50 \\
70 \\
150\end{array}$ & $\begin{array}{r}\mathbf{2 2} \\
88 \\
148 \\
105 \\
62\end{array}$ & $\begin{array}{r}12.6 \\
5.2 \\
6.8 \\
4.5 \\
5.2\end{array}$ & $\begin{array}{l}\text { 2. } 9 \\
\text { 2. } 5 \\
\text { 3. } 2 \\
\text { 3. } 9 \\
\text { 2. } 9\end{array}$ & $\begin{array}{r}8.2 \\
4.5 \\
6.2 \\
20 \\
8.2\end{array}$ \\
\hline+ & $\begin{array}{r}16.2 \\
9.7 \\
12.6 \\
9.7 \\
6.8\end{array}$ & $\begin{array}{l}28 \\
54 \\
40 \\
56 \\
58\end{array}$ & $\begin{array}{r}159 \\
95 \\
62 \\
70 \\
50\end{array}$ & $\begin{array}{l}140 \\
148 \\
148 \\
145 \\
400\end{array}$ & $\begin{array}{l}352 \\
285 \\
502 \\
875\end{array}$ & $\begin{array}{r}1.220 \\
1,700 \\
2,000 \\
655 \\
330\end{array}$ & $\begin{array}{l}153 \\
183 \\
138 \\
128 \\
112\end{array}$ & $\begin{array}{r}198 \\
308 \\
1.130 \\
530 \\
308\end{array}$ & $\begin{array}{l}27 \\
27\end{array}$ & $\begin{array}{r}6.8 \\
6.2 \\
6.8 \\
3.7 \\
11.6\end{array}$ & $\begin{array}{l}2.5 \\
2.4 \\
2.3 \\
2.3 \\
2.0\end{array}$ & $\begin{array}{l}8 . \\
3 . \\
5 . \\
3 .\end{array}$ \\
\hline $\begin{array}{l}18 \\
19 \\
20\end{array}$ & $\begin{array}{r}6.2 \\
7.4 \\
24.8 \\
6.8 \\
14.9\end{array}$ & $\begin{array}{l}47 \\
52 \\
48 \\
50 \\
40\end{array}$ & $\begin{array}{r}98 \\
115 \\
95 \\
86 \\
108\end{array}$ & $\begin{array}{l}1,410 \\
2,420 \\
1,900 \\
2,200 \\
2,640\end{array}$ & & $\begin{array}{l}352 \\
875 \\
375 \\
261 \\
201\end{array}$ & $\begin{array}{l}115 \\
120 \\
142 \\
138 \\
125\end{array}$ & $\begin{array}{l}655 \\
998 \\
425 \\
261 \\
162\end{array}$ & $\begin{array}{r}8 . \\
8 . \\
10 . \\
18 . \\
18 .\end{array}$ & $\begin{array}{l}6.2 \\
4.8 \\
4.5 \\
3.5 \\
2.9\end{array}$ & $\begin{array}{l}2.0 \\
2.2 \\
2.2 \\
1.8 \\
1.7\end{array}$ & $\begin{array}{l}3.9 \\
3.5 \\
3.9 \\
6.8\end{array}$ \\
\hline $\begin{array}{l}21 \\
22 \\
23 \\
24 \\
25\end{array}$ & $\begin{array}{r}6.8 \\
8.9 \\
16.2 \\
25 \\
14.9\end{array}$ & $\begin{array}{l}38 \\
50 \\
50 \\
54 \\
52\end{array}$ & $\begin{array}{l}102 \\
140 \\
145 \\
128 \\
108\end{array}$ & $\begin{array}{r}1,500 \\
2.420 \\
1,310 \\
622 \\
352\end{array}$ & 250 & $\begin{array}{l}180 \\
207 \\
450 \\
655 \\
308\end{array}$ & $\begin{array}{l}91 \\
77 \\
73 \\
64 \\
62\end{array}$ & $\begin{array}{r}128 \\
135 \\
110 \\
79 \\
66\end{array}$ & $\begin{array}{l}8 . \\
6 . \\
5 . \\
7 . \\
4 .\end{array}$ & $\begin{array}{l}2.7 \\
2.9 \\
2.5 \\
2.4 \\
2.0\end{array}$ & $\begin{array}{l}1.8 \\
2.2 \\
2.4 \\
2.2 \\
2.2\end{array}$ & $\begin{array}{r}9.7 \\
8.9 \\
5.7 \\
10.6 \\
22\end{array}$ \\
\hline $\begin{array}{l}26 \\
27 \\
28 \\
28 \\
30 \\
31\end{array}$ & $\begin{array}{r}7.4 \\
5.2 \\
6.2 \\
12.6 \\
17.5 \\
12.6\end{array}$ & $\begin{array}{l}54 \\
47 \\
58 \\
47 \\
38 \\
-\end{array}$ & $\begin{array}{l}159 \\
257 \\
590 \\
838 \\
530 \\
352\end{array}$ & $\begin{array}{l}277 \\
238 \\
189 \\
220 \\
257 \\
220\end{array}$ & 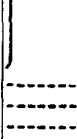 & $\begin{array}{r}217 \\
156 \\
128 \\
112 \\
95 \\
75\end{array}$ & $\begin{array}{r}56 \\
64 \\
66 \\
50 \\
48 \\
\end{array}$ & $\begin{array}{l}45 \\
54 \\
30 \\
11.6 \\
17.5 \\
24\end{array}$ & $\begin{array}{l}6.2 \\
5.2 \\
6.2 \\
8.2 \\
8.9\end{array}$ & $\begin{array}{l}2.2 \\
2.2 \\
7.4 \\
4.5 \\
3.9 \\
3.2\end{array}$ & $\begin{array}{r}2.0 \\
1.8 \\
14.9 \\
8.2 \\
5.2 \\
3.9\end{array}$ & $\begin{array}{r}10.6 \\
7.4 \\
10.6 \\
6.8 \\
4.5\end{array}$ \\
\hline
\end{tabular}

Nore.-Stage-discharge relation affected by ice Jan. 16 and Feb. 15 to Mar. 3; discharge estimated from study of observer's notes, weather records, and records of flow of near-by streams. Braced figures give mean discharge for period indicated. 
Monthly discharge of Conneaut Creek at Amboy, Ohio, for the year ending September 30, 1923

[Drainage area, 178 square miles]

\begin{tabular}{|c|c|c|c|c|c|}
\hline \multirow{2}{*}{ Month } & \multicolumn{4}{|c|}{ Discharge in second-feet } & \multirow{2}{*}{$\begin{array}{l}\text { Run-off in } \\
\text { inches }\end{array}$} \\
\hline & Maximum & Minimum & Mean & $\underset{\text { mile }}{\text { Per square }}$ & \\
\hline $\begin{array}{l}\text { October } \\
\text { November } \\
\text { December } \\
\text { January } \\
\text { February } \\
\text { March } \\
\text { April } \\
\text { May } \\
\text { June } \\
\text { July } \\
\text { August } \\
\text { September }\end{array}$ & $\begin{array}{r}25 \\
58 \\
838 \\
2,640 \\
1.310 \\
2.530 \\
1,800 \\
1,130 \\
148 \\
20 \\
14.9 \\
22\end{array}$ & \begin{tabular}{r|}
3.9 \\
8.9 \\
43 \\
132 \\
75 \\
48 \\
11.6 \\
4.5 \\
2.0 \\
1.7 \\
3.0
\end{tabular} & $\begin{array}{c}10.1 \\
41 \\
167 \\
751 \\
422 \\
589 \\
249 \\
200 \\
26.7 \\
5.42 \\
3.19 \\
7.63\end{array}$ & $\begin{array}{l}0.057 \\
.230 \\
.938 \\
4.22 \\
2.37 \\
3.31 \\
1.40 \\
1.12 \\
.150 \\
.030 \\
.018 \\
.043\end{array}$ & $\begin{array}{l}0.07 \\
.26 \\
1.08 \\
4.86 \\
2.47 \\
3.82 \\
1.86 \\
1.29 \\
.17 \\
.03 \\
.02 \\
.05\end{array}$ \\
\hline The year.... & 2,640 & 1.7 & 206 & 1.16 & 15.68 \\
\hline
\end{tabular}

Cattaradgus Creek at versaInLES, N. Y.

Location.-At three-span highway bridge in Versailles, Cattaraugus County, 21/4 miles above mouth of Clear Creek, 6 miles below Gowanda, and 8 miles above mouth of stream.

Drain age AREA. -467 square miles (measured on post-route map).

Records available.- September 23, 1910, to September 30; 1923, when station was discontinued.

GAGE.-Chain on upstream side of right span of bridge; read by Charles Wilson.

Discharge measurements.-Made from downstream. side of bridge or by wading.

Channel and control.-Bed composed of rocks and gravel; shifting.

ExTREMEs OF DISCHARGE.-Maximum open-water stage recorded during year, 9.20 feet at 5 p. m. March 4 (discharge, 11,700 second-feet); minimum discharge recorded, 39 second-feet several times in August.

1910-1923: Maximum open-water stage recorded, 12.3 feet during night of March 25, 1913 (discharge, about 30,000 second-feet); minimum discharge, that of August, 1923.

ICE.--Stage-discharge relation affected by ice.

A.ccuracy.-Stage-discharge relation not permanent; affected by ice from November to April. Gage read to half-tenths twice daily. Daily discharge throughout year ascertained by indirect method, applying mean daily effective gage height to rating table; corrections for obtaining effective gage heights determined from discharge measurements. Records below 500 second-feet fair; poor above.

Discharge measurements of Cattaraugus Creek at Versailles, N. Y., during the period October 1, 1922, to October 11, 1923

\begin{tabular}{|c|c|c|c|c|c|c|c|}
\hline Date & Made by- & $\begin{array}{c}\text { Gage } \\
\text { height }\end{array}$ & $\begin{array}{l}\text { Dis- } \\
\text { charge }\end{array}$ & Date & Made by- & $\begin{array}{c}\text { Gage } \\
\text { height }\end{array}$ & $\begin{array}{l}\text { Dis- } \\
\text { çharge }\end{array}$ \\
\hline $\begin{array}{l}\text { Nov. } 9 \\
\text { Jan. } 8 \\
\text { Feb. } 1\end{array}$ & $\begin{array}{l}\text { A. W. Harrington } \\
\text { B. F Howe Ho }\end{array}$ & $\begin{array}{r}\text { Eeet } \\
5.46 \\
\text { a } 5.44 \\
5.45\end{array}$ & $\begin{array}{r}\text { Sec. } f t . \\
513 \\
454 \\
509\end{array}$ & $\begin{array}{l}\text { Aug. } 17 \\
\text { Oct. } 11\end{array}$ & $\begin{array}{l}\text { A. W. Harrington } \\
\text { Lamson and Johnson.- }\end{array}$ & $\begin{array}{l}\text { Feet } \\
4.63 \\
4.72\end{array}$ & $\begin{array}{r}\text { Sec. } f t . \\
51.7 \\
84.2\end{array}$ \\
\hline
\end{tabular}

- Stage-discharge relation affected by ice. 
Daily discharge, in setond-feet, of Cattaraugus Creek at Versailles, N. Y., for the year ending September 30, 1923

\begin{tabular}{r|r|r|r|r|r|r|r|r|r|r|r|r}
\hline Day & Oct. & Nov. & Dec. & Jan. & Feb. & Mar. & Apr. & May & June & July & Aug. & Sept. \\
\hline & & & & & & & & & & & \\
\hline
\end{tabular}

Note.-Discharge, Nov. 27, Dec. 15 to Jan. 20, Feb. 6 to Mar. 3, and Mar. 29 to Apr. 2, determined from gage heights corrected for ice effect by means of two discharge measurements, study of weather records and gage-height graph, observer's notes, and comparison with record of Allegheny River at Red House.

Monthly discharge of Cattaraugus Creek at Versailles, $N$. Y., for the year ending September 30, 1923

[Drainage area, 467 square miles]

\begin{tabular}{|c|c|c|c|c|c|}
\hline \multirow{2}{*}{ Month } & \multicolumn{4}{|c|}{ Discharge in second-feet } & \multirow{2}{*}{$\begin{array}{l}\text { Run-off } \\
\text { in inches }\end{array}$} \\
\hline & Maximum & Minimum & Mean & $\begin{array}{c}\text { Per square } \\
\text { mile }\end{array}$ & \\
\hline $\begin{array}{l}\text { October } \\
\text { November } \\
\text { December } \\
\text { January } \\
\text { February } \\
\text { March } \\
\text { April } \\
\text { May } \\
\text { June } \\
\text { July } \\
\text { August }\end{array}$ & $\begin{array}{r}440 \\
550 \\
1,700 \\
3,800 \\
3,200 \\
8,000 \\
5,500 \\
3,200 \\
380 \\
550 \\
160 \\
110\end{array}$ & $\begin{array}{r}120 \\
160 \\
140 \\
200 \\
280 \\
340 \\
240 \\
150 \\
95 \\
70 \\
46 \\
55\end{array}$ & $\begin{array}{c}191 \\
247 \\
384 \\
800 \\
710 \\
1,830 \\
753 \\
457 \\
153 \\
119 \\
76.6 \\
79.3\end{array}$ & $\begin{array}{c}0.409 \\
.529 \\
.822 \\
1.71 \\
1.52 \\
3.92 \\
1.61 \\
.979 \\
.328 \\
.255 \\
.164 \\
.170\end{array}$ & $\begin{array}{r}0.47 \\
.59 \\
.95 \\
1.97 \\
1.58 \\
4.52 \\
1.80 \\
1.13 \\
.37 \\
.29 \\
.19 \\
.19\end{array}$ \\
\hline The year.... & 8,000 & 46 & 484 & 1.04 & 14.05 \\
\hline
\end{tabular}




\section{STREAMS TRIBUTARY TO LAKE ONTARIO}

IITTLE TONAWANDA CREEK AT IINDEN, N. Y.

Location.-At stone-arch highway bridge in Linden, Genesee County, 3 miles about junction with Tonawanda Creek.

Drainage area.-22 square miles (measured on topographic maps).

Records available.-July 8, 1912, to September 30, 1923.

GAGE.-Vertical staff on upstream side of right abutment; read by C. L. Schenck. Discharge measurememts.-Made by wading near gage.

Channel and control.-A standard Francis weir, 2.01 feet long and 8 inches high was reconstructed September 18,1920, under the upstream side of the bridge. When the water overtops this weir it flows over a 2-inch plank about 13 feet long, including the 2 feet of weir.

Extremes of Discharge.-Maximum stage recorded during year, 8.29 feet at 6 p. m. March 3 (discharge, 1,070 second-feet); minimum stage recorded, 0.23 foot at $6 \mathrm{p}$. m. October 5 and 9 a. $\mathrm{m}$. October 7 (discharge, 0.6 second-foot).

1912-1923: Maximum stage, 14.6 feet during the flood of April 22, 1916, determined by leveling from floodmarks (discharge, 2,400 second-feet). The discharge (2,500 second-feet) published in previous water-supply papers for the flood of May 10,1919, is too large; the revised determination of the maximum discharge for that date is 1,750 second-feet. Minimum discharge, 0.4 second-foot several times during September and October, 1921.

ICE.- Stage-discharge relation slightly affected by ice.

ACCURACY.- Stage-discharge relation changed at time of high water in March; affected by ice from December to March. Rating curves used before and after the change well defined below 800 second-feet. Gage read to hundredths twice daily. Daily discharge ascertained by applying mean daily gage height to rating table. Records good.

Discharge measurements of Little Tonawanda Creek at Linden, N. Y., during the year ending September 30, 1923

\begin{tabular}{|c|c|c|c|c|c|c|c|}
\hline Date & Made by- & $\begin{array}{c}\text { Gage } \\
\text { height }\end{array}$ & $\begin{array}{l}\text { Dis- } \\
\text { charge }\end{array}$ & Date & Made by- & $\begin{array}{c}\text { Gage } \\
\text { height }\end{array}$ & $\begin{array}{l}\text { Dis- } \\
\text { charge }\end{array}$ \\
\hline $\begin{array}{ll}\text { Nov. } & 8 \\
\text { Jan. } & 7 \\
\text { Feb. } & 3\end{array}$ & $\begin{array}{l}\text { A. W. Harrington } \\
\text { B. F. Howe }\end{array}$ & $\begin{array}{l}\text { Feet } \\
0.31 \\
a .91 \\
a .37\end{array}$ & $\begin{array}{r}\text { Sec.-ft. } \\
1.0 \\
7.4 \\
109\end{array}$ & $\begin{array}{l}\text { Apr. } 3 \\
\text { Aug. } 18\end{array}$ & $\begin{array}{l}\text { E. B. Shupe } \\
\text { A. W. Harrington. }\end{array}$ & $\begin{array}{l}\text { Feet } \\
1.44 \\
.405\end{array}$ & $\begin{array}{r}\text { Sec.-ft. } \\
38.3 \\
1.4\end{array}$ \\
\hline
\end{tabular}

a Stage-discharge relation affected by ice. 
Daily discharge, in second-feet, of Little Tonawanda Creek at Linden, N. Y., for the year ending September 30, 1923

\begin{tabular}{|c|c|c|c|c|c|c|c|c|c|c|c|c|}
\hline Day & Oct. & Nov. & Dec. & Jan. & Feb. & Mar. & Apr. & May & June & July & Aug. & Sept. \\
\hline $\begin{array}{l}1 \ldots \\
2 \ldots \\
3 \ldots \\
4 \ldots \\
5 . . .\end{array}$ & $\begin{array}{r}0.9 \\
.8 \\
.8 \\
.7 \\
.7\end{array}$ & $\begin{array}{l}1.0 \\
1.0 \\
1.1 \\
1.0 \\
1.0\end{array}$ & $\begin{array}{l}3.1 \\
4.5 \\
2.9 \\
2.6 \\
2.6\end{array}$ & $\begin{array}{r}11 \\
16 \\
14 \\
11 \\
9\end{array}$ & $\begin{array}{r}12 \\
48 \\
100 \\
65 \\
42\end{array}$ & $\begin{array}{r}19 \\
22 \\
550 \\
637 \\
154\end{array}$ & $\begin{array}{r}15 \\
23 \\
57 \\
135 \\
235\end{array}$ & $\begin{array}{l}12 \\
10 \\
8.4 \\
8.4 \\
7.8\end{array}$ & $\begin{array}{l}5 . \\
5 . \\
6 . \\
6 .\end{array}$ & $\begin{array}{r}4.5 \\
4.0 \\
3.8 \\
49 \\
11\end{array}$ & $\begin{array}{l}2.8 \\
2.7 \\
4.7 \\
2.9 \\
2.6\end{array}$ & $\begin{array}{l}1.1 \\
1.2 \\
1.1 \\
1.1 \\
1.2\end{array}$ \\
\hline $\begin{array}{r}6 .- \\
7= \\
8 .- \\
9- \\
10 .\end{array}$ & $\begin{array}{r}.7 \\
.7 \\
.9 \\
1.0 \\
1.8\end{array}$ & $\begin{array}{l}1.1 \\
1.1 \\
1.1 \\
1.1 \\
1.1\end{array}$ & $\begin{array}{l}2.4 \\
2.2 \\
3.3 \\
7.2 \\
4.2\end{array}$ & $\begin{array}{l}8 \\
8 \\
7 \\
7 \\
7\end{array}$ & $\begin{array}{l}32 \\
26 \\
20 \\
16 \\
14\end{array}$ & $\begin{array}{l}90 \\
62 \\
46 \\
39 \\
58\end{array}$ & $\begin{array}{r}112 \\
69 \\
69 \\
41 \\
34\end{array}$ & $\begin{array}{l}7.2 \\
6.8 \\
6.8 \\
9.2 \\
9.2\end{array}$ & $\begin{array}{c}6.2 \\
57 \\
135 \\
116 \\
36\end{array}$ & $\begin{array}{c}12 \\
7.8 \\
6.2 \\
5.3 \\
5.0\end{array}$ & $\begin{array}{l}2.3 \\
2.2 \\
2.3 \\
2.1 \\
2.0\end{array}$ & $\begin{array}{l}1.1 \\
1.0 \\
1.2 \\
1.4 \\
1.2\end{array}$ \\
\hline$=0$ & $\begin{array}{l}1.4 \\
1.1 \\
1.0 \\
1.0 \\
1.0\end{array}$ & $\begin{array}{l}1.1 \\
1.0 \\
1.0 \\
1.1 \\
1.4\end{array}$ & $\begin{array}{l}3.8 \\
2.8 \\
2.3 \\
2.3 \\
2.4\end{array}$ & $\begin{array}{l}7 \\
7 \\
6 \\
6 \\
8\end{array}$ & $\begin{array}{r}14 \\
10 \\
14 \\
7 \\
8\end{array}$ & $\begin{array}{r}74 \\
436 \\
135 \\
70 \\
58\end{array}$ & $\begin{array}{l}42 \\
61 \\
42 \\
29 \\
27\end{array}$ & $\begin{array}{c}8.8 \\
9.2 \\
10 \\
7.5 \\
18\end{array}$ & $\begin{array}{l}22 \\
14 \\
11 \\
8.8 \\
8.4\end{array}$ & $\begin{array}{l}4.8 \\
4.3 \\
4.0 \\
3.6 \\
4.0\end{array}$ & $\begin{array}{l}1.9 \\
1.8 \\
1.7 \\
1.7 \\
1.7\end{array}$ & $\begin{array}{l}1.1 \\
1.1 \\
1.1 \\
1.0 \\
1.0\end{array}$ \\
\hline 9 & $\begin{array}{r}1.1 \\
1.0 \\
.9 \\
.9 \\
.9\end{array}$ & $\begin{array}{l}1.2 \\
1.5 \\
1.4 \\
1.4 \\
2.0\end{array}$ & $\begin{array}{l}2 \\
2 \\
2 \\
2 \\
2\end{array}$ & $\begin{array}{r}7 \\
6 \\
6 \\
10 \\
11\end{array}$ & $\begin{array}{l}8 \\
7 \\
6 \\
6 \\
5\end{array}$ & $\begin{array}{r}516 \\
112 \\
112 \\
99 \\
58\end{array}$ & $\begin{array}{l}27 \\
23 \\
23 \\
23 \\
34\end{array}$ & $\begin{array}{l}77 \\
46 \\
46 \\
26 \\
19\end{array}$ & $\begin{array}{l}7.2 \\
6.8 \\
5.8 \\
5 . \\
5.0\end{array}$ & $\begin{array}{l}36 \\
8.0 \\
5.8 \\
5.2 \\
4.7\end{array}$ & $\begin{array}{l}1.7 \\
1.5 \\
1.4 \\
1.5 \\
1.4\end{array}$ & $\begin{array}{r}.9 \\
.9 \\
.9 \\
1.0 \\
1.3\end{array}$ \\
\hline 24 & $\begin{array}{l}1.0 \\
1.0 \\
1.2 \\
1.3 \\
1.1\end{array}$ & $\begin{array}{l}2.4 \\
2.2 \\
2.0 \\
2.5 \\
2.3\end{array}$ & $\begin{array}{l}2.2 \\
2.2 \\
2.3 \\
2.4 \\
2.6\end{array}$ & $\begin{array}{l}20 \\
34 \\
32 \\
24 \\
19\end{array}$ & $\begin{array}{l}5 \\
5 \\
5 \\
5 \\
5\end{array}$ & $\begin{array}{r}74 \\
108 \\
274 \\
78 \\
50\end{array}$ & $\begin{array}{l}23 \\
21 \\
34 \\
23 \\
18\end{array}$ & $\begin{array}{l}34 \\
26 \\
17 \\
13 \\
11\end{array}$ & $\begin{array}{l}4.7 \\
4.3 \\
4.3 \\
4.0\end{array}$ & $\begin{array}{l}4.3 \\
3.8 \\
3.6 \\
3.8 \\
3.8\end{array}$ & $\begin{array}{l}1.6 \\
1.9 \\
1.5 \\
1.5 \\
1.4\end{array}$ & $\begin{array}{r}1.4 \\
1.1 \\
1.1 \\
1.1 \\
.0\end{array}$ \\
\hline $\begin{array}{l}26 \\
27 \\
28 \\
30 \\
31\end{array}$ & $\begin{array}{l}1.0 \\
1.0 \\
1.0 \\
1.0 \\
1.0 \\
1.0\end{array}$ & $\begin{array}{l}2.0 \\
2.0 \\
1.9 \\
1.9 \\
1.8\end{array}$ & $\begin{array}{l}3.6 \\
5.2 \\
3.5 \\
3.8 \\
4.8 \\
4.8\end{array}$ & $\begin{array}{l}16 \\
15 \\
13 \\
11 \\
10 \\
10\end{array}$ & $\begin{array}{r}5 \\
10 \\
12 \\
\\
\end{array}$ & $\begin{array}{l}42 \\
35 \\
24 \\
24 \\
22 \\
22\end{array}$ & $\begin{array}{l}15 \\
13 \\
14 \\
14 \\
12\end{array}$ & $\begin{array}{c}10 \\
8.0 \\
7.2 \\
6.8 \\
6.2 \\
5.8\end{array}$ & $\begin{array}{c}13 \\
6.2 \\
5.3 \\
6.8 \\
5.2\end{array}$ & $\begin{array}{l}\text { 3. } 3 \\
3.0 \\
3.0 \\
\text { 3. } 0 \\
\text { 3. } 6 \\
3.2\end{array}$ & $\begin{array}{l}1.4 \\
1.3 \\
1.4 \\
1.4 \\
1.3 \\
1.2\end{array}$ & $\begin{array}{r}1.0 \\
.9 \\
.9 \\
.9 \\
.9 \\
. . .\end{array}$ \\
\hline
\end{tabular}

Note.-Discharge, Dec. 16-20, Jan 1 to Mar. 3, and Mar. 28-31, determined from gage heights corrected for ice effect by means of two discharge measurements, study of gage-height graph and weather records, observer's notes, and comparison with records of near-by streams.

Monthly discharge of Little Tonawanda Creek at Linden, N. Y., for the year ending September 30, 1923

[Drainage area, 22 square miles]

\begin{tabular}{|c|c|c|c|c|c|}
\hline \multirow{2}{*}{ Month } & \multicolumn{4}{|c|}{ Discharge in second-feet } & \multirow{2}{*}{$\begin{array}{c}\text { Run-off in } \\
\text { inches }\end{array}$} \\
\hline & Maximum & Minimuin & Mean & $\begin{array}{c}\text { Per square } \\
\text { mile }\end{array}$ & \\
\hline $\begin{array}{l}\text { October } \\
\text { November } \\
\text { December } \\
\text { January } \\
\text { February } \\
\text { March } \\
\text { April } \\
\text { May }\end{array}$ & $\begin{array}{r}1.8 \\
2.5 \\
7.2 \\
34 \\
100 \\
637 \\
235 \\
77 \\
135 \\
49 \\
4.7 \\
1.4\end{array}$ & \begin{tabular}{r|}
0.7 \\
1.0 \\
2 \\
6 \\
5 \\
19 \\
12 \\
5.8 \\
4.0 \\
3.0 \\
1.2 \\
.9
\end{tabular} & $\begin{array}{c}0.997 \\
1.49 \\
3.10 \\
12.1 \\
18.3 \\
132 \\
43.6 \\
16.1 \\
17.7 \\
7.34 \\
1.90 \\
1.07\end{array}$ & $\begin{array}{l}0.045 \\
.068 \\
.141 \\
.550 \\
.832 \\
6.00 \\
1.98 \\
.732 \\
.805 \\
.334 \\
.086 \\
.049\end{array}$ & $\begin{array}{r}0.05 \\
.08 \\
.16 \\
.65 \\
.87 \\
6.92 \\
7.21 \\
.84 \\
.90 \\
.39 \\
.10 \\
.05\end{array}$ \\
\hline The year.- & 637 & .7 & 21.4 & .973 & 13.20 \\
\hline
\end{tabular}


GENESEE RIVER AT SCIO, N. Y.

Location.-At steel highway bridge a quarter of a mile above Vandermark Creek, half a mile above Scio, Allegany County, and 1 mile above Knight Creek.

Drainage area. - 288 square miles (measured on map issued by United States Geological Survey; scale, 1:500,000).

Records available.-June 12, 1916, to September 30, 1923.

GAGE.-Vertical staff attached to downstream face of left abutment; read by Mrs. Margaret Potter.

Discharge measurements.-Made from downstream side of bridge or by wading.

Channel and control.-Coarse gravel; shifting occasionally.

ExTrEmes of Discharge.-Maximum stage recorded during year, 6.5 feet at 5.30 p. m. March 4 (discharge, 6,010 second-feet); minimum stage recorded, 0.10 foot several times during August and September (discharge, 16 secondfeet).

1916-1923: Maximum stage recorded, 9.1 feet at noon May 22, 1919 (discharge, 10,600 second-feet); minimum stage recorded, that of August and September, 1923.

ICE.-Stage-discharge relation affected by ice.

ACCURACY.-Stage-discharge relation changed presumably at time of high water March 3. Rating curve used before the change well defined between 20 and 2,000 second-feet; that used after the change fairly well defined between the same limits. Stage-discharge relation affected by ice from December to March. Gage read to hundredths twice daily. Daily discharge ascertained by applying mean daily gage height, corrected for backwater if necessary, to rating table. Records good except for estimated periods and for periods when stage-discharge relation was affected by ice or rubbish, for which they are fair.

Discharge measurements of Genesee River at Scio, N. Y., during the year ending September 30, 1928

\begin{tabular}{|c|c|c|c|c|c|c|c|}
\hline Date & Made by- & $\begin{array}{c}\text { Gage } \\
\text { height }\end{array}$ & $\begin{array}{l}\text { Dis- } \\
\text { charge }\end{array}$ & Date & Made by- & $\begin{array}{c}\text { Gage } \\
\text { height }\end{array}$ & $\begin{array}{l}\text { Dis- } \\
\text { charge }\end{array}$ \\
\hline $\begin{array}{l}\text { Nov. } 10 \\
\text { Jan. } 10 \\
\text { Feb. } 2\end{array}$ & $\begin{array}{l}\text { A. W. Harrington } \\
\text { B. F. How } 6\end{array}$ & $\begin{array}{c}\text { Feet } \\
0.51 \\
a 1.00 \\
-1.50\end{array}$ & $\begin{array}{c}\text { Sec. }-f t . \\
53.7 \\
135 \\
366\end{array}$ & $\begin{array}{l}\text { Mar. } 29 \\
\text { Aug. } 16\end{array}$ & $\begin{array}{l}\text { E. B. Shupe } \\
\text { A. W. Harring ton }\end{array}$ & $\begin{array}{l}\text { Feet } \\
1.45 \\
.25\end{array}$ & $\begin{array}{c}\text { Sec.-ft. } \\
384 \\
26.4\end{array}$ \\
\hline
\end{tabular}

a Stage-discharge relation affected by ice. 
Daily discharge, in second-feet, of Geneses River at Scio, N.Y., for the year ending September 30, 1923

\begin{tabular}{|c|c|c|c|c|c|c|c|c|c|c|c|c|}
\hline Day & Oct. & Nov. & Dec. & Jan. & Feb. & Mar. & Apr. & May & June & July & Aug. & Sept. \\
\hline $\begin{array}{l}1 \ldots \\
2 \ldots \\
3 \ldots \\
4 \ldots \\
5\end{array}$ & $\begin{array}{l}34 \\
33 \\
31 \\
29\end{array}$ & $\begin{array}{l}37 \\
37 \\
38 \\
37 \\
37\end{array}$ & $\begin{array}{r}140 \\
86 \\
80\end{array}$ & $\begin{array}{l}700 \\
500 \\
340 \\
280 \\
260\end{array}$ & $\begin{array}{l}850 \\
320 \\
260 \\
220 \\
220\end{array}$ & $\begin{array}{r}190 \\
200 \\
1,810 \\
5,060 \\
3,460\end{array}$ & $\begin{array}{r}340 \\
317 \\
317 \\
675 \\
4,310\end{array}$ & $\begin{array}{l}317 \\
275 \\
256 \\
256 \\
236\end{array}$ & $\begin{array}{l}200 \\
184 \\
158 \\
130 \\
148\end{array}$ & $\begin{array}{r}43 \\
42 \\
42 \\
538 \\
155\end{array}$ & $\begin{array}{l}59 \\
49 \\
58 \\
56 \\
47\end{array}$ & $\begin{array}{l}19 \\
17 \\
17 \\
30 \\
30\end{array}$ \\
\hline $\begin{array}{r}6 \\
7 \\
8 \\
8 \\
9 \\
10 \\
-\end{array}$ & $\begin{array}{l}88 \\
84\end{array}$ & $\begin{array}{l}38 \\
40 \\
44 \\
49 \\
46\end{array}$ & $\begin{array}{r}75 \\
80 \\
100 \\
160 \\
100\end{array}$ & $\begin{array}{l}220 \\
200 \\
170 \\
140 \\
140\end{array}$ & $\begin{array}{l}220 \\
180 \\
200 \\
200 \\
180\end{array}$ & $\begin{array}{r}1,610 \\
930 \\
860 \\
675 \\
675\end{array}$ & $\begin{array}{r}2,460 \\
1,420 \\
1,160 \\
860 \\
675\end{array}$ & $\begin{array}{l}236 \\
218 \\
236 \\
386 \\
386\end{array}$ & $\begin{array}{l}122 \\
180 \\
340 \\
218 \\
160\end{array}$ & $\begin{array}{r}108 \\
88 \\
68 \\
61 \\
59\end{array}$ & $\begin{array}{l}41 \\
36 \\
31 \\
28 \\
26\end{array}$ & $\begin{array}{l}27 \\
28 \\
51 \\
49 \\
33\end{array}$ \\
\hline- & $\begin{array}{r}113 \\
77 \\
62 \\
48 \\
43\end{array}$ & $\begin{array}{l}42 \\
40 \\
49 \\
37 \\
52\end{array}$ & $\begin{array}{l}70 \\
85 \\
48 \\
48 \\
55\end{array}$ & $\begin{array}{r}130 \\
110 \\
95 \\
95 \\
130\end{array}$ & $\begin{array}{l}180 \\
170 \\
140 \\
240 \\
140\end{array}$ & $\begin{array}{r}675 \\
1,810 \\
1,810 \\
930 \\
592\end{array}$ & $\begin{array}{l}620 \\
592 \\
538 \\
460 \\
410\end{array}$ & $\begin{array}{l}362 \\
795 \\
930 \\
648 \\
735\end{array}$ & $\begin{array}{r}106 \\
119 \\
98 \\
98 \\
79\end{array}$ & $\begin{array}{l}39 \\
37 \\
38\end{array}$ & $\begin{array}{l}26 \\
34\end{array}$ & $\begin{array}{l}28 \\
27 \\
25 \\
24 \\
23\end{array}$ \\
\hline $\begin{array}{l}16 \ldots \\
17 \\
18 \\
19 \\
20 \ldots\end{array}$ & $\begin{array}{l}40 \\
37 \\
38 \\
36 \\
35\end{array}$ & $\begin{array}{l}58 \\
56 \\
44 \\
48 \\
44\end{array}$ & $\begin{array}{l}60 \\
55 \\
50 \\
50 \\
50\end{array}$ & $\begin{array}{l}150 \\
110 \\
170 \\
170 \\
200\end{array}$ & $\begin{array}{l}130 \\
120 \\
120 \\
120 \\
140\end{array}$ & $\begin{array}{r}2,940 \\
1,710 \\
1,160 \\
1,160 \\
795\end{array}$ & $\begin{array}{l}386 \\
362 \\
317 \\
317 \\
296\end{array}$ & $\begin{array}{r}1,000 \\
860 \\
675 \\
592 \\
538\end{array}$ & $\begin{array}{l}72 \\
68\end{array}$ & $\begin{array}{l}34 \\
33 \\
33\end{array}$ & $\begin{array}{l}28 \\
27 \\
25 \\
22 \\
25\end{array}$ & $\begin{array}{l}26 \\
28 \\
22 \\
20 \\
21\end{array}$ \\
\hline $\begin{array}{l}21 \\
22 \\
24 \\
25\end{array}$ & $\begin{array}{r}31 \\
26 \\
32 \\
111 \\
81\end{array}$ & $\begin{array}{l}48 \\
43 \\
46 \\
43 \\
54\end{array}$ & $\begin{array}{l}50 \\
55 \\
55 \\
55 \\
60\end{array}$ & $\begin{array}{l}260 \\
900 \\
550 \\
480 \\
460\end{array}$ & $\begin{array}{l}120 \\
120 \\
140 \\
140 \\
150\end{array}$ & $\begin{array}{r}675 \\
795 \\
2,020 \\
1,420 \\
930\end{array}$ & $\begin{array}{l}256 \\
236 \\
296 \\
256 \\
236\end{array}$ & $\begin{array}{l}930 \\
592 \\
485 \\
410 \\
386\end{array}$ & $\begin{array}{l}44 \\
42\end{array}$ & $\begin{array}{l}28 \\
26 \\
24 \\
25 \\
30\end{array}$ & $\begin{array}{l}21 \\
22 \\
20 \\
19 \\
19\end{array}$ & $\begin{array}{l}23 \\
27 \\
25 \\
23 \\
22\end{array}$ \\
\hline $\begin{array}{l}26 \\
27 \\
28 \\
29 \\
30 \\
31\end{array}$ & $\begin{array}{l}56 \\
50 \\
48 \\
44 \\
46 \\
38\end{array}$ & $\begin{array}{l}44 \\
43 \\
43 \\
44 \\
44\end{array}$ & $\begin{array}{r}85 \\
95 \\
360 \\
300 \\
300 \\
340\end{array}$ & $\begin{array}{l}380 \\
280 \\
240 \\
220 \\
220 \\
220\end{array}$ & $\begin{array}{l}160 \\
190 \\
190 \\
-0-\end{array}$ & $\begin{array}{l}795 \\
648 \\
485 \\
386 \\
460 \\
362\end{array}$ & $\begin{array}{l}218 \\
200 \\
218 \\
296 \\
256\end{array}$ & $\begin{array}{l}340 \\
317 \\
275 \\
256 \\
236 \\
218\end{array}$ & $\begin{array}{l}52 \\
83 \\
51 \\
65 \\
56\end{array}$ & $\begin{array}{r}26 \\
26 \\
37 \\
42 \\
46 \\
106\end{array}$ & $\begin{array}{l}17 \\
17 \\
17 \\
22 \\
22 \\
20\end{array}$ & $\begin{array}{l}22 \\
22 \\
23 \\
54 \\
37\end{array}$ \\
\hline
\end{tabular}

Note.-Discharge for the following days estimated from comparison with records of flow of near-by streams: Oct. 5-8, Nov. 30, Dec. 1-3, 31, Feb. 3, 25, Mar. 21, June 10, 18-23, July 29, Aug. 9, 29, and Sept. 16. Discharge, Oct. 1-31, determined from gage heights corrected for backwater from rubbish by means of one discharge measurement and comparison with record of Genesee River at St. Helena. Discharge, Dec. 5 to Mar. 2, determined from gage heights corrected for ice effect by means of two discharge measurements, study of gage-height graph and weather records, observer's notes, and comparison with records of flow of near-by streams.

Monthly discharge of Genesee River at Scio, N.Y., for the year ending September 30,1923

[Drainage area, 288 square miles]

\begin{tabular}{|c|c|c|c|c|c|}
\hline \multirow{2}{*}{ Month } & \multicolumn{4}{|c|}{ Discharge in second-feet } & \multirow{2}{*}{$\begin{array}{c}\text { Run-off in } \\
\text { inches }\end{array}$} \\
\hline & Maximum & Minimum & Mean & $\begin{array}{c}\text { Per square } \\
\text { mile }\end{array}$ & \\
\hline $\begin{array}{l}\text { October } \\
\text { November } \\
\text { December } \\
\text { January } \\
\text { February } \\
\text { March } \\
\text { April } \\
\text { May } \\
\text { June } \\
\text { July } \\
\text { August } \\
\text { September }\end{array}$ & $\begin{array}{r}113 \\
58 \\
360 \\
900 \\
850 \\
5,060 \\
4,310 \\
1,000 \\
340 \\
538 \\
59 \\
54\end{array}$ & $\begin{array}{r}26 \\
37 \\
48 \\
95 \\
120 \\
190 \\
200 \\
218 \\
42 \\
24 \\
17 \\
17\end{array}$ & $\begin{array}{r}48.7 \\
44.2 \\
111 \\
275 \\
199 \\
1,230 \\
643 \\
464 \\
107 \\
65.6 \\
29.4 \\
27.4\end{array}$ & $\begin{array}{r}0.169 \\
.153 \\
.385 \\
.955 \\
.691 \\
4.27 \\
2.23 \\
1.61 \\
.372 \\
.228 \\
.102 \\
.095\end{array}$ & $\begin{array}{r}0.19 \\
.17 \\
.44 \\
1.10 \\
.72 \\
4.92 \\
2.49 \\
1.86 \\
.42 \\
.26 \\
.12 \\
.11\end{array}$ \\
\hline The year. & 5,060 & 17 & 271 & .941 & 12.80 \\
\hline
\end{tabular}


GEIESEE RIVER AT ST. HHLENA, N. Y.

Location.-At steel highway bridge in St. Helena, Wyoming County, $51 / 2$ miles below Portageville and site of proposed storage dam of New York State Conservation Commission and $91 / 2$ miles above mouth of Canaseraga Creek.

Drainage arma. - 992 square miles.

ReCoRds avaILABLe.-August 14, 1908, to September 30, 1923.

GAGE.-Stevens continuous water-stage recorder on left bank below bridge, installed September 28, 1917, and a chain gage on upstream side of the highway bridge installed August 14, 1908. Water-stage recorder inspected and chain gage read by Glenn Streeter.

Discharge measurements.-Made from upstream side of highway bridge or by wading.

Channel AND co NTRoL.-Gravel and rocks; shifting occasionally.

EXTRmmes of DIscharge.-Maximum open-water stage during year from waterstage recorder, 9.13 feet at 6 p. m. March 16 (discharge, 16,400 second-feet); minimum stage from water-stage recorder, 1.92 feet at 11 a. m. August 21 (discharge, 44 second-feet).

1908-1923: Maximum stage from water-stage recorder, 12.81 feet at 8 a. m. May 17, 1916 (discharge, ${ }^{2} 44,400$ second-feet); minimum stage recorded, 1.70 feet at $5 \mathrm{p}$. m. Ootober 5 and 8 a. m. October 17, 1913. (discharge, about 18 second-feet).

ICE.-Stage-discharge relation seriously affected by ice.

Accuracy.-Stage-discharge relation for medium and low stages changed presumably at time of high water April 5. Rating curve for chain gage used before the change fairly well defined between 150 and 2,500 second-feet. Rating curve for automatic gage used before the change and rating curve for automatic and chain gage used after the change fairly well defined between 30 and 30,000 second-feet. Stage-discharge relation affected by ice from December to March. Previous to June 19, when lower intake pipe was uncovered, chain-gage readings were used during periods when automatic gage was not functioning properly due to plugging of intake pipe. Daily discharge ascertained by applying to proper rating table mean daily gage height, determined by averaging the twice daily chain gage readings or by inspection of automatic record, except for days of considerable fluctuation, when the discharge is averaged for intervals of day. Records fair.

Discharge measurements of Genesee River at St. Helena, N.Y., during the year ending. September 30, 1923

\begin{tabular}{|c|c|c|c|c|c|c|c|}
\hline Date & Made by- & $\begin{array}{c}\text { Cagie } \\
\text { height }\end{array}$ & $\begin{array}{l}\text { Dis- } \\
\text { charge }\end{array}$ & Date & Made by- & $\begin{array}{c}\text { Gage } \\
\text { height }\end{array}$ & $\begin{array}{c}\text { Dis- } \\
\text { charge }\end{array}$ \\
\hline $\begin{array}{l}\text { Nov. } 8 \\
\text { Jan. } 11 \\
\text { Feb. } 5\end{array}$ & $\begin{array}{l}\text { A. W. Harrington. } \\
\text { B. F. Howe }\end{array}$ & $\begin{array}{c}\text { Feet } \\
2.56 \\
-3.33 \\
-4.41\end{array}$ & $\begin{array}{r}\text { Sec. } f t \\
201 \\
419 \\
1,840\end{array}$ & $\begin{array}{l}\text { Apr. } 2 \\
\text { Aug. } 18\end{array}$ & $\begin{array}{l}\text { E. B. Shupe } \\
\text { A. W. Harrington. }\end{array}$ & $\begin{array}{l}\text { Feet } \\
3.47 \\
1.94\end{array}$ & $\begin{array}{r}\text { Sec. fft. } \\
987 \\
46.3\end{array}$ \\
\hline
\end{tabular}

- Stage-discharge relation affected by ice.

Note-Measurements of Nov. 8 and Apr. 2 referred to chain gage; other measurements referred to aụtomatic gage.

2 Previously published as 43,500 sécond-feet. 
Daily discharge, in second-feet, of Genesee River at St. Helena, N. Y., for the year ending September, 30, 1923

\begin{tabular}{|c|c|c|c|c|c|c|c|c|c|c|c|c|}
\hline Day & Oct. & Nov. & Dec. & Jan. & Feb. & Mar. & Apr. & May & June & July & Aug.. & Sept. \\
\hline $\begin{array}{l}1 \ldots . \\
2 \ldots \\
3 . . . \\
5 . .\end{array}$ & $\begin{array}{l}115 \\
155 \\
155 \\
123 \\
132\end{array}$ & $\begin{array}{l}203 \\
198 \\
177 \\
167 \\
123\end{array}$ & $\begin{array}{r}203 \\
1,180 \\
785 \\
565 \\
608\end{array}$ & $\begin{array}{r}550 \\
1,600 \\
1,300 \\
900 \\
700\end{array}$ & $\begin{array}{r}800 \\
800 \\
1,700 \\
2,800 \\
1,800\end{array}$ & $\begin{array}{r}550 \\
800 \\
2,200 \\
4,600 \\
9,000\end{array}$ & $\begin{array}{r}950 \\
945 \\
1,090 \\
2,730 \\
10,400\end{array}$ & $\begin{array}{l}680 \\
680 \\
556 \\
518 \\
497\end{array}$ & $\begin{array}{l}350 \\
306 \\
285 \\
285 \\
322\end{array}$ & $\begin{array}{l}206 \\
166 \\
185 \\
518 \\
385\end{array}$ & $\begin{array}{l}362 \\
237 \\
202 \\
185 \\
185\end{array}$ & $\begin{array}{l}72 \\
75 \\
80 \\
68 \\
75\end{array}$ \\
\hline $\begin{array}{r}6 \\
7 .- \\
8 .- \\
9 \\
10\end{array}$ & $\begin{array}{r}115 \\
89 \\
85 \\
141 \\
158\end{array}$ & $\begin{array}{l}141 \\
167 \\
230 \\
208 \\
218\end{array}$ & $\begin{array}{l}592 \\
542 \\
556 \\
759 \\
776\end{array}$ & $\begin{array}{l}650 \\
550 \\
500 \\
460 \\
420\end{array}$ & $\begin{array}{r}1,400 \\
1,100 \\
900 \\
900 \\
800\end{array}$ & $\begin{array}{l}5,500 \\
3,200 \\
2,400 \\
2,000 \\
2,000\end{array}$ & $\begin{array}{l}7,490 \\
3,950 \\
2,760 \\
2,150 \\
1,820\end{array}$ & $\begin{array}{l}410 \\
410 \\
339 \\
497 \\
595\end{array}$ & $\begin{array}{l}362 \\
476 \\
638 \\
680 \\
556\end{array}$ & $\begin{array}{l}327 \\
252 \\
194 \\
211 \\
174\end{array}$ & $\begin{array}{r}185 \\
151 \\
144 \\
134 \\
94\end{array}$ & $\begin{array}{r}80 \\
80 \\
85 \\
97 \\
105\end{array}$ \\
\hline$=$ & $\begin{array}{l}485 \\
425 \\
354 \\
253 \\
177\end{array}$ & $\begin{array}{l}208 \\
230 \\
253 \\
253 \\
253\end{array}$ & $\begin{array}{l}600 \\
372 \\
349 \\
326 \\
320\end{array}$ & $\begin{array}{l}420 \\
420 \\
400 \\
400 \\
380\end{array}$ & $\begin{array}{l}750 \\
750 \\
750 \\
750 \\
850\end{array}$ & $\begin{array}{r}2,400 \\
7,000 \\
9,230 \\
3,700 \\
2,520\end{array}$ & $\begin{array}{l}1,690 \\
1,570 \\
1,320 \\
1,120 \\
1,040\end{array}$ & $\begin{array}{r}920 \\
1,260 \\
1,960 \\
1,240 \\
1,480\end{array}$ & $\begin{array}{l}448 \\
398 \\
301 \\
285 \\
327\end{array}$ & $\begin{array}{l}237 \\
137 \\
137 \\
130 \\
429\end{array}$ & $\begin{array}{r}111 \\
70 \\
102 \\
105 \\
105\end{array}$ & $\begin{array}{r}111 \\
108 \\
97 \\
91 \\
88\end{array}$ \\
\hline 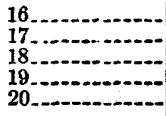 & $\begin{array}{l}203 \\
218 \\
218 \\
198 \\
132\end{array}$ & $\begin{array}{l}198 \\
218 \\
264 \\
218 \\
203\end{array}$ & $\begin{array}{l}300 \\
300 \\
280 \\
280 \\
280\end{array}$ & $\begin{array}{l}420 \\
440 \\
500 \\
550 \\
750\end{array}$ & $\begin{array}{l}850 \\
700 \\
550 \\
460 \\
400\end{array}$ & $\begin{array}{l}9,550 \\
7,810 \\
4,830 \\
4,830 \\
2,550\end{array}$ & $\begin{array}{l}970 \\
880 \\
840 \\
850 \\
840\end{array}$ & $\begin{array}{l}1,880 \\
1,750 \\
1,310 \\
1,040 \\
1,160\end{array}$ & $\begin{array}{l}285 \\
247 \\
252 \\
211 \\
185\end{array}$ & $\begin{array}{l}442 \\
275 \\
174 \\
147 \\
137\end{array}$ & $\begin{array}{r}100 \\
94 \\
82 \\
86 \\
79\end{array}$ & $\begin{array}{l}88 \\
75 \\
75 \\
75 \\
75\end{array}$ \\
\hline 23 & $\begin{array}{r}123 \\
85 \\
198 \\
253 \\
276\end{array}$ & $\begin{array}{l}235 \\
198 \\
203 \\
230 \\
224\end{array}$ & $\begin{array}{l}260 \\
280 \\
280 \\
300 \\
300\end{array}$ & $\begin{array}{l}1,000 \\
1,500 \\
2,600 \\
1,600 \\
1,200\end{array}$ & $\begin{array}{l}380 \\
340 \\
340 \\
340 \\
340\end{array}$ & $\begin{array}{l}2,220 \\
3,270 \\
6,970 \\
5,400 \\
2,840\end{array}$ & $\begin{array}{l}707 \\
820 \\
880 \\
970 \\
870\end{array}$ & $\begin{array}{r}2,080 \\
1,340 \\
1,020 \\
970 \\
820\end{array}$ & $\begin{array}{l}166 \\
147 \\
137 \\
140 \\
147\end{array}$ & $\begin{array}{l}111 \\
108 \\
117 \\
117 \\
137\end{array}$ & $\begin{array}{l}79 \\
83 \\
68 \\
94 \\
72\end{array}$ & $\begin{array}{l}137 \\
301 \\
162 \\
117 \\
117\end{array}$ \\
\hline $\begin{array}{l}26 \\
27 \\
28 \\
29 \\
30 \\
31\end{array}$ & $\begin{array}{l}354 \\
253 \\
264 \\
230 \\
208 \\
208\end{array}$ & $\begin{array}{l}230 \\
230 \\
208 \\
230 \\
198\end{array}$ & $\begin{array}{l}320 \\
400 \\
650 \\
950 \\
650 \\
550\end{array}$ & $\begin{array}{r}1,100 \\
900 \\
800 \\
800 \\
800 \\
800\end{array}$ & \begin{tabular}{r}
340 \\
420 \\
500 \\
\\
\hdashline \\
\end{tabular} & $\begin{array}{r}2,440 \\
2,010 \\
1.570 \\
1,140 \\
1,220 \\
986\end{array}$ & $\begin{array}{l}820 \\
595 \\
595 \\
556 \\
638\end{array}$ & $\begin{array}{l}680 \\
595 \\
518 \\
476 \\
435 \\
385\end{array}$ & $\begin{array}{l}185 \\
194 \\
228 \\
194 \\
270\end{array}$ & $\begin{array}{r}134 \\
124 \\
111 \\
88 \\
462 \\
580\end{array}$ & $\begin{array}{l}50 \\
52 \\
62 \\
65 \\
68 \\
72\end{array}$ & $\begin{array}{l}111 \\
111 \\
429 \\
233 \\
170\end{array}$ \\
\hline
\end{tabular}

NoTE.-Discharge for the following days when water-stage recorder not operating satisfactorily estimated from hydrograph and fragmentary automatic record: Oct. 1-4, Dee. 5, 26, 30, Jan. 7, Apr. 1, 2, 8, 9, 21, June 19-25, July 1-3, 7, 8, and Sept. 10-13. Chain-gage rendings used Oct. 1 to Dec. 4, Feb. 19 to.Mar. 3, April 24 to May 11, and May 24 to June 18; water-stage recorder not operating satisfactorily. Discharge Dec. 15 to Mar. 12, determined from gage heights correeted for ice effect by means of two discharge measurements, study of observer's notes, gage-height graph and weather records, and comparison with record of Genesee River at Scio.

Monthly discharge of Genesee River at St. Helena, N. Y., for the year ending September 30, 1923

[Drainage area, 992 square miles]

\begin{tabular}{|c|c|c|c|c|c|}
\hline \multirow{2}{*}{ Month } & \multicolumn{4}{|c|}{ Discharge in second-feet } & \multirow{2}{*}{$\begin{array}{c}\text { Run-off in } \\
\text { inches }\end{array}$} \\
\hline & Maximum & Minimum & Mean & $\begin{array}{c}\text { Per square } \\
\text { mile }\end{array}$ & \\
\hline $\begin{array}{l}\text { October } \\
\text { November } \\
\text { December } \\
\text { January } \\
\text { February } \\
\text { March } \\
\text { April } \\
\text { May } \\
\text { June } \\
\text { July } \\
\text { August } \\
\text { September }\end{array}$ & $\begin{array}{r}485 \\
264 \\
1,180 \\
2,600 \\
2.800 \\
9,550 \\
10,400 \\
2,080 \\
680 \\
580 \\
362 \\
429\end{array}$ & $\begin{array}{r}85 \\
123 \\
203 \\
380 \\
340 \\
550 \\
550 \\
556 \\
\quad 339 \\
137 \\
88 \\
\quad 50 \\
\quad 68 \\
\end{array}$ & $\begin{array}{r}206 \\
211 \\
481 \\
820 \\
815 \\
3,770 \\
1,760 \\
919 \\
300 \\
224 \\
115 \\
120\end{array}$ & $\begin{array}{l}0.208 \\
.213 \\
.485 \\
.827 \\
.822 \\
3.80 \\
1.77 \\
.926 \\
.302 \\
.226 \\
.116 \\
.121\end{array}$ & $\begin{array}{r}0.24 \\
.24 \\
.56 \\
.95 \\
.86 \\
4.38 \\
1.98 \\
1.07 \\
.34 \\
.26 \\
.13 \\
.14\end{array}$ \\
\hline The year & 10,400 & 50 & 814 & .821 & 11.15 \\
\hline
\end{tabular}




\section{GENESEE RIVER AT JONESS BRIDGR, NEAR MOUNT MORRIS, H. Y.}

Location.-At highway bridge known as Jones Bridge, $11 / 2$ miles below mouth of Canaseraga Creek, 13/4 miles above mouth of Beards Creek, 5 miles below Mount Morris, Livingston County, and 6 miles above Geneseo.

Drainage area.-1,400 square miles.

ReCoRds AVAILABLe.-May 22, 1903, to April 30, 1906; August 12, 1908, to - December 31, 1913; July 12, 1915, to September 30, 1923.

GAGE.-Gurley seven-day water-stage recorder on right bank; inspected by Theron S. Trewer. Prior to 1915 a chain gage fastened to upstream side of highway bridge was used. Datum of water-stage recorder, 540 feet New York State Conservation Commission datum, and 2.73 feet higher than that of chain gage.

Discharge measurements.-Made from footbridge erected on lower chord of upstream bridge truss or by wading.

Channel and control.-Sandy clay; fairly permanent in recent years.

EXTREMES OF DISCHARGE.- Maximum open-water stage during year from waterstage recorder, 19.47 feet at 8 p. m. April 5 (discharge, 12,200 second-feet); minimum stage from water-stage recorder, 0.20 foot from 7 to $9 \mathrm{p}$. $\mathrm{m}$. September 14 and 1 to $3 \mathrm{p}$. m. September 18 (discharge, 45 second-feet).

1903-1906, 1908-1913, and 1915-1923: Maximum stage recorded, 25.44 feet at noon May 17, 1916 (discharge, 55,100 second-feet); minimum stage, 2.7 feet (old datum) at 6 p. m. August 29, 1909 (discharge, about 18 secondfeet).

ICE.--Stage-discharge relation seriously affected by ice.

Regulation.-Operation of mills at Mount Morris causes considerable diurnal fluctuation during low-water period.

ACcURACY.- Stage-discharge relation practically permanent except as affected by ice from December to March. Rating curve well defined between 50 and 4,000 second-feet and fairly well defined between 4,000 and 20,000 secondfeet. Operation of water-stage recorder satisfactory except as indicated in footnote to daily-discharge table. Daily discharge ascertained by applying to rating table mean daily gage height determined by inspection of gageheight graph or for days of considerable fluctuation, by averaging discharge for intervals of day. Records good, except during periods of estimate, for which they are fair.

Discharge measurements of Genesee River at Jones Bridge, near Mount Morris, N. Y., during the year ending September 30,1923

\begin{tabular}{|c|c|c|c|c|c|c|c|}
\hline Date & Mada by- & $\begin{array}{c}\text { Gage } \\
\text { hoight }\end{array}$ & $\begin{array}{c}\text { Dis- } \\
\text { charge }\end{array}$ & Date & Made by- & $\begin{array}{c}\text { Gage } \\
\text { height }\end{array}$ & $\begin{array}{l}\text { Dis- } \\
\text { charge }\end{array}$ \\
\hline $\begin{array}{l}\text { Nov. } 6 \\
7 \\
\text { Jan. } 15 \\
\text { Feb. } 6\end{array}$ & $\begin{array}{l}\text { A. W. Harrington } \\
\text { B. F. Howe }\end{array}$ & $\begin{array}{l}\text { Fect } \\
1.33 \\
1.33 \\
64.63 \\
66.86\end{array}$ & $\begin{array}{r}\text { Sec. }-f t . \\
284 \\
283 \\
564 \\
1,640\end{array}$ & $\begin{array}{l}\text { Mar. } 27 \\
\text { Apr. } 7 \\
\text { Aug. } 19\end{array}$ & E. B. Shupe & \begin{tabular}{r|} 
Feet \\
5.64 \\
12.73 \\
.82
\end{tabular} & $\begin{array}{r}\text { Sec. }-f t \\
2,700 \\
7,800 \\
140\end{array}$ \\
\hline
\end{tabular}

- Stage-discharge relation affected by 100. 
Daily discharge, in second-feet, of Genesee River at Jones Bridge, near Mount Morris, N. Y., for the year ending September 30, 1923

\begin{tabular}{|c|c|c|c|c|c|c|c|c|c|c|c|c|}
\hline Day & Oct. & Nov. & Dec. & Jan. & Feb. & Mar. & Apr. & May & June & July & Aug. & Sept. \\
\hline 4 & $\begin{array}{l}215 \\
237 \\
241 \\
201 \\
196\end{array}$ & $\begin{array}{l}260 \\
264 \\
270 \\
262 \\
221\end{array}$ & & & & & $\begin{array}{l}1,460 \\
1,280 \\
1,460 \\
2,870 \\
9,350\end{array}$ & $\begin{array}{l}872 \\
872 \\
762 \\
708 \\
630\end{array}$ & $\begin{array}{l}494 \\
454 \\
398 \\
406 \\
427\end{array}$ & $\begin{array}{l}233 \\
210 \\
224 \\
248 \\
469\end{array}$ & $\begin{array}{l}605 \\
378 \\
286 \\
254 \\
218\end{array}$ & 80 \\
\hline $\begin{array}{l}6 \\
7 \\
7 \\
8 \\
9\end{array}$ & $\begin{array}{l}195 \\
175 \\
207 \\
232 \\
310\end{array}$ & $\begin{array}{l}270 \\
283 \\
294 \\
306 \\
334\end{array}$ & & & & 6,200 & $\begin{array}{r}11,200 \\
7,160 \\
4,860 \\
3,850 \\
2,800\end{array}$ & $\begin{array}{l}605 \\
545 \\
545 \\
605 \\
790\end{array}$ & $\begin{array}{l}423 \\
485 \\
580 \\
845 \\
790\end{array}$ & $\begin{array}{l}485 \\
321 \\
254 \\
257 \\
230\end{array}$ & $\begin{array}{l}227 \\
224 \\
210 \\
210 \\
145\end{array}$ & 114 \\
\hline & $\begin{array}{l}503 \\
605 \\
476 \\
406 \\
358\end{array}$ & $\begin{array}{l}362 \\
321 \\
321 \\
300 \\
293\end{array}$ & & & 1,110 & & $\begin{array}{l}2,390 \\
2,320 \\
2,130 \\
1,880 \\
1,580\end{array}$ & $\begin{array}{l}1,040 \\
1,040 \\
2,200 \\
2,010 \\
1,460\end{array}$ & $\begin{array}{l}427 \\
390 \\
358\end{array}$ & $\begin{array}{l}233 \\
218 \\
221 \\
227 \\
196\end{array}$ & $\begin{array}{l}142 \\
170 \\
122 \\
103 \\
148\end{array}$ & $\begin{array}{r}130 \\
130 \\
170 \\
74 \\
130\end{array}$ \\
\hline - & $\begin{array}{l}314 \\
310 \\
293 \\
280 \\
264\end{array}$ & $\begin{array}{l}293 \\
321 \\
336 \\
304 \\
321\end{array}$ & 640 & 1,120 & & $\begin{array}{l}7,520 \\
6,500 \\
4,060\end{array}$ & $\begin{array}{l}1,460 \\
1,460 \\
1,370 \\
1,280 \\
1,230\end{array}$ & $\begin{array}{l}2,000 \\
2,460 \\
1,940 \\
1,520 \\
1,280\end{array}$ & $\begin{array}{r}347 \\
293\end{array}$ & $\begin{array}{l}686 \\
710 \\
394 \\
304 \\
260\end{array}$ & $\begin{array}{l}138 \\
121 \\
106 \\
153 \\
108\end{array}$ & $\begin{array}{r}134 \\
101 \\
95 \\
96 \\
125\end{array}$ \\
\hline 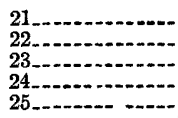 & $\begin{array}{l}254 \\
224 \\
239 \\
304 \\
432\end{array}$ & $\begin{array}{l}314 \\
293 \\
310 \\
310 \\
314\end{array}$ & & & & $\begin{array}{l}3,010 \\
3,360 \\
5,300 \\
7,700 \\
5,300\end{array}$ & $\begin{array}{l}1,230 \\
1,090 \\
1,120 \\
1,260 \\
1,060\end{array}$ & $\begin{array}{l}1,490 \\
2,390 \\
1,580 \\
1,200 \\
1,040\end{array}$ & $\begin{array}{l}257 \\
242 \\
230 \\
186 \\
216\end{array}$ & $\begin{array}{l}239 \\
196\end{array}$ & $\begin{array}{l}105 \\
106 \\
106 \\
110 \\
117\end{array}$ & $\begin{array}{l}126 \\
104 \\
314 \\
224 \\
188\end{array}$ \\
\hline ב- & $\begin{array}{l}436 \\
370 \\
332 \\
286 \\
286 \\
270\end{array}$ & 270 & & & & $\begin{array}{l}3,710 \\
2,800 \\
2,260 \\
1,520 \\
1,700 \\
1,760\end{array}$ & $\begin{array}{l}928 \\
818 \\
790 \\
790 \\
845\end{array}$ & $\begin{array}{l}928 \\
790 \\
680 \\
655 \\
570 \\
521\end{array}$ & $\begin{array}{l}260 \\
254 \\
264 \\
314 \\
293\end{array}$ & $\begin{array}{l}196 \\
135 \\
461\end{array}$ & $\begin{array}{r}158 \\
66 \\
98 \\
93 \\
79 \\
105\end{array}$ & $\begin{array}{l}169 \\
152 \\
236 \\
368 \\
224\end{array}$ \\
\hline
\end{tabular}

NorE.-Discharge for the following periods when recorder did not operate estimated by comparison with records of flow at other stations in the basin: Nov. 26 to Mar. 17, Mar. 20-26, 31, July 7, 8, 22-29, Aug. 12, 26, and Sept. 2-10.

Monthly discharge of Genesee River at Jones Bridge, near Mount Morris, N. Y., for the year ending September 30, 1923

[Drainage area, 1,400 square miles]

\begin{tabular}{|c|c|c|c|c|c|}
\hline \multirow{2}{*}{ Month } & \multicolumn{4}{|c|}{ Discharge in second-feet } & \multirow{2}{*}{$\begin{array}{c}\text { Run-off in } \\
\text { inches }\end{array}$} \\
\hline & Maximun & Minimum & Mean & $\begin{array}{c}\text { Per square } \\
\text { mile }\end{array}$ & \\
\hline $\begin{array}{l}\text { October } \\
\text { November } \\
\text { December } \\
\text { January } \\
\text { February } \\
\text { March } \\
\text { April } \\
\text { May } \\
\text { June } \\
\text { July } \\
\text { August } \\
\text { September }\end{array}$ & \begin{tabular}{|r|}
605 \\
362 \\
\\
\hdashline 11,200 \\
2,460 \\
845 \\
710 \\
605 \\
368
\end{tabular} & \begin{tabular}{|r|r|}
221 \\
221 \\
1,520 \\
790 \\
621 \\
186 \\
135 \\
66 \\
74
\end{tabular} & $\begin{array}{r}305 \\
301 \\
640 \\
1,120 \\
1,110 \\
5,220 \\
2,440 \\
1,150 \\
387 \\
282 \\
168 \\
145\end{array}$ & $\begin{array}{c}0.218 \\
.215 \\
.457 \\
.800 \\
.793 \\
3.73 \\
1.74 \\
.821 \\
.276 \\
.201 \\
.120 \\
.104\end{array}$ & $\begin{array}{r}0.25 \\
.24 \\
.53 \\
.92 \\
.83 \\
4.30 \\
1.94 \\
.95 \\
.31 \\
.23 \\
.14 \\
.12\end{array}$ \\
\hline The year.... & 11,200 & 66 & 1,110 & .798 & 10.76 \\
\hline
\end{tabular}


GEIESER RIVER AT DRIVING PARK AVENUE, ROCHESTER, N. Y.

Location.-In station No. 5 of Rochester Gas \& Electric Corporation, 400 feet above Driving Park Avenue Bridge, $1 \frac{1}{2}$ miles northwest of center of city of Rochester, Monroe County, and 5 miles above mouth.

Drainage area. $-2,460$ square miles.

Records AVAILABLE.-December 17, 1919, to September 30, 1923.

GAGE.-Gurley seven-day water-stage recorder in northwest corner of old power house; inspected by employee of the Rochester Gas \& Electric Corporation.

Discharge measurements.- Made from cable 2,000 feet below gage.

ChanNel and CONTROL.-Coarse gravel and large broken rock; practically permanent.

EXTREMEs of DISCHARGE.-Maximum stage during year from water-stage recorder, 11.20 feet at 3 p. m. March 13 (discharge, 19,300 second-feet); minimum stage is reached nearly every day during low-water period when power plant shuts down.

1919-1923: Maximum discharge recorded, about 26,000 second-feet at 2.30 p. m. March 17, 1920 (observed at Court Street dam).

ICE.-Stage-discharge relation not affected by ice.

Diversions.-The Barge Canal crosses the river near the southern line of the city of Rochester. It discharges water into Genesee River from Lake Erie and diverts water to the east for eanal purposes.

Regulation.-Daily discharge affected by storage for power purposes at Rochester and points upstream.

AccURACY.-Stage-discharge relation practically permanent. Rating curve fairly well defined between 20 and 20,000 second-feet. Operation of water-stage recorder satisfactory except as indicated in footnote to daily-discharge table Daily discharge ascertained by averaging the discharge for bihourly intervals of the day. Records fair.

Discharge measurements of Genesee River at Driving Park Avenue, Rochester, N.Y., during the year ending September 30, 1928

\begin{tabular}{|c|c|c|c|c|c|c|c|}
\hline Date & Made by- & $\begin{array}{c}\text { Gage } \\
\text { height }\end{array}$ & $\underset{\operatorname{charg} \theta}{\text { Dis* }}$ & Date & Made by- & $\begin{array}{c}\text { Gage } \\
\text { height }\end{array}$ & $\begin{array}{l}\text { Dis- } \\
\text { charge }\end{array}$ \\
\hline $\begin{array}{ll}\text { Nov. } & 4 \\
\text { Jan. } & 5\end{array}$ & $\begin{array}{l}\text { A. W. Harrington... } \\
\text { B. F. HOWe }\end{array}$ & $\begin{array}{c}\text { Feet } \\
2.84 \\
2.76 \\
3.76\end{array}$ & $\left\{\begin{array}{r}\text { Sec.ft. } \\
993 \\
887 \\
2,030\end{array}\right.$ & $\begin{array}{l}\text { Mar. 26 } \\
\text { Aug. } 19\end{array}$ & $\begin{array}{l}\text { Shupe and Davidson }{ }^{a}- \\
\text { A. W. Harrington... }\end{array}$ & $\begin{array}{c}\text { Feet } \\
5.81 \\
2.76\end{array}$ & $\begin{array}{r}\text { Sec.ft. } \\
5,150 \\
928\end{array}$ \\
\hline
\end{tabular}

- Engineer, Rochester Gas \& Electric Corporation. 
Daily discharge, in second-feet, of Genesee River at Driving Park Avenue, Rochester, N. Y., for the year ending September 30, 1923

\begin{tabular}{r|r|r|r|r|r|r|r|r|r|r|r|r}
\hline Date & Oct. & Nov. & Dec. & Jan. & Feb. & Mar. & Apr. & May & June & July & Aug. & Sept. \\
\hline & & & & & & & & & & & \\
\hline
\end{tabular}

NoTE.-Discharge for the following periods when water-stage recorder was not operating satisfactorily, estimated from fragmentary automatic record and comparison with record of Genesee River at Jones Bridge: Nov 6, 7, 10, 11, 13, Dec. 15, 16, Jan. 22, 24, Mar. 7, 8, 15, 29-31, Apr. 2-4, 14, 28, May 12, 21-26, June 2, July 3, 4, 26-28 Aug. 4, 24, 25, and Sept. 22.

Monthly discharge of Genesee River at Driving Park Avenue, Rochester, N. Y., for the year ending September 30, 1923

[Drainage area, 2,460 square miles]

\begin{tabular}{|c|c|c|c|c|c|}
\hline \multirow{2}{*}{ 、 Month } & \multicolumn{4}{|c|}{ Discharge in second-feet } & \multirow{2}{*}{$\begin{array}{l}\text { Run-off in } \\
\text { inches }\end{array}$} \\
\hline & Maximum & Minimum & Mean & $\begin{array}{c}\text { Per square } \\
\text { mile }\end{array}$ & \\
\hline $\begin{array}{l}\text { October } \\
\text { November } \\
\text { December } \\
\text { January } \\
\text { February } \\
\text { March } \\
\text { April } \\
\text { May } \\
\text { June } \\
\text { July } \\
\text { August } \\
\text { September }\end{array}$ & $\begin{array}{r}1,270 \\
1,050 \\
2,010 \\
2,880 \\
5,760 \\
18,300 \\
14,800 \\
3,770 \\
3,610 \\
1,640 \\
1,150 \\
990\end{array}$ & $\begin{array}{r}688 \\
678 \\
620 \\
1,010 \\
851 \\
1,100 \\
389 \\
1,430 \\
939 \\
802 \\
700 \\
686\end{array}$ & $\begin{array}{r}938 \\
909 \\
1,100 \\
1,590 \\
1,820 \\
9,050 \\
3,460 \\
2,200 \\
1,580 \\
1,050 \\
857 \\
822\end{array}$ & $\begin{array}{l}0.381 \\
.370 \\
.447 \\
.646 \\
.740 \\
3.68 \\
1.41 \\
.894 \\
.642 \\
.427 \\
.348 \\
.334\end{array}$ & $\begin{array}{r}0.44 \\
.41 \\
.52 \\
.74 \\
.77 \\
4.24 \\
1.57 \\
1.03 \\
.72 \\
.49 \\
.40 \\
.37\end{array}$ \\
\hline The year.... & 18,300 & 389 & 2,120 & .862 & 11. 70 \\
\hline
\end{tabular}

Note.-The figures on discharge and run-off given above do not represent the natural fiow from the drainage area on account of inflow and diversion at the crossing of the Barge Canal during the navigation season. 
Canaseraga CrEeK NEAR DANSVILLE, N. Y.

Location.-At highway bridge 1 mile west of Dansville, Livingston County, half a mile below mouth of Mill Brook and 22 miles above mouth.

Drainage AREA.-158 square miles (furnished by New York State Conservation Commission).

Records available.-July 21, 1910, to December 31, 1912; July 10, 1915, to June, 30, 1917; March 10, 1919, to June 16, 1919; March 17, 1920, to September 30, 1923.

GAGE.-Gurley seven-day water-stage recorder installed October 19, 1920, on left abutment. During winter a vertical staff at the same location is used because of unsatisfactory operation of water-stage recorder. Recorder inspected and staff gage read by Frank S. Fox.

Discharge measurements.-Made from downstream side of bridge or by wading.

Channel AND control.-Sand and gravel; shifting frequently.

Extremes of DISchaRgE.-Maximum stage recorded during year, 11.20 feet (staff gage reading) at $4 \mathrm{p} . \mathrm{m}$. March 4, (discharge, determined from logarithmic extension of rating curve, about 3,500 second-feet); minimum stage from water-stage recorder, 6.26 feet from $11 \mathrm{p} . \mathrm{m}$. September 2 to $5 \mathrm{a}$. m. September 3 (discharge, 14 second-feet).

1910-1912; 1915-1917; 1919-1923: Maximum stage recorded, 13 feet at 9.30 p. m. May 16, 1916 (discharge, determined from logarithmic extension of rating curve, roughly 6,600 second-feet); minimum discharge, 14 secondfeet September 10, 1921, and September 2 and 3, 1923.

ICE.-Stage-discharge relation affected by ice.

Accuracy.-Stage-discharge relation not permanent until after spring breakup when it became practically permanent for the remainder of the year; affected by ice from December to March. Rating curve used subsequent to March 3 fairly well defined between 10 and 1,000 second-feet. Daily discharge ascertained by applying to rating table mean daily gage height as observed or as determined by inspection of gage-height graph, or for days of considerable fluctuation, by averaging discharge for intervals of day; shifting-control method used October 1 to December 16. Records fair.

Discharge measurements of Canaseraga Creek near Dansville, $N, Y$., during the year ending September 30, 1923

\begin{tabular}{|c|c|c|c|c|c|c|c|}
\hline Date & Made by- & $\begin{array}{c}\text { Gage } \\
\text { height }\end{array}$ & $\begin{array}{c}\text { Dis- } \\
\text { charge }\end{array}$ & Date & Made by- & $\begin{array}{c}\text { Gage } \\
\text { height }\end{array}$ & $\begin{array}{c}\text { Dis- } \\
\text { charge }\end{array}$ \\
\hline $\begin{array}{l}\text { Oct. } 10 \\
\text { Nov. } 10 \\
\text { Jan. } 12\end{array}$ & $\begin{array}{l}\text { A. W. Harrington. } \\
\text { B. F. Howe }\end{array}$ & $\begin{array}{c}\text { Feet } \\
7.01 \\
6.80 \\
a 6.89\end{array}$ & $\begin{array}{r}\text { Sec.-ft. } \\
98.5 \\
54.6 \\
65.8\end{array}$ & $\begin{array}{l}\text { Feb. } 7 \\
\text { Mar. } 28 \\
\text { Aug. } 16\end{array}$ & $\begin{array}{l}\text { B. F. Howe } \\
\text { A. B. Shupe } \\
\text { A. Harrington }\end{array}$ & $\begin{array}{c}\text { Feet } \\
\times 7.26 \\
7.10 \\
6.35\end{array}$ & $\begin{array}{c}\text { Sec. }-f t . \\
114 \\
178 \\
21.8\end{array}$ \\
\hline
\end{tabular}

a stage-discharge relation affected by ice. 
Daily discharge, in second-feet, of Canaseraga Creek near Dansville, N. Y., for the year ending September 30, 1923

\begin{tabular}{|c|c|c|c|c|c|c|c|c|c|c|c|c|}
\hline Day & Oet. & Nov. & Dee. & Jan. & Feb. & Mar. & Apr. & May & June & July & Aug. & Sept. \\
\hline $\begin{array}{l}1 \\
2 \ldots-\ldots \\
3 \\
4 \ldots- \\
5 \ldots\end{array}$ & $\begin{array}{l}52 \\
52 \\
52 \\
50 \\
50\end{array}$ & $\begin{array}{l}46 \\
46 \\
46 \\
47 \\
46\end{array}$ & $\begin{array}{l}72 \\
95 \\
65 \\
58 \\
60\end{array}$ & $\begin{array}{r}240 \\
160 \\
120 \\
110 \\
95\end{array}$ & $\begin{array}{r}95 \\
280 \\
650 \\
340 \\
220\end{array}$ & $\begin{array}{r}90 \\
120 \\
1,400 \\
3,300 \\
1,110\end{array}$ & $\begin{array}{r}155 \\
155 \\
164 \\
320 \\
1,260\end{array}$ & $\begin{array}{l}94 \\
89 \\
83 \\
78 \\
78\end{array}$ & $\begin{array}{l}59 \\
59 \\
50 \\
50 \\
50\end{array}$ & $\begin{array}{l}37 \\
33 \\
30 \\
43 \\
36\end{array}$ & $\begin{array}{l}27 \\
24 \\
25 \\
25 \\
23\end{array}$ & $\begin{array}{l}18 \\
17 \\
17 \\
19 \\
21\end{array}$ \\
\hline $\begin{array}{r}6 \\
7 \\
8 \\
9 \\
10\end{array}$ & $\begin{array}{l}49 \\
52 \\
60 \\
70 \\
92\end{array}$ & $\begin{array}{l}50 \\
52 \\
52 \\
56 \\
55\end{array}$ & $\begin{array}{l}50 \\
47 \\
56 \\
80 \\
60\end{array}$ & $\begin{array}{l}85 \\
75 \\
70 \\
70 \\
65\end{array}$ & $\begin{array}{r}120 \\
100 \\
95 \\
80 \\
70\end{array}$ & $\begin{array}{l}440 \\
342 \\
270 \\
239 \\
221\end{array}$ & $\begin{array}{l}695 \\
440 \\
365 \\
286 \\
239\end{array}$ & $\begin{array}{r}78 \\
78 \\
68 \\
118 \\
126\end{array}$ & $\begin{array}{r}59 \\
59 \\
94 \\
135 \\
78\end{array}$ & $\begin{array}{l}34 \\
33 \\
30 \\
27 \\
25\end{array}$ & $\begin{array}{l}22 \\
21 \\
22 \\
22 \\
20\end{array}$ & $\begin{array}{l}22 \\
19 \\
37 \\
28\end{array}$ \\
\hline 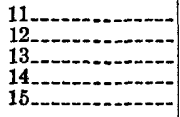 & $\begin{array}{l}92 \\
68 \\
63 \\
55 \\
52\end{array}$ & $\begin{array}{l}52 \\
50 \\
47 \\
46 \\
50\end{array}$ & $\begin{array}{l}95 \\
65 \\
65 \\
65 \\
65\end{array}$ & $\begin{array}{l}70 \\
65 \\
60 \\
64 \\
60\end{array}$ & $\begin{array}{l}70 \\
80 \\
80 \\
95 \\
95\end{array}$ & $\begin{array}{r}299 \\
1,180 \\
792 \\
365 \\
247\end{array}$ & $\begin{array}{l}232 \\
221 \\
196 \\
170 \\
155\end{array}$ & $\begin{array}{l}126 \\
164 \\
170 \\
135 \\
113\end{array}$ & $\begin{array}{l}64 \\
68 \\
50 \\
50 \\
54\end{array}$ & $\begin{array}{l}27 \\
26 \\
25 \\
24 \\
43\end{array}$ & $\begin{array}{l}19 \\
19 \\
18 \\
18 \\
19\end{array}$ & $\begin{array}{l}2 \\
2\end{array}$ \\
\hline $\begin{array}{l}16 \\
18 \\
18 \\
18 \\
20\end{array}$ & $\begin{array}{r}53 \\
+52 \\
+49 \\
49 \\
47\end{array}$ & $\begin{array}{l}50 \\
49 \\
47 \\
46 \\
46\end{array}$ & $\begin{array}{l}53 \\
42 \\
36 \\
36 \\
36\end{array}$ & $\begin{array}{r}75 \\
70 \\
100 \\
100 \\
130\end{array}$ & $\begin{array}{l}90 \\
80 \\
75 \\
70 \\
60\end{array}$ & $\begin{array}{r}1,980 \\
760 \\
520 \\
492 \\
320\end{array}$ & $\begin{array}{l}155 \\
140 \\
140 \\
140 \\
140\end{array}$ & $\begin{array}{l}196 \\
203 \\
140 \\
140 \\
113\end{array}$ & $\begin{array}{l}50 \\
50 \\
50 \\
50 \\
43\end{array}$ & $\begin{array}{r}316 \\
99 \\
68 \\
52 \\
42\end{array}$ & $\begin{array}{l}20 \\
19 \\
19 \\
18 \\
18\end{array}$ & 20 \\
\hline 21 & $\begin{array}{l}46 \\
45 \\
55 \\
78 \\
63\end{array}$ & $\begin{array}{l}45 \\
46 \\
45 \\
47 \\
45\end{array}$ & $\begin{array}{l}36 \\
36 \\
36 \\
38 \\
42\end{array}$ & $\begin{array}{l}160 \\
440 \\
200 \\
160 \\
110\end{array}$ & $\begin{array}{l}55 \\
50 \\
46 \\
34 \\
48\end{array}$ & $\begin{array}{l}286 \\
342 \\
825 \\
492 \\
320\end{array}$ & $\begin{array}{l}135 \\
126 \\
137 \\
124 \\
111\end{array}$ & $\begin{array}{l}221 \\
164 \\
140 \\
108 \\
101\end{array}$ & $\begin{array}{l}36 \\
36 \\
30 \\
30 \\
27\end{array}$ & $\begin{array}{l}36 \\
30 \\
28 \\
30 \\
48\end{array}$ & $\begin{array}{l}18 \\
20 \\
19 \\
18 \\
19\end{array}$ & $\begin{array}{l}25 \\
23\end{array}$ \\
\hline $\begin{array}{l}26 \\
27 \\
28 \\
29 \\
30\end{array}$ & $\begin{array}{l}58 \\
53 \\
52 \\
49 \\
47 \\
46\end{array}$ & $\begin{array}{l}43 \\
46 \\
45 \\
43 \\
43\end{array}$ & $\begin{array}{r}55 \\
90 \\
180 \\
110 \\
110 \\
95\end{array}$ & $\begin{array}{l}95 \\
90 \\
85 \\
80 \\
85 \\
80\end{array}$ & $\begin{array}{r}46 \\
55 \\
55 \\
-2 \\
-\end{array}$ & $\begin{array}{l}258 \\
203 \\
170 \\
170 \\
170 \\
155\end{array}$ & $\begin{array}{r}103 \\
99 \\
103 \\
116 \\
101\end{array}$ & $\begin{array}{l}89 \\
78 \\
68 \\
68 \\
68 \\
68\end{array}$ & $\begin{array}{l}54 \\
43 \\
59 \\
68 \\
50\end{array}$ & $\begin{array}{l}35 \\
30 \\
33 \\
30 \\
29 \\
\mathbf{3 0}\end{array}$ & $\begin{array}{l}19 \\
19 \\
20 \\
21 \\
19 \\
18\end{array}$ & $\begin{array}{l}23 \\
22 \\
22 \\
23 \\
23\end{array}$ \\
\hline
\end{tabular}

NoTE.-Discharge for the following days estimated or interpolated because of missing gage-height record: Dec. 11-13, Feb. 5, Apr. 30, May 1-6, and Sept. 20-25. Staff gage readings used Doc. 14 to Apr. 22 , and May 7 to June 3n, when' there was no record from water-stage reeorder. Discharge, Dee. 17 to Mar. 3 determined from gage heights corrected for ice effect by means of two diseharge measurements, study of observer's notes, gage-height graph, and weather records.

Monthly discharge of Canaseraga Creek near Dansville, N. Y., for the year ending September 30,1923

[Drainage area, 158 square miles]

\begin{tabular}{|c|c|c|c|c|c|}
\hline \multirow{2}{*}{ Month } & \multicolumn{4}{|c|}{ Discharge in second-feet } & \multirow{2}{*}{$\begin{array}{c}\text { Run-off in } \\
\text { inches }\end{array}$} \\
\hline & Maximum & Minimum & Mean & $\begin{array}{c}\text { Per square } \\
\text { mile }\end{array}$ & \\
\hline $\begin{array}{l}\text { October } \\
\text { November } \\
\text { December } \\
\text { January } \\
\text { February } \\
\text { March } \\
\text { April } \\
\text { May } \\
\text { June } \\
\text { July } \\
\text { August } \\
\text { September }\end{array}$ & $\begin{array}{r}92 \\
56 \\
180 \\
440 \\
650 \\
3,300 \\
1,260 \\
221 \\
135 \\
316 \\
27 \\
46\end{array}$ & $\begin{array}{l}45 \\
43 \\
36 \\
60 \\
34 \\
90 \\
99 \\
68 \\
27 \\
24 \\
18 \\
17\end{array}$ & $\begin{array}{r}56.5 \\
47.6 \\
65.5 \\
112 \\
116 \\
577 \\
231 \\
115 \\
55.2 \\
45.5 \\
20.3 \\
22.5\end{array}$ & $\begin{array}{l}0,358 \\
.301 \\
.415 \\
.709 \\
.734 \\
3.65 \\
1.46 \\
.728 \\
.349 \\
.288 \\
.128 \\
.142\end{array}$ & $\begin{array}{r}0,41 \\
.34 \\
.48 \\
.82 \\
.76 \\
4.21 \\
1.63 \\
.84 \\
.39 \\
.33 \\
.15 \\
.16\end{array}$ \\
\hline The year........... & 3,300 & 17 & 122 & .772 & 10.52 \\
\hline
\end{tabular}


KESHEQUA CREEK AT CRAIG COLONY, SONYEA, N. Y.

Location.-200 feet downstream from private bridge on grounds of Craig Colony at Sonyea, Livingston County, and $21 / 2$ miles above mouth.

Drainage AREA.-70 square miles (measured by New York State Conservation Commission).

Records Available.-October 31, 1917, to September 30, 1923, at present site; July 22, 1910, to December 31, 1912, at a site 200 feet upstream. August 29,1915 , to October 31,1917 , at a site 1 mile downstream.

GAGE.-Vertical staff in three sections on retaining wall on left bank just above the concrete dam for pumping plant of Craig Colony; read by A. J. Porter.

Discharge measurements.-Made from downstream side of private bridge above gage or by wading.

Controt.-Concrete dam with two crests of equal elevation separated by a trough 18 inches wide which serves as an intake for the Craig Colony pumping plant; permanent.

EXTREMES OF DISCHARGe.-Maximum stage recorded during year, 3.06 feet at 6 p. m. March 3 (discharge, 1,780 second-feet); minimum discharge, 0.7 second-foot at 5 p. m. August 24.

1917-1923: Maximum stage recorded, 5.9 feet at 10 a. m. May 22, 1919 (discharge not determined); minimum discharge, 0.7 second-foot August 20 , 1918, and August 24, 1923.

IcE.-Stage-discharge relation slightly affected by ice.

ACCURACY. - Stage-discharge relation practically permanent except as affected by ice from December to March and by sandbag on dam during August and September. Rating curve fairly well defined between 1 and 1,500 second-feet. Gage read to hundredths twice daily. Daily discharge ascertained by applying mean daily gage height to rating table. Records fair.

Discharge measurements of Keshequa Creek at Craig Colony, Sonyea, N. Y., during the year ending September 30, 1923

\begin{tabular}{|c|c|c|c|c|c|c|c|}
\hline Date & Made by- & $\begin{array}{c}\text { Gage } \\
\text { beight }\end{array}$ & $\begin{array}{l}\text { Dis- } \\
\text { charge }\end{array}$ & Date & Made by- & $\begin{array}{c}\text { Gage } \\
\text { height }\end{array}$ & $\begin{array}{l}\text { Dis- } \\
\text { charge }\end{array}$ \\
\hline $\begin{array}{l}\text { Oct. } 10 \\
\text { Nev. } 7 \\
\text { Jan. } 12\end{array}$ & $\begin{array}{l}\text { A. W. Harrington } \\
\text { B. F. Howe. }\end{array}$ & $\begin{array}{c}\text { Feet } \\
0.77 \\
.44 \\
.64\end{array}$ & $\begin{array}{c}\text { Sec. } . f t . \\
34.0 \\
7.89 \\
18.8\end{array}$ & $\begin{array}{l}\text { Feb. } 6 \\
\text { Aug. } 18\end{array}$ & $\begin{array}{l}\text { B. F. Howe } \\
\text { A. W. Harrington. }\end{array}$ & $\begin{array}{l}\text { Feet } \\
a .73 \\
\text { b. } 21\end{array}$ & $\begin{array}{l}\text { Sec.-ft. } \\
27.9 \\
1.31\end{array}$ \\
\hline
\end{tabular}

- Stage-discharge relation affected by ice.

- Stage-discharge relation affected by sand bag on control. 
Daily di charge, in second-feet, of Keshequa Creek at Craig Colony, Sonyea, N. Y., for the year ending September 30, 1923

\begin{tabular}{|c|c|c|c|c|c|c|c|c|c|c|c|c|}
\hline Day & Oct. & Nov. & Dec. & Jan. & Feb. & Mar. & Apr. & May & June & July & Aug. & Sept. \\
\hline $\begin{array}{l}2 \ldots \\
3 \ldots \\
4 \ldots \\
5 \ldots\end{array}$ & $\begin{array}{l}4.9 \\
4.4 \\
4.6 \\
4.6\end{array}$ & $\begin{array}{c}9.6 \\
6.7 \\
6.7 \\
10 \\
7.2\end{array}$ & $\begin{array}{c}9.6 \\
22 \\
9.1 \\
11 \\
12\end{array}$ & $\begin{array}{r}120 \\
85 \\
55 \\
40 \\
30\end{array}$ & $\begin{array}{r}35 \\
130 \\
440 \\
120 \\
55\end{array}$ & $\begin{array}{r}60 \\
90 \\
300 \\
1,070 \\
303\end{array}$ & $\begin{array}{c}6.2 \\
19 \\
41 \\
115 \\
730\end{array}$ & $\begin{array}{l}22 \\
17 \\
17 \\
17 \\
14\end{array}$ & $\begin{array}{l}9.6 \\
8.1 \\
7.6 \\
8.1 \\
9.1\end{array}$ & $\begin{array}{l}5.7 \\
4.1 \\
4.1 \\
8.1 \\
6.2\end{array}$ & $\begin{array}{l}3.4 \\
3.6 \\
3.2 \\
3.2 \\
2.8\end{array}$ & $\begin{array}{l}1.3 \\
1.2 \\
1.0 \\
.9 \\
1.5\end{array}$ \\
\hline $\begin{array}{r}6 \\
7 \\
8 \\
9\end{array}$ & $\begin{array}{l}4.1 \\
4.1 \\
5.2 \\
12 \\
23\end{array}$ & $\begin{array}{l}6.2 \\
9.1 \\
8.1 \\
9.6 \\
9.1\end{array}$ & $\begin{array}{l}8.6 \\
5.9 \\
12 \\
22 \\
14\end{array}$ & $\begin{array}{l}22 \\
11 \\
11 \\
11 \\
13\end{array}$ & $\begin{array}{l}36 \\
28 \\
19 \\
17 \\
14\end{array}$ & $\begin{array}{r}100 \\
72 \\
58 \\
60 \\
86\end{array}$ & $\begin{array}{r}258 \\
132 \\
110 \\
66 \\
58\end{array}$ & $\begin{array}{l}13 \\
12 \\
12\end{array}$ & $\begin{array}{l}7.2 \\
10 \\
14 \\
15 \\
11\end{array}$ & $\begin{array}{l}7.2 \\
4.1 \\
4.6 \\
3.6 \\
3.9\end{array}$ & $\begin{array}{l}3.0 \\
2.4 \\
1.7 \\
2.6 \\
2.1\end{array}$ & $\begin{array}{l}1.9 \\
3.2 \\
2.5 \\
2.0 \\
1.7\end{array}$ \\
\hline- & $\begin{array}{l}21 \\
11 \\
11 \\
8.1 \\
7.2\end{array}$ & $\begin{array}{l}8.1 \\
7.2 \\
7.2 \\
9.6 \\
9.1\end{array}$ & $\begin{array}{r}9.1 \\
12 \\
7.2 \\
7.6 \\
8.1\end{array}$ & $\begin{array}{l}14 \\
19 \\
14 \\
10 \\
11\end{array}$ & $\begin{array}{l}14 \\
16 \\
22 \\
24 \\
17\end{array}$ & $\begin{array}{r}150 \\
1,010 \\
391 \\
105 \\
68\end{array}$ & $\begin{array}{l}60 \\
50 \\
47 \\
40 \\
36\end{array}$ & $\begin{array}{l}25 \\
31 \\
44 \\
28 \\
37\end{array}$ & $\begin{array}{l}10 \\
7.6 \\
6.2 \\
6.2 \\
6.2\end{array}$ & $\begin{array}{r}3.6 \\
3.4 \\
3.0 \\
3.0 \\
13\end{array}$ & $\begin{array}{l}1.2 \\
1.5\end{array}$ & $\begin{array}{l}1.7 \\
1.7 \\
1.5 \\
1.1 \\
1.5\end{array}$ \\
\hline 80 & $\begin{array}{l}7.2 \\
9.6 \\
7.6 \\
8.1 \\
5.7\end{array}$ & $\begin{array}{l}10 \\
7.2 \\
7.2 \\
7.2 \\
6.2\end{array}$ & $\begin{array}{l}6 \\
4 \\
4 \\
4 \\
4\end{array}$ & $\begin{array}{l}14 \\
17 \\
22 \\
24 \\
36\end{array}$ & $\begin{array}{r}14 \\
10 \\
9 \\
7 \\
7\end{array}$ & $\begin{array}{r}780 \\
227 \\
126 \\
150 \\
51\end{array}$ & $\begin{array}{l}36 \\
31 \\
29 \\
27 \\
27\end{array}$ & $\begin{array}{l}46 \\
31 \\
29 \\
22 \\
19\end{array}$ & $\begin{array}{l}6.2 \\
5.7 \\
5.2 \\
5.2 \\
4.1\end{array}$ & $\begin{array}{c}236 \\
29 \\
12 \\
9.1 \\
7.2\end{array}$ & $\begin{array}{l}1.2 \\
1.9 \\
1.6 \\
1.3 \\
1.0\end{array}$ & $\begin{array}{l}1.9 \\
1.3 \\
2.1 \\
2.1\end{array}$ \\
\hline $\begin{array}{l}21 \\
22 \\
23 \\
24 \\
25 \\
25\end{array}$ & $\begin{array}{c}11 \\
5.9 \\
7.2 \\
18 \\
12\end{array}$ & $\begin{array}{c}8.6 \\
8.1 \\
8.1 \\
10 \\
9.1\end{array}$ & $\begin{array}{r}4 \\
4 \\
5 \\
7 \\
11\end{array}$ & $\begin{array}{r}65 \\
170 \\
120 \\
70 \\
44\end{array}$ & $\begin{array}{l}5 \\
5 \\
4 \\
3 \\
5\end{array}$ & $\begin{array}{r}68 \\
100 \\
298 \\
132 \\
51\end{array}$ & $\begin{array}{l}28 \\
26 \\
31 \\
27 \\
22\end{array}$ & $\begin{array}{l}34 \\
39 \\
27 \\
20 \\
18\end{array}$ & $\begin{array}{l}4.4 \\
3.4 \\
3.2 \\
3.4 \\
2.1\end{array}$ & $\begin{array}{l}4.6 \\
3.9 \\
3.6 \\
3.6 \\
7.6\end{array}$ & $\begin{array}{r}1.0 \\
1.5 \\
1.2 \\
.9 \\
1.2\end{array}$ & $\begin{array}{r}2.5 \\
25 \\
7.6 \\
5.2 \\
5.7\end{array}$ \\
\hline $\begin{array}{l}26 \\
27 \\
28 \\
29 \\
30 \\
31\end{array}$ & $\begin{array}{l}10 \\
9.1 \\
9.1 \\
6.7 \\
6.7 \\
6.2\end{array}$ & $\begin{array}{l}9.1 \\
9.6 \\
9.1 \\
9.1 \\
8.1\end{array}$ & $\begin{array}{l}20 \\
30 \\
22 \\
15 \\
11 \\
34\end{array}$ & $\begin{array}{l}38 \\
36 \\
26 \\
24 \\
19 \\
22\end{array}$ & $\begin{array}{r}6 \\
11 \\
29 \\
\hdashline\end{array}$ & $\begin{array}{l}61 \\
41 \\
31 \\
36 \\
41 \\
25\end{array}$ & $\begin{array}{l}18 \\
20 \\
17 \\
25 \\
21\end{array}$ & $\begin{array}{l}17 \\
12 \\
12 \\
11 \\
10 \\
10\end{array}$ & $\begin{array}{r}6.2 \\
9.6 \\
5.7 \\
12 \\
7.2\end{array}$ & $\begin{array}{l}6.2 \\
5.7 \\
4.1 \\
3.9 \\
3.6 \\
3.6\end{array}$ & $\begin{array}{l}1.5 \\
1.3 \\
1.2 \\
1.4 \\
1.6 \\
1.6\end{array}$ & $\begin{array}{l}3.0 \\
2.8 \\
3.2 \\
4.1 \\
2.8\end{array}$ \\
\hline
\end{tabular}

NotE.-Discharge for the following days when gage was not read, estimated, or interpolated: Dec. 10,15, Jan. 1, 23-25, A pril 14, July 11, A ug. 7, 29, and Sept. 8 and 9. Discharge, Dec. 16 to Mar. 3, determined from gage heights corrected for ice effect by means of two discharge measurements, study of observer's notes, gage-height graph and weather records, and comparison with records of Canaserage Creek near Dansville, Discharge, Aug. 12 to Sept. 30, determined from gage heights corrected for backwater effect from obstruction on dam by means of two discharge measurements.

Monthly discharge of Keshequa Creek at Craig Colony, Sonyea, N. Y., for the year ending September 30, 1923

[Drainage area, 70 square miles]

\begin{tabular}{|c|c|c|c|c|c|}
\hline \multirow{2}{*}{ Month } & \multicolumn{4}{|c|}{ Discharge in second-feet } & \multirow{2}{*}{$\begin{array}{l}\text { Run-ofi in } \\
\text { inches }\end{array}$} \\
\hline & Maximum & Minimum & Mean & $\begin{array}{c}\text { Per square } \\
\text { mile }\end{array}$ & \\
\hline \multirow[t]{2}{*}{$\begin{array}{l}\text { October } \\
\text { November } \\
\text { December. } \\
\text { January } \\
\text { February } \\
\text { March } \\
\text { April } \\
\text { May } \\
\text { June } \\
\text { July } \\
\text { August } \\
\text { September } \\
\text { The year }\end{array}$} & $\begin{array}{c}23 \\
10 \\
34 \\
170 \\
440 \\
1,070 \\
730 \\
46 \\
15 \\
236 \\
3.6 \\
25\end{array}$ & $\begin{array}{c}4.1 \\
6.2 \\
4 \\
10 \\
3 \\
25 \\
6.2 \\
10 \\
2.1 \\
3.0 \\
.9 \\
.9\end{array}$ & $\begin{array}{c}8.73 \\
8.34 \\
11.5 \\
39.1 \\
39.4 \\
198 \\
71.8 \\
22.3 \\
7.32 \\
13.6 \\
1.80 \\
3.19 \\
\end{array}$ & $\begin{array}{c}0.125 \\
.119 \\
.164 \\
.559 \\
.563 \\
2.83 \\
1.03 \\
.319 \\
.105 \\
.194 \\
.026 \\
.046 \\
\end{array}$ & $\begin{array}{r}0.14 \\
.13 \\
.19 \\
.64 \\
.59 \\
3.26 \\
1.15 \\
.37 \\
.12 \\
.22 \\
.03 \\
.05 \\
\end{array}$ \\
\hline & 1,070 & .9 & 35.5 & .507 & 6.89 \\
\hline
\end{tabular}




\section{CONESUS CREEK NEAR LAKRVILLE, N. Y.}

Location.-At highway bridge known locally as Millville Bridge, 11/2 miles north of Lakeville, Livingston County, and outlet of Conesus Lake.

Drainage AREa.-71 square miles (furnished by New York State Conservation Commission).

Records available.-November 13, 1919, to September 30, 1923.

GAGE.-Vertical staff bolted to upstream side of right abutment of bridge; read by W. B. Milliman.

Discharge measurements.-Made from highway bridge about a quarter of a mile downstream or by wading.

Channel and control.-A rectangular weir, 2.01 feet long and 0.67 foot high under upstream side of bridge. When the water overtops this weir it flows over a 2-inch plank 25.75 feet long, including the 2 feet of weir. The theoretical stage-discharge relation does not apply on account of leakage under the left abutment and around the right end of weir. During night of March 11, 1923, the weir was carried out by ice and débris from the lake; the control then became a 2 by 4 inch timber bolted to concrete apron under highway bridge.

Extremes of Discharge.-Maximum discharge recorded during year, 202 second-feet at 10 a. m. March 20; minimum discharge, about 3 second-feet December 15-21.

1919-1923: Maximum discharge recorded, that of March 20, 1923; minimum discharge, about 2.5 second-feet several times during October and November, 1921.

IcE.-Stage-discharge relation slightly affected by ice.

Diversions.- No water is diverted from Conesus Lake above the station.

ACCURACY.-Stage-discharge relation practically permanent, except as affected by ice from December to night of March 11, when weir was carried away by ice and débris. Rating curve for weir fairly well defined between 4 and 150 second-feet. Rating curve for temporary control fairly well defined between 3 and 150 second-feet. Gage read to hundredths twice daily. Daily discharge ascertained by applying mean daily gage height to rating table. Records fair.

Discharge measurements of Conesus Creek near Lakeville, N. Y., during the year ending September 30, 1923

\begin{tabular}{|c|c|c|c|c|c|c|c|}
\hline Date & Made by- & $\begin{array}{c}\text { Gage } \\
\text { height }\end{array}$ & $\begin{array}{c}\text { Dis- } \\
\text { charge }\end{array}$ & Date & Made by- & $\begin{array}{c}\text { Gage } \\
\text { height }\end{array}$ & $\begin{array}{l}\text { Dis- } \\
\text { charge }\end{array}$ \\
\hline $\begin{array}{l}\text { Oct. } 11 \\
\text { Nov. } 5 \\
\text { Jan. } 13\end{array}$ & $\begin{array}{l}\text { A. W. Harrington } \\
\text { B. F. Howe... }\end{array}$ & $\begin{array}{c}\text { Feet } \\
0.85 \\
.54 \\
a .98\end{array}$ & $\begin{array}{r}\text { Sec.-ft. } \\
13.1 \\
5.5 \\
22.5\end{array}$ & $\begin{array}{l}\text { Jan. } 31 \\
\text { Apr. } 4 \\
\text { Aug. } 19\end{array}$ & $\begin{array}{l}\text { B. F. Howe } \\
\text { A. B. Shupe } \\
\text { A. W. Harrington }\end{array}$ & $\begin{array}{r}\text { Fbe } \\
a_{1.11} .72 \\
-.16\end{array}$ & $\begin{array}{r}\text { Sec.-ft. } \\
30.4 \\
118 \\
14.0\end{array}$ \\
\hline
\end{tabular}

a Stage-discharge relation affected by ice. 
Daily discharge, in second-feet, of Conesus Creek near Lakeville, N. Y., for the year ending September 30, 1923

\begin{tabular}{|c|c|c|c|c|c|c|c|c|c|c|c|c|}
\hline Day & Oct. & Nov. & Dec. & Jan. & Feb. & Mar. & Apr. & May & June & July & Aug. & Sept. \\
\hline $\begin{array}{l}1 \\
1 \\
3 \\
4 \\
5 \\
5\end{array}$ & $\begin{array}{l}14 \\
15 \\
14 \\
15 \\
15\end{array}$ & $\begin{array}{l}4.4 \\
4.5 \\
5.2 \\
5.6 \\
5.4\end{array}$ & $\begin{array}{l}9.7 \\
6.0 \\
4.2 \\
4.0 \\
6.8\end{array}$ & $\begin{array}{r}7 \\
9 \\
16 \\
18 \\
18\end{array}$ & $\begin{array}{l}36 \\
34 \\
36 \\
36 \\
38\end{array}$ & $\begin{array}{l}36 \\
50 \\
65 \\
80 \\
88\end{array}$ & $\begin{array}{l}145 \\
122 \\
122 \\
115 \\
152\end{array}$ & $\begin{array}{l}74 \\
71 \\
69 \\
69 \\
66\end{array}$ & $\begin{array}{l}36 \\
34 \\
35 \\
38 \\
39\end{array}$ & & & $\begin{array}{l}12 \\
11 \\
12 \\
11 \\
10\end{array}$ \\
\hline $\begin{array}{r}6 \\
7 \\
8 \\
9 \\
9\end{array}$ & $\begin{array}{l}14 \\
14 \\
14 \\
15 \\
17\end{array}$ & $\begin{array}{l}6.0 \\
6.8 \\
7.6 \\
7.6 \\
8.8\end{array}$ & $\begin{array}{l}4.0 \\
3.7 \\
3.9 \\
4.4 \\
4.2\end{array}$ & $\begin{array}{l}18 \\
17 \\
18 \\
17 \\
18\end{array}$ & $\begin{array}{l}40 \\
38 \\
34 \\
32 \\
30\end{array}$ & $\begin{array}{r}94 \\
101 \\
101 \\
94 \\
101\end{array}$ & $\begin{array}{l}152 \\
152 \\
145 \\
152 \\
152\end{array}$ & $\begin{array}{l}64 \\
62 \\
62 \\
64 \\
61\end{array}$ & $\begin{array}{l}38 \\
47 \\
55 \\
50 \\
45\end{array}$ & 22 & 16 & $\begin{array}{l}11 \\
12 \\
13 \\
11 \\
11\end{array}$ \\
\hline $\begin{array}{l}11 \\
12 \\
13 \\
14 \\
15\end{array}$ & $\begin{array}{l}16 \\
16 \\
15 \\
15 \\
14\end{array}$ & $\begin{array}{l}8.5 \\
8.5 \\
7.6 \\
7.6 \\
7.0\end{array}$ & $\begin{array}{l}4.0 \\
4.0 \\
3.6 \\
3.4\end{array}$ & $\begin{array}{l}20 \\
24 \\
28 \\
18 \\
16\end{array}$ & $\begin{array}{l}28 \\
30 \\
28 \\
32 \\
36\end{array}$ & $\begin{array}{l}108 \\
176 \\
140 \\
138 \\
130\end{array}$ & $\begin{array}{l}138 \\
138 \\
130 \\
122 \\
115\end{array}$ & $\begin{array}{l}61 \\
61 \\
61 \\
59 \\
62\end{array}$ & $\begin{array}{l}40 \\
35 \\
33 \\
30\end{array}$ & & & $\begin{array}{l}10 \\
9.7 \\
9.7 \\
9.1 \\
9.7\end{array}$ \\
\hline $\begin{array}{l}16 \\
17 \\
18 \\
19 \\
20\end{array}$ & $\begin{array}{l}13 \\
13 \\
12 \\
12 \\
11\end{array}$ & $\begin{array}{l}6.6 \\
7.6 \\
6.6 \\
4.9 \\
4.8\end{array}$ & 3 & $\begin{array}{l}13 \\
13 \\
13 \\
16 \\
16\end{array}$ & $\begin{array}{l}32 \\
28 \\
28 \\
26 \\
24\end{array}$ & $\begin{array}{l}168 \\
168 \\
176 \\
184 \\
176\end{array}$ & $\begin{array}{l}108 \\
108 \\
108 \\
108 \\
101\end{array}$ & $\begin{array}{l}60 \\
61 \\
59 \\
56 \\
56\end{array}$ & & & $\begin{array}{l}13 \\
11\end{array}$ & $\begin{array}{l}9.7 \\
9.1 \\
8.4 \\
9.1 \\
9.7\end{array}$ \\
\hline $\begin{array}{l}21 \\
22 \\
24 \\
25\end{array}$ & $\begin{array}{l}10 \\
11 \\
12 \\
11 \\
11\end{array}$ & $\begin{array}{l}4.7 \\
4.8 \\
4.8 \\
4.3 \\
4.8\end{array}$ & $\begin{array}{l}\text { 3. } 5 \\
3.5 \\
\text { 3. } 7 \\
\text { 3. } 9\end{array}$ & $\begin{array}{l}22 \\
24 \\
26 \\
28 \\
26\end{array}$ & $\begin{array}{l}18 \\
16 \\
15 \\
15 \\
15\end{array}$ & $\begin{array}{l}160 \\
168 \\
168 \\
168 \\
168\end{array}$ & $\begin{array}{r}101 \\
94 \\
94 \\
94 \\
94\end{array}$ & $\begin{array}{l}56 \\
54 \\
53 \\
54 \\
54\end{array}$ & 24 & 30 & $\begin{array}{c}12 \\
9.7 \\
9.7 \\
10 \\
9.7\end{array}$ & $\begin{array}{l}9.7 \\
11 \\
12 \\
11 \\
11\end{array}$ \\
\hline $\begin{array}{l}26 \\
27 \\
28 \\
29 \\
30 \\
31\end{array}$ & $\begin{array}{r}12 \\
7.3 \\
5.6 \\
5.0 \\
4.6 \\
4.5\end{array}$ & $\begin{array}{l}4.9 \\
7.6 \\
4.7 \\
4.7 \\
4.6 \\
-9\end{array}$ & $\begin{array}{l}4.5 \\
4.9 \\
4.5 \\
4.1 \\
4 \\
5\end{array}$ & $\begin{array}{l}24 \\
22 \\
24 \\
28 \\
28 \\
36\end{array}$ & $\begin{array}{r}18 \\
24 \\
34 \\
-\end{array}$ & $\begin{array}{l}160 \\
160 \\
152 \\
145 \\
145 \\
138\end{array}$ & $\begin{array}{l}87 \\
82 \\
79 \\
79 \\
75\end{array}$ & $\begin{array}{l}56 \\
47 \\
44 \\
42 \\
41 \\
38\end{array}$ & & & $\begin{array}{l}11 \\
12 \\
13 \\
12 \\
13 \\
12\end{array}$ & $\begin{array}{l}10 \\
9.7 \\
9.7 \\
9.7 \\
9.1\end{array}$ \\
\hline
\end{tabular}

NoтE.-Discharge for the following periods when gage-height record was doubtful estimated by comparison with record of Keshequa Creek at Craig Colony Dec. 15-21, Mar. 13, June 15 to Aug. 18. Discharge, Dec. 30 to Mar. 4, determined from gage heights corrected for ice effect by means of two discharge measurements, study of observer's notes, gage-height graph and weather records and comparison with records of flow of near-by streams.

Monthly discharge of Conesus Creek near Lakeville, N. Y., for the year ending September 30, 1923

[Drainage area, 71 square miles]

\begin{tabular}{|c|c|c|c|c|c|}
\hline \multirow{2}{*}{ Month } & \multicolumn{4}{|c|}{ Discharge in second-feet } & \multirow{2}{*}{$\begin{array}{l}\text { Run-off in } \\
\text { inches }\end{array}$} \\
\hline & Maximum & Minimum & Moan & $\begin{array}{c}\text { Per square } \\
\text { mile }\end{array}$ & \\
\hline $\begin{array}{l}\text { October } \\
\text { November } \\
\text { December } \\
\text { January } \\
\text { February } \\
\text { March } \\
\text { April } \\
\text { May } \\
\text { June } \\
\text { July } \\
\text { August } \\
\text { September }\end{array}$ & $\begin{array}{r}17 \\
8.8 \\
9.7 \\
36 \\
40 \\
184 \\
152 \\
74 \\
55 \\
\hdashline 13\end{array}$ & $\begin{array}{r}4.5 \\
4.3 \\
7 \\
15 \\
36 \\
75 \\
38 \\
9.7 \\
8.4\end{array}$ & $\begin{array}{r}12.2 \\
6.05 \\
4.15 \\
20.0 \\
28.6 \\
132 \\
115 \\
58.0 \\
31.4 \\
26.1 \\
14.1 \\
10.4\end{array}$ & $\begin{array}{l}0.172 \\
.085 \\
.058 \\
.282 \\
.403 \\
1.86 \\
1.62 \\
.817 \\
.442 \\
.368 \\
.199 \\
.146\end{array}$ & $\begin{array}{r}0.20 \\
.09 \\
.07 \\
.33 \\
.42 \\
2.14 \\
1.81 \\
.94 \\
.49 \\
.42 \\
.23 \\
.16\end{array}$ \\
\hline The year ..... & 184 & -................ & 38.3 & .539 & 7.30 \\
\hline
\end{tabular}


CARADIOE LAKE OUTLET geaR HEMLOCK, N. $\mathbf{Y}$.

Location.-At outlet of Canadice Lake, Livingston County. Outlet flows into Genesee River through Canadice Lake outlet and Honeoye Creek.

Drainage area.-12.6 square miles, of which 1 square mile is lake surface. Records available.-April, 1903, to September 30, 1923.

GAGE.-Hook, in channel above weir.

Channel and control.-Outflow is measured over a standard thin-edged weir with a 5-foot crest and two end contractions so arranged with needle timbers at the ends that the length may be increased to 14.96 feet. No end contractions during high water. The weir crest stands 3.14 feet above the stream channel, which is artifioial with a plank bottom and vertical sides, and the crest is never submerged by backwater. Two additional rectangular gates, each 1 foot square with three complete contractions and a fourth incomplete contraction at the bottom.

Icw.-Stage-discharge relation not affected by ice as the pool above the weir is free from ice throughout the winter.

Diversions. - No water is diverted from Canadice Lake above the station.

REgULATion.-Outflow of lake is regulated by bulkhead and gates at dam above weir.

AcCURACY.-Stage-discharge relation permanent. Rating curve used is expressed by Francis formula. Corrections are made for velocity of approach for high stages. Gage read to hundredths once daily. Records good.

Cooperation.-Data collected, computed, and furnished for publication by the city engineer of Rochester.

Monthly discharge of Canädice Lake outlet near Hemlock, N. Y., for the year ending September 30, 1923

[Drainage area, 12.6 square miles]

\begin{tabular}{|c|c|c|c|c|c|}
\hline Month & $\begin{array}{c}\text { Mean } \\
\text { discharge }\end{array}$ & $\begin{array}{c}\text { Mean } \\
\text { elevation } \\
\text { of lake } \\
\text { above low } \\
\text { water } \\
\text { mark }\end{array}$ & Month & $\begin{array}{c}\text { Mean } \\
\text { discharge }\end{array}$ & $\begin{array}{l}\text { Mean } \\
\text { elevation } \\
\text { of lake } \\
\text { above } \\
\text { low } \\
\text { water } \\
\text { mark }\end{array}$ \\
\hline \multirow[t]{2}{*}{$\begin{array}{l}\text { October } \\
\text { November } \\
\text { December } \\
\text { January } \\
\text { February } \\
\text { March }\end{array}$} & \multirow[t]{2}{*}{$\begin{array}{r}\text { Sec.-ft. } \\
4.143 \\
.213 \\
6.516 \\
7.290 \\
10.858 \\
27.867 \\
29.988\end{array}$} & \multirow[t]{2}{*}{$\begin{array}{r}\text { Feet } \\
1.356 \\
1.204 \\
.943 \\
.758 \\
.985 \\
2.321 \\
2.257\end{array}$} & \multirow{2}{*}{$\begin{array}{l}\text { May } \\
\text { June } \\
\text { July } \\
\text { September } \\
\text { The year. }\end{array}$} & \begin{tabular}{|r|} 
Sec. $-f t$ \\
14.588 \\
6.479 \\
3.174 \\
2.781 \\
1.600
\end{tabular} & $\begin{array}{r}\text { Feet } \\
0.964 \\
.573 \\
. .247 \\
-.510 \\
-1.251\end{array}$ \\
\hline & & & & 9. 625 & 0.821 \\
\hline
\end{tabular}

-Nore.-Terminal water surface for the year was 3.14 feet lower than for the previous year, corresponding to a loss in storage of $89,483,164$ cubic feet, or a discharge of 2.837 second-feet for the year. This correction applied to the above gives 6.788 second-feet, equivalent to 0.539 second-foot per square mile or a run-off of 7.298 inches from the drainage area.

OWASCO LAKE OUTLET mear AUBURN, $\mathbf{N}, \mathbf{Y}$.

Location.-On farm of Charles H. Pearce, 2 miles below center of Auburn. Cayuga County, and 33/4 miles below State dam at outlet of Owasco Lake, Drainage AREA. - 206 square miles (measured on topographic maps.)

Records avarlable.-November 17, 1912, to September 30, 1923.

GAGE.-Gurley seven-day water-stage recorder on left bank; inspected by Mrs.

Charles H. Pearce. 
Discharge measurements.-Made from a cable directly opposite gage or by wading.

Channel and control.-The control is artificial and consists of a low concrete dam 100 feet long 25 feet below the gage. The elevation of crest of left half of dam is 1.28 feet gage datum; right half of dam is at elevation of $\mathbf{2 . 1 3}$ feet.

Extremes of DIScharge.-Maximum stage during year from water-stage recorder, 3.61 feet at 2 p. m. March 24 (discharge, 1,230 second-feet); minimum stage from water-stage recorder, 1.50 feet at $3.30 \mathrm{p}$. m. September 23 (discharge, 14 second-feet).

1912-1923: Maximum stage, 6.4 feet during period March 25-30, 1913, determined by leveling from floodmarks (discharge, 2,750 second-feet); minimum stage from water-stage recorder, 1.38 feet(effective) at $7 \mid p . m$. August 21, 1920 (discharge, 3.8 second-feet).

Ice.-Stage-discharge relation seldom affected by ice.

Diversions.-An average flow of about 10 second-feet is pumped from Owasco Lake for the municipal water supply of the city of Auburn. Proportion returning to stream above the gaging station is not known.

Regulation.- - Large diurnal fluctuation in flow during low-water periods due to operation of mills in the city of Auburn; seasonal flow regulated at the State dam.

AcCURACY.-Stage-discharge relation permanent except for possible slight backwater effect from ice during the winter and from aquatic growth during the late summer. Rating curve well defined below 1,700 second-feet. Operation of water-stage recorder satisfactory except as indicated in footnote to daily discharge table. Daily discharge ascertained by averaging the discharge for bihourly intervals of the day. Records good except for periods of estimate, for which they are fair.

Discharge measurements of Owasco Lake outlet near Auburn, N.Y., during the year ending September 30, 1923

\begin{tabular}{|c|c|c|c|c|c|c|c|}
\hline Date & Made by- & $\begin{array}{c}\text { Gage } \\
\text { height }\end{array}$ & $\begin{array}{l}\text { Dis- } \\
\text { charge }\end{array}$ & Date & Made by- & $\begin{array}{c}\text { Gage } \\
\text { height }\end{array}$ & $\begin{array}{l}\text { Dis- } \\
\text { charge }\end{array}$ \\
\hline $\begin{array}{l}\text { Nov. } 3 \\
\text { Jan. } 4 \\
30\end{array}$ & $\begin{array}{l}\text { A. W. Harrington } \\
\text { B. F. Howe }\end{array}$ & $\begin{array}{l}\text { Feet } \\
2.25 \\
2.39 \\
2.55\end{array}$ & $\begin{array}{r}\text { Sec.fft. } \\
18 \mathrm{i} \\
225 \\
318\end{array}$ & $\begin{array}{l}\text { Mar. } 25 \\
\text { Aug. } 20\end{array}$ & $\begin{array}{l}\text { E. B. Shupe } \\
\text { A.fW. Harrington...... }\end{array}$ & $\begin{array}{c}\text { Feet } \\
3.52 \\
2.18\end{array}$ & $\begin{array}{r}\text { Sec. ft. } \\
1,070 \\
14 \AA\end{array}$ \\
\hline
\end{tabular}


Daily discharge, in second-feet, of Owasco Lake outlet near Auburn, N. Y., for the year ending September 30, 1923

\begin{tabular}{|c|c|c|c|c|c|c|c|c|c|c|c|c|}
\hline Day & Oct. & Nov. & Dec. & Jan. & Feb. & Mar. & Apr. & May & June & July & Aug. & Sept. \\
\hline $\begin{array}{l}1 \ldots \\
2 \ldots \\
3 \ldots \\
4 \ldots \\
5 \ldots\end{array}$ & $\begin{array}{l}157 \\
192 \\
180 \\
181 \\
187\end{array}$ & $\begin{array}{l}142 \\
144 \\
136 \\
145 \\
118\end{array}$ & $\begin{array}{r}146 \\
164 \\
79 \\
146 \\
131\end{array}$ & $\begin{array}{l}140 \\
160\end{array}$ & $\begin{array}{l}291 \\
299 \\
347\end{array}$ & $\begin{array}{l}225 \\
223 \\
268 \\
324 \\
383\end{array}$ & $\begin{array}{l}788 \\
750 \\
640 \\
596 \\
764\end{array}$ & $\begin{array}{l}253 \\
235 \\
236 \\
239 \\
247\end{array}$ & $\begin{array}{l}196 \\
188 \\
188 \\
206 \\
191\end{array}$ & $\begin{array}{l}167 \\
193 \\
189 \\
168 \\
190\end{array}$ & $\begin{array}{l}193 \\
189 \\
204 \\
237 \\
143\end{array}$ & $\begin{array}{l}124 \\
107 \\
120 \\
133 \\
121\end{array}$ \\
\hline $\begin{array}{r}6 \\
7-- \\
8-. \\
9 \\
10\end{array}$ & $\begin{array}{l}169 \\
175 \\
156 \\
182 \\
201\end{array}$ & $\begin{array}{l}144 \\
135 \\
128 \\
133 \\
135\end{array}$ & $\begin{array}{l}133 \\
141 \\
141\end{array}$ & & $\begin{array}{l}341 \\
333\end{array}$ & $\begin{array}{l}427 \\
449 \\
464 \\
465 \\
464\end{array}$ & $\begin{array}{r}973 \\
1,020 \\
1,020 \\
985 \\
922\end{array}$ & $\begin{array}{l}230 . \\
254 \\
245 \\
236 \\
232\end{array}$ & $\begin{array}{l}199 \\
189 \\
201 \\
219 \\
200\end{array}$ & $\begin{array}{l}188 \\
196 \\
174 \\
210 \\
196\end{array}$ & $\begin{array}{l}206 \\
212 \\
190 \\
204 \\
186\end{array}$ & $\begin{array}{l}89 \\
90\end{array}$ \\
\hline 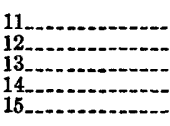 & $\begin{array}{l}184 \\
175 \\
176 \\
178 \\
154\end{array}$ & $\begin{array}{l}125 \\
116 \\
138 \\
135 \\
125\end{array}$ & & 140 & $\begin{array}{l}324 \\
325 \\
318 \\
304 \\
302\end{array}$ & $\begin{array}{l}450 \\
523 \\
538 \\
548 \\
554\end{array}$ & $\begin{array}{l}877 \\
803 \\
708 \\
644 \\
616\end{array}$ & $\begin{array}{l}228 \\
233 \\
228 \\
253 \\
358\end{array}$ & $\begin{array}{l}255 \\
416 \\
344 \\
290 \\
250\end{array}$ & $\begin{array}{l}193 \\
200 \\
201 \\
210 \\
192\end{array}$ & $\begin{array}{l}188 \\
148 \\
196 \\
185 \\
178\end{array}$ & 112 \\
\hline $\begin{array}{l}17 \\
18 \\
19 \\
20\end{array}$ & $\begin{array}{l}173 \\
174 \\
170 \\
179 \\
174\end{array}$ & $\begin{array}{r}130 \\
115 \\
134 \\
76 \\
121\end{array}$ & 130 & & $\begin{array}{l}302 \\
317 \\
299 \\
266 \\
218\end{array}$ & $\begin{array}{l}668 \\
790 \\
879 \\
931 \\
\mathbf{9 4 4}\end{array}$ & $\begin{array}{l}553 \\
528 \\
499 \\
442 \\
367\end{array}$ & $\begin{array}{l}470 \\
540 \\
538 \\
465 \\
416\end{array}$ & $\begin{array}{l}246 \\
239 \\
262 \\
229 \\
183\end{array}$ & $\begin{array}{l}222 \\
222 \\
209 \\
215 \\
220\end{array}$ & $\begin{array}{l}186 \\
181 \\
182 \\
156 \\
180\end{array}$ & $\begin{array}{r}106 \\
109 \\
99 \\
56 \\
96\end{array}$ \\
\hline 221 & $\begin{array}{l}170 \\
164 \\
193 \\
182 \\
175\end{array}$ & $\begin{array}{l}129 \\
130 \\
131 \\
130 \\
148\end{array}$ & & $\begin{array}{l}263 \\
299\end{array}$ & $\begin{array}{l}217 \\
232 \\
230 \\
240 \\
224\end{array}$ & $\begin{array}{r}905 \\
915 \\
989 \\
1,130 \\
1,130\end{array}$ & $\begin{array}{l}372 \\
346 \\
370 \\
338 \\
303\end{array}$ & $\begin{array}{l}422 \\
372 \\
363 \\
348 \\
333\end{array}$ & $\begin{array}{l}192 \\
191 \\
184 \\
174 \\
193\end{array}$ & $\begin{array}{l}220 \\
193 \\
227 \\
216 \\
209\end{array}$ & $\begin{array}{l}151 \\
144 \\
150 \\
150 \\
150\end{array}$ & $\begin{array}{r}112 \\
95 \\
74 \\
98 \\
98\end{array}$ \\
\hline $\begin{array}{l}26 \\
27 \\
28 \\
29 \\
30 \\
31\end{array}$ & $\begin{array}{l}168 \\
170 \\
166 \\
153 \\
173 \\
166\end{array}$ & $\begin{array}{l}106 \\
139 \\
142 \\
134 \\
129\end{array}$ & & 280 & \begin{tabular}{r}
233 \\
240 \\
232 \\
$\cdots$ \\
\hdashline$\ldots$. \\
$\ldots$.
\end{tabular} & $\begin{array}{r}1,120 \\
1,060 \\
991 \\
946 \\
878 \\
822\end{array}$ & $\begin{array}{l}285 \\
239 \\
259 \\
237 \\
255\end{array}$ & $\begin{array}{l}296 \\
274 \\
263 \\
202 \\
179 \\
200\end{array}$ & $\begin{array}{l}188 \\
174 \\
194 \\
184 \\
186\end{array}$ & $\begin{array}{l}217 \\
227 \\
211 \\
194 \\
225 \\
207\end{array}$ & $\begin{array}{l}136 \\
144 \\
114 \\
134 \\
118 \\
128\end{array}$ & $\begin{array}{l}86 \\
89 \\
85 \\
83 \\
81\end{array}$ \\
\hline
\end{tabular}

Note.-Discharge estimated for the following periods when water-stage recorder was not operating satisfactorily: Dec. 9, to Jan. 4, Jan. 6-20, 23-30, Feb. 4-8, July 20, 21, Aug. 20, 24, 25, and Sept. 7-14.

Monthly discharge of Owasco Lake outlet near Auburn, N. Y., for the year ending September 30,1923

[Drainage area, 206 square miles]

\begin{tabular}{|c|c|c|c|c|c|}
\hline \multirow{2}{*}{ Month } & \multicolumn{4}{|c|}{ Discharge in second-feet } & \multirow{2}{*}{$\begin{array}{l}\text { Run-off } \\
\text { in inches }\end{array}$} \\
\hline & Maximum & Minimum & Mean & $\begin{array}{c}\text { Per square } \\
\text { mile }\end{array}$ & \\
\hline $\begin{array}{l}\text { October } \\
\text { November } \\
\text { December. } \\
\text { January } \\
\text { February } \\
\text { March } \\
\text { April } \\
\text { May } \\
\text { June } \\
\text { July } \\
\text { August } \\
\text { September }\end{array}$ & \begin{tabular}{r|}
201 \\
148 \\
164 \\
347 \\
1,130 \\
1,020 \\
540 \\
416 \\
227 \\
237 \\
133
\end{tabular} & $\begin{array}{r}153 \\
76 \\
79 \\
217 \\
223 \\
237 \\
179 \\
174 \\
167 \\
114 \\
56\end{array}$ & $\begin{array}{l}174 \\
130 \\
131 \\
191 \\
289 \\
690 \\
583 \\
303 \\
218 \\
203 \\
170 \\
98.8\end{array}$ & $\begin{array}{c}0.845 \\
.631 \\
.636 \\
.927 \\
1.40 \\
3.35 \\
2.83 \\
1.47 \\
1.06 \\
.985 \\
.825 \\
.480\end{array}$ & $\begin{array}{r}0.97 \\
.70 \\
.73 \\
1.07 \\
1.46 \\
3.86 \\
3.16 \\
1.70 \\
1.18 \\
1.14 \\
.95 \\
.54\end{array}$ \\
\hline The year... & 1,130 & 56 & 265 & 1.29 & 17.46 \\
\hline
\end{tabular}




\section{FAST BRANCH OF FISH CREGK AT TABERG, N. Y.}

Location.-At steel highway bridge in village of Taberg, Oneida County, just below mouth of Furnace Creek and 23/4 miles above confluence of East and

West branches near Blossvale.

Drainage ARea.-188 square miles (measured on topographic maps).

Records avatlable.-April 1 to September 30, 1923.

GAGE.-Vertical staff attached to downstream face of left abutment; read by Alvin Thorn.

Discharge measurements.-Made from downstream side of highway bridge or by wading.

Channel and control.-Large and small boulders; shifting occasionally. Extremes of Discharge.-Maximum stage recorded during the period of record, 5.66 feet at 6.30 p. m. April 8 (discharge, about 5,180 second-feet); minimum stage, 0.57 foot at 11.40 a. m. August 21 (measured discharge, 19.8 second-feet).

ICE.-Stage-discharge relation slightly affected by ice during extremely cold weather.

Regulation.-Operation of mills upstream causes some diurnal fluctuation of flow during extremely low water.

AcCURACY.-Stage-discharge relation permanent during period. Rating curve fairly well defined between 20 and 1,500 second-feet; extended above. Gage read to tenths twice daily; accuracy doubtful. Daily discharge ascertained by applying mean daily gage height to rating table. Records poor because of doubtful gage-height record.

Discharge measurements of East Branch of Fish Creek at Taberg, N. Y., during the year ending September 30, 1923

\begin{tabular}{|c|c|c|c|c|c|c|c|}
\hline Date & Made by- & $\begin{array}{c}\text { Gage } \\
\text { height }\end{array}$ & $\begin{array}{c}\text { Dis- } \\
\text { charge }\end{array}$ & Date & Made by- & $\begin{array}{l}\text { Gage } \\
\text { height }\end{array}$ & $\begin{array}{c}\text { Dis- } \\
\text { charge }\end{array}$ \\
\hline $\begin{array}{l}\text { Mar. } 24 \\
\text { Apr. }{ }_{25}^{17}\end{array}$ & $\begin{array}{l}\text { Shupe, White, a and } \\
\text { Lamprecht a } \\
\text { Lamson and Harrington }\end{array}$ & $\begin{array}{l}\text { Feet } \\
3.54 \\
2.99 \\
3.74\end{array}$ & $\begin{array}{r}\text { Sec. } f t . \\
1,320 \\
868 \\
1,490\end{array}$ & $\begin{array}{r}\text { Aug. } 7 \\
21 \\
21 \\
\text { Sept. } 27\end{array}$ & \begin{tabular}{l} 
Harrington and White \\
A. W. Harrington....... \\
\hdashline
\end{tabular} & $\begin{array}{c}\text { Feet } \\
0.67 \\
.57 \\
.58 \\
.97\end{array}$ & $\begin{array}{r}\text { Sec.ft. } \\
33.7 \\
19.8 \\
21.0 \\
62.9\end{array}$ \\
\hline
\end{tabular}

- Engineer, Utica Gas \& Electric Co.

Daily discharge, in second-feet, of East Branch of Fish Creek at Taberg, N. Y., for the year ending September 30, 1923

\begin{tabular}{|c|c|c|c|c|c|c|c|c|c|c|c|c|c|}
\hline Day & Apr. & May & June & July & Aug. & Sept. & Day & Apr. & May & June & July & Aug. & Sept. \\
\hline $\begin{array}{l}1- \\
2= \\
3= \\
4= \\
5\end{array}$ & $\begin{array}{r}220 \\
220 \\
260 \\
850 \\
2,200\end{array}$ & $\begin{array}{r}1,200 \\
800 \\
700 \\
650 \\
600\end{array}$ & $\begin{array}{l}200 \\
190 \\
200 \\
190 \\
240\end{array}$ & $\begin{array}{l}220 \\
150 \\
110 \\
110 \\
100\end{array}$ & $\begin{array}{l}75 \\
60 \\
65 \\
65 \\
65\end{array}$ & $\begin{array}{l}55 \\
46 \\
46 \\
55 \\
46\end{array}$ & $\begin{array}{l}16 . \\
17 . \\
18 . \\
19 . \\
20 .\end{array}$ & $\begin{array}{r}1,200 \\
900 \\
750 \\
700 \\
1,400\end{array}$ & $\begin{array}{r}1,100 \\
1,700 \\
1,200 \\
800 \\
600\end{array}$ & $\begin{array}{l}160 \\
140 \\
130 \\
130 \\
110\end{array}$ & $\begin{array}{r}260 \\
260 \\
75 \\
60 \\
55\end{array}$ & $\begin{array}{l}40 \\
40 \\
30 \\
36 \\
44\end{array}$ & $\begin{array}{l}55 \\
55 \\
55 \\
75 \\
95\end{array}$ \\
\hline $\begin{array}{r}6 \\
7 \\
8 \\
9 \\
9 \\
10\end{array}$ & $\begin{array}{l}4,200 \\
3,800 \\
4,600 \\
3,000 \\
2,600\end{array}$ & $\begin{array}{l}480 \\
420 \\
360 \\
950 \\
950\end{array}$ & $\begin{array}{r}200 \\
190 \\
700 \\
1,200 \\
650\end{array}$ & $\begin{array}{l}80 \\
80 \\
80 \\
65 \\
65\end{array}$ & $\begin{array}{l}60 \\
46 \\
44 \\
40 \\
44\end{array}$ & $\begin{array}{r}46 \\
55 \\
260 \\
200 \\
180\end{array}$ & $\begin{array}{l}21 . \\
22 \\
23 \\
24 \\
25\end{array}$ & $\begin{array}{l}3,400 \\
4,000 \\
3,600 \\
2,400 \\
1,500\end{array}$ & $\begin{array}{r}1,000 \\
1,000 \\
650 \\
500 \\
460\end{array}$ & $\begin{array}{r}110 \\
100 \\
90 \\
80 \\
80\end{array}$ & $\begin{array}{l}55 \\
46 \\
46 \\
44 \\
44\end{array}$ & $\begin{array}{l}36 \\
65 \\
55 \\
46 \\
55\end{array}$ & $\begin{array}{l}380 \\
420 \\
220 \\
180 \\
110\end{array}$ \\
\hline $\begin{array}{l}11 \\
12 \\
13 \\
14\end{array}$ & $\begin{array}{l}2,600 \\
2,800 \\
2,400 \\
1,600 \\
1,500\end{array}$ & $\begin{array}{l}700 \\
600 \\
550 \\
440 \\
\mathbf{5 5 0}\end{array}$ & $\begin{array}{l}400 \\
280 \\
220 \\
190 \\
170\end{array}$ & $\begin{array}{l}60 \\
55 \\
55 \\
55 \\
55\end{array}$ & $\begin{array}{l}36 \\
34 \\
46 \\
36 \\
44\end{array}$ & $\begin{array}{r}110 \\
80 \\
75 \\
65 \\
60\end{array}$ & $\begin{array}{l}26 \\
27 \\
28 \\
29 \\
30 \\
31\end{array}$ & $\begin{array}{l}1,500 \\
1,500 \\
1,400 \\
2,000 \\
1,500\end{array}$ & $\begin{array}{l}360 \\
300 \\
260 \\
260 \\
220 \\
200\end{array}$ & $\begin{array}{l}280 \\
550 \\
280 \\
300 \\
280\end{array}$ & $\begin{array}{l}44 \\
46 \\
80 \\
75 \\
65 \\
75\end{array}$ & $\begin{array}{l}55 \\
46 \\
50 \\
80 \\
95 \\
75\end{array}$ & $\begin{array}{l}95 \\
80 \\
80 \\
95 \\
95\end{array}$ \\
\hline
\end{tabular}


Monthly discharge of East Branch of Fish Creek at Taberg, N. Y., for the year ending September 30, 1923

[Drainage area, 188 square miles]

\begin{tabular}{|c|c|c|c|c|c|}
\hline \multirow{2}{*}{ Month } & \multicolumn{4}{|c|}{ Discharge in second-feet } & \multirow{2}{*}{$\begin{array}{c}\text { Run-off in } \\
\text { inches }\end{array}$} \\
\hline & Maximum & Minimum & Mean & $\underset{\text { mile }}{\text { Per square }}$ & \\
\hline $\begin{array}{l}\text { April } \\
\text { May } \\
\text { June- } \\
\text { July } \\
\text { August } \\
\text { September }\end{array}$ & $\begin{array}{r}4,600 \\
1,700 \\
3,200 \\
260 \\
95 \\
420\end{array}$ & $\begin{array}{r}220 \\
200 \\
80 \\
44 \\
30 \\
46\end{array}$ & $\begin{array}{l}2,020 \\
663 \\
268 \\
86.1 \\
51.5 \\
116\end{array}$ & $\begin{array}{l}10.74 \\
3.53 \\
1.43 \\
.458 \\
.274 \\
.617\end{array}$ & $\begin{array}{r}11.98 \\
4.07 \\
1.60 \\
.53 \\
.32 \\
.69\end{array}$ \\
\hline
\end{tabular}

BLACK RIVER NEAR BOONVILLE, N. Y.

Location.-At highway bridge 1 mile above mouth of Sugar River, 2 miles northeast of Boonville, Oneida County, and 2 miles, by river, downstream from Hawkinsville.

DraINAGE AREA.-303 square miles (measured on topographic maps).

Records avallable.-February 16, 1911, to September 30, 1923.

GAGE.-Chain near center of left span, downstream side of bridge; staff gage on right abutment used for high-water readings; read by W. D. Charbonneau

Discharge measurements.-Made from a cable half a mile above gage or by wading near gage.

Channel and control.-Rough and full of boulders; permanent.

Extremes of Discharge.-Maximum stage recorded during year, 8.88, feet at 5 p. m. April 8 and 22 (discharge, 3,980 second-feet); minimum stage, 3.20 feet several times in August and September (discharge, 42 second-feet).

1911-1923: Maximum stage (determined by leveling from floodmark) about 12.5 feet during night of March 28, 1913 (discharge, about 10,000 second-feet); minimum stage, 2.40 feet at 5 p. m. August 26, 1918 (discharge, about 5 second-feet).

IcE.-Stage-discharge relation affected by ice.

Drversions.-Water is diverted at Forestport during the navigation season through the Forestport feeder, flowing west to a basin in Boonville. The Black River canal flows north from this basin, entering Black River at the foot of Lyons Falls. A spillway from the basin overflows into Mill Creek, a tributary of Black River. Water flowing through these two channels returns to the river below the gaging station, thus passing around it. The Black River canal also flows south from Boonville, passing out of the Black River drainage and entering the summit level of the Erie Canal (or Barge Canal) at Rome.

A continuous record of the amount of diversion through the Forestport feeder from Black River at Forestport during navigation season is published under "Forestport feeder near Boonville, N. Y." . A continuous record of the amount of diversion out of the Black River drainage ba $\mathrm{n}$ is published under "Black River canal (flowing south) near Boonville, N. Y." Th difference in discharge between these two records doubtless indicates very nearly the amount of water diverted around this station and returned to Black River.

$72038-26 \dagger$-wsp $564-7$ 
Regulation.-The State Pond at Forestport, 8 miles upstream, impounds 13,068,000 cubic feet; another State dam 11/2 miles upstream from Forestport provides a reservoir with a capacity of $213,440,000$ cubic feet and receives storage from headwater reservoirs with total capacity of about $1,800,000,000$ cubic feet, from which $1,397,000,000$ cubic feet may be drawn each year.

ACCURACr.--Stage-discharge relation practically permanent, except as affected by ice from December to April. Rating curve well defined between 35 and 2,800 second-feet and fairly well defined between 2,800 and 4,500 secondfeet. Gage read to hundredths twice daily. Daily discharge ascertained by applying mean daily gage height to rating table. Records good, except for period of ice effect, for which they are fair.

Discharge measurements of Black River near Boonville, N. Y., during the year ending September 30, 1928

\begin{tabular}{|c|c|c|c|c|c|c|c|}
\hline Date & Made by- & $\begin{array}{c}\text { Gage } \\
\text { height }\end{array}$ & $\begin{array}{c}\text { Dis- } \\
\text { charge }\end{array}$ & Date & Made by- & $\begin{array}{c}\text { Gage } \\
\text { height }\end{array}$ & $\begin{array}{l}\text { Dis- } \\
\text { charge }\end{array}$ \\
\hline $\begin{array}{l}\text { Jan. } 11 \\
\text { Feb. } 8 \\
\text { Mar. } 13 \\
\text { Apr. } 17\end{array}$ & $\begin{array}{l}\text { J. L. Lamson } \\
\text { A. W. Harrington. } \\
\text { J. L. Lamson } \\
\text { Lamson and Harrington }\end{array}$ & $\begin{array}{l}\text { Freet } \\
\text { a } 5.50 \\
\text { a } 5.40 \\
\text { a } 5.40 \\
6.65\end{array}$ & $\begin{array}{r}\text { Sec. ft. } \\
413 \\
292 \\
309 \\
1,450\end{array}$ & $\begin{array}{r}\text { June } 2 \\
\text { Aug. } \\
2 \\
2\end{array}$ & $\begin{array}{l}\text { J. L. Lamson } \\
\text { Johnson and Lamson. }\end{array}$ & $\begin{array}{l}\text { Feet } \\
4.89 \\
4.00 \\
4.00\end{array}$ & $\begin{array}{r}\text { Sec.-ft. } \\
371 \\
134 \\
138\end{array}$ \\
\hline
\end{tabular}

a Stage-discharge relation affected by ice.

Daily discharge, in second-feet, of Black River near Boonville, N. Y., for the year ending September 30, 1928

\begin{tabular}{|c|c|c|c|c|c|c|c|c|c|c|c|c|}
\hline $\mathrm{Da}$ & Oct. & Nov. & Dec. & Jan. & Feb. & Mar. & Apr. & May & June & July & Aug. & Sept. \\
\hline $\begin{array}{l}1 \\
2 \ldots \\
3 \\
4 \\
5\end{array}$ & $\begin{array}{l}\mathbf{1 4 5} \\
\mathbf{1 2 7} \\
119 \\
119 \\
\mathbf{1 2 7}\end{array}$ & $\begin{array}{l}127 \\
136 \\
145 \\
275 \\
227\end{array}$ & $\begin{array}{l}535 \\
535 \\
410 \\
390 \\
370\end{array}$ & $\begin{array}{r}950 \\
1,500 \\
1,400 \\
900 \\
800\end{array}$ & $\begin{array}{l}280 \\
280 \\
260 \\
260 \\
260\end{array}$ & $\begin{array}{l}180 \\
200 \\
200 \\
240 \\
260\end{array}$ & $\begin{array}{r}650 \\
600 \\
650 \\
1,200 \\
1,800\end{array}$ & $\begin{array}{l}2,860 \\
2,160 \\
1,640 \\
1,210 \\
1,060\end{array}$ & $\begin{array}{l}390 \\
370 \\
352 \\
352 \\
352\end{array}$ & $\begin{array}{l}275 \\
238 \\
290 \\
262 \\
238\end{array}$ & $\begin{array}{l}136 \\
136 \\
205 \\
127 \\
111\end{array}$ & $\begin{array}{l}60 \\
66 \\
51 \\
44 \\
44\end{array}$ \\
\hline $\begin{array}{r}6 \\
7-- \\
8- \\
9 \\
9 \\
10\end{array}$ & $\begin{array}{l}136 \\
145 \\
184 \\
205 \\
238\end{array}$ & $\begin{array}{l}184 \\
174 \\
164 \\
154 \\
145\end{array}$ & $\begin{array}{l}320 \\
335 \\
352 \\
410 \\
370\end{array}$ & $\begin{array}{l}600 \\
550 \\
460 \\
420 \\
400\end{array}$ & $\begin{array}{l}260 \\
260 \\
300 \\
300 \\
280\end{array}$ & $\begin{array}{l}280 \\
420 \\
380 \\
320 \\
280\end{array}$ & $\begin{array}{l}2,620 \\
3,240 \\
3,880 \\
3,880 \\
3,240\end{array}$ & $\begin{array}{r}990 \\
920 \\
\mathbf{8 5 5} \\
1,140 \\
1,370\end{array}$ & $\begin{array}{l}352 \\
320 \\
305 \\
630 \\
680\end{array}$ & $\begin{array}{l}216 \\
194 \\
184 \\
174 \\
164\end{array}$ & $\begin{array}{r}111 \\
104 \\
97 \\
84 \\
84\end{array}$ & $\begin{array}{r}53 \\
66 \\
145 \\
216 \\
164\end{array}$ \\
\hline (2) & $\begin{array}{r}227 \\
111 \\
78 \\
97 \\
111\end{array}$ & $\begin{array}{l}154 \\
164 \\
174 \\
184 \\
205\end{array}$ & $\begin{array}{l}335 \\
335 \\
320 \\
320 \\
300\end{array}$ & $\begin{array}{l}400 \\
380 \\
380 \\
380 \\
380\end{array}$ & $\begin{array}{l}280 \\
260 \\
260 \\
280 \\
280\end{array}$ & $\begin{array}{l}280 \\
280 \\
320 \\
360 \\
440\end{array}$ & $\begin{array}{l}3,360 \\
3,110 \\
2,980 \\
2,740 \\
2,270\end{array}$ & $\begin{array}{r}1,210 \\
1,140 \\
990 \\
1,060 \\
1,060\end{array}$ & $\begin{array}{l}370 \\
275 \\
238\end{array}$ & $\begin{array}{l}164 \\
154 \\
145 \\
136 \\
145\end{array}$ & $\begin{array}{l}97 \\
84 \\
78\end{array}$ & $\begin{array}{r}127 \\
90 \\
72 \\
56\end{array}$ \\
\hline a & $\begin{array}{r}136 \\
111 \\
90 \\
104 \\
104\end{array}$ & $\begin{array}{l}450 \\
370 \\
320 \\
305 \\
370\end{array}$ & $\begin{array}{l}300 \\
280 \\
280 \\
260 \\
240\end{array}$ & $\begin{array}{l}400 \\
360 \\
340 \\
360 \\
460\end{array}$ & $\begin{array}{l}260 \\
220 \\
220 \\
240 \\
240\end{array}$ & $\begin{array}{r}550 \\
1,800 \\
1,200 \\
1,200 \\
1,100\end{array}$ & $\begin{array}{l}1,740 \\
1,460 \\
1,210 \\
1,210 \\
1,290\end{array}$ & $\begin{array}{l}1,460 \\
3,490 \\
2,620 \\
1,840 \\
1,210\end{array}$ & $\begin{array}{l}216 \\
194 \\
174 \\
164 \\
164\end{array}$ & $\begin{array}{l}174 \\
184 \\
184 \\
127 \\
119\end{array}$ & $\begin{array}{l}70 \\
70 \\
63\end{array}$ & $\begin{array}{l}53 \\
51 \\
57 \\
60 \\
97\end{array}$ \\
\hline 21 & $\begin{array}{l}119 \\
111 \\
111 \\
305 \\
250\end{array}$ & $\begin{array}{l}335 \\
305 \\
335 \\
262 \\
227\end{array}$ & $\begin{array}{l}260 \\
260 \\
240 \\
240 \\
220\end{array}$ & $\begin{array}{l}600 \\
650 \\
750 \\
650 \\
600\end{array}$ & $\begin{array}{l}200 \\
200 \\
220 \\
200 \\
180\end{array}$ & $\begin{array}{r}950 \\
1,100 \\
1,300 \\
1,500 \\
1,700\end{array}$ & $\begin{array}{l}2,620 \\
3,880 \\
3,880 \\
3,750 \\
2,740\end{array}$ & $\begin{array}{l}990 \\
920 \\
795 \\
680 \\
605\end{array}$ & $\begin{array}{l}164 \\
184 \\
227 \\
216 \\
238\end{array}$ & $\begin{array}{r}111 \\
97 \\
90 \\
84 \\
111\end{array}$ & $\begin{array}{l}49 \\
42 \\
51 \\
63 \\
84\end{array}$ & $\begin{array}{l}184 \\
227 \\
174 \\
154 \\
127\end{array}$ \\
\hline $\begin{array}{l}26 \\
27 \\
28 \\
29 \\
30\end{array}$ & $\begin{array}{l}216 \\
194 \\
184 \\
174 \\
154 \\
145\end{array}$ & $\begin{array}{l}205 \\
227 \\
205 \\
227 \\
470\end{array}$ & $\begin{array}{l}260 \\
280 \\
300 \\
320 \\
300 \\
360\end{array}$ & $\begin{array}{l}550 \\
500 \\
460 \\
340 \\
300 \\
280\end{array}$ & $\begin{array}{l}180 \\
180 \\
190\end{array}$ & $\begin{array}{r}1,600 \\
1,300 \\
1,100 \\
1,000 \\
850 \\
700\end{array}$ & $\begin{array}{l}2,500 \\
2,380 \\
2,160 \\
3,490 \\
3,110\end{array}$ & $\begin{array}{l}558 \\
470 \\
430 \\
410 \\
352 \\
370\end{array}$ & $\begin{array}{l}410 \\
450 \\
450 \\
470 \\
390\end{array}$ & $\begin{array}{l}111 \\
119 \\
136 \\
127 \\
111 \\
119\end{array}$ & $\begin{array}{l}84 \\
84 \\
90 \\
97 \\
84 \\
65\end{array}$ & $\begin{array}{l}66 \\
44 \\
72 \\
65 \\
72\end{array}$ \\
\hline
\end{tabular}

Note-Discharge, Dec. 13 to Apr. 5, determined from gage heights corrected for ice effect from three discharge measurements, study of weather records and gage-height graph, observer's notes, and comparison with records of flow of near-by streams. 
Monthly discharge of Black River near Boonville, $N . Y$., for the year ending September 30,1923

[Drainage area, 303 square miles]

\begin{tabular}{|c|c|c|c|c|c|}
\hline \multirow{2}{*}{ Month } & \multicolumn{4}{|c|}{ Discharge in second-feet } & \multirow{2}{*}{$\underset{\substack{\text { Run-off in } \\
\text { inches }}}{ }$} \\
\hline & Maximum & Minimum & Mean & $\begin{array}{c}\text { Per square } \\
\text { milie }\end{array}$ & \\
\hline $\begin{array}{l}\text { October } \\
\text { November } \\
\text { December } \\
\text { January } \\
\text { February } \\
\text { March } \\
\text { April } \\
\text { May } \\
\text { June } \\
\text { July } \\
\text { August }\end{array}$ & $\begin{array}{r}305 \\
470 \\
535 \\
1,500 \\
300 \\
1,700 \\
3,880 \\
3,490 \\
680 \\
290 \\
205 \\
227\end{array}$ & $\begin{array}{r}78 \\
127 \\
220 \\
280 \\
180 \\
180 \\
600 \\
352 \\
164 \\
84 \\
42 \\
44\end{array}$ & $\begin{array}{c}151 \\
241 \\
324 \\
565 \\
244 \\
722 \\
2,450 \\
1,190 \\
344 \\
161 \\
88.2 \\
96.7\end{array}$ & $\begin{array}{l}0.498 \\
.795 \\
1.07 \\
1.86 \\
.805 \\
2.38 \\
8.09 \\
3.93 \\
1.14 \\
.531 \\
.291 \\
.319\end{array}$ & $\begin{array}{r}0.57 \\
.89 \\
1.23 \\
2.14 \\
.84 \\
2.74 \\
9.03 \\
4.53 \\
1.27 \\
.61 \\
.34 \\
.36\end{array}$ \\
\hline The year..... & 3,880 & 42 & 548 & 1.81 & 24.55 \\
\hline
\end{tabular}

Note.-Water diverted past this station by the Forestport feeder is not included in the above table.

BLACK RIVER AT WATERTOWN, N. Y.

Location.-At Vanduzee Street Bridge in Watertown, Jefferson County, 8 miles above mouth. No important tributary enters the river below this point.

Drainage area.-1,890 square miles (measured on topographic maps).

Records avarlable.-July 18, 1920, to September 30, 1923.

GAGE.-Gurley seven-day water-stage recorder on downstream side of right abutment; inspected by employees of Black River regulating district. Prior to September 3, 1921, a vertical staff at same location and an inclined staff on right bank 150 feet below, were used.

Discharge measurements.-Made from cable 150 feet below gage.

Channel and control.-Rocky and rough; practically permanent.

Extremes of Discharge.-Maximum stage recorded during year, 8.50 feet (staff gage reading) at 8.30 a. m. April 9 (discharge, 22,000 second-feet); minimum stage from water-stage recorder, 0.30 foot from 1 to $5 \mathrm{a} . \mathrm{m}$. August 6, (discharge, 155 second-feet).

1920-1923: Maximum stage recorded, 9.45 feet (staff gage reading) at 6 p. m. April 13, 1922 (discharge, 26,200 second-feet); minimum stage from water-stage recorder, that of August 6, 1923.

IcE.- Stage-discharge relation probably not affected by ice.

Diversions.- Water is diverted from Black River into the Forestport feeder at Forestport. A part of this water returns to the river through various spillways and through the Black River canal (flowing north); the rest passes out of the drainage basin through the Black River canal (flowing south), the record at the station on Black River canal (flowing south) near Boonville indicates the amount of this diversion. See also "Regulation" and "Diversions" in description of station on Black River near Boonville.

REGULATION.- Seasonal distribution of flow is regulated by Beaver River flow, Fulton Chain Lakes, Forestport reservoir, and other storage reservoirs in the upper part of the drainage basin. During medium and low water periods there is considerable diurnal fiuctuation in flow, caused by mills and power plants at Watertown and above. 
ACCURACY.-Stage-discharge relation practically permanent; probably not affected by ice. Rating curve well defined between 200 and 25,000 second-feet. Operation of water-stage recorder satisfactory except as indicated in footnote to daily-discharge table. Daily discharge ascertained by applying to rating table mean daily gage height determined by inspection of gage-height graph, or for days of considerable fluctuation, by averaging discharge for intervals of day. Records excellent, except for periods of estimate, for which they are fair.

Discharge measurements of Black River at Watertown, $N_{\text {, }} Y$. , during the year ending September 30, 1923

\begin{tabular}{|c|c|c|c|c|c|c|c|}
\hline Date & Made by- & $\begin{array}{c}\text { Gage } \\
\text { height }\end{array}$ & $\begin{array}{c}\text { Dis- } \\
\text { charge }\end{array}$ & Date & Made by- & $\begin{array}{c}\text { Gage } \\
\text { height }\end{array}$ & $\begin{array}{c}\text { Dis- } \\
\text { charge }\end{array}$ \\
\hline $\begin{array}{l}\text { Dec. } \quad 4 \\
\text { Jan. } 8 \\
\text { Feb. } 10 \\
\text { Apr. } 20\end{array}$ & $\begin{array}{l}\text { A. W. Harrington } \\
\text { J. L. Lamson } \\
\text { A. W. Harrington } \\
\end{array}$ & $\begin{array}{l}\text { Feet } \\
1.92 \\
2.58 \\
1.91 \\
4.50\end{array}$ & $\begin{array}{r}\text { Sec.-ft. } \\
1,590 \\
2,850 \\
1,730 \\
7,840\end{array}$ & $\begin{array}{lr}\text { July } & 29 \\
\text { Aug. } & 6 \\
& 6\end{array}$ & $\begin{array}{l}\text { Lamson and Johnson. } \\
\text { A. W. Harrington... }\end{array}$ & \begin{tabular}{c|} 
Feet \\
0.83 \\
1.19 \\
.47
\end{tabular} & $\begin{array}{r}\text { Sec.-ft. } \\
554 \\
822 \\
243\end{array}$ \\
\hline
\end{tabular}

Daily discharge, in second-feet, of Black River at Watertown, N. Y., for the year ending September 30,1923

\begin{tabular}{r|r|r|r|r|r|r|r|r|r|r|r|r}
\hline Day & Oct. & Nov. & Dec & Jan. & Feb. & Mar. & Apr. & May & June & July & A ug. & Sept. \\
\hline & & & & & & & & \\
\hline & & & & & & & & \\
\hline
\end{tabular}

Nota.-Discharge for the fcllowing periots when water-stage recorder was not operating satisfactorily estimated by comparison with records of flow of several tributary streams: Oct. 1, 7-13, 29, Nov. 12, Feb. 6-10, Mar. 18-21, Aug. 26 and 27. 
Monthly discharge of Black River at Watertown, N.Y., for the year ending September 30,1923

[Drainage area, 1,890 sq nare miles]

\begin{tabular}{|c|c|c|c|c|c|}
\hline \multirow{2}{*}{ Month } & \multicolumn{4}{|c|}{ Discharge in second-feet } & \multirow{2}{*}{$\begin{array}{c}\text { Run-off in } \\
\text { inches }\end{array}$} \\
\hline & Maximum & Minimum & Mean & $\underset{\text { mile }}{\text { Per square }}$ & \\
\hline $\begin{array}{l}\text { October } \\
\text { November } \\
\text { December } \\
\text { January } \\
\text { February } \\
\text { March } \\
\text { April } \\
\text { May } \\
\text { June } \\
\text { July } \\
\text { August } \\
\text { September }\end{array}$ & $\begin{array}{r}1,970 \\
2,220 \\
2,050 \\
4,660 \\
2,050 \\
9,250 \\
21,600 \\
11,200 \\
4,430 \\
1,750 \\
992 \\
1,450\end{array}$ & $\begin{array}{r}920 \\
1,170 \\
948 \\
1,540 \\
1,050 \\
1,210 \\
3,250 \\
2,390 \\
1,220 \\
606 \\
410 \\
484\end{array}$ & $\begin{array}{r}1,280 \\
1,560 \\
1,400 \\
2,540 \\
1,470 \\
4,080 \\
11,900 \\
6,440 \\
2,080 \\
1,090 \\
730 \\
919\end{array}$ & $\begin{array}{l}0.677 \\
.825 \\
.741 \\
1.34 \\
.778 \\
2.16 \\
6.30 \\
3.41 \\
1.10 \\
.577 \\
.386 \\
.486\end{array}$ & $\begin{array}{r}0.78 \\
.92 \\
.85 \\
1.54 \\
.81 \\
2.49 \\
7.03 \\
3.93 \\
1.23 \\
.67 \\
.44 \\
.54\end{array}$ \\
\hline The year & 21,600 & 410 & 2,950 & 1.56 & 21.23 \\
\hline
\end{tabular}

NoTE.-See "Regulation" and "Diversions" in station description.

FORESTPORT FEEDER NEAR BOONVILLE, N. Y.

Location.-Slope station at lower end of feeder, above point where it enters basin at Boonville, Oneida County.

Records avallable.-Occasional discharge measurements 1900 and 1905-1915 continuous record, October 30, 1915, to September 30, 1923.

GAGES.-Two Gurley seven-day graph water-stage recorders, with natural scale for gage height. Gage No. 1 is at downstream end of left abutment of steel highway bridge in village of Hawkinsville; gage No. 2 is on left bank; just below a farm bridge 1 mile above the basin at Boonville; they are 2.53 miles apart. These gages and the two in Black River canal (flowing south) near Boonville are set to the same datum. Recorders inspected by Fred Kesauer.

Discharge measurements.-Made from the steel highway bridge at gage No. 1 in Hawkinsville.

Determination of discharge.-Daily discharge determined by use of Chezy formula. The coefficient "C," computed from each current-meter measurement, is plotted on a curve showing the variation of " $\mathrm{C}$ " through the season. A smooth curve drawn through the plotted points shows the coefficients for intervening days. The other factors in the Chezy formula are obtained from gage-height records and cross section of the canal.

Diversions.--One spillway takes water from the Forestport feeder just below gage No. 2 and a second spillway takes water from the basin in Boonville. Both discharge into Mill Creek, which enters Black River below the Boonville gaging station. No spillway between gage No. 1 and gage No. 2. Other spillways in the feeder above gage No. 1 discharge into Black River above the gaging station. The sum of this record and the record for Black River near Boonville indicates the total run-off of the Black River basin above tnese gaging stations.

Regulation.-Flow in the feeder is regulated at the outlet of Forestport reservoir.

ICE.-There is usually some flow in the canal during the winter, and occasionaI current-meter measurements of the discharge have been made. 
ACCURACY.-Operation of water-stage recorders satisfactory except as indicated in footnote to daily-discharge table. Records good except when either recorder was not operating, when estimates of missing gage heights were made from comparison with other recorder or from a study of the slope relation. Records for such periods, fair.

Discharge measurements of Forestport feeder near Boonville, N. Y., during the year ending September 30, 1928

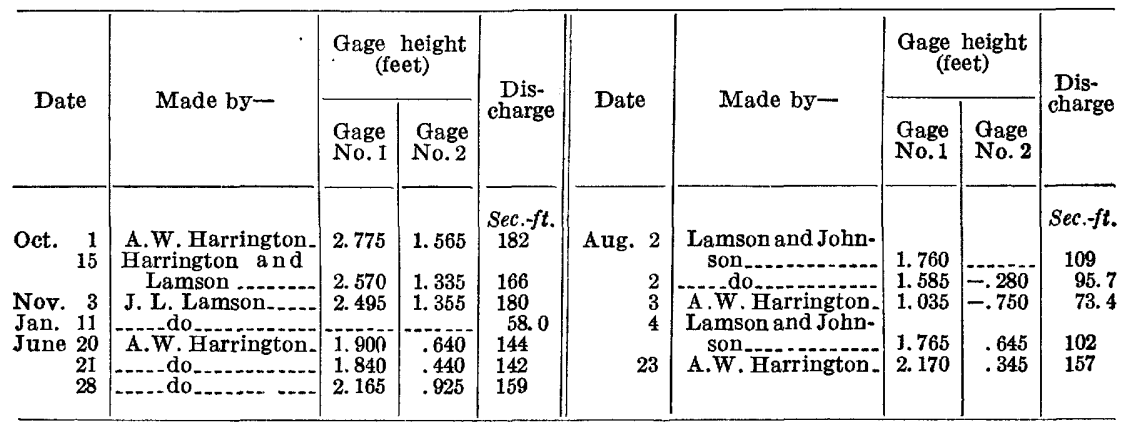

Daily discharge, in second-feet, of Forestport feeder near Boonville, N. Y., for the year ending September 30, 1923

\begin{tabular}{|c|c|c|c|c|c|c|c|c|c|c|c|c|c|}
\hline Dey & Oct. & Nor. & June & July & Aug. & Sept. & Day & Oet. & Nov. & June & July & Aug. & Sept. \\
\hline $\begin{array}{l}1 \\
2 \\
2 \\
3 \\
4 \\
4 \\
5 \\
-\ldots\end{array}$ & $\begin{array}{l}177 \\
176 \\
177 \\
176 \\
178\end{array}$ & $\begin{array}{l}\mathbf{1 6 9} \\
171 \\
170 \\
142 \\
155\end{array}$ & - & $\begin{array}{r}150 \\
130 \\
76 \\
130 \\
130\end{array}$ & $\begin{array}{r}111 \\
105 \\
78 \\
108 \\
124\end{array}$ & $\begin{array}{l}153 \\
156 \\
154 \\
152 \\
153\end{array}$ & $\begin{array}{l}16 \\
17= \\
18= \\
19= \\
20=\end{array}$ & $\begin{array}{l}173 \\
176 \\
180 \\
175 \\
178\end{array}$ & מ- & $\begin{array}{l}113 \\
131 \\
166 \\
165 \\
148\end{array}$ & $\begin{array}{l}131 \\
135 \\
125 \\
129 \\
133\end{array}$ & $\begin{array}{l}157 \\
157 \\
155 \\
158 \\
157\end{array}$ & $\begin{array}{l}152 \\
152 \\
164 \\
171 \\
170\end{array}$ \\
\hline $\begin{aligned} 6 & \ldots \\
7 & \cdots \\
8 & -\cdots \\
9 & \cdots \\
10 & \cdots\end{aligned}$ & $\begin{array}{l}177 \\
181 \\
186 \\
189 \\
190\end{array}$ & $\begin{array}{l}188 \\
165 \\
170 \\
196 \\
194\end{array}$ & $\begin{array}{l}138 \\
127\end{array}$ & $\begin{array}{l}130 \\
128 \\
122 \\
120 \\
118\end{array}$ & $\begin{array}{l}123 \\
122 \\
122 \\
131 \\
154\end{array}$ & $\begin{array}{l}156 \\
167 \\
169 \\
158 \\
161\end{array}$ & $\begin{array}{l}21 \ldots \\
22 \\
23 \\
24 \\
25\end{array} \ldots$ & $\begin{array}{l}179 \\
178 \\
184 \\
191 \\
188\end{array}$ & 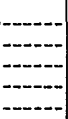 & $\begin{array}{l}144 \\
146 \\
149 \\
155 \\
162\end{array}$ & $\begin{array}{l}132 \\
132 \\
133 \\
134 \\
120\end{array}$ & $\begin{array}{l}156 \\
156 \\
157 \\
152 \\
150\end{array}$ & $\begin{array}{l}175 \\
173 \\
170 \\
167 \\
168\end{array}$ \\
\hline $\begin{array}{l}\text { II } \\
12 \\
13 \\
14 \\
15\end{array}$ & $\begin{array}{l}184 \\
166 \\
175 \\
175 \\
174\end{array}$ & $\begin{array}{l}198 \\
194 \\
192 \\
170 \\
\ldots . . .\end{array}$ & $\begin{array}{l}120 \\
114 \\
112 \\
112 \\
112\end{array}$ & $\begin{array}{l}124 \\
129 \\
134 \\
131 \\
132\end{array}$ & $\begin{array}{l}143 \\
147 \\
147 \\
156 \\
157\end{array}$ & $\begin{array}{l}152 \\
151 \\
153 \\
153 \\
152\end{array}$ & $\begin{array}{l}26 \\
27 \\
28 \\
29 \\
30 \\
31\end{array}$ & $\begin{array}{l}188 \\
184 \\
174 \\
166 \\
169 \\
169\end{array}$ & 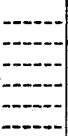 & $\begin{array}{l}160 \\
153 \\
154 \\
165 \\
147\end{array}$ & $\begin{array}{l}125 \\
121 \\
128 \\
125 \\
124 \\
124\end{array}$ & $\begin{array}{l}150 \\
142 \\
154 \\
151 \\
151 \\
152\end{array}$ & $\begin{array}{l}168 \\
169 \\
168 \\
166 \\
165\end{array}$ \\
\hline
\end{tabular}

NotE.-Discharge for the following periods when only one water-stage recorder was operating satisfact o rily determined from gage heights estimated by comparison with other recorder graph or from a study of the slope relation: June 12-19 and June 21 to Aug. 2.

Monthly discharge, in second-feet, of Forestport feeder near Boonville, N. Y., for the year ending September 30, 1923

\begin{tabular}{|c|c|c|c|c|c|c|c|}
\hline Month & Maximum & Minimum & Mean & Month & Maximum & Minimum & Mean \\
\hline $\begin{array}{l}\text { October } \\
\text { November 1-14 } \\
\text { June } 9-30\end{array}$ & $\begin{array}{l}191 \\
198 \\
166\end{array}$ & $\begin{array}{l}166 \\
142 \\
112\end{array}$ & $\begin{array}{l}178 \\
177 \\
141\end{array}$ & $\begin{array}{l}\text { July } \\
\text { August } \\
\text { September.......... }\end{array}$ & $\begin{array}{l}150 \\
158 \\
175\end{array}$ & $\begin{array}{r}76 \\
78 \\
151\end{array}$ & $\begin{array}{l}127 \\
141 \\
161\end{array}$ \\
\hline
\end{tabular}


BLACK RIVER CANAL (FLOWING SOUTH) NEAR BOONVILLE, N. Y.

Location.-Slope station in summit level of Black River canal, near Boonville, Oneida County.

RECoRdS AVATLABLE.-Occasional discharge measurements 1900 and 1905-1915; continuous record, September 16, 1915, to September 30, 1923.

GAGES.-Two Gurley seven-day graph water-stage recorders, 1.81 miles apart, with natural scale for gage heights. Gage No. 1 is on right bank (opposite towpath) about 50 feet downstream from collector's office in Boonville; gage No. 2 is on right bank (opposite towpath) about 300 yards above Lock 70 and 50 yards above spillway from the canal into Lansing Kill. These gages and the two gages in the Forestport feeder near Boonville are set to the same datum. Recorders inspected by Fred Kesauer.

Discharge measurements.-Made from the steel and concrete highway bridge in the village of Boonville, a short distance below gage No. 1.

Determination of Discharge.-Daily discharge determined by use of Chezy formula. The coefficient "C," computed from each current meter measurement, is plotted on a curve showing the variation of " $\mathrm{C}$ " through the season. A smooth curve drawn through the plotted points shows the coefficients for intervening days. The other factors in the Chezy formula are obtained from gage-height records and cross section of canal.

Drversions. - No diversion between gage No. 1 and gage No. 2. Records obtained at this station indicate the quantity of water diverted from the Black River basin into the Mohawk River basin.

REgUlation.-Flow in canal is regulated by operation of spillway and sluice gates at Lock 70 and also by discharge of Forestport feeder into the basin at Boonville.

ICE.-No flow in canal during winter.

ACCURACY.-Operation of water-stage recorders satisfactory except as indicated in footnote to daily-discharge table. Records good, except when either recorder is not operating, when estimates for missing gage heights are made from comparison with other recorder or from a study of the slope relation. Records for such periods, fair.

Discharge measurements of Black River canal (flowing south) near Boonville, N.Y., during the year ending September 30, 1923

\begin{tabular}{|c|c|c|c|c|c|c|c|c|c|}
\hline \multirow{2}{*}{ Date } & \multirow{2}{*}{ Made by- } & \multicolumn{2}{|c|}{$\underset{\text { (feet) }}{\text { Gage height }}$} & \multirow{2}{*}{$\begin{array}{l}\text { Dis- } \\
\text { charge }\end{array}$} & \multirow{2}{*}{ Date } & \multirow{2}{*}{ Made by- } & \multicolumn{2}{|c|}{$\begin{array}{l}\text { Gage height } \\
\text { (feet) }\end{array}$} & \multirow{2}{*}{$\begin{array}{c}\text { Dis- } \\
\text { charge }\end{array}$} \\
\hline & & $\begin{array}{l}\text { Gage } \\
\text { No. } 1\end{array}$ & $\begin{array}{l}\text { Gage } \\
\text { No.2 }\end{array}$ & & & & $\begin{array}{l}\text { Gage } \\
\text { No. } 1\end{array}$ & $\begin{array}{l}\text { Gage } \\
\text { No.2 }\end{array}$ & \\
\hline $\begin{array}{l}\text { Oct. } \begin{array}{r}1 \\
15 \\
\text { Nov. } 3 \\
\text { June } 20 \\
21 \\
28\end{array}\end{array}$ & $\begin{array}{l}\text { A.W.Harrington. } \\
\text { Lamson and Har- } \\
\text { rington. } \\
\text { J. L. Lamson } \\
\text { A. W. Harrington. } \\
\text { do } \\
\text { do }\end{array}$ & $\begin{array}{r}1.230 \\
1.035 \\
1.160 \\
.410 \\
.180 \\
.790\end{array}$ & $\begin{array}{r}0.770 \\
.825 \\
.930 \\
.220 \\
-.020 \\
.580\end{array}$ & $\begin{array}{r}\text { Sec.fft. } \\
145 \\
\\
145 \\
129 \\
117 \\
100 \\
112\end{array}$ & $\begin{array}{rr}\text { Aug. } & 3 \\
4 \\
4 \\
4 \\
23 \\
\text { Sept. } 27\end{array}$ & $\begin{array}{l}\text { Lamson and } \\
\text { Johnson. } \\
\text { Harrington and } \\
\text { Johnson } \\
\text { Joh ns on and } \\
\text { Lamson } \\
\text { A. W.Harrington. } \\
\end{array}$ & $\begin{array}{r}0.735 \\
.295 \\
-.420 \\
.030\end{array}$ & $\begin{array}{r}0.555 \\
.060 \\
-1.810 \\
-1.420\end{array}$ & \begin{tabular}{|c} 
Sec. $-f t$. \\
42.0 \\
97.2 \\
80.7 \\
100 \\
146
\end{tabular} \\
\hline
\end{tabular}


Daily discharge, in second-feet, of Black River canal (flowing south) near Boonville N. Y., for the year ending September 30, 1928

\begin{tabular}{|c|c|c|c|c|c|c|c|c|c|c|c|c|c|}
\hline Day & Oct. & Nov. & June & July & Aug. & Sept. & Day & Oet. & Nov. & June & July & Aug. & Sept. \\
\hline $\begin{array}{l}1 \\
2 \\
2 \\
3 \\
4 \\
5 \\
5\end{array}$ & $\begin{array}{l}146 \\
148 \\
149 \\
150 \\
150\end{array}$ & $\begin{array}{l}128 \\
130 \\
126 \\
123 \\
125\end{array}$ & 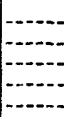 & $\begin{array}{r}122 \\
98 \\
40 \\
80\end{array}$ & $\begin{array}{l}66 \\
60 \\
40 \\
62 \\
83\end{array}$ & $\begin{array}{l}120 \\
129 \\
126 \\
127 \\
125\end{array}$ & $\begin{array}{l}16 \ldots \\
17 \ldots \\
18 \ldots \\
19 \\
20\end{array}$ & $\begin{array}{l}147 \\
145 \\
144 \\
147 \\
151\end{array}$ & & 117 & $\begin{array}{l}80 \\
90 \\
85 \\
81 \\
92\end{array}$ & $\begin{array}{l}113 \\
113 \\
113 \\
110 \\
112\end{array}$ & $\begin{array}{l}116 \\
114 \\
121 \\
131 \\
128\end{array}$ \\
\hline $\begin{array}{r}6 \\
7 \\
7 \\
8 \\
9 \\
9 \\
10\end{array}$ & $\begin{array}{l}150 \\
155 \\
146 \\
150 \\
147\end{array}$ & $\begin{array}{r}130 \\
126 \\
127 \\
126 \\
125\end{array}$ & $\mid-\ldots$ & 85 & $\begin{array}{r}84 \\
82 \\
81 \\
104 \\
108\end{array}$ & $\begin{array}{l}130 \\
114 \\
114\end{array}$ & $\begin{array}{l}21 \\
22 \\
23 \\
24 \\
25\end{array}$ & $\begin{array}{l}152 \\
153 \\
148 \\
142 \\
144\end{array}$ & 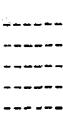 & $\begin{array}{l}114 \\
119 \\
117 \\
115 \\
109\end{array}$ & $\begin{array}{l}96 \\
93 \\
96 \\
81 \\
78\end{array}$ & $\begin{array}{l}115 \\
112 \\
109 \\
106 \\
104\end{array}$ & 135 \\
\hline $\begin{array}{l}11 \\
12 \\
13 \\
15\end{array}$ & $\begin{array}{l}149 \\
135 \\
139 \\
148 \\
145\end{array}$ & $\begin{array}{l}122 \\
125 \\
124 \\
110 \\
\ldots . .\end{array}$ & 95 & $\begin{array}{l}79 \\
80\end{array}$ & $\begin{array}{r}96 \\
99 \\
97 \\
107 \\
112\end{array}$ & $\begin{array}{l}110 \\
121 \\
129 \\
124 \\
120\end{array}$ & $\begin{array}{l}26 \\
27 \\
28 \\
29 \\
30 \\
31\end{array}$ & $\begin{array}{l}150 \\
147 \\
136 \\
127 \\
127 \\
128\end{array}$ & \begin{tabular}{ll}
$-\cdots-1$ \\
\hdashline$-\cdots$ \\
\hdashline$-\cdots$ \\
\hdashline$-\cdots$
\end{tabular} & $\begin{array}{r}122 \\
125 \\
103 \\
104 \\
106 \\
-\end{array}$ & $\begin{array}{l}84 \\
82 \\
80 \\
79 \\
83 \\
79\end{array}$ & $\begin{array}{l}103 \\
104 \\
107 \\
108 \\
109 \\
111\end{array}$ & $\begin{array}{l}140 \\
130 \\
118 \\
114\end{array}$ \\
\hline
\end{tabular}

Note-Discharge for the following periods when only one water-stage recorder was operating satisfactorily determined from gage heights estimated by comparison with other recorder graph or from a study of the slope relation: Oct. 11-14, 19-21, 24-27, Nov. 10, 11, 13, 14, June 9-19, July 2-13, 15, Aug. 2-4, 10-13, $15,16,23$, and Sept. $€-8,14$, and $19-26$.

Monthly discharge of Black River canal (flowing south) near Boonville, N. Y., for the year ending September 30, 1923

\begin{tabular}{|c|c|c|c|c|c|c|c|}
\hline \multirow{2}{*}{ Month } & \multicolumn{3}{|c|}{ Discharge in second-feet } & \multirow{2}{*}{ Month } & \multicolumn{3}{|c|}{ Discharge in second-feet } \\
\hline & Maximum & Minimum & Mean & & Maximum & Minimum & Mean \\
\hline $\begin{array}{l}\text { October } \\
\text { November } 1-14 \\
\text { Jufle } 9-30\end{array}$ & $\begin{array}{l}155 \\
130 \\
125\end{array}$ & $\begin{array}{l}127 \\
110\end{array}$ & $\begin{array}{l}145 \\
125 \\
104\end{array}$ & $\begin{array}{l}\text { July } \\
\text { August } \\
\text { September }\end{array}$ & $\begin{array}{l}122 \\
115 \\
140\end{array}$ & $\begin{array}{r}40 \\
40 \\
110\end{array}$ & $\begin{array}{r}84.6 \\
97.7 \\
129\end{array}$ \\
\hline
\end{tabular}

MOOSE RIVER AT MCKEEVER, N. Y.

Locatron.-Half a mile west of village of McKeever, Herkimer County, 2 miles below mouth of South Branch of Moose River, and 16 miles above junction of Black and Moose rivers at Lyons Falls.

Drainage area.-366 square miles (measured on topographic maps).

Records available.-May 28, 1922, to September 30, 1923. Comparable record from station at Moose River, 3 miles below, from June 5, 1900, to December 31, 1922.

GAGE.-Gurley seven-day graph water-stage recorder on left bank, just above Moose Head Inn and half a mile below dam of Iroquois Pulp \& Paper Co.; installed November 2, 1922. Prior to this date gage was a vertical staff at same location. Gage read and recorder inspected by R. D. Nash.

Discharge measurements.-Made from cable 250 feet above gage or by wading.

Channel and control.-Low-water control consists principally of coarse gravel and boulders; probably permanent.

Extremes of discharge.-1922-1923: Maximum stage recorded, 12.9 feet about 10 p. m. June 22, 1922 (discharge, about 10,000 second-feet); minimum stage from water-stage recorder, 1.49 feet from $9 \mathrm{p} . \mathrm{m}$. to midnight July 27, 1923 (discharge, 83 second-feet).

IcE.-Stage-discharge relation considerably affected by ice.

Regulation.-Flow regulated to a considerable extent for short periods at dam of Iroquois Pulp \& Paper Co., half a mile above. Seasonal distribution of flow affected by operation of State dam at Old Forge. This regulation indicated by record at station on Middle Branch of Moose River at Old Forge. 
Accuracr.- Stage-discharge relation permanent except as affected by ice from December to April. Rating curve well defined between 100 and 5,500 second-feet. Operation of recorder satisfactory except as indicated in footnote to daily-discharge table. Daily discharge ascertained by applying to rating table mean of twice daily staff gage readings or mean daily gage height determined by inspection of recorder graph. For days of considerable fluctuation, mean discharge is ascertained from recorder graph by averaging discharge for intervals of day. Records prior to November 2, good; open-water records after that date excellent except those for estimated periods which are fair; records for period of ice effect, fair.

Discharge measurements of Monse River at McKeever, N. Y., during the year end ing September 30, 1923

\begin{tabular}{|c|c|c|c|c|c|c|c|}
\hline Date & Made by- & $\begin{array}{c}\text { Gage } \\
\text { height }\end{array}$ & $\begin{array}{l}\text { Dis- } \\
\text { charge }\end{array}$ & Date & Made by- & $\begin{array}{c}\text { Gage } \\
\text { height }\end{array}$ & $\begin{array}{c}\text { Dis- } \\
\text { charge }\end{array}$ \\
\hline $\begin{array}{lr}\text { Oct. } & 2 \\
17 \\
17 \\
19 \\
22 \\
29 \\
29 \\
\text { Dec. } 5 \\
\text { Jan. } 12 \\
\text { Feb. } 13 \\
\text { Mar. } 23\end{array}$ & $\begin{array}{l}\text { A. W. Harrington } \\
\text { J. Lamson } \\
\text { A. do W. Harrington. } \\
\text { J. L. Lamson } \\
\text { A. W. Harrington. } \\
\text { J. L. Lamson }\end{array}$ & $\begin{array}{r}\text { Feet } \\
1.62 \\
1.95 \\
1.77 \\
1.97 \\
2.34 \\
1.91 \\
1.98 \\
2.60 \\
\text { a } 2.00 \\
\text { a } 2.97 \\
\text { a } 4.26\end{array}$ & $\begin{array}{r}\text { Sec.-ft. } \\
111 \\
177 \\
158 \\
195 \\
322 \\
215 \\
220 \\
462 \\
154 \\
319 \\
823\end{array}$ & $\begin{array}{lr}\text { Apr. } & 18 \\
19 \\
23 \\
24 \\
26 \\
27 \\
27 \\
\text { May } 29 \\
& 30 \\
\text { June } & 21 \\
\text { Aug. } & 3\end{array}$ & $\begin{array}{l}\text { Lamson and Harrington. } \\
\text { A. W. Harrington } \\
\text { Lamson and Harrington. } \\
\text { A. W. Harrington } \\
\text { J. L. Lamson } \\
\text { J. W. Harrington } \\
\text { Johnson and Lamson. }\end{array}$ & $\begin{array}{l}\text { Feet } \\
4.52 \\
4.39 \\
8.55 \\
7.27 \\
6.34 \\
6.48 \\
3.10 \\
2.73 \\
1.73 \\
1.77\end{array}$ & $\begin{array}{r}\text { Sec. }-f t . \\
1,360 \\
1,340 \\
5,060 \\
3,630 \\
2,870 \\
3,010 \\
656 \\
507 \\
143 \\
134\end{array}$ \\
\hline
\end{tabular}

a Stage-discharge relation affected by ice.

Daily discharge, in second-feet, of Moose River at McKeever, N. Y., for the year ending September 30, 1923

\begin{tabular}{r|r|r|r|r|r|r|r|r|r|r|r|r}
\hline Day & Oct & Nov. & Dec. & Jan. & Feb. & Mar. & Apr. & May & June & July & Aug. & Sept. \\
\hline & & & & & & & & & \\
\hline & & & & & & & \\
\hline & & & & & & & \\
\hline
\end{tabular}

Note.-Discharge estimated for following periods when water-stage recorder was not operating satisfactorily: Jan. 7, 13, Feb. 17, June 9-15, 17-19, and June 23 to July 7. Discharge, Dec. 17 to April 7, determined from gage heights corrected for ice effect from three discharge measurements and study of weather records. $72038-26 \dagger-w s P \quad 564-8$ 


\section{Monthly discharge of Moose River at McKeever, N. Y., for the year ending September 30, 1929}

[Drainage area, 366 square miles]

\begin{tabular}{|c|c|c|c|c|c|}
\hline \multirow{2}{*}{ Month } & \multicolumn{4}{|c|}{ Discharge in second-feet } & \multirow{2}{*}{$\begin{array}{l}\text { Run-off in } \\
\text { inches }\end{array}$} \\
\hline & Maximum & Minimum & Mean & $\underset{\text { mile }}{\text { Per square }}$ & \\
\hline $\begin{array}{l}\text { October } \\
\text { November } \\
\text { December } \\
\text { January } \\
\text { February } \\
\text { March } \\
\text { April } \\
\text { May } \\
\text { June } \\
\text { July } \\
\text { August } \\
\text { September }\end{array}$ & $\begin{array}{r}755 \\
584 \\
528 \\
1,500 \\
550 \\
1,300 \\
5,000 \\
3,960 \\
550 \\
171 \\
574\end{array}$ & $\begin{array}{l}124 \\
139 \\
144 \\
140 \\
180 \\
160 \\
460 \\
580 \\
226 \\
119 \\
100 \\
111\end{array}$ & $\begin{array}{r}353 \\
369 \\
319 \\
582 \\
312 \\
539 \\
2,770 \\
1,470 \\
429 \\
208 \\
126 \\
240 \\
\end{array}$ & $\begin{array}{l}0.964 \\
1.01 \\
.872 \\
1.59 \\
.852 \\
1.47 \\
7.57 \\
4.02 \\
1.17 \\
.568 \\
.344 \\
.656 \\
\end{array}$ & $\begin{array}{r}1.11 \\
1.13 \\
1.01 \\
1.83 \\
.89 \\
1.70 \\
8.45 \\
4.64 \\
1.30 \\
.65 \\
.40 \\
.73 \\
\end{array}$ \\
\hline The year.......... & 5,000 & 100 & 642 & 1.75 & 23.84 \\
\hline
\end{tabular}

Note.-See "Regulation" in station description.

MOOSE RIVER AT MOOSE RIVER, N. Y.

Location.-In Moose River, Lewis County, 3 miles downstream from McKeever, 5 miles below mouth of South Branch of Moose River and 13 miles above junction of Black and Moose rivers at Lyons Falls.

Drainage area. - 370 square miles (measured on topographic maps).

Records available.-June 5, 1900, to December 31, 1922, when station was discontinued. Comparable record from station at $\mathrm{McKeever,} 3$ miles above, beginning May 28, 1922.

GAGE.-Staff in two sections on left bank; read by W. D. Rinkle. Gage datum was lowered 0.17 foot February 28, 1903, and again 5.00 feet on January 1, 1913.

Discharge measurements.-Made from cable a short distance below gage or by wading.

Channel and control.-Cobblestones and boulders; fairly permanent. Current smooth; depth comparatively uniform. Ice and logs occasionally jam above the station on a small island.

Extremes of discharge.-Maximum stage recorded during period, October 1 to December 31, 1922, 7.3 feet at 8.30 a. $\mathrm{m}$. December 11 (discharge, 810 second-feet); minimum stage recorded, 5.39 feet at 9 a. m. October 4 (discharge, 124 second-feet).

1900-1922: Maximum stage recorded, 16.3 feet during the afternoon of March 27, 1913, determined by leveling from floodmarks (discharge, about 16,500 second-feet); minimum stage, 4.94 feet July 21, 23, and 25-27, 1913 (discharge, about 42 second-feet).

IcE.-Stage-discharge relation affected by ice.

Regulation.-A dam at McKeever, 3 miles upstream, is used for power and for the regulation of flow during log driving. Seasonal distribution of flow affected by operation of the State dam at Old Forge. This regulation is indicated by the record at station on Middle Branch of Moose River at Old Forge. 
Accuracy. - Stage-discharge relation permanent except as affected by.ice December 16-31. Rating curve well defined. Gage read to half-tenths once daily. Daily discharge ascertained by applying daily gage height to rating table. Records fair except during period of ice effect and for low stages when one daily reading of gage may not indicate the correct mean daily gage height, owing to fluctuations in stage. During such periods records are poor.

The following discharge measurement was made by $\mathrm{A}$. W. Harrington:

October 1, 1922: Gage-height, 5.88 feet; discharge, 263 second-feet.

Daily discharge, in second-feet, of Moose River at Moose River, N.Y., for the period October 1 to December 31, 1922

\begin{tabular}{|c|c|c|c|c|c|c|c|c|c|c|c|}
\hline Day & Oct. & Nov. & Dec. & Day & Oct. & Nov. & Dec. & Day & Oct. & Nov. & Dec. \\
\hline 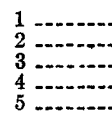 & $\begin{array}{l}216 \\
540 \\
259 \\
124 \\
202\end{array}$ & $\begin{array}{l}422 \\
189 \\
320 \\
422 \\
369\end{array}$ & $\begin{array}{l}320 \\
625\end{array}$ & $\begin{array}{l}11 \\
12 \\
13 \\
14 \\
15\end{array}$ & $\begin{array}{l}386 \\
441 \\
422 \\
404 \\
176\end{array}$ & $\begin{array}{l}352 \\
216 \\
400 \\
304 \\
164\end{array}$ & $\begin{array}{l}810 \\
369 \\
202 \\
304 \\
386\end{array}$ & $\begin{array}{l}21 \\
22 \\
23\end{array} 2$ & $\begin{array}{l}336 \\
352 \\
460 \\
441 \\
670\end{array}$ & $\begin{array}{l}500 \\
500 \\
500 \\
369 \\
369\end{array}$ & $\begin{array}{l}260 \\
240 \\
240 \\
220 \\
220\end{array}$ \\
\hline $\begin{array}{r}6 \\
7 \\
8 \\
8\end{array} \ldots$ & $\begin{array}{l}259 \\
259 \\
259 \\
336 \\
670\end{array}$ & $\begin{array}{l}216 \\
422 \\
520 \\
520 \\
440\end{array}$ & 380 & $\begin{array}{l}16 \\
17 \\
18 \\
19 \\
20\end{array}$ & $\begin{array}{l}422 \\
148 \\
164 \\
189 \\
289\end{array}$ & $\begin{array}{l}422 \\
625 \\
500 \\
176 \\
540\end{array}$ & $\begin{array}{l}360 \\
340 \\
320 \\
320 \\
300\end{array}$ & $\begin{array}{l}26 \\
27 \\
28 \\
29 \\
29 \\
30\end{array} \ldots$ & $\begin{array}{l}540 \\
500 \\
460 \\
230 \\
422 \\
280\end{array}$ & $\begin{array}{l}136 \\
500 \\
386 \\
369 \\
136\end{array}$ & $\begin{array}{l}220 \\
220 \\
220 \\
220 \\
260 \\
240\end{array}$ \\
\hline
\end{tabular}

Nore-Discharge Oct. 31, Nov. 10 and 13, and Dec. 3-9, estimated by comparison with record at McKeever; no gage-height record. Discharge, Dec. 16-31, determined from gage heights corrected for ice effect from study of weather records, observer's notes, and comparison with record at McKeever.

Monthly discharge of Moose River at Moose River, $N . Y$, for the period October 1 to December 31, 1922

[Drainage area, 370 square miles]

\begin{tabular}{|c|c|c|c|c|c|}
\hline \multirow{2}{*}{ Month } & \multicolumn{4}{|c|}{ Discharge in second-feet } & \multirow{2}{*}{$\underset{\text { Run-off in }}{\text { inches }}$} \\
\hline & Maximum & Minimum & Mean & $\begin{array}{c}\text { Per square } \\
\text { mile }\end{array}$ & \\
\hline $\begin{array}{l}\text { October } \\
\text { November } \\
\text { December }\end{array}$ & $\begin{array}{l}670 \\
625 \\
810\end{array}$ & $\begin{array}{l}124 \\
136 \\
164\end{array}$ & $\begin{array}{l}350 \\
377 \\
324\end{array}$ & $\begin{array}{l}0.946 \\
1.02 \\
.876\end{array}$ & $\begin{array}{l}1.09 \\
1.14 \\
1.01\end{array}$ \\
\hline
\end{tabular}

Note.-See "Regulation" in station description.

MIDDLE BRANCH OF MOOSE RIVER AT OLD FORGE, N. Y.

Location.-300 feet below highway bridge and 400 feet below State dam at Old Forge, Herkimer County.

Drainage AREA.-51.5 square miles (measured on topographic maps).

Records available.-November 9, 1911, to September 30, 1923.

GaGE.-Vertical staff on left bank 300 feet below highway bridge; read by Joseph Otis.

Discharge measurements.-Made from highway bridge or by wading near gage.

Channel and control.-Bed near gage composed of stone and gravel. Control is rock ledge about 200 feet below gage; practically permanent.

EXTREMes of DISCHARge.-Maximum stage recorded during year, 2.6 feet April 25 and 26 and May 1 (discharge, 225 second-feet); minimum stage, 0.82 foot from $5 \mathrm{p}$. $\mathrm{m}$. October 14 to $8 \mathrm{a}$. m. October 18 (discharge, 22 second-feet).

1911-1923: Maximum discharge recorded, 862 second-feet morning and afternoon March 23, 1921; minimum discharge, 16 second-feet several times in October and November, 1919. 
ICE.-Stage-discharge relation not affected by ice.

Regulation. - Flow controlled by gates at dam.

Accuracy. $\rightarrow$ Stage-discharge relation practically permanent except as affected by backwater from landslide near control from June to September. Rating curve well defined between 20 and 300 second-feet. Gage read to hun. dredths twice daily. Daily discharge ascertained by applying to .rating table mean daily gage height corrected for backwater effect when necessary. Records good.

Discharge measurements of Middle Branch of Moose River at Old Forge, N. Y., during the year ending September 30, 1923

\begin{tabular}{|c|c|c|c|c|c|c|c|}
\hline Date' & Made by- & $\begin{array}{c}\text { Gage } \\
\text { height }\end{array}$ & $\begin{array}{l}\text { Dis- } \\
\text { charge }\end{array}$ & Date & Made by- & $\begin{array}{c}\text { Gage } \\
\text { height }\end{array}$ & $\begin{array}{l}\text { Dis- } \\
\text { charge }\end{array}$ \\
\hline 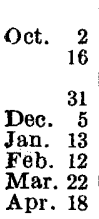 & $\begin{array}{l}\text { A. W. Harrington } \\
\text { Lamson and Harring- } \\
\text { ton } \\
\text { J. L. Lamson } \\
\text { A. W Harrington } \\
\text { J. L. Lamson } \\
\text { A. W. Harrington } \\
\text { J. L. Lamson } \\
\text { A. W. Harrington } \\
\text {.... }\end{array}$ & $\begin{array}{l}\text { Feet } \\
2.11 \\
.82 \\
1.71 \\
1.26 \\
1.08 \\
1.85 \\
1.35 \\
2.18\end{array}$ & $\begin{array}{l}\text { Sec. } f t . \\
139 \\
\\
22.4 \\
92.0 \\
48.3 \\
34.3 \\
105 \\
55.8 \\
149\end{array}$ & $\begin{array}{lr}\text { Apr. } & 23 \\
& 24 \\
& 26 \\
\text { May } & 29 \\
\text { June } & 2 \\
& 20 \\
\text { Aug. } & 3\end{array}$ & $\begin{array}{l}\text { Lamson and Harring- } \\
\text { ton } \\
\text { A. Wo Harrington } \\
\text { J. L. Lamson ... } \\
\text { A. Wo Harrington. } \\
\text { Lamson and Johnson. }\end{array}$ & $\begin{array}{l}\text { Feet } \\
2.43 \\
2.52 \\
2.50 \\
2.24 \\
1.50 \\
1.48 \\
\text { a } 1.39\end{array}$ & $\begin{array}{c}\text { Sec.ft. } \\
208 \\
199 \\
200 \\
168 \\
71.1 \\
69.9 \\
53.2\end{array}$ \\
\hline
\end{tabular}

- Backwater from landslide near control.

Daily discharge, in second-feet, of Middle Branch of Moose River at Old Forge, $N . Y$., for the year ending September 30, 1923

\begin{tabular}{|c|c|c|c|c|c|c|c|c|c|c|c|c|}
\hline Day & Oct. & Nov. & Dec. & Jan. & Feb. & Mar. & Apr. & May & June & July & Aug. & Sept. \\
\hline $\begin{array}{l}1- \\
2- \\
3- \\
4- \\
5\end{array}$ & $\begin{array}{l}150 \\
143 \\
143 \\
143 \\
143\end{array}$ & $\begin{array}{l}129 \\
116 \\
122 \\
116 \\
116\end{array}$ & $\begin{array}{l}50 \\
50 \\
50 \\
50 \\
50\end{array}$ & $\begin{array}{l}50 \\
50 \\
52 \\
43 \\
36\end{array}$ & $\begin{array}{r}64 \\
116 \\
116 \\
116 \\
116\end{array}$ & $\begin{array}{l}28 \\
28 \\
65 \\
65 \\
47\end{array}$ & $\begin{array}{l}57 \\
57 \\
57 \\
57 \\
58\end{array}$ & $\begin{array}{l}225 \\
207 \\
207 \\
198 \\
190\end{array}$ & $\begin{array}{l}71 \\
71 \\
71 \\
71 \\
71\end{array}$ & $\begin{array}{l}65 \\
65 \\
65 \\
60 \\
60\end{array}$ & $\begin{array}{l}55 \\
55 \\
55 \\
55 \\
55\end{array}$ & $\begin{array}{l}33 \\
33 \\
35 \\
35 \\
55\end{array}$ \\
\hline $\begin{array}{r}6 \\
7 \\
8 \\
9\end{array}$ & $\begin{array}{l}143 \\
143 \\
143 \\
143 \\
143\end{array}$ & $\begin{array}{r}116 \\
116 \\
81 \\
67 \\
67\end{array}$ & $\begin{array}{l}50 \\
50 \\
50 \\
50 \\
50\end{array}$ & $\begin{array}{l}36 \\
36 \\
36 \\
86 \\
36\end{array}$ & $\begin{array}{l}116 \\
116 \\
116 \\
110 \\
110\end{array}$ & $\begin{array}{l}33 \\
33 \\
33 \\
33 \\
33\end{array}$ & $\begin{array}{l}59 \\
67 \\
76 \\
76 \\
81\end{array}$ & $\begin{array}{r}190 \\
143 \\
92 \\
71 \\
71\end{array}$ & $\begin{array}{l}71 \\
71 \\
71 \\
71 \\
71\end{array}$ & $\begin{array}{l}60 \\
60 \\
60 \\
60 \\
60\end{array}$ & $\begin{array}{l}55 \\
50 \\
50 \\
50 \\
50\end{array}$ & $\begin{array}{l}55 \\
55 \\
55 \\
55 \\
55\end{array}$ \\
\hline $5^{-2}-2$ & $\begin{array}{r}143 \\
143 \\
68 \\
22 \\
22\end{array}$ & $\begin{array}{l}60 \\
48 \\
50 \\
47 \\
49\end{array}$ & $\begin{array}{l}50 \\
50 \\
50 \\
50 \\
50\end{array}$ & $\begin{array}{l}36 \\
36 \\
36 \\
36 \\
36\end{array}$ & $\begin{array}{l}110 \\
110 \\
110 \\
110 \\
110\end{array}$ & $\begin{array}{l}33 \\
33 \\
33 \\
33 \\
33\end{array}$ & $\begin{array}{r}81 \\
92 \\
98 \\
104 \\
104\end{array}$ & $\begin{array}{l}71 \\
71 \\
71 \\
71 \\
98\end{array}$ & $\begin{array}{l}71 \\
71 \\
71 \\
71 \\
71\end{array}$ & $\begin{array}{l}60 \\
55 \\
55 \\
55 \\
55\end{array}$ & $\begin{array}{l}50 \\
50 \\
50 \\
46 \\
42\end{array}$ & $\begin{array}{l}55 \\
75 \\
75 \\
75 \\
75\end{array}$ \\
\hline $\begin{array}{l}18 \\
19 \\
20\end{array}$ & $\begin{array}{r}22 \\
22 \\
76 \\
166 \\
166\end{array}$ & $\begin{array}{l}47 \\
48 \\
49 \\
49 \\
49\end{array}$ & $\begin{array}{l}50 \\
50 \\
50 \\
50 \\
49\end{array}$ & $\begin{array}{r}36 \\
36 \\
76 \\
129 \\
129\end{array}$ & $\begin{array}{r}110 \\
110 \\
110 \\
104 \\
60\end{array}$ & $\begin{array}{l}47 \\
57 \\
56 \\
57 \\
59\end{array}$ & $\begin{array}{l}136 \\
158 \\
168 \\
150 \\
150\end{array}$ & $\begin{array}{l}150 \\
190 \\
190 \\
190 \\
190\end{array}$ & $\begin{array}{l}71 \\
71 \\
71 \\
71 \\
70\end{array}$ & $\begin{array}{l}55 \\
55 \\
55 \\
55 \\
55\end{array}$ & $\begin{array}{l}42 \\
42 \\
38 \\
34 \\
32\end{array}$ & $\begin{array}{l}75 \\
75 \\
75 \\
75 \\
75\end{array}$ \\
\hline 22 & $\begin{array}{l}166 \\
166 \\
158 \\
150 \\
143\end{array}$ & $\begin{array}{l}49 \\
49 \\
49 \\
49 \\
49\end{array}$ & $\begin{array}{l}50 \\
50 \\
50 \\
50 \\
50\end{array}$ & $\begin{array}{r}129 \\
69 \\
29 \\
29 \\
29\end{array}$ & $\begin{array}{l}29 \\
28 \\
28 \\
28 \\
28\end{array}$ & $\begin{array}{l}59 \\
57 \\
57 \\
57 \\
57\end{array}$ & $\begin{array}{l}150 \\
166 \\
190 \\
207 \\
225\end{array}$ & $\begin{array}{l}190 \\
190 \\
190 \\
190 \\
182\end{array}$ & $\begin{array}{l}69 \\
69 \\
70 \\
65 \\
65\end{array}$ & $\begin{array}{l}55 \\
55 \\
55\end{array}$ & $\begin{array}{l}31 \\
31 \\
31 \\
31 \\
31\end{array}$ & $\begin{array}{l}80 \\
90 \\
90 \\
90 \\
80\end{array}$ \\
\hline $\begin{array}{l}26 \\
27 \\
28 \\
29 \\
30 \\
31\end{array}$ & $\begin{array}{r}136 \\
136 \\
129 \\
98 \\
92 \\
92\end{array}$ & $\begin{array}{r}49 \\
49 \\
+\quad 49 \\
49 \\
49\end{array}$ & $\begin{array}{l}50 \\
50 \\
50 \\
50 \\
50 \\
50\end{array}$ & $\begin{array}{l}29 \\
29 \\
29 \\
29 \\
29 \\
29\end{array}$ & \begin{tabular}{r}
28 \\
28 \\
28 \\
\hdashline \\
\hdashline \\
\end{tabular} & $\begin{array}{l}58 \\
57 \\
59 \\
59 \\
59 \\
57\end{array}$ & $\begin{array}{l}225 \\
207 \\
190 \\
207 \\
207\end{array}$ & $\begin{array}{l}182 \\
174 \\
174 \\
166 \\
166 \\
110\end{array}$ & $\begin{array}{l}65 \\
65 \\
65 \\
65 \\
65\end{array}$ & $\begin{array}{l}55 \\
55 \\
55 \\
55\end{array}$ & $\begin{array}{l}31 \\
31 \\
31 \\
31 \\
31 \\
33\end{array}$ & $\begin{array}{l}75 \\
80 \\
80 \\
80 \\
80\end{array}$ \\
\hline
\end{tabular}

Note.-Discharge June 23 to Aug. 19, and Sept. 5 to 30 determined from gage heights corrected tor backwater effect from landslide near control, by means of two discharge measurements and stuciy o: water surface alevations above old Forge dam. 
Monthly discharge of Middle Branch of Moose River at Old Forge, N. Y., for the year ending September 30,1923

[Drainage area 51.5 square miles]

\begin{tabular}{|c|c|c|c|c|c|}
\hline \multirow{2}{*}{ Month } & \multicolumn{4}{|c|}{ Discharge in second-feet } & \multirow{2}{*}{$\begin{array}{l}\text { Run-off in } \\
\text { inches }\end{array}$} \\
\hline & Maximum & Minimum & Mean & $\begin{array}{c}\text { Per square } \\
\text { mile }\end{array}$ & \\
\hline $\begin{array}{l}\text { October } \\
\text { November } \\
\text { December } \\
\text { January } \\
\text { February } \\
\text { March } \\
\text { April } \\
\text { May } \\
\text { July } \\
\text { August } \\
\text { Septem }\end{array}$ & $\begin{array}{r}166 \\
129 \\
50 \\
129 \\
116 \\
65 \\
225 \\
225 \\
71 \\
65 \\
55 \\
90\end{array}$ & $\begin{array}{l}22 \\
47 \\
49 \\
29 \\
28 \\
28 \\
57 \\
71 \\
65 \\
55 \\
31 \\
33\end{array}$ & $\begin{array}{l}121 \\
67.7 \\
50.0 \\
47.0 \\
84.5 \\
47.6 \\
128 \\
155 \\
69.4 \\
57.3 \\
41.9 \\
67.4\end{array}$ & $\begin{array}{l}2.35 \\
1.31 \\
.971 \\
.913 \\
1.64 \\
.924 \\
2.49 \\
3.01 \\
1.35 \\
1.11 \\
.814 \\
1.31\end{array}$ & $\begin{array}{r}2.71 \\
1.46 \\
1.12 \\
1.05 \\
1.71 \\
1.07 \\
2.78 \\
3.47 \\
1.51 \\
1.28 \\
.94 \\
1.46\end{array}$ \\
\hline The year.... & 225 & 22 & 78.0 & 1.51 & 20.56 \\
\hline
\end{tabular}

Note.-The above figures do not necessarily represent the natural flow from the basin, because of artificial storage in Fulton Chain of Lakes.

BEAVER RIVER AT STATE DAM, NEAR BEAVER RIVER, N. Y.

Location.-At concrete storage dam at outlet of Beaver River Flow, $71 / 2$ miles west of Beaver River post office, Herkimer County, and 7 miles above Beaver Lake at Number Four.

DRAINAGE AREA.-176 square miles (measured on topographic maps).

Records available.-May 11, 1908, to September 30, 1923.

$\mathrm{G}_{\mathrm{AGE}}$.- -Elevation of water surface in the reservoir is determined by a staff gage in two sections on the west corner of the gate house; read by James C. Dunbar, gate tender. The mean elevation of the crest of the spillway is at gage height 16.96 feet. Width of sluice gate openings determined by measuring on the gate stems the distance they have been raised.

Discharge measurements.-Made from a temporary footbridge at the moutfir of the outlet tunnel, below the gates. Discharge over the spillway has not been measured.

Determination of Discharge.-Records include the discharge through one or more of four 4-foot circular sluice gates, when opened, the discharge over the spillway, and the discharge through the logway at the west end of the spillway. The sluice gates have been rated by current-meter measurements made at different lake elevations, but no measurements have been made of the discharge over the spillway or through the logway. Theoretic coefficients based on the Cornell experiments ${ }^{3}$ have been used to compute ratings for the spillway and logway.

Extremes of STAGE.- Maximum elevation of water surface in reservoir recorded during year, 19.0 feet May 18 and 19; minimum elevation recorded, 1.1 feet, several times October 13 to 22 .

1908-1923: Maximum elevation of water surface in reservoir, 19.85 feet at 3.15 p. m. June 23,1922 ; minimum elevation, that of October, 1922.

EXtremes of Discharge.--Maximum daily discharge during year, 2,400 secondfeet April 23 and 24; minimum daily discharge, practically zero April $\mathbf{9}$ and 10 . 
1908-1923: Maximum discharge, 3,380 second-feet April 12, 1922; mini. mum discharge, practically zero during periods when gates were closed and there was no discharge over spillway.

REgULATION.-At ordinary stages the discharge of Beaver River is completely regulated by the operation of the sluice gates.

ACCURACY.- Stage-discharge relation permanent; probably not affected by ice. Rating curves for sluice gates fairly well defined. Lake gage read to halftenths once daily. The accuracy of computations depends to a large extent on the care with which the gates were set to the recorded openings. Records fair.

Discharge measurements of Beaver River at State dam, near Beaver River, N. Y., during the year ending September 30, 1928

[Made by Johnson and Lamson]

\begin{tabular}{|c|c|c|c|c|c|c|c|c|c|}
\hline \multirow{2}{*}{ Date } & \multicolumn{2}{|c|}{ Gate } & \multirow{2}{*}{$\begin{array}{c}\text { Lake } \\
\text { gage } \\
\text { height }\end{array}$} & \multirow{2}{*}{ Discharge } & \multirow{2}{*}{ Date } & \multicolumn{2}{|r|}{ Gate } & \multirow{2}{*}{$\begin{array}{c}\text { Lake } \\
\text { gage } \\
\text { height }\end{array}$} & \multirow{2}{*}{ Discharge } \\
\hline & No. & Opening & & & & No. & Opening & & \\
\hline $\begin{array}{ll}\text { July } & 31 \\
31 & \\
& 31 \\
& 31\end{array}$ & $\begin{array}{l}1 \\
1 \\
1 \\
1\end{array}$ & $\begin{array}{r}\text { Inches } \\
12 \\
24 \\
36 \\
48\end{array}$ & $\begin{array}{l}\text { Fect } \\
13.80 \\
13.80 \\
13.80 \\
13.75\end{array}$ & \begin{tabular}{c|} 
Sec. $f t$. \\
79.2 \\
147 \\
217 \\
264
\end{tabular} & $\begin{array}{l}\text { July } 31 \\
\text { Aug. } 1 \\
\\
1 \\
1\end{array}$ & $\begin{array}{l}2 \\
2 \\
3 \\
4\end{array}$ & $\begin{array}{r}\text { Inehes } \\
12 \\
24 \\
24 \\
24\end{array}$ & $\begin{array}{l}\text { Feet } \\
13.75 \\
13.70 \\
13.70 \\
13.70\end{array}$ & $\begin{array}{c}\text { Sec. } f t . \\
77.8 \\
145 \\
146 \\
149\end{array}$ \\
\hline
\end{tabular}

Monthly discharge of Beaver River at State dam, near Beaver River, N. Y., for the year ending September 30, 1923

[Drainage area, 176 square miles]

\begin{tabular}{|c|c|c|c|c|c|}
\hline \multirow{2}{*}{ Month } & \multicolumn{4}{|c|}{ Discharge in second-feet } & \multirow{2}{*}{$\begin{array}{l}\text { Run-off } \\
\text { in inches }\end{array}$} \\
\hline & Maximum & Minimum & Mean & $\begin{array}{c}\text { Per square } \\
\text { mile }\end{array}$ & \\
\hline $\begin{array}{l}\text { October } \\
\text { November } \\
\text { December } \\
\text { January } \\
\text { February } \\
\text { March } \\
\text { April } \\
\text { May } \\
\text { June } \\
\text { July } \\
\text { August } \\
\text { September }\end{array}$ & $\begin{array}{r}130 \\
180 \\
140 \\
200 \\
140 \\
240 \\
2,400 \\
1,600 \\
460 \\
260 \\
200 \\
240 \\
\end{array}$ & $\begin{array}{r}80 \\
95 \\
100 \\
100 \\
90 \\
90 \\
100 \\
160 \\
180 \\
170 \\
100 \\
\end{array}$ & $\begin{array}{r}94.5 \\
128 \\
125 \\
168 \\
106 \\
156 \\
1,100 \\
625 \\
267 \\
205 \\
175 \\
179 \\
\end{array}$ & $\begin{array}{l}0.537 \\
.727 \\
.710 \\
.955 \\
.602 \\
.886 \\
6.25 \\
3.55 \\
1.52 \\
1.16 \\
.994 \\
1.02 \\
\end{array}$ & $\begin{array}{r}0.62 \\
.81 \\
.82 \\
1.10 \\
.63 \\
1.02 \\
6.97 \\
4.09 \\
1.70 \\
1.34 \\
1.15 \\
1.14\end{array}$ \\
\hline The year.- & 2,400 & $-\ldots$ & 278 & 1.58 & 21.39 \\
\hline
\end{tabular}

NorE -The above figures do not represent the natural flow of the river on account of regulation at he dam. 
BEAVER RIVER AT EAGLE FALLS, NEAR NUMBER FOUR, N. Y.

Locatron.-500 feet below Eagle Falls power plant of Northern New York Utilities (Inc.) 21/2 miles below Beaver Lake, 4 miles north of Number Four, Lewis County, and 9 miles below State dam at outlet of Beaver River Flow.

Drainage AREA.-230 square miles (measured on topographic maps).

Records available.-August 21, 1921, to September 30, 1923.

GAGE.-Gurley seven-day water-stage recorder on left bank; inspected by employees of Northern New York Utilities (Inc.).

Discharge MEASUREMENTs.-Made from a cable over tailrace and river channel, 300 feet above gage or by wading.

Channel and control.-Boulders and large broken rock; shifts occasionally.

EXTREMES of DISCHARge.-Maximum stage during year from water-stage recorder, 5.70 feet from 6 to 9 p.m. April 23 (discharge, 3,090 second-feet); minimum stage from water-stage recorder, 0.22 foot at noon December 17 (discharge, 5.8 second-feet).

1921-1923: Maximum stage from water-stage recorder, 7.30 feet at 3.30 p.m. April 13, 1922 (discharge, 4,980 second-feet); minimum stage from water-stage recorder, that of December 17, 1922.

ICE.-Stage-discharge relation not affected by ice.

Regulation.-Seasonal flow is regulated by storage in Beaver River Flow 9 miles above. Diurnal flow regulated at dam at foot of Beaver Lake according to needs of power plant. Some regulation in other ponds and lakes in drainage area.

ACCURACY.-Stage-discharge relation changed presumably at time of high water April 23. Rating curve used October 1 to April 23 fairly well defined between 10 and 3,500 second-feet; curve used subsequent to April 23 well defined between 100 and 1,000 second-feet. Operation of water-stage recorder satisfactory except as indicated in footnote to daily-discharge table. Daily discharge ascertained by applying to rating table mean daily gage height determined by inspection of gage-height graph, or for days of considerable fluctuation, by averaging discharge for intervals of day. Records good. 
Discharge measurements of Beaver River at Eagle Falls, near Number Four, N. Y., during the year ending September 30, 1923

\begin{tabular}{|c|c|c|c|c|c|c|c|}
\hline Date & Mada by- & $\begin{array}{c}\text { Gage } \\
\text { height }\end{array}$ & $\begin{array}{c}\text { Dis- } \\
\text { charge }\end{array}$ & Date & Made by- & $\begin{array}{c}\text { Gage } \\
\text { height }\end{array}$ & $\begin{array}{l}\text { Dis- } \\
\text { charge }\end{array}$ \\
\hline $\begin{array}{l}\text { Oct. } 17 \\
\text { Jan. } 9 \\
\text { Apr. } 22\end{array}$ & $\begin{array}{l}\text { A. W. Harrington..... } \\
\text { J. L. Lamson }\end{array}$ & $\begin{array}{l}\text { Feet } \\
1.40 \\
2.08 \\
4.89\end{array}$ & $\begin{array}{r}\text { Sec-ft. } \\
128 \\
291 \\
2,150\end{array}$ & $\begin{array}{l}\text { July } 30 \\
\text { Aug. } 1\end{array}$ & Lamson and Johnson. & $\begin{array}{l}\text { Feet } \\
1.96 \\
1.69\end{array}$ & $\begin{array}{r}\text { Sec-ft. } \\
276 \\
194\end{array}$ \\
\hline
\end{tabular}

Daily discharge, in second-feet, of Beaver River at Eagle Falls, near Number Four, N. Y., for the year ending September 30,1923

\begin{tabular}{r|r|r|r|r|r|r|r|r|r|r|r|r}
\hline Date & Oct. & Nov. & Dec. & Jan. & Feb. & Mar. & Apr. & May & June & July & Aug. & Sept. \\
\hline & & & & & & & & & & & & \\
\hline
\end{tabular}

Note.-Discharge estimated for the following days when water-stage recorder was not operating satis factorily: Oct. $22,23,29,30$, Nov. 18, 24, 25, Dec. 20, 22-24, Jan. 4-6, 19, Mar. 7, 8, May 31, June 1, and Aug. 25 and 26 . 
Monthly discharge of Beaver River at Eagle Falls, near Number Four, N. Y., for the year ending September $30,19 \% 3$

[Drainage area, 230 square miles]

\begin{tabular}{|c|c|c|c|c|c|}
\hline \multirow{2}{*}{ Month } & \multicolumn{4}{|c|}{ Discharge in second-feet } & \multirow{2}{*}{$\begin{array}{l}\text { Run-off } \\
\text { in inches }\end{array}$} \\
\hline & Maximum & Minimum & Mean & $\begin{array}{c}\text { Per square } \\
\text { mile }\end{array}$ & \\
\hline $\begin{array}{l}\text { October } \\
\text { November } \\
\text { December } \\
\text { January } \\
\text { February } \\
\text { March } \\
\text { April } \\
\text { May } \\
\text { June } \\
\text { July } \\
\text { August } \\
\text { September }\end{array}$ & $\begin{array}{r}176 \\
269 \\
226 \\
326 \\
223 \\
437 \\
2,980 \\
1,920 \\
798 \\
381 \\
286 \\
323\end{array}$ & $\begin{array}{r}11 \\
16 \\
14 \\
23 \\
20 \\
20 \\
185 \\
270 \\
118 \\
37 \\
37 \\
29\end{array}$ & $\begin{array}{r}104 \\
169 \\
149 \\
233 \\
137 \\
228 \\
1,270 \\
1,010 \\
377 \\
238 \\
194 \\
186\end{array}$ & $\begin{array}{c}0.452 \\
.735 \\
.648 \\
1.01 \\
.596 \\
.991 \\
5.52 \\
4.39 \\
1.64 \\
1.03 \\
.843 \\
.809\end{array}$ & $\begin{array}{r}0.52 \\
.82 \\
.75 \\
1.16 \\
.62 \\
1.14 \\
6.16 \\
5.06 \\
1.83 \\
1.19 \\
.97 \\
.90\end{array}$ \\
\hline The year..... & 2,980 & 11 & 358 & 1.56 & 21. 12 \\
\hline
\end{tabular}

NoTe. - The above figures do not necessarily represent the natural flow from the basin because of artificial storage, mainly in Stillwater reservoir and Beaver Lake.

\section{STREAMS TRIBUTARY TO ST. LAWRENCE RIVER}

\section{EAST BRANCH OF OSWEGATCHIE RIVER AT CRANBERRY LAKE, N. Y.}

Location. - In village of Cranberry Lake, St. Lawrence County, 500 feet below concrete dam at outlet of Cranberry Lake, and 101/2 miles above Newton Falls.

Drainage area.-144 square miles (measured on topographic maps).

Records available.-May 5 to September 30, 1923. Comparable records at station at Newton Falls, 101/2 miles below, October 6, 1912, to May 4, 1923.

GaGE.-Slope gage on left bank; read by Herbert Dean.

Discharge measurements.-Made from cable about 200 feet below gage or by wading.

Channel and Control.-Large boulders and gravel; probably permanent.

Extremes of DISCHARGE.--Maximum stage recorded during the period May 5 to September $30,1923,6.63$ feet at 8 a. $\mathrm{m}$. June 11 (discharge, 1,030 second-feet). The minimum stage is reached when gates in dam are closed and when there is no discharge over the spillway (discharge, practically zero).

ICE.-Stage-discharge relation probably not affected by ice.

REgulation.-Discharge is regulated by operation of sluice gates at Cranberry Lake dam.

ACCURACY.- Stage-discharge relation practically permanent. Rating curve well defined between 40 and 350 second-feet. Gage read to half-tenths once daily. Daily discharge ascertained by applying daily gage height to rating table. Records good except on days when sluice gates in dam above are operated, when one gage reading a day may not give the true mean daily gage height. 
Discharge measurements of East Branch of Oswegatchie River at Cranberry Lake, $N$. Y., during the year ending September 30, 1923

\begin{tabular}{|c|c|c|c|c|c|c|c|}
\hline Date & Made by- & $\begin{array}{c}\text { Gage } \\
\text { height }\end{array}$ & $\begin{array}{c}\text { Dis- } \\
\text { charge }\end{array}$ & Date & Made by- & $\begin{array}{c}\text { Gage } \\
\text { height }\end{array}$ & $\begin{array}{c}\text { Dis- } \\
\text { charge }\end{array}$ \\
\hline $\begin{array}{r}\text { Mar. } 15 \\
15 \\
15 \\
16 \\
16 \\
16 \\
16\end{array}$ & $\begin{array}{l}\text { Lamson and Johnson } a \\
- \text { do } \\
- \text { do }\end{array}$ & $\begin{array}{c}\text { Feet } \\
3.58 \\
4.00 \\
4.20 \\
3.65 \\
4.04 \\
4.22 \\
4.28\end{array}$ & $\begin{array}{r}\text { Sec.-ft. } \\
51.2 \\
92.8 \\
119 \\
57.8 \\
93.1 \\
123 \\
131\end{array}$ & $\begin{array}{r}\text { Mar. } 16 \\
\text { June } 26 \\
26 \\
26 \\
27 \\
27 \\
\text { July } 27\end{array}$ & $\begin{array}{l}\text { Lamson and Johnsona. } \\
\text { A. W. Harrington } \\
\text { J. L. Lamson. do } \\
\text { do }\end{array}$ & $\begin{array}{c}\text { Feet } \\
4.24 \\
4.18 \\
4.74 \\
4.75 \\
5.15 \\
4.23 \\
4.96\end{array}$ & $\begin{array}{r}\text { Sec.-ft. } \\
126 \\
124 \\
217 \\
225 \\
328 \\
124 \\
284\end{array}$ \\
\hline
\end{tabular}

afEngineer, Board of Black River Regulating District.

Daily discharge, in second-feet, of East Branch of Oswegatchie River at Cranberry Lake, N. Y., for the period May 5 to September 30, 1923

\begin{tabular}{|c|c|c|c|c|c|c|c|c|c|c|c|}
\hline Day & May & June & July & Aug. & Sept. & Day & May & Jnue & July & Aug. & Sept. \\
\hline $\begin{array}{l}1 \\
2 \\
2 \\
3 \\
4 \\
5\end{array}-\ldots$ & 119 & $\begin{array}{l}201 \\
180 \\
180 \\
161 \\
161\end{array}$ & $\begin{array}{l}190 \\
190 \\
190 \\
190 \\
200\end{array}$ & $\begin{array}{l}287 \\
274 \\
274 \\
274 \\
315\end{array}$ & $\begin{array}{l}301 \\
301 \\
301 \\
301 \\
301\end{array}$ & $\begin{array}{l}16 \\
17 \\
19 \\
20\end{array}$ & $\begin{array}{l}119 \\
127 \\
127 \\
127 \\
127\end{array}$ & $\begin{array}{l}224 \\
119 \\
119 \\
119 \\
119\end{array}$ & $\begin{array}{l}301 \\
301 \\
301 \\
287 \\
287\end{array}$ & $\begin{array}{l}301 \\
301 \\
301 \\
301 \\
\mathbf{3 0 1}\end{array}$ & $\begin{array}{l}301 \\
301 \\
301 \\
301 \\
287\end{array}$ \\
\hline 10 & $\begin{array}{l}119 \\
119 \\
119 \\
119 \\
119\end{array}$ & $\begin{array}{l}161 \\
180 \\
201 \\
511 \\
700\end{array}$ & $\begin{array}{l}212 \\
212 \\
212 \\
212 \\
212\end{array}$ & $\begin{array}{l}315 \\
315 \\
315 \\
315 \\
301\end{array}$ & $\begin{array}{l}301 \\
301 \\
301 \\
301 \\
301\end{array}$ & $\begin{array}{l}21 \ldots \\
22- \\
23- \\
24- \\
25 .\end{array}$ & $\begin{array}{l}390 \\
390 \\
301 \\
390 \\
301\end{array}$ & $\begin{array}{l}119 \\
119 \\
119 \\
144 \\
144\end{array}$ & $\begin{array}{l}287 \\
287 \\
287 \\
287 \\
287\end{array}$ & $\begin{array}{l}301 \\
301 \\
301 \\
301 \\
301\end{array}$ & $\begin{array}{l}287 \\
287 \\
287 \\
287 \\
287\end{array}$ \\
\hline $\begin{array}{l}11 \\
12 \\
14 \\
15\end{array}$ & $\begin{array}{l}119 \\
119 \\
119 \\
119 \\
119\end{array}$ & $\begin{array}{r}\mathbf{1}, \mathbf{0 1 0} \\
\mathbf{3 3 0} \\
330 \\
224 \\
\mathbf{2 2 4}\end{array}$ & $\begin{array}{l}301 \\
301 \\
301 \\
301 \\
301\end{array}$ & $\begin{array}{l}301 \\
301 \\
301 \\
301 \\
301\end{array}$ & $\begin{array}{l}301 \\
301 \\
301 \\
301 \\
301\end{array}$ & $\begin{array}{l}26 \\
27 \\
28 \\
30 \\
31\end{array}$ & $\begin{array}{l}301 \\
301 \\
105 \\
201 \\
201 \\
201\end{array}$ & $\begin{array}{l}180 \\
224 \\
201 \\
190 \\
190\end{array}$ & $\begin{array}{l}287 \\
274 \\
287 \\
287 \\
287 \\
287\end{array}$ & $\begin{array}{l}287 \\
301 \\
301 \\
301 \\
301 \\
301\end{array}$ & $\begin{array}{l}287 \\
287 \\
287 \\
287 \\
287\end{array}$ \\
\hline
\end{tabular}

Nore.-Discharge, June 10 and July 5, estimated because of doubtful gage-height record.

Monthly discharge of East Branch of Oswegatchie River at Cranberry Lake, N. Y., for the period May 5 to September 30, 1923

[Drainage area, 144 square miles]

\begin{tabular}{|c|c|c|c|c|c|}
\hline \multirow{2}{*}{ Month } & \multicolumn{4}{|c|}{ Discharge in second-feet } & \multirow{2}{*}{$\begin{array}{c}\text { Run-off in } \\
\text { inches }\end{array}$} \\
\hline & Maximum & Minimum & Mean & $\begin{array}{c}\text { Per square } \\
\text { mile }\end{array}$ & \\
\hline $\begin{array}{l}\text { May } 5-31 \\
\text { June } \\
\text { July } \\
\text { August } \\
\text { September }\end{array}$ & $\begin{array}{r}390 \\
1,010 \\
301 \\
315 \\
301\end{array}$ & $\begin{array}{l}105 \\
119 \\
190 \\
274 \\
287\end{array}$ & $\begin{array}{l}186 \\
236 \\
263 \\
300 \\
296\end{array}$ & $\begin{array}{l}1.29 \\
1.64 \\
1.83 \\
2.08 \\
2.06\end{array}$ & $\begin{array}{l}1.30 \\
1.83 \\
2.11 \\
2.40 \\
2.30\end{array}$ \\
\hline
\end{tabular}

Nore. - The monthly discharge does not necessarily represent the natural flow from the basin because of artifical storage in Cranberry Lake. 
EAST BRANCH OF OSWEGATCHIE RIVER AT NEWTON FALLS, N. $Y$.

Locatron.--In village of Newton Falls, St. Lawrence County, 600 feet below lower dam of Newton Falls Paper Co., 4 miles above mouth of Little River, and $101 / 2$ miles below outlet of Cranberry Lake.

Drainage area.-170 square miles (measured on topographic maps); previously published as 166 square miles.

Records available.-October 6, 1912, to May 4, 1923, when station was discontinued because of backwater from Browns Falls dam.

GAGE.- - Vertical staff on left bank; read by Henry Van Waldick. Datum lowered 1 foot on July 28, 1920.

Discharge measurements.--Made from cable 50 feet above gage or by wading.

Channel and control.-Small boulders and rock; covered with waste from pulp mill; practically permanent.

Extremes of DISCHARge.- Maximum stage recorded during the period October 1, 1922, to May 4, 1923, 4.28 feet at 4.30 p.m. November 6 (discharge, 737 second-feet); minimum stage is reached nearly every Sunday in low-water period, when paper mills shut down.

1912-1923: Maximum stage recorded, 6.1 feet (old datum) at 5.15 p.m. March 28, 1913 (discharge, 2,200 second-feet).

ICE.-Stage-discharge relation affected by ice only for a short time during extremely cold weather.

Regulation.-Considerable diurnal fluctuation in flow caused by operation of paper mills at Newton Falls. Seasonal flow largely controlled by storage at Cranberry Lake.

AccoracY.-Stage-discharge relation practically permanent; probably not affected by ice. Rating curve well defined between 40 and 1,000 second-feet. Gage read to tenths twice daily. Daily discharge ascertained by applying mean daily gage height to rating table, except for days of great fluctuation due to closing down of power plant, when discharge is averaged for intervals of day. Records only fair, as mean daily gage height is obtained from only two readings and may be considerably in error on account of artificial regulation.

Discharge measurements of East Branch of Oswegatchie River at Newton Falls, N. Y., during the period October 1, 1922, to May 4, 1923

\begin{tabular}{|c|c|c|c|}
\hline Date & Made by- & $\begin{array}{c}\text { Gage } \\
\text { height }\end{array}$ & Discharge \\
\hline $\begin{array}{l}\text { Oct. } 18 \\
\text { Jan. } 28 \\
\text { Mar. } 17\end{array}$ & $\begin{array}{l}\text { A. W. Harrington } \\
\text { J. L. Lamson } \\
\text { Lamson and Johnsona }\end{array}$ & $\begin{array}{r}F \text { Feet } \\
3.17 \\
1.36 \\
2.41\end{array}$ & $\begin{array}{c}\text { Sec. } . f t . \\
364 \\
42.4 \\
205\end{array}$ \\
\hline
\end{tabular}

- Engineer, Board of Black River Regulating District 
Daily discharge, in second-feet, of East Branch of Oswegatchie River at Newton Falls, N. Y., for the period October 1, 1922, to May 4, 1923.

\begin{tabular}{|c|c|c|c|c|c|c|c|c|}
\hline Day & Oct. & Nov. & Dec. & Jan. & Feb. & Mar. & Apr. & May \\
\hline (1) & $\begin{array}{l}286 \\
309 \\
286 \\
263 \\
309\end{array}$ & $\begin{array}{l}411 \\
357 \\
383 \\
321 \\
298\end{array}$ & $\begin{array}{l}321 \\
274 \\
298 \\
321 \\
333\end{array}$ & $\begin{array}{l}357 \\
263 \\
286 \\
298 \\
263\end{array}$ & $\begin{array}{r}274 \\
298 \\
263 \\
110 \\
69\end{array}$ & $\begin{array}{r}55 \\
168 \\
198 \\
\cdot \quad 44 \\
55\end{array}$ & $\begin{array}{r}64 \\
168 \\
188 \\
383 \\
321\end{array}$ & $\begin{array}{l}321 \\
333 \\
357 \\
309\end{array}$ \\
\hline $\begin{array}{l}6 \\
8 \\
8\end{array}$ & $\begin{array}{l}286 \\
333 \\
263 \\
357 \\
321\end{array}$ & $\begin{array}{l}563 \\
499 \\
411 \\
357 \\
383\end{array}$ & $\begin{array}{l}298 \\
321 \\
263 \\
309 \\
159\end{array}$ & $\begin{array}{r}357 \\
159 \\
.286 \\
309 \\
286\end{array}$ & $\begin{array}{l}208 \\
219 \\
219 \\
168 \\
208\end{array}$ & $\begin{array}{r}198 \\
116 \\
58 \\
39 \\
58\end{array}$ & $\begin{array}{l}263 \\
219 \\
188 \\
357 \\
357\end{array}$ & 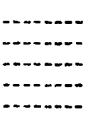 \\
\hline 1 & $\begin{array}{l}309 \\
298 \\
298 \\
263 \\
309\end{array}$ & $\begin{array}{l}321 \\
150 \\
274 \\
274 \\
321\end{array}$ & $\begin{array}{l}321 \\
357 \\
298 \\
321 \\
333\end{array}$ & $\begin{array}{r}39 \\
55 \\
150 \\
49 \\
150\end{array}$ & $\begin{array}{r}168 \\
75 \\
132 \\
168 \\
124\end{array}$ & $\begin{array}{r}44 \\
141 \\
188 \\
159 \\
178\end{array}$ & $\begin{array}{l}321 \\
357 \\
321 \\
208 \\
116\end{array}$ & 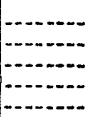 \\
\hline 16 & $\begin{array}{l}298 \\
263 \\
357 \\
411 \\
309\end{array}$ & $\begin{array}{l}357 \\
357 \\
263 \\
333 \\
309\end{array}$ & $\begin{array}{l}263 \\
150 \\
230 \\
241 \\
357\end{array}$ & $\begin{array}{l}168 \\
178 \\
178 \\
178 \\
168\end{array}$ & $\begin{array}{r}159 \\
49 \\
58 \\
44 \\
178\end{array}$ & $\begin{array}{r}188 \\
188 \\
55 \\
88 \\
150\end{array}$ & $\begin{array}{l}208 \\
298 \\
309 \\
298 \\
321\end{array}$ & 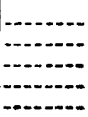 \\
\hline 21 & $\begin{array}{l}357 \\
333 \\
309 \\
321 \\
321\end{array}$ & $\begin{array}{l}274 \\
557 \\
469 \\
321 \\
383\end{array}$ & $\begin{array}{l}357 \\
274 \\
150 \\
150 \\
252\end{array}$ & $\begin{array}{r}58 \\
81 \\
132 \\
168 \\
298\end{array}$ & $\begin{array}{r}69 \\
168 \\
188 \\
108 \\
150\end{array}$ & $\begin{array}{r}219 \\
219 \\
274 \\
263 \\
69\end{array}$ & $\begin{array}{r}321 \\
81 \\
333 \\
298 \\
286\end{array}$ & (n) \\
\hline 30 & $\begin{array}{l}357 \\
357 \\
298 \\
321 \\
411 \\
411\end{array}$ & $\begin{array}{l}263 \\
274 \\
263 \\
321 \\
309\end{array}$ & $\begin{array}{l}309 \\
321 \\
333 \\
333 \\
298 \\
298\end{array}$ & $\begin{array}{l}263 \\
241 \\
100 \\
120 \\
263 \\
263\end{array}$ & $\begin{array}{l}168 \\
178 \\
188 \\
-18\end{array}$ & $\begin{array}{r}88 \\
81 \\
132 \\
241 \\
178 \\
168\end{array}$ & $\begin{array}{l}321 \\
298 \\
357 \\
141 \\
298\end{array}$ & 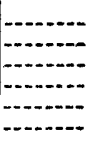 \\
\hline
\end{tabular}

Note.-Discharge Jan. 28, 29, and Feb. 4 determined by averaging discharge for intervals of day; paper mills shut down, causing large diurnal fluctuation in flow.

Monthly discharge of East Branch of Oswegatchie River at Newton Falls, N. Y., for the period October 1, 1922, to May 4, 1923

[Drainage area, 170 square miles]

\begin{tabular}{|c|c|c|c|c|c|}
\hline \multirow{2}{*}{ Month } & \multicolumn{4}{|c|}{ Discharge in second-feet } & \multirow{2}{*}{$\begin{array}{c}\text { Run-off in } \\
\text { inches }\end{array}$} \\
\hline & Maximum & Minimum & Mean & $\underset{\text { mile }}{\text { Per square }}$ & \\
\hline $\begin{array}{l}\text { October } \\
\text { November } \\
\text { December } \\
\text { January } \\
\text { February } \\
\text { March } \\
\text { April } \\
\text { May } \mathrm{i}-4\end{array}$ & $\begin{array}{l}411 \\
563 \\
357 \\
357 \\
298 \\
274 \\
383 \\
357\end{array}$ & $\begin{array}{r}263 \\
150 \\
150 \\
39 \\
44 \\
39 \\
64 \\
309\end{array}$ & $\begin{array}{l}320 \\
339 \\
285 \\
199 \\
157 \\
139 \\
267 \\
330\end{array}$ & $\begin{array}{l}1.88 \\
1.99 \\
1.68 \\
1.17 \\
.924 \\
.818 \\
1.57 \\
1.94\end{array}$ & $\begin{array}{r}2.17 \\
2.22 \\
1.94 \\
1.35 \\
.96 \\
.94 \\
1.95 \\
.29\end{array}$ \\
\hline
\end{tabular}

Note.-The above flgures do not necessarily represent the natural flow from the basin because of artincial storage, mainly in Cranberry Lake. 


\section{OSWEGATCHIE RIVER NEAR HEUVELTON, N, Y.}

Locatron.-21/2 miles above Heuvelton, St. Lawrence County, 3 miles below Rensselaer Falls, and 7 miles above mouth of Indian River (outlet of Black Lake).

Drainage AREA.-967 square miles (measured on topographic maps); previously published as 961 square miles.

Records available.-June 23, 1916, to September 30, 1923.

GAGE.-Gurley seven-day water-stage recorder on right bank; inspeeted by George B. Todd.

Discharge measurements.--Made from cable 20 feet below gage or by wading Channel and Control.-Solid rock; practically permanent.

EXTREmES of Discharge.-Maximum stage during year from water-stage. recorder, 6.89 feet at 5.30 p. $\mathrm{m}$. April 10 (discharge, 9,920 second-feet); minimum stage from water-stage recorder, 0.82 foot at noon August 8 (discharge, 280 second-feet).

1916-1923: Maximum stage from water-stage recorder, 7.6 feet from 9 a. m. to noon March 30, 1917 (discharge, 11,700 second-feet); minimum stage from water-stage recorder, 0.81 foot from 2 to $4 \mathrm{a}$. m. September 30 , 1921 (discharge, 274 second-feet).

ICE.-Stage-discharge relation slightly affected by ice during extremely cold periods.

Regulation.-During low water there is some diurnal fluctuation due to operation of mills at Rensselaer Falls and above. Seasonal flow regulated by storage in Cranberry Lake.

ACCURACY.-Stage-discharge relation practically permanent; probably not affected by ice. Rating curve well defined between 400 and 12,000 second-feet. Operation of water-stage recorder satisfactory except as indicated in footnote to daily discharge table. Daily discharge ascertained by applying to rating table mean daily gage height determined by inspection of recorder graph, or for days of considerable fluctuation, by averaging discharge for intervals of day. Records good, except for periods of estimate, for which they are fair.

Discharge measurements of Oswegatchie River near Hermslton, N. Y., during the year ending September 30, 1923

\begin{tabular}{|c|c|c|c|}
\hline Date & Made by- & $\begin{array}{c}\text { Gage } \\
\text { height }\end{array}$ & $\begin{array}{c}\text { Dis- } \\
\text { charge }\end{array}$ \\
\hline $\begin{array}{l}\text { Jan. } 27 \\
\text { Apr. } 21 \\
\text { July } 24\end{array}$ & $\begin{array}{l}\text { J. L. Lamson. } \\
\text { A. W. Harrington } \\
\text { Lamson and Johnson }\end{array}$ & $\begin{array}{l}\text { Feet } \\
1.72 \\
2.68 \\
1.02\end{array}$ & $\begin{array}{r}\text { Sec. ft. } \\
937 \\
2,090 \\
388\end{array}$ \\
\hline
\end{tabular}


Daily discharge, in second-feet, of Oswegatchie River near Heuvelton, N.Y., for the year ending September 30,1923

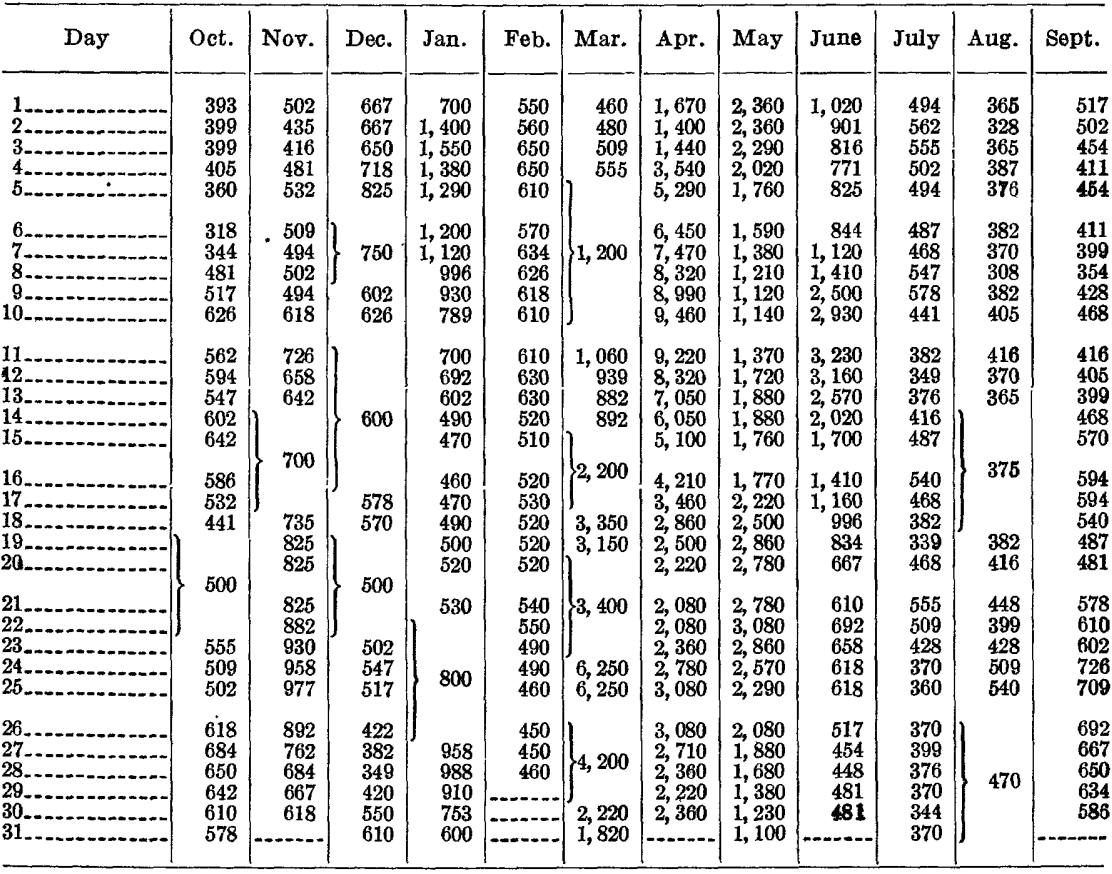

Note.-Discharge for following periods when water-stage recorder was not operating satisfactorily es timated by comparison with records of flow of nearby streams: Oct. 19-22, Nov. 13-18, 26, 27, Dec. 6-16 19-22, 28-31, Jan. 4-6, 14-26, 31, Feb. 1-4, 9, 10, 12-28, Mar. 1, 2, 5-10, 12, 13, 15-23, 26-29, May 18, 19, Jul. , 1. Aug. 12-19, Aug. 26 to Sept. 1.

Monthly. discharge of Oswegatchie River near Heuvelton, N.Y., for the year endin, September 30, 1923

[Drainage area, 967 square miles]

\begin{tabular}{|c|c|c|c|c|c|}
\hline \multirow{2}{*}{ Month } & \multicolumn{4}{|c|}{ Discharge in second-feet } & \multirow{2}{*}{$\begin{array}{l}\text { Run-off } \\
\text { in inches }\end{array}$} \\
\hline & Maximum & Minimum & Mean & $\begin{array}{l}\text { Per square } \\
\text { mile }\end{array}$ & \\
\hline $\begin{array}{l}\text { October } \\
\text { November } \\
\text { December } \\
\text { January } \\
\text { February } \\
\text { March } \\
\text { April } \\
\text { May } \\
\text { June } \\
\text { July } \\
\text { August }\end{array}$ & $\begin{array}{r}684 \\
977 \\
825 \\
1,550 \\
650 \\
6,250 \\
9,460 \\
3,080 \\
3,230 \\
578 \\
540 \\
726\end{array}$ & $\begin{array}{r}318 \\
416 \\
349 \\
460 \\
450 \\
460 \\
1,400 \\
1,100 \\
448 \\
339 \\
308 \\
354\end{array}$ & $\begin{array}{r}519 \\
680 \\
582 \\
822 \\
553 \\
2,360 \\
4,340 \\
1,960 \\
1,220 \\
445 \\
408 \\
527\end{array}$ & $\begin{array}{l}0.537 \\
.703 \\
.602 \\
.850 \\
.572 \\
2.44 \\
4.49 \\
2.03 \\
1.26 \\
.460 \\
.422 \\
.545\end{array}$ & $\begin{array}{r}0.62 \\
.78 \\
.66 \\
.98 \\
.60 \\
2.81 \\
5.01 \\
2.34 \\
1.41 \\
.53 \\
.49 \\
.61 \\
\end{array}$ \\
\hline The year .. & 9,460 & 308 & 1,200 & 1.24 & 16.87 \\
\hline
\end{tabular}

Note-The above figures do not necessarily represent the natural flow from the bsain because of art ificial storage mainly in Cranberry Lake. 


\section{WEST BRATCH OF OSWEGATCHIE RIVER NEAR HARRISVILLE, N. Y.}

Location.-At highway bridge near Geers Corners, 4 miles downstream from Harrisville, Lewis County, and 16 miles above confluence of east and west branches near Talcville.

Drainage area.-256 square miles (measured on topographic maps); previously published as 245 square miles.

Records AVAILABLe.-July 1, 1916, to September 30, 1923.

GAGE.-Vertical staff in three sections on right bank; section graduated from 0.0 to 3.3 feet 25 feet below bridge, and two sections graduated from 3.3 to 10.1 feet on downstream side of bridge abutment. Gage read by Frank Osborne.

Discharge meastrements.-Made from cable 200 feet above gage from downstream side of highway bridge or by wading.

Channel and control.-Rocky and rough; practically permanent.

Extremes of Discharge.-Maximum stage recorded during year, 7.2 feet at 6 p. m. April 7 (discharge, 3,740 second-feet); minimum stage, 0.90 foot several times during period August 10-19 (discharge, 27 second-feet).

1916-1923: Maximum stage recorded, 8.1 feet at 6.30 a. m. and $6 \mathrm{p.m}$. March 28, 1917 (discharge, 4,880 second-feet); minimum discharge, that of August 10-19, 1923.

IC®.-Stage-discharge relation only slightly affected by ice during extremely cold - periods.

Regdlation.-During low water there is some diurnal fluctuation in flow caused by operation of pulp mill at Harrisville.

ACCURACX. - Stage-discharge relation practically permanent; not seriously affected by ice. Rating curve fairly well defined between 50 and 4,000 second-feet. Gage read to half-tenths twice daily. Daily discharge ascertained by applying mean daily gage height to rating table. Records good except those for low stages, which, owing to diurnal fluctuation, are only fair. ।

Discharge measurements of West Branch of Oswegatchie River near Harrisvilte N. Y., during the year ending September 30, 1923

\begin{tabular}{|c|c|c|c|c|c|c|c|}
\hline Date & Made by- & $\begin{array}{c}\text { Gage } \\
\text { height }\end{array}$ & $\begin{array}{c}\text { Dis- } \\
\text { charge }\end{array}$ & Date & Made by- & $\begin{array}{c}\text { Gage } \\
\text { height }\end{array}$ & $\begin{array}{l}\text { Dis- } \\
\text { charge }\end{array}$ \\
\hline $\begin{array}{l}\text { Oct. } 18 \\
\text { Mar. } 18 \\
\text { Apr. } 20\end{array}$ & $\begin{array}{l}\text { A. W. Harrington. } \\
\text { J. L. Lamson } \\
\text { do }\end{array}$ & $\begin{array}{c}\text { Feet } \\
1.41 \\
a 3.70 \\
3.34\end{array}$ & $\begin{array}{c}\text { Sec. }-f t . \\
76.3 \\
739 \\
637\end{array}$ & $\begin{array}{l}\text { June } 27 \\
\text { July } 28\end{array}$ & $\begin{array}{l}\text { A. W. Harrington } \\
\text { Lamson and Johnson }\end{array}$ & $\begin{array}{l}\text { Feet } \\
1.57 \\
1.52\end{array}$ & $\begin{array}{r}\text { Sec. ft. } \\
100 \\
101\end{array}$ \\
\hline
\end{tabular}

a Stage-discharge relation slightly affected by ice. 
Daily discharge, in second-feet, of West Branch of Oswegatchie River near Harrisville, N. Y., for the year ending September 30, 1923

\begin{tabular}{|c|c|c|c|c|c|c|c|c|c|c|c|c|}
\hline Day & Oct. & Nov. & Dec. & Jan. & Feb. & Mar. & Apr. & May & June & July & Aug. & Sept. \\
\hline $\begin{array}{l}1 \ldots \\
2 \ldots \\
3 \ldots \\
4 \ldots \\
5 \ldots\end{array}$ & $\begin{array}{l}48 \\
48 \\
52 \\
45 \\
40\end{array}$ & $\begin{array}{r}106 \\
106 \\
98 \\
106 \\
114\end{array}$ & $\begin{array}{l}142 \\
163 \\
163 \\
210 \\
235\end{array}$ & $\begin{array}{l}222 \\
438 \\
535 \\
535 \\
495\end{array}$ & $\begin{array}{l}174 \\
186 \\
186 \\
222 \\
222\end{array}$ & $\begin{array}{l}114 \\
123 \\
142 \\
235 \\
351\end{array}$ & $\begin{array}{r}438 \\
384 \\
456 \\
915 \\
1,640\end{array}$ & $\begin{array}{r}1,090 \\
970 \\
860 \\
665 \\
575\end{array}$ & $\begin{array}{l}290 \\
262 \\
248 \\
210 \\
210\end{array}$ & $\begin{array}{l}210 \\
163 \\
152 \\
142 \\
106\end{array}$ & $\begin{array}{l}56 \\
56 \\
60 \\
60 \\
72\end{array}$ & $\begin{array}{l}72 \\
75 \\
65 \\
60 \\
56\end{array}$ \\
\hline $\begin{array}{l}6 \\
7 \\
8 \\
9\end{array}$ & $\begin{array}{r}40 \\
40 \\
68 \\
90 \\
106\end{array}$ & $\begin{array}{l}142 \\
180 \\
186 \\
186 \\
186\end{array}$ & $\begin{array}{l}123 \\
186 \\
163 \\
186 \\
186\end{array}$ & $\begin{array}{l}438 \\
384 \\
351 \\
290 \\
262\end{array}$ & $\begin{array}{l}198 \\
198 \\
174 \\
152 \\
186\end{array}$ & $\begin{array}{l}384 \\
290 \\
351 \\
351 \\
305\end{array}$ & $\begin{array}{l}2,790 \\
3,740 \\
3,520 \\
3,300 \\
2,890\end{array}$ & $\begin{array}{l}495 \\
456 \\
402 \\
456 \\
665\end{array}$ & $\begin{array}{l}210 \\
222 \\
276 \\
575 \\
970\end{array}$ & $\begin{array}{l}98 \\
78 \\
82 \\
82 \\
65\end{array}$ & $\begin{array}{l}42 \\
39 \\
36 \\
40 \\
36\end{array}$ & $\begin{array}{r}62 \\
68 \\
72 \\
82 \\
163\end{array}$ \\
\hline $\begin{array}{l}13 \\
145\end{array}$ & $\begin{array}{r}106 \\
106 \\
123 \\
123 \\
82\end{array}$ & $\begin{array}{l}174 \\
152 \\
142 \\
142 \\
152\end{array}$ & $\begin{array}{l}174 \\
163 \\
186 \\
186 \\
163\end{array}$ & $\begin{array}{l}248 \\
210 \\
210 \\
186 \\
152\end{array}$ & $\begin{array}{l}163 \\
163 \\
123 \\
106 \\
123\end{array}$ & $\begin{array}{l}290 \\
276 \\
290 \\
290 \\
290\end{array}$ & $\begin{array}{l}2,130 \\
1,960 \\
1,800 \\
1,640 \\
1,490\end{array}$ & $\begin{array}{l}760 \\
760 \\
760 \\
710 \\
620\end{array}$ & $\begin{array}{l}970 \\
760 \\
575 \\
419 \\
305\end{array}$ & $\begin{array}{r}65 \\
62 \\
65 \\
72 \\
106\end{array}$ & $\begin{array}{l}34 \\
36 \\
45 \\
42 \\
48\end{array}$ & $\begin{array}{r}132 \\
142 \\
123 \\
106 \\
90\end{array}$ \\
\hline $\begin{array}{l}16 \\
178 \\
18 \\
190\end{array}$ & $\begin{array}{r}123 \\
90 \\
87 \\
90 \\
90\end{array}$ & $\begin{array}{l}262 \\
320 \\
276 \\
262 \\
248\end{array}$ & $\begin{array}{l}163 \\
123 \\
123 \\
123 \\
106\end{array}$ & $\begin{array}{l}152 \\
152 \\
163 \\
142 \\
152\end{array}$ & $\begin{array}{l}123 \\
132 \\
142 \\
123 \\
123\end{array}$ & $\begin{array}{l}438 \\
665 \\
810 \\
860 \\
860\end{array}$ & $\begin{array}{r}1,210 \\
1,030 \\
810 \\
710 \\
665\end{array}$ & $\begin{array}{r}665 \\
810 \\
1,030 \\
1,150 \\
1,090\end{array}$ & $\begin{array}{l}248 \\
222 \\
198 \\
163 \\
163\end{array}$ & $\begin{array}{r}65 \\
62 \\
114 \\
82 \\
78\end{array}$ & $\begin{array}{l}45 \\
40\end{array}$ & $\begin{array}{l}90 \\
65 \\
50 \\
62 \\
65\end{array}$ \\
\hline $\begin{array}{l}21 \\
23 \\
24 \\
25\end{array}$ & $\begin{array}{r}98 \\
98 \\
98 \\
186 \\
248\end{array}$ & $\begin{array}{l}305 \\
351 \\
305 \\
262 \\
235\end{array}$ & $\begin{array}{r}123 \\
90 \\
90 \\
98 \\
106\end{array}$ & $\begin{array}{l}235 \\
290 \\
336 \\
305 \\
351\end{array}$ & $\begin{array}{l}114 \\
1 \% 3 \\
123 \\
123 \\
132\end{array}$ & $\begin{array}{r}760 \\
760 \\
1,030 \\
1,490 \\
1,560\end{array}$ & $\begin{array}{r}665 \\
915 \\
1,420 \\
1,640 \\
1,490\end{array}$ & $\begin{array}{r}1,030 \\
1,030 \\
.970 \\
915 \\
760\end{array}$ & $\begin{array}{l}142 \\
142 \\
123 \\
106 \\
123\end{array}$ & $\begin{array}{l}78 \\
98\end{array}$ & $\begin{array}{l}42 \\
48\end{array}$ & $\begin{array}{l}163 \\
320 \\
305 \\
235 \\
198\end{array}$ \\
\hline $\begin{array}{l}26 \\
27 \\
28 \\
30 \\
31\end{array}$ & $\begin{array}{l}186 \\
174 \\
174 \\
152 \\
123 \\
106\end{array}$ & $\begin{array}{l}186 \\
186 \\
186 \\
174 \\
163\end{array}$ & $\begin{array}{l}123 \\
114 \\
123 \\
132 \\
123 \\
106\end{array}$ & $\begin{array}{l}351 \\
305 \\
262 \\
210 \\
210 \\
186\end{array}$ & $\begin{array}{r}123 \\
123 \\
123 \\
\\
-1 .- \\
\end{array}$ & $\begin{array}{r}1,350 \\
1,090 \\
860 \\
760 \\
575 \\
535\end{array}$ & $\begin{array}{r}1 ; 280 \\
970 \\
860 \\
915 \\
1,030 \\
\\
\end{array}$ & $\begin{array}{l}665 \\
535 \\
456 \\
351 \\
320 \\
290\end{array}$ & $\begin{array}{l}114 \\
132 \\
123 \\
142 \\
: 22\end{array}$ & $\begin{array}{l}62 \\
68 \\
68 \\
98 \\
56 \\
60\end{array}$ & $\begin{array}{l}75 \\
60 \\
62 \\
87 \\
87 \\
82\end{array}$ & $\begin{array}{r}123 \\
106 \\
106 \\
98 \\
90\end{array}$ \\
\hline
\end{tabular}

Norn,-Discharge estimated Nov. 7; no gage-height record.

Monthly discharge of West Branch of Oswegatchie River near Harrisville, $N$. Y., for the year ending September 30, 1923

[Drainage area, 256 square miles]

\begin{tabular}{|c|c|c|c|c|c|}
\hline \multirow{2}{*}{ Month } & \multicolumn{4}{|c|}{ Discharge in second-feet } & \multirow{2}{*}{$\begin{array}{l}\text { Run-off in } \\
\text { inches }\end{array}$} \\
\hline & Maximum & Minimium & Mean & $\begin{array}{c}\text { Per square } \\
\text { mile }\end{array}$ & \\
\hline $\begin{array}{l}\text { October } \\
\text { November } \\
\text { December. } \\
\text { January } \\
\text { February } \\
\text { March } \\
\text { April } \\
\text { May } \\
\text { June } \\
\text { July } \\
\text { August }\end{array}$ & $\begin{array}{r}248 \\
351 \\
235 \\
535 \\
222 \\
1,560 \\
3,740 \\
1,150 \\
970 \\
210 \\
87 \\
320\end{array}$ & $\begin{array}{r}40 \\
98 \\
90 \\
142 \\
106 \\
114 \\
384 \\
290 \\
106 \\
50 \\
30 \\
50\end{array}$ & $\begin{array}{l}105 \\
196 \\
145 \\
283 \\
150 \\
596 \\
1,490 \\
720 \\
296 \\
87.5 \\
52.8 \\
115\end{array}$ & $\begin{array}{c}0.410 \\
.766 \\
.566 \\
1.10 \\
.586 \\
2.33 \\
5.82 \\
2.81 \\
1.16 \\
.342 \\
.206 \\
.449\end{array}$ & $\begin{array}{r}0.47 \\
.85 \\
.65 \\
1.27 \\
.61 \\
2.69 \\
6.49 \\
3.24 \\
1.29 \\
.39 \\
.24 \\
.50\end{array}$ \\
\hline The year. ........ & 3,740 & 30 & 353 & 1.38 & 18.69 \\
\hline
\end{tabular}


RAQUETTE RIVER AT PIERCEFIELD, N. $\mathbf{Y}$.

Locatron.-Half a mile below dam of International Paper Co. at Piercefield, St. Lawrence County, and three-quarters of a mile above head of Black Rapids.

Drainage area.--723 square miles (all but 16 square miles measured on topographic maps).

Records available.-August 20, 1908, to September 30, 1923.

GAGE.-Stevens continuous water-stage recorder installed October 22, 1912, on left bank; inspected by employee of International Paper Co.

Discharge measurements.- - Made from a cable three-quarters of a mile below gage.

Channel and control.-Channel opposite gage is a deep pond with no perceptible velocity. Control is at head of Black Rapids.

EXTREMES OF DISCHARGE.- Maximum stage during year from water-stage recorder, 9.63 feet at 9.30 a. m. April 30 (discharge, 4,600 second-feet); minimum stage from water-stage recorder, 1.50 feet from 4 to $10 \mathrm{a} . \mathrm{m}$. O ctober 8 (discharge 36 second-feet).

1908-1923: Maximum stage from water-stage recorder, 11.82 feet from 6 to 8 p. m. April 17, 1922 (discharge, 7,580 second-feet); minimum stage from water-stage recorder, 0.85 foot at $11 \mathrm{a} . \mathrm{m}$. September 2, 1913 (discharge, about 10 second-feet).

Ice.-Stage-discharge relation slightly affected by ice.

REGULATION.-Large diurnal fluctuation in flow during low and medium stages caused by operation of paper mill. Numerous lakes in upper part of drainage basin afford considerable storage, most of which is so controlled that the effect on the seasonal distribution of flow is large.

ACCURACY.-Stage-discharge relation practically permanent except as affected by ice from December to April. Rating curve well defined between 50 and 5,000 second-feet. Operation of water-stage recorder satisfactory except as indicated in footnote to daily-discharge table. Daily discharge ascertained by applying to rating table mean daily gage height determined by inspection of recorder graph, or for days of considerable fluctuation, by averaging discharge for intervals of day. Records good except for period of ice effect for which they are fair.

Discharge measurements of Raquette River at Piercefield, N.Y., during the year ending September 30, 1923

\begin{tabular}{|c|c|c|c|c|c|c|c|}
\hline Date & Made by- & $\begin{array}{c}\text { Gage } \\
\text { height }\end{array}$ & $\begin{array}{l}\text { Dis- } \\
\text { charge }\end{array}$ & Date & Made by- & $\begin{array}{c}\text { Gage } \\
\text { height }\end{array}$ & $\begin{array}{c}\text { Dis- } \\
\text { charge }\end{array}$ \\
\hline $\begin{array}{l}\text { Oct. } 31 \\
\text { Mar. } 22 \\
\text { April } 19 \\
\end{array}$ & \begin{tabular}{c} 
J. L. Lamson. \\
\\
\hdashline do do \\
- do
\end{tabular} & $\begin{array}{r}\text { Seet } \\
4.00 \\
64.50 \\
8.49 \\
9.28\end{array}$ & $\begin{array}{r}\text { Sec. }- \text { ft. } \\
471 \\
557 \\
3,250 \\
4,070\end{array}$ & $\begin{array}{ll}\text { May } & 30 \\
& 31 \\
\text { July } & 14 \\
& 15\end{array}$ & $\begin{array}{l}\text { J. L. Lamson } \\
\text { A. E. Johnson } \\
\text { Lamson and Johnson. }\end{array}$ & $\begin{array}{l}\text { Feet } \\
6.92 \\
7.40 \\
4.45 \\
2.46\end{array}$ & $\begin{array}{r}\text { Sec. }-f t . \\
1,980 \\
2,440 \\
648 \\
132\end{array}$ \\
\hline
\end{tabular}

¿Stage-discharge relation affected by ice. 
Daily discharge, in second-feet, of Raquette River at Piercefield, N.Y., for the year ending September 30, 1923

\begin{tabular}{|c|c|c|c|c|c|c|c|c|c|c|c|c|}
\hline Day & Oet. & Nov. & Dec. & Jan. & Feb. & Mar. & Apr. & May & June & July & Aug. & Sept. \\
\hline $\begin{array}{l}1 \ldots \\
2 \ldots \\
3 \ldots \\
4 \ldots \\
5 \ldots\end{array}$ & $\begin{array}{l}175 \\
284 \\
398 \\
311 \\
203\end{array}$ & $\begin{array}{r}425 \\
221 \\
84 \\
258 \\
198\end{array}$ & $\begin{array}{l}470 \\
470 \\
247 \\
367 \\
485\end{array}$ & $\begin{array}{l}500 \\
550 \\
550 \\
600 \\
700\end{array}$ & $\begin{array}{l}550 \\
550 \\
500 \\
280 \\
440\end{array}$ & $\begin{array}{l}420 \\
420 \\
300 \\
150 \\
340\end{array}$ & $\begin{array}{r}420 \\
650 \\
850 \\
950 \\
1,200\end{array}$ & $\begin{array}{l}4,450 \\
4,450 \\
4,340 \\
4,230 \\
4,120\end{array}$ & $\begin{array}{l}\mathbf{2}, 170 \\
\mathbf{2}, 050 \\
1,670 \\
1,870 \\
1,800\end{array}$ & $\begin{array}{r}469 \\
724 \\
1,130 \\
299 \\
416\end{array}$ & $\begin{array}{l}394 \\
435 \\
485 \\
330 \\
142\end{array}$ & $\begin{array}{r}354 \\
182 \\
75 \\
289 \\
290\end{array}$ \\
\hline $\begin{array}{r}6 \\
7 \\
8 \\
9 \\
10\end{array}$ & $\begin{array}{r}323 \\
140 \\
60 \\
324 \\
455\end{array}$ & $\begin{array}{l}320 \\
425 \\
410 \\
306 \\
358\end{array}$ & $\begin{array}{l}470 \\
470 \\
485 \\
547 \\
300\end{array}$ & 900 & $\begin{array}{l}550 \\
550 \\
550 \\
550 \\
500\end{array}$ & $\begin{array}{l}480 \\
500 \\
480 \\
460 \\
300\end{array}$ & $\begin{array}{l}1,100 \\
1,300 \\
1,500 \\
2,000 \\
2,200\end{array}$ & $\begin{array}{l}3,800 \\
3,800 \\
3,700 \\
3,600 \\
3,500\end{array}$ & & $\begin{array}{l}565 \\
840 \\
265 \\
430 \\
600\end{array}$ & $\begin{array}{l}287 \\
326 \\
230 \\
230 \\
222\end{array}$ & $\begin{array}{r}146 \\
85 \\
69 \\
65 \\
75\end{array}$ \\
\hline 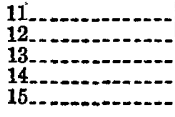 & $\begin{array}{l}403 \\
226 \\
243 \\
331 \\
127\end{array}$ & $\begin{array}{l}434 \\
205 \\
297 \\
433 \\
440\end{array}$ & $\begin{array}{l}420 \\
550 \\
600 \\
550 \\
500\end{array}$ & 650 & $\begin{array}{l}280 \\
420 \\
500 \\
550 \\
500\end{array}$ & $\begin{array}{l}170 \\
320 \\
440 \\
480 \\
500\end{array}$ & $\begin{array}{l}2,600 \\
2,800 \\
3,000 \\
3,300 \\
3,500\end{array}$ & $\begin{array}{l}3,400 \\
3,300 \\
3,110 \\
3,200 \\
3,110\end{array}$ & & $\begin{array}{l}582 \\
582 \\
582 \\
565 \\
269\end{array}$ & $\begin{array}{r}166 \\
73 \\
285 \\
372 \\
210\end{array}$ & $\begin{array}{r}69 \\
163 \\
372 \\
398 \\
197\end{array}$ \\
\hline - & $\begin{array}{r}307 \\
351 \\
89 \\
70 \\
316\end{array}$ & $\begin{array}{l}440 \\
308 \\
371 \\
231 \\
333\end{array}$ & $\begin{array}{l}480 \\
240 \\
400 \\
550 \\
460\end{array}$ & $\begin{array}{l}700 \\
650\end{array}$ & $\begin{array}{l}500 \\
480 \\
200 \\
200 \\
380\end{array}$ & $\begin{array}{l}550 \\
550 \\
280 \\
360 \\
500\end{array}$ & $\begin{array}{l}3,600 \\
3,500 \\
3,500 \\
3,300 \\
3,200\end{array}$ & $\begin{array}{l}3,110 \\
3,110 \\
3,200 \\
3,200 \\
3,110\end{array}$ & $\begin{array}{l}1,700 \\
1,070 \\
1,700 \\
1,560 \\
1,400\end{array}$ & $\begin{array}{l}397 \\
548 \\
548 \\
530 \\
515\end{array}$ & $\begin{array}{r}58 \\
67 \\
69 \\
75 \\
298\end{array}$ & $\begin{array}{r}72 \\
68 \\
62 \\
68 \\
302\end{array}$ \\
\hline 221 & $\begin{array}{l}410 \\
204 \\
182 \\
357 \\
372\end{array}$ & $\begin{array}{l}455 \\
440 \\
455 \\
455 \\
440\end{array}$ & $\begin{array}{l}480 \\
500 \\
420 \\
130 \\
160\end{array}$ & $\begin{array}{l}300 \\
550 \\
700 \\
550 \\
550\end{array}$ & $\begin{array}{l}440 \\
440 \\
420 \\
300 \\
150\end{array}$ & $\begin{array}{l}500 \\
550 \\
550 \\
550 \\
280\end{array}$ & $\begin{array}{l}3,300 \\
3,400 \\
3,700 \\
4,010 \\
4,120\end{array}$ & $\begin{array}{l}3,200 \\
3,200 \\
3,110 \\
3,020 \\
2,930\end{array}$ & $\begin{array}{r}1,250 \\
1,130 \\
1,130 \\
439 \\
462\end{array}$ & $\begin{array}{l}515 \\
227 \\
392 \\
515 \\
515\end{array}$ & $\begin{array}{r}425 \\
123 \\
56 \\
69 \\
66\end{array}$ & $\begin{array}{r}425 \\
398 \\
143 \\
75 \\
295\end{array}$ \\
\hline $\begin{array}{l}26 \\
27 \\
28 \\
29 \\
30 \\
31\end{array}$ & $\begin{array}{r}207 \\
199 \\
91 \\
86 \\
336 \\
433\end{array}$ & $\begin{array}{l}236 \\
390 \\
582 \\
530 \\
470\end{array}$ & $\begin{array}{l}460 \\
550 \\
550 \\
550 \\
550 \\
180\end{array}$ & $\begin{array}{l}600 \\
600 \\
300 \\
440 \\
550 \\
600\end{array}$ & $\begin{array}{r}280 \\
360 \\
420 \\
-2 \\
\end{array}$ & $\begin{array}{l}400 \\
550 \\
650 \\
650 \\
800 \\
900\end{array}$ & $\begin{array}{l}4,230 \\
4,230 \\
4,230 \\
4,230 \\
4,450\end{array}$ & $\begin{array}{l}2,840 \\
2,570 \\
2,750 \\
2,570 \\
2,410 \\
2,410\end{array}$ & $\begin{array}{r}582 \\
815 \\
600 \\
1,000 \\
1,190 \\
\end{array}$ & $\begin{array}{l}530 \\
530 \\
500 \\
236 \\
392 \\
500\end{array}$ & $\begin{array}{r}76 \\
71 \\
66 \\
70 \\
70 \\
162\end{array}$ & $\begin{array}{l}398 \\
410 \\
341 \\
220 \\
126\end{array}$ \\
\hline
\end{tabular}

Nотk-Discharge estimated for following days when water-stage recorder was not operating satisfactorily: Jan. 7-18, 26-28, 30, 31, Feb. 1-7, Mar. 9 and 10. Disoharge, Dec. 10 to Apr. 13, determined from gage heights corrected for ice effect by means of one discharge measurement, study of gage-height graph, and weather records.

Monthly discharge of Raquette River at Piercefield, N. Y., for the year ending September 30,1923

[Drainage area, 723 square miles]

\begin{tabular}{|c|c|c|c|c|c|}
\hline \multirow{2}{*}{ Month } & \multicolumn{4}{|c|}{ Discharge in second-feet } & \multirow{2}{*}{$\begin{array}{c}\text { Run off in } \\
\text { inches }\end{array}$} \\
\hline & Maximum & Minimum & Mean & $\underset{\text { mile }}{\text { Per square }}$ & \\
\hline $\begin{array}{l}\text { October } \\
\text { November } \\
\text { December } \\
\text { January } \\
\text { February } \\
\text { March } \\
\text { April } \\
\text { May } \\
\text { June } \\
\text { July } \\
\text { August } \\
\text { September }\end{array}$ & $\begin{array}{r}455 \\
582 \\
600 \\
900 \\
550 \\
900 \\
4,450 \\
4,450 \\
2.170 \\
1,130 \\
485 \\
425\end{array}$ & $\begin{array}{r}60 \\
84 \\
130 \\
300 \\
150 \\
150 \\
420 \\
2,410 \\
439 \\
227 \\
56 \\
62\end{array}$ & $\begin{array}{r}258 \\
365 \\
438 \\
603 \\
423 \\
464 \\
2,810 \\
3,320 \\
1,460 \\
507 \\
194 \\
208\end{array}$ & $\begin{array}{r}0.357 \\
.505 \\
.606 \\
.834 \\
.585 \\
.642 \\
3.89 \\
4.59 \\
2.02 \\
.701 \\
.268 \\
.288\end{array}$ & $\begin{array}{r}0.41 \\
.56 \\
.70 \\
.96 \\
.61 \\
.74 \\
4.34 \\
5.29 \\
2.25 \\
.81 \\
.31 \\
.32\end{array}$ \\
\hline The year... & 4,450 & 56 & 921 & 1.27 & 17.30 \\
\hline
\end{tabular}

L NotE.-See paragraph on "Regulation" 


\section{ST. REGIS RIVER AT BRASHER CENTER, $\mathbf{N}$. $\mathrm{Y}$.}

Location.-600 feet above steel highway bridge in Brasher Center, St: Lawrence County, 6 miles below junction of East and West branches of St. Regis River at Winthrop, 7 miles above mouth of Deer River, and 15 miles above mouth.

Drainage AREa.-621 square miles (measured on post-route map).

Records available.-August 22, 1910, to November 10, 1917, and January 1, 1919, to September 30, 1923.

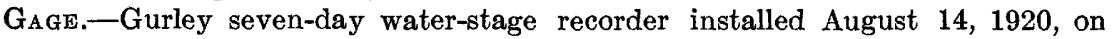
left bank; inspected by Alfred Berry. Datum same as that of staff gage with inclined and vertical sections used June 24, 1916, to August 14, 1920. A chain gage on downstream side of bridge, at independent datum, was used August 22, 1910, to June 23, 1916.

Discharge MeasUREMENTs.-Made from cable at gage or by wading.

Channel and control.--Rock ledge, small boulders, and coarse gravel; practically permanent.

Extremes of discharge.-Maximum stage during year from water-stage recorder, 9.75 feet at 6 a. m. April 9 (discharge, 6,430 second-feet); minimum stage from water-stage recorder, 5.72 feet at midnight August 16 (discharge, 138 second-feet).

1910-1923: Maximum stage recorded, 9.1 feet (old datum) at 7 a. m. March 27, 1914 (discharge, 16,200 second-feet); minimum stage recorded, 5.25 feet at 5 p. m. August 8, 1917 (discharge, about 34 second-feet).

ICE.--Stage-discharge relation affected by ice.

ACCURACY. - Stage-discharge relation practically permanent, except as affected by ice. Rating curve well defined between 200 and 6,000 second-feet. Operation of water-stage recorder satisfactory except as indicated in footnote to daily-discharge table. Daily discharge ascertained by applying to rating table mean daily gage height determined by inspection of recorder graph, or for days of considerable fluctuation, by averaging discharge for intervals of day. Records good except for periods of ice effect or estimate, for which they are fair.

Discharge measurements of St. Regis River at Brasher Center, N.Y., during the year ending September 30, 1928

\begin{tabular}{|c|c|c|c|c|c|c|c|}
\hline Date & Made by- & $\begin{array}{c}\text { Gage } \\
\text { height }\end{array}$ & $\begin{array}{c}\text { Dis- } \\
\text { charge }\end{array}$ & Date & Made by- & $\begin{array}{c}\text { Gage } \\
\text { height }\end{array}$ & $\begin{array}{l}\text { Dis- } \\
\text { charge }\end{array}$ \\
\hline 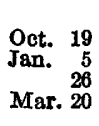 & \begin{tabular}{l} 
A. W. Harrington.... \\
J. L. Lamson \\
\\
\hdashline do
\end{tabular} & $\begin{array}{l}\text { Feet } \\
5.90 \\
6.46 \\
\times 6.44 \\
\times 7.33\end{array}$ & $\begin{array}{r}\text { Sec.ft. } \\
210 \\
651 \\
427 \\
1,010\end{array}$ & $\begin{array}{l}\text { Apr. } 21 \\
\text { June } 25 \\
\text { July } 23\end{array}$ & $\begin{array}{l}\text { A.W. Harrington } \\
\text { A.E. Johnson. }\end{array}$ & $\begin{array}{l}\text { Feet } \\
7.57 \\
6.55 \\
5.92\end{array}$ & $\begin{array}{r}\text { Sec. ft. } \\
2,140 \\
818 \\
207\end{array}$ \\
\hline
\end{tabular}

a Stage-discharge relation affected by ice. 
Daily discharge, in second-feet, of St. Regis River at Brasher Center, N. Y., for the year ending September 30, 1923

\begin{tabular}{|c|c|c|c|c|c|c|c|c|c|c|c|c|}
\hline$D$ & et. & Nov. & Dee. & Jan. & Feb. & Mar. & Apr. & May & Jane & July & Aug. & Sept. \\
\hline $\begin{array}{l}1 \\
2 \\
3 \\
4 \\
5\end{array}$ & $\begin{array}{l}184 \\
184 \\
179 \\
184 \\
179\end{array}$ & $\begin{array}{l}412 \\
372 \\
326 \\
388 \\
465\end{array}$ & $\begin{array}{l}605 \\
800 \\
894 \\
870 \\
850\end{array}$ & $\begin{array}{r}700 \\
1,200 \\
800 \\
700 \\
600\end{array}$ & $\begin{array}{l}380 \\
400 \\
440 \\
460 \\
420\end{array}$ & $\begin{array}{l}280 \\
280 \\
300 \\
440\end{array}$ & $\begin{array}{r}950 \\
900 \\
1,110 \\
2,400 \\
3,200\end{array}$ & $\begin{array}{l}2,920 \\
2,500 \\
2,100 \\
1,820 \\
1,650\end{array}$ & $\begin{array}{r}492 \\
474 \\
456 \\
538 \\
2,470\end{array}$ & 858 & $\begin{array}{l}195 \\
190 \\
185 \\
179 \\
170\end{array}$ & $\begin{array}{l}202 \\
192 \\
188 \\
179 \\
197\end{array}$ \\
\hline $\begin{array}{r}6 \\
7 \\
8 \\
9 \\
10\end{array}$ & $\begin{array}{l}184 \\
202 \\
270 \\
492 \\
567\end{array}$ & $\begin{array}{l}675 \\
685 \\
576 \\
615 \\
705\end{array}$ & $\begin{array}{l}800 \\
750 \\
650 \\
550 \\
500\end{array}$ & $\begin{array}{l}600 \\
550 \\
500 \\
480 \\
400\end{array}$ & $\begin{array}{l}420 \\
400 \\
400 \\
400 \\
360\end{array}$ & & $\begin{array}{l}3,600 \\
4,000 \\
4,400 \\
5,180 \\
3,820\end{array}$ & $\begin{array}{l}1,520 \\
1,380 \\
1,240 \\
1,210 \\
1,240\end{array}$ & $\begin{array}{l}2,920 \\
3,260 \\
4,640 \\
5,400 \\
3,720\end{array}$ & 520 & $\begin{array}{l}174 \\
170 \\
162 \\
154 \\
158\end{array}$ & $\begin{array}{l}166 \\
192 \\
170 \\
170 \\
215\end{array}$ \\
\hline 5 & $\begin{array}{l}529 \\
412 \\
326 \\
284 \\
259\end{array}$ & $\begin{array}{l}695 \\
625 \\
576 \\
447 \\
483\end{array}$ & $\begin{array}{l}420 \\
420 \\
320 \\
300 \\
280\end{array}$ & $\begin{array}{l}400 \\
380 \\
360 \\
340 \\
280\end{array}$ & $\begin{array}{l}340 \\
340 \\
320\end{array}$ & & $\begin{array}{l}3,820 \\
4,330 \\
4,120 \\
3,530 \\
3,260\end{array}$ & $\begin{array}{l}1,280 \\
1,490 \\
1,580 \\
1,460 \\
1,280\end{array}$ & $\begin{array}{l}2,740 \\
2,180 \\
1,730 \\
1,390 \\
1,140\end{array}$ & $\begin{array}{l}319 \\
298\end{array}$ & $\begin{array}{l}150 \\
150 \\
154 \\
150 \\
146\end{array}$ & $\begin{array}{l}284 \\
284 \\
248 \\
226 \\
215\end{array}$ \\
\hline$\cdots-\cdots$ & $\begin{array}{l}242 \\
232 \\
215 \\
220 \\
232\end{array}$ & $\begin{array}{l}605 \\
665 \\
625 \\
586 \\
635\end{array}$ & $\begin{array}{l}280 \\
280 \\
300 \\
280 \\
260\end{array}$ & $\begin{array}{l}280 \\
280 \\
300 \\
300 \\
300\end{array}$ & & & $\begin{array}{l}3,000 \\
2,500 \\
2,030 \\
1,820 \\
1,480\end{array}$ & $\begin{array}{l}1,390 \\
1,980 \\
2,100 \\
2,020 \\
1,760\end{array}$ & $\begin{array}{l}978 \\
822 \\
685 \\
510 \\
429\end{array}$ & $\begin{array}{l}291 \\
284 \\
270 \\
259 \\
248\end{array}$ & $\begin{array}{l}146 \\
146 \\
154 \\
158 \\
162\end{array}$ & $\begin{array}{l}210 \\
202 \\
202 \\
197 \\
202\end{array}$ \\
\hline $\begin{array}{l}21 \\
22 \\
24 \\
25\end{array}$ & $\begin{array}{l}237 \\
259 \\
277 \\
364 \\
456\end{array}$ & $\begin{array}{l}846 \\
870 \\
705 \\
615 \\
615\end{array}$ & $\begin{array}{l}300 \\
320 \\
340 \\
340 \\
320\end{array}$ & $\begin{array}{l}320 \\
550 \\
600 \\
500 \\
500\end{array}$ & 300 & $\begin{array}{l}1,100 \\
1,300 \\
3,600 \\
2,800 \\
1,800\end{array}$ & $\begin{array}{l}2,260 \\
3,820 \\
5,290 \\
4,860 \\
3,920\end{array}$ & $\begin{array}{l}2,000 \\
2,260 \\
1,900 \\
1,560 \\
1,270\end{array}$ & $\begin{array}{l}372 \\
340 \\
380 \\
558 \\
685\end{array}$ & $\begin{array}{l}237 \\
237 \\
232 \\
215 \\
215\end{array}$ & $\begin{array}{l}170 \\
188 \\
206 \\
232 \\
226\end{array}$ & $\begin{array}{l}226 \\
264 \\
438 \\
465 \\
429\end{array}$ \\
\hline $\begin{array}{l}26 \\
27 \\
28 \\
29 \\
29 \\
30 \\
31 \\
\end{array}$ & $\begin{array}{l}538 \\
548 \\
529 \\
474 \\
429 \\
404\end{array}$ & $\begin{array}{l}529 \\
492 \\
520 \\
483 \\
510\end{array}$ & $\begin{array}{l}320 \\
320 \\
280 \\
240 \\
240 \\
260\end{array}$ & $\begin{array}{l}420 \\
420 \\
420 \\
400 \\
400 \\
380\end{array}$ & & $\begin{array}{r}1,600 \\
1,300 \\
1,200 \\
1,100 \\
1,000 \\
950\end{array}$ & $\begin{array}{l}3,170 \\
2,740 \\
2,500 \\
2,660 \\
3,000\end{array}$ & $\begin{array}{r}1,100 \\
900 \\
834 \\
766 \\
655 \\
567\end{array}$ & $\begin{array}{r}625 \\
766 \\
954 \\
1,080 \\
1,090 \\
\end{array}$ & $\begin{array}{l}215 \\
220 \\
215 \\
210 \\
205 \\
200\end{array}$ & $\begin{array}{l}188 \\
174 \\
215 \\
202 \\
202\end{array}$ & $\begin{array}{l}356 \\
305 \\
277 \\
264 \\
254\end{array}$ \\
\hline
\end{tabular}

NOTE.-Discharge for the following periods when water-stage recorder was not operating satisfactorily estimated by comparison with recoids of flow of near-by streams: Jan. 3-5, 7, 18, 19, 21, 22, Feb. 1,2,14-28, Mar. 1, 2, 5-16, 23, 26-30, Apr. 1,2,4-6, May 25, 29-31, June 1, 4, 6-8, 11-15, 21, 22, 24, 25, 29, July $1-13,17-20$, and July 28 to Aug. 3. Discharge, Dec. 5 to Apr. 8, determined from gage heights corrected for ice effect by means of three discharge measurements, study of gage-height graph and weather records, and comparison with records of flow of near-by streams.

Monthly discharge of St. Regis River at Brasher Center, N. Y., for the year ending Seplember 30, 1923

[Drainage area, 621 square miles]

\begin{tabular}{|c|c|c|c|c|c|}
\hline \multirow{2}{*}{ Month } & \multicolumn{4}{|c|}{ Discharge in second-feet } & \multirow{2}{*}{$\underset{\text { inches }}{\substack{\text { Run-off i n }\\
}}$} \\
\hline & Maximum & Minimum & Mean & $\begin{array}{c}\text { Per square } \\
\text { mile }\end{array}$ & \\
\hline $\begin{array}{l}\text { October } \\
\text { November } \\
\text { December. } \\
\text { January } \\
\text { February } \\
\text { March } \\
\text { April } \\
\text { May } \\
\text { June } \\
\text { July } \\
\text { August } \\
\text { September }\end{array}$ & $\begin{array}{r}567 \\
870 \\
894 \\
1,200 \\
460 \\
3,600 \\
5,290 \\
2.920 \\
5,400 \\
858 \\
232 \\
465\end{array}$ & $\begin{array}{r}179 \\
326 \\
240 \\
280 \\
280 \\
900 \\
567 \\
340 \\
200 \\
146 \\
166\end{array}$ & $\begin{array}{r}326 \\
578 \\
442 \\
473 \\
342 \\
1,100 \\
3,120 \\
1,540 \\
1,460 \\
370 \\
176 \\
247\end{array}$ & $\begin{array}{c}0.525 \\
.931 \\
.712 \\
.762 \\
.551 \\
1.77 \\
5.02 \\
2.48 \\
2.35 \\
.596 \\
.283 \\
.398\end{array}$ & $\begin{array}{r}0.61 \\
1.04 \\
.82 \\
.88 \\
.57 \\
2.04 \\
5.60 \\
2.86 \\
2.62 \\
.69 \\
.33 \\
.44\end{array}$ \\
\hline The year & 5.400 & 146 & 847 & 1. 36 & 18.50 \\
\hline
\end{tabular}




\section{RIOHELIEU RIVER ÁT FORT MONTGOMERY, ROUSES POINT, N. Y.}

Location.-Inside fort, three-eighths of a mile south of international boundary, half a mile above head of Richelieu River, outlet of Lake Champlain, and 1 mile northeast of Rouses Point, Clinton County.

Drainage area.-7,870 square miles, including 436 square miles of water surface (from annual report of New York State Engineer and Surveyor).

Records available.- - 1875 to September 30, 1923.

GAGE.-Distance to water surface from reference point on curb of well inside the fort measured by a staff, graduated to feet and tenths, during portion of year. At other times a temporary staff gage, set by water level, fastened to a pile just below the Rutland railroad bridge, and about 25 feet from shore, was read, and readings reduced to datum of gage at the fort. Gages read by Thomas Bourke. Elevation of gage zero, 92.50 feet above mean sea level.

Extremes of STAGk.-Maximum elevation recorded during year, 98.7 feet at 10 a. m. May 3; minimum elevation recorded, 92.9 feet at 10 a. m. September 21 .

1869-1923: Maximum elevation recorded, 103.28 feet April, 1869; minimum elevation recorded, 91.9 feet November 13, 1908.

Cooperation.-Gage heights observed under direction of the Corps of Engineers of the United States Army and reported monthly to the United States Geological Survey.

Daily gage height, in feet, of Richelieu River at Fort Montgomery, Rouses Point, N. Y., for the year ending September 30,1923

\begin{tabular}{|c|c|c|c|c|c|c|c|c|c|c|c|c|}
\hline I & Oct. & Nov. & Dec. & Jan. & Feb. & Mar. & Apr. & May & June & July & Aug. & Sept. \\
\hline $\begin{array}{l}1 \ldots \\
2 \ldots \\
3 \ldots \\
4 \ldots \\
5 \ldots\end{array}$ & $\begin{array}{l}1.15 \\
1.15 \\
1.05 \\
1.1 \\
.95\end{array}$ & $\begin{array}{l}0.55 \\
.65 \\
.6 \\
.6 \\
.6\end{array}$ & $\begin{array}{l}0.9 \\
.4 \\
.7 \\
.6 \\
1.05\end{array}$ & $\begin{array}{l}0.6 \\
.65 \\
.85 \\
.95 \\
1.1\end{array}$ & $\begin{array}{l}1.2 \\
1.2 \\
1.15 \\
1.15 \\
1.15\end{array}$ & $\begin{array}{l}1.05 \\
1.1 \\
1.1 \\
1.15 \\
1.15\end{array}$ & $\begin{array}{l}2.35 \\
2.35 \\
2.4 \\
2.85 \\
3.1\end{array}$ & $\begin{array}{l}6.1 \\
6.15 \\
6.2 \\
6.15 \\
6.05\end{array}$ & $\begin{array}{l}4.55 \\
4.4 \\
4.3 \\
4.25 \\
4.2\end{array}$ & $\begin{array}{l}3.05 \\
3.0 \\
2.95 \\
2.95 \\
2.85\end{array}$ & $\begin{array}{l}1.75 \\
1.65 \\
1.65 \\
1.55 \\
1.5\end{array}$ & $\begin{array}{l}0.8 \\
.9 \\
.85 \\
.8 \\
.9\end{array}$ \\
\hline $\begin{array}{r}6 \\
7 \\
8 \\
9 \\
9\end{array}$ & $\begin{array}{l}.95 \\
1.0 \\
1.1 \\
1.0 \\
.95\end{array}$ & $\begin{array}{l}.55 \\
.8 \\
.65 \\
.65 \\
.75\end{array}$ & $\begin{array}{l}.85 \\
.8 \\
.6 \\
.5 \\
.5\end{array}$ & $\begin{array}{l}1.05 \\
1.05 \\
1.05 \\
1.05 \\
1.05\end{array}$ & $\begin{array}{l}1.1 \\
1.1 \\
1.1 \\
1.1 \\
1.05\end{array}$ & $\begin{array}{l}1.15 \\
1.15 \\
1.15 \\
1.15 \\
1.15\end{array}$ & $\begin{array}{l}3.6 \\
4.15 \\
4.6 \\
4.85 \\
5.15\end{array}$ & $\begin{array}{l}6.0 \\
5.95 \\
5.95 \\
5.9 \\
5.8\end{array}$ & $\begin{array}{l}4.15 \\
4.25 \\
4.4 \\
4.25 \\
4.45\end{array}$ & $\begin{array}{l}2.75 \\
2.65 \\
2.65 \\
2.7 \\
2.6\end{array}$ & $\begin{array}{l}1.45 \\
1.65 \\
1.35 \\
1.35 \\
1.35\end{array}$ & $\begin{array}{l}.8 \\
.95 \\
.9 \\
.7 \\
.65\end{array}$ \\
\hline 50 & $\begin{array}{l}1.0 \\
1.3 \\
.95 \\
1.3 \\
1.35\end{array}$ & $\begin{array}{l}.85 \\
.45 \\
.5 \\
.8 \\
.5\end{array}$ & $\begin{array}{l}.45 \\
.55 \\
.5 \\
.5 \\
.5\end{array}$ & $\begin{array}{l}1.1 \\
1.1 \\
1.15 \\
1.2 \\
1.3\end{array}$ & $\begin{array}{l}1.05 \\
1.05 \\
1.05 \\
1.1 \\
1.1\end{array}$ & $\begin{array}{l}1.15 \\
1.15 \\
1.2 \\
1.2 \\
1.2\end{array}$ & $\begin{array}{l}5.3 \\
5.35 \\
5.45 \\
5.45 \\
5.6\end{array}$ & $\begin{array}{l}5.7 \\
5.5 \\
5.5 \\
5.4 \\
5.4\end{array}$ & $\begin{array}{l}4.55 \\
4.4 \\
4.45 \\
4.25 \\
4.3\end{array}$ & $\begin{array}{l}2.55 \\
2.4 \\
2.35 \\
2.3 \\
2.25\end{array}$ & $\begin{array}{l}1.3 \\
1.25 \\
1.2 \\
1.35 \\
1.15\end{array}$ & $\begin{array}{l}.7 \\
.75 \\
.7 \\
.7 \\
.65\end{array}$ \\
\hline 0 & $\begin{array}{r}.95 \\
1.05 \\
.95 \\
.95 \\
.85\end{array}$ & $\begin{array}{l}.5 \\
.85 \\
.6 \\
.5 \\
.6\end{array}$ & $\begin{array}{l}.5 \\
.45 \\
.45 \\
.45 \\
.45\end{array}$ & $\begin{array}{l}1.3 \\
1.3 \\
1.3 \\
1.3 \\
1.3\end{array}$ & $\begin{array}{l}1.1 \\
1.1 \\
1.1 \\
1.1 \\
1.1\end{array}$ & $\begin{array}{l}1.2 \\
1.25 \\
1.4 \\
1.45 \\
1.5\end{array}$ & $\begin{array}{l}5.6 \\
5.5 \\
5.5 \\
5.45 \\
5.4\end{array}$ & $\begin{array}{l}5.45 \\
5.3 \\
5.35 \\
5.35 \\
5.4\end{array}$ & $\begin{array}{l}4.25 \\
4.1 \\
4.1 \\
4.1 \\
3.9\end{array}$ & $\begin{array}{l}2.15 \\
2.15 \\
2.15 \\
2.15 \\
2.1\end{array}$ & $\begin{array}{l}1.2 \\
1.2 \\
1.2 \\
1.6 \\
1.05\end{array}$ & $\begin{array}{l}.6 \\
.55 \\
.6 \\
.4 \\
.5\end{array}$ \\
\hline $\begin{array}{l}21-22 \\
23 \\
24 \\
25\end{array}$ & $\begin{array}{l}.8 \\
.8 \\
1.3 \\
.75 \\
1.1\end{array}$ & $\begin{array}{l}.6 \\
.6 \\
.7 \\
.55 \\
.45\end{array}$ & $\begin{array}{l}.45 \\
.5 \\
.5 \\
.5 \\
.5\end{array}$ & $\begin{array}{l}\text { 1. } 3 \\
1.3 \\
1.35 \\
1.4 \\
1.4\end{array}$ & $\begin{array}{l}1.1 \\
1.1 \\
1.1 \\
1.1 \\
1.1\end{array}$ & $\begin{array}{l}1.6 \\
1.65 \\
1.7 \\
1.95 \\
2.1\end{array}$ & $\begin{array}{l}5.35 \\
5.4 \\
5.35 \\
5.55 \\
5.55\end{array}$ & $\begin{array}{l}5.4 \\
5.4 \\
5.35 \\
5.25 \\
5.2\end{array}$ & $\begin{array}{l}\text { 3. } 7 \\
3.6 \\
\text { 3. } 55 \\
3.5 \\
3.4\end{array}$ & $\begin{array}{l}2.15 \\
1.95 \\
1.9 \\
2.05 \\
1.9\end{array}$ & $\begin{array}{r}1.15 \\
.95 \\
.95 \\
.95 \\
.95\end{array}$ & $\begin{array}{l}.4 \\
.5 \\
.65 \\
.65 \\
.6\end{array}$ \\
\hline $\begin{array}{l}26 \\
27 \\
28 \\
29 \\
30 \\
31\end{array}$ & $\begin{array}{l}.55 \\
.5 \\
.5 \\
.55 \\
.55 \\
.55\end{array}$ & $\begin{array}{l}.45 \\
.85 \\
.6 \\
.55 \\
.5\end{array}$ & $\begin{array}{l}.5 \\
.5 \\
.5 \\
.55 \\
.6 \\
.6\end{array}$ & $\begin{array}{l}\text { 1. } 35 \\
1.3 \\
1.3 \\
1.25 \\
1.25 \\
1.2\end{array}$ & $\begin{array}{r}1.05 \\
1.05 \\
1.05 \\
\end{array}$ & $\begin{array}{l}2.15 \\
2.3 \\
2.4 \\
2.4 \\
2.35 \\
2.35\end{array}$ & $\begin{array}{l}5.85 \\
5.75 \\
5.75 \\
5.85 \\
6.0\end{array}$ & $\begin{array}{l}5.05 \\
4.95 \\
4.85 \\
4.7 \\
4.65 \\
4.55\end{array}$ & $\begin{array}{l}3.4 \\
3.3 \\
3.2 \\
3.15 \\
3.1 \\
\end{array}$ & $\begin{array}{l}1.8 \\
1.95 \\
1.7 \\
1.7 \\
1.7 \\
1.85\end{array}$ & $\begin{array}{c}.95 \\
.95 \\
1.25 \\
.85 \\
.9 \\
.9\end{array}$ & $\begin{array}{l}.65 \\
.75 \\
.65 \\
.65\end{array}$ \\
\hline
\end{tabular}

${ }^{4}$ Hoyt, J. C., U. S. Geol. Supply Water-Supply Paper 97, p. 340. 
LAKE CHAMPLAIN AT BURLINGTON, VT.

Location.-On south side of roadway leading to dock of Champlain Transportation Co., at foot of King Street, Burlington, Chittenden County.

Records avallable.--May 1, 1907, to September 30, 1923.

GAGE.-Staff:- Comparisons of gage readings indicate that zero of gage at Burlington is at practically the same elevation as that of gage at Fort Montgomery, 92.5 feet above mean sea level. Gage read by employee of the Champlain Transportation Co.

Extremes of Discharge.-Maximum stage recorded during year, 6.53 feet May 3; minimum stage recorded, 0.74 foot December 23 .

1907-1923: Maximum stage recorded, 8.22 feet April 19, 1922; minimum stage, -0.25 foot December $4,1908$.

IcE.-Wider parts of Lake Champlain not usually frozen over until the latter part of January. Occasionally closure does not occur until February, and in some years it lasts only for a few days. The northern end of the lake above the outlet is usually covered with ice from the middle of December to the middle of April.

Accuracy.-Gage read to hundredths at irregular intervals. Gage readings made when the lake is rough subject to inaccuracies due to wave action.

Cooperation.-Gage-height record furnished by D. A. Loomis, general manager of the Champlain Transportation Co.

Daily gage height, in feet, of Lake Champlain at Burlington, Vt., for the year ending September 30, 1928

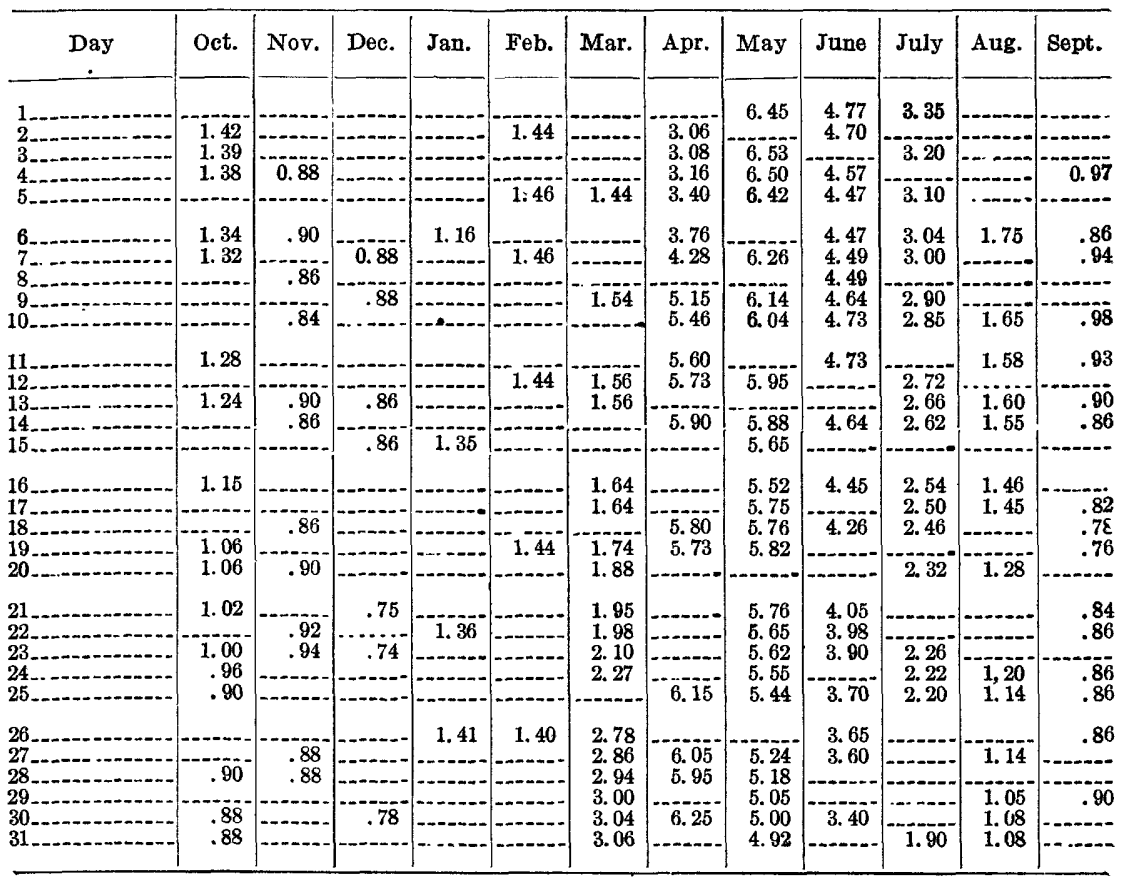


SARANAC RIVER NEAR PLATTSBURG, N, Y.

LocATIon.-At Indian Rapids power plant (formerly known as Lozier dam) of Plattsburg Gas \& Electric Co., 6 miles above mouth at Plattsburg, Clinton County.

Drainage area. -607 square miles (measured on topographic maps).

Records avallable.-March 27, 1903, to September 30, 1923.

GAGES.-Gage showing elevation of water surface above intake to power plant is a Gurley seven-day graph water-stage recorder installed November 12, 1919 , on retaining wall above power house on right side of river. Before that date the crest gage was a vertical staff on the angle of the wing wall at the end of the racks. Datum raised 0.76 foot August 20, 1906. Tailrace gage, a vertical staff spiked to timber-work dike between tailrace and river and about 50 feet below power house. Records of kilowatt output are obtained at half hour intervals. Inclined staff gage at cable station, a quarter of a mile below dam. Gages and watt meters read by power-house operators.

Discharge measurements.-Made from a cable at head of Indian Rapids, a quarter of a mile below dam, or by wading under cable or in tailrace.

Determination of Drscharge.-Records include the discharge over concrete spillway which has been rated by current-meter measurements; the discharge through two power units equipped with 300-kilowatt generators which have also been rated by current-meter measurements; and the discharge through two 5-foot waste gates when open, the rating for which is theoretical.

Extremes of DIscharge.-Maximum mean daily discharge during year, 3,250 second-feet June 9; minimum mean daily discharge, 65 second-feet January 7.

1903-1923: Maximum mean daily discharge recorded, 6,410 second-feet April 20, 1914; minimum mean daily discharge recorded, 15 second-feet. August 4, 1908.

IcE.-The crest of the spillway is kept free from ice so that the stage-discharge relation is not affected.

RegULATION.-The lakes and ponds on the main stream and tributaries above the station comprise a water surface area of about 25.5 square miles. The actual storage afforded by these reservoirs has been largely increased by the State dam at lower Saranac Lake, the operation of which affects distribution of flow during the year.

Accuracy. - Stage-discharge relation permanent. Spillway rating curve fairly well defined between 100 and 5,000 second-feet; turbine rating fairly well defined throughout. Operation of water-stage recorder satisfactory. Discharge over the spillway ascertained by averaging discharge for intervals of day. Discharge through the turbines ascertained by applying to their ratings the mean kilowatt output and head for periods of run. Records fair.

Cooperation.--Record of power-plant operation furnished by Plattsburg Gas \& Electric Co. 
Discharge measurements of Saranac River near Plattsburg, N. Y., during the year ending September 30,1923

[Made by Lamson and Johnson]

\begin{tabular}{|c|c|c|c|c|c|c|}
\hline & Date & $\begin{array}{c}\text { Gage } \\
\text { height }\end{array}$ & $\begin{array}{l}\text { Unit } \\
\text { No. }\end{array}$ & Output & Head & $\begin{array}{l}\text { Dis- } \\
\text { charge }\end{array}$ \\
\hline July & 18 & $\begin{array}{r}\begin{array}{r}\text { Feet } \\
0.78 \\
.42\end{array} \\
\\
\end{array}$ & $\begin{array}{r}2 \\
2 \\
2 \\
2 \\
1 \\
1 \\
1\end{array}$ & \begin{tabular}{r}
\multicolumn{1}{c}{$\begin{array}{c}\text { Kilo- } \\
\text { watts }\end{array}$} \\
\hdashline 200 \\
150 \\
100 \\
50 \\
150 \\
100 \\
50
\end{tabular} & $\begin{array}{c}\text { Feet } \\
15.32 \\
15.21 \\
15.40 \\
15.54 \\
15.24 \\
15.37 \\
15.72\end{array}$ & $\begin{aligned} & \text { Sec. } f t: \\
& \text { a } 388 \\
& \text {. } 158 \\
& \text { b } 288 \\
& \text { b } 248 \\
& \text { b } 220 \\
& \text { b } 182 \\
& \text { b } 250 \\
& \text { b } 229 \\
& \text { b } 190\end{aligned}$ \\
\hline
\end{tabular}

a Discharge over spillway only. $\quad b$ Measurement made in tailrace; spillway excluded.

Daily discharge, in second-feet, of Saranac River near Plattsburg, N. Y., for the year ending September 30, 1923

\begin{tabular}{|c|c|c|c|c|c|c|c|c|c|c|c|c|}
\hline Day & oct & Nor. & Dec. & Jan. & Feb. & Mar. & Apr. & May & June & July & Aug. & Sept. \\
\hline $\begin{array}{l}1 \ldots \\
2 . . . \\
3 \ldots \\
4 \ldots \\
5 \ldots\end{array}$ & 200 & $\begin{array}{l}395 \\
395 \\
335 \\
340 \\
248\end{array}$ & $\begin{array}{l}204 \\
194 \\
295 \\
560 \\
485\end{array}$ & $\begin{array}{l}350 \\
570 \\
455 \\
440 \\
470\end{array}$ & $\begin{array}{l}310 \\
315 \\
315 \\
142 \\
335\end{array}$ & $\begin{array}{l}300 \\
300 \\
380 \\
325 \\
395\end{array}$ & $\begin{array}{r}234 \\
440 \\
495 \\
920 \\
1,400\end{array}$ & $\begin{array}{l}1,960 \\
1,800 \\
1,640 \\
1,420 \\
1,340\end{array}$ & $\begin{array}{l}830 \\
600 \\
355 \\
660 \\
690\end{array}$ & $\begin{array}{l}530 \\
590 \\
660 \\
610 \\
610\end{array}$ & $\begin{array}{l}390 \\
370 \\
460 \\
345 \\
405\end{array}$ & $\begin{array}{l}315 \\
305 \\
335\end{array}$ \\
\hline $\begin{array}{r}6 \\
7 \\
8 \\
9 \\
10\end{array}$ & 248 & $\begin{array}{l}360 \\
230 \\
250 \\
305 \\
265\end{array}$ & $\begin{array}{l}380 \\
290 \\
380 \\
500 \\
370\end{array}$ & $\begin{array}{c}28 a \\
65 \\
330 \\
190 \\
244\end{array}$ & $\begin{array}{l}148 \\
196 \\
300 \\
290 \\
290\end{array}$ & $\begin{array}{l}265 \\
310 \\
345 \\
305 \\
186\end{array}$ & $\begin{array}{l}2,320 \\
2,650 \\
1,980 \\
2,280 \\
2,260\end{array}$ & $\begin{array}{l}1,280 \\
1,200 \\
1,120 \\
1,060 \\
1,040\end{array}$ & $\begin{array}{l}1,240 \\
1,980 \\
2,400 \\
3,250 \\
2,750\end{array}$ & $\begin{array}{l}510 \\
530 \\
370 \\
580 \\
480\end{array}$ & $\begin{array}{l}495 \\
500 \\
490 \\
465 \\
370\end{array}$ & $\begin{array}{l}325 \\
300 \\
365 \\
310 \\
180\end{array}$ \\
\hline ....... & $\begin{array}{l}345 \\
400 \\
365 \\
370 \\
265\end{array}$ & $\begin{array}{l}228 \\
194 \\
330 \\
182 \\
246\end{array}$ & $\begin{array}{l}470 \\
385 \\
400 \\
280 \\
390\end{array}$ & $\begin{array}{l}275 \\
315 \\
265 \\
144 \\
275\end{array}$ & $\begin{array}{l}410 \\
415 \\
300\end{array}$ & $\begin{array}{l}345 \\
500 \\
485 \\
460 \\
430\end{array}$ & $\begin{array}{l}2,320 \\
2,950 \\
2,850 \\
2,180 \\
1,900\end{array}$ & $\begin{array}{r}990 \\
930 \\
1,060 \\
985 \\
1,040\end{array}$ & $\begin{array}{l}2,400 \\
2,100 \\
1,640 \\
1,440 \\
1,220\end{array}$ & $\begin{array}{l}550 \\
420 \\
415 \\
285 \\
415\end{array}$ & $\begin{array}{l}300 \\
242 \\
305 \\
420 \\
320\end{array}$ & $\begin{array}{l}246 \\
290 \\
238 \\
206 \\
168\end{array}$ \\
\hline 20 & $\begin{array}{l}440 \\
350 \\
355 \\
325 \\
370\end{array}$ & $\begin{array}{l}172 \\
270 \\
244 \\
164 \\
154\end{array}$ & $\begin{array}{l}360 \\
196 \\
360 \\
265 \\
265\end{array}$ & $\begin{array}{l}216 \\
192 \\
260 \\
325 \\
280\end{array}$ & $\begin{array}{l}420 \\
335 \\
465\end{array}$ & $\begin{array}{l}510 \\
630 \\
540 \\
590 \\
390\end{array}$ & $\begin{array}{l}1,720 \\
1,520 \\
1,300 \\
1,080 \\
1,100\end{array}$ & $\begin{array}{l}1,320 \\
1,700 \\
1,740 \\
1,560 \\
1,380\end{array}$ & $\begin{array}{r}1,120 \\
830 \\
850 \\
710 \\
700\end{array}$ & $\begin{array}{l}340 \\
385 \\
435 \\
410 \\
485\end{array}$ & $\begin{array}{l}320 \\
280 \\
265 \\
228 \\
325\end{array}$ & $\begin{array}{l}218 \\
204 \\
280 \\
400\end{array}$ \\
\hline 24 & $\begin{array}{l}375 \\
355 \\
345 \\
170 \\
340\end{array}$ & $\begin{array}{l}164 \\
200 \\
190 \\
164 \\
192\end{array}$ & $\begin{array}{r}375 \\
290 \\
196 \\
90 \\
186\end{array}$ & $\begin{array}{l}170 \\
440 \\
400 \\
275 \\
295\end{array}$ & $\begin{array}{l}440 \\
430 \\
390 \\
320 \\
285\end{array}$ & $\begin{array}{r}510 \\
510 \\
750 \\
1,680 \\
920\end{array}$ & $\begin{array}{l}2,200 \\
3,050 \\
3,000 \\
2,550 \\
2,200\end{array}$ & $\begin{array}{l}1,460 \\
1,440 \\
1,360 \\
1,180 \\
1,140\end{array}$ & $\begin{array}{l}650 \\
630 \\
560 \\
485 \\
560\end{array}$ & $\begin{array}{l}385 \\
315 \\
440 \\
390 \\
455\end{array}$ & $\begin{array}{l}370 \\
248 \\
246 \\
226 \\
192\end{array}$ & $\begin{array}{l}450 \\
550 \\
280 \\
206 \\
335\end{array}$ \\
\hline $\begin{array}{l}26 \\
27 \\
28 \\
29 \\
30 \\
31\end{array}$ & $\begin{array}{r}380 \\
255 \\
310 \\
83 \\
206 \\
320\end{array}$ & $\begin{array}{l}110 \\
220 \\
204 \\
192 \\
214\end{array}$ & $\begin{array}{l}370 \\
380 \\
188 \\
198 \\
246 \\
224\end{array}$ & $\begin{array}{l}320 \\
270 \\
178 \\
400 \\
375 \\
305\end{array}$ & \begin{tabular}{r}
300 \\
385 \\
285 \\
\hdashline \\
\end{tabular} & $\begin{array}{l}670 \\
440 \\
375 \\
440 \\
485 \\
445\end{array}$ & $\begin{array}{l}2,060 \\
1,960 \\
1,820 \\
2,100 \\
2,140 \\
\end{array}$ & $\begin{array}{r}1,080 \\
760 \\
980 \\
910 \\
940 \\
950\end{array}$ & $\begin{array}{l}560 \\
630 \\
620 \\
610 \\
650 \\
\end{array}$ & $\begin{array}{l}460 \\
380 \\
410 \\
228 \\
570 \\
430\end{array}$ & $\begin{array}{l}285 \\
220 \\
280 \\
275 \\
400 \\
415\end{array}$ & $\begin{array}{l}260 \\
285 \\
250 \\
255 \\
222\end{array}$ \\
\hline
\end{tabular}

Nore.-Mean discharge, Oct. 1-9, estimated by comparison with record of Ausable River at Ausabl Forks; power plant shut down for repairs, pond drained and total discharge passed through waste gates. 
Monthly discharge of Saranac River near Plattsburg, N.Y., for the year ending September 30, 1923

[Drainage area, 607 square miles]

\begin{tabular}{|c|c|c|c|c|c|}
\hline \multirow{2}{*}{ Month } & \multicolumn{4}{|c|}{ Discharge in second-feet } & \multirow{2}{*}{$\begin{array}{c}\text { Run-off in } \\
\text { inches }\end{array}$} \\
\hline & Maximum & Minimum & Mean & $\underset{\text { Per square }}{\text { mile }}$ & \\
\hline $\begin{array}{l}\text { October } \\
\text { November } \\
\text { December } \\
\text { January } \\
\text { February } \\
\text { March } \\
\text { April } \\
\text { May } \\
\text { June } \\
\text { July } \\
\text { August } \\
\text { September }\end{array}$ & $\begin{array}{r}440 \\
395 \\
560 \\
570 \\
500 \\
1,680 \\
3,050 \\
1,960 \\
3,250 \\
660 \\
500 \\
550\end{array}$ & $\begin{array}{r}83 \\
110 \\
90 \\
65 \\
142 \\
186 \\
234 \\
760 \\
355 \\
228 \\
192 \\
168\end{array}$ & $\begin{array}{r}283 \\
239 \\
315 \\
302 \\
328 \\
491 \\
1,900 \\
1,250 \\
1,120 \\
454 \\
337 \\
290\end{array}$ & $\begin{array}{l}0.466 \\
.394 \\
.519 \\
.498 \\
.540 \\
.809 \\
3.13 \\
2.06 \\
1.85 \\
.748 \\
.555 \\
.478\end{array}$ & $\begin{array}{r}0.54 \\
.44 \\
.60 \\
.57 \\
.56 \\
.93 \\
3.49 \\
2.38 \\
2.06 \\
.86 \\
.64 \\
.53\end{array}$ \\
\hline The year ... & 3,250 & 65 & 609 & 1.00 & 13.60 \\
\hline
\end{tabular}

NOTE.-See paragraph on "Regulation."

WEST BRANCH OF AUSABLE RIVER NEAR NEWMAN, N. Y.

Locatron.-On farm formerly owned by James Dudley, 4 miles northeast of Newman, Essex County, and 4 miles below Lake Placid.

Drainage area. - 116 square miles (measured on topographic maps).

Records aVarlable.-June 7, 1916, to December 31, 1917, and July 15, 1919; to September 30, 1923.

GAGE.-Staff, in two sections, on right bank; lower section, inclined, graduated

from 1.4 to 8.65 feet; upper section, vertical, graduated from 8.7 to 11 feet; read by Mrs. Ethel Fuller.

Discharge MEASUREMENTs.-Made from cable 300 feet above gage or by wading. Channel and control.-Solid rock; permanent.

ExTrEmes of DISCHARGE.-Maximum stage recorded during year, 6.41 feet at 8 a. m. April 22 (discharge, 2,750 second-feet); minimum stage recorded, 1.90 feet at 2 p. m. February 4 (discharge, 8 second-feet).

1916-17; 1919-1923: Maximum open-water stage recorded, 8.22 feet at 7 a. m. April 12, 1922 (discharge, about 6,300 second-feet); minimum stage recorded, 1.60 feet at 7.30 p. m. September 13, 1920, caused by closing gates in dam (discharge, practically zero).

Ice.--Stage-discharge relation usually affected by ice.

ACCURACY.- Stage-discharge relation practically permanent except as affected by ice from December to April. Rating curve fairly well defined between 30 and 1,000 second-feet; extended beyond these limits. Gage read to quarter-tenths twice daily, except December 10 to April 21, when it was read only once a day. Daily discharge ascertained by applying mean daily gage height to rating table. Records only fair, as mean daily gage height, determined from one or two gage readings, is subject to error owing to fluctuations in stage caused by operation of dams upstream.

$72038-26 \dagger$-wSP 564-9 
Discharge measurements of West Branch of Ausable River near Newman, N.Y., during the year ending September 30,1923

\begin{tabular}{|c|c|c|c|c|c|c|c|}
\hline Date & Madeby- & $\begin{array}{c}\text { Gage } \\
\text { height }\end{array}$ & $\begin{array}{l}\text { Dis- } \\
\text { charge }\end{array}$ & Date & Made by- & $\begin{array}{c}\text { Gage } \\
\text { height }\end{array}$ & $\begin{array}{c}\text { Dis- } \\
\text { charge }\end{array}$ \\
\hline 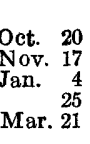 & \begin{tabular}{l} 
A.W. Harrington..... \\
J. L. Lamson \\
\hdashline \\
\hdashline
\end{tabular} & \begin{tabular}{c|} 
Feet \\
2.52 \\
2.79 \\
a2. \\
a 2. 26 \\
a 3.08
\end{tabular} & $\begin{array}{r}\text { Sec.ft. } \\
46.7 \\
98.7 \\
87.7 \\
44.4 \\
71.9\end{array}$ & $\begin{array}{ll}\text { May } & 2 \\
& 2 \\
& 26 \\
\text { July } & 16\end{array}$ & $\begin{array}{l}\text { E.B. Shupe } \\
\text { A.W. Ho Harrington } \\
\text { J.L. Lamson }\end{array}$ & $\begin{array}{l}\text { Feet } \\
3.84 \\
3.79 \\
3.25 \\
2.55\end{array}$ & \begin{tabular}{|c} 
Sec. $-f t$. \\
396 \\
380 \\
197 \\
52.6
\end{tabular} \\
\hline
\end{tabular}

a Stage-discharge relation affected by ice.

Daily discharge, in second-feet, of West Branch of Ausable River near Newman, N.Y., for the year ending September 30,1923

\begin{tabular}{|c|c|c|c|c|c|c|c|c|c|c|c|c|}
\hline Day & Oct. & Nov. & Dec. & Jan. & Feb. & Mar. & Apr. & May & June & July & Aug. & Sept. \\
\hline $\begin{array}{l}1 \ldots \\
2 \ldots \\
3 \ldots \\
4 \ldots \\
5 \ldots\end{array}$ & $\begin{array}{l}37 \\
34 \\
33 \\
34 \\
34\end{array}$ & $\begin{array}{l}44 \\
46 \\
61 \\
61 \\
73\end{array}$ & $\begin{array}{r}73 \\
130 \\
82 \\
100 \\
76\end{array}$ & $\begin{array}{r}90 \\
260 \\
160 \\
140 \\
70\end{array}$ & $\begin{array}{r}30 \\
38 \\
30 \\
8 \\
15\end{array}$ & $\begin{array}{r}50 \\
38 \\
48 \\
110 \\
85\end{array}$ & $\begin{array}{l}110 \\
130 \\
400 \\
480 \\
700\end{array}$ & $\begin{array}{l}534 \\
395 \\
395 \\
395 \\
534\end{array}$ & $\begin{array}{r}100 \\
67 \\
67 \\
100 \\
208\end{array}$ & $\begin{array}{r}142 \\
100 \\
87 \\
94 \\
87\end{array}$ & $\begin{array}{l}36 \\
37 \\
38 \\
35 \\
31\end{array}$ & $\begin{array}{l}37 \\
34 \\
36 \\
34 \\
35\end{array}$ \\
\hline $\begin{array}{r}6 \\
7 \\
8 \\
9 \\
9 \\
10 \\
-\end{array}$ & $\begin{array}{l}46 \\
39 \\
46 \\
94 \\
67\end{array}$ & $\begin{array}{r}64 \\
76 \\
113 \\
130 \\
87\end{array}$ & $\begin{array}{l}44 \\
66 \\
76 \\
87 \\
66\end{array}$ & $\begin{array}{l}36 \\
32 \\
32 \\
32 \\
32\end{array}$ & $\begin{array}{l}20 \\
15 \\
13 \\
11 \\
10\end{array}$ & $\begin{array}{l}50 \\
50 \\
36 \\
40 \\
36\end{array}$ & $\begin{array}{r}1,300 \\
1,060 \\
1,140 \\
830 \\
485\end{array}$ & $\begin{array}{l}395 \\
333 \\
353 \\
732 \\
700\end{array}$ & $\begin{array}{r}1,230 \\
1,020 \\
1,140 \\
975 \\
763\end{array}$ & $\begin{array}{l}80 \\
56 \\
66\end{array}$ & $\begin{array}{l}28 \\
29 \\
34 \\
40 \\
29\end{array}$ & $\begin{array}{r}31 \\
31 \\
40 \\
208 \\
130\end{array}$ \\
\hline 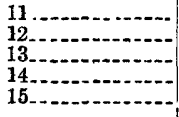 & $\begin{array}{l}90 \\
78 \\
61 \\
54 \\
64\end{array}$ & $\begin{array}{l}78 \\
67 \\
90 \\
64 \\
75\end{array}$ & $\begin{array}{l}46 \\
58 \\
46 \\
40 \\
34\end{array}$ & $\begin{array}{l}32 \\
32 \\
32 \\
32 \\
30\end{array}$ & $\begin{array}{l}11 \\
18 \\
19 \\
22 \\
26\end{array}$ & $\begin{array}{l}40 \\
24 \\
65 \\
36 \\
40\end{array}$ & $\begin{array}{l}586 \\
700 \\
417 \\
353 \\
333\end{array}$ & $\begin{array}{l}353 \\
240 \\
258 \\
294 \\
224\end{array}$ & $\begin{array}{l}439 \\
353 \\
313 \\
240 \\
179\end{array}$ & $\begin{array}{l}42 \\
41 \\
44\end{array}$ & $\begin{array}{l}28 \\
29 \\
30 \\
35 \\
31\end{array}$ & $\begin{array}{r}100 \\
76 \\
57 \\
52 \\
47\end{array}$ \\
\hline 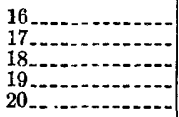 & $\begin{array}{l}72 \\
67 \\
54 \\
54 \\
51\end{array}$ & $\begin{array}{r}166 \\
87 \\
82 \\
98 \\
142\end{array}$ & $\begin{array}{l}28 \\
24 \\
26 \\
28 \\
28\end{array}$ & $\begin{array}{l}28 \\
26 \\
24 \\
22 \\
28\end{array}$ & $\begin{array}{l}26 \\
22 \\
24 \\
20 \\
24\end{array}$ & $\begin{array}{r}120 \\
230 \\
260 \\
70 \\
60\end{array}$ & $\begin{array}{l}275 \\
130 \\
120 \\
179 \\
153\end{array}$ & $\begin{array}{r}865 \\
1,520 \\
641 \\
534 \\
374\end{array}$ & $\begin{array}{r}100 \\
109 \\
120 \\
94 \\
83\end{array}$ & $\begin{array}{l}52 \\
70 \\
58 \\
44 \\
42\end{array}$ & $\begin{array}{l}31 \\
29 \\
29 \\
30 \\
28\end{array}$ & $\begin{array}{l}44 \\
45 \\
42 \\
36 \\
38\end{array}$ \\
\hline | & $\begin{array}{r}48 \\
44 \\
58 \\
130 \\
105\end{array}$ & $\begin{array}{r}153 \\
90 \\
90 \\
73 \\
56\end{array}$ & $\begin{array}{l}32 \\
36 \\
40 \\
46 \\
60\end{array}$ & $\begin{array}{l}50 \\
40 \\
60 \\
40 \\
46\end{array}$ & $\begin{array}{l}20 \\
24 \\
20 \\
15 \\
15\end{array}$ & $\begin{array}{r}75 \\
90 \\
440 \\
480 \\
200\end{array}$ & $\begin{array}{r}1,630 \\
2,210 \\
1,630 \\
763 \\
510\end{array}$ & $\begin{array}{l}395 \\
374 \\
313 \\
240 \\
208\end{array}$ & $\begin{array}{r}58 \\
61 \\
67 \\
111 \\
130\end{array}$ & $\begin{array}{l}38 \\
35 \\
45\end{array}$ & $\begin{array}{l}35 \\
57 \\
56 \\
46 \\
33\end{array}$ & $\begin{array}{r}179 . \\
130 \\
94 \\
73 \\
76\end{array}$ \\
\hline $\begin{array}{l}26 \\
27 \\
28 \\
29 \\
30 \\
31\end{array}$ & $\begin{array}{l}94 \\
90 \\
61 \\
48 \\
54 \\
51\end{array}$ & $\begin{array}{l}56 \\
61 \\
46 \\
58 \\
56\end{array}$ & $\begin{array}{l}50 \\
40 \\
36 \\
28 \\
28 \\
40\end{array}$ & $\begin{array}{l}40 \\
36 \\
24 \\
22 \\
20 \\
24\end{array}$ & $\begin{array}{r}30 \\
60 \\
55 \\
-0 \\
-0 \\
-0\end{array}$ & $\begin{array}{l}200 \\
190 \\
150 \\
130 \\
110 \\
110\end{array}$ & $\begin{array}{r}439 \\
417 \\
670 \\
1,740 \\
900\end{array}$ & $\begin{array}{l}208 \\
179 \\
153 \\
142 \\
100 \\
101\end{array}$ & $\begin{array}{r}92 \\
130 \\
120 \\
240 \\
258\end{array}$ & $\begin{array}{l}42 \\
42 \\
45 \\
38 \\
37 \\
38\end{array}$ & $\begin{array}{l}30 \\
34 \\
47 \\
90 \\
60 \\
48\end{array}$ & $\begin{array}{l}61 \\
46 \\
46 \\
48 \\
38\end{array}$ \\
\hline
\end{tabular}

Note.-Discharge interpolated Jan. 29 and Feb. 14; nogage-height record. Discharge Dec. 14 to April 6 determined from gage heights corrected for ice effect by means of three discharge measurements; study of observer's notes, gage-height graph, and weather records; and comparison with records of Ausable River at Ausable Forks, N. Y. 
Monthly discharge of West Branch of Ausable River near Newman, N. Y., for the year ending September 30,1923

[Drainage area, 116 square miles]

\begin{tabular}{|c|c|c|c|c|c|}
\hline \multirow{2}{*}{ Month } & \multicolumn{4}{|c|}{ Discharge in second-feet } & \multirow{2}{*}{$\begin{array}{l}\text { Run-off } \\
\text { in inches }\end{array}$} \\
\hline & Maximum & Minimum & Mean & $\underset{\text { mile }}{\text { Per square }}$ & \\
\hline $\begin{array}{l}\text { October } \\
\text { November } \\
\text { December } \\
\text { January } \\
\text { February } \\
\text { March } \\
\text { April } \\
\text { May } \\
\text { June } \\
\text { July } \\
\text { August } \\
\text { September }\end{array}$ & $\begin{array}{r}130 \\
166 \\
130 \\
260 \\
60 \\
480 \\
2,210 \\
1,520 \\
1,230 \\
142 \\
90 \\
208 \\
\end{array}$ & $\begin{array}{r}33 \\
44 \\
24 \\
20 \\
8 \\
24 \\
110 \\
100 \\
58 \\
35 \\
28 \\
31 \\
\end{array}$ & $\begin{array}{r}61.0 \\
81.4 \\
51.4 \\
50.7 \\
22.9 \\
120 \\
693 \\
403 \\
299 \\
58.9 \\
37.8 \\
64.8 \\
\end{array}$ & $\begin{array}{l}0.526 \\
.702 \\
.443 \\
.437 \\
.197 \\
1.03 \\
5.97 \\
3.47 \\
2.58 \\
.508 \\
.326 \\
.559 \\
\end{array}$ & $\begin{array}{r}0.61 \\
.78 \\
.51 \\
.50 \\
.21 \\
1.19 \\
6.66 \\
4.00 \\
2.88 \\
.59 \\
.38 \\
.62 \\
\end{array}$ \\
\hline (n) & 2,210 & 8 & 162 & 1.40 & 18.93 \\
\hline
\end{tabular}

AUSABLE RIVER AT AUSABLE FORKS, N. Y.

Location.-In Ausable Forks, Clinton County, immediately below junction of East and West branches and 15 miles above mouth of river.

Drainage area.-444 square miles (measured on topographic maps).

Records avarlable.-August 17, 1910, to September 30, 1923.

GAGE.-Chain on left bank 1,000 feet below junction of East and West branches; read by A. S. Baker.

Discharge measurements.-Made from a cable $1 \frac{1}{2}$ miles below gage or by wading either near cable or a short distance above gage.

Channer and controu.- Stone and gravel; occasionally shifting. Channel divided by an island opposite the gage.

Extremes of Discharge.-Maximum stage recorded during year, 6.60 feet at 8 a. m. April 29 (discharge, 6,490 second-feet); minimum stage recorded, 3.12 feet at 5 p. m. July 27 and August 12 (discharge, 24 second-feet).

1910-1923: Maximum stage recorded, 10.2 feet in the evening of March 27, 1913 (discharge, roughly 25,000 second-feet); minimum stage, 3 feet at 7 a. m. July 21, 1912 (discharge, practically zero).

IcE.-Stage-discharge relation slightly affected by ice.

Accoracy.-Stage-discharge relation practically permanent after October 19 except as affected by ice from December to March. Rating curve fairly well defined between 175 and 3,000 second-feet. Gage read to hundredths twice daily. Daily discharge ascertained by applying mean daily gage height to rating table; shifting-control method used October 1-19. Records only fair as mean daily gage height, determined from two gage readings, is subject to error owing to fluctuations in stage caused by operation of power plants upstream.

Discharge measurements of Ausable River at Ausable Forks, N. Y., during the year ending September 30, 1923

\begin{tabular}{|c|c|c|c|c|c|c|c|}
\hline Date & Made by- & $\begin{array}{l}\text { Gage } \\
\text { height }\end{array}$ & $\begin{array}{l}\text { Dis- } \\
\text { charge }\end{array}$ & Date & Made by- & $\begin{array}{c}\text { Gage } \\
\text { height }\end{array}$ & $\begin{array}{l}\text { Dis- } \\
\text { charge }\end{array}$ \\
\hline Oct. 20 & A. W. Harrington. & Feet & $\begin{array}{l}\text { Sec.-ft. } \\
247 \\
\end{array}$ & Jan. 24 & J. & $\begin{array}{l}\text { Feet } \\
\text { a.59 }\end{array}$ & Sec.-ft. \\
\hline $\begin{array}{l}\text { Nov. } 18 \\
\text { Jan. } 3\end{array}$ & J. L. Lamson & $\begin{array}{r}3.58 \\
3.68 \\
\\
3.82\end{array}$ & $\begin{array}{l}193 \\
302 \\
333\end{array}$ & $\begin{array}{ll}\text { May } & 1 \\
& 26 \\
\text { July } & 17\end{array}$ & A. W. Harrington & $\begin{array}{l}\text { 4. } 70 \\
\text { 4.08 } \\
3.56\end{array}$ & $\begin{array}{r}1,810 \\
670 \\
207\end{array}$ \\
\hline
\end{tabular}

- Stage-discharge relation affected by ice. 
Daily discharge, in second-feet, of Ausable River at Ausable Forks, N. Y., for the year ending September 30, 1923

\begin{tabular}{r|r|r|r|r|r|r|r|r|r|r|r|r}
\hline Day & Oct. & Nov. & Dec. & Jan. & Feb. & Mar. & Apr. & May & June & July & Aug. & Sept. \\
\hline & & & & & & & & & & & \\
\hline
\end{tabular}

Note.-Discharge Dec. 12 to Mar. 24 determined from gage heights corrected for ice effect by means of $t w 0$ discharge measurements and study of gage-height graph, weather records, and observer's notes.

Monthly discharge of Ausable River at Ausable Forks, N. Y., for the year ending September 30, 1923

[Drainage area, 444 square miles]

\begin{tabular}{|c|c|c|c|c|c|}
\hline \multirow{2}{*}{ Month } & \multicolumn{4}{|c|}{ Discharge in second-feet } & \multirow{2}{*}{$\begin{array}{c}\text { Run-off in } \\
\text { inches }\end{array}$} \\
\hline & Maximum & Minimum & Mean & $\begin{array}{c}\text { Per square } \\
\text { mile }\end{array}$ & \\
\hline $\begin{array}{l}\text { October } \\
\text { November } \\
\text { December } \\
\text { January } \\
\text { February } \\
\text { March } \\
\text { April } \\
\text { May } \\
\text { June } \\
\text { July } \\
\text { August } \\
\text { September }\end{array}$ & $\begin{array}{r}372 \\
426 \\
354 \\
340 \\
170 \\
1,500 \\
5,030 \\
4,750 \\
\mathbf{3}, 320 \\
362 \\
153 \\
\mathbf{7 0 2} \\
\end{array}$ & $\begin{array}{r}88 \\
164 \\
98 \\
140 \\
46 \\
110 \\
264 \\
379 \\
189 \\
58 \\
28 \\
46\end{array}$ & $\begin{array}{r}225 \\
268 \\
169 \\
175 \\
137 \\
445 \\
2,000 \\
1,230 \\
833 \\
154 \\
99.4 \\
193\end{array}$ & $\begin{array}{l}0.507 \\
.604 \\
.381 \\
.394 \\
.309 \\
1.00 \\
4.50 \\
2.77 \\
1.88 \\
.347 \\
.224 \\
.435\end{array}$ & $\begin{array}{r}0.58 \\
.67 \\
.44 \\
.45 \\
.32 \\
1.15 \\
5.02 \\
3.19 \\
2.10 \\
.40 \\
.26 \\
.49\end{array}$ \\
\hline The year... & 5,030 & 28 & 493 & 1.11 & 15.07 \\
\hline
\end{tabular}




\section{LAKE GEORGE AT ROGERS ROCK, N. Y.}

Location.-At boathouse in a small bay on north side of steamboat landing at Rogers Rock, Essex County.

Records available.-July 10, 1913, to September 30, 1923.

GAGE.- Vertical staff gage fastened to a pile in the back end of the boathouse.

Datum 3.15 feet below crest of dam at outlet of lake. During the winter a temporory vertical staff gage located at Hoopers dock is used. Gage read once daily to hundredths by an employee of the International Paper Co. A study of gage heights at Rogers Rock and Glen Island stations indicates that the datum of the Rogers Rock gage is about 4.9 feet above that of the gage at Glen Island.

Extremes of STAGE.-Maximum stage recorded during year, 4.20 feet May 17 and 22; minimum stage recorded, 1.06 feet December 29.

1913-1923: Maximum stage recorded, 5.07 feet April 18, 1922; minimum stage recorded, that of December 29,1.922.

REgolation.--The elevation of lake surface is regulated by the operation of gates and wheels at the dam at the outlet of the lake at Ticonderoga.

Cooperation.-Gage-height record furnished by Mr. C. S. Colson, hydraulic engineer, International Paper Co.

Daily gage height, in feet, of Lake George at Rogers Rock, N. Y., for the year ending September 30, 1923

\begin{tabular}{|c|c|c|c|c|c|c|c|c|c|c|c|c|}
\hline Day & ct. & Nov. & Dec. & Jan. & Feb. & Aar. & Apr. & May & June & July & Aug. & Sept. \\
\hline$\cdots$ & $\begin{array}{l}2.48 \\
2.46 \\
2.42 \\
2.38 \\
2.32\end{array}$ & $\begin{array}{l}1.88 \\
1.84 \\
1.82 \\
1.80 \\
1.78\end{array}$ & $\begin{array}{l}1.4 \\
1.4 \\
1.4\end{array}$ & $\begin{array}{l}1.4 \\
1.4 \\
1.5\end{array}$ & & & & & & & $\begin{array}{l}28 \\
25 \\
22\end{array}$ & 2.7 \\
\hline & $\begin{array}{l}2.40 \\
2.36 \\
2.34 \\
2.32 \\
2.38\end{array}$ & $\begin{array}{l}1.74 \\
1.72 \\
1.72 \\
1.76 \\
1.78\end{array}$ & $\begin{array}{l}1.38 \\
1.36 \\
1.36 \\
1.34 \\
1.28\end{array}$ & $\begin{array}{l}1.50 \\
1.54 \\
1.50 \\
1.54 \\
1.52\end{array}$ & & & & & & & $\begin{array}{r}3.15 \\
3.10 \\
3.18\end{array}$ & 2.64 \\
\hline & & $\begin{array}{l}1.76 \\
1.74 \\
1.72 \\
1.70 \\
1.74\end{array}$ & $\begin{array}{l}1.26 \\
1.28 \\
1.26 \\
1.28 \\
1.24\end{array}$ & $\begin{array}{l}1.54 \\
1.56 \\
1.58 \\
1.60 \\
1.62\end{array}$ & & & & & & & & \\
\hline & & $\begin{array}{l}1.72 \\
1.70 \\
1.68 \\
1.64 \\
1.60\end{array}$ & $\begin{array}{l}1.26 \\
1.26 \\
1.28 \\
1.27 \\
1.28\end{array}$ & $\begin{array}{l}\text { 1. } 64 \\
1.64\end{array}$ & & & & & & & & \\
\hline & $\begin{array}{l}2.12 \\
2.10 \\
2.20 \\
2.08 \\
2.04\end{array}$ & $\begin{array}{l}1.60 \\
1.58 \\
1.56 \\
1.54 \\
1.54\end{array}$ & $\begin{array}{l}1.2 \\
1.2 \\
1.2 \\
1.2 \\
1.2\end{array}$ & & $\begin{array}{l}\text { 1. } 62 \\
\text { 1. } 60 \\
1.60 \\
1.58 \\
1.60\end{array}$ & $\begin{array}{l}1.84 \\
1.88 \\
1.90 \\
1.96 \\
2.00\end{array}$ & & & & & & $\begin{array}{l}2 . \\
2 . \\
2 .\end{array}$ \\
\hline$\cdots$ & $\begin{array}{l}2.00 \\
1.98 \\
1.94 \\
1.92 \\
1.88 \\
1.90\end{array}$ & $\begin{array}{l}1.52 \\
1.50 \\
1.52 \\
1.50 \\
1.48\end{array}$ & $\begin{array}{l}1.22 \\
1.16 \\
1.10 \\
1.06 \\
1.18 \\
1.28\end{array}$ & $\begin{array}{l}1.62 \\
1.60 \\
1.60 \\
1.58 \\
1.62\end{array}$ & $\begin{array}{l}1.6 \\
1.5 \\
1.5\end{array}$ & $\begin{array}{l}2.10 \\
2.04 \\
2.01 \\
2.02 \\
2.04\end{array}$ & $\begin{array}{l}3.55 \\
3.58 \\
3.60 \\
3.80 \\
4.00\end{array}$ & & & $\begin{array}{l}\text { 3. } 30 \\
3.28 \\
3.30 \\
3.28\end{array}$ & $\begin{array}{l}2.78 \\
2.76 \\
2.78 \\
2.78 \\
2.76 \\
2.74\end{array}$ & $\begin{array}{l}2 . \\
2 . \\
2 . \\
2 . \\
2 .\end{array}$ \\
\hline
\end{tabular}




\section{IAKE GEORGE AT GLEN ISLAND, NEAR BOLTON IANDING, N. Y.}

Location.-100 feet east of dock on northeast side of Glen Island, 2 miles northeast of Bolton Landing; reached by boat from Bolton Landing.

Records available.-September 4, 1919, to September 30, 1923.

GAGE.-Slope gage, anchored to solid rock ledge, graduated to half-tenths from 6 to 10.4 feet, installed November 21, 1922; previous to this, a vertical castiron staff gage, reading from 6 to 10 feet, fastened to a 2 by 8 inch oak plank, spiked to the dock 100 feet west, was used. During winter a temporary vertical staff gage, attached to dock at Bolton Landing, has been used. Gage read twice daily to quarter-tenths by Jay Taylor, ranger. A comparative study of gage heights at the Glen Island and Rogers Rock stations indicates that the datum of the Glen Island gage is about 4.9 feet below that of the gage at Rogers Rock.

Extremes of STAGE.-Maximum stage recorded during year, 9.1 feet May 2124; minimum stage recorded, 6 feet December 24-27.

1919-1923: Maximum stage recorded, 9.9 feet April 15-18, 1922; minimum stage recorded, that of December $\dot{2} 4-27,1922$.

REGULATION.-The elevation of lake surface is regulated by the operation of gates and wheels at the dam at the outlet of the lake at Ticonderoga.

Cooperation.-Gage-height record furnished by State of New York Conservation Commission.

Daily gage height, in feet, of Lake George at Glen Island, near Bolton Landing, N. Y., for the year ending September 30, 1923

\begin{tabular}{|c|c|c|c|c|c|c|c|c|c|c|c|c|}
\hline $\mathrm{D}$ & Oct. & Nov. & Dec. & Jan. & Feb. & Mar. & Apr. & May & June & July & Aug. & Sept. \\
\hline $\begin{array}{l}1 \ldots \\
2 \ldots \\
3 \\
4 \\
5 \ldots\end{array}$ & $\begin{array}{l}7.45 \\
7.4 \\
7.4 \\
7.4 \\
7.35\end{array}$ & $\begin{array}{l}6.75 \\
6.75 \\
6.75 \\
6.75 \\
6.75\end{array}$ & $\begin{array}{l}6.35 \\
6.35 \\
6.35 \\
6.35 \\
6.35\end{array}$ & $\begin{array}{l}6.25 \\
6.3 \\
6.35 \\
6.4 \\
6.4\end{array}$ & $\begin{array}{l}6.6 \\
6.6 \\
6.6 \\
6.65 \\
6.65\end{array}$ & $\begin{array}{l}6.55 \\
6.6 \\
6.6 \\
6.6 \\
6.6\end{array}$ & $\begin{array}{l}7.1 \\
7.15 \\
7.3 \\
7.35 \\
7.4\end{array}$ & & $\begin{array}{l}8.9 \\
8.85 \\
8.85 \\
8.85 \\
8.8\end{array}$ & $\begin{array}{l}8.55 \\
8.5 \\
8.5 \\
8.5 \\
8.5\end{array}$ & $\begin{array}{l}8.25 \\
8.25 \\
8.25 \\
8.25 \\
8.25\end{array}$ & $\begin{array}{l}7.65 \\
7.65 \\
7.65 \\
7.65 \\
7.6\end{array}$ \\
\hline $\begin{array}{r}6 \\
7 \\
8 \\
9 \\
9 \\
10 \ldots\end{array}$ & $\begin{array}{l}7.35 \\
7.3 \\
7.3 \\
7.3 \\
7.25\end{array}$ & $\begin{array}{l}6,75 \\
6.75 \\
6.75 \\
6.7 \\
6.7\end{array}$ & $\begin{array}{l}6.35 \\
6.35 \\
6.25 \\
6.25 \\
6.25\end{array}$ & $\begin{array}{l}6.45 \\
6.45 \\
6.5 \\
6.5 \\
6.5\end{array}$ & $\begin{array}{l}6.7 \\
6.7 \\
6.7 \\
6.7 \\
6.7\end{array}$ & $\begin{array}{l}6.6 \\
6.65 \\
6.65 \\
6.65 \\
6.65\end{array}$ & $\begin{array}{l}7.45 \\
7.55 \\
7.65 \\
7.75 \\
7.85\end{array}$ & & & & $\begin{array}{l}8.2 \\
8.15 \\
8.1 \\
8.1 \\
8.1\end{array}$ & $\begin{array}{l}7.6 \\
7.55 \\
7.55 \\
7.6 \\
7.6\end{array}$ \\
\hline $5 \ldots$ & $\begin{array}{l}7.25 \\
7.2 \\
7.2 \\
7.2 \\
7.1\end{array}$ & $\begin{array}{l}6.7 \\
6.7 \\
6.7 \\
6.65 \\
6.65\end{array}$ & $\begin{array}{l}6.25 \\
6.25 \\
6.25 \\
6.25 \\
6.2\end{array}$ & $\begin{array}{l}6.55 \\
6.6 \\
6.6 \\
6.6 \\
6.6\end{array}$ & $\begin{array}{l}6.7 \\
6.7 \\
6.7 \\
6.7 \\
6.7\end{array}$ & $\begin{array}{l}6.65 \\
6,65 \\
6.65 \\
6.7 \\
6.75\end{array}$ & & & & & $\begin{array}{l}8.05 \\
8.05 \\
8.0 \\
8.0 \\
8.0\end{array}$ & $\begin{array}{l}7.6 \\
7.55 \\
7.55 \\
7.5 \\
7.5\end{array}$ \\
\hline 9 & $\begin{array}{l}7.1 \\
7.05 \\
7.0 \\
7.0 \\
6.95\end{array}$ & $\begin{array}{l}6.65 \\
6.65 \\
6.65 \\
6.65 \\
6.65\end{array}$ & $\begin{array}{l}6.2 \\
6.2 \\
6.2 \\
6.2 \\
6.2\end{array}$ & $\begin{array}{l}6.6 \\
6.5 \\
6.5 \\
6.5 \\
6.5\end{array}$ & $\begin{array}{l}6 . \\
6 . \\
6 . \\
6 . \\
6 .\end{array}$ & & & & & & $\begin{array}{l}8.0 \\
7.9 \\
7.9 \\
7.9 \\
7.85\end{array}$ & $\begin{array}{l}7.4 \\
7.4 \\
7.4 \\
7.35 \\
7.45\end{array}$ \\
\hline 5 & $\begin{array}{l}6.95 \\
6.95 \\
6.95 \\
7.0 \\
6.95\end{array}$ & $\begin{array}{l}6.6 \\
6.55 \\
6.55 \\
6.55 \\
6.5\end{array}$ & $\begin{array}{l}6.1 \\
6.05 \\
6.05 \\
6.0 \\
6.0\end{array}$ & $\begin{array}{l}6.5 \\
6.5 \\
6.5 \\
6.5 \\
6.6\end{array}$ & $\begin{array}{l}6.7 \\
6.7 \\
6.7 \\
6.7 \\
6.65\end{array}$ & $\begin{array}{l}6.9 \\
6.95 \\
7.0 \\
7.0\end{array}$ & $\begin{array}{l}8.45 \\
8.45 \\
8.45 \\
8.5\end{array}$ & $\begin{array}{l}9.1 \\
9.1 \\
9.05\end{array}$ & $\begin{array}{l}8 . \\
8 . \\
8 . \\
8 .\end{array}$ & & $\begin{array}{l}7.8 \\
7.75 \\
7.75 \\
7.75 \\
7.7\end{array}$ & $\begin{array}{r}7.45 \\
7.45 \\
\\
\end{array}$ \\
\hline 30 & $\begin{array}{l}6.9 \\
6.9 \\
6.85 \\
6.8 \\
6.75 \\
6.75\end{array}$ & $\begin{array}{l}6.5 \\
6.45 \\
6.45 \\
6.45 \\
6.4\end{array}$ & $\begin{array}{l}6.0 \\
6.0 \\
6.25 \\
6.25 \\
6.25 \\
6.25\end{array}$ & $\begin{array}{l}6.6 \\
6,6 \\
6.6 \\
6.6 \\
6.6 \\
6.6\end{array}$ & $\begin{array}{l}6.65 \\
6.6 \\
6.55 \\
\end{array}$ & $\begin{array}{l}7.0 \\
7.05 \\
7.05 \\
7.05 \\
7.1 \\
7.1\end{array}$ & $\begin{array}{l}8.55 \\
8.6 \\
8.75 \\
8.8 \\
8.85\end{array}$ & $\begin{array}{l}9.0 \\
8.95 \\
8.95 \\
8.95 \\
8.95 \\
8.9\end{array}$ & $\begin{array}{l}8.65 \\
8.6 \\
8.6 \\
8.6 \\
8.55\end{array}$ & $\begin{array}{l}8.3 \\
8.3 \\
8.25 \\
8.25 \\
8.25 \\
8.25\end{array}$ & $\begin{array}{l}7.65 \\
7.6 \\
7.75 \\
7.75 \\
7.7 \\
7.7\end{array}$ & 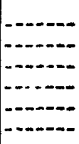 \\
\hline
\end{tabular}

Note.-Mean daily gage heights Apr. 28 and Aug. 2-4 estimated by hydrograph comparison with record of Lake George at Rogers Rock; gage-height record either faulty or missing. No gage-height record Sept. 23-30. Readings from Oct. 22 to A pr. 27 made on temporary staff gage at Bolton Landing reduced to datum of Glen Island gage. 
WINOOSKI RIVER AT MONTPELIER, VT.

Location.-1 mile downstream from Central Vermont Railway station in Montpelier, Washington County, three-eighths mile above mouth of Dog River, and 11/4 miles below mouth of North Branch.

Drainage area.-420 square miles.

Records available.-May 19, 1909, to September 30, 1923, when station was discontinued.

GAGE.-Water-stage recorder on right bank, installed July 4, 1914; gage height referred to datum by means of a hook gage inside well; outside staff gage is used for auxiliary readings. Recorder inspected by L. D. Smith.

Discharge measurements.-Made from cable or by wading.

Channel and control. - Channel deep and fairly uniform in section at the gage. Control is formed by sharply defined rock outcrop 500 feet below gage.

ExtREMES of DISCHARge.-Maximum open-water stage during year from waterstage recorder, 11.43 feet at 11 a. m. April 30 (discharge, by extension of rating curve, 9,100 second-feet); minimum stage during year from waterstage recorder, 2.82 feet at $7 \mathrm{a}$. $\mathrm{m}$. June 27 (discharge, from extension of rating curve, 53 second-feet).

1909-1923: Maximum stage determined by leveling from floodmarks preserved on building near present gage, 17.31 feet, April 7, 1912 (discharge, estimated 20,200 second-feet); minimum stage from water-stage recorder, 2.58 feet, September 30, 1921 (discharge, from extension of rating curve, 6 secondfeet).

IcE-Stage-discharge relation affected by ice during winter. Daily discharge ascertained by means of gage heights, current-meter measurements, observer's notes, and climatic records.

Regulation.-Operation of power plants on main stream and tributaries above station causes diurnal fluctuation in stage.

ACcURACY. - Stage-discharge relation changed March 20, probably due to use of dynamite in breaking up ice on control. Rating curve used prior to March 20 well defined between 30 and 7,500 second-feet; curve used after that date fairly well defined between 200 and 2,500 second-feet. Operation of waterstage recorder satisfactory except for periods indicated in footnote to dailydischarge table. Daily discharge December 19 to April 5 determined by applying to rating table mean daily gage height corrected for effect of ice; daily discharge during remainder of year ascertained by use of discharge integrator. Records good.

Discharge measurements of Winooski River at Montpelier, Vt., during the year ending September 30, 1923

\begin{tabular}{|c|c|c|c|c|c|c|c|}
\hline Date & Made by- & $\begin{array}{c}\text { Gage } \\
\text { height }\end{array}$ & $\begin{array}{c}\text { Dis- } \\
\text { charge }\end{array}$ & Date & Made by- & $\begin{array}{c}\text { Gage } \\
\text { haight }\end{array}$ & $\begin{array}{c}\text { Dis- } \\
\text { charge }\end{array}$ \\
\hline $\begin{array}{l}\text { Feb. } 5 \\
\text { Apr. } 24\end{array}$ & H.F. Hill, jr.... & $\begin{array}{l}\text { Feet } \\
04.85 \\
5.96\end{array}$ & $\begin{array}{r}\text { Sec.-ft. } \\
319 \\
910\end{array}$ & June 22 & W. E. Armistrong & $\begin{array}{l}\text { Feet } \\
\text { 3. } 90\end{array}$ & Sec. ftt. \\
\hline June 20 & W.E. Armstrong. & 3.70 & & & & & \\
\hline
\end{tabular}

a Stage-discharge relation aflected by ice. 
Daily discharge, in second-feet, of Winooski River at Montpelier, Vt., for the year ending September 30,1923

\begin{tabular}{|c|c|c|c|c|c|c|c|c|c|c|c|c|}
\hline $\mathrm{De}$ & Oct. & Nov. & Dec. & Jan. & Feb. & Mar. & Apr & May & June & July & Aug. & Sept. \\
\hline $\begin{array}{l}1 .- \\
2 . . \\
3 . . \\
4 .- \\
5 .-\end{array}$ & $\begin{array}{r}72 \\
110 \\
108 \\
106 \\
110\end{array}$ & $\begin{array}{l}136 \\
142 \\
140 \\
134 \\
124\end{array}$ & $\begin{array}{l}220 \\
360 \\
200 \\
235 \\
255\end{array}$ & $\begin{array}{r}600 \\
1,050 \\
880 \\
740 \\
620\end{array}$ & $\begin{array}{l}220 \\
240 \\
220 \\
210 \\
240\end{array}$ & $\begin{array}{l}210 \\
200 \\
195 \\
220 \\
260\end{array}$ & $\begin{array}{r}440 \\
390 \\
370 \\
1,720 \\
4,350\end{array}$ & $\begin{array}{l}3,210 \\
1,890 \\
1,460 \\
1,240 \\
1,010\end{array}$ & $\begin{array}{l}292 \\
278 \\
248 \\
274 \\
494\end{array}$ & $\begin{array}{l}150 \\
210 \\
150 \\
110 \\
190\end{array}$ & $\begin{array}{r}150 \\
140 \\
130 \\
120 \\
80\end{array}$ & $\begin{array}{r}105 \\
65 \\
85 \\
130 \\
90\end{array}$ \\
\hline $\begin{array}{r}6 \ldots . \\
7 \\
8 \ldots \\
9 \ldots \\
10 \ldots\end{array}$ & $\begin{array}{l}110 \\
112 \\
114 \\
290 \\
320\end{array}$ & $\begin{array}{l}136 \\
138 \\
154 \\
340 \\
320\end{array}$ & $\begin{array}{l}188 \\
188 \\
205 \\
205 \\
162\end{array}$ & $\begin{array}{l}500 \\
420 \\
420 \\
440 \\
420\end{array}$ & $\begin{array}{l}240 \\
230 \\
220 \\
220 \\
210\end{array}$ & $\begin{array}{l}240 \\
230 \\
210 \\
190 \\
170\end{array}$ & $\begin{array}{l}7,430 \\
4,610 \\
4,420 \\
3,690 \\
2,440\end{array}$ & $\begin{array}{r}960 \\
850 \\
770 \\
840 \\
1,200\end{array}$ & $\begin{array}{r}1,060 \\
720 \\
1,040 \\
1,450 \\
1,120\end{array}$ & $\begin{array}{l}185 \\
145 \\
110 \\
170 \\
140\end{array}$ & $\begin{array}{l}120 \\
115 \\
120 \\
125 \\
100\end{array}$ & $\begin{array}{l}215 \\
185 \\
105 \\
100 \\
120\end{array}$ \\
\hline 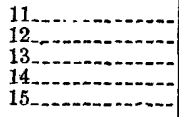 & $\begin{array}{l}345 \\
245 \\
186 \\
150 \\
100\end{array}$ & $\begin{array}{l}200 \\
170 \\
182 \\
162 \\
172\end{array}$ & $\begin{array}{l}205 \\
200 \\
190 \\
205 \\
198\end{array}$ & $\begin{array}{l}380 \\
370 \\
350 \\
350 \\
360\end{array}$ & $\begin{array}{l}220 \\
230 \\
230 \\
240 \\
230\end{array}$ & $\begin{array}{l}180 \\
180 \\
190 \\
190 \\
180\end{array}$ & $\begin{array}{l}2,360 \\
3,060 \\
2,280 \\
1,670 \\
1,440\end{array}$ & $\begin{array}{r}1,010 \\
845 \\
960 \\
745 \\
700\end{array}$ & $\begin{array}{l}675 \\
488 \\
400 \\
340 \\
304\end{array}$ & $\begin{array}{l}135 \\
120 \\
110 \\
100 \\
200\end{array}$ & $\begin{array}{r}110 \\
85 \\
304 \\
242 \\
205\end{array}$ & $\begin{array}{l}110 \\
110 \\
110 \\
105 \\
110\end{array}$ \\
\hline $0_{0}$ & $\begin{array}{l}156 \\
150 \\
154 \\
150 \\
142\end{array}$ & $\begin{array}{l}260 \\
225 \\
188 \\
198 \\
280\end{array}$ & $\begin{array}{l}210 \\
170 \\
205 \\
220 \\
230\end{array}$ & $\begin{array}{l}350 \\
310 \\
260 \\
260 \\
260\end{array}$ & $\begin{array}{l}230 \\
230 \\
220 \\
240 \\
260\end{array}$ & $\begin{array}{l}240 \\
380 \\
400 \\
380 \\
330\end{array}$ & $\begin{array}{l}1,290 \\
1,180 \\
1,120 \\
1,080 \\
1,250\end{array}$ & $\begin{array}{r}1,160 \\
1,670 \\
920 \\
800 \\
675\end{array}$ & $\begin{array}{l}278 \\
260 \\
288 \\
242 \\
220\end{array}$ & $\begin{array}{l}440 \\
274 \\
210 \\
185 \\
140\end{array}$ & $\begin{array}{r}170 \\
150 \\
110 \\
90 \\
130\end{array}$ & $\begin{array}{l}110 \\
120 \\
140 \\
120 \\
145\end{array}$ \\
\hline 24 & $\begin{array}{l}130 \\
104 \\
148 \\
425 \\
285\end{array}$ & $\begin{array}{l}450 \\
315 \\
235 \\
205 \\
176\end{array}$ & $\begin{array}{c}230 \\
200 \\
190 \\
155 \\
155\end{array}$ & $\begin{array}{l}260 \\
320 \\
330 \\
270 \\
300\end{array}$ & $\begin{array}{l}220 \\
220 \\
210 \\
200 \\
200\end{array}$ & $\begin{array}{r}295 \\
295 \\
1,050 \\
2,400 \\
1,700\end{array}$ & $\begin{array}{l}3,140 \\
3,230 \\
2,810 \\
1,790 \\
1,340\end{array}$ & $\begin{array}{r}780 \\
1,130 \\
795 \\
610 \\
560\end{array}$ & $\begin{array}{l}205 \\
210 \\
185 \\
115 \\
180\end{array}$ & $\begin{array}{l}130 \\
100 \\
160 \\
130 \\
120\end{array}$ & $\begin{array}{l}125 \\
120 \\
115 \\
115 \\
115\end{array}$ & $\begin{array}{l}215 \\
290 \\
215 \\
190 \\
145\end{array}$ \\
\hline $\begin{array}{l}26 \\
27 \\
28 \\
28 \\
30\end{array}$ & $\begin{array}{l}235 \\
210 \\
178 \\
148 \\
162 \\
152\end{array}$ & $\begin{array}{l}164 \\
205 \\
180 \\
205 \\
150 \\
---\end{array}$ & $\begin{array}{l}170 \\
165 \\
165 \\
175 \\
195 \\
260\end{array}$ & $\begin{array}{l}260 \\
240 \\
220 \\
240 \\
240 \\
250\end{array}$ & $\begin{array}{r}220 \\
220 \\
210 \\
-2 \\
\end{array}$ & $\begin{array}{r}1,200 \\
960 \\
760 \\
620 \\
560 \\
500\end{array}$ & $\begin{array}{r}1,340 \\
1,100 \\
1,230 \\
5,130 \\
6,230 \\
-\cdots\end{array}$ & $\begin{array}{l}500 \\
440 \\
430 \\
380 \\
340 \\
335\end{array}$ & $\begin{array}{l}190 \\
160 \\
160 \\
160 \\
190\end{array}$ & $\begin{array}{l}160 \\
160 \\
140 \\
215 \\
242 \\
180\end{array}$ & $\begin{array}{r}85 \\
135 \\
130 \\
110 \\
140 \\
125\end{array}$ & $\begin{array}{l}120 \\
125 \\
185 \\
270 \\
100\end{array}$ \\
\hline
\end{tabular}

NoTE-Operation of water-stage recorder unsatisfactory Nov, 27-29, Dec, 30 to Jan. 2, July 18, and Sept. 6-30; discharge during these periods estimated from observer's readings and by comparison with records of flow of tributary streams.

Monthly discharge of Winooski River at Montpelier, Vt., for the year ending September 30,1923

[Drainage area, 420 square miles]

\begin{tabular}{|c|c|c|c|c|c|}
\hline \multirow{2}{*}{ Month } & \multicolumn{4}{|c|}{ Discharge in second-feet } & \multirow{2}{*}{$\begin{array}{l}\text { Run-off in } \\
\text { inches }\end{array}$} \\
\hline & Maximum & Minimum & Mean & $\begin{array}{l}\text { Per square } \\
\text { mile }\end{array}$ & \\
\hline $\begin{array}{l}\text { October } \\
\text { November } \\
\text { December } \\
\text { January } \\
\text { February } \\
\text { March } \\
\text { April } \\
\text { May } \\
\text { June } \\
\text { July } \\
\text { August } \\
\text { September }\end{array}$ & $\begin{array}{r}425 \\
450 \\
360 \\
1,050 \\
260 \\
2,400 \\
7,430 \\
3,210 \\
1,450 \\
440 \\
304 \\
290\end{array}$ & $\begin{array}{r}72 \\
124 \\
155 \\
220 \\
200 \\
170 \\
370 \\
335 \\
115 \\
100 \\
80 \\
65\end{array}$ & $\begin{array}{r}174 \\
203 \\
204 \\
396 \\
224 \\
488 \\
2,460 \\
942 \\
408 \\
168 \\
133 \\
141\end{array}$ & $\begin{array}{c}0.414 \\
.483 \\
.486 \\
.943 \\
.533 \\
1.16 \\
5.86 \\
2.24 \\
.971 \\
.400 \\
.317 \\
.336\end{array}$ & $\begin{array}{r}0.48 \\
.54 \\
.56 \\
1.09 \\
.56 \\
1.34 \\
6.54 \\
2.58 \\
1.08 \\
.46 \\
.36 \\
.37\end{array}$ \\
\hline The year........ & 7,430 & 65 & 494 & 1.18 & 15.96 \\
\hline
\end{tabular}


MOLLYS BROOK IEAR MARSHFIELD, VT.

Location.-At head of Mollys Falls, one-fourth mile above confluence with Winooski River, and 1 mile from Marshfield, Washington County.

Drainage AREa.-24 square miles (from surveys by engineers of Montpelier \& Barre Light \& Power Co.).

Records available.-August 11, 1920, to September 30, 1923, when station was discontinued.

GaGE.-Inclined staff on right bank; vertical high-water section on left bank; read by Carroll George.

Discharge measurements.-Made from cable or by wading.

Channel and control.-Bed covered with gravel and alluvial deposits. Control is well defined at head of Mollys Falls; probably permanent.

EXTREMES OF DISCHARGE.-Maximum stage recorded during year, 6.3 feet at 4.30 p. m. April 30 (discharge, by extension of rating curve, 495 second-feet); minimum stage, 1.30 feet at $5.30 \mathrm{p}$. m. August 18 (discharge, 4.5 secondfeet).

1920-1923: Maximum discharge recorded, 680 second-feet April 12, 1922; minimum discharge, 2.2 second-feet September 15, 1921 (by extension of rating curve).

ICE.-Ice forms at the gage and on rocks at the control; stage-discharge relation somewhat affected.

Regulation.--Storage in Peacham Pond has some effect on the distribution of flow.

Accuracy. - Stage-discharge relation changed about October 1, 1922, owing to reconstruction of bridge at control. Rating curve well defined between 5 and 200 second-feet. Gage read to hundredths twice daily. Daily discharge ascertained by applying'to rating table mean daily gage height corrected for effect of ice during winter. Records good.

Discharge measurements of Mollys Brook near Marshfield, Vt., during the year ending September 30, 1923

\begin{tabular}{|c|c|c|c|c|c|c|c|}
\hline Date & Made by- & $\begin{array}{c}\text { Gage } \\
\text { height }\end{array}$ & $\begin{array}{c}\text { Dis- } \\
\text { charge }\end{array}$ & Date & Made by- & $\begin{array}{c}\text { Gage } \\
\text { height }\end{array}$ & $\begin{array}{l}\text { Dis- } \\
\text { charge }\end{array}$ \\
\hline $\begin{array}{lr}\text { Feb. } & 5 \\
\text { Apr. } & 25 \\
\text { May } & 2 \\
& 2\end{array}$ & $\begin{array}{l}\text { W. E. Armstrong } \\
\text { H. F. Hill, jr }\end{array}$ & $\begin{array}{l}\text { Feet } \\
1.68 \\
3.21 \\
3.91 \\
3.87\end{array}$ & $\begin{array}{c}\text { Sec. } f t . \\
15.2 \\
97 \\
164 \\
158\end{array}$ & $\begin{array}{lr}\text { May } & 2 \\
& 2 \\
\text { June } & 21 \\
21\end{array}$ & $\begin{array}{l}\text { H. F. Eill, je. } \\
\text { W. E. Armstrong }\end{array}$ & $\begin{array}{l}\text { Feet } \\
\text { 3. } 82 \\
\text { 3. } 80 \\
1.80 \\
1.78\end{array}$ & $\begin{array}{c}\text { Sec.-ft. } \\
154 \\
150 \\
18.3 \\
17.1\end{array}$ \\
\hline
\end{tabular}

$72038-26 \dagger-$ WSP $564-10$ 
Daily discharge, in second-feet, of Mollys Brook near Marshfield, Vt., for the year ending September 30, 1923

\begin{tabular}{|c|c|c|c|c|c|c|c|c|c|c|c|c|}
\hline Day & Oct. & Nov. & Dec. & Jan. & Feb. & Mar. & Apr. & May & Jnne & July & Aug. & Sept. \\
\hline $\begin{array}{l}1 \\
2 \\
3 \\
4 \\
5\end{array}$ & $\begin{array}{l}13 \\
12 \\
11 \\
10 \\
13\end{array}$ & $\begin{array}{l}13 \\
13 \\
12 \\
12 \\
12\end{array}$ & $\begin{array}{l}20 \\
25 \\
17 \\
18 \\
15\end{array}$ & $\begin{array}{l}31 \\
80 \\
54 \\
43 \\
35\end{array}$ & $\begin{array}{l}16 \\
16 \\
16 \\
15 \\
15\end{array}$ & $\begin{array}{l}18 \\
19 \\
17 \\
16 \\
15\end{array}$ & $\begin{array}{l}19 \\
18 \\
22 \\
66 \\
96\end{array}$ & $\begin{array}{r}233 \\
153 \\
123 \\
100 \\
88\end{array}$ & $\begin{array}{l}25 \\
23 \\
23 \\
23 \\
35\end{array}$ & $\begin{array}{l}13 \\
12 \\
13 \\
17 \\
24\end{array}$ & $\begin{array}{c}12 \\
11 \\
12 \\
12 \\
9.6\end{array}$ & $\begin{array}{l}12 \\
12 \\
11 \\
11 \\
10\end{array}$ \\
\hline $\begin{array}{r}6 .- \\
7 .- \\
8 . \\
9 .- \\
10 . .\end{array}$ & $\begin{array}{l}14 \\
13 \\
27 \\
27 \\
38\end{array}$ & $\begin{array}{l}12 \\
12 \\
13 \\
33 \\
19\end{array}$ & $\begin{array}{l}12 \\
12 \\
13 \\
15 \\
14\end{array}$ & $\begin{array}{l}29 \\
27 \\
24 \\
20 \\
19\end{array}$ & $\begin{array}{l}15 \\
14 \\
14 \\
15 \\
14\end{array}$ & $\begin{array}{l}13 \\
12 \\
12 \\
12 \\
13\end{array}$ & $\begin{array}{l}300 \\
210 \\
233 \\
209 \\
153\end{array}$ & $\begin{array}{l}76 \\
88 \\
76 \\
73 \\
96\end{array}$ & $\begin{array}{l}57 \\
40 \\
48 \\
66 \\
45\end{array}$ & $\begin{array}{l}14 \\
13 \\
12\end{array}$ & $\begin{array}{l}23 \\
27 \\
25 \\
20 \\
18\end{array}$ & $\begin{array}{c}9.3 \\
9.0 \\
8.8 \\
23 \\
13\end{array}$ \\
\hline.- & $\begin{array}{l}33 \\
20 \\
17 \\
15 \\
13\end{array}$ & $\begin{array}{l}17 \\
15 \\
14 \\
10 \\
21\end{array}$ & $\begin{array}{l}13 \\
14 \\
14 \\
13 \\
12\end{array}$ & $\begin{array}{l}16 \\
15 \\
14 \\
15 \\
16\end{array}$ & $\begin{array}{l}13 \\
13 \\
13 \\
13 \\
13\end{array}$ & $\begin{array}{l}13 \\
13 \\
10 \\
10 \\
11\end{array}$ & $\begin{array}{r}153 \\
174 \\
133 \\
96 \\
80\end{array}$ & $\begin{array}{l}76 \\
73 \\
73 \\
60 \\
57\end{array}$ & $\begin{array}{l}35 \\
29 \\
27 \\
25 \\
24\end{array}$ & $\begin{array}{l}12 \\
12 \\
19 \\
25 \\
35\end{array}$ & $\begin{array}{l}17 \\
16 \\
54 \\
31 \\
16\end{array}$ & $\begin{array}{r}11 \\
9.6 \\
10 \\
8.5 \\
7.0\end{array}$ \\
\hline $\begin{array}{l}16 \\
17 \\
18 \\
19 \\
20\end{array}$ & $\begin{array}{l}13 \\
16 \\
15 \\
15 \\
15\end{array}$ & $\begin{array}{l}20 \\
15 \\
16 \\
19 \\
23\end{array}$ & $\begin{array}{r}11 \\
11 \\
11 \\
10 \\
9\end{array}$ & $\begin{array}{l}15 \\
14 \\
14 \\
16 \\
18\end{array}$ & $\begin{array}{l}13 \\
13 \\
13 \\
13 \\
13\end{array}$ & $\begin{array}{l}21 \\
29 \\
25 \\
19 \\
15\end{array}$ & $\begin{array}{l}66 \\
60 \\
54 \\
51 \\
70\end{array}$ & $\begin{array}{l}73 \\
73 \\
54 \\
48 \\
43\end{array}$ & $\begin{array}{l}21 \\
23 \\
21 \\
19 \\
18\end{array}$ & $\begin{array}{l}29 \\
19 \\
12 \\
12 \\
12\end{array}$ & $\begin{array}{l}10 \\
7.7 \\
4.9 \\
7.0 \\
8.5\end{array}$ & $\begin{array}{l}7.0 \\
6.7 \\
6.5 \\
6.7 \\
6.5\end{array}$ \\
\hline (2) & $\begin{array}{l}14 \\
13 \\
15 \\
33 \\
19\end{array}$ & $\begin{array}{l}31 \\
20 \\
15 \\
16 \\
18\end{array}$ & $\begin{array}{l}13 \\
13 \\
13 \\
12 \\
12\end{array}$ & $\begin{array}{l}19 \\
29 \\
21 \\
18 \\
16\end{array}$ & $\begin{array}{l}13 \\
14 \\
14 \\
16 \\
18\end{array}$ & $\begin{array}{l}13 \\
15 \\
27 \\
60 \\
\mathbf{5 1}\end{array}$ & $\begin{array}{l}221 \\
300 \\
272 \\
133 \\
105\end{array}$ & $\begin{array}{l}57 \\
66 \\
45 \\
38 \\
38\end{array}$ & $\begin{array}{l}17 \\
17 \\
15 \\
15 \\
15\end{array}$ & $\begin{array}{l}12 \\
12 \\
17\end{array}$ & $\begin{array}{l}6.7 \\
13 \\
17 \\
15 \\
14\end{array}$ & $\begin{array}{l}45 \\
17 \\
10 \\
12 \\
12\end{array}$ \\
\hline $\begin{array}{l}26 \\
27 \\
28 \\
30 \\
31\end{array}$ & $\begin{array}{l}21 \\
18 \\
17 \\
14 \\
14 \\
13\end{array}$ & $\begin{array}{l}15 \\
14 \\
13 \\
13 \\
14 \\
-\end{array}$ & $\begin{array}{l}13 \\
13 \\
12 \\
12 \\
11 \\
12\end{array}$ & $\begin{array}{l}15 \\
14 \\
14 \\
14 \\
14 \\
16\end{array}$ & $\begin{array}{l}17 \\
17 \\
19\end{array}$ & $\begin{array}{l}45 \\
33 \\
27 \\
24 \\
23 \\
20\end{array}$ & $\begin{array}{r}80 \\
80 \\
88 \\
360 \\
495\end{array}$ & $\begin{array}{l}33 \\
33 \\
29 \\
29 \\
27 \\
25\end{array}$ & $\begin{array}{l}13 \\
15 \\
15 \\
16 \\
15\end{array}$ & $\begin{array}{l}14 \\
18 \\
15 \\
13\end{array}$ & $\begin{array}{l}13 \\
16 \\
15 \\
19 \\
15 \\
14\end{array}$ & $\begin{array}{c}10 \\
9.6 \\
12 \\
14 \\
12 \\
\end{array}$ \\
\hline
\end{tabular}

Note.-Stage-discharge relation affected by ice Jan. 7-30 and Feb. 18 to Mar. 23; discharge based on gage heights corrected for effect of ice.

Monthly discharge of Mollys Brook near Marshfield, Vt., for the year ending September 30, 1923

[Drainage area, 24 square miles]

\begin{tabular}{|c|c|c|c|c|c|}
\hline \multirow{2}{*}{ Month } & \multicolumn{4}{|c|}{ Discharge in second-feet } & \multirow{2}{*}{$\begin{array}{l}\text { Run-off } \\
\text { in inches }\end{array}$} \\
\hline & Maximum & Minimum & Mean & $\begin{array}{c}\text { Per square } \\
\text { mile }\end{array}$ & \\
\hline $\begin{array}{l}\text { October } \\
\text { November } \\
\text { December } \\
\text { January } \\
\text { February } \\
\text { March } \\
\text { April } \\
\text { May } \\
\text { June } \\
\text { July } \\
\text { August } \\
\text { September }\end{array}$ & $\begin{array}{r}38 \\
33 \\
25 \\
80 \\
19 \\
60 \\
495 \\
233 \\
66 \\
35 \\
54 \\
45\end{array}$ & $\begin{array}{r}10 \\
10 \\
9 \\
14 \\
13 \\
10 \\
18 \\
25 \\
13 \\
12 \\
4.9 \\
6.5\end{array}$ & $\begin{array}{r}17.5 \\
16.3 \\
13.4 \\
22.7 \\
14.6 \\
21.0 \\
147 \\
69.5 \\
26.0 \\
16.2 \\
16.1 \\
11.7\end{array}$ & $\begin{array}{l}0.729 \\
.679 \\
.558 \\
.946 \\
.608 \\
.875 \\
6.12 \\
2.90 \\
1.08 \\
.675 \\
.671 \\
.488\end{array}$ & $\begin{array}{r}0.84 \\
.76 \\
.64 \\
1.09 \\
.63 \\
1.01 \\
6.83 \\
3.34 \\
1.20 \\
.78 \\
.77 \\
.54\end{array}$ \\
\hline The year. & 495 & 4.9 & 32.6 & 1.36 & 18.43 \\
\hline
\end{tabular}




\section{JAIL BRAYCH ${ }^{5}$ AT EAST BARRE, VT.}

Location.-At ruins of old dam one-fourth mile above highway bridge in East Barre, Washington County, and 3 miles above confluence with Stevens Branch.

Drainage area.-About 38 square miles, including 13 square miles tributary to Orange Brook reservoir (see "Diversions").

Records available.--August 14,1920 , to September 30, 1923, when station was discontinued.

GAGE.-Inclined staff gage on left bank; read by George J. Dobbs.

Discharge measurements.--Made from cable or by wading.

Channel and control.-Bed covered with rocks and boulders. Control formed by granite blocks and foundation of old dam near gage.

Extremes of Discharge.-Maximum stage recorded during year ending September $30,1922,8.38$ feet at $6 \mathrm{p} . \mathrm{m}$. April 10 (discharge, by extension of rating curve, 1,350 second-feet); minimum discharge, 2.1 second-feet at 7 a. $\mathrm{m}$. October 8. Maximum open-water stage recorded during year ending September $30,1923,7$ feet? at 7 a. $\mathrm{m}$. April 5 (discharge, by extension of rating curve, 830 second-feet); minimum stage, 2.43 feet September 7 and 8 (discharge, by extension of rating curve, 0.6 second-foot).

1920-1923: Maximum stage recorded, that of April 10, 1922; minimum discharge, by extension? of rating curve, 0.5 second-foot September 11, 1921 . ICE.-Ice forms somewhat affected.

Diversions.-Water is diverted from about 13 square miles tributary to Orange Brook reservoir, and used for municipal supply of Barre. No records available as to quantity diverted or amount wasted back into Jail Branch.

Accuracy.--Stage-discharge relation changed during high water of April 10, 1922. Rating curve used previous to the change well defined between 1 and 60 second-feet, and by measurements at 859 and 873 second-feet. Rating curve used subsequent to the date of change well defined between 5 and 350 second-feet. Gage read to hundredths twice daily. Daily discharge ascertained by applying mean daily gage height to rating table except as shown in footnote to table of daily discharge. Records good.

Discharge measurements of Jail Branch at East Barre, Vt., during the year ending September 30, 1923

\begin{tabular}{|c|c|c|c|c|c|c|c|}
\hline Date & Made by- & $\underset{\text { height }}{\text { Gage }}$ & $\begin{array}{c}\text { Dis- } \\
\text { charge }\end{array}$ & Date & Made by- & $\begin{array}{c}\text { Gage } \\
\text { height }\end{array}$ & $\begin{array}{l}\text { Dis- } \\
\text { charge }\end{array}$ \\
\hline $\begin{array}{lr}\text { Feb. } & 5 \\
\text { Apr. } & 25 \\
\text { May } & 1 \\
& 1\end{array}$ & $\begin{array}{l}\text { W. E. Armstrong.... } \\
\text { H. F. Hill, jr... }\end{array}$ & $\begin{array}{l}\text { Feet } \\
3.08 \\
4.21 \\
\text { 4.99 } \\
\text { 4.92 }\end{array}$ & $\begin{array}{c}\text { Sec. } f t . \\
17.8 \\
147 \\
295 \\
282\end{array}$ & $\begin{array}{r}\text { May } 1 \\
\text { June 21 } \\
21\end{array}$ & $\begin{array}{l}\text { H. F. Hill, Jr. } \\
\text { W. E. Armstrong. }\end{array}$ & $\begin{array}{l}\text { Feet } \\
4.88 \\
2.78 \\
2.78\end{array}$ & $\begin{array}{c}\text { Sec. } f t . \\
256 \\
7.1 \\
6.1\end{array}$ \\
\hline
\end{tabular}

"Previously published as "Jail Brook." 
Daily discharge, in second-feet, of Jail Bianch at East Barre, Vt., for the years ending September 30, 1922 and 1923

\begin{tabular}{|c|c|c|c|c|c|c|c|c|c|c|c|c|}
\hline Day & Oct. & Nov. & Dec. & Jan. & Feb. & Mar. & Apr. & May & June & July & Aug. & Sept. \\
\hline $\begin{array}{l}1 \ldots \\
2 \ldots \\
3 \ldots \\
4 \ldots \\
5 \ldots\end{array}$ & $\begin{array}{l}\text { 8. } 6 \\
4.6 \\
3.0 \\
4.6 \\
4.6\end{array}$ & $\begin{array}{l}13 \\
41 \\
28 \\
20 \\
21\end{array}$ & $\begin{array}{r}41 \\
60 \\
635 \\
127 \\
98\end{array}$ & $\begin{array}{l}14 \\
13 \\
13 \\
11 \\
15\end{array}$ & $\begin{array}{r}8 \\
10 \\
12 \\
14 \\
13\end{array}$ & $\begin{array}{r}9 \\
9 \\
10 \\
10 \\
11\end{array}$ & $\begin{array}{l}175 \\
127 \\
123 \\
127 \\
175\end{array}$ & $\begin{array}{r}70 \\
73 \\
72 \\
95 \\
217\end{array}$ & $\begin{array}{l}12 \\
14 \\
48 \\
71 \\
29\end{array}$ & $\begin{array}{r}59 \\
110 \\
87 \\
153 \\
83\end{array}$ & $\begin{array}{l}4.6 \\
43 \\
13 \\
14 \\
12\end{array}$ & $\begin{array}{l}9.4 \\
7.8 \\
6.2 \\
4.8 \\
4.6\end{array}$ \\
\hline $\begin{array}{r}6 \\
7 \ldots \ldots \\
8 \\
9 \\
10 \\
10\end{array}$ & $\begin{array}{l}\text { 3. } 0 \\
3.0 \\
4.0 \\
\text { 8. } \\
7.4\end{array}$ & $\begin{array}{l}13 \\
13 \\
12 \\
23 \\
14\end{array}$ & $\begin{array}{l}86 \\
80 \\
78 \\
80 \\
98\end{array}$ & $\begin{array}{l}12 \\
11 \\
11 \\
11 \\
11\end{array}$ & $\begin{array}{l}10 \\
11 \\
11 \\
10 \\
10\end{array}$ & $\begin{array}{r}47 \\
280 \\
590 \\
475 \\
237\end{array}$ & $\begin{array}{r}187 \\
225 \\
870 \\
635 \\
1,140\end{array}$ & $\begin{array}{r}246 \\
153 \\
153 \\
97 \\
73\end{array}$ & $\begin{array}{l}29 \\
46 \\
21 \\
16 \\
21\end{array}$ & $\begin{array}{l}50 \\
34 \\
37 \\
50 \\
30\end{array}$ & $\begin{array}{c}8.3 \\
153 \\
137 \\
31 \\
18\end{array}$ & $\begin{array}{l}8.6 \\
8.3 \\
7.8 \\
5.0 \\
4.4\end{array}$ \\
\hline 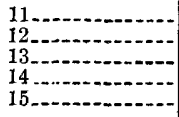 & $\begin{array}{l}12 \\
67 \\
33 \\
18 \\
9.5\end{array}$ & $\begin{array}{l}21 \\
18 \\
20 \\
23 \\
20\end{array}$ & $\begin{array}{l}80 \\
66 \\
54 \\
41 \\
35\end{array}$ & $\begin{array}{l}12 \\
14 \\
13 \\
13 \\
11\end{array}$ & $\begin{array}{r}10 \\
10 \\
10 \\
9 \\
8\end{array}$ & $\begin{array}{l}162 \\
127 \\
127 \\
237 \\
310\end{array}$ & $\begin{array}{l}770 \\
980 \\
413 \\
298 \\
320\end{array}$ & $\begin{array}{l}66 \\
59 \\
50 \\
47 \\
43\end{array}$ & $\begin{array}{l}43 \\
54 \\
23 \\
15 \\
16\end{array}$ & $\begin{array}{l}23 \\
20 \\
21 \\
16 \\
19\end{array}$ & $\begin{array}{l}14 \\
11 \\
10 \\
8.9 \\
8.3\end{array}$ & $\begin{array}{l}4.0 \\
12 \\
27 \\
12 \\
53\end{array}$ \\
\hline $\begin{array}{l}9 \\
0\end{array}$ & $\begin{array}{r}5.6 \\
5.0 \\
5.0 \\
4.0 \\
16\end{array}$ & $\begin{array}{r}20 \\
26 \\
200 \\
460 \\
430\end{array}$ & $\begin{array}{r}41 \\
106 \\
605 \\
138 \\
74\end{array}$ & $\begin{array}{l}11 \\
13 \\
11 \\
12 \\
13\end{array}$ & $\begin{array}{l}8 \\
8 \\
8 \\
8 \\
8\end{array}$ & $\begin{array}{r}225 \\
200 \\
150 \\
98 \\
67\end{array}$ & $\begin{array}{l}277 \\
342 \\
413 \\
266 \\
217\end{array}$ & $\begin{array}{r}38 \\
34 \\
69 \\
153 \\
78\end{array}$ & $\begin{array}{l}16 \\
23 \\
48 \\
92 \\
47\end{array}$ & $\begin{array}{c}12 \\
15 \\
12 \\
9.4 \\
7.8\end{array}$ & $\begin{array}{l}7.5 \\
8.3 \\
7.5 \\
7.2 \\
7.0\end{array}$ & $\begin{array}{l}84 \\
24 \\
18 \\
14 \\
11\end{array}$ \\
\hline 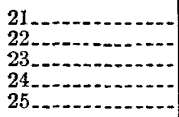 & $\begin{array}{c}60 \\
14 \\
9.5 \\
10 \\
12\end{array}$ & $\begin{array}{r}106 \\
60 \\
54 \\
54 \\
106\end{array}$ & $\begin{array}{l}60 \\
54 \\
47 \\
41 \\
35\end{array}$ & $\begin{array}{l}13 \\
13 \\
11 \\
11 \\
11\end{array}$ & $\begin{array}{l}10 \\
10 \\
10 \\
10 \\
13\end{array}$ & $\begin{array}{r}63 \\
50 \\
60 \\
82 \\
119\end{array}$ & $\begin{array}{l}180 \\
145 \\
129 \\
121 \\
110\end{array}$ & $\begin{array}{l}48 \\
41 \\
38 \\
33 \\
24\end{array}$ & $\begin{array}{r}72 \\
256 \\
171 \\
61 \\
41\end{array}$ & $\begin{array}{r}8.3 \\
8.0 \\
8.3 \\
11 \\
7.8\end{array}$ & $\begin{array}{r}6.7 \\
7.2 \\
4.4 \\
5.3 \\
14\end{array}$ & $\begin{array}{r}8.9 \\
7.5 \\
7.2 \\
9.7 \\
14\end{array}$ \\
\hline $\begin{array}{l}26 \ldots \ldots \\
27 \\
28 \\
29 \\
31 \ldots \\
31 \ldots \\
\ldots\end{array}$ & $\begin{array}{l}15 \\
13 \\
14 \\
11 \\
10 \\
7.4\end{array}$ & $\begin{array}{l}74 \\
74 \\
89 \\
81 \\
50\end{array}$ & $\begin{array}{l}29 \\
24 \\
21 \\
19 \\
17 \\
15\end{array}$ & $\begin{array}{r}11 \\
10 \\
8 \\
8 \\
8 \\
8\end{array}$ & $\begin{array}{r}11 \\
10 \\
10 \\
-10 \\
-1\end{array}$ & $\begin{array}{l}256 \\
442 \\
671 \\
954 \\
406 \\
205\end{array}$ & $\begin{array}{r}113 \\
121 \\
95 \\
76 \\
71\end{array}$ & $\begin{array}{l}31 \\
25 \\
22 \\
19 \\
18 \\
15\end{array}$ & $\begin{array}{r}26 \\
18 \\
77 \\
198 \\
137 \\
\end{array}$ & $\begin{array}{l}7.0 \\
7.8 \\
5.3 \\
5.0 \\
4.6 \\
4.8\end{array}$ & $\begin{array}{r}207 \\
25 \\
18 \\
15 \\
13 \\
10\end{array}$ & $\begin{array}{r}12 \\
8.0 \\
7.2 \\
6.2 \\
5.8\end{array}$ \\
\hline $\begin{array}{l}1922 \\
1 \\
2 \\
3 \\
4 \\
4 \\
5\end{array}$ & $\begin{array}{l}5.8 \\
6.7 \\
7.2 \\
6.2 \\
5.5\end{array}$ & $\begin{array}{l}10 \\
11 \\
12 \\
12 \\
11\end{array}$ & $\begin{array}{l}29 \\
29 \\
21 \\
27 \\
21\end{array}$ & $\begin{array}{r}73 \\
200 \\
115 \\
84 \\
64\end{array}$ & $\begin{array}{l}18 \\
17 \\
18 \\
19 \\
21\end{array}$ & $\begin{array}{l}14 \\
14 \\
15 \\
24 \\
33\end{array}$ & $\begin{array}{r}38 \\
34 \\
42 \\
260 \\
830\end{array}$ & $\begin{array}{l}320 \\
236 \\
162 \\
137 \\
121\end{array}$ & $\begin{array}{l}20 \\
15 \\
12 \\
11 \\
18\end{array}$ & $\begin{array}{l}6.7 \\
5.5 \\
3.8 \\
3.4 \\
4.4\end{array}$ & $\begin{array}{l}11 \\
10 \\
4.8 \\
3.0 \\
2.6\end{array}$ & $\begin{array}{r}1.6 \\
1.1 \\
.9 \\
1.2 \\
1.1\end{array}$ \\
\hline $\begin{array}{r}6 \\
7 \\
8 \\
9 \\
9 \\
10 \ldots\end{array}$ & $\begin{array}{l}6.7 \\
7.2 \\
16 \\
30 \\
27\end{array}$ & $\begin{array}{r}12 \\
14 \\
50 \\
121 \\
84\end{array}$ & $\begin{array}{l}16 \\
18 \\
18 \\
17 \\
18\end{array}$ & $\begin{array}{l}54 \\
48 \\
43 \\
39 \\
31\end{array}$ & $\begin{array}{l}19 \\
18 \\
15 \\
14 \\
14\end{array}$ & $\begin{array}{l}27 \\
22 \\
21 \\
18 \\
17\end{array}$ & $\begin{array}{l}710 \\
463 \\
514 \\
365 \\
207\end{array}$ & $\begin{array}{r}105 \\
94 \\
73 \\
110 \\
198\end{array}$ & $\begin{array}{r}51 \\
73 \\
81 \\
111 \\
101\end{array}$ & $\begin{array}{l}4.0 \\
4.4 \\
4.2 \\
3.4 \\
3.4\end{array}$ & $\begin{array}{l}2.5 \\
2.6 \\
2.5 \\
2.5 \\
1.9\end{array}$ & $\begin{array}{r}.9 \\
.6 \\
2.5 \\
35 \\
9.4\end{array}$ \\
\hline 13 & $\begin{array}{c}37 \\
21 \\
15 \\
11 \\
9.7\end{array}$ & $\begin{array}{l}24 \\
22 \\
17 \\
12 \\
14\end{array}$ & $\begin{array}{l}18 \\
23 \\
25 \\
24 \\
24\end{array}$ & $\begin{array}{l}28 \\
27 \\
28 \\
31 \\
39\end{array}$ & $\begin{array}{l}14 \\
14 \\
15 \\
15 \\
14\end{array}$ & $\begin{array}{l}16 \\
16 \\
15 \\
14 \\
14\end{array}$ & $\begin{array}{l}277 \\
320 \\
189 \\
121 \\
110\end{array}$ & $\begin{array}{r}129 \\
129 \\
129 \\
113 \\
92\end{array}$ & $\begin{array}{l}42 \\
23 \\
21 \\
15\end{array}$ & $\begin{array}{l}3.0 \\
2.6 \\
2.5 \\
2.0 \\
5.3\end{array}$ & $\begin{array}{c}2.0 \\
2.6 \\
20 \\
5.0 \\
3.0\end{array}$ & $\begin{array}{l}5.3 \\
3.2 \\
3.0 \\
2.6 \\
2.6\end{array}$ \\
\hline $\begin{array}{l}16 \\
17 \\
18 \\
20 \\
20\end{array}$ & $\begin{array}{l}9.7 \\
13 \\
12 \\
9.4 \\
9.2\end{array}$ & $\begin{array}{l}18 \\
17 \\
16 \\
18 \\
19\end{array}$ & $\begin{array}{l}20 \\
13 \\
11 \\
11 \\
10\end{array}$ & $\begin{array}{l}31 \\
31 \\
31 \\
33 \\
35\end{array}$ & $\begin{array}{l}14 \\
14 \\
13 \\
13 \\
14\end{array}$ & $\begin{array}{r}16 \\
21 \\
145 \\
145 \\
57\end{array}$ & $\begin{array}{l}121 \\
108 \\
105 \\
101 \\
145\end{array}$ & $\begin{array}{r}137 \\
121 \\
72 \\
53 \\
47\end{array}$ & $\begin{array}{l}17 \\
17 \\
15 \\
18 \\
13\end{array}$ & $\begin{array}{r}75 \\
24 \\
12 \\
9.2 \\
9.2\end{array}$ & $\begin{array}{l}2.6 \\
2.0 \\
2.8 \\
2.5 \\
1.1\end{array}$ & $\begin{array}{l}1.7 \\
1.4 \\
1.4 \\
1.3 \\
1.2\end{array}$ \\
\hline $\begin{array}{l}21 \\
22 \\
24 \\
25\end{array}$ & $\begin{array}{l}9.7 \\
9.7 \\
10 \\
42 \\
20\end{array}$ & $\begin{array}{l}18 \\
16 \\
17 \\
16 \\
18\end{array}$ & $\begin{array}{l}9.7 \\
11 \\
14 \\
14 \\
14\end{array}$ & $\begin{array}{l}39 \\
98 \\
72 \\
52 \\
39\end{array}$ & $\begin{array}{l}14 \\
14 \\
14 \\
14 \\
14\end{array}$ & $\begin{array}{r}37 \\
34 \\
145 \\
280 \\
200\end{array}$ & $\begin{array}{l}320 \\
365 \\
365 \\
189 \\
153\end{array}$ & $\begin{array}{r}72 \\
104 \\
58 \\
43 \\
34\end{array}$ & $\begin{array}{l}8.6 \\
7.5 \\
7.0 \\
5.3 \\
5.5\end{array}$ & $\begin{array}{l}5.3 \\
3.8 \\
5.0 \\
10 \\
13\end{array}$ & $\begin{array}{l}1.4 \\
1.0 \\
1.1 \\
1.0 \\
1.1\end{array}$ & $\begin{array}{l}6.7 \\
9.4 \\
5.8 \\
14 \\
11\end{array}$ \\
\hline $\begin{array}{l}26 \\
27 \\
28 \\
29 \\
30 \\
31\end{array}$ & $\begin{array}{l}17 \\
14 \\
12 \\
12 \\
12 \\
12\end{array}$ & $\begin{array}{l}16 \\
14 \\
14 \\
14 \\
15\end{array}$ & $\begin{array}{l}16 \\
17 \\
14 \\
14 \\
14 \\
14 \\
14\end{array}$ & $\begin{array}{l}35 \\
25 \\
21 \\
19 \\
18 \\
18\end{array}$ & $\begin{array}{l}14 \\
15 \\
15 \\
- \\
-\end{array}$ & $\begin{array}{r}145 \\
115 \\
92 \\
66 \\
50 \\
44\end{array}$ & $\begin{array}{l}129 \\
121 \\
342 \\
710 \\
594\end{array}$ & $\begin{array}{l}29 \\
26 \\
25 \\
26 \\
25 \\
23\end{array}$ & $\begin{array}{l}5.8 \\
5.5 \\
5.5 \\
6.2 \\
7.5 \\
\\
\end{array}$ & $\begin{array}{c}14 \\
8.3 \\
13 \\
5.8 \\
33 \\
14\end{array}$ & $\begin{array}{r}1.2 \\
1.1 \\
5.0 \\
18 \\
5.5 \\
2.3\end{array}$ & $\begin{array}{l}10 \\
11 \\
9.4 \\
9.4 \\
8.6\end{array}$ \\
\hline
\end{tabular}

Note.-Stage-discharge relation affected by ice Dec. 7-13, 15, 21-31, 1921, Jan. 1-3, and Feb. 2 to Mar. 7, 1922, Jan. 2-25, Mar. 18, 24-27, and Apr. 4, 1923; discharge based on gage heights corrected for effect of ice. Daily discharge, Apr. 11 to Sept. 30, 1922, supersedes that published in Water-Supply Paper 544. 
Monthly discharge, in second-feet, of Jail Branch at East Barre, Vt., for the years ending September 30, 1922 and 1923

\begin{tabular}{|c|c|c|c|c|c|c|}
\hline \multirow{2}{*}{ Month } & \multicolumn{3}{|c|}{$1921-22$} & \multicolumn{3}{|c|}{$1922-23$} \\
\hline & Maximum & Minimum & Mean & Maximum & Minimum & Meane \\
\hline $\begin{array}{l}\text { October } \\
\text { November } \\
\text { December } \\
\text { January } \\
\text { February } \\
\text { Mareh } \\
\text { April } \\
\text { May } \\
\text { June } \\
\text { July } \\
\text { August } \\
\text { September }\end{array}$ & $\begin{array}{r}67 \\
460 \\
635 \\
15 \\
14 \\
954 \\
1,140 \\
246 \\
256 \\
153 \\
207 \\
84\end{array}$ & $\begin{array}{r}3.0 \\
12 \\
15 \\
8 \\
8 \\
9 \\
71 \\
15 \\
12 \\
4.6 \\
4.4 \\
4.0\end{array}$ & $\begin{array}{l}13.0 \\
72.8 \\
96.3 \\
11.5 \\
10.0 \\
216 \\
308 \\
71.0 \\
58.0 \\
29.9 \\
27.4 \\
13.7\end{array}$ & $\begin{array}{r}42 \\
121 \\
29 \\
200 \\
21 \\
280 \\
830 \\
320 \\
111 \\
75 \\
20 \\
35\end{array}$ & $\begin{array}{c}5.5 \\
10 \\
9.7 \\
18 \\
13 \\
14 \\
34 \\
23 \\
5.3 \\
2.0 \\
1.0 \\
.6\end{array}$ & $\begin{array}{r}14.1 \\
22.4 \\
17.6 \\
48.4 \\
15.2 \\
60.4 \\
278 \\
98.2 \\
27.3 \\
10.1 \\
4.05 \\
5.78\end{array}$ \\
\hline The year. & 1,140 & 3.0 & 77.5 & 830 & .6 & 50.1 \\
\hline
\end{tabular}

Nore, - Monthly discharge April to September, 1922, and yearly discharge, 1922, supersedes that pubished in Water-Supply Paper 544.

\section{LAMOILLE RIVER AT CADYS FALLS, VT.}

Location.--One-fourth mile below power plant of Morrisville Electric Light \& Power Co., at what was formerly known as Cadys Falls, 2 miles downstream from village of Morrisville, Lamoille County.

Drain Age AREA.-280 square miles.

Records Available.-September 4, 1913, to September 30, 1923, when station was discontinued.

$\mathrm{G}_{\mathrm{AGE}}$--Friez water-stage recorder in gage house on right bank, one-fourth mile below highway bridge at Cadys Falls. Gage heights are referred to gage datum by means of a hook gage inside the well; an outside staff gage is used for auxiliary readings. Recorder inspected by N. E. Cobleigh.

Discharge measUREMENTs.-Made from cable or by wading.

Channel. and control.-Channel smooth gravel; well-defined gravel control 500 feet downstream from gage.

Extremes of Discharge.-Maximum stage recorded during year from waterstage recorder, 9.46 feet at 2 p. m. April 30 (discharge, 5,820 second-feet); discharge practically nil at various times in October (water held back by dam).

1913-1923: Maximum stage recorded, 11.63 feet October 1, 1920 (discharge, from extension of rating curve, 8,730 second-feet); discharge practically nil at various times when water was held back by dam.

ICE.-River freezes over at gage during extremely cold weather, but control usually remains partly open.

Regulatron.-Dams at Cadys Falls and Morrisville have some effect on the distribution of flow. There are several lakes and ponds in the basin, but these have little if any regulation for storage.

Accuracy.-Stage-discharge relation practically permanent, except when affected by ice. Rating curve well defined. Operation of water-stage recorder satisfactory except for short periods mentioned in footnote to daily discharge table. Daily discharge for open-water period ascertained by discharge integrator. Open-water records excellent; winter records fair. 
Discharge measurements of Lamoille River at Cadys Falls, Vt., during the year ending September 30, 1923

[Made by H. F. Hill, jr.]

\begin{tabular}{r|r|r|r}
\hline & Date & $\begin{array}{r}\text { Gage } \\
\text { height }\end{array}$ & $\begin{array}{r}\text { Dis- } \\
\text { charge }\end{array}$ \\
\hline Feb. 6 Feet & Sec.ft. \\
187 \\
3,290
\end{tabular}

- Stage-discharge relation affected by ice.

Daily discharge, in second-feet, of Lamoille River at Cadys Falls, Vt., for the year ending September 30, 1923

\begin{tabular}{|c|c|c|c|c|c|c|c|c|c|c|c|c|}
\hline Day & Oct. & Nov. & Dec. & Jan. & Feb. & Mar. & Apr. & May & June & July & Aug. & Sept. \\
\hline $\begin{array}{l}1 \\
2 . \\
3 . \\
4 \\
5\end{array}$ & $\begin{array}{r}64 \\
114 \\
102 \\
100 \\
98\end{array}$ & $\begin{array}{r}190 \\
160 \\
134 \\
140 \\
50\end{array}$ & $\begin{array}{l}156 \\
290 \\
150 \\
210 \\
196\end{array}$ & $\begin{array}{r}230 \\
1,000 \\
630 \\
470 \\
350\end{array}$ & $\begin{array}{r}182 \\
166 \\
85 \\
30 \\
96\end{array}$ & $\begin{array}{r}130 \\
110 \\
64 \\
10 \\
44\end{array}$ & $\begin{array}{r}320 \\
310 \\
265 \\
870 \\
1,740\end{array}$ & $\begin{array}{r}2,500 \\
1,600 \\
1,100 \\
870 \\
690\end{array}$ & $\begin{array}{r}195 \\
195 \\
90 \\
130 \\
530\end{array}$ & $\begin{array}{r}150 \\
170 \\
190 \\
90 \\
135\end{array}$ & $\begin{array}{r}120 \\
120 \\
115 \\
90 \\
65\end{array}$ & $\begin{array}{r}100 \\
65 \\
110 \\
100 \\
110\end{array}$ \\
\hline $\begin{array}{r}6 .- \\
7 .- \\
8- \\
9- \\
10 .-\end{array}$ & $\begin{array}{r}90 \\
164 \\
48 \\
190 \\
210\end{array}$ & $\begin{array}{l}132 \\
134 \\
136 \\
136 \\
136\end{array}$ & $\begin{array}{r}220 \\
186 \\
186 \\
186 \\
90\end{array}$ & $\begin{array}{l}290 \\
250 \\
240 \\
230 \\
230\end{array}$ & $\begin{array}{r}105 \\
105 \\
96 \\
125 \\
145\end{array}$ & $\begin{array}{r}52 \\
60 \\
220 \\
200 \\
150\end{array}$ & $\begin{array}{l}4,000 \\
3,200 \\
2,950 \\
2,600 \\
1,840\end{array}$ & $\begin{array}{l}545 \\
475 \\
460 \\
430 \\
540\end{array}$ & $\begin{array}{r}980 \\
750 \\
870 \\
1,960 \\
1,000\end{array}$ & $\begin{array}{l}170 \\
190 \\
110 \\
130 \\
120\end{array}$ & $\begin{array}{l}180 \\
140 \\
120 \\
120 \\
115\end{array}$ & $\begin{array}{r}95 \\
95 \\
130 \\
70 \\
165\end{array}$ \\
\hline $\begin{array}{l}13 \\
14 \\
15\end{array}$ & $\begin{array}{r}198 \\
196 \\
194 \\
162 \\
52\end{array}$ & $\begin{array}{l}100 \\
120 \\
156 \\
156 \\
180\end{array}$ & $\begin{array}{l}180 \\
170 \\
170 \\
162 \\
130\end{array}$ & $\begin{array}{r}210 \\
220 \\
210 \\
70 \\
172\end{array}$ & $\begin{array}{r}66 \\
180 \\
180 \\
160 \\
150\end{array}$ & $\begin{array}{r}96 \\
110 \\
115 \\
130 \\
140\end{array}$ & $\begin{array}{l}1,840 \\
2,200 \\
1,660 \\
1,260 \\
1,060\end{array}$ & $\begin{array}{l}640 \\
365 \\
270 \\
485 \\
400\end{array}$ & $\begin{array}{l}570 \\
360 \\
290 \\
250 \\
230\end{array}$ & $\begin{array}{r}110 \\
125 \\
45\end{array}$ & $\begin{array}{r}110 \\
40 \\
75 \\
155 \\
90\end{array}$ & $\begin{array}{l}125 \\
120 \\
120 \\
14 \\
15\end{array}$ \\
\hline $\begin{array}{l}16 \ldots \\
17 . . \\
18 . \\
19 . \\
20 \ldots\end{array}$ & $\begin{array}{l}116 \\
136 \\
152 \\
128 \\
100\end{array}$ & $\begin{array}{l}230 \\
200 \\
194 \\
150 \\
245\end{array}$ & $\begin{array}{r}160 \\
50 \\
116 \\
140 \\
150\end{array}$ & $\begin{array}{l}172 \\
166 \\
160 \\
141 \\
141\end{array}$ & $\begin{array}{r}105 \\
96 \\
62 \\
98 \\
120\end{array}$ & $\begin{array}{r}125 \\
94 \\
115 \\
320 \\
320\end{array}$ & $\begin{array}{l}920 \\
820 \\
740 \\
650 \\
770\end{array}$ & $\begin{array}{r}570 \\
1,500 \\
830 \\
560 \\
395\end{array}$ & $\begin{array}{l}195 \\
180 \\
200 \\
195 \\
175\end{array}$ & $\begin{array}{l}100 \\
115 \\
115 \\
115 \\
105\end{array}$ & $\begin{array}{r}80 \\
100 \\
125 \\
95 \\
160\end{array}$ & $\begin{array}{l}95 \\
200 \\
135 \\
100 \\
120\end{array}$ \\
\hline $\begin{array}{l}21 \\
22 \\
23 \\
24 \\
25\end{array}$ & $\begin{array}{r}84 \\
15 \\
80 \\
150 \\
200\end{array}$ & $\begin{array}{l}345 \\
290 \\
230 \\
215 \\
215\end{array}$ & $\begin{array}{r}106 \\
106 \\
104 \\
17 \\
24\end{array}$ & $\begin{array}{l}138 \\
191 \\
231 \\
153 \\
141\end{array}$ & $\begin{array}{r}125 \\
120 \\
115 \\
125 \\
86\end{array}$ & $\begin{array}{r}360 \\
350 \\
330 \\
1,340 \\
1,240\end{array}$ & $\begin{array}{l}2,750 \\
3,600 \\
3,700 \\
1,740 \\
1,160\end{array}$ & $\begin{array}{l}460 \\
600 \\
420 \\
340 \\
340\end{array}$ & $\begin{array}{l}170 \\
175 \\
150 \\
120 \\
170\end{array}$ & $\begin{array}{l}115 \\
110 \\
125 \\
170 \\
145\end{array}$ & $\begin{array}{r}125 \\
90 \\
110 \\
110 \\
125\end{array}$ & $\begin{array}{l}27 \\
34 \\
18 \\
18 \\
12\end{array}$ \\
\hline $\begin{array}{l}26 \\
28 \\
29 \\
31 \\
31\end{array}$ & $\begin{array}{l}188 \\
180 \\
188 \\
100 \\
182 \\
210\end{array}$ & $\begin{array}{r}80 \\
170 \\
194 \\
184 \\
100\end{array}$ & $\begin{array}{r}85 \\
98 \\
106 \\
180 \\
174 \\
53\end{array}$ & $\begin{array}{l}141 \\
147 \\
109 \\
172 \\
185 \\
191\end{array}$ & $\begin{array}{r}120 \\
135 \\
125 \\
\end{array}$ & $\begin{array}{l}810 \\
610 \\
495 \\
405 \\
395 \\
225\end{array}$ & $\begin{array}{r}920 \\
950 \\
1,040 \\
4,100 \\
4,650\end{array}$ & $\begin{array}{l}290 \\
195 \\
225 \\
230 \\
165 \\
200\end{array}$ & $\begin{array}{l}190 \\
225 \\
205 \\
240 \\
195\end{array}$ & $\begin{array}{r}115 \\
155 \\
165 \\
70 \\
85 \\
130\end{array}$ & $\begin{array}{r}90 \\
115 \\
125 \\
110 \\
140 \\
115\end{array}$ & $\begin{array}{l}150 \\
200 \\
190 \\
190 \\
140\end{array}$ \\
\hline
\end{tabular}

Nore.-Stage-discharge relation affected by ice Jan. 4 to Mar. 23; discharge for this period based on gage heights corrected for effect of ice. 
Monthly discharge of Lamoille River at Cadys Falls, Vt., for the year ending September 30,1928

[Drainage area, 280 square miles]

\begin{tabular}{|c|c|c|c|c|c|}
\hline \multirow{2}{*}{ Month } & \multicolumn{4}{|c|}{ Discharge in second-feet } & \multirow{2}{*}{$\begin{array}{l}\text { Run-off } \\
\text { in inches }\end{array}$} \\
\hline & Maximum & Minimum & Mean & $\begin{array}{c}\text { Per square } \\
\text { mile }\end{array}$ & \\
\hline $\begin{array}{l}\text { October } \\
\text { November } \\
\text { December } \\
\text { January } \\
\text { February } \\
\text { March } \\
\text { April } \\
\text { May } \\
\text { June } \\
\text { July } \\
\text { August } \\
\text { September }\end{array}$ & $\begin{array}{r}210 \\
345 \\
290 \\
1,000 \\
182 \\
1,340 \\
4,650 \\
2,500 \\
1,960 \\
190 \\
180 \\
340\end{array}$ & $\begin{array}{r}15 \\
50 \\
17 \\
70 \\
30 \\
10 \\
265 \\
165 \\
90 \\
45 \\
40 \\
65\end{array}$ & $\begin{array}{r}135 \\
170 \\
140 \\
238 \\
118 \\
296 \\
1,820 \\
603 \\
373 \\
126 \\
112 \\
144\end{array}$ & $\begin{array}{l}0.482 \\
.607 \\
.500 \\
.850 \\
.421 \\
1.06 \\
6.50 \\
2.15 \\
1.33 \\
.450 \\
.400 \\
.514\end{array}$ & $\begin{array}{r}0.56 \\
.68 \\
.58 \\
.98 \\
.44 \\
1.22 \\
7.25 \\
2.48 \\
1.48 \\
.52 \\
.46 \\
.57\end{array}$ \\
\hline The year. & 4,650 & 10 & 355 & 1.27 & 17.22 \\
\hline
\end{tabular}

NOTE.-See paragraph on "Regulation."

\section{GREEN RIVER AT GARFIELD, VT.}

Location.-At site of old dam above highway bridge at Garfield village, town of Hyde Park; Lamoille County. Green River is tributary to Lamoille River 4 miles east of Morrisville.

Drainage AREa.-About 20 square miles.

Records available.-January 23, 1915, to March 16, 1921, and December 3, 1922, to September 30, 1923.

GAGE.-Inclined staff on left bank in pool back of weir; read by P. M. Trescott.

Discharge measurements.-Made at footbridge half a mile downstream from weir or at old bridge half a mile above weir.

Channel and control.-Sharp-crested compound weir, installed December, 1922; length of crest at gage height 0.00 is 9.0 feet; at gage height 0.83 foot length of crest is increased 11.17 feet. A pool of considerable size is formed by the old mill dam on which the weir is built; at ordinary stages the velocity of approach to the weir is very small.

EXTREMES OF DISCHARGE.- - Maximum stage recorded during the period December 3, 1922, to September 30,1923, 4.07 feet at 9 a. m. April 22 (discharge, by extension of rating curve, 586 second-feet); minimum stage recorded during period, 0.11 foot at 5 p. m. August 11 and 5 p. m. August 12 (discharge, 2.2 second-feet).

1915-1921; 1922-1923: Maximum discharge (determined from high-water marks and extension of rating curve), 710 second-feet on April 12, 1919; minimum discharge recorded, 2.2 second-feet August 11 and 12, 1923.

IcE.-Weir kept clear of ice during winter; stage-discharge relation not affected by ice.

REgULATION.-An old timber dam 2 miles upstream affects flow to some extent. Leakage through dam exceeds low-water flow. During prolonged low stages the surface of water in pond (103 acres) falls below crest of dam; subsequent increased flow into pond is retained until water again flows over crest, when the increased flow is apparent at gaging station. 
AccuRAcr.--Stage-discharge relation permanent since weir was rebuilt, in December, 1922. Rating curve based on weir formula, $Q=3.33 \mathrm{LH}^{1.5}$ with corrections determined from current-meter measurements and with logarithmic extension above gage height 1.5 feet. Gage read to hundredths twice daily. Daily discharge ascertained by applying mean daily gage height to rating table. Records below 130 second-feet good; a higher stages weir is flooded and records are somewhat uncertain.

Discharge measurements of Green River at Garfield, Vt., during the year ending September 30, 1923

[Made by H. F. Hill, jr.]

\begin{tabular}{|c|c|c|c|c|c|}
\hline Date & $\begin{array}{l}\text { Gage } \\
\text { height }\end{array}$ & $\begin{array}{l}\text { Dis- } \\
\text { charge }\end{array}$ & Date & $\begin{array}{l}\text { Gage } \\
\text { height }\end{array}$ & $\begin{array}{l}\text { Dis- } \\
\text { charge }\end{array}$ \\
\hline 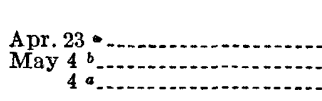 & $\begin{array}{l}\text { Feet } \\
3.36 \\
1.26 \\
1.26\end{array}$ & $\begin{array}{r}\text { Sec. fft. } \\
444 \\
52 \\
60\end{array}$ & May 44 & $\begin{array}{l}\text { Feet } \\
1.24 \\
1.23\end{array}$ & Sec-ft. ${ }_{51}$ \\
\hline
\end{tabular}

- Made at footbridge half a mile below gage

b Made at old bridga half a mile above gage.

Daily discharge, in second-feet, of Green River at Garfield, Vt., for the year ending September 30, 1923

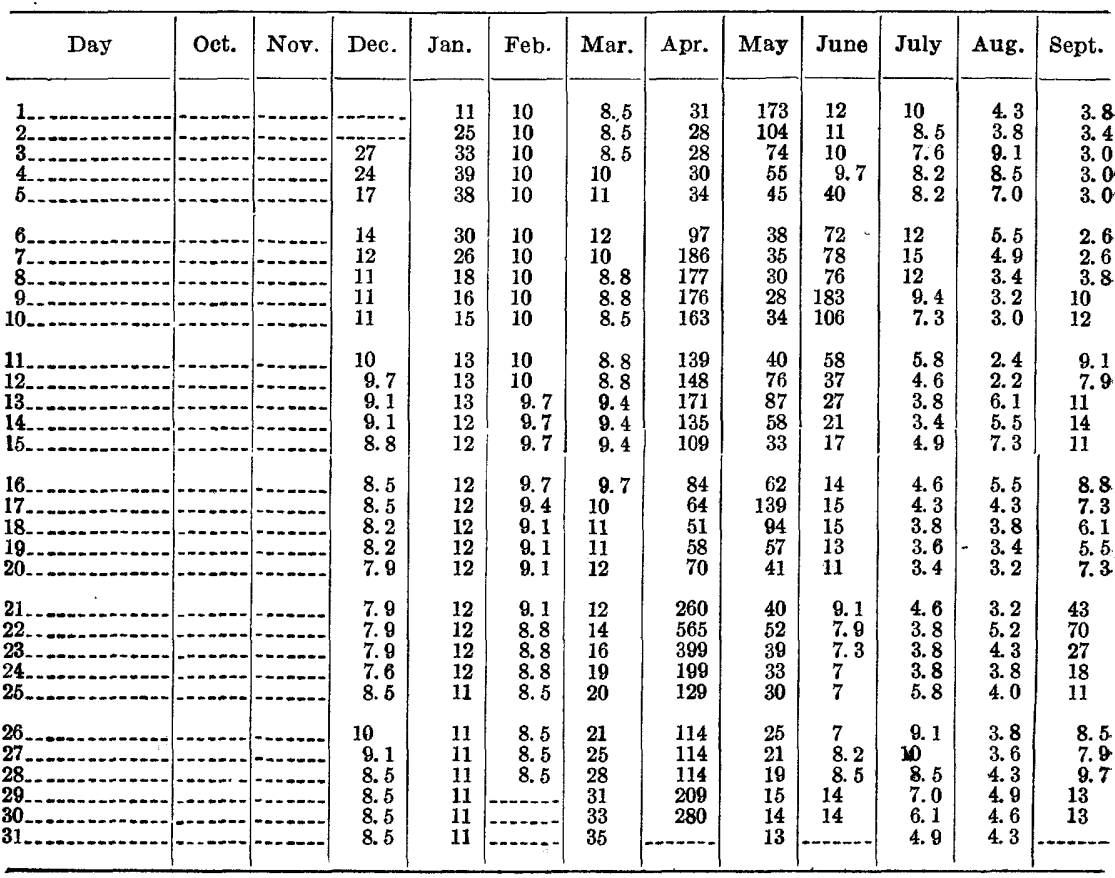


Monthly discharge, in second-feet, of Green River at Garfield, Vt., for the year ending September 30, 1923

\begin{tabular}{|c|c|c|c|c|c|c|c|}
\hline Month & Maximum & Minimum & Mean & Month & Maximum & Minimum & Mean \\
\hline $\begin{array}{l}\text { December 3-31. } \\
\text { January } \\
\text { February } \\
\text { March } \\
\text { April }\end{array}$ & $\begin{array}{r}27 \\
39 \\
10 \\
35 \\
565\end{array}$ & $\begin{array}{c}7.6 \\
11 \\
8.5 \\
8.5 \\
28\end{array}$ & $\begin{array}{r}10.6 \\
16.1 \\
9.5 \\
14.4 \\
145\end{array}$ & $\begin{array}{l}\text { May..... } \\
\text { June } \\
\text { July } \\
\text { August } \\
\text { September. }\end{array}$ & $\begin{array}{l}173 \\
183 \\
15 \\
9.1 \\
70\end{array}$ & $\begin{array}{l}13 \\
7.3 \\
3.4 \\
2.2 \\
2.6\end{array}$ & $\begin{array}{r}51.8 \\
30.5 \\
6.7 \\
4.6 \\
11.9\end{array}$ \\
\hline
\end{tabular}

Note.-See paragraph on "Regulation."

\section{MISSISQUOI RIVER NEAR RICHFORD, VT.}

Location.-3 miles downstream from Richford, Franklin County, and mouth of North Branch and 2 miles above mouth of Trout River.

Drainage AREA.-445 square miles.

Records available.-May 22, 1909, to December 3, 1910, and June 26, 1911, to September 30, 1923, when station was discontinued.

GAGE.-Gurley water-stage recorder on left bank, one-fourth mile above highway bridge; inspected by Harry Jenne. Chain gage on highway bridge used from June 26, 1911, to July 31, 1915 . From May 22, 1909, to December 3, 1910, gage was just below plant of the Sweat-Comings Co. in Richford.

Discharge measurements.-Made from highway bridge or by wading.

Channel and control.-Channel deep, banks not subject to overflow; stream bed composed of gravel, boulders and ledge rock; control is sharply defined by rock outcrop 100 feet below gage.

Extremes of Discharge.-Maximum stage during year from water-stage recorder, 14.38 feet at $8 \mathrm{p} . \mathrm{m}$. April 7 (discharge, by extension of rating curve, 13,000 second-feet); minimum stage, from water-stage recorder, 1.94 feet at 2 a. m. October 2 (discharge, 20 second-feet, water held back by dams).

1911-1923: Maximum discharge, that of April 7, 1923; minimum discharge, about 8 second-feet July 14, 1911 (water held back by dams).

ICE:-Stage-discharge relation usually affected by ice from December to March; discharge determined from gage heights corrected for effect of ice by means of current-meter measurements, observer's notes, and weather records.

Regulation.-Considerable daily fluctuation at low stages caused by operation of power plants at Richford.

AccuracY.- Stage-discharge relation practically permanent except when affected by ice. Rating curve fairly well defined below 6,000 second-feet. Operation of water-stage recorder generally satisfactory. Daily discharge ascertained by applying to rating table mean daily gage height determined by inspection of recorder sheets, with corrections for effect of ice during the winter or, for days having large variation in stage, by averaging discharge of 12 two-hour periods. Open-water records good; winter records fair.

Discharge measurements of Missisquoi River near Richford, Vt., during the year ending September 30, 1923

\begin{tabular}{|c|c|c|c|}
\hline Date & Made by- & $\begin{array}{c}\text { Gage } \\
\text { height }\end{array}$ & $\begin{array}{c}\text { Dis- } \\
\text { charge }\end{array}$ \\
\hline $\begin{array}{l}\text { Oct } 30 \\
\text { Feb. } \\
\text { Apr. } 21\end{array}$ & $\begin{array}{l}\text { W. E. Armstrong } \\
\text { H. Fo Hiil, jr... }\end{array}$ & $\begin{array}{l}\text { Feet. } \\
2.99 \\
\times 3.96 \\
7.50\end{array}$ & $\begin{array}{r}\text { Sec. } f t \\
250 \\
181 \\
4,080\end{array}$ \\
\hline
\end{tabular}

atage-discharge relation affected by ice. 
Daily discharge, in second-feet, of Missisquoi River near Richford, Vt., for year ending September 30, 1923

\begin{tabular}{|c|c|c|c|c|c|c|c|c|c|c|c|c|}
\hline Day & Oct. & Nov. & Dec. & Jan. & Feb. & Mar. & Apr. & May & June & July & Aug. & Sept. \\
\hline $\begin{array}{l}1 \\
2 \\
3 \\
3 \\
4 \\
5\end{array}$ & $\begin{array}{r}88 \\
73 \\
70 \\
75 \\
132\end{array}$ & $\begin{array}{l}221 \\
191 \\
188 \\
194 \\
185\end{array}$ & $\begin{array}{l}460 \\
650 \\
580 \\
530 \\
460\end{array}$ & $\begin{array}{r}260 \\
1,100 \\
1,000 \\
680 \\
480\end{array}$ & $\begin{array}{l}175 \\
190 \\
195 \\
200 \\
200\end{array}$ & $\begin{array}{l}150 \\
150 \\
150 \\
170 \\
180\end{array}$ & $\begin{array}{r}860 \\
830 \\
1,700 \\
3,000 \\
4,500\end{array}$ & $\begin{array}{l}6,720 \\
4,160 \\
2,340 \\
1,760 \\
1,520\end{array}$ & $\begin{array}{l}407 \\
355 \\
282 \\
275 \\
523\end{array}$ & $\begin{array}{l}460 \\
339 \\
279 \\
282 \\
300\end{array}$ & $\begin{array}{r}112 \\
102 \\
90 \\
86 \\
84\end{array}$ & $\begin{array}{r}127 \\
110 \\
86 \\
88 \\
73\end{array}$ \\
\hline 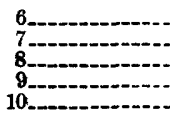 & $\begin{array}{l}140 \\
140 \\
152 \\
215 \\
206\end{array}$ & $\begin{array}{l}\mathbf{1 9 4} \\
\mathbf{2 0 0} \\
\mathbf{1 7 9} \\
206 \\
233\end{array}$ & $\begin{array}{l}470 \\
480 \\
500 \\
500 \\
400\end{array}$ & $\begin{array}{l}380 \\
330 \\
290 \\
300 \\
290\end{array}$ & $\begin{array}{l}195 \\
180 \\
190 \\
200 \\
200\end{array}$ & $\begin{array}{l}210 \\
230 \\
230 \\
200 \\
180\end{array}$ & $\begin{array}{r}7,220 \\
11,000 \\
11,500 \\
10,700 \\
5,400\end{array}$ & $\begin{array}{r}1,320 \\
1,170 \\
960 \\
890 \\
960\end{array}$ & $\begin{array}{l}2,640 \\
1,970 \\
1,520 \\
3,610 \\
2,840\end{array}$ & $\begin{array}{l}339 \\
424 \\
391 \\
296 \\
265\end{array}$ & $\begin{array}{l}77 \\
68 \\
73 \\
50 \\
55\end{array}$ & $\begin{array}{r}88 \\
86 \\
68 \\
197 \\
185\end{array}$ \\
\hline 15 & $\begin{array}{l}224 \\
233 \\
215 \\
167 \\
147\end{array}$ & $\begin{array}{l}359 \\
403 \\
446 \\
375 \\
451\end{array}$ & $\begin{array}{l}310 \\
280 \\
260 \\
240 \\
230\end{array}$ & $\begin{array}{l}270 \\
260 \\
240 \\
240 \\
230\end{array}$ & $\begin{array}{l}195 \\
195 \\
200 . \\
210 \\
220\end{array}$ & $\begin{array}{l}175 \\
175 \\
170 \\
170 \\
190\end{array}$ & $\begin{array}{l}4.710 \\
4,820 \\
4,820 \\
4,600 \\
3,830\end{array}$ & $\begin{array}{r}1,030 \\
1,100 \\
1,360 \\
995 \\
890\end{array}$ & $\begin{array}{r}1,240 \\
800 \\
686 \\
565 \\
500\end{array}$ & $\begin{array}{l}254 \\
206 \\
170 \\
162 \\
155\end{array}$ & $\begin{array}{l}58 \\
68 \\
84 \\
86 \\
90\end{array}$ & $\begin{array}{l}188 \\
155 \\
160 \\
167 \\
147\end{array}$ \\
\hline-1 & $\begin{array}{l}157 \\
137 \\
132 \\
130 \\
135\end{array}$ & $\begin{array}{l}645 \\
532 \\
419 \\
415 \\
560\end{array}$ & $\begin{array}{l}220 \\
220 \\
210 \\
210 \\
200\end{array}$ & $\begin{array}{l}230 \\
240 \\
210 \\
210 \\
210\end{array}$ & $\begin{array}{l}200 \\
190 \\
185 \\
185 \\
180\end{array}$ & $\begin{array}{l}220 \\
380 \\
520 \\
600 \\
560\end{array}$ & $\begin{array}{l}2,150 \\
1,800 \\
1,640 \\
1,600 \\
1,600\end{array}$ & $\begin{array}{l}2,640 \\
5,280 \\
3,610 \\
2,060 \\
1,400\end{array}$ & $\begin{array}{l}469 \\
478 \\
433 \\
343 \\
307\end{array}$ & $\begin{array}{l}152 \\
140 \\
152 \\
140 \\
135\end{array}$ & $\begin{array}{r}97 \\
105 \\
84 \\
71 \\
88\end{array}$ & $\begin{array}{l}125 \\
140 \\
135 \\
162 \\
147\end{array}$ \\
\hline $\begin{array}{l}23 \\
24 \\
25\end{array}$ & $\begin{array}{l}130 \\
137 \\
157 \\
424 \\
505\end{array}$ & $\begin{array}{l}960 \\
860 \\
650 \\
550 \\
478\end{array}$ & $\begin{array}{l}200 \\
210 \\
210 \\
210 \\
210\end{array}$ & $\begin{array}{l}220 \\
280 \\
300 \\
270 \\
250\end{array}$ & $\begin{array}{l}180 \\
195 \\
180 \\
185 \\
190\end{array}$ & $\begin{array}{r}500 \\
560 \\
600 \\
1,700 \\
1,950\end{array}$ & $\begin{array}{l}3,940 \\
7,090 \\
7,610 \\
5,400 \\
3,060\end{array}$ & $\begin{array}{r}1,240 \\
1,440 \\
1,130 \\
1,030 \\
860\end{array}$ & $\begin{array}{l}272 \\
237 \\
221 \\
182 \\
179\end{array}$ & $\begin{array}{l}137 \\
137 \\
135 \\
117 \\
115\end{array}$ & $\begin{array}{l}107 \\
331 \\
227 \\
176 \\
188\end{array}$ & $\begin{array}{l}478 \\
536 \\
387 \\
261 \\
209\end{array}$ \\
\hline $\begin{array}{l}26 \\
27 \\
28 \\
29 \\
30 \\
31\end{array}$ & $\begin{array}{l}514 \\
482 \\
343 \\
272 \\
240 \\
230\end{array}$ & $\begin{array}{l}451 \\
491 \\
428 \\
355 \\
339\end{array}$ & $\begin{array}{l}210 \\
220 \\
220 \\
220 \\
220 \\
220\end{array}$ & $\begin{array}{l}250 \\
230 \\
210 \\
230 \\
200 \\
180\end{array}$ & $\begin{array}{l}170 \\
170 \\
155\end{array}$ & $\begin{array}{r}1,850 \\
1,600 \\
1,400 \\
1,100 \\
960 \\
880\end{array}$ & $\begin{array}{l}2,060 \\
1,970 \\
2,190 \\
4,930 \\
7,610\end{array}$ & $\begin{array}{l}800 \\
625 \\
575 \\
523 \\
424 \\
399\end{array}$ & $\begin{array}{l}254 \\
428 \\
523 \\
536 \\
590\end{array}$ & $\begin{array}{l}125 \\
130 \\
132 \\
145 \\
117 \\
112\end{array}$ & $\begin{array}{l}170 \\
137 \\
105 \\
157 \\
145 \\
135\end{array}$ & $\begin{array}{l}182 \\
279 \\
303 \\
343 \\
351\end{array}$ \\
\hline
\end{tabular}

Note--Stage-discharge relation affected by ice Dec.1 to Apr. 5; daily discharge for days when gage was read based on gage heights corrected for effect of ice. No gage-height record Nov. 9, 10, Jan. 30, Feb 4-6, Feb. 22 to Mar. 3, Mar. 11-15, 21, 22, Mar. 31 to Apr. 3, Apr. 13-15, 18-20, and May 17 and 18; daily discharge estimated by hydrograph comparisons and meteorological data.

Monthly discharge of Missisquoi River near Richford, Vt., for the year ending September 30, 1923

[Drainage area, 445 square miles]

\begin{tabular}{|c|c|c|c|c|c|}
\hline \multirow{2}{*}{ Month } & \multicolumn{4}{|c|}{ Discharge in second-feet } & \multirow{2}{*}{$\begin{array}{c}\text { Run-off in } \\
\text { inches }\end{array}$} \\
\hline & Maximum & Minimum & Mean & $\begin{array}{c}\text { Per square } \\
\text { mile }\end{array}$ & \\
\hline $\begin{array}{l}\text { October } \\
\text { November } \\
\text { December } \\
\text { January } \\
\text { February } \\
\text { March } \\
\text { April } \\
\text { May } \\
\text { June } \\
\text { July } \\
\text { August } \\
\text { September }\end{array}$ & $\begin{array}{r}514 \\
960 \\
650 \\
1,100 \\
220 \\
1,950 \\
11,500 \\
6,720 \\
3,610 \\
460 \\
331 \\
536\end{array}$ & $\begin{array}{r}70 \\
179 \\
200 \\
180 \\
155 \\
150 \\
830 \\
399 \\
179 \\
112 \\
50 \\
68\end{array}$ & $\begin{array}{r}206 \\
405 \\
315 \\
325 \\
190 \\
584 \\
4,470 \\
1,650 \\
789 \\
208 \\
113 \\
199\end{array}$ & $\begin{array}{r}0.463 \\
.910 \\
.708 \\
.730 \\
.427 \\
1.31 \\
10.0 \\
3.71 \\
1.77 \\
.467 \\
.254 \\
.447\end{array}$ & $\begin{array}{r}0.53 \\
1.02 \\
.82 \\
.84 \\
.44 \\
1.51 \\
11.16 \\
4.28 \\
1.98 \\
.54 \\
.29 \\
.50\end{array}$ \\
\hline The year..... & 11,500 & 50 & 786 & 1.77 & 23. 91 \\
\hline
\end{tabular}


CLYDE RIVER AT WEST DERBY (NEWPORT), VT.

Location.-Just below plant of Newport Electric Light Co. at West Derby (Newport), Orleans County, 1 mile above mouth of river.

Drainage ARea.-150 square miles.

Records available.-May 25, 1909, to September 30, 1919, and May 24, 1920, to September 30, 1923.

GAGEs. - Water-stage recorder on right bank; referenced to gage datum by hook gage inside well; chain gage fastened to tree is used for auxiliary readings. Recorder inspected by F. R. Sherwell.

Discharae measurements.--Made by wading near gage or from highway bridge one-half mile downstream.

Channel and control.-Stream bed rough and irregular; covered with boulders and ledge rock; fall of river rapid for some distance below gage.

EXTREMes of DISCHARGe.-Maximum stage during year from water-stage recorder, 4.58 feet at $11 \mathrm{p}$. $\mathrm{m}$. April 1 (discharge, from extension of rating curve, 2,310 second-feet); minimum discharge practically nil at $8 \mathrm{a}$. m. December 25 (water held back by dams).

1909-1923: High water of March 25-30, 1913, reached maximum stage of 5.8 feet, as determined from high-water marks (discharge, about 6,300 second-feet); minimum discharge practically nil at various times when water was held back by dams.

ICE,-River usually remains open at control; stage-discharge relation seldom affected.

Regulation.-FIow at ordinary stages fully controlled by two dams at West Derby; distribution of flow affected also by several dams above West Derby. Seymour Lake and several small ponds in the basin afford a large amount of natural storage, but at the present time there is little if any artificial regulation at these ponds.

AcCurAcy.-Stage-discharge relation permanent during year. Rating curve fairly well defined. Operation of water-stage recorder satisfactory except for periods indicated in footnote to daily-discharge table. Daily discharge ascertained by applying mean daily gage height to rating table. Records good when water-stage recorder was in operation.

Discharge measurements of Clyde River at West Derby (Newport), Vt., during the year ending September 30, 1923

\begin{tabular}{|c|c|c|c|c|c|c|c|}
\hline Date & Made by- & $\begin{array}{c}\text { Gage } \\
\text { height }\end{array}$ & $\begin{array}{c}\text { Dis- } \\
\text { charge }\end{array}$ & Date & Made by- & $\begin{array}{c}\text { Gage } \\
\text { height }\end{array}$ & $\begin{array}{c}\text { Dis- } \\
\text { charge }\end{array}$ \\
\hline Oct. $\begin{array}{r}28 \\
28 \\
29\end{array}$ & $\begin{array}{l}\text { W. E. Armstrong } \\
\text { do }\end{array}$ & $\begin{array}{l}\text { Feet } \\
2.20 \\
2.18 \\
2.18\end{array}$ & $\begin{array}{r}\text { Sec. } f t \\
93 \\
86 \\
.83\end{array}$ & 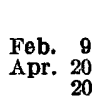 & $\begin{array}{l}\text { W. E. Armstrong } \\
\text { H. F. Hill, jr }\end{array}$ & $\begin{array}{l}\text { Feet } \\
2.25 \\
2.87 \\
2.87\end{array}$ & $\begin{array}{r}\text { Sec.-ft } \\
109 \\
471 \\
475\end{array}$ \\
\hline
\end{tabular}


Daily discharge, in second-feet, of Clyde River at West Derby (Newport), Vt., for the year ending September 30, 1923

\begin{tabular}{|c|c|c|c|c|c|c|c|c|c|c|c|c|}
\hline Day & Oct. & Nov. & Dec. & Jan. & Feb. & Mar. & Apr. & May & June & July & Aug. & Sept. \\
\hline $\begin{array}{l}1 \ldots \\
2 \ldots \\
3 \ldots \\
4 \ldots \\
5 \ldots\end{array}$ & & $\begin{array}{l}68 \\
70 \\
58 \\
76 \\
60\end{array}$ & $\begin{array}{r}142 \\
79 \\
73 \\
133 \\
133\end{array}$ & & 85 & $\begin{array}{l}48 \\
50 \\
68 \\
58 \\
89\end{array}$ & $\begin{array}{l}202 \\
182 \\
197 \\
232 \\
212\end{array}$ & $\begin{array}{l}2,100 \\
2,080 \\
1,800 \\
1,400 \\
1,150\end{array}$ & $\begin{array}{l}227 \\
217 \\
202 \\
173 \\
173\end{array}$ & $\begin{array}{l}142 \\
125 \\
121 \\
125 \\
117\end{array}$ & $\begin{array}{l}76 \\
76 \\
76 \\
85 \\
66\end{array}$ & $\begin{array}{l}89 \\
66 \\
68 \\
82 \\
82\end{array}$ \\
\hline 6. & 55 & $\begin{array}{r}70 \\
79 \\
101 \\
109 \\
46\end{array}$ & $\begin{array}{r}117 \\
117 \\
113 \\
93 \\
50\end{array}$ & & $\begin{array}{l}121 \\
125\end{array}$ & $\begin{array}{l}64 \\
62 \\
58 \\
54 \\
70\end{array}$ & $\begin{array}{r}316 \\
474 \\
950 \\
1,080 \\
1,080\end{array}$ & $\begin{array}{l}990 \\
870 \\
790 \\
622 \\
660\end{array}$ & $\begin{array}{l}187 \\
248 \\
316 \\
374 \\
394\end{array}$ & $\begin{array}{l}151 \\
164 \\
178 \\
155 \\
151\end{array}$ & $\begin{array}{l}76 \\
68 \\
70 \\
73 \\
68\end{array}$ & $\begin{array}{l}73 \\
66 \\
58 \\
52 \\
82\end{array}$ \\
\hline 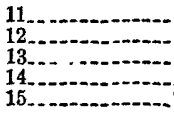 & $\begin{array}{l}54 \\
48\end{array}$ & $\begin{array}{l}40 \\
48 \\
56 \\
58 \\
66\end{array}$ & $\begin{array}{l}73 \\
89 \\
89 \\
85 \\
85\end{array}$ & & $\begin{array}{r}117 \\
113 \\
93 \\
89 \\
109\end{array}$ & $\begin{array}{l}40 \\
58 \\
64 \\
73 \\
64\end{array}$ & $\begin{array}{r}1,040 \\
1,040 \\
970 \\
900 \\
820\end{array}$ & $\begin{array}{l}631 \\
631 \\
622 \\
603 \\
593\end{array}$ & $\begin{array}{l}384 \\
387 \\
360 \\
328 \\
286\end{array}$ & $\begin{array}{l}142 \\
125 \\
121 \\
101 \\
113\end{array}$ & $\begin{array}{l}70 \\
50 \\
70 \\
70 \\
68\end{array}$ & $\begin{array}{r}82 \\
76 \\
79 \\
93 \\
109\end{array}$ \\
\hline 16 & $\begin{array}{l}70 \\
62 \\
68 \\
58 \\
60\end{array}$ & $\begin{array}{l}64 \\
60 \\
54 \\
56 \\
64\end{array}$ & $\begin{array}{l}93 \\
17 \\
31 \\
39 \\
52\end{array}$ & & $\begin{array}{r}105 \\
113 \\
64 \\
85 \\
169\end{array}$ & $\begin{array}{r}68 \\
66 \\
56 \\
85 \\
113 \\
113\end{array}$ & $\begin{array}{l}730 \\
641 \\
565 \\
532 \\
498\end{array}$ & $\begin{array}{l}593 \\
593 \\
574 \\
565 \\
557\end{array}$ & $\begin{array}{l}248 \\
243 \\
212 \\
202 \\
178\end{array}$ & $\begin{array}{l}93 \\
82 \\
97 \\
79 \\
85\end{array}$ & $\begin{array}{l}73 \\
73 \\
82 \\
52 \\
85\end{array}$ & $\begin{array}{r}70 \\
97 \\
93 \\
97 \\
105\end{array}$ \\
\hline $\begin{array}{l}21 \\
22 \\
24 \\
25\end{array}$ & $\begin{array}{l}56 \\
46 \\
64 \\
68 \\
68\end{array}$ & $\begin{array}{l}101 \\
113 \\
109 \\
105 \\
109\end{array}$ & $\begin{array}{l}60 \\
58 \\
58 \\
26 \\
26\end{array}$ & & $\begin{array}{r}169 \\
129 \\
109 \\
97 \\
54\end{array}$ & $\begin{array}{l}137 \\
178 \\
173 \\
133 \\
121\end{array}$ & $\begin{array}{r}548 \\
641 \\
930 \\
1,170 \\
1,170\end{array}$ & $\begin{array}{l}507 \\
474 \\
445 \\
407 \\
394\end{array}$ & $\begin{array}{r}164 \\
151 \\
137 \\
121 \\
70\end{array}$ & $\begin{array}{r}79 \\
89 \\
101 \\
79 \\
76\end{array}$ & $\begin{array}{r}89 \\
89 \\
89 \\
93 \\
101 \\
101\end{array}$ & $\begin{array}{l}109 \\
121 \\
129 \\
125 \\
133\end{array}$ \\
\hline $\begin{array}{l}26 \\
27 \\
28 \\
29 \\
30 \\
31\end{array}$ & $\begin{array}{r}62 \\
73 \\
105 \\
82 \\
85 \\
93\end{array}$ & $\begin{array}{l}101 \\
121 \\
105 \\
113 \\
105\end{array}$ & 62 & 100 & $\begin{array}{r}54 \\
60 \\
46 \\
-2 \\
-2\end{array}$ & \begin{tabular}{r|}
85 \\
82 \\
133 \\
178 \\
178 \\
178
\end{tabular} & $\begin{array}{r}1,060 \\
950 \\
770 \\
990 \\
1,500 \\
\end{array}$ & $\begin{array}{l}367 \\
347 \\
310 \\
292 \\
275 \\
243\end{array}$ & $\begin{array}{r}82 \\
89 \\
89 \\
109 \\
109 \\
\end{array}$ & $\begin{array}{l}76 \\
79 \\
89 \\
66 \\
85 \\
79\end{array}$ & $\begin{array}{l}52 \\
93 \\
82 \\
89 \\
89 \\
97\end{array}$ & $\begin{array}{l}133 \\
129 \\
129 \\
160 \\
142\end{array}$ \\
\hline
\end{tabular}

Note.-Water-stage recorder not in operation Oct. 1-13, Dec. 27 to Feb. 8, Mar. 29, 30, Sept. 6-8, 26 and 27; discharge estimated.

Monthly discharge of Clyde River at West Derby (Newport), Vt., for the year ending September 30, 1923

[Drainage area, 150 square miles]

\begin{tabular}{|c|c|c|c|c|c|}
\hline \multirow{2}{*}{ Month } & \multicolumn{4}{|c|}{ Discharge in second-feet } & \multirow{2}{*}{$\begin{array}{l}\text { Run-off } \\
\text { in inches }\end{array}$} \\
\hline & Maximum & Minimum & Mean & $\begin{array}{c}\text { Per square } \\
\text { mile }\end{array}$ & \\
\hline $\begin{array}{l}\text { October } \\
\text { November } \\
\text { December } \\
\text { January } \\
\text { February } \\
\text { March } \\
\text { April } \\
\text { May } \\
\text { June } \\
\text { July } \\
\text { August } \\
\text { September }\end{array}$ & $\begin{array}{r}105 \\
121 \\
142 \\
169 \\
178 \\
1,500 \\
2,100 \\
394 \\
178 \\
101 \\
160\end{array}$ & $\begin{array}{r}46 \\
40 \\
17 \\
-46 \\
40 \\
182 \\
243 \\
70 \\
66 \\
50 \\
52\end{array}$ & $\begin{array}{r}62.5 \\
79.5 \\
80.4 \\
124 \\
96.4 \\
93.0 \\
746 \\
725 \\
216 \\
109 \\
77.1 \\
97.6\end{array}$ & $\begin{array}{c}0.417 \\
.530 \\
.536 \\
.827 \\
.643 \\
.620 \\
4.97 \\
4.83 \\
1.44 \\
.737 \\
.514 \\
.651\end{array}$ & $\begin{array}{r}0.48 \\
.59 \\
.62 \\
.95 \\
.67 \\
.71 \\
5.55 \\
5.57 \\
1.61 \\
.85 \\
.59 \\
.73\end{array}$ \\
\hline The year. & 2,100 & 17 & 209 & 1.39 & $\overline{18.92}$ \\
\hline
\end{tabular}

Nots.-See paragraph on "Regulation." 


\section{MISCELLANEOUS DISCHARGE MEASUREMENTS}

Discharge measurements of streams in the St. Lawrence River. drainage basin at points other than regular gaging stations, made during the year ending September 30,1923, are listed in the following table:

Miscellaneous discharge measurements in the St. Lawrence River drainage basin during the year ending September 30, 1923

\begin{tabular}{|c|c|c|c|c|}
\hline Date & Stream & $\begin{array}{l}\text { Tributary to or divert- } \\
\text { ing from- }\end{array}$ & Locality & $\begin{array}{l}\text { Dis- } \\
\text { charge }\end{array}$ \\
\hline Oct. & Maumee River & Lake Eri & Napoleon Ohic & Sec.-ft. \\
\hline $\begin{array}{l}27 \\
\text { May } 11 \\
\text { Aug. } 2\end{array}$ & 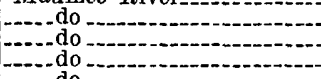 & 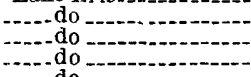 & 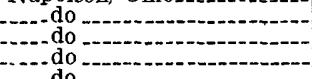 & $\begin{array}{l}394 \\
693 \\
667\end{array}$ \\
\hline $\begin{array}{ll}\text { May } & 16 \\
\text { Aug. } & 3 \\
\text { Oct. } & 4\end{array}$ & $\begin{array}{l}\text { Ottawa River } \\
\text { Miami and Erie Canal..... }\end{array}$ & $\begin{array}{l}\text { Auglaize River } \\
\text { Maumee River... }\end{array}$ & $\begin{array}{l}\text { Kalida, Ohio } \\
\text { Florida, Ohio } \\
\text { Napoleon, Ohio }\end{array}$ & $\begin{array}{r}613 \\
56 \\
201 \\
14\end{array}$ \\
\hline $\begin{array}{rr} & 27 \\
\text { May } & 11 \\
\text { Aug. }\end{array}$ & 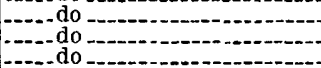 & \begin{tabular}{l}
$-d 0_{0}$ \\
\hdashline do
\end{tabular} & $\begin{array}{l}\text { do } \\
\text { do }\end{array}$ & 31 \\
\hline May 16 & Sandusky River. & Lake Erie-_. & Tiffin, ohio... & 5,520 \\
\hline
\end{tabular}




\section{STREAM-GAGING STATIONS AND PUBLICATIONS RELATING TO WATER RESOURCES}

\section{INTRODUCTION}

Investigation of water resources by the United States Geological Survey has consisted in large part of measurements of the volume of flow of streams and studies of the conditions affecting that flow, but it has comprised also investigation of such closely allied subjects as irrigation, water storage, water powers, ground waters, and quality of waters. Most of the results of these investigations have been published in the series of water-supply papers, but some have appeared in the monographs, bulletins, professional papers, and annual reports.

The results of stream-flow measurements are now published annually in 12 parts, each part covering an area whose boundaries coincide with natural drainage features as indicated below:

Part I. North Atlantic slope basins (St. John River to York River).

II. South Atlantic slope and eastern Gulf of Mexico basins (James River to the Mississippi).

III. Ohio River basin.

IV. St. Lawrence River basin.

V. Upper Mississippi River and Hudson Bay basins.

VI. Missouri River basin.

VII. Lower Mississippi River basin.

VIII. Western Gulf of Mexico basins.

IX. Colorado River basin.

X. Great Basin.

XI. Pacific slope basins in California.

XII. North Pacific slope basins, in three parts:

A, Pacific slope basins in Washington and upper Columbia River basin.

B, Snake River basin.

C, Lower Columbia River basin and Pacific slope basins in Oregon. 


\section{HOW GOVERNMENT REPORTS MAY BE OBTAINED OR CONSULTED}

Water-supply papers and other publications of the United States Geological Survey containing data in regard to the water resources of the United States may be obtained or consulted as indicated below.

1. Copies may be purchased at nominal cost from the Superintendent of Documents; Government Printing Office, Washington, D. C., who will, on application, furnish lists giving prices.

2. Sets of the reports may be consulted in the libraries of the principal cities of the United States.

3. Complete sets are available for consultation in the local offices of the water-resources branch of the Geological Survey, as follows:

Boston, Mass., 2500 Customhouse.

Albany, N. Y., 704 Journal Building.

Trenton, N. J., Statehouse.

Charlottesville, Va., care of University of Virginia.

Asheville, N. C., 316 Jackson Building.

Chattanooga, Tenn., 37 Municipal Building.

Columbus, Ohio, Engineering Experiment Station, Ohio State University .

Chicago, Ill., 950 Transportation Building.

Madison, Wis., care of Railroad Commission of Wisconsin.

Ames, Iowa, State Highway Commission Building.

Rolla, Mo., Rolla Building, School of Mines and Metallurgy.

Topeka, Kans., 23 Federal Building.

Helena, Mont., 45-46 Federal Building.

Denver, Colo., 403 Post Office Building.

Salt Lake City, Utah, 313 Federal Building.

Idaho Falls, Idaho, 228 Federal Building.

Boise, Idaho, Federal Building.

Tacoma, Wash., 404 Federal Building.

Portland, Oreg., 606 Post Office Building.

San Francisco, Calif., 303 Customhouse.

Los Angeles, Calif,, 600 Federal Building.

Tucson, Ariz., room 106, College of Law Building, University of Arizona.

Austin, Tex., State Capitol.

Honolulu, Hawaii, 25 Capitol Building.

A list of the Geological Survey's publications may be obtained by apply to the Director, United States Geological Survey, Washington, D. C.

\section{STREAM-FLOW REPORTS}

Stream-flow records have been obtained at about 5,600 points in the United Slates, and the data obtained have been published in the reports tabulated below. 
Stream-flow data in reports of the United States Geological Survey

[A=Annual Report; B=Bulletin; $W=$ Water-Supply Paper]

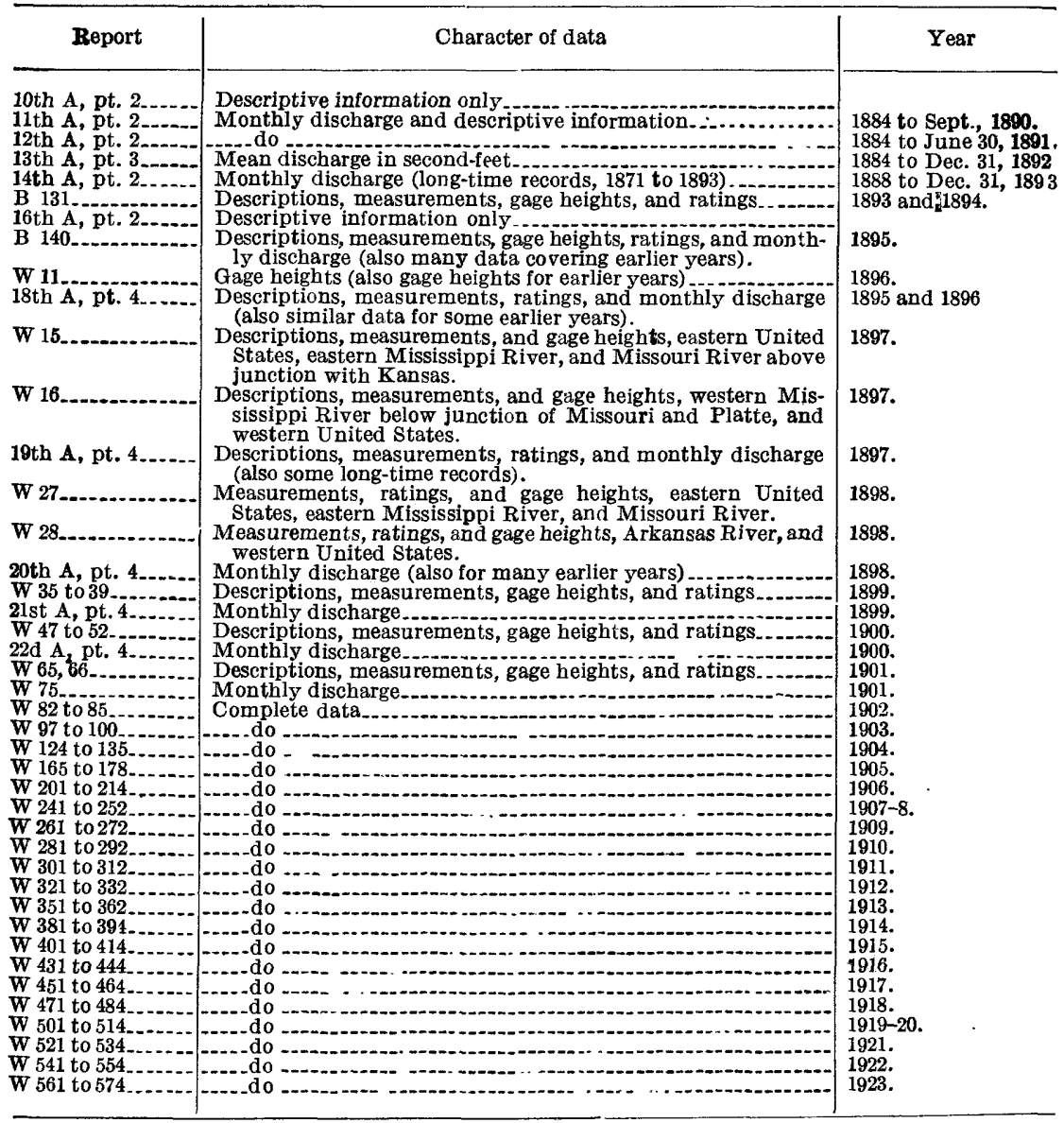

The records at most of the stations discussed in these reports extend over a series of years, and miscellaneous measurements at many points other than regular gaging stations have been made each year. An index of the reports containing records obtained prior to 1904 has been published in Water-Supply Paper 119 .

The following table gives, by years and drainage basins, the numbers of the papers on surface-water supply published from 1899 to 1923. The data for any particular station will be found in the reports covering the years during which the station was maintained. For example, data for Machias River at Whitneyville, Maine, 1903 to 1921 , are published in Water-Supply Papers 97, 124, 165, 201, 241, $261,281,301,321,351,381,401,431,451,471,501$, and 521, which contain records for the New England streams from 1903 to 1921. Results of miscellaneous measurements are published by drainage basins. 


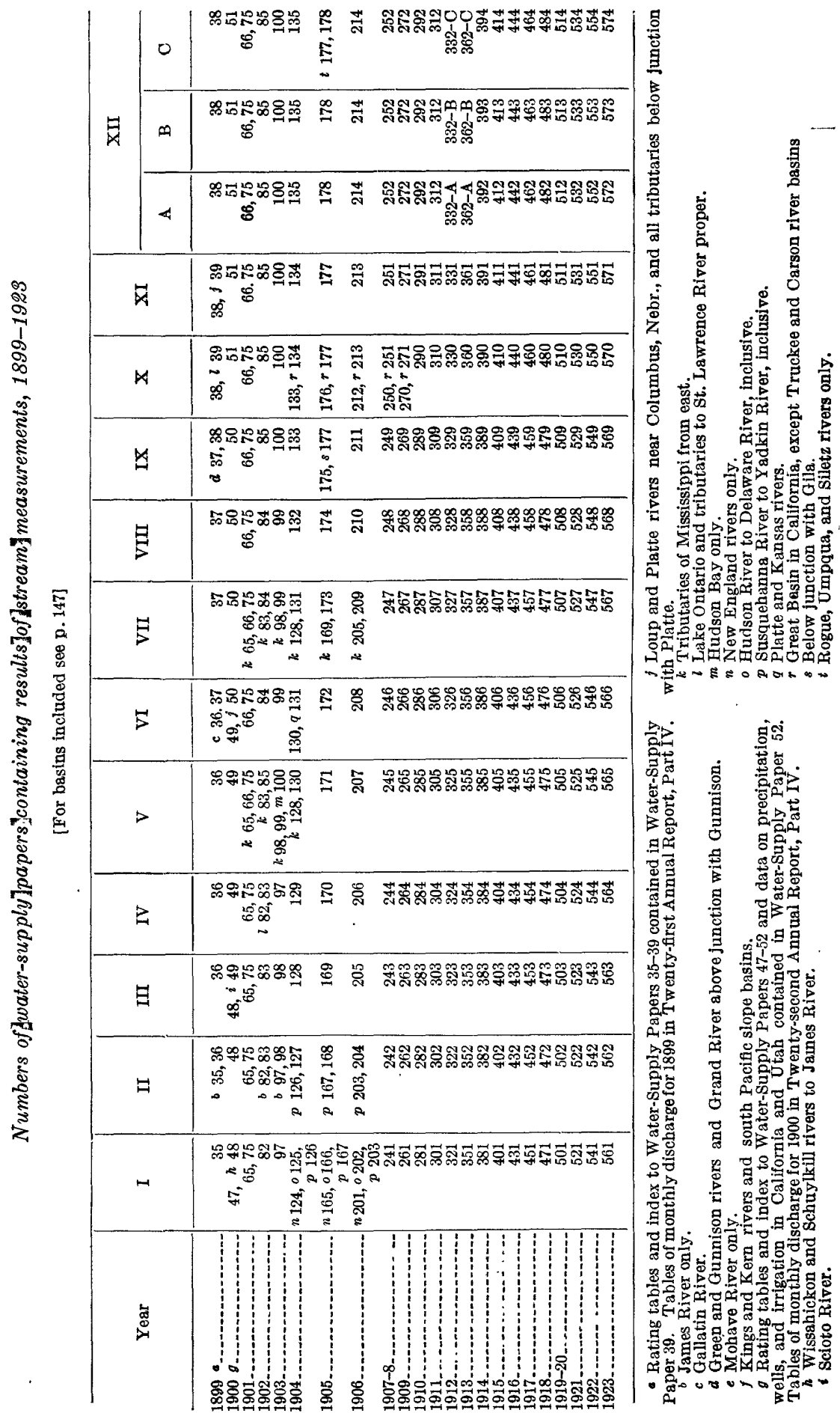


In these papers and in the following lists the stations are arranged in downstream order. The main stem of any river is determined by measuring or estimating its drainage area - that is, the headwater stream having the largest drainage area is considered the continuation of the main stream, and local changes in name and lake surface are disregarded. All stations from the source to the mouth of the main stem of the river are presented first, and the tributaries in regular order from source to mouth follow, the streams in each tributary basin being listed before those of the next basin below.

In exception to this rule the records for Mississippi River are given in four parts, as indicated on page 147, and the recards for large lakes are presented in order of streams around the rim of the lake.

\section{PRINCIPAL STREAMS}

The St. Lawrence River basin includes streams which drain into the Great Lakes and St. Lawrence River. The principal streams flowing directly or indirectly into Lake Superior from the United States are St. Louis, Ontonagon, Dead, and Carp rivers; streams flowing into Lake Michigan are Escanaba, Menominee, Peshtigo, Oconto, Fox, St. Joseph, and Grand rivers; into Lake Huron flow Thunder Bay, Ausable, Rifle, and Saginaw rivers; into Lake Erie flow Huron, Maumee, Sandusky, Black, and Cuyahoga rivers. Streams flowing into Lake Ontario are Genesee, Oswego, Salmon, and Black rivers. The St. Lawrence receives Oswegatchie and Raquette rivers, Richelieu River (the outlet of Lake Champlain), and St. Francis River, whose principal tributary, Clyde River, reaches it through Lake Memphremagog. The streams of this basin drain wholly or in part the States of Illinois, Indiana, Michigan, Minnesota, New York, Ohio, Pennsylvania, Vermont, and Wisconsin.

In addition to the list of gaging stations and annotated list of publications relating specifically to the section, this part contains brief references to reports published by State and other organizations. (See p. 163.)

\section{GAGING STATIONS}

Note.-Dash following a date indicates that station was being maintained September 30, 1923. Period after date indicates discontinuance.

Streams tributary to Lake Superior:

Brule River at mouth, Minn., 1911.

Devil Track River at mouth, Minn., 1911.

Cascade River at mouth, Minn., 1911.

Poplar River at Lutsen, Minn., 1911-1917.

Beaver Bay River at Beaver Bay, Minn., 1911-1914.

St. Louis River near Cloquet, Minn., 1903.

St. Louis River near Thomson, Minn., 1909-1915.

Whiteface River at Meadowlands, Minn., 1909-1912.

Whiteface River below Meadowlands, Minn., 1912-1917.

Cloquet River at Independence, Minn., 1909-1917. 
Streams tributary to Lake Superior-Continued.

Aminicon River near Aminicon Falls, Wis., 1914-1916.

Brule River near Brule, Wis., 1914-1917.

Bad River near Odanah, Wis., 1914-1922.

Montreal River at Ironwood, Mich., 1918-1922.

West Branch of Montreal River at Gile, Wis., 1918-

Ontonagon River near Rockland, Mich., 1903.

Sturgeon River near Sidnaw, Mich., 1912-1915.

Perch River near Sidnaw, Mich., 1912-1915.

Dead River near Negaunee, Mich., 1902-3.

Dead River at Forestville, Mich., 1898-1902.

Carp River near Marquette, Mich., 1902-3.

Streams tributary to Lake Michigan:

Escanaba River near Escanaba, Mich., 1903-1915.

Brule River (head of Menominee River) near Florence, Wis., 1914-1916.

Menominee River at Twin Falls, near Iron Mountain, Mich., 1914-

Menominee River near Iron Mountain, Mich., 1902-1914.

Menominee River at Lower Quinnesec Falls, Wis., 1898-99.

Menominee River at Koss, Mich., 1907-1909; 1914.

Menominee River below Koss, Mich., 1913-

Iron River near Iron River, Mich., 1900-1905.

Pine River near Florence, Wis., 1914-

Pike River at Amberg., Wis., 1914-

Peshtigo River at High Falls, near Crivitz, Wis., 1912-

Peshtigo River near Crivitz, Wis., 1906-1909.

Peshtigo River at Crivitz, Wis., 1906.

Oconto River near Gillett, Wis., 1906-1909; 1914-

Oconto River at Stiles, Wis. 1906.

Fox River at Berlin, Wis., 1898-

Fox River at Omro, Wis., 1902-3.

Fox River at Oshkosh, Wis., 1902.

Fox River at Wrightstown, Wis., 1902-1904.

Fox River at Rapide Croche dam, near Wrightstown, Wis., 1896-

Wolf River at Keshena, Wis., 1907-1909; 1911-

Wolf River at White House Bridge, near Shawano, Wis., 1906-7.

Wolf River at Darrows Bridge, near Shawano, Wis., 1906.

Wolf River at New London, Wis., 1913-

Wolf River at Northport, Wis., 1905.

Wolf River at Winneconne, Wis., 1902-3.

West Branch of Wolf River at Neopit, Wis., 1911-1917.

Embarrass River near Embarrass, Wis., 1919-

Little Wolf River at Royalton, Wis., 1914-

Little Wolf River near Northport, Wis., 1907-1910.

Waupaca River near Waupaca, Wis., 1917-

Waupaca River near Weyauwega, Wis., 1916-17.

Fond du Lac River, West Branch (head of Fond du Lac River), at Fond du Lac, Wis., 1903.

East Branch of Fond du Lac River at Fond du Lac, Wis., 1903.

Sheboygan River near Sheboygan, Wis., 1916-

Milwaukee River near Milwaukee, Wis., 1914-

Little Calumet River at Harvey, Ill., 1916-

St. Joseph River at Mendon, Mich., 1902-1905.

St. Joseph River near Buchanan, Mich., 1901-1906.

Fawn River at White Pigeon, Mich., 1903-4. 
Streams tributary to Lake Michigan-Continued.

Kalamazoo River near Allegan, Mich., 1901-1907.

Reeds Springs near Albion, Mich., 1904-1906.

Grand River at North Lansing, Mich., 1901-1906.

Grand River at Grand Rapids, Mich., 1901-1918.

Red Cedar River at Agricultural College, Mich., 1902-3.

Crockery Creek at Slocums Grove, Mich., 1902-3.

Muskegon River at Newaygo, Mich., 1901-1906.

Manistee River near Sherman, Mich., 1903-1916.

Boardman River at Traverse City, Mich., 1904.

Streams tributary to Lake Huron:

Thunder Bay River near Alpena, Mich., 1901-1908.

Au Sable River near Lovells, Mich., 1908-1914.

Au Sable River at Bamfield, Mich., 1902-1913.

Rifle River near Sterling, Mich., 1905-1908.

Rifle River at Omer, Mich., 1902-3.

Shiawassee River (head of Saginaw River):

Flint River at Flint, Mich., 1903-4.

Cass River at Frankenmuth, Mich., 1908-9.

Cass River at Bridgeport, Mich., 1908.

Tittabawassee River at Freeland, Mich., 1903-1909; 1912-

Streams tributary to Lake Erie:

Huron River at Dover, Mich., 1904.

Huron River at Dexter, Mich., 1904-1916.

Huron River at Barton, Mich., 1914-

Huron River at Geddes, Mich., 1904-1914.

Huron River at French Landing, Mich., 1904-5.

Huron River at Flat Rock, Mich., 1904-1922.

Maumee River at Antwerp, Ohio, 1921-

Maumee River near Sherwood, Ohio, 1903-1906.

Maumee River at Waterville, Ohio, 1898-1901; 1921-

St. Marys River at Fort Wayne, Ind., 1905-6.

St. Joseph River at Fort Wayne, Ind., 1905-6.

Tiffin River near Stryker, Ohio, 1921-

Tiffin River near Defiance, Ohio, 1903-1906.

Auglaize River near Fort Jennings, Ohio, 1921-

Auglaize River near Defiance, Ohio, 1903; 1915-

Ottawa River at Lima, Ohio, 1902-3.

Blanchard River at Ottawa, Ohio, 1902-3.

Blanchard River at Glandorf, Ohio, 1921-

Miami and Erie Canal at Waterville, Ohio, 1921-

Sandusky River near Upper Sandusky, Ohio, 1921-

Sandusky River near Mexico, Ohio, 1898-1900; 1923-

Sandusky River at Fremont, Ohio, 1898-1901.

Black River near Elyria, Ohio, 1903-1906.

East Branch of Black River at Elyria, Ohio, 1922-

Cuyahoga River at Old Portage, Ohio, 1921-

Cuyahoga River at Brecksville, Ohio, 1923-

Cuyahoga River at Independence, Ohio, 1903-1906; 1921-1923.

Cuyahoga River at Cleveland, Ohio, 1903.

Ohio Canal feeder at Brecksville, Ohio, 1923-

Ohio Canal at Indepedence, Ohio, 1921-1923.

Grand River near Madison, Ohio, 1922-

Conneaut Creek at Amboy, Ohio, 1922-

Cattaraugus Creek at Versailles, N. Y., 1910-1923. 
Streams tributary to Lake Ontario:

Niagara River:

Tonawanda Creek:

Little Tonawanda Creek at Linden, N. Y., 1912-

Barge Canal at Lock 30, Macedon, N. Y., 1919-1920.

Barge Canal at Lock 32, Pittsford, N. Y., 1919-1921.

Genesee River at Scio, N. Y., 1916-

Genesee River at St. Helena, N. Y., 1908-

Genesee River at Mount Morris, N. Y., 1905-1909.

Genesee River at Jones Bridge, near Mount Morris, N. Y., 1903-1906; 1908$1913 ; 1915-$

Genesee River at Rochester, N. Y., 1904-1919.

Genesee River at Driving Park Avenue, Rochester, N. Y., 1919-

Canaseraga Creek near Dansville, N. Y., 1910-1912; 1915-1917; 1919-

Canaseraga Creek at Cumminsville. N. Y., 1917-1919.

Canaseraga Creek at Groveland Station, N. Y., 1915-1920.

Canaseraga Creek at Shakers Crossing, N. Y., 1915-1922.

Keshequa Creek at Sonyea, N. Y., 1910-1912; 1917-

Keshequa Creek near Sonyea, N. Y., 1915-1917.

Conesus Creek near Lakeville, N. Y., 1919-

Hemlock Lake at Hemlock, N. Y., 1894-1902.

Canadice Lake outlet near Hemlock, N. Y., 1903-

Honeoye Creek at East Rush, N. Y., 1903-1906.

Seneca River (head of Oswego River) at Baldwinsville, N. Y., 1898-1908.

Oswego River at Fulton, N. Y., 1900; 1902.

Oswego River at Battle Island, above Minetto, N, Y., 1900-1906.

Oswego River at high dam, near Oswego, N. Y., 1897-1901.

Seneca Lake at Geneva, N. Y., 1905-6.

Cayuga Lake at Ithaca, N. Y., 1905-1908.

Fall Creek near Ithaca, N. Y., 1908-9.

Owasco Lake outlet near Auburn, N. Y., 1912-

Skaneateles Lake at Skaneateles, N. Y., 1890-91.

Skaneateles Lake outlet at Willow Glen, N. Y., 1892-1908.

Skaneateles Lake outlet at Jordan, N. Y., 1890-1892.

Onondaga Lake outlet at Long Branch, N. Y., 1904.

West Branch of Onondaga Creek at South Onondaga, N. Y., 1916-1918.

Fish Creek, East Branch (through Oneida Lake, head of Oneida River), at Point Rock, N. Y., 1898-99.

Fish Creek, East Branch, at Taberg, N. Y., 1923-

Oneida River at Brewerton, N. Y., 1899.

Oneida River at Oak Orchard, near Euclid, N. Y., 1902-1909.

Oneida River at Caughdenoy, N. Y., 1910-1913

West Branch of Fish Creek at McConnelsville, N. Y., 1898-1901. Oneida Creek at Kenwood, N. Y., 1898-1900.

Chittenango Creek at Chittenango, N. Y., 1901-1906.

Chittenango Creek at Bridgeport, N. Y.,1898-1901.

Salmon River at Stillwater Bridge, near Redfield, N. Y., 1911-1913.

Salmon River near Pulaski, N. Y., 1900-1908; 1910-1914.

Orwell Brook near Altmar, N. Y.,1911-1916.

Black River near Boonville, N. Y., 1911-

Black River near Felts Mills, N. Y., 1902-1913.

Black River at Black River, N. Y., 1917-1920.

Black River at Huntingtonville dam, near Watertown, N. Y., 1897-1901. 
Streams tributary to Lake Ontario-Continued.

Black River at Watertown, N. Y., 1920-

Forestport feeder near Boonville, N. Y., 1915-

Black River canal (flowing south) near Buonville, N. Y., 1915-

Moose River at McKeever, N. Y., 1922-

Moose River at Moose River, N. Y., 1900-1922.

Middle Branch of Moose River at Old Forge, N. Y., 1911-

Beaver River at State dam, near Beaver River, N. Y., 1908-

Beaver River at Eagle Falls, near Number Four, N. Y., 1921-

Beaver River at Croghan, N. Y., 1901-1903.

Streams tributary to St. I,awrence River:

Oswegatchie River, East Branch (head of Oswegatchie River), at Cranberry Lake, N. Y., 1923-

Oswegatchie River, East Branch, at Newton Falls, N. Y., 1912-1923.

Oswegatchie River near Heuvelton, N. Y., 1916-

Oswegatchie River near Ogdensburg, N. Y., 1903-1916.

West Branch of Oswegatchie River near Harrisville, N. Y., 1916-

Raquette River at Raquette Falls, near Coreys, N. Y., 1908-1912.

Raquette River at Piercefield, N. Y., 1908-

Raquette River at South Colton, N. Y., 1904.

Raquette River at Massena Springs, N. Y., 1903-1916.

Bog River near Tupper Lake, N. Y., 1908-1912.

St. Regis River at Brasher Center, N. Y., 1910-1917; 1919-

Deer River at Brasher Iron Works (railroad station), Ironton, N. Y., 1912-1916.

Chateaugay River near Chateaugay, N. Y., 1908.

Richelieu River at Fort Montgomery, Rouses Point, N. Y., 1875-

Lake Champlain at Burlington, Vt., 1907-

Big Chazy River at Mooers, N. Y., 1908.

Saranac River at Saranac Lake, N. Y., 1902-3.

Saranac River near Plattsburg, N. Y.,1903-

Ausable River, West Branch (head of Ausable River), near Newman, N. Y., 1916-1917; 1919-

Ausable River at Ausable Forks N. Y., 1910-

Ausable River at Keeseville, N. Y., 1904; 1908.

Boquet River at Willsboro, N. Y., 1904; 1908.

Lake George at Rogers Rock, N. Y. 1913-

Lake George at Glen Island, near Bolton Landing, N. Y., 1919-

Lake George outlet at Ticonderoga, N. Y., 1904-5.

Poultney River at Fairhaven, Vt., 1908.

Mettawee River at Whitehall, N. Y. 1908.

Otter Creek at Middlebury, Vt., 1903-1907; 1910-1920.

East Creek near Rutland, Vt., 1911-1913.

Winooski River above Stevens Branch, near Montpelier, Vt., 19091914.

Winooski River at Montpelier, Vt., 1909-1923.

Winooski River at Richmond, Vt., 1903-1907; 1910.

Winooski River near Winooski, Vt., 1903.

Mollys Brook near Marshfield, Vt., 1920-1923.

Stevens Branch:

Jail Branch at East Barre, Vt., 1920-1923. 
Streams tributary to St. Lawrence River-Continued.

Richelieu River tributaries-Continued.

Lake Champlain tributaries-Continued.

Winooski River tributaries-Continued.

North Branch (Worcester Branch) of Winooski River at Montpelier, Vt., 1909-1914.

Dog River at Northfield, Vt., 1909-r920.

Dog River near Montpelier Junction, Vt., 1910.

Mad River near Moretown, Vt., 1910.

Waterbury (Little) River near Waterbury, Vt., 1910.

Huntington River at Jonesville, Vt., 1910

Lamoille River at Morrisville, Vt., 1909-10.

Lamoille River at Cadys Falls, Vt., 1913-1923.

Lamoille River at Johnson, Vt., 1910-1913.

Lamoille River at West Milton, Vt., 1903.

Green River at Garfield, Vt., 1915-1921; 1922-

Missisquoi River at Richford, Vt., 1909-10.

Missisquoi River near Richford, Vt., 1911-1923.

Missisquoi River at Swanton, Vt., 1903.

St. Francis River (by way of Lake Memphremagog and Magog River):

Clyde River at West Derby (Newport), Vt., 1909- 


\section{REPORTS ON WATER RESOURCES OF THE ST. LAWRENCE RIVER BASIN}

\section{PUBLICATIONS OF THE UNITED STATES GEOLOGICAL SURVEY \\ WATER-SUPPLY PAPERS}

Water-supply papers may be purchased (at price quoted below)from the Superintendent of Documents, Washington, D. C. An asterisk $\left(^{*}\right)$ indicates that the report is out of print. Water-supply papers are of octavo size.

*21. Wells of northern Indiana, by Frank Leverett. 1899.82 pp., 2 pls. (Continued in No. 26.)

Discusses, by counties, the glacial deposits and the sources of well water; gives many well sections.

24. Water resources of the State of New York, Part I, by G. W. Rafter. 1899. 99 pp., 13 pls. 15c.

*25. Water resources of the State of New York, Part II, by G. W. Rafter. 1899. 100 pp. 12 pls.

No. 24 contains descriptions of the principal rivers of New York and their more important tributaries and data on temperature, precipitation, evaporation, and stream flow.

No. 25 contains discussion of water-storage projects on Genesee and Hudson Rivers, power development at Niagara Falls, description and early history of State canals, and a chapter on the use and value of the water powers of the streams and canals; also brief discussion of the water yield of sand areas of Long Island.

*26. Wells of southern Indiana (continuation of No. 21), by Frank Leverett. 1899. $64 \mathrm{pp}$.

Discusses, by counties, the glacial deposits and the sources of well water; contains many well sections.

*30. Water resources of the Lower Peninsular of Michigan, by A. C. Lane. 1899. 97 pp., 7 pls.

Describes lake and river transportation and navigation, water powers and domestic water supplies; discusses climate, topography, geology, and well waters; compares quality and quan tity of waters.

31. Lower Michigan mineral waters, by A. C. Lane. 1899.97 pp., 4 pls. $10 \mathrm{c}$.

Treats of economic value of mineral waters and discussion and classification of analyses; contains analyses of waters of Lake Superior and of smaller lakes and rivers and of well waters from various geologic formations; also sanitary condition of drinking waters.

*57. Preliminary list of deep borings in the United States, Part I (Alabama-Mon tana), by N. H. Darten. 1902.60 pp. $\quad$ (See No. 149.)

*61. Preliminary list of deep borings in the United States, Part II (NebraskaW yoming), by N. H. Darton. $1902.67 \mathrm{pp}$.

Nos. 57 to 61 contain information as to depth, diameter, yield, and head of water in borings more than 400 feet deep; under head "Remarks" give information concerning temperature, quality of water, purposes of boring, etc. The lists are arranged by States, and the States are arranged alphabetically. A second, revised, edition was published in 1905 as Water-Supply Paper 149 (q. v.).

1 For stream measurement reports, see tables on pp. 149 and 150

$72038-26 \dagger-$ WSP $564-11$ 
91. The natural features and economic development of the Sandusky, Maumee, Muskingum, and Miami drainage areas in Ohio, by B. H. and M. S. Flynn. 1904. 130 pp. 10c.

Describes the topography, geology, and soils of the areas, and discusses stream flow, dams, water powers, and public water supplies.

*102. Contributions to the hydrology of eastern United States, 1903; M. L. Fuller, geologist in charge. 1904. 522 pp.

Contains brief reports on wells and'springs of Minnesota and of lower Michigan. The report comprises tabulated well records giving information as to location, owner, depth yield, head, etc., supplemented by notes as to elevation above sea, materials penetrated, temperature, use and quality; many miscellaneous analyses.

*103. A review of the laws forbidding pollution of inland waters in the United States, by E. B. Goodell. 1904.120 pp. Superseded by 152.

Cites statutory restrictions of water pollution.

*110. Contributions to the hydrology of eastern United States, 1904; M. L. Fuller, geologist in charge. 1905.211 pp., 5 pls.

Contains:

Water resources of the Watkins Glen quadrangle, New York, by Ralph S. Tarr; pp. 134-140. Discusses the use of the surface and underground waters for municipal supplies and their quality as indicated by examination of Sixmile and Fall creeks, and sanitary analyses of well water at Ithaca.

New artesian water supply at Ithaca, New York, by F. L. Whitney, pp. 55-64.

*114. Underground waters of eastern United States; M. L.-Fuller, geologist in charge. 1905.285 pp., 18 pls.

Contains brief reports as follows:

Minnesota, by C. W. Hall; Wisconsin district, by, Alfred R. Schultz; Lower Michigan; Illinois, by Frank Leverett; Indiana, by Frank Leverett; New York, by F. B. Weeks; Ohio, by Frank Leverett.

Each of these reports describes briefly the topography of the area, the relation of the geology to the water supplies, and gives list of pertinent publications; lists also principal mineral springs.

121. Preliminary report on the pollution of Lake Champlain, by M. O. Leighton. 1905. 119 pp., 13 pls. 20 c.

Describes the lake and principal inflowing streams and discusses the characteristics of the water and the wastes resulting from the manufacturing processes by which the waters are polluted. Discusses also the effect of mill waste on algae, bacteria, and fish.

*122. Relation of the law to underground waters, by D. W. Johnson. 1905. $55 \mathrm{pp}$.

Cites legislative acts relating to ground waters in Michigan and Wisconsin.

144. The normal distribution of chlorine in the natural waters of New York and New England, by D. D. Jackson. 1905. 31 pp., 5 pls. $10 \mathrm{c}$.

Discusses common salt in coast and inland waters, salt as an index to pollution of streams and wells, the solutions and methods used in chlorine determinations, and the use of the normal chlorine map; gives charts and tables for chlorine in the New England States and New York.

*145. Contributions to the hydrology of eastern United States, 1905; M. L. Fuller, geologist in charge. 1905.220 pp., 6 pls.

Contains three brief reports pertaining chiefly to areas in the St. Lawrence River basin:

Two unusual types of artesian flow, by Myron L. Fuller. Describes (1) artesian flows from uniform, unconfined sand on Long Island, N. Y., and in Michigan; and (2) flow from jointed upper portions of limestone and other rocks in southeastern Michigan.

Water resources of the Catatonk area, New York, by E. M. Kindle. Deseribes topography and geology of areas southeast of Finger Lake region, New York, including part of city of Ithaca; discusses briefly the artesian wells of Ithaca, the quality of the spriug water at several small towns, and of the streams used for municipal supplies and for power.

A ground-water problem in southeastern Michigan, by Myron L. Fuller. Discusses cause of failure of wells in certain areas in southeastern Michigan in 1904 and the applications of the conclusions to other regions. 
*147. Destructive floods in the United States in 1904, by E. C. Murphy and others 1905. 206 pp., 18 pls.

Describes flood on Grand River, Mich. (from report of R. E. Horton), discussing streams, pre cipitation, temperature, discharge, damage, and prevention of future damage.

149. Preliminary list of deep borings in the United States, second edition, with additions, by N. H. Darton. 1905.175 pp. $10 \mathrm{c}$.

Gives by States (and within the States by counties) the location, depth, diameter, yield, height of water, and other features of wells 400 feet or more in depth; includes all wells listed in Water-Supply Papers 57 to 61; mentions also primcipal publications relating to deep borings.

*152. A review of the laws forbidding pollution of inland waters in the United States (second edition), by E. B. Goodell. $1905.140 \mathrm{pp}$.

Cites statutory restrictions of water pollution in Illinois, Indiana, Michigan, Minnesota, New York, Ohio, Pennsylvania, Vermont, and Wisconsin.

*156. Water powers of northern Wisconsin, by L. S. Smith. 1906.145 pp., 5. pls.

Describes, by river systems, the drainage geology, topography, rainfall, and run-off, waterpowers and dams.

*160. Underground-water papers, 1906; M. L. Fuller, geologist in charge. 1906. 104 pp., 1 pl.

Contains brief report entitled "Flowing well districts in the eastern part of the northern peninsula of Michigan," by Frank Leverett.

*162. Destructive floods in the United States in 1905, with a discussion of flood discharge and frequency and an index toflood literature, by E. C. Murphy and others. 1906.105 pp., 4 pls.

Contains accounts of floods on Sixmile Creek and Cayuga Inlet, N. Y. (in 1857, 1901, and 1905), and on Grand River, Mich., and estimate of flood discharge and frequency for Genesee River; gives index to literature on floods in American streams.

182. Flowing wells and municipal water supplies in the southern portion of the southern peninsula of Michigan, by Frank Leverett and others. 1906. 292 pp., 5 pls. $50 \mathrm{c}$.

183. Flowing wells and municipal water supplies in the middle and northern portions of the southern peninsula of Michigan, by Frank Leverett and others. 1907. 393 pp., 5 pls. 50c.

Water-Supply Papers 182 and 183 deseribe in general the geographic features, water-bearing formations, drainage, quality of water, and subterranean-water temperature, and give details concerning water supplies by counties. The report contains many analyses.

193. The quality of surface waters in Minnesota, by R. B. Dole and F. F. Wesbrook. 1907. 171 pp., 7 pls. $25 \mathrm{c}$.

Describes by river basins the topography, geology, and soils, the industrial and municipal pollution of the streams, and gives notes on the municipaiities; contains many analyses.

*194. Pollution of Illinois and Mississippi rivers by Chicago sewage (a digest of the testimony taken in the case of the State of Missouri $v$. the State of Illinois and the Sanitary District of Chicago), by M. O. Leighton. 1907. 369. pp., 2 pls.

scope indicated by amplification of title. 
*236. The quality of surface water in the United States: Part I, Analyses of waters east of the one hundredth meridian, by R. B. Dole. 1909. 123 pp.

Describes collection of samples, method of examination, preparation of solutions, accuracy of estimates, and expression of analytical results; gives results of analyses of waters of Lake Superior and Lake Michigan, Kalamazoo and Grand rivers, Lake Huron, Lake Erie, Maumee River and St. Lawrence and Oswegatchie rivers.

239. The quality of the surface waters of Illinois, by W. D. Collins. 1910. 94 pp., 3 pls. $10 \mathrm{c}$.

Discusses the natural and economic features that determine the character of the streams, describes the larger drainage basins and the methods of collecting and analyzing the samples of water, and discusses each river in detail with reference to its source, course, and quality of water; includes short chapters on municipal supplies and industrial uses.

254. The underground waters of north-central Indiana, by S. R. Capps, with a chapter on the chemical character of the waters, by R. B. Dole. 1910. 279 pp., 7 pls. $40 \mathrm{c}$.

Describes relief, drainage, vegetation, soils and crops, industrial development, geologic formations; sources, movements, occurrence, and volume of ground water; methods of well construction and lifting devices; discusses in detail, for each county, surface features and drainage, geology, and ground water, city, village, and rural supplies, and gives record of wells and analyses of water. Discusses also, under chemical character, methods of analyses and expression of results, mineral constituents, effects of the constituents on waters for domestic, industrial, and medicinal uses, methods of purification and chemical composition; many analyses and field assays.

364. 'Water analyses from the laboratory of the United States Geological Survey, tabulated by F. W. Clarke, chief chemist. $1914.40 \mathrm{pp}$. $5 \mathrm{c}$.

Contains analyses of water from Caledonia spring, New York, and from the Quincy mine, Mich.

417. Profile surveys of rivers in Wisconsin, prepared under the direction of $\mathrm{W}$. H. Herron, acting chief geographer. $1917.16 \mathrm{pp} ., 32$ pls. $45 \mathrm{c}$.

Contains brief description of general features of drainage of Wisconsin and of the rivers surveyed, but consists chiefly of maps showing "not only the outlines of the river banks, the islands, the position of rapids, falls, shoals, and existing dams, and the crossings of all ferries and roads, but the contours of banks to an elevation high enough to indicate the possibility of using the stream."

424. Surface waters of Vermont, by C. H. Pierce. 1917. 218 pp., 14 pls. 25c. Gives gaging-station descriptions and stream-flow records for streams in the St. Lawrence and Connecticut River basins. Contains a gazateer of streams.

\section{ANNUAL REPORTS}

Each of the papers contained in the annual reports was also issued in separate form.

Annual reports may be purchased (at the price quoted below) from the Superintendent of Documents, Washington, D. C. An asterisk (*) indicates that the report is out of print.

Fourteenth Annual Report of the United States Geological Survey, 1892-93, J. W. Powell, Director. 1893. (Pt. II, 1894.) 2 parts. Pt. II. Accompanying papers, $\mathrm{xx}, 597 \mathrm{pp} ., 73 \mathrm{pls}$. \$2.10. Contains:

${ }^{*}$ The potable waters of eastern United States, by J. W. MeGee, pp. 1 to 47. Discusses: cistern water, stream waters, and ground waters, including mineral springs and artesian wells.

Seventeenth Annual Report of the United States Geological Survey, 1895-96, Charles D. Walcott, Director. 1896. 3 parts in 4 vols, *Pt. II. Economic geology and hydrography, xxv, 864 pp., 113 pls. Contains:

*The water resources of Illinois, by Frank Leverett, pp. 695-849, pls. 108-113. Describes the physical features of the State, and the drainage basins, including Illinois, Des Plaines, Kankakee, Fox, nlinois-Vermilion, Spoon, Mackinaw, and Sangamon rivers, Macoupin Creek, Rock River, tributaries of the Mississippi in western Illinois, Kaskaskia, Big Muddy, and tributaries of the Wabash; discusses the rainfall and run-off, navigable waters and water powers, the wells supplying water for rural districts, and artesian wells; contains tabulated artesian well data and water analyses. 
Eighteenth Annual Report, United States Geological Survey, 1896-97, Charles D. Walcott, Director. 1897. 5 parts in 6 volumes. Pt. IV. Hydrography, x, 756 pp., 102 pls. $\$ 1.75$. Contains:

*The water resources of Indiana and Ohio, by Frank Leverett, pp. 419-560, pls. 33-37. Describes Wabash, Whitewater, Great Miami, Little Miami, Scioto, Hocking Muskingum, and Beaver rivers and lesser tributaries of the Ohio in Indiana and Ohio, the streams discharging into Lake Erie and Lake Michigan, and streams flowing to the Upper Mississippi through the Illinois; discusses shallow and drift wells, the flowing wells from the drift and deeper artesian wells, and gives records of wells at many of the cities; describes the mineral springs and gives analyses. of the waters, contains also tabulated lists of cities using surface waters for waterworks, and of cities and villages using shallow and deep well waters; discusses the source and quality of the city and village supplies, and gives precipitation tables for various points.

Nineteenth Annual Report of the United States Geological Survey, 1897-98, Charles D. Walcott, Director. 1898. (Pts. II, III, and V, 1899.) 6 parts in 7 volumes and separate case for maps with Pt. V. Pt. IV. Hydrography. \$1.85. Contains:

*The rock waters of Ohio, by Edward Orton, pp. 633-717, pls. 71-73. Describes the principal geologic formations of Ohio and the waters from the different strata; discusses the flowing wells at various points and the artesian wells of the deep preglacial channels in Allen, Auglaize, and Mercer counties; discusses city and village supplies; gives analyses of waters from various formations.

\section{MONOGRAPHS}

Monographs may be purchased (at price quoted below) from the Superintendent of Documents, Washington, D. C. An asterisk (*) indicates that the report is out of print. Monographs are of quarto size.

41. Glacial formations and drainage features of the Erie and Ohio basins, by Frank Leverett. 1902.802 pp., 26 pls. $\$ 1.75$.

Treats of an area extending westward from Genesee Valley in New York across northwestern Pennsly vania and Ohio, central and southern Indiana, and southward from Lakes Ontario and Erie to Allegheny and Ohio rivers.

\section{PROFESSIONAL PAPERS}

Professional papers may be purchased (at price quoted below) from the Superintendent of Documents, Washington, D. C. An asterisk (*) indicates that the report is out of print. Professional papers are of quarto size.

*135. The composition of the river and lake waters of the United States, by F. W. Clarke. 1924. iv, 199 pp.

Gives analyses of the water of the Great Lakes and many of their tributaries.

\section{BULLETINS}

Bulletins may be purchased (at price quoted below) from the Superintendent of Documents, Washington, D. C. An asterisk (*) indicates that the report is out of print. Bulletins are of octavo size.

*264. Record of deep-well drilling for 1904, by M. L. Fuller, E. F. Lines, and A. C. Veatch. 1905. $106 \mathrm{pp}$.

Discusses the importance of accurate well records to the driller, to owners of oil, gas, and water wells, and to the geologist; describes the general methods of work; gives tabulated records of wells in Illinois, Indiana, Michigan, Minnesota, New York, Ohio, Pennsylvania, and Wisconsin, and detailed record of wells in Onondaga County, N. Y., and Hancock and Wood counties, Ohio. These wells were selected because they gave definite stratigraphic information.

*298. Record of deep-well drilling for 1905 , by M. L. Fuller and Samuel Sanford. 1906. $299 \mathrm{pp}$.

Gives an account of progress in the collection of well records and samples; contains tabulated records of wells in Illinois, Indiana, Michigan, Minnesota, New York, Ohio, Pennsylvania, Vermont, and Wisconsin, and detailed records of wells in Cook County, Ill.; Erie County, N. Y.; Ottawa, Sandusky, and Summit counties, Ohio; and Manitowoc County, Wis. The wells of which detailed sections are given were selected because they afford valuable stratigraphic information 


\section{GEOLOGIC FOLIOS}

Under the plan adopted for the preparation of a geologic map of the United States the entire area is divided into small quadrangles, bounded by certain meridians and parallels, and these quadrangles, which number several thousand, are separately surveyed and mapped. ${ }^{2}$ The unit of survey is also the unit of publication, and the maps and description of each quadrangle are issued in the form of a folio. When all the folios are completed they will constitute the Geologic Atlas of the United States.

A folio is designated by the name of the principal town or of a prominent natural feature within the quadrangle. Each folio includes maps showing the topography, geology, underground structure, and mineral deposits of the area mapped and several pages of descriptive text. The text explains the maps and describes the topographic and geologic features of the country and its mineral products. The topographic map shows roads, railroads, waterways, and, by contour lines, the shapes of the hills and valleys and the height above sea level of all points in the quadrangle. The areal-geology map shows the distribution of the various rocks at the surface. The structural-geology map shows the relations of the rocks to one another underground. The economic-geology map indicates the location of mineral deposits that are commercially valuable. The artesian-water map shows the depth of underground-water horizons. Economicgeology and artesian-water maps are included in folios if the conditions in the areas mapped warrant their publication. The folios are of special interest to students of geography and geology and are valuable as guides in the development and utilization of mineral resources.

Folios 1 to 163 , inclusive, are published in only one form (18 by 22 inches), called the library edition. Some of the folios that bear numbers higher than 163 are published also in an octavo edition ( 6 by 9 inches). Owing to a fire in the Geological Survey building May 18, 1913, the stock of geologic folios was more or less damaged by fire and water, but 80 or 90 per cent of the folios are usable. They will be sold at the uniform price of 5 cents each, with no reduction for wholesale orders. This rate applies to folios in stock from 1 to 184, inclusive except reprints), also to the library edition of Folio 186. The library edition of Folios 185, 187, and higher numbers sells for 25 cents a copy, except that some folios which contain an unusually large amount of matter sell at higher prices. The octavo edition of Folio 185 and higher numbers sells for 50 cents a copy, except Folio 193, which sells for 75 cents a copy. A discount of 40 per cent is allowed on an order for folios or for folios together with topographic maps amounting to $\$ 5$ or more at the retail rate.

All the folios contain descriptions of the drainage of the quadrangles. The folios in the following list contain also brief discussions of the underground waters in connection with the economic resources of the areas and more or less information concerning the utilization of the water resources.

An asterisk $\left(^{*}\right)$ indicates that the stock of the folio is exhausted.

*81. Chicago, Illinois-Indiana.

Describes an area embracing not only the immediate site of the city but adjacent parts of Cook, Dupage, and Will counties, Ill.; gives an account of the water power, discusses the quality of the waters, and gives analyses of waters from artesian wells; gives also a list of papers relating to the geology and paleontology of the area.

*140. Milwaukee special, Wisconsin.

Gives analyses of spring waters and of artesian water in Milwaukee; also tabulated data concerning wells.

Index maps showing areas in the St. Lawrence basin covered by topographic maps and by geologic folios will be mailed on receipt of request addressed to the Director U. S. Geological Survey, Washington D. C. 
155. Ann Arbor, Mich. 25c.

Discusses the present lakes, the lakes of the glacial period, and under "Economic geology" the water resources, including the use of the rivers for power and of the underground waters, shallow and artesian, for city and village supplies; discusses the quality of the waters, and gives details by townships.

*169. Watkins Glen-Catatonk, New York.

Includes discussion of water supply at Ithaca.

190. Niagara, N. Y. 50c. field edition. ${ }^{3}$

Gives analyses of mineral water from well at Akron; discusses briefly the municipal supplies of Buffalo, Niagara Falls, Tonawanda, La Salle, and Youngstown, and the use of Niagara River for power development.

205. Detroit, Mich. 50c. either edition.

Discusses surface and ground waters; gives mineral analyses of water from Lake Huron, from rivers near Detroit, and from salt wells.

\section{MISCELLANEOUS REPORTS}

Other Federal bureaus and State and other organizations have from time to time published reports relating to the water resources of the various sections of the country. Notable among those pertaining to the St. Lawrence River basin are the reports of the Chief of Engineers, United States Army, the State Geological Survey of Illinois, the Illinois Water-Supply Commission, the Rivers and Lakes Commission of Illinois, the New York State Conservation Commission, the New York State Water-Supply Commission, the New York State engineer and surveyor, the Vermont State geologist, and the water-power report of the Tenth Census (vol. 16). The following reports deserve special mention:

The mineral content of Illinois waters, by Edward Bartow, J. A. Udden, S. W. Parr, and George T. Palmer: Illinois State Geol. Survey Bull. 10, 1909.

Chemical and biological survey of waters of Illinois, by Edward Bartow: Univ. Illinois Pubs. 3, 6, 7, 1906-1909.

Chemical survey of the waters of Illinois, report for the years 1897-1902, by A. W. Palmer, with report on geology of Illinois as related to its water supply, by Charles W. Rolfe: Univ. Illinois Pub.

Diversion of the waters of the Great Lakes by way of the Sanitary and Ship canal of Chicago: A brief of the facts and issues, by Lyman E. Cooley, Chicago, 1913.

The State of Missouri $v$. the State of Illinois and the Sanitary district of Chicago, before Frank S. Bright, commissioner of the Supreme Court of the United State, 1904.

The mineral waters of Indiana, their location, origin, and character, by W. S. Blatchley: Indiana Dept. Geology and Nat. Res. Twenty-sixth Ann. Rept., 1901.

Reports of the water resources investigation of Minnesota, by the State Drainage Commission, 1909-1912.

Water powers of Wisconsin, by L. S. Smith: Wisconsin Geol. and Nat. Hist. Survey Bull. 20, 1908.

First report of the Railroad Commission of Wisconsin to the legislature on water powers, 1915. 
Second report of the Railroad Commission of Wisconsin on water powers 1914-1923.

Reexamination of Fox River, Wis.: 67th Cong., 2d sess., H. Doc. 146., 1922. Special report of the Vermont Water Resources Commission to the legislature of 1921.

Diversion of water from the Great Lakes and Niagara River: Report of Corps of Engineers, 1921.

Hydrology of the State of New York, by George W. Rafter: New York State Mus. Bull. 85, 1905.

Many of these reports can be obtained from the various commissions, and probably all can be consulted in the public libraries of the larger cities. 


\section{AREAS AND PUBLICATIONS COVERED}

[A=Annual Report; $\mathbf{M}=$ Monograph; $\mathbf{B}=$ Bulletin; $\mathbf{P}=$ Professional papers; $\mathbf{W}=\mathbf{W}$ ater-supply paper; G $\mathbf{F}=$ Geologic folio]

Artesian waters: Essential conditions _. . . . . . . . . . . . . . . . W 114

Chemical analyses ${ }^{4}$ : Methods and interpretation ........... W 236, 364; P 135

Engineering methods.................... W 110

Floods . . . . . . . .

Illinois: Quality of waters.............. A 17 ii; W 194, 236, 239; G F 81

Surface waters...................... A 17 ii; W 236, 239; G F 81

Underground waters......... A $17 \mathrm{ii}$; B 264, 298; W 57, 114, 149; G F 81

Indiana: Quality of waters...................... A 18 iv; W 236, 254

Surface waters............................. W 147; M 41; G F 81

Underground waters..................... A 18 iv; B 264, 298; W 21, 26, $57,114,149,254$; G F 81

Legal aspects: Surface waters............................... W 152

Underground waters . . .

Michigan: Quality of waters........ W 30, 31, 102, 182, 183, 236; G F 155, 205

Surface waters.......................... W 30, 147, 162; G F 155, 205

Underground waters....................... B 264, 298; W 30,31, 57, $102,114,145,149,160,182,183$; G F 155, 205

Mineral springs: Analyses......................... A 14 ii; W 364

Origin, distribution, etc............ A 14 ii

Lists . . . . . .

Minnesota: Quality of waters........... W 193, 236

Surface waters _...

Underground waters.................... B 264, 298; W 57, 102, 114, 149

Motions of ground waters...... W 110

New York: Quality of waters............. W 110, 144, 145, 236; G F 169, 190

Surface waters............... M 41; W 24, 25, 147, 162, 187; G F 169, 190

Underground waters.......................... B 264, 298; W 61, 102 ,

$110,114,145,149 ;$ G F 169,190

Ohio: Quality of waters................. A 18, iv; 19, iv; W 179, 236, 259

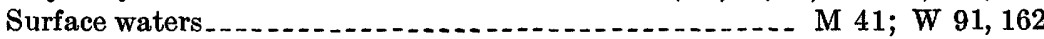

Underground waters........... A 18 iv, 19 iv; B 264, 298; W 91, 114, 149

Pennsylvania: Surface waters............................. W 147, 162

Underground waters................ B 264, 298; W 110, 114, 145, 149

Pollution:

By sewage...... W 194

Laws forbidding

Indices of $\ldots \ldots$. 144,160

Sanitation; quality of waters; pollution; sewage irrigation...... W 103. 110,

$114,121,145,152,160,194,236$

S wage disposal and purification .................. W 194

4 Many analyses of river, spring, and well waters are scattered through publications, as noted in abstracts 
Underground water: Legal aspects. W 122

Methods of utilization W 114

Pollution W $110,145,160$

Vermont: Quality of waters W 144, 236

Surface water W 187,424

Underground waters

Wisconsin: Quality of waters

W 102, 110, 114, 149

Profile surveys

Surface waters

G F 140

Underground waters

W 156; G F 140

B 264,298 ; W $61,114,145,149$; G F 140 


\section{STREAMS ON WHICH GAGING STATIONS HAVE BEEN MAINTAINED}

Arninicon River, Wis

Auglaize River, Ohio

Au Sable River, Mich

Ausable River, N. Y

Ausable River, West Branch, N. $\mathrm{Y}$

Bad River, Wis

Barge Canal, N. Y.

Beaver Bay River, Minn

Beaver River, N. Y

Big Chazy River, N. Y

Black River canal, N. Y.......

Black River, East Branch, Ohio_

Black River, N. Y

Black River, Ohio

Blanchard River, Ohio

Boardman River, Mich........

Bog River, N. Y

Boquet River, N. Y.........

Brule River, Minn.

Brule River, Wis. (Tributary to Lake Michigan) ...........

Brule River, Wis. (Tributary

to. Lake Superior)

Calumet River, Little, Ill.......

Canadice outlet, N. Y

Canaseraga Creek, N. Y.......

Carp River, Mich............

Cascade River, Minn.........

Cass River, Mich

Cattaragus Creek, N. Y.

Cayuga Lake, N. Y

Champlain Lake, Vt..........

Chateaugay River, N. Y

Chazy River, Big, N. Y.

Chittenango Creek, N. Y

Cloquet River, Minn

Clyde River, Vt.

Conesus Creek, N. Y

Conneaut Creek, Ohio

Crockery Creek, Mich

Cuyahoga River, Ohio

Dead River, Mich

\begin{tabular}{|c|c|c|}
\hline & & \\
\hline 52 & Def & \\
\hline 153 & evil Track River, Minn. & 51 \\
\hline 153 & Dog River, Vt..... & 156 \\
\hline 155 & $\begin{array}{l}\text { East Branch or Fork. See name } \\
\text { of main stream. }\end{array}$ & \\
\hline 155 & East Creek, Vt & 155 \\
\hline 152 & s River, Wi & 152 \\
\hline 154 & Escanaba River, Mich. & 152 \\
\hline 151 & Creek, N. Y... & 154 \\
\hline 155 & ch. & 152 \\
\hline 155 & $Y_{-}$ & 154 \\
\hline 155 & t Branch, N. Y & 154 \\
\hline 53 & Flint River, Mich & 153 \\
\hline 54 & d du Lac River, East Branch, & \\
\hline 53 & Wis & 152 \\
\hline 53 & du Lac River, Wes & \\
\hline 153 & anch, Wis & 152 \\
\hline 155 & ler, N. Y $\ldots \ldots$ & 15 \\
\hline 155 & Wis & 152 \\
\hline \multirow[t]{2}{*}{151} & ver, N. Y & 154 \\
\hline & re, N. Y $\ldots$ & 155 \\
\hline \multirow[t]{2}{*}{152} & lich_ & 153 \\
\hline & 10............. & 15 \\
\hline 52 & $--2-1-2--2-2$ & 156 \\
\hline 52 & N. Y & 154 \\
\hline 54 & N. Y & 15 \\
\hline 54 & $e r, V t \ldots$ & 156 \\
\hline 52 & ich.. & 153 \\
\hline 1 & Ir & 152 \\
\hline 53 & 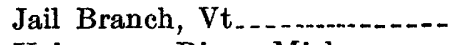 & 155 \\
\hline 53 & r, Mich & 153 \\
\hline 54 & $Y_{-\ldots}$ & 154 \\
\hline 155 & $\mathrm{n}, \mathrm{Vt}$ & 155 \\
\hline 55 & T. $Y_{n} \ldots$ & 155 \\
\hline 55 & itlet, N. Y & 155 \\
\hline 54 & $V t_{-} \ldots$ & 156 \\
\hline 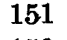 & River, Ill.. & 152 \\
\hline 56 & & 156 \\
\hline 154 & reek, N. Y. & 154 \\
\hline 53 & Wis $\ldots$ & 152 \\
\hline 53 & - & 156 \\
\hline & & 153 \\
\hline & Maumee $\mathrm{R}$ & 153 \\
\hline
\end{tabular}


Menominee River, Mich., Wis..

Mettawee River, N. Y ........

Miami and Erie Canal, Ohio...

Middle Branch or Fork. See name of main stream.

Milwaukee River, Wis

Missisquoi River, Vt. . . . . . . . . .

Mollys Brook, Vt

Montreal River, Mich..........

Montreal River, West Branch, Wis

Moose River, N. Y

Moose River, Middle Branch, N. $\mathrm{Y}$

Muskegon River, Mich

Niagara River, N. Y

Oconto River, Wis

Ohio Canal, Ohio

Ohio Canal feeder, Ohio

Oneida Creek, N. Y

Oneida River, N. Y

Onondaga Creek, West Branch, N. Y.

Onondaga Lake outlet, N. Y...

Ontonagon River, Mich.......

Orwell Brook, N. Y

Oswegatchie River, N. Y

Oswegatchie River, East Branch N. Y

Oswegatchie River, West Branch N. Y .

Oswego River, N. Y

-Ottawa River, Ohio

Otter Creek, Vt

Owasco Lake outlet, N. Y .....

Perch River, Mich

Peshtigo River, Wis

Pike River, Wis

Pine River, Wis

Poplar River, Minn...........

Poultney River, Vt
Page

152

155

153

152

156

155

152

152

155

155

153

154

152

153

153

154

154

154

154

152

154

155

155

155

154

153

155

154

152

152

152

152

151

155
Raquette River, N. Y

Red Cedar River, Mich

155

153

153

155

153

156

153

152

151

153

155

154

153

155

154

154

152

153

Skaneateles Lake and outlet, N. $\mathrm{Y}$

154

155

152

153

153

153

154

154

156

152

151

155

Winooski River, Vt.............

Winooski Biver, North Branch

Branch Vt..................

Winooski River, Worcester

Branch, Vt.............

Wolf River, Little, Wis........ 152

Wolf River, West Branch, Wis.

152

Wolf $\cdot$ River, Wis

152 


\section{INDEX}

A

Accuracy of data and results, degrees of ....... 4-5

Acre-foot, definition of.

Amberg, Wis., Pike River at.

2

Amboy, Ohio, Conneaut Creek at........... 69-71

Antwerp, Ohio, Maumee River at........... 42-43

Appropriations, record of ................... 1

Areas and publications covered........... 165

Auburn, N. Y., Owasco Lake outlet near... 89-91

Auglaize River near Defiance, Ohio......... 50-51 near Fort Jennings, Ohio . ............... 48-49

Ausable Forks, N. Y., Ausable River at.-- 127-128 Ausable River at Ausable Forks, N. Y. ... 127-128

West Branch of, near Newman, N. Y ... 125-127 Au water-stage recorder, plate showing...... 2

B

Barton; Mich., Huron River at.............. 40-41 Beaver River at Eagle Falls, near Number Four, N. Y

at State dam, near Beaver River, N. Y. 105-106

Beaver River Power Corporation and Northern New York Utilities (Inc.),

1 cooperation by ..................

6

Berlin, Wis., Fox River at.................. 19-21

Black River at Watertown, N. Y............ 95-97

East Branch of, at Elyria, Ohio _...... 58-59

near Boonville, N. Y . ................. 93-95

Black River canal near Boonville, N. Y .... 99-100

Black River Regulating District, cooperation

by . . . . . 5- 6

Blanchard River at Glandorf, Ohio ......... 51-52

Bolton Landing. N. Y., Lake George near.... 130

Boonville, N. Y., Black River canal near -.. 99-100

Black River near................ 93-95

Forestport feeder near ..................... 97-98

Brasher Center, N. Y., St. Regis River at. 119-120

Brecksville, Ohio, Cuyahoga River at...... 61-62

Ohio, Ohio Canal feeder at .............. 64-65

Bridge measurement, typical gaging station for, plate showing

Burlington, Vt., Lake Champlain at........ 122

Cadys Falls, vt., Lamoille River at ...... 137-139 Canadice Lake outlet near Hemlock, N. Y . $\quad 89$ Canaseraga Creek near Dansville, N. Y . .... 83-84 Cattaraugus Creek at Versailles, N. Y....... 71-72 Charles T. Middlebrook, cooperation by... 6 Clyde River at West Derby, Vt........... 143-144 Commission for the Improvement of Oswegatchie River, cooperation by ....... 6

Computations, results of, accuracy of....... 4-5

Conesus Creek near Lakeville, N. Y ........ 87-88

Conneaut Creek at Amboy, Ohio .......... 69-71

Control, definition of ..................... 2-3

Cooperation, record of.
Cranberry Lake, N. Y., East Branch of Oswegatchie River at.......... 109-110

Crivitz, Wis., Peshtigo River near........... 16-17

Current meters, Price, plate showing ........ 2

Cuyahoga River at Brecksville, Ohio........ 61-62 at Independence, Ohio................ 62-64 at old Portage, Ohio................... 59-61

\section{D}

Dansville, N. Y., Canaseraga Creek near.... 83-84

Data, accuracy of ........................ 4-5 explanation of . $3-4$

Defiance, Ohio, Auglaize River near........ 50-51

\section{$\mathbf{E}$}

East Barre, Vt., Jail Branch at ............. 135-137 Elyria, Ohio, East Branch of Black River at 58-59 Embarrass River near Embarrass, Wis..... 27-28

\section{F}

Fish Creek, East Branch of, at Taberg, N. Y.. 92-93 Florence, Wis., Pine River near. ........... 12-13 Forestport feeder near Boonville, N. Y...... 97-98 Fort Jennings, Ohio, Auglaize River near ... 48-49 Fox River at Berlin, Wis. .................... 19-21 at Rapide Croche dam, near Wrightstown, Wis.......................... 21-22 Freeland, Mich., Tittabawassee River at..... 39-40

\section{G}

Gaging stations, list of

Gaging stations, typical, plates showing ..... 2 Garfield, vt., Green River at............. 139-141 Genesee River at Driving Park Avenue, Rochester, N. Y . . . ............. 81-82 at Jones Bridge, near Mount Morris, N. Y . . . . 79-80 at St. Helena, N. Y at Scio, N. Y Gile, Wis., West Branch of Montreal River at. $\quad 7-8$ Gillett, Wis., Oconto River near.......... 18-19 Glandorf, Ohio, Blanchard River at......... 51-52 Grand River near Madison, Ohio_.......... 67-69 Green River at Garfield, vt.............. 139-141 Grosbach, H. E., work of ................. 6 Gurley water-stage reeorder, plate showing .. $\quad 2$

\section{H}

Harrington, A. H., and assistants, work of... Harrisville, N. Y., West Branch of Oswegatchie River near .................. 115-116

Hemlock, N. Y., Canadice Lake outlet near _- 89 Heuvelton, N. Y., Oswegatchie River near - 113-114 Horton, A. H., and assistant, work of ......... 6 Huron River at Barton, Mich ............... 40-41 
Illinois, cooperation by .

Independence, Ohio, Cuyahoga River at ..... 62-64 Ohio Canal at. ...................... 65-67

International Paper Co., cooperation by..... 6 Iron Mountain, Mich., Menominee River near.

$\mathrm{J}$

Jail Branch at East Barre, Vt. $135-137$

\section{K}

Kalida, Ohio, Ottawa River at

Keshena, Wis., Wolf River at............... 23-24

Keshequa Creek at Craig Colony, Sonyea,

$$
\text { N. Y - }
$$
$85-86$

Koss, Mich., Menominee River below....... 10-11

\section{$\mathbf{L}$}

Lake Champlain at Burlington, Vt.......... 122

Lake Erie, streams tributary to............... 40-72

Lake George at Glen Island, near Bolton Landing, N. $\mathrm{Y}$.

at Rogers Rock, N. Y

Lake Huron, streams tributary to . ........... 39-40

Lake Michigan, streams tributary to. . . . . . . 8- 8-38

Lake Ontario, streams tributary to . . . . . . . 73-109

Lake Superior, streams tributary to ......... 7-8

Lakeville, N. Y., Conesus Creek near. . ..... 87-88

Lamoille River at Cadys Falls, Vt . . . ..... 137-139

Lee, Lasley, and assistants, work of .......

Linden, N. Y., Little Tonawanda Creek at.- 73-74

Little Calumet River at Harvey, Ill......... 37-38

Little Tonawanda Creek at Linden, N. Y.. 73-74

Little Wolf River at Royalton, Wis......... 29-30

\section{M}

Madison, Ohio, Grand River near. $67-69$

Marshfield, vt., Mollys Brook near...... 133-134

Maumee River at Antwerp, Ohio........... 42-43

at Napoleon, Ohio...................... 145

at Waterville, Ohio ..................... 44-45

MeKeever, N. Y., Moose River at........ 100-102

Menominee River at Twin Falls, near Iron Mountain, Mich..... 8-10

below Koss, Mich ......................... 10-11

Mexico, Ohio, Sandusky River near.......... 56-57

Miami and Eris Canal at Napoleon, Ohio... 145 at Waterville, Ohio........................ $53-54$

Milwaukee River near Milwaukee, Wis..... 35-36

Missisquoi River near Richford, Vt........ 141-142

Mollys Brook near Marshfield, Vt.......... 133-134

Montpelier \& Barre Light \& Power Co., cooperation by ..................... 6

Montpelier, Vt., Winooski River at...... 131-132

Montreal River, West Branch of, at Gile, $W$ is ................................. 7-8

Moose River at McKeever, N. Y .......... 100-102 Middle Branch of, at Old Forge, N. Y. 103-105 at Moose River, N. Y................ 102-103

Mount Morris, N. Y., Genesee River near.- 79-80 Mussey, O. D., work of .

\section{$\mathbf{N}$}

Napoleon, Ohio, Maumee River at

$\mathrm{N}_{\mathrm{ew}}$ London, Wis., Wolf River at

Wis., Wolf River at_._...... 25-26

Newman, N. Y., West Branch of Ausable River near. . . . . . . .

Newport Electric Light Co., cooperation by.

Newton Falls, N. Y., East Branch of Oswegatchie River at

6

New York \& Pennsylvamia Co., cooperation by ..................................

New York, cooperation by ................. 5 Number Four, N. Y., Beaver River near _- 107-109

Oconto River near Gillett, Wis............... 18-19 Ohio Canal at Independence, Ohio ........... 65-67 Ohio Canal feeder at Brecksville, Ohio...... 64-65 Ohio, cooperation by ......................... 5 Old Forge, N. Y., Middle Branch of Moose River at.......................... 103-105 Old Portage, Ohio, Cuyahoga River at...... 59-61 Oswegatehie River, East Branch of, at Cranberry Lake, N. Y 109-110

at Newton Falls, N. Y............. 111-112

near Heuvelton, N. Y .................. 113-114

West Branch of, near Harrisville, N. Y. 115-116 Ottawa River at Kalida, Ohio............... ' 145 Owasco Lake outlet near Auburn, N. Y..... 89-91

\section{$\mathbf{P}$}

Peshtigo River at High Falls, near Crivitz,

Wis . ............................... 16-17

Pierce, C. H., and assistants, work of........ 6

Piercefield, N. Y., Raquette River at...... 117-118

Pike River at Amberg, Wis .................. 14-15

Pine River near Florence, Wis................. 12-13

Plattsburg Gas \& Electric Co., cooperation by 6

Plattsburg, N. Y., Saranac River near.... 123-125

Price current meters, plate showing ......... 3

Publications, information concerning......147-151,

157-164

obtaining or consulting of.................. 148

on stream flow, list of ...................... 149

\section{$\mathbf{R}$}

Raquette River at Piercefield, N. Y........ 117-118 Richelieu River at Fort Montgomery, Rouses Point, N. Y

Richford, Vt., Missisquoi River near....... 141-142 Rochester Gas \& Electric Corporation, cooperation by

Rochester, N. Y., cooperation by............. 5

Genesee River at. $\ldots . . . . . . . . . . . . . . . . . . .81-82$

Rogers Rock, N. Y., Lake George at......... 129 Rouses Point, N. Y., Richelieu River at...- 121 Royalton, Wis., Little Wolf River at........ 29-30 Run-off in inches, definition of............... 2

\section{S}

St. Helena, N. Y., Genesee River at........ 77-78 St. Lawrence River, streams tributary to .- 109-144 St. Regis River at Brasher center, N. Y ... 119-120 Sandusky River at Tiffin, Ohio........... 145 near Mexico, Ohio........................ 56-57 near Upper Sandusky, Ohio.............. 54-56 Saranac River near Plattsburg, N. Y ......- 123-125 Scio, N. Y., Genesee River at............... 75-76 Second-feet, definition of. 
Second-feet per square mile, definition of.... Sheboygan River near Sheboygan, Wis..... 33-34

Sonyea, N. Y., Keshequa Creek at.......... 85-86

Soulé, S. B., and assistants, work of........ 6

Stage-discharge relation, definition of......... 2

Stevens water-stage recorder, plate showing. Stream-gaging stations and publications relating to water resources, information concerning. 147-164

Streams on which gaging stations have been maintained, list of. 167

Stryker, Ohio, Tiffin River near........... 46-47

\section{$\mathbf{T}$}

Taberg, N. Y., East Branch of Fish Creek at. 92-93 Terms, definition of 2-3

Tiffin, Ohio, Sandusky River at............. 145

Tiffin River near Stryker, Ohio.............. 46-47

Tittabawassee River at Freeland, Mich. $39-40$

\section{U}

United States Engineer Corps, cooperation by

Upper Sandusky, Ohio, Sandusky River near 54-56 Utica Gas \& Electric Co., cooperation by...
V

Page

Vermont, cooperation by

Versailles, N. Y., Cattaraugus Creek at..... 71-72

\section{$\mathbf{W}$}

Wading measurement, typical gaging station

$$
\text { for, plate showing. }
$$

Water-stage recorders, plate showing ........ 2

Watertown, N. Y., Black River at .......... 95-97

Waterville, Ohio, Maumee River at......... 44-45 Miami and Erie Canal at.................. 53-54

Waupaca River near Waupaca, Wis........ 31-32

West Derby, Vt., Clyde River at........... 143-144

Winooski River at Montpelier, Vt......... 131-132

Wisconsin, cooperation by

Wolf River at Keshena, Wis.................. 23-24 at New London, Wis................. 25-26

Work, authorization of ....................... 1 division of.................................. 6 scope of ................................. 1-2

Wrightstown, Wis., Fox River near......... 21-22 2

Zero flow, point of, definition of............ 